Universität Szeged

Philosophische Fakultät

Promotionskolleg Sprachwissenschaft - Germanistische Linguistik

\title{
Die Selbstständigkeit der Substantivvalenz
}

\author{
Dissertation \\ zur Erlangung des akademischen Grades PhD in germanistischer Linguistik \\ an der Universität Szeged
}

Vorgelegt von:

Ágnes Sántáné-Túri

Wissenschaftlicher Betreuer:

Prof. em. Dr. Péter Bassola DSc

Szeged 



\section{Danksagung}

An dieser Stelle möchte ich meinem Doktorvater, Prof. em. Dr. Péter Bassola herzlich danken, der meine Arbeit schon von den allerersten Schritten an begleitet, betreut, unermüdlich und immer wohlwollend unterstützt und weder Zeit noch Energie gescheut hat, wenn es um die Besprechung wichtiger Fragen ging.

Dr. habil. Petra Szatmári und Dr. habil. Pál Uzonyi, den Opponenten meiner vorliegenden Dissertation, danke ich besonders für ihre wertvollen Bemerkungen zur ersten Fassung meiner Arbeit, die mich zum weiteren Nachdenken motiviert und somit auch neue Gedankengänge angeregt haben.

Ferner danke ich Prof. em. Dr. Sándor Albert, dass er den Vorsitz bei meinen Prüfungen übernommen und mich während der Vorbereitung auf die Verteidigung meiner Dissertation mit wichtigen Ratschlägen unterstützt hat.

Mein aufrechter Dank gilt auch Prof. em. Dr. Árpád Bernáth, Dr. Csilla Bernáth, Prof. em. Dr. Károly Csúri und Dr. Csilla Mihály, die mich immer tatkräftig unterstützt und ermutigt haben, meine wissenschaftlichen Pläne nie aufzugeben.

Danken möchte ich außerdem Dr. habil. Attila Bombitz für die ersten Publikationsmöglichkeiten und für seine ständige Diskussionsbereitschaft, egal ob es sich um Literatur, Kultur, Arbeit oder Freizeit handelt.

Herzlich danke ich auch Dr. György Scheibl, dank dessen Einführungskurs im ersten Semester meiner Studienzeit die Syntax ihren Schrecken für mich verloren hat, und mit dem ich seitdem schon sehr viele lebhafte Diskussionen über theoretische und praktische Fragen führen durfte, die mich immer vorangebracht haben.

Des Weiteren möchte ich mich bei Christoph Beeh bedanken, der nicht nur Teile der vorliegenden Arbeit, sondern auch weitere frühere Beiträge von mir in Obhut genommen und meine Texte immer mit größter Sorgfalt korrekturgelesen hat.

Für ihr Anfeuern und ihre herzliche Unterstützung danke ich Krisztina Pöltl-Deák und ihrer Familie.

Schließlich gilt ein unendlich großer Dank meiner Familie: Meiner Mutter, die seit meiner Kindheit immer mutig und geduldig genug war, mir die Freiheit zu lassen, meinen eigenen Weg zu finden, und meinem Mann und unserem Sohn, ohne die ich die vorliegende Arbeit wahrscheinlich nie beendet hätte. 



\section{Inhaltsverzeichnis}

1. Einleitung

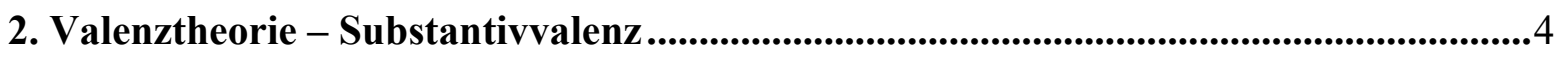

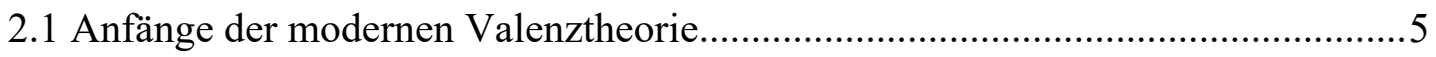

2.2 Eine Valenzdefinition als Ausgangspunkt .................................................... 10

2.3 Drei Phasen der Substantivvalenzforschung nach Helbig (1992) ...................... 11

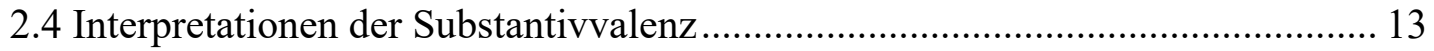

2.4.1 Substantivvalenz - vererbt oder sui generis? ................................... 13

2.4.2 Substantivvalenz - Sui generis, aber auch vererbt? ........................... 14

2.4.3 Substantivvalenz im Lichte (allgemeiner) linguistischer Arbeiten ...... 19

2.5 Komplemente und Supplemente in der allgemeinen Valenztheorie und in der

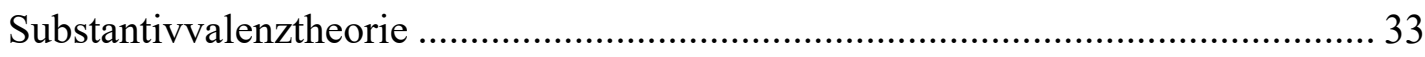

2.5.1. Das Problem der Unterscheidung zwischen Komplementen und Supplementen I.

2.5.2 Das Problem der Unterscheidung zwischen Komplementen und

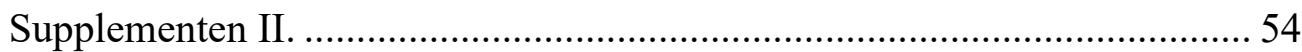

2.5.3 Obligatorische Komplemente beim Substantiv? .............................. 63

2.5.4 Zur Nomenklatur der Substantivkomplemente in unterschiedlichen

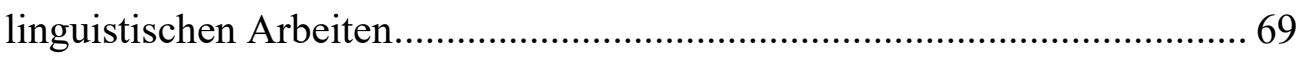

2.6 Grundlagen des eigenen multidimensionalen Substantivvalenzkonzepts ........... 72

2.6.1 Logisch-semantische und formale Valenzbeziehungen ..................... 73

2.5.4 Zum Bestimmungsverfahren und zur Klassifizierung der

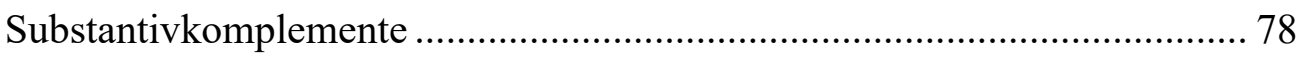

3. FVG / SVG im Rahmen der Untersuchung der Substantivvalenz .............................. 81

3.1 FVG / SVG - Ein ontologisches bzw. terminologisches Problem aus der

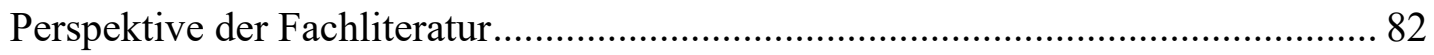

3.2 Valenzbeschreibungen von FVG / SVG .................................................... 102

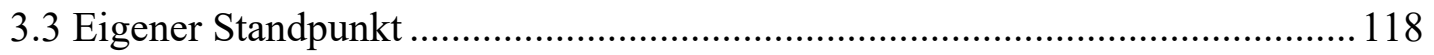

4. Korpusbasierte Untersuchung der Valenz ausgewählter Substantive ....................... 121

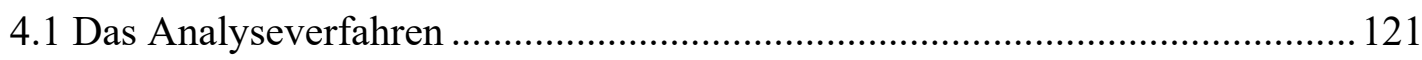

4.1.1 Fragestellungen und Vorgehensweise ............................................. 121

4.1.2 Zur Auswahl der untersuchten Substantive..................................... 122 
4.1.3 Zur Auswertung und Darstellung der korpusanalytischen Daten und Ergebnisse

4.2 Die untersuchten Substantive im Korpus .................................................... 126

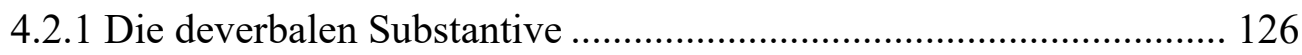

4.2.1.1 Das Substantiv Übersetzung ........................................ 126

4.2.1.2 Das Substantiv Ausdruck ................................................ 131

4.2.1.3 Das Substantiv Verspätung............................................ 135

4.2.2 Das deadjektivische Substantiv Freiheit ........................................ 137

4.2.3 Die nicht-abgeleiteten Substantive ................................................ 141

4.2.3.1 Das Substantiv Problem ..................................................... 141

4.2.3.2 Das Substantiv Ursache .................................................... 143

4.3 Zusammenfassung der Ergebnisse der korpusbasierten Untersuchung der

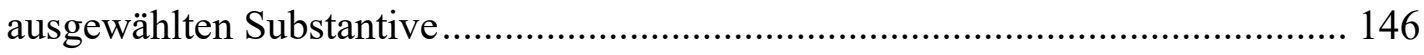

5. Schlussfolgerungen und Ausblick .......................................................................................... 149

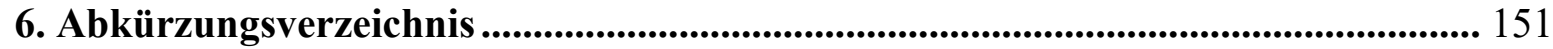

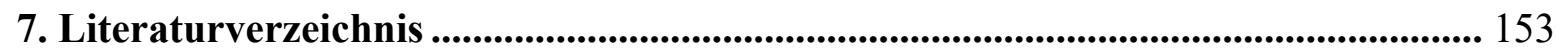

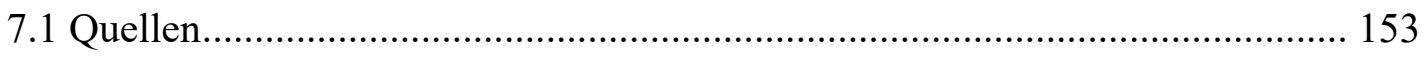

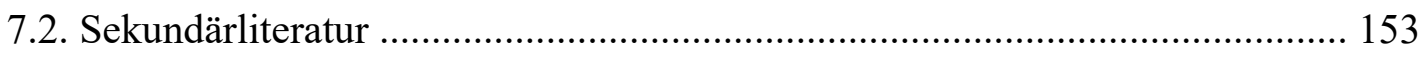

7.3 Zitierte bzw. verwendete eigene Publikationen............................................ 160

8. Anhang ........................................................................................................................................... 162

8.1 Belege für Übersetzung ..................................................................... 162

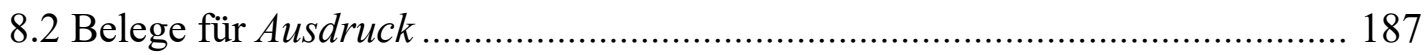

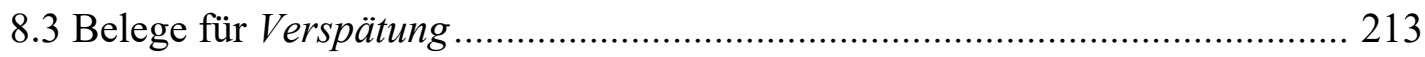

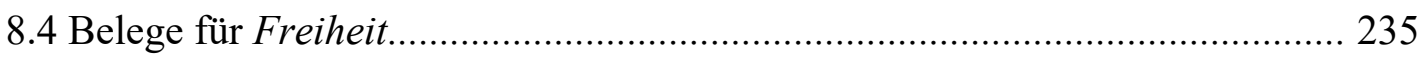

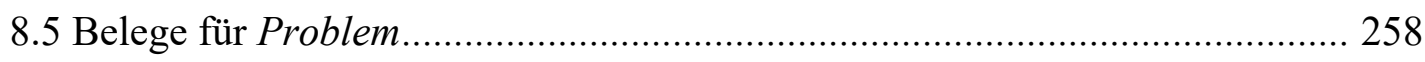

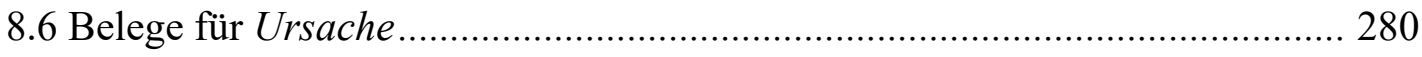




\section{Einleitung}

Vorliegende Arbeit befasst sich mit der Beschreibung der Substantivvalenz als einem eigenständigen System. Die Substantivvalenztheorie ist ein Teilbereich der Valenztheorie, die innerhalb des sowieso an Diskussionen nicht armen Forschungszweigs oft infrage gestellt und zu einem aus der Valenz der Verben (und Adjektive) ableitbaren, sekundären Phänomen herabgesetzt wird, was mich - neben meiner wissenschaftlichen Überzeugung von der Selbstständigkeit der Substantivvalenz - zusätzlich zu deren Untermauerung beizutragen motiviert hat.

Meine Arbeit gliedert sich in fünf Hauptkapitel, von denen sich das zweite der Behandlung theoretischer Fragen der Substantivvalenz widmet. Dabei halte ich es für wichtig, die Substantivvalenz eingebettet in den Kontext der allgemeinen Valenztheorie darzustellen, deswegen spreche ich zuerst in Kap. 2.1 - von den Anfängen der modernen Valenztheorie bei Tesnière ausgehend - die Frage an, ob die Substantivvalenz auch schon in das Tesnière'sche Konzept integrierbar ist. Das ist nämlich ein Problem, das zwar nicht allzu oft, dafür aber umso uneinheitlicher in der Forschung betrachtet wird.

Kap. 2.2 und 2.3 dienen der stufenweisen Überleitung von den in Kap. 2.1 thematisierten valenzhistorischen Grundlagen über eine heute eingenommene Definition der Valenz und der Beschreibung der Entwicklung der Substantivvalenzforschung schon zum Gegenstand der Substantivvalenz. In Kap. 2.4 werden in fünf Unterkapiteln Fragen der Interpretierbarkeit der Substantivvalenz erörtert. Kap. 2.4.1 schildert verschiedene Ansätze zur Deutung der Substantivvalenz, während sich Kap. 2.4.2 eingehend mit der sui-generis-Interpretation der Substantivvalenz auseinandersetzt. Im Rahmen dieser Erörterungen fasse ich auch meine Ansicht über die Selbstständigkeit der Valenz der Substantive zusammen und stelle auch Zusammenhänge zwischen bestimmten Deutungsansätzen klar. Kap. 2.4 .3 bietet einen kritischen Einblick in die Substantivvalenzbeschreibung unterschiedlicher linguistischer Arbeiten.

Eine der wichtigsten Streitfragen der allgemeinen Valenztheorie wird in Kap. 2.5 in vier Unterkapiteln detaillierter diskutiert. Die Unterscheidung von valenzgebundenen und nichtvalenzgebundenen Dependentien war und ist ein heiß diskutiertes Thema der Forschung, wobei die Entscheidung dieser Frage in Bezug auf nominale Valenzträger aus mehrerlei Sicht besonders problematisch erscheint. Im Anschluss an diese Überlegungen stelle ich in Kap. 2.6 die wichtigsten Aspekte meines multidimensionalen Konzepts zur Unterscheidung von Substantivkomplementen und -supplementen dar, das als Grundlage der korpusanalytischen 
Untersuchungen von Kap. 4 dienen und zugleich durch Erkenntnisse dieser Analysen auch weiter verfeinert werden soll.

Zur Untermauerung der sui-generis-Interpretation der Substantivvalenz und auch zur Beantwortung der Frage, wie weit die Selbstständigkeit dieses Systems reicht bzw. wie sie im Sprachgebrauch „ertappt“ werden kann, finde ich die Untersuchung dessen sehr relevant, ob und wenn ja, wie Argumentrealisierungen von valenten Substantiven Unterschiede aufweisen - abhängig davon, ob sie in einer festen Verbindung mit einem sog. Stützverb, als Teil eines Stützverbgefüges, vorkommen oder ohne Stützverb realisiert werden. Aus diesem Grund befasst sich Kap. 3 mit dem sprachlichen Phänomen der sog. Stützverbgefüge (SVG) und / oder Funktionsverbgefüge (FVG).

Dabei ist wichtig hervorzuheben, dass ich schon die Beschreibung von FVG bzw. SVG an sich ein riesengroßes Unternehmen finde, das im Rahmen der primär valenzorientierten und auf die Substantivvalenz fokussierenden Arbeit keinesfalls vollständig durchgeführt werden kann. Mir geht es in dieser Hinsicht in erster Linie darum, die Problematik so zu umreißen, dass ich dadurch zu einer fundierten Arbeitsdefinition von SVG gelange, die ebenfalls als Grundlage der korpusbasierten Analysen von Kap. 4 dienen kann. Dieser Zielsetzung folgt in Kap. 3.1 die Thematisierung dessen, inwieweit die Unterscheidung $F V G$ vs. $S V G$ ein rein terminologisches oder aber ein ontologisches Problem darstellt. Aus der Sicht der vorliegenden Arbeit ist die Charakterisierung der Valenzeigenschaften von FVG bzw. SVG besonders von Belang. Wie aus Kap. 3.2 ersichtlich wird, gehen die Ansichten in der Forschung auch diesbezüglich auseinander. Die schon erwähnte eigene Arbeitsdefinition von SVG, ich verbleibe nämlich im Rahmen meiner Arbeit bei der Verwendung dieses Terminus, wird in Kap. 3.3 dargeboten.

Aufbauend auf den theoretischen Erkenntnissen von Kap. 2 und 3, setzt sich Kap. 4 zum Ziel, die Substantivvalenz auf Grundlage korpusbasierter Untersuchungen möglichst vielfältig und adäquat darzustellen. Da ich die Substantivvalenz in ihrer Selbstständigkeit beschreiben und dabei Beweise und eventuelle Grenzen dieser Selbstständigkeit entdecken will, halte ich es für wichtig, in meinen Analysen sowohl deverbale als auch deadjektivische und nichtabgeleitete Substantive miteinzubeziehen. Somit können eventuelle Unterschiede und Ähnlichkeiten im Valenzverhalten der abgeleiteten und nicht-abgeleiteten Substantive erforscht werden, die Anhaltspunkte dafür bieten können, als wie selbstständig oder eben wie sekundär das System der Substantivvalenz zu betrachten ist. Wie oben bereits erwähnt, finde ich dabei auch die Frage wichtig, wie sich Substantive, die auch in SVG-Konstruktionen vorkommen, in und außerhalb dieser Konstruktionen in ihren Valenzeigenschaften beschreiben lassen. Das finde ich einerseits deswegen interessant, weil die vorliegenden Untersuchungen zu SVG bzw. 
FVG fast ausschließlich verborientiert sind. Andererseits will ich zeigen, mit welchen Einschränkungen SVG zur Ableitung der Valenzeigenschaften der in ihnen enthaltenen Substantive geeignet sind.

Die in Kap. 4 analysierten 600 Belege wurden dem Deutschen Referenzkorpus mit Hilfe von COSMAS, dem Online-Recherche- und Analysesystem des Leibniz-Instituts für deutsche Sprache, entnommen. Die Fragestellungen und Vorgehensweise der Untersuchung bzw. die detaillierte Begründung der Auswahl der sechs untersuchten Substantive finden sich in den Kap. 4.1.1-4.1.3. All die 600 Belege, die die Grundlage für die in den sechs Unterkapiteln von Kap. 4.2 dargestellten Ergebnisse bedeuten, werden in ihrer Form unverändert und mit den eigenen Analysedaten ergänzt im Anhang zur Verfügung gestellt. Die eruierten Daten und deren Auswertungen werden in Kap. 4.2 thematisiert und ebenfalls dort in Form von Tabellen auch quantitativ auswertbar und einzeln überprüfbar dargeboten. ${ }^{1}$ Die wichtigsten Ergebnisse der korpusbasierten Untersuchung werden in Kap. 4.3 zusammengefasst.

In Kap. 5 runde ich meine Arbeit durch die Schlussfolgerungen ab, die die Teilergebnisse meiner theoretischen Erörterungen und Korpusanalysen in Bezug auf die Hauptuntersuchungsfrage nach der Selbstständigkeit der Substantivvalenz zu einem geordneten Ganzen zusammenfassen. Ferner gebe ich noch einen Ausblick auf Fragen, die den Gedankengang meiner Arbeit fortführen und dadurch zur Vervollkommnung meines Konzepts beitragen könnten.

\footnotetext{
${ }^{1}$ Alle in der Arbeit, darunter auch in den Tabellen, verwendeten Abkürzungen werden im Abkürzungsverzeichnis in Kap. 6 aufgeführt.
} 


\section{Valenztheorie - Substantivvalenz}

In diesem Kapitel behandle ich Probleme der Substantivvalenztheorie, die in der Fachliteratur am meisten diskutiert werden. Diese Fragen sind - meines Erachtens - die Interpretation der Substantivvalenz, die Problematik der Unterscheidung zwischen valenzbedingten und valenzunabhängigen Dependentien bei substantivischen Valenzträgern und die Diskussion über die Möglichkeit von obligatorischen Substantivkomplementen².

Ich gehe zuerst von einer kurzen Thematisierung der Anfänge der modernen Valenztheorie bei Tesnière (vgl. Kap. 2.1) aus und nähere mich Schritt für Schritt dem Bereich der Substantivvalenz. So gebe ich in Kap. 2.2 ein Beispiel für eine Valenzdefinition, in die - im Gegensatz zu Tesnières Valenzauffassung - auch die Idee der Substantivvalenz ohne Einschränkungen integrierbar ist. Dabei spreche ich auch die terminologische Vielfalt in Bezug auf die Bezeichnung von valenzbedingten und valenzunabhängigen Dependentien an bzw. deute die Problematik der Unterscheidung der von ihnen markierten Erweiterungstypen an, die ich jedoch erst in Kap. 2.5 eingehender thematisiere.

In Kap. 2.3 gebe ich in Anlehnung an Helbig (1992) einen kurzen Überblick über die bisherige Substantivvalenzforschung. Das Ziel dieses Kapitels liegt vor allem darin, zu zeigen, welchen Weg die Forschung bis vor kurzem zurückgelegt hat, denn dadurch werden z. T. schon die wichtigsten unterschiedlichen Auffassungen über die Substantivvalenz erkenntlich.

Mit der Beschreibung der verschiedenen Substantivvalenzinterpretationen befasse ich mich in Kap. 2.4.1. Bei der Charakterisierung der Ansätze dient mir Teubert (2003) als Wegweiser, wobei ich aber auch meine Ansicht darstelle, der zufolge zwischen zweien der von Teubert (2003) beschriebenen drei Ansätze eine nähere Verwandtschaft angenommen werden kann. In Kap. 2.4.2 biete ich nach der kritischen Darstellung von Teuberts (2003) Argumenten, mit denen er ein selbstständiges Substantivvalenzsystem begründet, einen ersten Definitionskern meiner Deutung der Substantivvalenz an. Im Anschluss daran nehme ich in Kap. 2.4.3 die Substantivvalenzdarstellung einiger linguistischen Arbeiten kritisch unter die Lupe.

In Kap. 2.5 befasse ich mich mit dem Fragenkomplex der Unterscheidung zwischen valenzbedingten und valenzunabhängigen Dependentien. Dabei erörtere ich zuerst in Kap. 2.5.1 wie dieses Problem im Allgemeinen in der Fachliteratur erscheint. Hier ist festzustellen, dass die „umfassenden“ Beiträge, die sich mit dieser Frage beschäftigen, meistens nur den Bereich der Verbvalenz abdecken. Trotzdem halte ich es für nützlich, einen Überblick auch über diese

\footnotetext{
${ }^{2}$ Wie ich unten in Kap. 2.2 erkläre, verstehe ich unter Komplement ein valenzbedingtes Dependens.
} 
„allgemeine“ Betrachtung des Problems zu geben, denn dadurch kann man - meines Erachtens - einen guten Einblick in diesen Fragenkomplex gewinnen. Mit einem möglichst detaillierten Bild der allgemeinen Probleme möchte ich ersichtlich machen, wie diverse Betrachtungen dieser Frage schon vorgeschlagen worden sind. Neben der (kritischen) Darstellung der unterschiedlichen Theorien, betrachte ich bestimmte Fragestellungen, bei denen es sich meiner Meinung nach - auch aus der Perspektive der Substantivvalenz anbietet, auch wenn es in der referierten Fachliteratur nicht getan wird. Nach dieser - z. T. allgemeinen Problemdarstellung fasse ich in Kap. 2.5.2 zusammen, wie das Problem dieser Unterscheidung in bestimmten linguistischen Arbeiten speziell beim Substantiv erscheint. An mehreren Stellen ergänze ich diese Darstellung mit meinen Überlegungen zu den jeweiligen Theorien. In Kap. 2.5.3 behandle ich noch die Frage der Möglichkeit von obligatorischen Komplementen beim Substantiv, die in der Forschung vielleicht noch umstrittener ist als die Substantivvalenz selbst. Kap. 2.5.4 befasst sich ebenfalls mit der Problematik der Substantivkomplemente, indem darin unterschiedliche Ansätze zu ihrer Klassifizierung und Bezeichnung unter die Lupe genommen werden.

Schließlich lege ich in Kap. 2.6 mein multidimensionales Konzept zur Beschreibung der Substantivvalenz dar, das als Grundlage für die korpusanalytische Untersuchung in Kap. 4 dient.

\subsection{Anfänge der modernen Valenztheorie}

Es ist, wie ich weiter unten noch näher erläutern werde, umstritten, ob die Idee der Substantivvalenz mit dem Tesnière'schen Valenzbegriff vereinbar ist. Dennoch halte ich es für wichtig, im Vorfeld meiner Ausführungen über die Substantivvalenz, Tesnières Tätigkeit und Werk zumindest kurz anzusprechen, denn der französische Linguist gilt als „Begründer der modernen Dependenzgrammatik [..] und der mit dieser forschungshistorisch stark verbundenen Valenztheorie“ (Ágel / Fischer 2010: 250). ${ }^{3}$ Hier können nur ganz wenige, jedoch aus der Sicht der vorliegenden Arbeit höchstrelevante Gedanken aus der von Engel stammenden deutschen Übersetzung seines posthum erschienenen Hauptwerks Éléments de syntaxe structurale, dt. Grundzüge der strukturalen Syntax (Tesnière 1980) thematisiert werden.

\footnotetext{
${ }^{3}$ Zwar fängt die Geschichte der modernen Valenztheorie mit Tesnière an, die Wurzeln des Valenzgedankens aber lassen sich weit in die Antike zurückverfolgen (vgl. z. B. Polenz 1988: 54; Ágel / Fischer 2010: 262).
} 
Tesnière (1980: $31 \mathrm{ff}$.) zufolge basiert die abstrakte Ordnung des Satzes auf zwei Prinzipien, nämlich auf dem der „linearen Ordnung“ und dem der „strukturalen Ordnung“. Unter der strukturalen Ordnung versteht er die Relation der im Satz vorkommenden Wörter untereinander, wobei die konkrete Realisierung des Satzes und die Reihenfolge der einzelnen Elemente nicht beachtet werden. Die lineare Ordnung meint dagegen gerade die konkrete Realisierung des Satzes. Tesnière (1980: 49ff.) ist ferner der Ansicht, dass die strukturale und die semantische Ebene auseinandergehalten werden sollen, wobei die „strukturale Syntax“ sich mit den abstrakten Ordnungsprinzipien der strukturalen Ordnung zu beschäftigen hat.

Von den drei Grundrelationen ${ }^{4}$ der Syntax bei Tesnière ist aus der Perspektive des Valenzbegriffes die Konnexion von Bedeutung, die die hierarchische Verbindung von Wörtern des Satzes untereinander meint und somit mit der Relation der Dependenz gleichzusetzten ist (vgl. Vater 1978: 299 Anm. 6). Dementsprechend geht Tesnière (1980: 25ff.) davon aus, dass in einem Satz wie Alfred spricht ${ }^{5}$, neben dem (vom Verb) abhängigen Element, d. h. dem Dependens Alfred und dem diesem übergeordneten Regens spricht ein drittes Element, nämlich die Konnexion des Regens und Dependens zu beschreiben ist, wodurch überhaupt der Satz entsteht. Während Tesnière (1980: 28) zufolge von einem Regens mehrere Dependentien abhängig sein können, kann ein Dependens nur von einem Regens regiert werden. Unter Nexus versteht er das „Bündel“ eines Regens mit all seinen Dependentien. Daraus folgt, dass Regentien nexusbildende Fähigkeit besitzen. An dieser Stelle finde ich wichtig, auf ein terminologisches Problem hinzuweisen, was auch bei Ágel (2000, 36 Anm. 31) deutlich wird: Der von Engel als Nexus übersetzte Terminus kann weitgehend mit dem von Knoten identifiziert werden, der ferner ,[...] im Großen und Ganzen dem modernen Phrasenbegriff [entspricht] (wenn man davon absieht, dass Tesnière selbst den Begriff nicht ganz konsequent verwendet)“. Tesnière (1980: 63ff., Zitat von 64) unterscheidet zwischen vollen und leeren Wörtern, wobei erstere eine semantische Funktion haben, während letztere „ohne semantische Funktion“ „als bloße grammatische Hilfsmittel“ zu betrachten sind. ${ }^{6}$ Er rechnet Verben, Substantive, Adjektive und Adverbien zu den vollen Wörtern und betrachtet genau diese als nexusbildend (vgl. Tesnière 1980: 72ff.).

Tesnière (1980: 31) nimmt sowohl im Falle der Konnexionen als auch in dem der Nexus Hierarchien an, d. h., dass es Nexus und Konnexionen auf mehreren Ebenen eines Satzes geben

\footnotetext{
${ }^{4}$ Zur Junktion und Translation, die die weiteren zwei Relationen der strukturalen Syntax darstellen, vgl. den zweiten und dritten Teil der Grundzüge (Tesnière 1980: 216ff. und 248ff.).

${ }^{5}$ Beispiel von Tesnière (1980: 25ff.)

${ }^{6}$ Wie auch Ágel (2000: 36) darauf hinweist, stimmt diese Unterscheidung nahezu gänzlich mit der zwischen Autound Synsemantika überein.
} 
kann. Die Abhängigkeiten innerhalb eines Satzes können in Form eines Stemmas abgebildet werden, das so die „strukturale Ordnung“ des Satzes visualisiert. Die „Folge von Lauten oder Phonemen“, die in der Parole realisiert werden, nennt er ,gesprochene Kette“ (Tesnière 1980: 32). Sowohl für diese als auch für deren Verschriftung sieht er ihre „lineare Ordnung“ als wichtiges Charakteristikum an. Stemmata entstehen durch die Umwandlung der linearen in die strukturale Ordnung. Da er - wie oben schon erwähnt - die semantische als eine unabhängige Ebene postuliert - findet Tesnière (1980: 55ff.) neben dem Begriff des Nexus die Einführung von Nukleus notwendig, der neben dem strukturalen Nexus weitere Funktionen, darunter v. a. semantische, in sich trägt.

Der Zentralnexus, ein verbaler Nexus, ist bei ihm mit dem Satz selbst gleichzusetzen, der jedoch mit einem substantivischen, adjektivischen und / oder adverbialen Nexus verbunden sein kann. Den verbalen Nexus vergleicht Tesnière (1980: 93f.) „mit einem kleinen Drama“. Zum Geschehen dieses Dramas gehören meist Akteure und Umstände, die er im Rahmen der strukturalen Syntax als Aktanten und Angaben bezeichnet. ${ }^{7}$ Ferner vertritt er die Auffassung, dass Aktanten immer durch Substantive oder deren Äquivalente und Angaben durch Adverbien oder deren Äquivalente realisiert werden.

Bezüglich des Untersuchungsgegenstandes meiner Arbeit ist von großem Belang, wie Tesnière (1980: 128ff.) den substantivischen Nexus beschreibt. Dabei ist wichtig, dass er dem Substantiv generell die Fähigkeit zuschreibt, ,als Sitz eines Bündels von Konnexionen zu fungieren“" (Tesnière 1980: 128). Allerdings hebt er von den Dependentien des Substantivs das Attribut hervor, das meistens durch Adjektive, seltener durch Substantive oder Nebensätze zum Ausdruck gebracht wird. Im Vergleich der verbalen und substantivischen Regentien zueinander stellt Tesnière den wichtigen Unterschied fest, demzufolge Verben höchstens drei Aktanten haben könnten, während von Substantiven unbegrenzt viele Attribute abhängen können.

Den Valenzbegriff führt Tesnière (1980: 161ff.) zur Charakterisierung des Verbs ein und erklärt ihn mit einem Vergleich aus dem Bereich der Chemie, wonach ein Verb genauso wie ein Atom eine bestimmte Anzahl von Häkchen hat, durch die es andere Elemente an sich binden kann, die im Falle des Verbs seine Aktanten sind. Unter Valenz des Verbs versteht er die Anzahl der vom Verb regierten Aktanten, d. h. der Begriff hat bei Tesnière eindeutig einen quantitativen Charakter. Ich halte es noch für wichtig darauf hinzuweisen, dass bei ihm auch die Grundidee der Unterscheidung zwischen Valenzpotenz und Valenzrealisierung erkannt werden kann, was durch das folgende Zitat verdeutlicht werden soll: „Es ist im übrigen nie

\footnotetext{
${ }^{7}$ Zum Begriffspaar Aktanten vs. Angaben vgl. unten Kap. 2.2 und 2.5.
} 
erforderlich, daß alle Valenzen eines Verbs durch ihren jeweiligen Aktanten belegt sind und damit das Verb sozusagen saturiert ist. Gewisse Valenzen können unbesetzt oder frei bleiben.“ (Tesnière 1980: 161)

Nach dieser kurzen Darstellung der Tesnière'schen Dependenzgrammatik und des darauf basierenden Valenzbegriffs wird - meines Erachtens - ersichtlich, wie viele bis jetzt immer noch dominierende Begriffe und Fragen der Valenztheorie ihre Wurzeln bei Tesnière haben. Dies alles hat einen großen Einfluss darauf, ob seinem System entsprechend Substantive überhaupt als Valenzträger in Frage kommen. Explizit wird diese Frage von Tesnière (1980) nicht thematisiert. Dementsprechend kann man - meines Erachtens - diesbezüglich nur deduktiv vorgehen und versuchen, seine dependenzgrammatischen Erörterungen und seinen Valenzbegriff in diese Richtung weiterzudenken, wodurch man sich jedoch z. T. auf unsicheren Boden begibt. Wie sehr das der Fall ist, sollen einige unterschiedliche, einander zum Teil entgegengesetzte Annahmen aus der Forschung demonstrieren.

Askedal (2003: 90ff., v. a. 92) - zum Beispiel - sieht Valenz bei Tesnière auf „lexikalische Vollverben“ beschränkt. Ähnlich gehen Ágel / Fischer (2010: 274) davon aus, dass „,[Tesnière] nichtverbale Wörter als Valenzträger ausschließt“.

Vater (1978b: 270) dagegen steht auf dem Standpunkt, dass sich Tesnière zwar in den Grundzügen auf „die Valenz von Verben konzentriert [hat]“, aber in der von ihm beschriebenen Hierarchie der Konnexionen jedem Regens eine Valenz zugesprochen werden könne. Vater macht ferner an gleicher Stelle eine - meines Erachtens - interessante und zugleich problematische Feststellung die Substantivvalenz betreffend, indem er im System von Tesnière die Valenz des Substantivs mit dessen Fähigkeit gleichsetzt, „Determinantien oder Adjektive zu regieren“. Wenn wir beachten, dass Tesnière zufolge die Valenz die Anzahl der Aktanten, d. h. der valenzbedingten Erweiterungen bestimmen soll, ist Vaters obige Erklärung der Substantivvalenz schon aus der Sicht fraglich, dass von Substantiven theoretisch beliebig viele attributive Adjektive abhängen können. Außerdem ist auch die Frage diskussionsbedürftig, ob Determinantien tatsächlich als valenzbedingte Dependentien von Substantiven klassifiziert werden können. ${ }^{8}$

Engel, der - wie oben schon angesprochen - die Grundzüge von Tesnière ins Deutsche übersetzt hat, vertritt ebenfalls die Auffassung, dass ,an keiner Stelle des fast 700 Seiten umfassenden Hauptwerkes [...] sich ein Hinweis darauf [findet], daß Valenz am Verb exemplifiziert, nicht auf andere Wortarten übertragbar sei“ (Engel 1996: 58). Die Beispiele der

8 Diese Frage nehme ich unten (vgl. Kap. 2.5.2), im Zusammenhang mit Engels (2009b) Substantivvalenzbeschreibung noch einmal auf. 
nichtverbalen Sätze zeigen - seiner Meinung nach -, dass nicht nur Verben, sondern auch Nomina, Adjektive und Adverbien als „Zentralknoten fungieren können“, woraus gefolgert werden könne, dass „Tesnière ganz nahe daran war, auch diesen Wortarten Valenz zuzubilligen, (...)“. Im Rahmen seiner Kritik an Valenzgrammatiken bzw. -theorien, denen zufolge Valenz lediglich auf verbale Valenzträger beschränkt werden solle, formuliert er an einer anderen Stelle Folgendes: „Wer solches behauptet, darf sich nicht auf Tesnière berufen, der dergleichen nie gesagt, auch nie praktiziert hat, der freilich seine Grammatiklehre namentlich am Verb und seinen Satelliten demonstriert hat" (Engel 2009b: 73; meine Hervorhebungen Á. S.-T.).

Ich bin der Ansicht, dass zwar die Substantivvalenz explizit nicht Teil des Valenzkonzepts von Tesnière (1980) ist, aber auf der Grundlage seiner dependenzgrammatischen Überlegungen teilweise in sein System integrierbar ist. Die Tatsache, dass er - wie oben schon angesprochen - den Substantiven eine Aktantenrolle zuschreibt, kann - im Gegensatz zur oft zu beobachtenden Praxis in der Forschung - nicht eindeutig als Argument gegen den Gedanken der Substantivvalenz gedeutet werden, da sich diese Rollenzuordnung auch auf den verbalen Nexus beschränken lässt, was nicht automatisch bedeutet, dass in einem anderen Rahmen, $d . h$. auf einer anderen strukturalen Ebene betrachtet, Substantive nicht als Valenzträger fungieren könnten. Ich finde es wichtig, dass in der Dependenzgrammatik von Tesnière Substantive über eine nexusbildende Fähigkeit verfügen und unter ihren - theoretisch - unbegrenzt vielen Attributen Adjektive, Substantive und Relativsätze angenommen werden. Ich bin nämlich der Auffassung, dass sowohl adjektivische als auch substantivische Attribute teilweise valenzbedingte Erweiterungen realisieren (vgl. v. a. Kap. 2.6). Dennoch ist die Annahme einer Substantivvalenz im Beschreibungssystem von Tesnière - meines Erachtens - problematisch, wenn - entsprechend seiner Definition der Verbvalenz - durch die Valenz rein quantitative Angaben gemacht werden sollen, denn beim Substantiv kann es z. B. von attributiven Adjektiven tatsächlich unbegrenzt viele geben.

Zusammenfassend lässt sich - von meinem Standpunkt aus - feststellen, dass der Gedanke der Substantivvalenz auf der Grundlage der Dependenz- und Valenztheorie von Tesnière nicht auszuschließen ist, aber auch nicht ohne Einwände ins Tesnière'sche Valenzkonzept integrierbar ist. Deswegen stelle ich im nächsten Kapitel - als weiteren Schritt der Annäherung an das Thema meiner Arbeit - eine heute üblichere Definition der Valenz dar, in der nicht nur Verben als potenzielle Valenzträger betrachtet werden. Außerdem schneide ich noch eine Frage an, die sich schon bei Tesnière als problematisch erwiesen hat und es immer noch ist, nämlich die Einteilung der Dependentien in valenzgebundene und valenzunabhängige Elemente. 


\subsection{Eine Valenzdefinition als Ausgangspunkt}

Als weitere Einleitung zur Thematisierung des Phänomens der Substantivvalenz behandle ich hier eine allgemeine Valenzdefinition, in die - im Gegensatz zur Tesnière'schen - auch die Vorstellung der Substantivvalenz ohne Einschränkungen integriert werden kann. Dieser Forderung entspricht die folgende Begriffserklärung von Dürr / Schlobinski (2006: 116), der zufolge man unter Valenz ,die Fähigkeit von Wörtern [versteht], Leerstellen zu eröffnen und die Besetzung dieser Leerstellen zu regeln“. In dieser Deutung wird Valenz nämlich nicht nur dem Verb zugeschrieben, sondern sie wird als „Fähigkeit von Wörtern“ betrachtet. ${ }^{9}$

Eine problematische Frage war schon bei Tesnière die - oben bereits erwähnte Unterscheidung zwischen valenzgebundenen und valenzunabhängigen Konstituenten. Die erste Gruppe wird bei ihm als Gruppe der Aktanten, die zweite als die der Angaben bezeichnet. In meinem Substantivvalenzkonzept (vgl. v. a. Kap. 2.6 und Kap. 4) verwende ich dagegen für valenzgebundene Dependentien den Terminus Komplemente für valenzunabhängige den von Supplementen, wobei ich jedoch beim kritischen Überblick der (Substantiv)valenzforschung (vgl. Kap. 2.4-2.5) eventuell ihre Synonyme benutze, wenn sie in den unterschiedlichen Ansätzen als Termini gebraucht werden. In Bezug auf die häufigsten, in der Fachliteratur anzutreffenden Begriffspaare für diese Unterscheidung findet sich folgender tabellarische Überblick bei Storrer (2003: 766, Tab. 54.1):

$\begin{array}{ll}\text { actants } & \text { circonstants } \\ \text { Ergänzungen } & \text { Angaben } \\ \text { Aktanten } & \text { Zirkumstanten } \\ \text { Mitspieler } & \text { Umstandsbestimmungen } \\ \text { Valenzpartner } & \text { (freie) Angaben } \\ \text { Komplemente } & \text { Supplemente } \\ \text { Argumente } & \text { Adjunkte } \\ \text { (engl.) complement } & \text { (engl.) supplement } \\ \text { (engl.) argument } & \text { (engl.) adjunct }\end{array}$

Diese Zweiteilung kann in einem ersten Schritt in eine Dreiteilung überführt werden, indem bei den valenzbedingten Dependentien obligatorische und fakultative Ergänzungen ${ }^{10}$

\footnotetext{
9 Zur ganzen Wahrheit gehört jedoch, dass Dürr / Schlobinski (2006: 116) trotz der obigen Interpretation der Valenz in ihrer Deskripitive[n] Linguistik davon ausgehen, dass ,diese Fähigkeit vor allem Verben auszeichnet“. In der Hinsicht halte ich noch für wichtig, Bußmanns (2008: 769; meine Hervorhebung Á.S.-T.) Definition der Valenz anzusprechen, wonach ,V[alenz] [...] die Eigenschaft eines Lexems (z. B. eines Verbs, Adjektivs, Substantivs) ist, seine syntaktischen Umgebungen vorzustrukturieren, indem es anderen Konstituenten im Satz Bedingungen bezüglich ihrer grammatischen Eigenschaften auferlegt.“ In dieser Deutung wird Valenz nämlich explizit auch als „Eigenschaft“ des Substantivs anerkannt. Bei näherer Betrachtung dieser Definition wird jedoch klar, dass sie andererseits jedoch wesentlich enger gefasst ist als die von Dürr / Schlobinski (2006: 116), da sie das Phänomen der Valenz auf syntaktische Aspekte eingrenzt.

10 Wie Dürscheid $(2000,112)$ darauf hinweist, sind diese Unterbegriffe in dieser Form erstmals bei Helbig / Schenkel in ihrem Wörterbuch zur Valenz und Distribution deutscher Verben vorzufinden.
} 
voneinander unterschieden werden. ${ }^{11}$ Die Idee dieser Weiterdifferenzierung ist auch bei Tesnière erkenntlich, indem er - wie schon oben thematisiert - davon ausgeht, dass nicht immer alle Valenzen des Verbs realisiert werden. Mit der Dreiteilung wird aber das Problem der Unterscheidung zwischen valenzbedingten und valenzunabhängigen Dependentien nicht gelöst. Es sind schon viele operationelle Tests zur Klärung dieser Frage vorgeschlagen worden, aber keiner von ihnen führt zum eindeutigen Ergebnis. Es muss schon hier darauf hingewiesen werden, dass bei der Gruppierung der Dependentien eines Valenzträgers - meines Erachtens oft keine eindeutigen Entscheidungen getroffen werden können. Da eine der bedeutenden Fragestellungen von Kap. 2 gerade in dieser Problematik, in einer der Zentralfragen auch im Bereich der Substantivvalenz, eingebettet ist, befasse ich mich hier nicht näher mit diesem Problembereich, sondern widme den Unterscheidungsschwierigkeiten im Allgemeinen und speziell beim Substantiv je ein eigenes Unterkapitel (vgl. Kap. 2.5.1 und 2.5.2).

Nach diesen allgemeinen Themen des Valenzbegriffs und der Valenztheorie gehe ich im nächsten Unterkapitel schon auf den Bereich der Substantivvalenz ein. Als Erstes führe ich dabei die Schwierigkeiten ihrer Forschung in Anlehnung an Helbig (1992) vor.

\subsection{Drei Phasen der Substantivvalenzforschung nach Helbig (1992) $)^{12}$}

Helbig (1992: 114f.) teilt in seinem Buch Probleme der Valenz- und Kasustheorie „die Forschung der Valenz der Substantive“ in drei Abschnitte. Ich nehme diese Beschreibung als Grundlage dafür, mich Schritt für Schritt den tatsächlichen und (immer noch) aktuellen Problemfragen der Substantivvalenz bzw. ihrer Forschung zu nähern. In diesem Hinblick ist meiner Meinung nach - die dritte Phase ${ }^{13}$ von großer Bedeutung, dementsprechend werden mir ihre Fragestellungen als Anregung für umfassendere Darstellungen der angesprochenen Probleme dienen.

Zuerst sei aber - wie auch bei Helbig - kurz auf die beiden ersten Phasen der Substantivvalenzforschung hingewiesen. Für die erste Phase gilt, dass die deverbalen und deadjektivischen Substantive mit den bei der Verbvalenz schon eingenommenen Methoden

\footnotetext{
${ }^{11}$ Wie sich unten in Kap. 2.5.1 bzw. 2.5.2 zeigen wird, gibt es auch den Vorschlag zu einer Vierteilung und noch weitere für einen fließenden Übergang zwischen mehreren Komplement- und Supplementklassen.

${ }^{12}$ Hier muss darauf hingewiesen werden, dass dieser Einteilung auch in einem früheren Artikel von Helbig (1986) zu begegnen ist, in dem er umstrittene Fragen der Substantivvalenz behandelt. Da es keine Unterschiede betreffend diese Darstellung zwischen diesem Artikel und der Monographie von Helbig gibt, recherchiere ich diese Frage nur auf der Grundlage der letzteren, d. h. Helbig (1992: 114f.).

${ }^{13}$ Dieser Phase wird auch bei Helbig (1992) eine deutlich größere Aufmerksamkeit geschenkt als der ersten und der zweiten.
} 
untersucht und beschrieben wurden. Dabei ,aber wurde der (ungerechtfertigte) Eindruck erweckt, als seien alle Substantive auf diese Weise beschreibbar“ (Helbig 1992: 114; Hervorhebung von Helbig).

In der nächsten Phase wurde erkannt, dass viele Substantive durch Transformationen aus Verben oder Adjektiven ableitbar sind, wodurch auch ihre Valenz sich erklären lässt. Deswegen wurde davon ausgegangen, dass nur Nominalisierungen, aber auch von ihnen nicht alle, syntaktische Valenz haben können. ${ }^{14}$ Helbig stellt noch im Zusammenhang mit dieser Beschränkung die These von Sandberg ${ }^{15}$ dar, nach der nur die nicht-lexikalisierten Verbalsubstantive, die die Bedeutung des Ausgangsverbs bewahrt haben, im gleichen Sinne wie die Verben syntaktisch valent sind. ${ }^{16}$

In der dritten Phase sind mehrere frühere Hypothesen der Substantivvalenz ins Wanken geraten und die mit ihnen zusammenhängenden Fragen wurden neu aufgenommen. Zu diesen Themen gehören die Annahme, dass „die Substantive (im Unterschied zu den Verben und Adjektiven) in der Regel nur fakultative Aktanten haben (...)“ (Helbig 1992: 114); die Problematik der Unterscheidung zwischen Ergänzungen und Angaben bei Substantiven und die der Interpretation der Substantivvalenz. Diese Probleme stellen - meines Erachtens - die Zentralfragen im Bereich der Substantivvalenzforschung dar, deswegen bemühe ich mich in den weiteren Teilen von Kap. 2 um ihre möglichst vielseitige Thematisierung.

Zuerst setze ich mich dabei mit (den) unterschiedlichen Interpretationsmöglichkeiten der Substantivvalenz in der Forschung auseinander, denn ich kann mich erst den Problembereichen innerhalb der Substantivvalenz zuwenden, nachdem ich den Betrachtungsrahmen klargestellt habe. Deswegen stelle ich in Kap 2.4.1 zuerst die wichtigsten Richtungen der Beschreibung des Substantivvalenzsystems dar, um dann auch meine Stellungnahme zum Problem formulieren zu können (vgl. Kap. 2.4.2). Im Anschluss daran gebe ich noch einige weitere Beispiele dafür, wie das Phänomen der Substantivvalenz in verschiedenen linguistischen Arbeiten - vor allem in Grammatiken - behandelt wird (vgl. Kap. 2.4.3).

\footnotetext{
${ }^{14}$ Diese Deutung der Substantivvalenz wird in Unterkapitel 2.4.1 unter dem Begriff Nominalisierungsansatz nochmals angesprochen (vgl. dazu Teubert 2003: 30ff., v. a. 32ff.).

${ }^{15}$ Helbig (1992: 114) beruft sich dabei auf die folgenden Arbeiten von Sandberg: Sandberg, Bengt (1976): Die neutrale en-Ableitung in der deutschen Gegenwartssprache. Göteborg.; und Sandberg (1979), von denen Sandberg (1979) unten auch ich thematisiere (vgl. Kap. 2.5.3).

${ }^{16}$ Das Warten ist z. B. ein nicht-lexikalisiertes Verbalsubstantiv, während z. B. die Bitte als lexikalisiert gilt. Wie auch im späteren Teil meiner Arbeit deutlich wird, bin ich der Auffassung, dass auch Bitte über syntaktische Valenz verfügt, so stellen in jmds. Bitte an jmdn. um etw. jmds., an jmdn. und um etw. Komplemente des Substantivs dar (zur detaillierten Beschreibung der Valenzstruktur von Bitte vgl. z. B. Bassola et al. 2003: 64ff.).
} 


\subsection{Interpretationen der Substantivvalenz}

\subsubsection{Substantivvalenz - vererbt oder sui generis?}

In Anlehnung an Teubert (2003: 827ff.) lassen sich in der Forschung drei Ansätze zur Beschreibung der Substantivvalenz unterscheiden. Von diesen drei Deutungen sehe ich zwei als miteinander enger verbundene an, wodurch sich die Zahl der Grundideen - meines Erachtens - auf zwei reduzieren lässt. ${ }^{17}$

Der sog. Nominalisierungsansatz hat sich als erste Interpretationsweise schon in der Anfangsphase der Substantivvalenzforschung entwickelt und wird v. a. in der Generativen Grammatik auch heute noch vertreten (vgl. Teubert 2003: 827ff.). Dieser Auffassung zufolge wird nur den Nominalisierungen syntaktische Valenz zugeschrieben und die Valenzstruktur eines solchen Substantivs wird als regelhafte Übertragung ${ }^{18}$ der Valenzstruktur des Basisverbs für erklärbar gehalten. ${ }^{19}$ Wie auch durch die unteren Erörterungen $\mathrm{zu}$ den beiden anderen Ansätzen ersichtlich werden soll, setzt sich dieser Ansatz - meiner Meinung nach - deutlich von den restlichen zwei ab. Ähnlich sehen Ágel/Fischer (2010:274) zwischen dem Nominalisierungsansatz auf der einen und der Stützverbgefüge- und sui-generis-Interpretation auf der anderen Seite einen deutlichen Unterschied, indem sie feststellen, dass ,,von denen nur der Nominalisierungsansatz davon ausgeht, dass es keine genuine Substantivvalenz gebe, (...)“.

Im Stützverbgefüge-Ansatz dient als Basis die Beobachtung, dass Substantive, die in Funktionsverbgefügen vorkommen, auch als selbstständige Substantive dieselben Konstruktionen aufweisen wie in den Verbindungen mit Funktionsverben. Demnach könne die Valenz eines Substantivs aus entsprechenden Funktionsverbgefügen erklärt werden (vgl. Teubert 2003: 829f.). Den dritten Ansatz stellt die sui-generis-Auffassung des Substantivvalenzsystems dar (vgl. Teubert 2003: 830), der zufolge die Valenzpotenz ein selbständiges und kein von der verbalen oder adjektivischen Valenz stammendes Merkmal der Wortklasse Substantiv ist. Für die enge Verbindung der beiden letzten Ansätze scheint mir die folgende Feststellung von - dem eher zu den Vertretern des Stützverbgefüge-Ansatzes gehörenden - Stein (1996: 200) einen Beweis darzustellen:

\footnotetext{
${ }_{17}$ Dadurch erklärt sich, warum im Titel dieses Unterkapitels nicht drei, sondern nur zwei Begriffe mit der Substantivvalenz in Verbindung gesetzt werden.

${ }^{18}$ Darauf bezieht sich vererbt in der Zwischenüberschrift.

${ }^{19}$ Diese Deutungsweise kann aber die Valenzstruktur von vielen Substantiven, darunter eventuell auch von einigen Nominalisierungen, nicht erklären (vgl. dazu Teubert 2003: 827ff.). Hier thematisiere ich die Probleme dieser Theorie jedoch nicht eingehender, denn bei der Begründung dessen, warum ich einen anderen Ansatz für plausibel halte, werden die wichtigsten von diesen Problemen - auch wenn nur indirekt - angedeutet (vgl. unten Kap 2.4.2).
} 
Bei den Substantiven hat sich gezeigt, dass es nicht ausreicht, diejenigen Rollen zu beschreiben, die bei isolierter Verwendung des Substantivs syntaktisch realisierbar sind, da auch latente Argumente aus dem zugrunde liegenden Verb in Verb-Substantiv-Verbindungen relevant werden können.

Diese Argumentation legt - so wie ich das sehe - die Vermutung nahe, dass beim Substantiv die „nicht-latenten“ Argumente zum Substantiv selbst gehören und so das Substantiv zumindest teilweise - über eigene Valenz verfügt.

Auch das spricht - meiner Meinung nach - für die Kompatibilität der beiden Auffassungen, dass im Deutsch - ungarischen Wörterbuch zur Substantivvalenz (Bassola et al. 2003 und 2012), in dem die Autoren die Substantivvalenz als ein System sui generis betrachten, ,als Ausgangsbasis für die Eruierung der Valenzen auf Konstruktionen mit ,Stützverb' - ,verbe support' und dem betreffenden Substantiv zurückgegriffen“ wird (Bassola / Kubczak / László 2003, 179).

Nach dieser Darstellung der verschiedenen Substantivvalenzansätze erörtere ich im nächsten Unterkapitel - ausgehend von Teuberts sui-generis-Auffassung - meine Deutung der Substantivvalenz.

\subsubsection{Substantivvalenz - Sui generis, aber auch vererbt?}

Der Titel dieses Unterkapitels mag auf den ersten Blick komisch erscheinen, aber durch meine Überlegungen zur Ontologie der Substantivvalenz soll schließlich klar werden, was diese etwas konfus wirkende Formulierung andeutet.

Als Ausgangspunkt für die Formulierung meines Beschreibungsrahmens der Substantivvalenz betrachte ich die Substantivvalenzinterpretation von Teubert. Sowohl in seiner Monographie Valenz des Substantivs aus dem Jahre 1979 als auch in einem seiner Artikel aus dem Jahre 2003 gibt es dieselben ${ }^{20}$ drei Kriterien für die Untermauerung einer Deutung der Substantivvalenz als ein System sui generis (vgl. Teubert 1979: 13 bzw. 73ff.; 2003: 830). Diese drei Beweisgründe fasse ich im Folgenden einzeln zusammen und nehme sie kritisch unter die Lupe. Betrachten wir zuerst das folgende Argument von Teubert:

1) Es gibt valente Substantive, die - zumindest synchronisch betrachtet - nicht als abgeleitet gelten.

\footnotetext{
${ }^{20}$ Bei der Formulierung eines Kriteriums gibt es in den beiden Arbeiten einen Unterschied und auch die Beispiele sind nicht immer identisch, aber ich werde auf diese Differenzen (soweit sie Bedeutung für meine Ausführungen haben) unten, an entsprechenden Stellen dieses Unterkapitels eingehen.
} 
Als Beispiele für solche nicht abgeleiteten valenten Substantive führt er u. a. die folgenden auf: Straße nach Rom, Vorrat an Erdöl (Teubert 1979: 13); Vater, Flasche, Debatte (Teubert 2003: 830). Bei einigen dieser Beispiele bin ich der Meinung, dass sie nicht unbedingt die überzeugendsten Beweise für das oben genannte Kriterium darstellen. So kann bei Straße nach Rom zwar eine engere semantische Zusammengehörigkeit zwischen dem Bezugssubstantiv und seiner Erweiterung entdeckt werden, es ist aber doch fraglich, ob die Präpositionalphrase als Realisierung eines Arguments ${ }^{21}$ des Substantivs zu betrachten und somit als Ergänzung zu deuten ist. ${ }^{22}$ Während ich bei einem Bezugssubstantiv wie der Weg ein Direktivum als Ergänzung plausibel finde, ist meiner Intuition nach bei einem Substantiv wie die Straße eher ein Situativ als Argument im Denotat verankert. Meines Erachtens kann man nach Rom hier höchstens als eine weniger typische Ergänzung ${ }^{23}$ betrachten.

Mit Flasche liegt - in meinen Augen - ein ähnlicher Problemfall vor. Unter den Stoffergänzungen kommt dieses Wort in dem Beispiel eine Flasche Merseburger Bieres (Teubert 1979: 86 bzw. 2003: 830) vor, wobei hier der Genitivus partitivus - meiner Meinung nach - zwar wiederum als Ergänzung, aber ebenfalls als eine weniger typische eingestuft werden soll. Schließlich gilt Debatte im Deutschen nicht als Ableitung, es wurde aber aus einem französischen Deverbativum (débat) entlehnt (vgl. Kluge 183; DHW 210), wodurch es sich meines Ermessens - als wenig stichhaltiger Beweis für Beweisgrund 1) erweist. Von den oben zitierten Beispielen finde ich Vorrat an Erdöl besonders treffend, da es eindeutig ein nicht abgeleitetes Substantiv darstellt, dessen Argument durch das Präpositionalattribut realisiert wird. Als ähnliche Beispiele könnten u. a. Appetit auf oder Lust auf / zu / an genannt werden.

Als weiteren Grund dafür, dass er die Substantivvalenz als ein System sui generis interpretiert, gibt Teubert Folgendes an:

2) Valente Substantive können Ergänzungen haben, die bei den Verben keine Entsprechungen haben.

In Bezug auf dieses Argument ist in Teuberts Monographie ein kleiner Widerspruch bzw. Ungenauigkeit zu entdecken. So führt er bei der ersten Erwähnung des obigen Arguments als Beispiel für eine solche Ergänzung den Genitivus partitivus auf (vgl. Teubert 1979: 13), der

\footnotetext{
21 Ich verwende den Terminus Argument, wie er in der formalen Logik definiert wird, und verstehe dementsprechend die Leerstellen eines Prädikats bzw. einer Funktion darunter (vgl. Bußmann 2008: 56).

${ }^{22} \mathrm{Zu}$ meiner Sicht der Unterscheidbarkeit von Komplementen und Supplementen von Substantiven vgl. unten Kap. 2.6. So viel kann jedoch vorausgeschickt werden, dass ich bei der Beurteilung dessen, ob eine Substantiverweiterung als Komplement oder als Supplement zu klassifizieren ist, entscheidend finde, ob sie ein Argument des Bezugssubstantivs ausdrückt.

${ }^{23}$ Mit weniger typischen Ergänzungen meine ich Ergänzungen, die zu den Peripheriebereichen der Großmenge von Ergänzungen gerechnet werden sollen (vgl. Kap. 2.5.3).
} 
jedoch bei der detaillierten Beschreibung der Ergänzungsklassen (vgl. Teubert 1979: 88ff.) unter den 17 Ergänzungsklassen gar nicht erscheint. Das hat - meines Erachtens - auch seinen Grund, der sich auch mit Hilfe von Teuberts (2003) späterem Beitrag gut erklären lässt. In diesem Artikel trifft er nämlich terminologische Verfeinerungen, wobei er zwischen Arguments-, Ergänzungs- und Attributsklassen unterscheidet (vgl. Teubert 2003: 825ff.). Ihm zufolge gibt es auf der semantischen Ebene Argumentsklassen, die sowohl durch Ergänzungen als auch durch Angaben realisiert werden könnten. Attributsklassen versteht Teubert (2003: 825) als „rein syntaktisch definiert“, während Ergänzungsklassen Argumente seien, die durch Attributsklassen realisiert sind, die nur von bestimmten Substantivklassen abhängig sein können und somit subklassenspezifisch sind. Es sei hier angemerkt, dass ich zwar mit diesen Erklärungen von Teubert nicht ganz einverstanden bin (vgl. dazu v. a. Kap. 2.5.1 die Erörterung des Subklassenspezifikproblems und Kap. 2.6), aber die Grundidee einer solchen begrifflichen und terminologischen Unterscheidung dennoch für wichtig halte. Es ist - meines Erachtens nämlich von großer Bedeutung klarzustellen, dass bestimmte Attributsklassen (d. h. Realisierungsformen von Attributen) abhängig von ihrer Distribution, d.h. von ihrem Bezugssubstantiv, eventuell unterschiedliche Ergänzungsklassen oder sogar Angaben realisieren.

Dementsprechend ist aber der Genitivus partitivus gar nicht als Ergänzung, sondern als Attributsklasse zu betrachten. So kommt er in dem oben schon angesprochenen Beispiel eine Flasche Merseburger Bieres als konkrete sprachliche Realisierung der Stoffergänzung vor, die wohl als eine Ergänzungsklasse betrachtet werden kann, die keine Entsprechung unter den verbalen Ergänzungen hat. Im Beitrag von Teubert (2003: 830) wird neben der Stoffergänzung auch die Personenergänzung (z. B. Peters Vater, ihre Nachbarin) als eine Ergänzungsklasse ohne Entsprechung im verbalen oder adjektivischen Bereich angeführt. Als dritte ausschließlich beim Substantiv vorkommende Ergänzungsklasse nennt Teubert (2003, 830) noch die Benennungsergänzung mit den Beispielen die Regierung Brandt, das Wort Askese. Zu dieser Ergänzung gehört Teubert (1979: z. B. 90) zufolge die Attributsklasse Apposition.

Im Zusammenhang mit der Betrachtung der Apposition als Realisierungsform der Benennungsergänzung, halte ich es für wichtig, einen kurzen Exkurs zu machen. Appositionen können nämlich in Subklassen eingeteilt werden. So unterscheidet z. B. Eroms (2000: 300ff.) einerseits weitere Appositionen - wie z. B. in dem Ausdruck Vizepräsident Fröhlich, dieser allzeit zu Scherzen aufgelegte Kollege ${ }^{24}$-, die er als „,verkürzte Prädikationen“ kennzeichnet

\footnotetext{
${ }^{24}$ Das Beispiel stammt von Eroms (2000: 300; meine Hervorhebung Á. S.-T.).
} 
(Eroms 2000: 300). Im Gegensatz dazu sind Eroms (2001: 301) zufolge engere Appositionen wie z. B. in Frau Maier ${ }^{25}$, ,gänzlich anders zu beurteilende syntaktische Erscheinung[en]“. Meines Erachtens ist auch der Ergänzungsstatus der beiden Appositionstypen - wenn auch nicht „gänzlich anders“ aber - unterschiedlich zu bewerten. Weitere Appositionen als eigene Prädikationen drücken kein Argument des Bezugssubstantivs aus und so können sie - meiner Meinung nach - nicht als Ergänzungen klassifiziert werden. Die engeren Appositionen aber, wie auch die Beispiele für die Benennungsergänzung bei Teubert, sind Fälle, bei denen überlegt werden kann bzw. soll, ob sie Vertreter von Ergänzungen sind. So bin ich der Meinung, dass von den Beispielen, die Teubert aufführt, die Apposition in der Nominalphrase die Regierung Brandt eindeutig als Ergänzung betrachtet werden kann, da es ein - agensähnliches - Argument ausdrückt. Dagegen finde ich die Klassifizierung der Apposition als Ergänzung in dem Beispiel das Wort Askese problematisch, da es eher mit dem Denotat selbst als mit einem Argument des Bezugswortes identifiziert werden sollte (vgl. dazu den Terminus Quasiargumente v. a. in Kap. 2.6.1). Deswegen bin ich der Meinung, dass auch die engeren Appositionen nicht eindeutig als Ergänzungen oder zumindest nicht als in gleichem Maße typische Ergänzungen klassifiziert werden können. Diesen kleinen Exkurs habe ich für nötig gehalten, weil dadurch gezeigt werden konnte, dass auch innerhalb einer auf der Oberfläche einheitlich scheinenden Attributsklasse bei der Beurteilung des Ergänzungs- bzw. Angabestatus des konkreten Attributs Differenzierungen von Bedeutung sein können. ${ }^{26}$

Zur Untermauerung der sui-generis-Deutung der Substantivvalenz nennt Teubert noch ein Argument, dessen Formulierung in den beiden hier referierten Arbeiten jedoch bestimmte Unterschiede aufweist:

3a), ,...] verbale Ergänzungen [lassen sich] nicht systematisch und durch generelle Regeln beschreibbar in substantivische Ergänzungen überführen (z. B.: jemandem helfen / Hilfe für jemand [sic], aber: jemandem danken / Dank an jemanden)“ (Teubert 1979, 13; Hervorhebungen von Teubert)

\footnotetext{
${ }^{25}$ Das Beispiel wurde Eroms (2000: 300; meine Hervorhebung Á. S.-T.) entnommen.

${ }^{26}$ Zur Kritik an der Klassifizierung engere vs. weitere Appostionen und gleichzeitig zur differenzierten Beurteilung des Ergänzungs- vs. Angabestatus der betreffenden Erweiterungen vgl. Engel (2009a: 449ff. bzw. 292ff.).
} 
3b) „... [Es] gibt $[\ldots]$ bei den Ergänzungen abgeleiteter Substantive oft nicht prognostizierbare Ausnahmen von der allgemeinen Tendenz ihrer syntaktischen Realisierung. Deverbale ${ }^{27}$ Akkusativobjekte werden beispielsweise nicht immer durch genitivische NGn [Nominalgruppen, Á. S.-T.] realisiert: der Hass auf Peter, die Achtung vor Julia, die Einladung an Maria." (Teubert 2003: 830; Hervorhebungen von Teubert)

Die Differenzen zwischen den beiden Formulierungsweisen sind - meiner Ansicht nach - mit der oben schon thematisierten begrifflichen und terminologischen Verfeinerung zu erklären, wobei Teubert (2003: 825ff.) in seiner späteren Valenzbeschreibung zwischen den drei Ebenen der Arguments-, Ergänzungs- und Attributsklassen unterscheidet. Ich bin der Meinung, dass diese formale, (morphologisch-)syntaktische Variationsmöglichkeit bzw. jede Art der Idiosynkrasie der Valenz abgeleiteter Substantive von speziellen substantivischen Eigenschaften zeugen, die vielleicht keine selbstständige aber zumindest eine von der verbalen (und adjektivischen) Valenz sich unterscheidende Substantivvalenz begründen.

Zusammenfassend zu den hier dargestellten drei Argumenten von Teubert sei Folgendes festgestellt: Ich bin mit den Feststellungen 1) und 2) einverstanden und finde sie zur Begründung einer sui-generis-Interpretation der Substantivvalenz geeignet. Meine obigen kritischen Bemerkungen betreffen nicht das Wesen der Argumente, sondern nur bestimmte Beispiele, die von Teubert zu ihrer Demonstration dargestellt worden sind. Dem Beweisgrund, den ich unter 3a) bzw. 3b) behandelt habe, stimme ich ebenfalls zu, auch wenn ich da der Meinung bin, dass er - wie ich oben gerade thematisiert habe - nicht ohne Weiteres als Argument für eine selbstständige Substantivvalenz betrachtet werden kann.

Generell gehe ich davon aus, dass auch bei Substantiven eine „selbstständige“ (d. h. nicht aus Ableitung resultierende) Valenz angenommen werden kann. Darüber hinaus gibt es auch zahlreiche deverbale und deadjektivische Substantive, die ihre Argumentstruktur von ihrem Basiswort geerbt haben, aber selbst bei ihnen kann es spezielle Einschränkungen geben, die auch dieser „abgeleiteten Substantivvalenz“ eine spezielle Art verleihen. ${ }^{28}$ So bin ich der

\footnotetext{
${ }^{27}$ Hier möchte ich darauf hinweisen, dass die Terminologie von Teubert an einigen Stellen einer Erklärung bedarf. Er verwendet z. B. „deverbal“ und „denominal“ zum Ausdruck der Abhängigkeit von einem Verb oder einem Substantiv, „deverbativ“ für eine Ableitung aus einem Verb (vgl. dazu Teubert 1979: 27). In meiner Arbeit könnte die komplette Übernahme dieser Ausdrucksweise manchmal zu nicht eindeutigen, gar störenden Formulierungen führen. Deswegen kommen bei mir - wenn nötig - entsprechende Paraphrasierungen oder äquivalente Termini vor: z. B. verbale Valenz statt deverbale Valenz. Ich verwende außerdem - im Gegensatz zu Teubert - deverbal in der Bedeutung aus einem Verb abgeleitet.

Im obigen Zitat sind also mit dem Ausdruck "deverbale Akkusativobjekte“ die vom Verb abhängigen Akkusativobjekte gemeint.

${ }^{28}$ Dazu gehören einerseits die oben unter Punkt 3) angesprochenen formalen Idiosynkrasien, aber andererseits auch Beispiele wie das deverbale Substantiv die Tat, wo ein Argument des Basiswortes, hier das Akkusativobjekt von tun, beim Substantiv - im Allgemeinen - nicht realisiert werden kann, da es eigentlich durch das Substantiv selbst ausgedrückt wird.
} 
Meinung, dass die Substantivvalenz im Grunde genommen als ein System sui generis zu betrachten ist, in dem aber an mehreren Punkten verbale und adjektivische „Erbschaften“ eine mitbestimmende Rolle haben, wodurch sich auch der Titel dieses Unterkapitels erklären lässt. Ein ähnlicher Gedanke kann auch bei Teubert $(2003,830)$ erkannt werden, indem er Folgendes feststellt: „Die Substantivvalenz als eigenständiges System zu beschreiben, bedeutet nicht, die Augen vor den offensichtlichen Analogien und Entsprechungen zur Valenz der Verben und Adjektive zu verschließen.“

Bevor ich in Kap. 2.6 mein multidimensionales Konzept der Substantivvalenz darstelle, sei hier vorerst ein erster Definitionskern meiner Interpretation der Substantivvalenz dargeboten. Die Valenz des Substantivs ist - meiner Meinung nach - die Fähigkeit bestimmter abgeleiteter und nicht-abgeleiteter Substantive, Leerstellen für andere Elemente im Satz bereitzustellen, deren Besetzung vom jeweiligen substantivischen Valenzträger semantisch determiniert und formal in unterschiedlichem Maße bestimmt wird.

In Kap. 2.6. gehe ich detaillierter darauf ein, welche Valenzbeziehungen ich in der Substantivvalenz voraussetze und wie diese bei der Unterscheidung von Substantivkomplementen und -supplementen zur Geltung kommen. Ziel der korpusbasierten Untersuchungen in Kap. 4 wird in erster Linie sein, durch die Analyse sowohl abgeleiteter als auch nicht-abgeleiteter Substantive $\mathrm{zu}$ beleuchten, welche Ähnlichkeiten und/oder Unterschiede sie in ihrem Valenzverhalten aufweisen und welche Besonderheiten für sie alle gleichermaßen gelten. Dies alles soll zur Untermauerung einer sui-generis-Deutung der Substantivvalenz beitragen.

Nach diesen grundlegenden Erörterungen zur Frage der Ontologie der Substantivvalenz befasse ich mich im nächsten Unterkapitel mit der Substantivvalenzbeschreibung in einigen linguistischen Arbeiten.

\subsubsection{Substantivvalenz im Lichte (allgemeiner) linguistischer Arbeiten}

Die oben in Kap. 2.4.2 bereits thematisierten wichtigsten Grundgedanken von Teubert (1979 und 2003) im Zusammenhang mit der sui-generis-Interpretation der Substantivvalenz geraten bei Helbig (1992: 118ff.) in die Kritik. ${ }^{29}$ Zum einen meint er, dass das oben als drittes

\footnotetext{
${ }^{29}$ Genau genommen können von Helbig (1992) natürlich nur die Gedanken von Teubert betrachtet werden, die er in seiner Monographie (Teubert 1979) formulierte, dabei - wie gerade im vorangehenden Unterkapitel erklärt wurde - stimmen die Grundgedanken im Beitrag von Teubert aus dem Jahre 2003 mit denen in seiner Monographie überein.
} 
aufgeführte Kriterium kein neues ist. Ferner geht er davon aus, dass die eventuell auftauchenden Unterschiede (wie z. B. im Falle von jmdm helfen vs. Hilfe für jmdn), ,morphosyntaktischer Art“ sind und „die tiefer liegenden Valenz- und (semantischen) Kasusverhältnisse“ (Helbig 1992: 120) nicht beeinflussen. ${ }^{30}$ Dementsprechend könnten sie kein Argument gegen die Deutung der Substantivvalenz als Ableitung aus der verbalen Valenz darstellen.

Er bezweifelt zudem, dass es sich bei den Substantivergänzungen, die keine Entsprechungen im verbalen Bereich haben, tatsächlich um syntaktische Aktanten handelt. Als wesentlicher Kritikpunkt gegen die sui-generis-Auffassung von Teubert, genauer gegen Teuberts Beweisführung, führt Helbig (1992: 120) auf, „daß die meisten Klassen [Ergänzungsklassen, Á. S.-T.] durch Beispiele belegt sind, die in der Tat deverbale oder deadjektivische Bildungen sind“, wodurch die sui-generis-Auffassung der Substantivvalenz - ihm zufolge - „etwas fragwürdig, mindestens aber voreilig“" erscheint.

Die Bedeutung dieses letzten, scheinbar gewichtigen Gegenarguments ist jedoch - meiner Meinung nach - mindestens aus zweierlei Gründen zu relativieren. Helbig selbst bringt zwar keine Beispiele für die kritisierten Belegbeispiele von Teubert (1979), wenn man aber die Ergänzungsklassen von Teubert (1979: 88ff) unter die Lupe nimmt, begegnet man tatsächlich vielen Beispielen, die auf den ersten Blick als rein deverbale oder deadjektivische Substantive betrachtet werden könn(t)en. Etwas Wichtiges ist aber - meines Erachtens - bei Helbig außer Acht gelassen worden, nämlich dass den lexikalisierten deadjektivischen und deverbalen Substantiven eine andere Interpretation gebührt als den nicht-lexikalisierten. Die Bedeutung der lexikalisierten abgeleiteten Substantive hat sich von der ihrer Basiswörter entfernt. Sie ist nicht einfach daraus herzuleiten und so ist bei ihrer Valenzstruktur eine Eigenständigkeit bzw. Unabhängigkeit anzunehmen. Viele der Helbig zufolge anfechtbaren Beispiele von Teubert (1979: 88ff.) sind - meiner Ansicht nach - lexikalisierte Ableitungen (wie z. B. die Prüfung, die Arbeit, das Risiko, der Besuch, die Lage, die Dauer), wodurch das Gegenargument von Helbig an Gewicht verliert. Ebenfalls sprechen - meines Erwägens - Beispiele von Teubert wie der Autor, der Hunger, der Vater, die Ehe, die nicht einmal diachron abgeleitet gelten, ergänzt durch zahlreiche synchron nicht mehr als Ableitungen erkennbare valente Substantivbeispiele

\footnotetext{
${ }^{30}$ Wie sich unten in Kap. 2.6 im Rahmen der Darstellung meines multidimensionalen Konzepts auch zeigen wird, bin ich mit dem Primat der Semantik in Bezug auf die Unterscheidung von Substantivkomplementen und -supplementen einverstanden. Dennoch kann ich Helbigs obiger Feststellung nicht ganz zustimmen, da ich der Meinung bin, dass bei der Valenzbeschreibung auch formale Aspekte mitbetrachtet werden sollen, auch wenn sie keine primär entscheidende Rolle bei der Klassifizierung der Attribute spielen. So vertrete ich die Auffassung, dass die Besonderheiten der Realisierungsformen der Substantivkomplemente gerade für die Eigenartigkeit der Substantivvalenz sprechen.
} 
wie der Brief oder die These, deutlich gegen die Kritik von Helbig (zur Etymologie der Beispiele vgl. z. B. DHW).

Sommerfeldt / Schreiber (1977: 9ff.) vertreten im Wörterbuch zur Valenz und Distribution der Substantive, dem ersten deutschen Substantivvalenzwörterbuch, die Auffassung, dass die Valenz nur ausgehend von der Bedeutung des Valenzträgers betrachtet werden kann, dementsprechend schreiben sie der semantischen Seite eine primäre und der formalen eine sekundäre Rolle zu. Da sie die Bedeutung als Grundlage der Valenz auffassen, gehen sie davon aus, dass Valenz generell den Autosemantika zugeschrieben werden kann, woraus auch folgt, dass sie auch beim Substantiv anzunehmen ist. In Bezug auf die Substantivvalenz weisen sie aber auch darauf hin, ,[d]aß es bei dieser Wortart eine Reihe von Besonderheiten gibt“ (Sommerfeldt/Schreiber 1977: 11), die sie an späterer Stelle in Bezug auf die Leerstellenbesetzung und der Stellungsregularitäten der Attribute auch detaillierter erörtern. Entsprechend ihres semantisch fundierten Valenzbegriffs fassen sie auf Satzebene nicht das Vollverb, sondern das Prädikat als Valenzträger, da in nominalen Prädikaten die Bedeutung nicht von der Kopula, sondern vom nominalen (d. h. adjektivischen oder substantivischen) Teil des Prädikats getragen wird (Sommerfeldt / Schreiber 1977: 10).

Im Vorwort des Wörterbuches erklären sie, dass sie sich auf die Beschreibung von Substantiven fokussieren, „die Eigenschaften und Relationen bezeichnen, also deverbale und deadjektivische Wörter“ (Sommerfeldt / Schreiber 1977: 7). Diese Fokussierung wird darauf folgend dadurch begründet, dass sie in erster Linie „solche Substantive“ in ihren Valenzeigenschaften charakterisieren wollen, „,bei denen man von einer logisch-semantischen und einer klaren syntaktischen Valenz sprechen kann“ (Sommerfeldt / Schreiber 1977: 14). Daraus wird ersichtlich, dass sie solche Valenzmerkmale primär bei abgeleiteten Substantiven voraussetzen. Valenzbedingte Dependentien eines valenten Substantivs nennen sie Aktanten, während sie für valenzunabhängige Dependentien eines Substantivs den Terminus freie Attribute verwenden (vgl. Sommerfeldt / Schreiber 1977: 36). Bezüglich der Untersuchung der Realisierung der Substantivaktanten ist folgende Feststellung besonders von Belang:

Wir wenden uns, wenn wir die Valenzsphäre des Substantivs im engen Sinne betrachten der Substantivgruppe zu. Das schließt nicht aus, daß auch andere Satzglieder (Subjekt, Objekt ...) Leerstellen eines Substantivs besetzen können. Andererseits können Aktanten fehlen, wenn Elemente des Kontextes Eindeutigkeit herbeiführen. (Sommerfeldt/Schreiber 1977: 18; Auslassung von Sommerfeldt / Schreiber)

Sommerfeldt / Schreiber (1977: 31f.) befassen sich auch mit der Frage der Weglassung fakultativer Substantivaktanten. Als eine der wichtigsten Bedingungen solcher Weglassungen 
sehen sie den Fall, in dem die betreffenden Aktanten durch den Kontext deutlich gemacht werden. Dabei bringen sie auch Beispiele dafür, wenn ein Aktant eines valenten Substantivs außerhalb der jeweiligen Nominalphrase und zwar durch ein Element realisiert wird, das morphosyntaktisch gesehen gar nicht vom valenten Substantiv bestimmt wird. Dabei könne dieses Element innerhalb des betreffenden Satzes, aber auch in einem vorangehenden oder nachfolgenden Satz vorkommen. ${ }^{31}$ So sehen sie im Satz Das sozialistische Lager trägt zur Festigung des Friedens (...) bei. (Sommerfeldt/Schreiber 1977: 32, Auslassung von Sommerfeldt / Schreiber) im Subjekt des Satzes (das sozialistische Lager) einen Aktanten des Substantivs Festigung, der jedoch dependentiell nicht als Regens von das sozialistische Lager betrachtet werden kann. Die Klassifizierung solcher formal nicht vom Valenzträger bestimmten Elemente als Aktanten des Valenzträgers ist in der oben thematisierten Auffassung von Sommerfeldt / Schreiber (1977) begründet, wonach Valenz primär semantisch zu interpretieren ist.

Im Zusammenhang mit der Eliminierung fakultativer Substantivaktanten heben sie ein wichtiges Merkmal hervor, das valente Substantive mit valenten Verben bzw. Adjektiven gemeinsam haben: Es gibt „Fälle, in denen nicht jede Leerstelle durch ein spezielles sprachliches Mittel besetzt wird“ (Sommerfeldt/Schreiber 1977: 32). So können bei bestimmten Substantiven als Valenzträger Substantive im Plural mehrere Argumente durch eine Aktantenrealisierung zum Ausdruck bringen. Eines der Beispiele, die Sommerfeldt / Schreiber (1977: 32f.) dafür anführen, ist das Adjektiv ähnlich und das daraus abgeleitete Substantiv Ähnlichkeit. So werden in den Beispielen ${ }^{32}$ Die beiden Familienmitglieder sind sich sehr ähnlich. bzw. die Ähnlichkeit der Familienmitglieder oder ihre Ähnlichkeit in den markierten pluralischen Aktanten zwei Aktanten vereinigt, die in den folgenden Konstruktionen jeweils einzeln realisiert werden: Der_ Sohn $\underline{\text { S }}$ ist dem Vater sehr

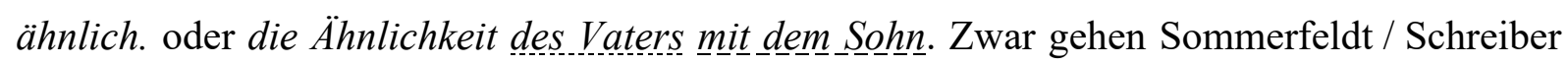
(1977: 33) nicht darauf ein, aber ich bin der Meinung, dass die Nominalphrase die Ähnlichkeit zwischen Vater und Sohn, die zusammen mit den weiteren Beispielen für Ähnlichkeit aufgezählt wird, etwas anders bewertet werden sollte als die obigen, mit zwei Aktantenrealisierungen oder mit einem pluralischen Substantiv als Aktanten, der für zwei Argumente steht. In dieser

\footnotetext{
${ }^{31}$ An diesem Punkt ist - meines Erachtens - eine wichtige Parallele zwischen der Annahme solcher Elemente und Hölzners (2007: v. a. 165ff.) transphrastischen Argumentrealisierungen (durch ein „Partnerwort im Satz“ oder in Form einer Argumentrealisierung im weiteren Kontext) zu entdecken (zu Hölzners (2007) multidimensionalem Substantivvalenzkonzept vgl. unten in Kap. 2.5.2).

${ }^{32}$ Die Beispiele wurden Sommerfeldt / Schreiber (1977: 32f.) entnommen, die Hervorhebungen stammen von mir (Á. S.-T.).
} 
Nominalphrase werden nämlich in dem Präpositionalattribut, das - formal gesehen - einen Aktanten darstellt, zwei Argumente durch zwei, von der Präposition zwischen abhängige koordinierte, jeweils singularische Substantive realisiert.

Sommerfeldt / Schreiber (1977: 19ff.) betrachten einzeln die Attributarten ${ }^{33}$, die in den von ihnen untersuchten Substantivklassen Aktanten realisieren können. Dabei bin ich zwar damit einverstanden, dass all die aufgezählten Realisierungsformen (nämlich Substantive im Genitiv, präpositionale Substantive, Possessivpronomina, relative Adjektive, Infinitivgruppen und Nebensätze) tatsächlich potenzielle Realisierungen von Aktanten darstellen, ich finde aber mehrere der Beispiele von Sommerfeldt / Schreiber problematisch. So rechnen sie - um nur ein entsprechendes Beispiel zu erwähnen - die unterstrichenen Possessiva in das Buch von meinem besten Freund und das Geburtshaus von Schiller genauso $\mathrm{zu}$ den Beispielen für Präpositionalattribute, die Aktanten darstellen sollen, wie z. B. die Präpositionalattribute in die

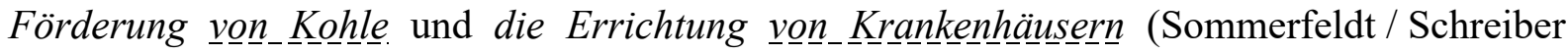
1977: 21; meine Hervorhebungen, Á. S.-T.). In meinen Augen sind in den obigen Beispielen die beiden ersten Präpositionalattribute mit von keine Aktanten, da sie keine Argumente der jeweiligen Bezugssubstantive zum Ausdruck bringen, während die beiden letzteren tatsächlich Leerstellen der Kopfnomina besetzen und somit als Aktanten zu bezeichnen sind.

Unter „speziellen Fragen der Valenz“ lenken Sommerfeldt / Schreiber (1977: 28ff.) u. a. darauf die Aufmerksamkeit, dass im Falle von polysemen Wörtern zu den einzelnen Bedeutungsvarianten eventuell unterschiedliche Valenzstrukturen gehören können. Ferner befassen sie sich mit der Frage des Verhältnisses zwischen der Valenz deverbaler und deadjektivischer, d. h. abgeleiteter Substantive und der Valenz ihrer Basiswörter. In diesem Zusammenhang halten sie die Transformation der zum jeweiligen abgeleiteten substantivischen Valenzträger gehörenden Nominalgruppe in einen Aussagekernsatz mit dem entsprechenden Basiswort, die einerseits dazu dienen soll, die Funktion der einzelnen Attribute zu verdeutlichen, ${ }^{34}$ andererseits sollen dadurch Ähnlichkeiten und Unterschiede der Aktantenrealisierungen des substantivischen Valenzträgers und seines Basiswortes leichter zu erkennen sein.

Bei der Beschreibung der Stellungsregularitäten der Attribute sehen Sommerfeldt / Schreiber (1977: 34ff.) die Nominalphrase durch das Kopfnomen, das die

\footnotetext{
${ }^{33}$ In Anlehnung an Teubert (2003) würde ich sie als Attributsklassen bezeichnen.

${ }^{34}$ Dies verdeutlichen Sommerfeldt / Schreiber (1977: 29) am Beispiel der Nominalgruppe die Beschreibung des Mannes. Lässt sich die Nominalgruppe in den Kernsatz Man beschreibt den Mann. transformieren, bezeichnet der Genitivaktant des Mannes das Ziel von Beschreibung. Dagegen bringt er den Täter zum Ausdruck, wenn die Nominalgruppe in den Kernsatz Der Mann beschreibt ... überführt werden kann.
} 
„Mitte“ darstellt, in ein Vor- und ein Nachfeld geteilt. Sie charakterisieren sowohl im Vorfeld als auch im Nachfeld die „,normale Reihenfolge der Attribute“, bei deren Bestimmung - ihnen zufolge - Wortart und Wortform entscheidend sind. Außerdem weisen sie auf einen direkten Zusammenhang zwischen der Enge der semantischen Bindung eines Attributs ans Kopfnomen und dessen (nahe) Stellung zu demselben innerhalb der Nominalphrase hin. Schließlich erklären sie, dass die Verlegung von Attributen von ihrer normalen Position der kommunikativen Funktion ihrer Hervorhebung dient.

Wolf (1982) fokussiert sich in seinem Report Probleme einer Valenzgrammatik des Deutschen überwiegend auf die Verbvalenz bzw. auch auf sprach- und grammatiktheoretischen Fragen der Valenz, aber er widmet der Beschreibung der Substantiv- und Adjektivvalenz ein eigenes Kapitel (vgl. Wolf 1982: 54ff.). Hier konzentriere ich mich - entsprechend des Gegenstandes des vorliegenden Kapitels - in erster Linie auf die Darstellung seiner Thesen bezüglich der Substantivvalenz, der aber die kurze Erörterung seiner allgemeinen Valenzdeutung vorausgeschickt werden muss.

Bei der Grundlegung seiner Valenzauffassung plädiert Wolf (1982: 11ff.) dafür, dass Semantik und Logik nicht miteinander vermischt werden dürfen. Ferner vertritt er die Auffassung, dass die Logik ,,als Hilfswissenschaft“ unentbehrlich für die Syntax ist, wobei sie ,allerdings den Bedürfnissen und speziellen Fragestellungen der Sprachwissenschaft angepasst werden [muss]“ (Wolf 1982: 17). Die Termini Prädikat und Argument rechnet er der Logik zu (vgl. Wolf 1982: 14) und hebt in Bezug auf die Argumente etwas sehr Wichtiges hervor:

Zahl und Klasse der Argumente sind von der (logischen) Semantik der Prädikate abhängig, woraus hervorgeht, dass die 'Argumente' keine kategorialen, sondern relationale Einheiten sind. Vergleichbar den Satzgliedern, können wir nur von einem 'Argument zu', nicht von einem 'Argument an sich's sprechen. (Wolf 1982: 13f.)

Was die Beschreibung der Propositionsbildung bzw. des Prozesses der Spracherzeugung betrifft, schlägt er ein Vier-Ebenen-Modell vor (vgl. Abb. 1).

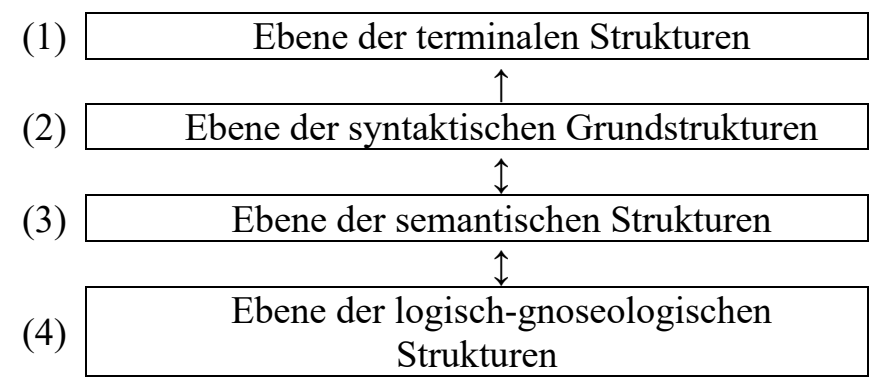

Abb. 1: Wolfs (1982: 18) Modell von Zuordnungsbeziehungen 
Zwar sind in Wolfs Modell Logik und Semantik deutlich voneinander getrennt (vgl. Ebene 3 und 4), aber zugleich sind sie auch gegenseitig voneinander abhängig. Ebene 2) kann ,als Ebene der hierarchischen Strukturen“ (Wolf 1982: 23) mit Tesnières (1980) strukturaler Ordnung gleichgesetzt werden, wobei Wolf auf dieser Ebene nicht nur mit Dependenz- sondern auch mit Konstituenzbeziehungen rechnet. Außerdem muss hervorgehoben werden, dass in Wolfs (1982: 25) Modell „die Syntax semantisch fundiert ist“. Zu Ebene 1) sollen Transformationen führen, wodurch die konkrete Äußerung herbeigeführt wird.

Wolf (1982: 28ff.) schreibt der Valenz in seinem Modell eine sehr wichtige Rolle zu, und betrachtet sie als eine Art Rektion, die semantisch bedingt und auf drei Ebenen zu beschreiben ist. Dementsprechend unterscheidet er die Ebenen der syntaktischen, der semantischen und der logischen Valenz. In Bezug auf die Verb- bzw. nominale Valenz, worunter Wolf (1982: 58ff.) die Substantiv- und Adjektivvalenz versteht, hält er es für wichtig eine Unterscheidung zu treffen und zwar in dem Sinne, dass ihm zufolge die Verbvalenz satz-, während die nominale Valenz satzgliedkonstituierend sei. Diese Differenzierung will er auch terminologisch zum Ausdruck bringen, und entscheidet sich dafür, valenzbedingte und valenzunabhängige Dependentien im Falle von verbalen bzw. nominalen Valenzträgern unterschiedlich zu bezeichnen. Demgemäß verwendet er bei der Beschreibung der Verbvalenz die Termini Ergänzungen vs. Angaben, ${ }^{35}$ während er im nominalen Bereich das Begriffspaar Adjunkte vs. freie Attribute gebraucht. Ferner erörtert er, dass diese Art Unterscheidung in der Darstellung

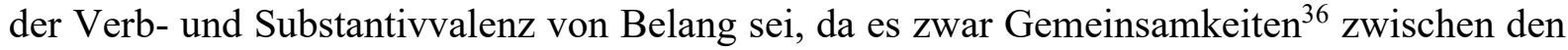
beiden Phänomenen gibt, dennoch aber die Unterschiede nicht zu übersehen seien. Dies zeige sich einerseits darin, welchen Einfluss sie -ihm zufolge - auf die Satzkonstitution haben und andererseits darin, dass ,[...] die Valenz des Substantivs einen größeren Spielraum bei der morphologischen Realisierung der Adjunkte [erlaubt]“ (Wolf 1982: 60).

Wolf (1982: 56) geht davon aus, dass Substantive auf Grund ihrer Valenzeigenschaften in drei Gruppen einzuteilen sind: „(1) Avalente Substantive ('Konkreta'), (2) Valente nicht-

\footnotetext{
${ }^{35}$ Ergänzungen charakterisiert Wolf (1982: 38ff.) als dependentiell mit dem Verb verbundene, unmittelbare Satelliten des Verbs, denen auf Ebene 4 generell Argumente zugeordnet werden können. Dagegen hebt er bezüglich der Angaben hervor, dass sie auf Ebene 4 nicht mit Argumenten verbunden werden können, sondern als eigene Propositionen zu betrachten sind, die ,weiter[e] unmittelbar[e] Konstituenten des Satzes“ (Wolf 1982: 48) darstellten. Da aber Ergänzungen und Angaben in der Form ihrer sprachlichen Realisierungen (d. h. morphologisch-syntaktisch) viele Gemeinsamkeiten haben, ist ihre Unterscheidung - auch Wolf zufolge - oft problematisch.

${ }^{36}$ Als eine wichtige Gemeinsamkeit zwischen der Verbvalenz und der nominalen Valenz (also nicht nur der Substantiv-, sondern auch der Adjektivvalenz) hebt Wolf (1982: 65) hervor, dass alle Valenzträger auf der Ebene der logisch-gnoseologischen Strukturen „ein Prädikat [repräsentieren]“.
} 
abgeleitete Substantive ('Relationalia') $[\mathrm{sic}]^{37}$ (3) Valente Ableitungen ('Abstrakta', 'nomina agentis $\left.{ }^{6}\right)^{\text {“ }}{ }^{38}$ Dies bedeutet also, dass er Valenz nicht nur bei abgeleiteten, sondern auch bei nicht-abgeleiteten Substantiven annimmt, wobei er die Auffassung vertritt, dass die Valenz abgeleiteter Substantive die Valenzverhältnisse ihrer Basiswörter widerspiegelt, wodurch die Transformation der einzelnen Nominalgruppen in Sätze mit dem entsprechenden Basiswort als Prädikat dabei behilflich sein kann, Adjunkte und freie Attribute voneinander zu trennen. Die Verwendbarkeit dieser Methode zur Unterscheidung von valenzabhängigen und valenzunabhängigen Erweiterungen bewertet er bei nicht-abgeleiteten Substantiven jedoch grundlegend anders:

Hier ist es schwierig, wenn nicht unmöglich, adäquate Paraphrasen zu finden, doch erscheint dies gar nicht notwenig [sic], weil in der Regel die Weglaßprobe in isolierten Sätzen ausreichen dürfte: *Der Redner bekräftigte den guten Zweck. *Der Prediger sprach über den Sinn. *Wir kommen zum Kern. *Ihr mißfällt die Art. In allen diesen Sätzen sind die Valenzstellen des verbalen Kerns besetzt, trotzdem sind die Sätze nicht grammatisch. (Wolf 1982: 55f.; Hervorhebungen von Wolf)

Diese Sichtweise finde ich einerseits besonders interessant, andererseits aber auch problematisch. Interessant ist sie, weil Wolf im Falle der nicht-abgeleiteten Substantive von sinn- und syntaktisch notwendigen Komplementen / Adjunkten auszugehen scheint, wobei worauf in Kap. 2.5.3 noch detaillierter eingehen werde - die Existenz von obligatorischen Substantivkomplementen in der Fachliteratur sehr umstritten ist und nur von relativ wenigen Autoren für möglich gehalten wird. Ferner bin ich der Meinung, dass Wolfs als Einschränkung interpretierbare Vorsicht, die seiner Formulierung bezüglich der Eignung dieses Tests zur Unterscheidung von Adjunkten und freien Attributen zu entnehmen ist, mehr als begründet ist. Wolf (1982: 56; meine Hervorhebungen, Á. S.-T.] bekundet demnach die Ansicht, dass ,[...] die Weglaßprobe in isolierten Sätzen [in der Regel] ausreichen dürfte“. Zum einen finde ich die obigen Beispiele, die - Wolf zufolge - alle gleichermaßen ungrammatisch sein sollen, unterschiedlich akzeptabel. Zum anderen gibt es - meines Erachtens - zahlreiche Beispiele für

\footnotetext{
${ }^{37}$ An der markierten Stelle fehlt ein Komma in Wolfs (1982: 56) Aufzählung.

${ }^{38}$ Es sei hier angemerkt, dass Wolf sich bei der weiteren Charakterisierung dieser Gruppen an einer Stelle vertippt zu haben scheint:

(2) und (3) sind kondensierte Propositionen bzw. Prädikate davon, sind deshalb, wenn sie isoliert stehen, ungesättigt und eröffnen deshalb Leerstellen. Wenn Konkreta attribuiert werden oder wenn zu Substantiven der Klassen (2) oder (3) valenzabhängige Attribute hinzutreten, dann können wir diese Satzgliedteile als eingebetette Proposition ansehen: Das grüne Haus gefällt mir $\leftarrow$ Das Haus ist grün. Es gefällt mir. Die hinterlistige Ermordung Cäsars erregte Aufsehen $\leftarrow$ Die Ermordung Cäsars war hinterlistig. Sie erregte Aufsehen. (Wolf 1982: 56f.; Fettdruck von mir, Á. S.-T.)

Wie übrigens auch Wolfs Beispielen zu entnehmen ist, sind es - im Gegensatz zu dem, was im obigen Zitat steht - gerade valenzunabhängige Attribute, die als eingebettete Propositionen betrachtet werden können (vgl. auch z. B. Wolf 1982: 58 ).
} 
valente nicht-abgeleiteten Substantive deren Komplemente auch in isolierten Sätzen ohne Weiteres weggelassen werden können, wie z. B. Über die Ursache wissen sie noch nichts. ${ }^{39}$

In der Deutschen Grammatik von Helbig / Buscha (2018) wird von einem Valenzbegriff ausgegangen, in dem die Fähigkeit der Valenz in erster Linie dem Verb zugeschrieben wird, wobei jedoch eine Hierarchie von Valenzbeziehungen angenommen wird, „,bei der die Verben als primäre, die anderen Wortklassen (vor allem das prädikative Adjektiv, aber auch vereinzelt das prädikative Substantiv) als sekundäre Valenzträger angesehen werden“ (Helbig / Buscha 2018: 516; Hervorhebungen von Helbig / Buscha). Obwohl demzufolge sowohl das Adjektiv als auch das Substantiv sekundäre Valenzträger sind, stellen Helbig / Buscha (2018: 528f.) die syntaktische Valenz von Substantiven in vielen Punkten den Valenzeigenschaften von Verben und Adjektiven gleichermaßen gegenüber. Generell gehen sie davon aus, dass „zumeist“ deverbale und deadjektivische Substantive über Valenz verfügen, deren Valenzeigenschaften „,in der Regel“ mit denen ihrer Basiswörter übereinstimmen. Wie diesen Merkmalen zu entnehmen ist, betonen Helbig / Buscha die sekundäre, abgeleitete Art der Substantivvalenz, auch wenn sie - beiläufig - einräumen, dass diese Charakterisierung nicht ohne Einschränkungen auf jedes valente Substantiv gilt. Bezüglich der Aktanten (d. i. Komplemente) von Substantiven geben sie ebenfalls eine etwas vage Beschreibung, indem sie feststellen, dass sie ,,in der Regel“ fakultativ sind (zur Problematik der Obligatheit der Substantivergänzungen vgl. unten Kap. 2.5.3).

Helbig / Buscha (2018: 529f.) unterscheiden auf Grund der Aktantenzahl der Substantive vier Klassen von Substantivgruppen: Substantive ohne Aktanten (z. B. das Schneien), ${ }^{40}$ die mit einem Aktanten (z. B. seine Reise nach Paris), die mit zwei Aktanten (z. B. die Reise des Wissenschaftlers nach Brasilien) und die mit drei Aktanten (z. B. die Überreichung der Zeugnisse an die Abiturienten durch den Schulleiter). Ich bin der Meinung, dass diese Klassifikation von Helbig / Buscha gar nicht eindeutig und an mehreren Punkten problematisch ist. So wird aus der obigen Aufzählung klar, dass sie Reise einmal unter den Substantiven angeben, die einen Aktanten haben, dann erscheint es aber auch unter den Substantiven mit

\footnotetext{
${ }^{39}$ Für weitere Beispiele vgl. v. a. im Anhang unter Punkt 8.5 und 8.6 die Belege mit nicht-abgeleiteten valenten Substantiven, in denen die Substantive komplementlos realisiert sind.

${ }^{40}$ Alle Beispiele, die im Zusammenhang mit der Valenzauffassung von Helbig / Buscha angegeben werden, werden Helbig / Buscha (2018: 529ff.) entnommen, da sie zur kritischen Darstellung ihrer Thesen grundlegend wichtig sind.
} 
zwei Aktanten, wobei in beiden Beispielen zwar dieselben Argumente ${ }^{41}$, aber syntaktischmorphologisch teilweise anders realisiert ${ }^{42}$ erscheinen.

Durch die Einbettung dieser Substantivgruppen in Sätze mit einem Kopulaverb entstehen Helbig/Buscha (2018: 530ff.) zufolge Satzmodelle, in denen die valenten Substantive zusammen mit einem Kopulaverb das Prädikativum bilden, wobei der strukturell gar nicht in die Nominalphrase gehörige Subjektsnominativ auch als ein Aktant des prädikativen Substantivs interpretiert wird. Zwar gibt es in der Forschung nur wenige Vertreter für eine Deutung der Substantivvalenz, derzufolge Argumente des Substantivs nicht nur innerhalb der betreffenden Nominalphrase oder in Form von Nebensätzen realisiert werden können, dennoch finde ich eine solche These absolut vertretbar (vgl. z. B. Hölzner 2007; Sántáné-Túri 2014 bzw. unten Kap. 2.6).

Die Beispiele von Helbig / Buscha sind - meines Erachtens - dennoch sehr problematisch und zeugen von einer verworrenen Deutung der Substantivvalenz. Um dies zu demonstrieren, stehe hier nur ein Beispielsatz von Helbig / Buscha (2018: 531): Das wichtigste Ereignis im Jahre 1945 war die Befreiung Europas vom Hitlerfaschismus durch die Anti-Hitler-Koalition. In diesem Satz stelle Befreiung einen Valenzträger mit vier Aktanten dar, wobei der erste Aktant durch den Subjektsnominativ, der zweite durch das Genitivattribut, der dritte und vierte durch je ein Präpositionalattribut realisiert seien. Ich bin der Meinung, dass es verkehrt ist, die Konstituente das wichtigste Ereignis zu den Aktanten des Substantivs zu rechnen. Ich finde die Funktion dieser Konstituente als erster Aktant unerklärbar, da es gar kein Argument des Valenzträgers realisiert. Stattdessen bin ich der Meinung, dass in diesem Beispielsatz drei Valenzen von Befreiung realisiert sind, von denen durch die Anti-Hitler-Koalition als erster Aktant, Europas als zweiter Aktant und vom Hitlerfaschismus als dritter Aktant interpretiert werden können.

Ähnlich wie Helbig / Buscha (2018) beharrt Eisenberg (2006), trotz einiger von ihm selbst in Erwägung gezogenen oder zumindest erwähnten Gegenargumenten, auf die sekundäre, abgeleitete Art der Substantivvalenz. In seinem Grundriss der deutschen Grammatik, genauer in dessen zweitem Band mit dem Untertitel Der Satz, stellt er klar, dass er kein multidimensionales oder Mehrebenenmodell der Valenz verwendet und Valenz mit syntaktischer Valenz gleichsetzt, wobei selbst dieser Begriff „sehr komplex“ ist (Eisenberg

\footnotetext{
${ }^{41}$ Es sind das Agens und ein Direktivum.

${ }^{42}$ In dem Beispiel seine Reise nach Paris stehen die Aktanten in Form eines Possessivpronomens und eines Präpositionalattributs, während in dem Beispiel die Reise des Wissenschaftlers nach Brasilien außer dem Präpositional- ein Genitivattribut vorkommt.
} 
2006: 58). Er verwendet die Termini Ergänzungen und Komplemente als Synonyme. Bei der Beschreibung der Verbvalenz erklärt er seine Ansicht, wonach ein Komplement „eine bestimmte sprachliche Form in einer bestimmten syntaktischen Funktion“ darstellt (Eisenberg 2006: 61) und die Komplementstruktur dementsprechend die möglichen Ergänzungen eines Valenzträgers samt ihren sprachlichen Formen und syntaktischen Funktionen abzubilden hat. In der Argumentstruktur wird dies - Eisenberg (2006: 75) zufolge - durch die Angabe der in den einzelnen Komplementpositionen realisierten semantischen Rollen, ${ }^{43}$ d. h. der Argumente ergänzt. Eisenberg (2006: 261ff.) thematisiert die Frage der Substantivvalenz im Zusammenhang mit der Beschreibung von Präpositionalattributen, und zwar aus dem Grunde, dass er der Ansicht ist, dass Genitiv- oder Präpositionalattribute Anlass dafür geben könnten, bei Substantiven genauso über Valenz zu sprechen wie bei Verben und Adjektiven. Schrittweise erklärt er jedoch seine entgegengesetzte Auffassung und sucht diese schließlich durch vier Argumente zu unterstützen:

Substantive haben nicht Valenz im selben Sinne wie Verben und Adjektive, weil (1) Substantive nicht syntaktisch nach der Stellenzahl subkategorisierbar sind, (2) eine Unterscheidung zwischen Komplementen und Adjunkten nicht möglich ist, (3) eine Unterscheidung zwischen fakultativen und obligatorischen Attributen nicht möglich ist und (4) die NGr [Nominalgruppe; Á. S.-T.] nicht wie der Satz durch Argumentpositionen des Kerns strukturiert ist. (Eisenberg 2006: 268)

Bei dieser Beweisführung muss vor Augen gehalten werden, dass Eisenberg - wie ich oben schon angesprochen habe - Valenz als syntaktische Valenz versteht. Ferner hängt die Beurteilung Eisenbergs obiger Argumente gegen eine selbstständige oder gleichberechtigte Substantivvalenz - meines Erachtens - teilweise deutlich davon ab, ob man beim Substantiv obligatorische Komplemente für möglich hält. Da ich der Frage der Obligatorik von Substantivkomplementen unten ein eigenes Subkapitel (vgl. Kap. 2.5.3) widme, sei hier vorerst nur festgestellt, dass es bezüglich dieser Problematik unterschiedliche Ansätze gibt. Entsprechend der kontroversen Beurteilung dieses Themas lasse ich hier die Frage meinerseits noch unbeantwortet und beachte bei der Analyse von Eisenbergs Argumentation beide Möglichkeiten.

Bei der Annahme obligatorischer Substantivkomplemente kann der dritte Beweisgrund gleich widerlegt werden und auch die weiteren drei geraten deutlich ins Wanken. Wenn man nämlich von der Existenz obligatorischer Substantivkomplemente ausgeht, müsste zumindest die Gruppe der Substantive mit diesen Komplementen abgegrenzt werden, was schon zu einer, wenn auch bescheidenen, Subkategorisierung nach Stellenzahl führen würde. Durch ihre

\footnotetext{
${ }^{43}$ Zur Frage, welche semantischen Rollen er in seiner Grammatik unterscheidet vgl. Eisenberg (2006: 76).
} 
Obligatorik und der damit verbundenen semantischen und zugleich formalen Eigenschaften sind die betreffenden Erweiterungen eindeutig als Komplemente identifizierbar und ferner ist die Nominalphrase zumindest durch diese Argumentpositionen in ihrer Struktur (vor)bestimmt, wodurch sowohl das zweite als auch das vierte Argument von Eisenberg an Gültigkeit verlieren.

Schließt man sich dagegen den Ansätzen an, die obligatorische Substantivkomplemente abstreiten, trifft das dritte Argument von Eisenberg vollkommen zu, wobei es jedoch - meiner Meinung nach - dennoch nicht als Beweisgrund gegen eine selbstständige Substantivvalenz bewertet werden kann. Wenn man nämlich für Substantive gelten lässt, dass sie nur fakultative Komplemente haben können, sollte man bei der Beurteilung der Legitimität eines selbstständigen Substantivvalenzsystems in erster Linie die Valenzpotenz und nicht die Valenzrealisierung beachten. So können die potenziellen Valenzstrukturen der valenten Substantive dargestellt werden. Danach wäre es - im Gegensatz dazu, was in Eisenbergs erstem Argument behauptet wird - möglich, Substantive syntaktisch nach ihrer potenziellen Stellenzahl in Untergruppen einzuteilen.

Betreffend das zweite Argument von Eisenberg (2006: 266ff.) bleibt noch zu thematisieren, dass er davon ausgeht, dass Attribute weder als Komplemente noch als Adjunkte betrachtet werden können, da sie Modifikatoren sind, die als solche keine Komplementpositionen besetzen und in ihrer Zahl unbegrenzt sind. Daraus folgt für ihn auch eine Relativierung der Theorie, wonach bei abgeleiteten Substantiven über Argumentvererbung die Rede sein könnte. Da er - wie oben schon erklärt - Argumente als in bestimmten Komplementpositionen realisierte semantische Rollen interpretiert, kann es - seines Erachtens - bei den Substantiven keine Argumentvererbung, sondern lediglich Vererbung von semantischen Rollen geben. An diesem Punkt halte ich es für wichtig hervorzuheben, dass ich einen so strikt definierten Komplementbegriff, wie den von Eisenberg (2006: 61), auch im verbalen Bereich nicht problemlos vertretbar finde (vgl. z. B. das Problem der (Nicht-)Obligatorik und der formalen Diversität von Situativkomplementen bei Verben wie wohnen). Dementsprechend betrachte ich das zweite Argument als Gegenbeweis ausschließlich für eine selbstständige Substantivvalenz nicht geeignet.

Was das vierte Argument von Eisenberg betrifft, ist dem zwar im Sinne von Eisenbergs Argumentdefinition zuzustimmen, aber wie ich schon teilweise erklärt habe und wie sich unten noch aus meiner Interpretation des Terminus Argument herausstellen wird (vgl. Kap. 2.6), finde ich gerade diese Art der Argumentbestimmung fraglich. Außerdem vertrete ich die Auffassung, 
dass bei der Realisierung ihrer Valenz auch die Substantive die Strukturierung der Nominalgruppe stark beeinflussen. ${ }^{44}$

Eisenberg (2006: 263ff.) gibt bei der Thematisierung der Substantivvalenzfrage eine detaillierte Beschreibung „[z]um Verhältnis von Attributen und verbalen Argumenten“, die nicht zuletzt dem Zweck dienen sollte, Regelmäßigkeiten der Ableitbarkeit der Valenz deverbaler Substantive zu zeigen. Dementsprechend hebt Eisenberg (2006: 265) z. B. im Falle des Präpositionalattributs hervor, dass bei nicht-lexikalisierten deverbalen Substantiven in überwiegendem Anteil die „direkte Übertragung von Präpositionalobjekten auf Attribute“ zu beobachten sei. Es wird jedoch bei jeder Attributgruppe zugleich klar, dass Idiosynkrasien auch bei abgeleiteten Substantiven häufig vorkommen. Diesbezüglich räumt Eisenberg (2006: 265) ein, dass zwar Nominalisierungen als Derivate sowohl semantisch als auch syntaktisch mit ihren Basiswörtern verwandt sind, aber zugleich „Substantive in eigenem Recht [sind] und [...] sich jederzeit mehr oder weniger weit von der Basis entfernen [können]“. Ferner weist er darauf hin, dass auch Substantive, die - wenigstens synchron betrachtet - als nicht-abgeleitet oder idiomatisiert bezeichnet werden können, „lexikalische Rektionseigenschaften“ aufweisen können. Dies alles zeugt - in meinen Augen - von der Relevanz der Betrachtung der Substantivvalenz als ein System sui generis.

Anknüpfend ans Phänomen der Präpositionalattribute, die den eigentlichen Ausgangspunkt von Eisenbergs (2006) Überlegungen zur Substantivvalenz bedeuten, will ich kurz auf einen Beitrag von Schierholz (2004) eingehen, den ich für meine Arbeit aus mehrerlei Sicht relevant und wichtig finde. Ich bin mit Schierholz (2004: 80) in der Hinsicht absolut einverstanden, dass er die Bedeutung und Notwendigkeit der korpusbasierten Untersuchung von sprachlichen Erscheinungen, darunter auch von Valenzerscheinungen betont. Die Feststellungen im Beitrag bezüglich sog. Rektionssubstantive basieren dementsprechend auf der Auswertung von Korpusdaten. Dabei ist mit Rektionssubstantiv ein Substantiv gemeint, „das eine oder mehrere Präpositionen regieren kann“ (Schierholz 2004: 82).

In die Untersuchung wurden sowohl abgeleitete als auch nicht-abgeleitete Rektionssubstantive miteinbezogen, wobei sich der Anteil der nicht-abgeleiteten Substantive mit $55 \%$ als „relativ hoch“ (Schierholz 2004: 88) erwiesen hat. Außerdem finde ich die folgende Bewertung von Schierholz (2004: 91; meine Hervorhebung Á. S.-T.) in Bezug auf Präpositionalattribute von deverbalen und deadjektivischen Rektionssubstantiven in dem untersuchten Korpus bedeutend: „Gemessen an der Gesamtzahl der Rektionssubstantive

\footnotetext{
${ }^{44}$ Es hängt nämlich von der potenziellen Valenzstruktur des jeweiligen Substantivs ab, welche und wie viele Komplemente mit ihm zusammen vorkommen können.
} 


\section{kommt die Übernahme der von den Basislexemen regierten Präpositionen nicht oft vor.“45}

Schließlich kommt Schierholz (2004:94) zu der Konklusion, dass die Selektion der Präpositionen von Rektionssubstantiven idiosynkratisch erfolgt und auch im Falle von abgeleiteten Substantiven nicht mit Hilfe der Annahme von morphosyntaktischer Vererbung erklärt werden kann. All die hier dargestellten Ergebnisse von Schierholz (2004) untermauern - so wie ich das sehe - die sui-generis-Interpretation der Substantivvalenz.

Engel (2009a: 89) definiert Valenz als „subklassenspezifische Rektion“ und vertritt die Auffassung, dass sie nicht nur dem Verb, sondern auch Adjektiven, Nomina, Pronomina und Präpositionen zugesprochen werden kann. ${ }^{46}$ Betreffend die Substantivvalenz geht Engel (2009a: 287; meine Hervorhebungen Á. S.-T.) - im Unterschied zu z. B. Eisenberg (2006) oder Helbig / Buscha (2018) - davon aus, dass „,[v]iele Nomina [...], ähnlich dem Verb, eine eigene Valenz [haben], die die Nomenergänzungen festlegt“", wobei er - ähnlich wie Teubert (1979 und 2003) alle Nomenergänzungen als fakultativ bezeichnet. In den Beschreibungen der verschiedenen Ergänzungsarten kommen in seiner Grammatik neben deverbalen und deadjektivischen Substantiven oft zahlreiche nicht-abgeleitete Substantive als Valenzträger vor, was - meiner Meinung nach - dafür spricht, dass er die Substantivvalenz (zumindest z. T.) als ein System sui generis interpretiert.

Engel (2009a: 289ff.; 2009b: 84ff.) beschreibt die Satelliten des Nomens entsprechend ihrer Stellung in der „Linearstruktur der Nominalphrase“ in zwei große Gruppen eingeteilt, nämlich in die des linken und die des rechten Feldes. Hier geht es nicht darum, alle von ihm unterschiedenen Ergänzungs- und Angabetypen zu thematisieren (zur Frage der Klassifizierung der Substantivergänzungen bei Engel 2009a vgl. unten Kap. 2.5.4), vielmehr will ich Aspekte seiner Erörterungen ansprechen, die ich aus der Sicht des Substantivvalenzdiskurses für interessant bzw. wichtig halte. Dabei beachte ich neben der oben schon zitierten Neubearbeitung seiner Deutschen Grammatik (Engel 2009a) auch seine Syntax der deutschen Gegenwartssprache (2009b). Zur Bestimmung der Nomenergänzungen macht Engel entsprechend seiner obigen Valenzdefinition - aus dem Kriterium der Subklassenspezifik Gebrauch, wobei er aber semantische und formale Merkmale zugleich beachtet. Das ist z. B. deutlich daran zu erkennen, dass er Attribute wie die Relativsätze, Präpositiv- und

\footnotetext{
${ }^{45}$ Diese Feststellung betrachte ich auch aus der Sicht als besonders interessant, da sie im Kontrast zu Eisenbergs (2006: 265) oben angesprochener Bewertung von Präpositionalattributen von deverbalen Substantiven steht.

46 Der Genauigkeit halber muss angesprochen werden, dass Engels - meines Erachtens etwas ungenaue Formulierung (2009a: 89; meine Hervorhebung Á. S.-T.) andeutet, dass er sogar auch noch im Falle von anderen Wortarten Valenz annimmt: „Ergänzungen gibt es nicht nur zum Verb, sondern auch zu anderen Wortklassen, besonders zu Adjektiven, Nomina, Pronomina und Präpositionen.“
} 
Genitivattribute, die rein formal gesehen keine subklassenspezifischen Nomenerweiterungen sind, differenziert ansieht und verschiedene Untertypen von ihnen bestimmt, die teils Ergänzungen, teils Angaben darstellen.

In Bezug auf die Nomenergänzungen findet sich eine - meines Erachtens - wichtige Charakterisierung bei Engel (2009b: 92), indem er feststellt, dass sie ,[...] je als Argument des Nomens [fungieren]“. Ferner sei hier noch angemerkt, dass in der Syntax von Engel (2009b: 90f.) das Determinativ als einziger obligatorischer Satellit des Nomens geschildert wird, wobei jedoch Determinative in Form des Nullartikels auf der Oberfläche oft nicht augenfällig sind. Dennoch ist es interessant, dass durch diese Klassifizierung der Determinative eine Gruppe obligatorischer Angaben angenommen wird, wofür es selten Beispiele in der Fachliteratur gibt.

Nachdem ich hier an einigen konkreten Arbeiten gezeigt habe, wie die unterschiedlichen Deutungsmöglichkeiten der Substantivvalenz sich in linguistischen Abhandlungen durchsetzen, werde ich in den kommenden zwei Unterkapiteln die Frage der Unterscheidung zwischen Komplementen und Supplementen generell und darin eingebettet die Problematik der Trennung zwischen Komplementen und Supplementen beim Substantiv thematisieren.

\subsection{Komplemente und Supplemente in der allgemeinen Valenztheorie und in der Substantivvalenztheorie}

\subsubsection{Das Problem der Unterscheidung zwischen Komplementen und Supplementen I.}

Wie ich schon im einleitenden Teil von Kap. 2 erklärt habe, behandle ich in diesem Unterkapitel Arbeiten, die zwar die betreffende Problematik thematisieren, aber nicht speziell in Bezug auf Substantive. Ich werde dennoch an mehreren Punkten die dargestellten Ansätze auch im Hinblick auf ihre Verwendbarkeit im Bereich der Substantivvalenz abwägen.

Ähnlich wie in den meisten Arbeiten, die sich mit der Frage der Unterscheidung zwischen Komplementen und Supplementen beschäftigen, äußert auch Varnhorn (1986) in ihrem Beitrag Kritik an unterschiedlichen Verfahren, die zu dieser Differenzierung verwendet werden. Sie „plädiert für eine neue Definition von Valenz, die als Ergänzung allein diejenigen Satzglieder gelten läßt, die nur bei bestimmten Elementen einer Wortklasse auftreten können und / oder nur bei Wortsubklassen obligatorisch sind“ (Varnhorn 1986: 1). In dieser Definition wird die Subklassenspezifik von Ergänzungen als ihr Unterscheidungsmerkmal betrachtet, was jedoch nicht als grundlegend neu betrachtet werden kann, da dieses Kriterium z. B. auch von Engel 
zur Differenzierung von Ergänzungen und Angaben dient, dessen Ansichten auch Varnhorn (1986: 1ff.) thematisiert.

Im Zusammenhang mit Varnhorns Definition ist noch anzumerken, dass sie so formuliert ist, dass sie nicht nur den Begriff der Verbvalenz involviert, sondern einen allgemeinen Valenzbegriff andeutet. Das kann - meines Erachtens - den Eindruck erwecken, dass die Feststellungen im Artikel bei allen valenten Wörtern ihre Gültigkeit haben. Ich glaube, dass Varnhorn selbst diesen Eindruck verstärken möchte, indem sie die Ausdrücke „Valenz“, „Ergänzung“ und „Angabe“ meistens ohne weitere Spezifizierung verwendet. In Bezug auf den Betrachtungsrahmen gibt es an einer Stelle eine Einschränkung: „Im Folgenden setze ich mich besonders mit dem Mannheimer und dem Leipziger Verfahren zur Unterscheidung von (Verb-)Ergänzungen und (Verb-)Angaben auseinander“ (Varnhorn 1986: 1; meine Hervorhebungen Á.S.-T.). Im Gegensatz zum breit gefassten Valenzbegriff und dem erweckten Eindruck wird aber an mehreren Stellen in der Position des Valenzträgers - wenn er extra genannt wird - immer nur das Verb erwähnt und auch unter den Beispielen finden wir keine anderen Valenzträger.

Die Zielsetzung von Varnhorn (1986: 1; meine Hervorhebung Á. S.-T.) erscheint mir - wenn auch verständlich - etwas unrealistisch. Sie geht nämlich davon aus, dass nach der Kritik der untersuchten Verfahren durch die Neuformulierung der Valenz-, Ergänzungs- und Angabedefinitionen ein ,zweifelsfreies und praktikables Unterscheidungsverfahren“ beschreibbar wird.

Hier geht es nicht darum, Varnhorns Kritikpunkte bezüglich der Unterscheidungsverfahren detailliert wiederzugeben, vielmehr fokussiere ich auf ihre wichtigsten Feststellungen, die aus der Sicht meiner Darstellungen von größerer Bedeutung sind. Varnhorn (1986: 3f.) wendet gegen bestimmte Unterscheidungskriterien ein, dass bei ihrer Anwendung die Beurteilung häufig intuitiv ist. ${ }^{47}$ Sie bezeichnet auch solche Tests als unbefriedigend, die auf Sprecherkompetenz basieren, da sie - ihrer Meinung nach - gerade in Zweifelsfällen zu besonders widersprüchlichen Ergebnissen führen können. Diesem Argument ist - meines Erachtens - zuzustimmen, ${ }^{48}$ trotzdem darf die Rolle der Intuition bei anderen, objektiv(er) erscheinenden Tests nicht unterschätzt werden. So ist u. a. auch die Einschätzung von Weglassbarkeit, die später auch von Varnhorn (1986: 10) als Unterscheidungskriterium

\footnotetext{
${ }^{47}$ z. B. beim Eliminierungstest; vgl. Varnhorn (1986: 4)

48 So zeigt z. B. auch Storrer (2003: 777) im Zusammenhang mit Heringers Assoziationsexperiment, dass Sprecherurteile gerade bei solchen Erweiterungen sehr unterschiedlich ausfallen können, die als problematisch gelten.
} 
vorgeschlagen wird, nicht ganz unabhängig von der individuellen Entscheidung des Untersuchenden.

Varnhorn (1986:9) bezeichnet die syntaktische Valenz,,als die Eigenschaft von Wortsubklassen, nur mit einer bestimmten Zahl und Art von Gliedern kombinierbar zu sein und / oder nur mit einer bestimmten Zahl und Art von Gliedern kombiniert werden zu müssen“. In dieser Definition ist sowohl die quantitative als auch die qualitative Seite der Valenz involviert und neben den fakultativen Ergänzungen werden auch die obligatorischen miteinbezogen. Die Angaben werden als nicht subklassenspezifische Erweiterungen, die nur fakultativ sein können, beschrieben. Ferner soll hervorgehoben werden, dass Varnhorn (1986: 9) zufolge bei der Bestimmung der Kombinierbarkeit von Elementen nur syntaktische Restriktionen beachtet und semantische außer Acht gelassen werden sollen.

Als Kriterien der Unterscheidung zwischen Ergänzungen und Angaben nennt sie das Kommutationskriterium und die Weglassbarkeit der betreffenden Erweiterung (vgl. Varnhorn 1986: 10f). Bei der Kommutationsprobe wird - ähnlich wie beim Substitutionstest - ermittelt, ob das fragliche Element mit beliebigen Verben kombiniert werden kann oder nicht. Beim negativen Ausgang ${ }^{49}$ des Tests bezeichnet sie die Erweiterung als Ergänzung. Im Falle eines positiven Ergebnisses der Kommutationsprobe soll nach Varnhorns Meinung noch getestet werden, ob die Erweiterung in der Phrase weglassbar ist oder nicht. Elemente, die sich beim betreffenden Verb als nicht weglassbar erweisen, betrachtet sie ebenfalls als Ergänzungen.

Ich bin der Meinung, dass Varnhorn mit dem obigen Unterscheidungsverfahren tatsächlich eine praktikable Methode dargestellt hat, die aber - im Gegensatz zu ihrer Zielsetzung und ihrer Annahme - keinesfalls zweifelsfrei verwendet werden kann. Ich denke, dass z. B. im Bereich der Substantivvalenz erhebliche Probleme bei der Verwendung dieser Methode auftreten können. So gelten z. B. Genitivattribute, wenn semantische Faktoren nicht beachtet werden, ohne weitere Differenzierung, immer als mit beliebigen Substantiven kombinierbar. Außerdem sind sie auch stets weglassbar. Dies sollte Varnhorn zufolge so gedeutet werden, dass Genitivattribute immer Angaben sind, eine Annahme, die zwar Vertreter in der Forschung hat,

\footnotetext{
${ }^{49}$ Unter „,negativem Ausgang“ der Kommutationsprobe verstehe ich den Fall, in dem sich die Erweiterung als nicht mit „,beliebigen“ Verben kombinierbar erweist.
} 
meines Erachtens jedoch eindeutig kritikwürdig ist. ${ }^{50}$ Darüber hinaus finde ich die Beurteilung der Kommutierbarkeit und Weglassbarkeit von Konstituenten in vielen Fällen auch intuitiv. ${ }^{51}$

Ein anderes Beispiel für die Kritik an den Unterscheidungsversuchen zwischen Ergänzungen und Angaben findet sich bei Welke (1988: 21ff.), der in seiner Einführung in die Valenz- und Kasustheorie (1988) davon ausgeht, dass die Unterscheidung zwischen Ergänzungen und Angaben von Unschärfen und Widersprüchen behindert wird, die daraus resultieren, dass die inhaltlichen Kriterien, nach denen die Klassifizierung der abhängigen Elemente erfolgen sollte, nicht deutlich genug herausgearbeitet worden sind.

An dieser Stelle können nur die Endergebnisse von Welkes ausführlicher Analyse dargestellt werden. Er stellt fest, dass im Hintergrund der verschiedenen operationellen Tests, die zur Unterscheidung von Ergänzungen und Angaben - und darunter auch noch zwischen obligatorischen und fakultativen Ergänzungen - verwendet werden, drei Kriterien zu entdecken sind: Das Kriterium der Sinnnotwendigkeit, der Subklassenspezifik und der Determiniertheit. ${ }^{52}$ Er zieht die Schlussfolgerung, dass Widersprüche in der Beschreibung von Ergänzungen und Angaben dann vermieden werden können, wenn man entweder das Kriterium der Sinnnotwendigkeit oder die Kriterien der Determiniertheit und der Subklassenspezifik als primär betrachtet. Als primäres Entscheidungskriterium verwirft er aber die Sinnnotwendigkeit, weil durch ihre vorrangige Verwendung mehrere Untertypen der Ergänzungen ${ }^{53}$ zu den Angaben gerechnet werden würden. Dementsprechend bestimmt er die Determiniertheit und die Subklassenspezifik als vorrangige Merkmale, wobei er aber auch noch unter den beiden eine Rangordnung aufstellt: „Da das Kriterium der Subklassenspezifik in einigen Fällen ${ }^{54}$ nicht zutrifft, ist das entscheidende Kriterium die Determiniertheit“ (Welke 1988: 52). Auf Grund dessen sind bei ihm Angaben determinierend und nicht subklassenspezifisch, während Ergänzungen als determiniert und subklassenspezifisch gelten. Die Sinnnotwendigkeit dient bei

\footnotetext{
${ }^{50}$ Dementsprechend sollte das Genitivattribut in Empfang der Gäste, als Angabe / Supplement eingestuft werden, obwohl es - meiner Ansicht nach - als Argumentrealisierung des Substantivs und somit als Ergänzung / Komplement zu klassifizieren ist. Zur Frage des Komplement- bzw. Supplementstatus von Genitivattributen vgl. auch Kap. 2.5.2 und 2.5.3.

${ }^{51}$ Vgl. dazu z. B. Storrers (2003: 771f.) Überlegungen zum - mit der Kommutationsprobe eng verwandten Substitutionstest und zur Weglassprobe.

${ }_{52}^{52}$ Determiniertheit wird hier nicht syntaktisch, sondern im prädikatenlogischen Sinne verstanden.

${ }^{53}$ Davon wären Welke (1988: 46) zufolge ,,die fakultativen Ergänzungen im engeren Sinne“ und die „durch Modalisierung und Kontrast möglichen relativ obligatorischen Ergänzungen“ betroffen (zu den Begriffen fakultative Ergänzungen im engeren Sinne bzw. durch Modalisierung und Kontrast mögliche relativ obligatorische Ergänzungen vgl. Welke 1988: 26ff.).

${ }^{54}$ Als Beispiel für einen solchen Fall erwähnt er an einer früheren Stelle das Subjektnomen bei den Verben, das ihm zufolge - auf Grund der Subklassenspezifik als Angabe zu klassifizieren wäre (vgl. Welke 1988: 37). Zur Problematisierung der Subklassenspezifikfrage beim Subjekt - Prädikat vgl. auch Uzonyi (2004: 236f.).
} 
ihm dazu, zwischen obligatorischen ${ }^{55}$ und fakultativen Ergänzungen und auch zwischen obligatorischen und fakultativen Angaben zu unterscheiden, wodurch bei ihm eine in der Valenztheorie nicht übliche Vierteilung entsteht. Ich halte es aber für wichtig anzumerken, dass Welke (1988: 46 und 52) zwar die Kategorie der obligatorischen Angaben einführt, aber keine Beispiele dafür gibt, sondern nur so viel anmerkt, dass obligatorische Angaben sehr selten sind. ${ }^{56}$ Zur Trennung zwischen Ergänzungen und Angaben bewertet Welke also nur die Kriterien der Determiniertheit und der Subklassenspezifik als nötig. Zwar weist er an einigen Stellen darauf hin, dass nicht nur Verben, sondern auch andere Wörter Valenzträger sein können, ${ }^{57}$ aber er behandelt ausschließlich Beispiele mit Verben und er spricht auch immer nur von Verbkomplementen. ${ }^{58}$ Ich überlege hier deswegen kurz, ob das von ihm neuermittelte Kriteriensystem auch bei den Substantiven eingesetzt werden könnte.

Subklassenspezifik kann - wie z. B. Teubert (1979) gezeigt hat - auch in der Substantivvalenz angenommen werden. ${ }^{59}$ Ihre Ermittlung kann aber unter Umständen - z. B. bei Genitiverweiterungen oder Präpositionalattributen - problematisch sein bzw. verlangt eine differenzierte, nicht nur formal fokussierte, sondern auch semantisch interpretierte Subklassenspezifikdefinition. Ähnlich verhält es sich mit dem Kriterium der Determiniertheit, das in manchen Fällen auch schwierig zu handhaben sein kann. Die Bestimmung der Determiniertheit kann nicht nur beim Substantiv mit Schwierigkeiten verbunden sein, sondern sie ist es im Allgemeinen. ${ }^{60}$ Das Kriterium der Sinnnotwendigkeit kann auch im Bereich der Substantivvalenz als Unterscheidungsmerkmal verwendet werden, denn auch valente Substantive verfügen über Argumente, zu deren Ermittlung dieses Kriterium dienen kann. Es sei hier nur am Rande erwähnt, dass dieses Kriterium - trotz seiner Anwendbarkeit im substantivischen Bereich - in der Funktion, die ihm Welke (1988) zuweist, dennoch problematisch sein kann. Es dient nämlich - wie oben schon erklärt -, Welke zufolge, primär nicht der Unterscheidung zwischen Ergänzungen und Angaben, sondern der zwischen obligatorischen und fakultativen Dependentien. Es gibt jedoch - wie ich schon oben angedeutet habe bzw. unten noch ausführlicher erörtern werde (vgl. Kap. 2.4.3 bzw. 2.5.3) - gegensätzliche

\footnotetext{
${ }^{55}$ Im Zusammenhang mit den obligatorischen Ergänzungen merkt Welke (1988: 52) an, dass er - im Gegensatz zu Helbig - auch die kontextuell weglassbaren Ergänzungen zu ihnen rechnet.

${ }^{56}$ Wie ich oben, in Kap. 2.4.3 schon angesprochen habe, ist bei Engel (2009b: 90f.) ebenfalls die Idee von obligatorischen Angaben zu entdecken, der Determinative so klassifiziert.

${ }^{57}$ z. B. „Das Verb bzw. das Wort von dem die Valenz ausgeht, heißt Valenzträger“ (Welke 1988: 22; meine Hervorhebung Á. S.-T.).

${ }^{58}$ z. B. „Ergänzungen sind determinierte Verbkomplemente, Angaben sind determinierende Verbkomplemente“ (Welke 1988: 51; meine Hervorhebungen Á. S.-T.).

${ }^{59} \mathrm{vgl}$. dazu unten Kap. 2.5.2

${ }^{60}$ Auch Welke (1988: 52) weist darauf hin, dass es Fälle geben kann, in denen ,eine Entscheidung hinsichtlich der Determiniertheit schwerfällt“".
} 
Meinungen darüber, ob Substantive obligatorische Ergänzungen haben können. Wenn man davon ausgeht, dass es bei den Substantiven keine obligatorischen Ergänzungen gibt, wäre die Sinnnotwendigkeit, Welkes (1988) Unterscheidungsmodell entsprechend verwendet, im substantivischen Bereich nur eingeschränkt angebracht. Dementsprechend sollte nämlich das Kriterium der Sinnnotwendigkeit im Falle einer Ablehnung der Möglichkeit von obligatorischen Ergänzungen beim Substantiv nur bei den Attributen eingesetzt werden, die mit Hilfe der beiden anderen Kriterien schon als Angaben ermittelt worden sind. ${ }^{61}$

Einen umfassenden Überblick der verschiedenen möglichen bzw. verwendeten Verfahren zur Unterscheidung von Ergänzungen und Angaben bietet Storrer (2003), wobei sie ihre Untersuchung auf die verbale Valenz der deutschen Gegenwartssprache beschränkt (vgl. Storrer 2003: 766).

Nach der kurzen Behandlung der Tesnière'schen Theorie und der - ihres Erachtens - darin verankerten Widersprüchlichkeiten spricht Storrer (2003: 764f.) die Entwicklungen der Valenztheorie an, die mit der Diskussion über die Probleme des Modells von Tesnière verbunden sind und die zur Ausbildung immer differenzierterer Modelle geführt haben. Sie teilt diese Modelle in zwei Klassen, nämlich in Mehrebenenmodelle und in mehrdimensionale Modelle ein, wobei Mehrebenenmodelle als solche $\mathrm{zu}$ charakterisieren sind, in denen die Oberflächenerscheinungen der Valenz aus „tieferliegenden“ Strukturebenen erklärt werden können. Währenddessen werden in mehrdimensionalen Modellen mehrere verschiedene Valenzrelationen angenommen, die gleichzeitig existieren, voneinander unabhängig aufdeckbar sind, aber unter denen unterschiedliche Implikationsbeziehungen bestehen.

Auf Grund der zur Unterscheidung von Ergänzungen und Angaben verwendeten Daten teilt Storrer (2003: 768) diese Differenzierungsmethoden in Kompetenztests auf der einen und in korpusorientierte Verfahren auf der anderen Seite ein. Während in den Kompetenztests der Untersuchende „selbstkonstruierte Beispiele“ betrachtet, werden in den korpusorientierten Verfahren aus authentischen Sprachmaterialien zusammengestellte Korpora analysiert. Es gibt sowohl unter den Kompetenztests als auch unter den korpusorientierten Verfahren gleichfalls einfache und auch komplexe Verfahren. In der Forschung wurden zuerst die einfachen Unterscheidungsverfahren entwickelt und allein oder parallel verwendet, später kam es zur Entwicklung von komplexen Verfahren, bei denen einfache Tests nacheinander verwendet als Filter funktionieren.

\footnotetext{
${ }^{61}$ Es sei an dieser Stelle dahingestellt, dass mir die Kategorie von obligatorischen Angaben schwer nachvollziehbar erscheint.
} 
Storrer (2003: 768; meine Hervorhebung Á. S.-T.) stellt im Zusammenhang mit dem Fragenkomplex der Differenzierung zwischen Ergänzungen und Angaben etwas - meiner Ansicht nach - sehr Wichtiges fest: „Wenn es zur E-A-Abgrenzung [Ergänzung-AngabeAbgrenzung; Á. S.-T.] bislang überhaupt eine einheitliche Forschungsmeinung gibt, so kann als deren kleinster gemeinsamer Nenner die Annahme gelten, dass die Valenz ein syntaktischer Reflex semantisch-kognitiver Strukturen ist"“.

Diese Voraussetzung führt dazu, dass der Argumenthaftigkeit eine zentrale Rolle bei der Valenzbeschreibung zukommt. Dementsprechend werden bei Storrer (2003: 768ff.) unter den kompetenzorientierten einfachen Tests zuerst diejenigen Methoden - mit all ihren Schwächen und Einschränkungen - vorgestellt, die zur Ermittlung dieses Kriteriums dienen bzw. dienen sollten. Darauf folgt die ebenso eingehende Behandlung derjenigen einfachen kompetenzorientierten Tests, die zur Beurteilung der formalen Spezifizität und der syntaktischen Notwendigkeit verwendet werden. Die Begründung dafür, warum gerade die Ermittlung der Kriterien von Argumenthaftigkeit, der formalen Spezifizität und der syntaktischen Notwendigkeit von ihr untersucht werden, ist in der folgenden Feststellung von Storrer (2003: 768) zu erkennen: „Die meisten Modelle nehmen Bezug auf mindestens eine der drei Valenzrelationen, die Jacobs (1994 (...)) als Argumenthaftigkeit (ARG), als formale Spezifizität (FOSP) und als syntaktische Notwendigkeit (NOT) bezeichnet hat".

An dieser Stelle will ich all die Tests, die Storrer behandelt, nicht einzeln thematisieren, denn eine solche Darstellung würde das Ziel dieses Unterkapitels - nämlich einen globalen Überblick über die allgemeinen Probleme der Unterscheidung zwischen Komplementen und Supplementen zu schaffen - weit überschreiten. Stattdessen sollen hier weitere, aus der Sicht meiner Arbeit wichtige Aspekte und Gedanken aus dem Artikel von Storrer hervorgehoben werden. ${ }^{62}$

Unter den korpusorientierten Verfahren erwähnt Strorrer (2003, 775) eins, das - meines Erachtens - sehr interessant ist, und - im Gegensatz zu seiner bei Storrer beschriebenen Verwendungsweise - nicht nur bei der Analyse von Sprachbelegen aus früheren Sprachperioden nützlich sein kann. Diese korpusorientierte Methode hat Ágel (1988) bei der Analyse solcher Belege verwendet, die aus dem Frühneuhochdeutschen stammen und bei denen die Relation der formalen Spezifizität nicht erwiesen werden konnte. Bei diesen Belegen wurde mit Hilfe von Frequenzanalysen über den Ergänzungs- oder Angabestatus der Erweiterungen

\footnotetext{
${ }^{62}$ Die Behandlung der komplexen Unterscheidungsverfahren bei Storrer (2003: 773f) stelle ich hier nicht dar, denn bei ihr werden nur zwei solche Verfahren kurz angesprochen, von denen ich den in der GDS beschriebenen komplexen Test in diesem Unterkapitel noch extra thematisieren werde.
} 
entschieden: Wenn Elemente ihrer Kategorie in mehr als 70\% der vorkommenden Ausdrücke als Erweiterung des betreffenden Verbalkomplexes vorkamen, wurde diese Erweiterung als Ergänzung klassifiziert, sonst als Angabe. Ich glaube, dass ein solcher zusätzlicher Test auch bei Substantiven praktiziert werden könnte, bei denen mit Hilfe von anderen Methoden nicht (eindeutig genug) darüber entschieden werden kann, ob ihr jeweiliges Attribut als Ergänzung oder als Angabe zu bewerten ist.

Storrer (2003: 776) setzt sich auch mit der Veränderung der Auffassung darüber auseinander, ob die Einteilung der abhängigen Konstituenten dichotomisch - d.h. in die beiden Klassen: Ergänzungen und Angaben - oder anders erfolgen sollte. Bei den nicht dichotomischen Klassifizierungen unterscheidet sie zwei Typen: die Valenzstufung und die Valenzquantifizierung. Für die Valenzstufung ist die Einteilungsweise der Dependentien in der GDS, die ich unten in diesem Unterkapitel noch behandeln werde, ein treffendes Beispiel. Bei Valenzstufungen (vgl. Storrer 2003: 776f.) werden nach verschiedenen Kriterien „typischere“ Ergänzungen und Angaben als zentral bzw. zum Kernbereich der Ergänzungen und Angaben gehörig, weniger typische als peripher bezeichnet. Storrer lenkt im Zusammenhang mit diesen Modellen die Aufmerksamkeit darauf, dass die Einstufung der Elemente, dadurch dass es bei solchen Unterscheidungsverfahren kein einheitliches Kriterium der Entscheidung gibt, als intuitiv bezeichnet werden kann, die auf der Grundlage von verschiedenen theoretischen Hypothesen vollzogen wird.

Bei der Valenzquantifizierung wird nach einem einheitlichen Kriterium die Stärke der Verbindung zwischen dem Valenzträger und seiner Erweiterung ermittelt (vgl. Storrer 2003: 777f.). $\mathrm{Zu}$ dieser Vorgehensweise kann das von Heringer (1986) durchgeführte Assoziationsexperiment gerechnet werden, bei dem mit Hilfe der Sprachkompetenz von Probanden, diese Verbindungsstärke festgestellt wurde. Ich bin der Meinung, dass eine solche Methode, die ja auf Sprecherkompetenz aufbaut, ebenfalls intuitiv ist. In Bezug auf diese Unterscheidungsweise hebt Storrer etwas hervor, was ich auch sehr wichtig finde: Fälle, die sich bei der Verwendung anderer Methoden als problematisch erweisen, werden auch von den befragten Probanden unterschiedlich eingestuft, und sind damit auch bei dieser Methode problematisch.

Bei der Valenzstufung gelten zuerst alle Dependentien eines Valenzträgers als valenzgebundene Elemente. Es können aber auch hier die Klassen von Ergänzungen und Angaben eingeführt werden, wenn ein bestimmter Schwellenwert angenommen wird. Dieser Schwellenwert soll die Grenze angeben, die zwischen Ergänzungen und Angaben anzunehmen ist. Wenn der Anteil derjenigen Probanden, die die betreffende Erweiterung beim untersuchten 
Valenzträger assoziiert haben, über diesem Wert liegt, soll sie als Ergänzung eingestuft werden. Wie auch Storrer darauf hinweist, können mehrere Schwellenwerte festgestellt werden, und damit ist die Einbindung der Methode auch in mehrstufige ${ }^{63}$ Modelle möglich. Es ist aber sehr wichtig, darauf hinzuweisen, dass die Bestimmung von Schwellenwerten wiederum intuitiv ist, denn diese Grenzen sind keine messbaren Größen, sondern werden immer vom Untersuchenden bestimmt. Storrer (2003: 778; meine Hervorhebungen Á. S.-T.) stellt zusammenfassend fest, dass ,[d]er E-A-Abgrenzung [...] eine Intuition zugrunde[liegt], die in jeder Lexikon- oder Grammatiktheorie neu präzisiert werden muss“. Sie geht darüber hinaus davon aus, dass, entsprechend dem jeweiligen Anwendungsbereich - auch bei Annahme mehrerer Valenzrelationen - bestimmte Valenzrelationen herausgegriffen werden können. ${ }^{64}$

Als wichtiges und eigentlich erstes Beispiel für die von Storrer (2003) mehrdimensionale, in der Fachliteratur jedoch oft multidimensional ${ }^{65}$ genannten Modelle ist das Konzept von Jacobs zu erwähnen, das er im Laufe der Zeit mehrmals überarbeitet hat. Eine seiner diesbezüglich grundlegenden Arbeiten ist seine Kontra Valenz, die er ursprünglich 1986 verfasste, als er sich im Rahmen einer Lehrveranstaltung zahlreichen Fragen und Problemen in Bezug auf die Interpretation des Valenzbegriffs konfrontiert sah. Seine Bemühungen, den Begriff zu erklären mündeten jedoch in eine Kritik an der „Valenzmisere“(Jacobs 1994: 5) und führten damit eher in eine „Begriffsdestruktion“ (Jacobs 1994: 2), was sich auch im Titel seiner Abhandlung äußert. Es ist jedoch darauf hinzuweisen, dass Jacobs seine Arbeit vorerst unveröffentlicht bleiben ließ, bis er 1994 den ursprünglichen Text, mit einem neuen Schlusskapitel ergänzt, publizierte.

In der hinzugefügten „Nachschrift“ (Jacobs 1994: 69ff.) revidiert er zwar nicht das Grundkonzept, dennoch mehrere wichtige Elemente seiner originären Vorstellung, wodurch er - auch seiner Einschätzung zufolge - den von ihm zuerst stark angefochtenen Valenzbegriff teilweise rehabilitiert. Außerdem schließt er diesen letzten Teil mit einer weiteren relativierenden Bemerkung betreffend den Kernteil seiner Arbeit ab: „Zweifel habe ich heute

\footnotetext{
${ }^{63}$ Der Terminus mehrstufig wurde hier Storrer (2003: 778) entnommen. Damit werden Modelle bezeichnet, in denen statt der dichotomischen Einteilung in Ergänzungen und Angaben eine komplexere Unterscheidung zu finden ist, wobei zentrale und periphere Unterklassen von Ergänzungen und Angaben angenommen werden. Diese Modelle wurden oben unter dem Begriff von Valenzstufung schon erwähnt.

${ }^{64}$ Auch ich gehe davon aus, dass es Fälle geben kann, in denen bestimmte Valenzrelationen nicht beachtet zu werden brauchen. So zum Beispiel ist die Frage, ob man alle drei von Welke (1988) festgestellten Kriterien bei der Untersuchung von valenten Substantiven verwenden sollte, davon abhängig, ob man obligatorische Erweiterungen beim Substantiv überhaupt für möglich hält (vgl. oben in diesem Unterkapitel).

${ }^{65}$ Es sei hier angemerkt, dass z. B. bei Fischer (2010), dessen Ansatz in diesem Kapitel weiter unten noch eingehender thematisiert wird, ein ganz anderes Begriffspaar für die obige Unterscheidung zu finden ist als bei Storrer (2003). Der Bezeichnung Mehrebenenmodell entspricht bei Fischer (2010:241) die Kategorie Schichtenmodell, während multidimensionale Modelle bei ihm Standardmodelle genannt werden.
} 
auch an zahlreichen Detailanalysen der vorangehenden Kapitel“" (Jacobs 1994: 72), wobei er jedoch nur ein konkretes Beispiel für diese ihm im Nachhinein fragwürdig erscheinenden Fälle bringt. Auf Grund dieser abschließenden Überlegungen von Jacobs finde ich zweckmäßig, anstatt seine forschungskritischen Erörterungen bzw. seine Ausführungen zu den einzelnen, von ihm vorgeschlagenen Valenzrelationen in all ihren Einzelheiten darzustellen, mich auf eine Zusammenfassung seines revidierten Grundkonzepts zu konzentrieren.

Den Grund für die von ihm als „Valenzmisere“ bezeichnete Lage der Valenzforschung zur Zeit der Entstehung des ersten Teils seiner Kontra Valenz sieht Jacobs (1994: 5ff.) darin, dass in der vorangehenden Zeit Valenz generell als einheitliches Phänomen betrachtet wurde, obwohl es - seines Erachtens - vielmehr als Sammelbegriff für unterschiedliche, voneinander unabhängige syntagmatische Relationen zu deuten ist. Jacobs (1994: 14ff.) bestimmt zuerst sieben solche Beziehungen, die er schließlich auf die vier Relationen von Notwendigkeit, Beteiligtheit, Argumenthaftigkeit und Spezifizität oder Subklassenspezifik reduziert. Seiner Definition entsprechend liegt Notwendigkeit im Falle einer Konstituente dann vor, wenn sie nicht weggelassen werden kann, ohne dass dadurch der Satz bei Bedeutungskonstanz ungrammatisch wird. Die Beziehung der Beteiligtheit erläutert Jacobs (1994: 16f.) auch durch einen Vergleich mit Tesnières - oben schon angesprochenen - Dramenmetapher, wobei Beteiligtheit bei den Elementen vorliegt, die in Tesnières Terminologie als Aktanten zu bezeichnen sind, d. h. an dem verbalen Geschehen oder Zustand beteiligt sind.

Die Relation der Argumenthaftigkeit versteht Jacobs (1994: 17ff.) so, dass sie gegeben ist, wenn eine Konstituente ein Argument in einer Prädikation darstellt. Dabei muss - meiner Meinung nach - eine spätere Erklärung Jacobs‘ (1994: 59) unbedingt hervorgehoben werden. So interpretiere er die Beziehung der Argumenthaftigkeit sofern anders als sie generell in der Forschung gedeutet wird, als er nicht voraussetzt, dass diese Relation vom Kopf ausgehen sollte. Dementsprechend betrachtet er in dem Beispiel Leider singt Peter ,das Adverbial als höheres Prädikat [...], als dessen Argument das Verb fungiert“. Es sei hier zuerst nur kurz angemerkt, dass ich eine solche Deutung der Argumenthaftigkeit problematisch und zur Unterscheidung von valenzgebundenen und nicht-valenzgebundenen Elementen nicht geeignet finde. Auf die Erörterung dieser Problematik werde ich weiter unten im Zusammenhang mit einem späteren Beitrag von Jacobs (2003) und dem darin vertretenen Argumentbegriff detaillierter eingehen.

Die vierte Relation der Subklassenspezifik, die Jacobs im Hauptteil seiner Arbeit noch deutlich kritisiert, erhält ihre bestimmende Rolle in seinem Valenzkonzept erst in der Nachschrift, indem er die im Vorangehenden etablierte Unterscheidung zwischen den 
Relationen der formalen und inhaltlichen Spezifizität ${ }^{66}$ zurücknimmt und durch die syntagmatische Beziehung der Subklassenspezifik ersetzt. In Bezug auf die Subklassenspezifik stellt Jacobs (1994: 72) fest, dass ,diese Beziehung nicht nur vom Verb zu seinen Begleitern, sondern auch in umgekehrter Richtung verlaufen kann“. Seiner Ansicht nach kann im Falle eines Begleiters dessen Ergänzungs- oder Angabestatus nicht dadurch bestimmt werden, ob Subklassenspezifik vorliegt oder nicht, sondern dadurch, ,in welcher Richtung sie verläuft und welche Art von Merkmalen sie betrifft" (Jacobs 1994: 72). Dementsprechend werden im Falle von Ergänzungen die Merkmalsforderungen vom verbalen Kopf dem Begleiter auferlegt.

Jacobs (1994: 71) trifft in der Nachschrift von Kontra Valenz eine - meines Erachtens wesentliche Verfeinerung, wonach die Valenzbeziehungen zwar unabhängig, aber doch prototypisch gemeinsam vorhanden sind. Dadurch gewinnt - ihm zufolge - auch der Valenzbegriff, den er im Hauptteil seiner Arbeit zu einem „Sammelbegriff für mehrere inhaltlich verschiedene Konzepte“ degradierte, der „als solcher von geringem sprachwissenschaftlichen Interesse“ sei (Jacobs 1994: 4), ihre Relevanz für linguistische Untersuchungen teilweise zurück.

Vor der Thematisierung des oben schon erwähnten späteren Artikels von Jacobs finde ich wichtig, noch ein wenig beim Begriff der Subklassenspezifik zu verweilen, da Subklassenspezifik in vielen Valenzansätzen als eine der bestimmenden oder sogar als die entscheidende Valenzrelation schlechthin betrachtet wird. Uzonyi (2004) setzt sich in seinem Beitrag mit der Frage des Verhältnisses von Valenz und Subklassenspezifik auseinander. Dabei weist er auch auf das Problem hin, dass Subklassenspezifik in der Fachliteratur nicht einheitlich definiert wird, (Uzonyi 2004: 233f.) was - seines Erachtens - zum Teil auch auf Uneinheitlichkeiten der Wortartenklassifizierungen zurückgeführt werden kann. Ferner hebt er - meiner Meinung nach - wohlbegründet hervor, dass wir bei der Bestimmung bzw. Annahme von Subklassen „,nicht über Klassen und Subklassen von vollständigen Lexemen sprechen sollten, sondern über die in Sememe zerlegten Lexeme (d. h. Lexemteile)“(Uzonyi 2004: 234). Das ist damit zu erklären, dass Lexeme verschiedene Lesarten haben können, mit denen sehr häufig auch unterschiedliche Valenzstrukturen verbunden sind, wodurch es besonders sinnvoll erscheint, nicht Lexeme als Einheiten, sondern „die in Sememe zerlegten Lexeme“ bei der Valenzuntersuchung zu betrachten.

Besonders bedeutend finde ich Uzonyis (2004: 234f.) Erörterungen in Bezug auf nicht kasusregierte Konstituenten, wie z. B. Adverbialien, indem er zeigt, dass solche Dependentien

\footnotetext{
${ }^{66}$ und deren Varianten DEFOSP und DEINSP (vgl. Jacobs 1994: 25f.)
} 
zuerst auf Grund ihrer semantischen Gemeinsamkeiten charakterisierbar sind. Darauffolgend lässt sich auch die begrenzte Menge formaler Merkmale ermitteln, die für die jeweilige Gruppe der Dependentien typisch ist. Dadurch erfährt der Subklassenspezifikbegriff eine - meines Ermessens - sehr wichtige und nötige Erweiterung. Die so interpretierte Subklassenspezifik ist auch geeignet z. B. im nominalen Bereich attributive Adjektive in sich zu fassen und sie damit unter den mit ihr verbundenen Valenzbegriff zu fassen. Dies halte ich deswegen von Belang, weil - wie ich unten ${ }^{67}$ noch thematisieren werde - attributive Adjektive von den meisten Autoren - im Gegensatz z. B. zu meiner Auffassung - eindeutig, und oft mit einem Hinweis auf ihre nicht-subklassenspezifische Art, als nicht-valenzgebunden eingestuft werden.

Wie oben schon angesprochen, geht Jacobs (1994) - im Gegensatz zur allgemein verbreiteten Auffassung in der Valenzforschung - davon aus, dass die Beziehung der Subklassenspezifik sowohl vom Regens zum Dependens als auch vom Dependens zum Regens wirken kann. Im Zusammenhang mit der Frage der „Subklassenspezifik von Subjekt und Prädikat" spricht auch Uzonyi (2004: 236f.) die Zweiseitigkeit der Subklassenspezifik an, indem er erklärt, dass in der Verbindung des finiten Verbs mit dem Subjekt als finitem Nomen (im Sinne von Ágel 1996) beide Parteien einander Werte zuweisen: Das finite Verb wird durch das Subjekt in seiner Person definiert, während das Subjekt vom finiten Verb den Kasus Nominativ zugeteilt bekommt. Dies führt jedoch dazu, wie Uzonyi (2004: 237) hervorhebt, dass ein ausschließlich auf der Subklassenspezifik aufbauender Valenzbegriff Hierarchien der Dependenzbeziehungen voraussetzen soll, damit in einer Verbindung wie der oben erwähnten die beiden Konstituenten nicht gegenseitig „Ergänzungen voneinander werden“, sondern eindeutig eine von ihnen als Valenzträger identifiziert wird.

Nach diesem kurzen, aber - meines Erachtens - sehr wichtigen Exkurs zum Phänomen der Subklassenspezifik kehre ich noch zu theoretischen Überlegungen von Jacobs zurück, wobei ich einen Beitrag von ihm aus dem Jahre 2003 in erster Linie wegen dessen Argumentbegriff thematisieren will. In dieser Arbeit stellt Jacobs (2003) ${ }^{68}$ [d]ie Problematik der Valenzebenen dar und beschäftigt sich - im Gegensatz zu Storrers obiger Darstellung - nicht nur mit der

\footnotetext{
${ }^{67}$ Vgl. v. a. meine Bemerkungen zur Frage der Bewertung des Komplement- und Supplementstatus von attributiven Adjektiven in Kap. 2.6, aber auch meine Überlegungen zu Teuberts (1979) Klassifizierung von Genitiv- und Präpositionalattributen in Kap. 2.5.2.

68 Jacobs (2003: 379ff.) unterscheidet in seinem Beitrag Die Problematik der Valenzebenen zwei Ebenen der Valenz, nämlich die kategoriale und die semantische Valenz, und auf beiden Ebenen nimmt er verschiedene Dimensionen der Valenz an. Zur kategorialen Valenz rechnet er Realisierungs- und Merkmalsforderungen, während zur semantischen Valenz Relat- und verschiedene inhaltliche Forderungen gehören. Wie aus dieser Aufzählung ersichtlich wird, unterscheiden sich die von Jacobs (2003) in diesem Beitrag beschriebenen Dimensionen teilweise von den Valenzrelationen, die er in seinem oben thematisierten Kontra Valenz (Jacobs 1994) festgehalten hat.
} 
Verbvalenz, sondern mit der Valenz im Allgemeinen. Er versucht sogar „die Einbindung von Valenzphänomenen in eine umfassende Sprachtheorie“ (Jacobs 2003:398) vorzubereiten, wodurch noch verständlicher wird, warum er auf keinen Fall den Betrachtungsrahmen auf die Valenz einer Wortart einengen kann. ${ }^{69}$

Betreffend die „langwierige und fruchtlose Debatte über Diagnoseverfahren“, die zur Unterscheidung von Ergänzungen und Angaben dienen sollen, behauptet Jacobs (2003: 395), dass ,der unglückliche Verlauf dieser Debatte weitgehend darauf zurückzuführen [ist], dass die Multidimensionalität der Valenz(bindung), ${ }^{70}$ insbesondere die Unabhängigkeit der Dimensionen verkannt wurde“.${ }^{71}$

Ich halte es für sehr wichtig hervorzuheben, dass bei Jacobs (2003) der Begriff Argument weiter gefasst ist, als es sonst in der Fachliteratur gewöhnlich ist. Jacobs (2003: 386) definiert diesen Begriff folgendermaßen: „Die Argumente eines Valenzträgers VT in einem Satz S sind jene Dependentien von VT, die ein Relat in der semantischen Valenz von VT spezifizieren, indem sie eine zusätzliche Information über das Relat geben“.

Jacobs (2003: 387) unterscheidet dabei referentielle (R-)Relatpositionen und nichtreferentielle (Non-R-) Relatpositionen. Erstere nehmen die Sachverhalte auf, auf die sich der Valenzträger bezieht und die zumindest zum Teil damit identifiziert werden. Non-RRelatpositionen werden dagegen nicht unbedingt und, wenn überhaupt, durch zusätzliche vom Valenzträger abhängige Konstituenten ausgedrückt. Um die Begriffe deutlicher zu machen, stehe hier ein Beispiel von Jacobs (2003: 387f.): In $x$ ist Tochter von $y$ sei $x$ die R-Relatposition und $y$ die Non-R-Relatposition. Jacobs nimmt zwei Typen von Argumenten an: Argumente, die Non-R-Relatpositionen des betreffenden Valenzträgers spezifizieren, nennt er Identifizierer, während R-Relatpositionen des Valenzträgers von Modifizierer spezifiziert werden, die den zweiten Typ von Argumenten darstellen. Dementsprechend sei von Gerda in dem Satz Die Tochter von Gerda freut sich ein Identifizierer, während in der Küche in Die Tochter in der Küche freut sich einen Modifizierer darstelle. ${ }^{72}$

\footnotetext{
${ }^{69}$ Er könnte seine Thematisierungen nur in dem Falle auf die Valenz einer bestimmten Wortart beschränken, wenn er davon ausgehen würde, dass nur bei dieser einen Wortklasse von Valenz die Rede sein kann. Denn zur „Einbindung von Valenzphänomenen in eine umfassende Sprachtheorie“ sollen alle Bereiche abgedeckt werden, die von der Erscheinung erfasst werden. Wie aus dem Artikel hervorgeht, nimmt Jacobs (2003: z. B. 391) auch Substantiv- und Adjektivvalenz an.

${ }^{70}$ Der Wortteil ist auch bei Jacobs (2003: 395) in Klammern gesetzt.

${ }^{71}$ Wie auch Jacobs (2003: 395) darauf hinweist, ist er in seiner oben gerade thematisierten Kontra Valenz. (Jacobs 1994) auf diese Feststellung gekommen.

${ }^{72}$ Die Beispielsätze wurden Jacobs (2003: 387) entnommen, auch weil sie aus der Sicht der kritischen Darstellung des Argumentbegriffs von Jacobs (2003) von Belang sind.
} 
Was den Argumentbegriff von Jacobs (2003) von dem in der Fachliteratur üblichen unterscheidet, ist, dass bei ihm auch Modifizierer als Argumente bewertet werden. ${ }^{73}$ Modifizierer werden nämlich in der Forschung gewöhnlich als Dependentien eines Valenzträgers betrachtet, die nicht valenziell vom Valenzträger bestimmt und damit zu den Angaben $^{74} \mathrm{zu}$ rechnen sind. Bei Jacobs (2003: 388) sollten damit nur solche Konstituenten eines sprachlichen Konstrukts als Angaben bewertet werden, mit denen „Operationen auf der Proposition oder der Illokution" ausgedrückt werden. ${ }^{75}$

Er gibt zwei Gründe dafür an, warum - seines Erachtens - auch Modifizierer als Argumente der Valenzträger und damit als Ergänzungen betrachtet werden sollen. Zum einen spezifizieren Modifizierer - so wie auch die Identifizierer - Relate, die vom Valenzträger eingeführt wurden. Zum anderen vertritt er die Meinung, dass Modifizierer bezüglich ihres Verhaltens bei vielen grammatischen Generalisierungen wie die Identifizierer zu charakterisieren und nicht mit den Konstituenten zu vergleichen sind, die in seinem System nicht als Argumente bezeichnet werden können.

Aus dieser Betrachtungsweise der Modifizierer folgt - meines Erachtens -, dass Angaben ausschließlich auf Satzebene möglich sind. Damit wäre z. B. die Substantivvalenz auf der semantischen Ebene bei jedem Substantiv möglich. Ich gehe aber davon aus, dass eine solche allzu breit gefasste (semantische) Valenz(ebene) vielleicht im Bereich der Verbvalenz, nicht aber z. B. in dem der Substantivvalenz verkraftet werden kann. Diese z. T. intuitive Feststellung von mir kann mit folgenden Argumenten unterstützt und dadurch objektiviert werden: Die eventuelle Kluft zwischen R-Relatpositionen und Non-R-Relatpositionen finde ich bei den Verben im Allgemeinen kleiner als er bei vielen Substantiven sein kann. So hat die Situationsstelle, die bei Verben von Modifizierern spezifiziert wird, immer noch engeren semantischen Zusammenhang sowohl mit Handlungen, als auch mit Zuständen und Ereignissen $^{76}$ als z. B. die semantische Verbindung in der Nominalphrase die Tochter in der Küche zwischen dem Modifizierer in der Küche und dem Substantiv Tochter ist. Ich gehe deswegen davon aus, dass in einer Assoziationsprobe Probanden mit viel größerer Häufigkeit

\footnotetext{
73 Wie auch Jacobs (2003: 396) an einer späteren Stelle darauf hinweist, kann der in der Fachliteratur eingenommene Argumentbegriff mit dem Terminus Identifizierer bei ihm gleichgesetzt werden.

74 Jacobs (2003: 388) verwendet an diesem Punkt den Terminus Angaben nicht, sondern spricht von „Dependentien, die tatsächlich keine Argumente sind“.

${ }^{75}$ Jacobs (2003:388) führt dafür folgende Beispiele auf: epistemische, evaluative und Sprechakt-Adverbiale (wie u. a. vermutlich, leider, offen gesagt).

76 Handlungen, Zustände und Ereignisse decken die drei wichtigsten Bedeutungsbereiche ab, die mit der Wortklasse Verb verbunden werden können.
} 
Temporalbestimmungen mit dem Verb erblicken ${ }^{77}$ verknüpfen würden als Lokalbestimmungen mit dem Substantiv Tochter. ${ }^{78}$ Noch deutlicher ist der Unterschied, wenn wir ein nichtrelationales Substantiv - wie z. B. Hund - betrachten. Entsprechend des Argumentbegriffs, bzw. der neu festgestellten Grenze der Ergänzungsmenge zu den Nicht-Ergänzungen bei Jacobs (2003), wäre die Präpositionalphrase unter dem Tisch in der Hund unter dem Tisch als Ergänzung zu klassifizieren. Eine solche allzu weit gefasste (semantische) Valenz(ebene) ist aber - meines Ermessens - bei Substantiven nicht anwendbar. ${ }^{79}$

Wie also ersichtlich wurde, bedeutet der Terminus Ergänzung bei Jacobs (2003) nicht dasselbe, was er gewöhnlich in der Fachliteratur bezeichnet. Darüber hinaus kann die unterschiedliche Verwendung der Termini von Ergänzung und Komplement bei Jacobs (2003) als weitere - diesmal eher nur terminologische - Abweichung von der in der Fachliteratur eingenommenen Bezeichnungsweise hervorgehoben werden. Während die beiden Begriffe in der Fachliteratur gewöhnlich als Synonyme vorkommen, werden sie von Jacobs (2002: 390f.) etwas anders interpretiert: Als Ergänzungen bezeichnet er alle - seiner Auffassung nach valenzgebundenen ${ }^{80}$ Elemente. Der Terminus Komplement bezieht sich bei ihm auf eine Untergruppe der Ergänzungen, zu der solche abhängigen Konstituenten gehören, die beim jeweiligen Valenzträger notwendig oder von ihm formregiert sind.

Ich halte es für sehr wichtig, auf noch eine Feststellung von Jacobs (2003: 385) näher einzugehen, denn sie kann mit dem von mir vertretenen Substantivvalenzbegriff nicht einwandfrei vereinbart werden. Er gibt nämlich unter den Beispielen für Fälle, bei denen der Kasus einer abhängigen Konstituente nicht über KR-Valenz bestimmt wird, auch „den Genitiv postnominaler Attribute“ (z. B. das Haus der Begegnung) an. Jacobs ist der Meinung, dass bei diesen Erweiterungen der Kasus des abhängigen Elements ,über die Position in der Konstituentenstruktur" festgelegt wird. Ich gehe dagegen davon aus, dass es Fälle gibt, in denen ein Genitiv von einem dependentiell übergeordneten Substantiv valenziell determiniert und

\footnotetext{
${ }^{77}$ Ich gebe deswegen die Verbindung des Verbs erblicken mit einer Temporalbestimmung als Beispiel an, weil Jacobs (2003: z. B. 386) zufolge in Peter erblickt jetzt die Tür das Adverb jetzt-als Modifizierer des Valenzträgers - auch als Ergänzung bewertet werden sollte.

${ }^{78}$ Ich bezweifle sogar, dass Lokalbestimmungen überhaupt bei diesem Substantiv assoziiert wären, um aber behaupten zu können, dass Lokalbestimmungen mit dem Substantiv „Tochter“ nicht assoziiert werden, sollte eine Assoziationsprobe für diesen „Valenzträger“ mit genug Probanden durchgeführt werden.

${ }^{79}$ Bei verbalen Valenzträgern halte ich - entsprechend meinen oben thematisierten Ansichten bezüglich der semantischen Verbindung zwischen verbalen R-Relatpositionen und Non-R-Relatpositionen - für vorstellbar, dass - zumindest bestimmte Teilgruppen der - Modifizierer als periphere, weniger typische Ergänzungen betrachtet werden.

${ }^{80}$ Jacobs (2003: 390) gibt alle möglichen syntagmatischen Beziehungen an, die aus der - von ihm beschriebenen - Unabhängigkeit der Valenzdimensionen ableitbar sind. Er nennt diese Beziehungen zusammenfassend Valenzbindungsrelationen (vgl. Jacobs 2003: 391). Wenn eine Konstituente in einer dieser Relationen zu einer anderen Konstituente steht, gilt sie, Jacobs zufolge, als valenzgebunden.
} 
auch in seiner Form als Genitiv bestimmt wird. So kann z. B. der Schüler in die Begrüßung der Schüler durch den Lehrer bei gleichzeitiger Benennung von Agens und Patiens nur das letztere ausdrücken. Deswegen bin ich der Meinung, dass die Genitive postnominaler Attribute nicht eindeutig als Dependentien betrachtet werden können, deren Kasus aus der „Position in der Konstituentenstruktur" folgt. ${ }^{81}$

Als weiteres und aus der Sicht der vorliegenden Arbeit bedeutendes multidimensionales Valenzkonzept ist das in der GDS entworfene Konzept zu erwähnen. Dem Problem der Unterscheidung von Komplementen und Supplementen wird in der GDS (1027ff.) ein eigenes Unterkapitel (vgl. Kap. E2 2.1) gewidmet. Zwar geht es in der betreffenden Einheit nur um den verbalen Bereich, aber die Verfasser nehmen nicht nur beim Verb, sondern u. a. auch beim Substantiv und Adjektiv Valenz an, und behandeln sie als multidimensionales Konzept. Da im Rahmen des Kapitels E2 2.1 nur die Verbvalenz vor Augen gehalten wird, sind die Termini Komplemente und Supplemente als Verbkomplemente und Verbsupplemente zu verstehen. Supplemente werden dabei als „Teile des Diktums außerhalb der Elementarproposition“, Komplemente als „Teile des Ausdrucks der Elementarproposition“"82 definiert (GDS 1029).

In Bezug auf die Kriterien und Testverfahren, die bei der Unterscheidung von Komplementen und Supplementen zur Verwendung kommen können, findet sich eine - meines Erachtens - sehr wichtige Feststellung in der GDS: „Entsprechend dieser Multidimensionalität stehen keine einheitlichen, für alle Fälle zutreffenden Kriterien und Testverfahren zur Verfügung“. Im Gegensatz also z. B. zu Varnhorns (1986) oben angesprochener Zielsetzung und Annahme gehen die Verfasser der GDS - gemäß ihrer Sichtweise der Valenz als multidimensionalem Konzept - davon aus, dass kein Unterscheidungskriterium bzw. -testverfahren allein zuverlässig sein kann. ${ }^{83}$

Die Basis des multidimensionalen Valenzkonzepts bildet die Annahme, dass bei den Valenzrelationen zum einen morphosyntaktische, zum anderen semantische oder semantischpragmatische betrachtet werden müssen (vgl. GDS 1030f.). Die morphosyntaktischen Valenzrelationen werden in der GDS Formrelationen, die semantischen oder semantischpragmatischen Bedeutungsrelationen genannt. Die Verfasser vertreten dabei die Ansicht, dass

\footnotetext{
${ }^{81}$ Ein wichtiges Bemühen von Jacobs (2003) in seinem Artikel ist ferner die Formulierung von Valenzgesetzen, auf die ich hier jedoch nicht eingehe, da sie eher schon sprachtheoretische Betrachtungen sind und dementsprechend nicht in den Rahmen meiner Untersuchungen passen.

${ }^{82} \mathrm{Zu}$ den Termini Diktum und Elementarproposition vgl. GDS (597ff.).

${ }^{83}$ Natürlich hängt diese Ansicht der Verfasser der GDS auch damit zusammen, dass sie ein multidimensionales Valenzkonzept als Ausgangsbasis nehmen. Ich glaube aber, dass Varnhorns (1986) Zielsetzung schon allein deswegen nicht ganz realistisch ist, weil - wie ich schon oben thematisiert habe - die Rolle der Intuition bei der Beurteilung des valenziellen Status einer Konstituente einkalkuliert werden sollte.
} 
für eine Differenzierung zwischen Komplementen und Supplementen, die oft mit Schwierigkeiten verbunden ist, nur die eine oder nur die andere Relation meistens nicht ausreicht. Die große Zweiteilung der Valenzrelationen entspricht hier in ihrem Grundgedanken der bei Jacobs (2003), wo die KR- und SR-Valenzebene voneinander unterschieden werden. ${ }^{84}$

$\mathrm{Zu}$ den Formrelationen werden in der GDS Fixiertheit, Rektion, Konstanz und Kasustransfer gerechnet, von denen sich Konstanz und Kasustransfer nur auf bestimmte Dependentien beziehen, die in Form von Präpositionalphrasen realisiert werden (vgl. GDS 1031ff.). Unter den Bedeutungsrelationen werden Sachverhaltsbeteiligung, Perspektivierung, Sachverhaltskontextualisierung und autonome Kodierung betrachtet (vgl. GDS 1038ff.). Hier geht es - ähnlich wie bei den obigen Darstellungen der verschiedenen Theorien - nicht darum, die Detailfragen der Relationen im Einzelnen zu thematisieren, stattdessen sollen weitere Merkmale des multidimensionalen Konzepts der GDS hervorgehoben werden, die aus der Sicht meiner Arbeit von größerer Bedeutung sind.

Sowohl bei den Formrelationen als auch bei den Bedeutungsrelationen werden sog. Komplement-Fürsprecher ${ }^{85}$ bzw. als ihre Gegenteile auch sog. Komplement-Gegenspieler angegeben (vgl. GDS 1037 und 1041). Betreffend die Unterscheidung zwischen Komplementen und Supplementen wird in der GDS (1041) Folgendes festgestellt: „Um zu einer tragfähigen Abgrenzung zwischen Komplementen und Supplementen zu kommen, sind entsprechend der Grundvorstellung dieser Grammatik [der GDS; Á. S.-T.]-die Relationen der Formseite mit denen der Bedeutungsseite in Beziehung zu setzen. “Eine (solche) Verbindung der verschiedenen Valenzrelationen finde ich auch besonders wichtig. ${ }^{86}$

Bei der Ermittlung der „Beziehungen zwischen Form- und Bedeutungsrelationen“, werden in der GDS (1041ff.) ein prototypischer und ein markierter Fall voneinander unterschieden. Beim prototypischen Fall besteht „Konvergenz zwischen der Form- und der Bedeutungsseite“

\footnotetext{
${ }^{84}$ Ich halte es aber für wichtig, darauf hinzuweisen, dass bei Jacobs (2003: 390ff.) die Ansicht vertreten wird, dass die Dimensionen innerhalb der beiden Ebenen voneinander unabhängig sind. Sie können zwar in bestimmten Fällen zusammenhängen, aber sie müssen nicht. In der GDS (1037ff.) werden die Bedeutungsrelationen als voneinander nicht unabhängig bezeichnet, und auch bei den Formrelationen, von denen die meisten als voneinander unabhängig charakterisiert werden, gibt es Fälle, in denen bestimmte Formrelationen zusammenhängen.

${ }_{85}$ Bei den Formrelationen werden +Rektion, +Konstanz, +Kasustransfer, ++/+Fixiertheit als KomplementFürsprecher angegeben, bei den Bedeutungsrelationen gelten +Sachverhaltsbeteiligung, ++/+ Perspektivierung, autonome Kodierung als solche. (Die Symbole,,+++- können folgendermaßen paraphrasiert werden: , ${ }^{+“}$ eingeschränkt zutreffend, ,,++“ uneingeschränkt zutreffend, ,," nicht zutreffend.)

${ }^{86}$ Vgl. dazu auch mein Konzept zur Unterscheidung zwischen Komplementen und Supplementen beim Substantiv unten in Kap. 2.6.
} 
(GDS 1041). ${ }^{87}$ Im Gegensatz dazu divergieren in markierten Fällen die Bedeutungs- und Formrelationen zumindest partiell. Auch solche markierten Fälle werden behandelt, bei denen eine absolute Divergenz der Bedeutungs- und Formrelationen vorhanden ist, d. h. bei den formseitigen oder bei den bedeutungsseitigen Relationen werden Komplement-Fürsprecher ermittelt, während auf der anderen Seite nur Komplement-Gegenspieler festgestellt werden. In solchen Fällen sei es „eine Frage der Gewichtung“ (GDS 1043), ob man der Seite Vorrang zuweist, an der Komplement-Fürsprecher erwiesen werden konnten, und dementsprechend die betreffende Konstituente als Komplement einstuft oder eben umgekehrt, und sie dadurch als Supplement klassifiziert. Hier wird also deutlich, dass schon bei der theoretischen Betrachtung des Problems mit Fällen gerechnet werden muss, bei denen die Entscheidung über den Komplement- oder Supplementstatus einer abhängigen Konstituente von der subjektiven Entscheidung des Untersuchenden abhängig ist. Dazu kommen noch - meines Erachtens - die Fälle, in denen bei den Tests Unsicherheiten auftreten können, die wiederum der Intuition eine Rolle zukommen lassen.

In der GDS (1064) werden zwei Gründe angegeben, warum die Unterscheidung zwischen Komplementen und Supplementen problematisch werden kann. Zum einen gibt es Fälle, bei denen Schwierigkeiten schon bei der Beurteilung bestimmter Valenzaspekte auftreten. ${ }^{88}$ Zum anderen können - wie gerade thematisiert - die Relationen auf der Formseite in Widerspruch zu denen auf der Bedeutungsseite stehen (oder umgekehrt), was die Entscheidung wiederum erschwert. Als Antwort auf diese Unsicherheiten werden in der GDS die Klassen der Komplemente und der Supplemente nicht als einheitlich aufgefasst, sondern als derart, dass zwischen Kern und Peripherie unterschieden werden kann. Eine solche Lösung halte ich zwar für plausibel und dem Wesen des Problems entsprechend, aber es muss betont werden, dass sich die bis dahin problematischen Fälle auch dabei als mehr oder weniger problematisch erweisen. Die Einstufung derjenigen Dependentien, die sich an der angenommenen Grenze zwischen Kern- und Peripheriebereich befinden, bzw. überhaupt die Feststellung der Grenzen bleibt nämlich weiterhin unsicher.

Bei dem Ermittlungsverfahren, das in der GDS (1043ff.) beschrieben wird, werden drei Tests praktiziert, die in bestimmter Reihenfolge nacheinander verwendet werden sollen. Mit

\footnotetext{
87 Das bedeutet, dass im prototypischen Fall zusammen mit formseitigen Komplement-Fürsprechern bedeutungsseitige Komplement-Fürsprecher vorkommen, bzw. mit formseitigen Komplement-Gegenspielern auf der Bedeutungsseite ebenfalls Komplement-Gegenspieler verbunden sind.

${ }^{88}$ Als Beispiel dafür wird in der GDS (1064) u. a. die Frage der Konstanz erwähnt.
} 
Hilfe dieser Tests wird die jeweilige Konstituente ${ }^{89}$ nach den oben thematisierten Relationen, die als Komplement-Fürsprecher oder als Komplement-Gegenspieler gelten, untersucht. Als erster Filter wird der Reduktionstest verwendet, bei dem die Elemente, die sich zur formalen (d. h. syntaktischen) Vollständigkeit der Phrase als notwendig erweisen, als Komplemente des Kernbereichs kategorisiert werden. Die Konstituenten, die syntaktisch nicht für notwendig befunden werden, werden dem zweiten Filter, dem Folgerungstest, ${ }^{90}$ unterzogen. Die Dependentien, bei denen dieser Test negativ verläuft, werden als Supplemente eingestuft. Bei den übrigen Konstituenten wird zwischen zwei Fällen unterschieden: Bei dem einen verläuft der Test uneingeschränkt positiv, bei dem anderen eingeschränkt positiv. ${ }^{91}$ Die beiden gelten nach dem Folgerungstest als eingeschränkte oder uneingeschränkte Komplementkandidaten und durchlaufen zur Entscheidung den dritten Filter, den Anschlusstest. ${ }^{92}$

Diejenigen, die nach dem Folgerungstest als eingeschränkte Komplementkandidaten gelten und bei denen der Anschlusstest negativ verläuft, werden der Klasse der Supplemente zugerechnet. ${ }^{93}$ Bei ihnen muss aber angemerkt werden, dass diese Gruppe von der Unterklasse der Supplemente unterschieden werden muss und auch wird, deren Vertreter schon mit Hilfe des Folgerungstests als eindeutige Supplemente ${ }^{94}$ eingestuft worden sind. Die Elemente dieser Gruppe, die den Anschlusstest mit negativem Ergebnis durchlaufen haben, galten nämlich nach dem Folgerungstest noch als eingeschränkte Komplementkandidaten. Hier wird wiederum deutlich, dass es auch bei einer graduellen Einstufung Fälle geben kann, die nicht eindeutig sind. Das wird auch aus der Formulierung in der GDS (1059; meine Hervorhebungen Á. S.-T.) im Zusammenhang mit dieser Phrasenklasse deutlich, wenn über sie geschrieben wird, dass die Elemente, die zu ihr gehören, „, (...) den insgesamt gegebenen Übergangsbereich zu den

\footnotetext{
${ }^{89}$ Die zu untersuchenden und zu klassifizierenden Ausdrücke werden in der GDS als Phrasenklassen bezeichnet. Ich werde im Folgenden diese Bezeichnung mit den von mir verwendeten Ausdrücken, wie Erweiterungen, Dependentien oder (abhängige) Konstituenten, synonym verwenden.

${ }^{90}$ Mit dem Folgerungstest wird die Impliziertheit der betreffenden Konstituente (d. h. ob auf sie - bei ihrer Weglassung aus der Phrase - mit einem indefiniten Ausdruck gefolgert werden kann) untersucht. Wenn sie nur in bestimmten Kontexten oder in bestimmten Verwendungsweisen des Phrasenkopfes als impliziert gilt, besteht sie den Folgerungstest eingeschränkt positiv, wenn sie in allen Fällen impliziert wird, durchläuft sie diesen Test mit uneingeschränkt positivem Ergebnis. Wenn sie beim jeweiligen Regens nicht impliziert werden kann, geht der Folgerungstest für sie mit negativem Ergebnis aus.

${ }^{91}$ vgl. dazu die obige Anmerkung zum Folgerungstest

${ }^{92}$ Der Anschluss-Test wird auch als ,und-das-Test“ bezeichnet (vgl. Storrer 2003: 774). Damit wird getestet, ob die betreffende Konstituente nach ihrer Weglassung aus der Phrase mit und das an die reduzierte Phrase angeschlossen werden kann.

${ }^{93}$ Als Beispiel für eine solche Erweiterung ist in der GDS (1058f., das Beispiel stammt von 1058) u. a. Folgendes zu finden: Ich habe für sie den Koffer getragen.

${ }^{94}$ Als Beispiele für solche „eindeutigen“ Supplemente werden in der GDS (1048) u. a. die illokutionsbezogenen Ausdrücke und der ethische Dativ erwähnt.
} 
adverbialen Supplementen am deutlichsten [markieren]“ und dass „sie (...) hier den Supplementen zugeschlagen [werden]“.

Die weiteren Dependentien werden abhängig von ihrer Bewertung beim Folgerungs- und Anschlusstest als Elemente von Phrasenklassen eingestuft, die entweder zum Kernbereich oder zur Peripherie der Komplemente gehören. Auf sie will ich nicht näher eingehen, denn ihre Klassifizierung erscheint mir weniger problematisch zu sein, als die Bewertung der von mir oben angesprochenen „Übergangsklasse“.$^{95}$

Schließlich sei in diesem Unterkapitel noch auf Fischers (2001) Arbeit eingegangen, in der er auch zum Konzept von Jacobs und der GDS Stellung nimmt. Fischer (2001: 242ff.) gibt einen knappen Überblick unterschiedlich angelegter Valenzansätze aus der Forschungsgeschichte. Dabei vertritt er - meines Erachtens absolut berechtigt - die Auffassung, dass „kategoriale Valenzdefinitionen“, die Satzgliedern unabhängig von der konkreten Konstruktion, d. h. vom konkreten Regens, auf Grund ihrer Kategorie Ergänzungsoder Angabestatus zuweisen, die relationale Art von Valenz außer Acht lassen und somit „prinzipiell kritisch zu betrachten“ (Fischer 2001: 242) sind. In diesem Zusammenhang befasst er sich auch mit dem Problem von Subklassenspezifik als definitorischem Merkmal von Valenzgebundenheit und plädiert dafür, dass Subklassenspezifik zwar als ,häufig auftretender kategorialer Valenzeffekt“" (Fischer 2001:243) in Valenzbeschreibungen ihre Daseinsberechtigung habe, aber keinesfalls zur Definition von Valenz geeignet sei.

Wie oben schon angedeutet, befasst er sich auch mit der „Neuorientierung der Valenzforschung“ (Fischer 2001: 241), die auf Jacobs“ (1994) Kontra Valenz folgte und wodurch multidimensionale Valenzkonzepte entstanden und $\mathrm{zu}$ dominierenden Ansätzen geworden sind. Von den multidimensionalen Valenzmodellen widmet Fischer (2001: 246ff.) der Präsentation des Konzepts von Jacobs ${ }^{96}$ und des Modells in der GDS je einen eigenen Abschnitt. An diesem Punkt konzentriere ich mich auf seine Gesamtbewertung der beiden Konzepte und gehe dabei auf seine diesbezüglichen Ausführungen nicht detailliert ein, da ich beide Modelle selber oben schon beschrieben habe.

\footnotetext{
95 Einen guten Überblick über den Ablauf des Filterverfahrens und über die Klassifizierung der dadurch entstehenden Phrasenklassen bietet Abbildung 3 auf Seite 1059 in der GDS.

Es ist noch hervorzuheben, dass nach der Beschreibung des Testverfahrens alle Phrasenklassen charakterisiert werden, wobei alle Komplement-Fürsprecher und / oder alle Komplement-Gegenspieler angegeben werden, durch die die Elemente der jeweiligen Klasse charakterisiert werden können (vgl. GDS 1060ff.).

${ }^{96}$ Bei der kritischen Darstellung des multidimensionalen Valenzmodells von Jacobs (1994 und 2003) verweist Fischer (2001) außer auf die Pionierarbeit Kontra Valenz ebenfalls auf seinen neueren Beitrag Die Problematik der Valenzebenen (Jacobs 2003), auf den auch ich in meiner Arbeit referenziere, und auf das multidimensionale Valenzmodell von Blume (2000: v. a. 15ff.), das das Konzept von Jacobs zur Grundlage hat, es jedoch in mehreren Punkten weiterentwickelt.
} 
Bezüglich Jacobs` Begleiterbindungsbeziehungen ist er der Ansicht, dass keine von ihnen „eine korrekte Präzisierung des Valenzbegriffs darstellt, wenn mehr zweifelsfreie Ergänzungen $^{97}$ (...) berücksichtigt werden“ (Fischer 2001:246). Die Relation der Argumentativität und die der inhaltlichen Spezifizität findet er jedoch „,bei geeigneter Fassung“ zur Trennung von zweifelsfreien Ergänzungen und Angaben tauglich. Im Zusammenhang mit dem multidimensionalen Valenzmodell der GDS hebt Fischer (2001:259) als wichtiges Positivum hervor, dass darin ,alle Dimensionen für die Beschreibung von Valenz relevant sind“. Dennoch sollte - seines Erachtens - verdeutlicht werden, dass die einzelnen Dimensionen nicht gleichberechtigt sind. Dabei plädiert er dafür, dass einzig die Dimension der Sachverhaltsbeteiligung geeignet sei, über den Ergänzungs- oder Angabestatus einer Konstituente zu beurteilen.

Dieser Gedanke führt schon zu Fischers (2001: 259ff.) Konzept einer „monokriterialen Abgrenzung von Ergänzungen und Angaben“ über, in dem Sachverhaltsbeteiligung als einzige Relation der Abgrenzung von Ergänzungen dient. Bezüglich der Sachverhaltsbeteiligung lenkt Fischer (2001:260f.) die Aufmerksamkeit darauf, dass diese Beziehung ,partiell indeterminiert" ist. Daraus folgt, dass in Fällen der Indeterminiertheit eine betreffende Konstituente sowohl als Ergänzung als auch als Angabe klassifiziert werden könne, wobei jedoch die Interpretation der Gesamtstruktur gleichbleibe. Als Beispiel für zweierlei analysierbare Konstituenten behandelt er die dativischen Dependentien in Sätzen wie „Ich trage ihr das Fahrrad in den Keller.“ oder „Mir ist die Vase zerbrochen.“ (Fischer 2001: 260; Hervorhebungen von Fischer). Er verweist in diesem Zusammenhang auch auf die GDS, der zufolge die Relation der Sachverhaltsbeteiligung für solche Konstituenten nicht gelte, wobei seines Erachtens - bei Annahme einer (gerade) in der GDS eingeführten „schwachen Sachverhaltsbeteiligung“ auch eine entgegengesetzte Entscheidung getroffen werden könne. Nichtdestotrotz findet er am wichtigsten, dass in Konstruktionen wie diese die Analyse der jeweiligen Konstituenten entweder als Ergänzung oder als Angabe keinen Einfluss auf die Interpretation des Satzes selbst habe. Ergänzend zu Fischers Überlegungen über die potenzielle Indeterminiertheit von Sachverhaltsbeteiligung soll noch angemerkt werden, dass er die Auffassung vertritt, dass diese Beziehung kein graduelles Phänomen sei: Entweder liegt sie vor oder nicht, oder sie ist indeterminiert.

\footnotetext{
${ }^{97}$ Im Rahmen seiner Arbeit betrachtet Fischer (2001: 241) als zweifelsfreie Ergänzungen „solche, die in gängigen Valenzwörterbüchern aufgeführt werden“. Dazu gehören - seines Erachtens - die Kasusergänzungen, darunter auch das Subjekt; präpositionale Ergänzungen, deren Präposition fest und inhaltsleer ist; adverbiale Ergänzungen (wie z. B. das Direktivum beim Verb fahren) und prädikative Ergänzungen.
} 
Mit der Modifizierung in Gegenstands- bzw. Qualitätskonstitution sieht Fischer (2001: 261) die Relation der Sachverhaltsbeteiligung auch auf Nomina und Adjektive anwendbar. Ferner findet er besonders wichtig, zwischen Aspekten der Valenzdefinition und der Valenzbeschreibung zu unterscheiden. Dementsprechend betrachtet er die Relation der Sachverhaltsbeteiligung als definitorisches Merkmal, während - seiner Ansicht nach - formale Charakteristika von Ergänzungen und Angaben zur Valenzbeschreibung gehören. Daraus folgt auch, dass er „Valenz im Grunde nur als etwas Semantisches“ deutet, „das allenfalls syntaktische Effekte hat" (Fischer 2001:265). Er stellt auch klar, dass die Idee der Sachverhaltsbeteiligung schon in der Dramenmetapher von Tesnière zu erkennen ist, wobei jedoch Fischer (2001:265) im Vergleich zu seinem eigenen Konzept als Unterschied hervorhebt, dass „Tesnière [..] [...] die Sachverhaltsbeteiligten als an bestimmte Ausdrucksformen gebunden an[sah]“.

Nachdem ich in diesem Unterkapitel mehrere linguistische Arbeiten, in denen das Problem der Unterscheidung zwischen Komplementen und Supplementen erörtert wird, kritisch und oft auch aus der Perspektive der Substativvalenz betrachtet habe, verbleibe ich auch im nächsten Unterkapitel beim Thema dieser Differenzierung, wobei ich jedoch solche Arbeiten oder Teile von Arbeiten unter die Lupe nehme, bei denen diese Problematik mehr oder weniger eingehend, aber speziell beim Substantiv behandelt wird.

\subsubsection{Das Problem der Unterscheidung zwischen Komplementen und Supplementen II.}

Die Frage der Unterscheidung zwischen Komplementen und Supplementen - wie es schon aus dem vorangehenden Unterkapitel ersichtlich werden sollte - ist ein zentrales, zugleich aber auch umstrittenes Thema in der Valenztheorie. Genauso ist es im Bereich der Substantivvalenz. Dieser Problematik kommt auch in Teuberts (1979) Monographie Valenz des Substantivs (1979) eine große Bedeutung zu. Er versucht sich bei der Abgrenzung von Ergänzungen und Angaben beim Substantiv vor allem an ,anwendungsorientierte[n] Gesichtspunkte zu orientieren“ (Teubert 1979: 34). Im Zusammenhang mit dem globalen Problem der Differenzierung zwischen Ergänzungen und Angaben vertritt er eine Ansicht, mit der auch ich weitgehend einverstanden bin: „Eine theoretisch allseits befriedigende Abgrenzung von Ergänzung und Angabe, sei es im verbalen oder nominalen Bereich, die gleichzeitig die 
syntaktischen Gegebenheiten und die Forderungen der Satzsemantik erfüllt, gibt es nicht.“ (Teubert 1979: 35) ${ }^{98}$

Wie auch Helbig darauf hinweist (1992: 116ff.), äußert sich Teubert (1979) im Bereich der Substantivvalenz dagegen, dass die syntaktischen Kriterien von Notwendigkeit und Subklassenspezifik aus der verbalen Valenzbeschreibung auf die nominale unverändert übertragen werden. Teubert (1979: 37) ist der Meinung, dass das Kriterium der Notwendigkeit - da es bei den Substantiven keine obligatorischen Ergänzungen gibt ${ }^{99}$ - im nominalen Bereich nicht funktionieren kann. Stattdessen schlägt er vor, bei der Abgrenzung von (fakultativen) Ergänzungen und Angaben zu kontrollieren, ob das vom Bezugssubstantiv abhängige Element „die Funktion einer Argumentstelle in einer durch den Nominalkomplex ausgedrückten Prädikation hat" (Teubert 1979: 38). Wenn dies der Fall ist, kann - seiner Ansicht nach - von einer Ergänzung gesprochen werden, sonst handelt es sich bei dem abhängigen Element um eine Angabe, die eine eigene Prädikation darstellt.

Man kann - meines Erachtens - mit Helbig (1992: 117) einverstanden sein, wenn er Teubert dafür kritisiert, dass er das Kriterium der Notwendigkeit „einseitig“ versteht. Helbig (1992: 117) formuliert diese einseitige Verstehensweise folgendermaßen: „Wegfall des Gliedes führt zur Ungrammatikalität“. Er betont auch, dass das ,,in dieser Einseitigkeit weder für das Verb noch für das Substantiv gilt"“. Dazu muss aber angemerkt werden, dass Teubert (1979: 35; meine Hervorhebung Á. S.-T.) selbst explizit das Kriterium nicht so einseitig formuliert, wenn er sagt: „Wird ein Satz durch das Weglassen eines Satzglieds ungrammatisch oder erhält er eine strukturell andere Bedeutung, so wird dieses Satzglied als Ergänzung bezeichnet". Bei den Beispielen aber, an denen er die Unverwendbarkeit des Unterscheidungskriteriums der Notwendigkeit demonstrieren will, hält er tatsächlich nur die Grammatikalität der Sätze vor Augen (vgl. Teubert 1979: 37).

Teubert (1979: 38ff.) hält schließlich das Kriterium der eigenen Prädikation und das der Subklassenspezifik als wegweisende Merkmale bei der Trennung zwischen Ergänzungen und Angaben fest. Aber er weist zugleich darauf hin, dass bei den Substantiven auch das Kriterium der Subklassenspezifik nicht völlig zuverlässig sei. Er betrachtet im Zusammenhang mit dem Problem der Verwendbarkeit der Subklassenspezifik zwei Fälle: Genitiv- und Präpositionalattribute.

\footnotetext{
${ }^{98}$ Der Akzent liegt dabei für mich auf „allseits“. Ferner finde ich - wie ich schon in Kap. 2.5.1 erklärt habe - die Verbindung von semantischen und syntaktischen Kriterien wichtig.

${ }^{99}$ Zumindest in seinem Konzept nicht. Zur weiteren Thematisierung dieser Frage vgl. unten Kap. 2.5.3.
} 
In Bezug auf die Genitivattribute erklärt er, dass z. B. der Genitivus possessivus in dieser Rolle ,[...] nicht spezifisch für eine bestimmte Subklasse von Substantiven [ist] und [...] somit als Angabe gewertet werden [muß]“ (Teubert 1979: 39). Es gibt dagegen Nominalphrasen, in denen ein Genitivattribut z. B. in der Rolle einer Agentivergänzung auftritt, wie in der erweiterten Nominalphrase Peters Brief, ${ }^{100}$ die als der Brief, den Peter geschrieben hat umschrieben werden kann. Ein weiteres Beispiel für eine genitivische Substantivergänzung ist der sächsische Genitiv in Peters Vater, der als Personenergänzung klassifiziert wird. Diese Entscheidung erklärt Teubert (1979: 112; meine Hervorhebung Á. S.-T.) ausgehend von der Bedeutung des Bezugssubstantivs: „Personenergänzungen hängen $\mathrm{ab}$ von relationalen Personenbezeichnungen, aus deren Bedeutung sich die jeweilige Spezifik der Beziehung ergibt".

Den zweiten Problemfall stellen - bezüglich des Kriteriums der Subklassenspezifik Teubert zufolge die Präpositionalattribute dar. Zuerst betrachtet er nur Ausdrücke, in denen solche Attribute als Angaben zu klassifizieren sind, wie z. B. in der Tisch aus Polen oder der Tisch mit einer Mahagonyplatte (sic) ${ }^{101}$. Zwar können solche Präpositionalattribute nicht uneingeschränkt mit beliebigen Substantiven vorkommen, aber die Bezugssubstantive, von denen sie abhängen können, sind - seiner Ansicht nach - nicht zu einer Substantivsubklasse zusammenzufassen. Deswegen bezeichnet er sie als Angaben. In Bezug auf die Präpositionalattribute stellt er nur so viel fest, dass die präpositional angeschlossenen Substantivangaben nicht subklassenspezifisch sind. Ohne weitere Thematisierung der Frage führt er jedoch unter den Ergänzungsklassen zahlreiche Beispiele für Präpositionalattribute als Ergänzungen auf (vgl. Teubert 1979: 88ff.).

Auf Grund der obigen Ausführungen bin ich der Meinung, dass nicht die von Teubert als Problemfälle der Bestimmung der Subklassenspezifik behandelten Attributstypen das eigentliche Problem darstellen, sondern der Subklassenspezifikbegriff selbst, der Teuberts Untersuchungen zugrunde liegt. Rein formal, d. h. morphologisch-syntaktisch betrachtet, sind tatsächlich weder Genitiv- noch Präpositionalattribute subklassenspezifisch. Wenn wir jedoch noch einen Aspekt in Betracht ziehen - wie Teubert dies im Zusammenhang mit den Personenergänzungen auch tut -, lassen sich diese Probleme relativ zuverlässig beseitigen. Dieser Aspekt ist die Semantik des Bezugssubstantivs und die seines Attributs: Wenn nämlich das Genitiv- oder Präpositionalattribut ein Argument des Substantivs realisiert, ist es als

\footnotetext{
${ }^{100}$ Beispiel von Teubert (1979: 38)

${ }^{101}$ Die Beispiele wurden Teubert (1979: 41) entnommen.
} 
Ergänzung zu bezeichnen. ${ }^{102}$ An dieser Stelle halte ich es für wichtig anzusprechen, dass durch diese Vorgehensweise der Subklassenspezifikbegriff - ähnlich dem in Kap. 2.5.1 schon thematisierten erweiterten Subklassenspezifikbegriff von Uzonyi (2004) - eine morphologischsemantische Fundierung erhält.

Betreffend das Problem der Genitivattribute finde ich hier einen kurzen Exkurs nötig. In Grammatiken werden nämlich verschiedene Typen des Genitivs unterschieden, bezüglich dieser Typen gibt es aber in der Fachliteratur keine Einheit. Das kann - meiner Meinung nach - dadurch erklärt werden, dass in den verschiedenen Darstellungen die semantischen Merkmale der Wörter unterschiedlich differenziert beachtet werden und so die einzelnen Kategorisierungen auf einigermaßen unterschiedliche Grundlagen aufbauen. Ich bin deswegen der Meinung, dass man bei der Thematisierung der Frage, wann ein Genitivattribut als Komplement $\mathrm{zu}$ betrachten ist, nicht darauf beharren sollte, den einzelnen Genitivtypen entweder Komplement- oder Supplementstatus zuzuweisen, sondern eher eine allgemein verwendbare Methode, wie z. B. die oben thematisierte, vorschlagen sollte, wobei die Beurteilung der Subklassenspezifik des jeweiligen Genitivattributs erst nach der Ermittlung der Argumente des Bezugssubstantivs erfolgt. ${ }^{103}$ Bei einer solchen Untersuchung der Genitive lassen sich tatsächlich verschiedene Typen des Genitivs ermitteln, unter denen es schon subklassenspezifische gibt. So wird ersichtlich, dass mit der Einbeziehung der semantischen Seite die Subklassenspezifik als ein Kriterium der Unterscheidung zwischen Komplementen und Supplementen auch bei Genitivattributen verwendet werden kann.

Es kann festgestellt werden, dass Teubert (1979) zwar versucht hat, die Kriterien zu klären, die für eine Unterscheidung zwischen Ergänzungen und Angaben des Substantivs entscheidend sein sollen, aber bei ihm ist schließlich der ausführlichen Beschreibung der einzelnen Ergänzungs- (vgl. Teubert 1979: 81ff.) und Angabeklassen (vgl. Teubert 1979: $145 f f$.) eine viel größere Rolle zugekommen. Im Zusammenhang mit Teuberts (1979) Klassifizierung der Ergänzungen, die von bestimmten Substantivsubklassen abhängen können, stellt Helbig (1992: 120) unter anderem Folgendes fest:

\footnotetext{
${ }^{102}$ In diesem Zusammenhang ist - meines Erachtens - Helbig (1992:117) zuzustimmen, der der Ansicht ist, dass das Kriterium der Subklassenspezifik und das von Teubert formulierte Kriterium der eigenen Prädikation „nur verschiedene Aspekte des gleichen Sachverhalts“ sind. Er führt dazu Folgendes aus: „Subkategorisierende Elemente gehören als Argumente zur semantischen Repräsentation des Substantivs, nicht-subkategorisierende Glieder dagegen (als eigene Prädikationen) nicht“" (Helbig 1992: 118).

Ferner ist noch Hölzners (2007: 152) Ansicht anzusprechen, wonach in Teuberts (1979) Kriterium der eigenen Prädikation die Grundidee der Argumenthaftigkeit zu erkennen ist.

${ }^{103}$ Mit diesem Vorschlag stelle ich keinesfalls die Existenz verschiedener Genitivtypen in Frage, sondern weise nur darauf hin, dass die teilweise uneinheitlichen Kategoriennamen und Kategorien nicht zur primären Grundlage der Thematisierung des Problems der Unterscheidung zwischen genitivischen Substantivkomplementen vs. -supplementen gemacht werden sollten.
} 
Bei den vorgestellten denominalen Ergänzungsklassen fällt (...) auf, daß es sich bei der Bezeichnung um ein Amalgam von Kasusetiketten (z. B. Agentiv, Objektiv), von semantischdenotativen Klassen (z. B. Personen-, Stoff-, Sachergänzung) und von Satzgliedumschreibungen (z. B. Gleichsetzung) handelt.

Dieser Feststellung ist - meines Erachtens - zuzustimmen. Die etwas uneinheitliche Systematik bei der Bezeichnungsverteilung lässt sich - in meinen Augen - teilweise dadurch erklären, dass bei der Analyse sowohl semantische als auch syntaktische Aspekte eine Rolle spielen sollen.

Obwohl er in seinem Buch 17 Ergänzungsklassen und 9 Angabeklassen voneinander unterschieden und detailliert beschrieben hat, äußert Teubert (1979: 42) betreffend die Lösung des Problemkreises seine Skepsis: „Weder die Einteilung der Attribute in Ergänzung und Angabe noch deren Abgrenzung sind in dieser Arbeit besonders originell und elegant gelöst worden“. Er vertritt aber die Ansicht, dass „(...) die extensionale Definition der Subklassen durch Aufzählung der Substantive, die diese bestimmte Ergänzungskategorie an sich binden können, unproblematisch [bleibt]“. Das zu verwirklichen ist - wie auch Teubert darauf hinweist - die Aufgabe eines Substantivvalenzwörterbuches.

Teubert (1979: 43) betont noch im Zusammenhang mit der Differenzierung zwischen Ergänzungen und Angaben beim Substantiv, dass auch auf diesem Gebiet - wie bei anderen Teilen der Sprachbeschreibung - „operationelle Kriterien nie ausreichen“. Er vertritt darüber hinaus eine Meinung, die der von mir in Kap. 2.5.1 schon geäußerten Auffassung entspricht. Danach wird bei den Argumentationen bezüglich der Bestimmung von Ergänzungen und Angaben eine „aufgeklärte Intuition“ 104 immer ein unentbehrliches Berufungselement bleiben.

Während bei Teubert (1979) eine eindeutig dichotomische Klassifizierung der vom Bezugssubstantiv abhängigen Elemente zu finden ist, gibt es in der GDS eine differenziertere Einteilung, wobei bei den Komplementen vom Kernbereich bis zur Peripherie mehrere Unterklassen unterschieden werden. Bei der Darstellung der Nominalphrase werden in der GDS zuerst durch Komplemente (GDS 1968ff., v. a. 1975ff.) und darauffolgend durch Supplemente erweiterte Nominalphrasen (GDS 1987ff.) beschrieben.

Im Rahmen der Ausführungen zur Unterscheidung zwischen Komplementen und Supplementen des Substantivs lenken die Verfasser die Aufmerksamkeit auf etwas - meines Erachtens - sehr Wichtiges: „(...) [hier $]^{105}$ [wird] die Darstellung von Syntax und Semantik

\footnotetext{
${ }^{104}$ Den Ausdruck ,,aufgeklärte Intuition“ übernimmt Teubert von Emons, der in Bezug auf die Valenzbeschreibung englischer Prädikatsverben festgestellt hat, dass man ,nicht ohne Berufung auf eine aufgeklärte Intuition auskommen können“ wird. (Zitat aus: Emons, Rudolf (1974): Valenzen englischer Prädikatsverben. Tübingen (= Linguistische Arbeiten 22), 105, zitiert nach Teubert (1979: 43)).

${ }^{105}$ Damit sind die Nominalphrase und der Bereich der Substantivvalenz gemeint.
} 
nicht getrennt, da semantische Überlegungen zur Argumentstruktur für die syntaktischen Ausführungen eine zentrale Rolle spielen“(GDS 1969; meine Hervorhebungen Á. S.-T.).

Darüber hinaus wird die Frage betrachtet, wie die für das Verb festgestellten Valenzrelationen und Tests ${ }^{106}$ im Bereich des Substantivs verwendet werden können (vgl. GDS 1969ff.). Hier ist es wichtig zu betonen, dass - ähnlich wie bei Teubert (1979) - auch in der GDS (1970) davon ausgegangen wird, dass unter Substantivattributen ,so gut wie keine obligatorischen Elemente“" anzunehmen sind. Daraus folgt, dass die Relation Fixiertheit bei substantivischen Valenzträgern entfällt. Die Verfasser modifizieren auch die Definitionen von Rektion ${ }^{107}$ und Konstanz, ${ }^{108}$ und bei den Bedeutungsrelationen heben sie die Wichtigkeit der Argumentselektion hervor, wobei sie der Ansicht sind, dass im nominalen Bereich noch die Relation der Sortenselektion ${ }^{109}$ ergänzend verwendet werden soll (vgl. GDS 1969f.).

Im Zusammenhang mit der Argumentselektion wird festgestellt, dass sie beim Substantiv nicht dichotomisch beurteilt werden kann. Die Verfasser unterscheiden drei theoretische Möglichkeiten bezüglich der Frage, inwiefern ein Attribut argumentselegiert ist. Von diesen drei theoretischen Möglichkeiten können sie zwei mit Belegen untermauern: Wenn das abhängige Element ,als ein bestimmtes Argument“ (GDS 1974) zu interpretieren ist, liegt ihrer Meinung nach - starke Argumentselektion vor, wie z. B. in Lust auf Erdbeeren. ${ }^{110}$ Den anderen belegbaren Fall bezeichnen sie als schwache Argumentselektion, bei der das betreffende Attribut , (...) neben einer oder mehreren Lesarten als Argument noch eine ,freie

\footnotetext{
${ }^{106} \mathrm{Zu}$ diesen Tests vgl. die Darstellung der Frage beim Verb in meiner Arbeit in Kap. 2.5.1.

${ }^{107}$ Der Substitutionstest, der in der GDS zur Ermittlung dieser Relation dient, wird folgendermaßen definiert: „Wird durch Änderung der Form, insbesondere des Kasus oder der Präposition, einer Konstituenten (sic) einer komplexen Nominalphrase (die nicht das Kopfnomen ist) die gesamte Phrase ungrammatisch, so ist diese Konstituente im starken Sinne regiert (und folglich ein nicht-peripheres Nomenkomplement)“"(GDS 1971). Der wichtigste Unterschied im Vergleich zur Interpretation der Rektion beim Verb liegt darin, dass beim Verb davon ausgegangen wird, dass bei der Rektion die Form des abhängigen Elements von einer Paradigmenkategorie des Verbs bestimmt wird (vgl. GDS 1034f.).

${ }_{108}$ Diese modifizierte Definition der Konstanz lautet wie folgt: „Ist ein Austausch der Präposition ohne Bedeutungsänderung oder bei gleicher Sortenselektion möglich, so ist die Präpositionalphrase im schwachen Sinne regiert (konstant)" (GDS 1971). Im Gegensatz dazu wird die Relation der Konstanz im verbalen Bereich unabhängig von der Bedeutungsseite betrachtet und so verstanden, dass die jeweilige Präposition beim betreffenden Verb durch eine Paradigmenkategorie dieses Verbs „unabänderlich“ determiniert ist (vgl. GDS 1035ff.).

${ }^{109}$ Diese Relation wird in der GDS (1970) im Sinne von Jacobs interpretiert, d. h. er „legt sortale Eigenschaften der Argumente fest“.

${ }^{110}$ Das Beispiel wurde der GDS (1974) entnommen.
} 
Lesart' [hat]" (GDS 1974), wie z. B. in der Nominalphrase Besuch der alten Dame ${ }^{111}$ (GDS 1974)..$^{112}$

Nach diesen Betrachtungen kommen die Verfasser der GDS (1974) auf die Feststellung, dass „nur stark argumentselegierte Nominalphrasen als Nomenkomplemente“ behandelt werden sollten, wobei zusätzlich auch die Sortenselektion als Unterscheidungskriterium fungieren kann. Die graduelle Art der Klassifizierung der Attribute in der GDS ist u. a. dadurch zu demonstrieren, dass z. B. bei der Untersuchung von Präpositionalphrasen fünf Klassen unterschieden werden, von denen zwei zu den Supplementen und drei zu den Komplementen gerechnet werden. Attribute, bei denen keine oder nur schwache Argumentselektion vorliegt, werden auch unter den Präpositionalattributen als Supplemente bezeichnet. ${ }^{113}$ Unter den Komplementen - bei denen, wie oben schon erwähnt, immer starke Argumentselektion gegeben sein muss - wird bei den peripheren Komplementen neben der Bedeutungsrelation der starken Argumentselektion kein weiteres Kriterium genannt, ${ }^{114}$ während bei halbzentralen Komplementen abgeschwächte Formselektion vorliegen und bei zentralen Komplementen die Formrelation von Konstanz bestehen muss. ${ }^{115}$

Bezüglich der Klassifizierung der Komplemente und Supplemente des Substantivs in der GDS sei hier schließlich noch die Einstufung von Ausdrücken wie z. B. Tochter reicher Eltern kurz angesprochen. Dieses Genitivattribut wird in der GDS (2030) als Genitivus auctoris gewertet und als Supplement eingestuft. Wie ich oben schon im Zusammenhang mit der Darstellung von Teuberts (1979) Klassifizierung angesprochen habe, gibt es die Möglichkeit, solche Genitiverweiterungen als Ergänzungen einzustufen. Der Genitivus in dem Ausdruck der Kopf des Politikers - gewertet als Genitivus possessoris - wird in der GDS (2030) ebenfalls den Supplementen zugerechnet. Bei solchen Genitiverweiterungen bin ich der Meinung, dass

\footnotetext{
${ }^{111}$ Beispiel aus der GDS (1974). Mit den Interpretationsweisen dieser Nominalphrase beschäftigen sich die Verfasser ausführlicher (vgl. GDS 1972ff.). Die oben zitierte Nominalphrase kann zwei solche Lesarten haben, in denen das Attribut als Argument (einmal als Genitivus subjectivus und einmal als Genitivus objectivus realisiert) zu interpretieren ist. Als ein Beispiel für eine freie Lesart wird die Umschreibung der Besuch des Kaisers in Rostock, von dem die alte Dame schwärmt dargestellt. Bei einer solchen Interpretation ist der Genitiv nicht als Argument des Substantivs Besuch zu deuten und stellt - in der GDS - dementsprechend ein Supplement dar.

112 Die dritte theoretische Möglichkeit, für die die Verfasser der GDS kein Beispiel finden konnten, wäre ihnen zufolge, dass das Attribut des Substantivs als „,irgendein Argument“ zu deuten ist, aber keine „freie“ Lesart hat (GDS 1974).

${ }^{113}$ Dafür gibt es folgende Beispiele in der GDS (1976): Skandal wegen der Affäre (keine Argumentselektion); Bild von Picasso, Einladung für Tanja (schwache Argumentselektion).

${ }^{114}$ Hier soll Folgendes angemerkt werden: Bei der Ermittlung von Präpositionalphrasen, die als Supplemente einzustufen sind, wird ihre Kommutierbarkeit mit als Supplemente klassifizierten Genitivphrasen vorgeschlagen. Ausdrücke wie Einladung an Tanja können zwar mit solchen Genitiven kommutiert werden, sie verfügen aber nur über eine objectivus-Lesart, was der GDS zufolge beweist, dass sie stark argumentselegiert sind. Sie werden deswegen nicht mehr zu den Supplementen, sondern zu den peripheren Komplementen gerechnet (vgl. GDS 1975). ${ }^{115}$ Es gibt u. a. folgende Beispiele für die einzelnen Unterklassen in der GDS (1977): Einladung an den Beirat (peripher); Ausflug nach Deidesheim (halbzentral); Befehl zum Antreten (zentral).
} 
mit Recht die Frage aufgeworfen werden kann, ob sie - im Gegensatz zu ihrer Klassifizierung in der GDS - als Komplemente betrachtet werden sollten: sie besetzen nämlich eine Argumentstelle des Substantivs. Diese Frage werde ich in Kap. 2.5.3 bei der Darstellung der Untersuchungen von Tamássy-Bíró (v. a. 1997 und 1998a; aber auch 1998b) bzw. in Kap. 2.6 bei der Erörterung meines multidimensionalen Konzepts zur Unterscheidung der Attribute noch einmal ansprechen.

Am Ende der Erörterungen zur Unterscheidung von Substantivkomplementen und supplementen in der Fachliteratur gehe ich noch auf die bedeutende Substantivvalenzmonographie von Hölzner (2007) ein, der die Stellung der Substantivvalenz innerhalb der Valenzforschung ähnlich kritisch bewertet wie ich. Dabei weist er auch auf Jacobs“ (1994) Einschätzung der früheren Lage der Valenzforschung als „Valenzmisere“ hin. Dabei ist er der Meinung, dass die dadurch intensiver gewordene Diskussion der Valenzforschung zwar nur genutzt hat, der Beschreibung der Substantive jedoch immer noch nicht genug Aufmerksamkeit zuteilwird. Er sieht dies z. T. dadurch begründet, dass die Substantivvalenz von vielen als etwas Sekundäres betrachtet wird, was aus den Valenzeigenschaften der (entsprechenden) Verben und Adjektiven regelhaft ableitbar wäre und dementsprechend nicht zusätzlich untersucht werden sollte. Als weiteren Kritikpunkt an der Substantivvalenzforschung - darunter auch an den meisten lexikographischen Arbeiten in diesem Bereich - führt er auf, dass ein Großteil der Ausführungen nicht (ausreichend) korpusbasiert fundiert ist, wodurch ihre Aussagekraft infrage gestellt werden kann bzw. sollte (vgl. Hölzner 2007: v. a. 108).

Hölzner (2007: 111ff.) teilt ferner Jacobs“ (1994) Auffassung, nach der aus der Valenzmisere multidimensionale Modelle einen Ausweg bieten können. Solche Beschreibungsansätze sind aber in Abhandlungen über die Substantivvalenz - mit Ausnahme der GDS - kaum anzutreffen. In Anlehnung an Jacobs‘ (2003) späteren Beitrag über [d]ie Problematik der Valenzebenen baut Hölzner sein multidimensionales Substantivvalenzkonzept auf den zwei semantischen Valenzdimensionen Argumenthaftigkeit und Inhaltsspezifik und auf den zwei syntaktischen (kategorialen) Valenzdimensionen syntaktische Notwendigkeit und Formspezifik auf, wobei er - meines Erachtens absolut begründet ${ }^{116}$ - die Auffassung vertritt, dass sie „größtenteils für die Anwendung im Bereich der Substantivvalenz expliziert und modifiziert werden müssen“ (Hölzner 2007: 113).

\footnotetext{
${ }^{116}$ Vgl. dazu auch meine kritische Darstellung von Jacobs` (2003) betreffendem Beitrag in Kap. 2.5.1, in deren Fokus jedoch nicht gerade die einzelnen Valenzdimensionen stehen, sondern Jacobs ${ }^{`}$ Annäherung der Frage der Unterscheidung von Komplementen und Supplementen.
} 
Ohne hier Hölzners Konzept detailliert darstellen zu wollen, sollen hier die aus der Sicht meiner Untersuchung wichtigsten Züge seiner Arbeit angesprochen werden. Ihm zufolge können nicht nur abgeleitete Substantive über Valenz verfügen, für ihn ist aus dieser Sicht vielmehr die von Behaghel (1923) vorgenommene Unterscheidung in ,absolute“ und „relative“ Substantive ausschlaggebend, da dadurch, dass relative Substantive „Argumentstellen eröffnen“ (Hölzner 2007: 153), diese auch als Valenzträger zu bezeichnen sind. Aus dieser Folgerung wird deutlich, dass Hölzner die Argumenthaftigkeit als ausreichendes Kriterium für die Zuweisung des Komplementstatus betrachtet.

Ferner beschreibt er fünf Stufen der Formspezifik, wobei die erste Stufe Konstruktionen charakterisiert, in denen das valente Substantiv idiosynkratisch die Form seiner Erweiterung bestimmt $^{117}$, während die fünfte Stufe als entgegengesetzter Pol des Kontinuums für Fälle steht, in denen das valente Substantiv keinen Einfluss auf die Form seiner Erweiterung hat (vgl. Hölzner 2007: 143ff.). Entsprechend seinen Grundlagen in Jacobs“ (1994 und 2003) multidimensionalem Valenzmodell, geht Hölzner (2007: 146ff.) in seinem Substantivvalenzkonzept von der Autonomie der oben aufgeführten vier Dimensionen aus, wobei er auch die „Implikationsbeziehungen NOT $\rightarrow$ ARG, FOSP $\rightarrow$ ARG, INSP $\rightarrow$ ARG“ zwischen den betreffenden Dimensionen annimmt (Hölzner 2007: 147).

Es soll noch hervorgehoben werden, dass Hölzner (2007: v. a. 163ff. bzw. 285ff.) die Argumentrealisierungen in zwei große Gruppen, nämlich in intra- und transphrastische, einteilt. Dabei gehören zur ersteren Gruppe die Komplemente, die innerhalb der Nominalphrase realisiert werden, während mit letzteren die Argumentrealisierungen gemeint sind, die entweder als „Partnerwort im Satz“ oder außerhalb des jeweiligen Satzes im Kontext anzutreffen sind. Das bedeutet zugleich, dass alle transphrastisch realisierten Argumente zur fünften Stufe der Formspezifik gehören, d. h., dass ,[i] $]$ hre Form [...] [...] sich dem Rektionspotential des Bezugssubstantivs entzieht“ (Hölzner 2007: 146). Die Betrachtung von Substantivkomplementen außerhalb des jeweiligen Satzes, in dem der Valenzträger realisiert wird, ist ein selten praktiziertes Verfahren in der Fachliteratur, was - meines Erachtens größtenteils durch die obige Feststellung erklärt werden kann, da Elemente, die nicht unter dem Rektionspotential des betreffenden Valenzträgers stehen, zumindest syntaktisch gesehen nicht zur Valenz gerechnet werden.

Nach dieser kritischen Darstellung verschiedener Ansätze zur Differenzierung zwischen Substantivkomplementen und -supplementen thematisiere ich im nächsten Unterkapitel noch

${ }^{117}$ Hölzner (2007: 143) führt Appetit auf als Beispiel dafür auf. 
eine besondere Frage bezüglich der substantivischen Komplemente, indem ich Ansätze zu der Problematik erörtere, ob beim Substantiv obligatorische Komplemente angenommen werden können oder nicht.

\subsubsection{Obligatorische Komplemente beim Substantiv?}

In den vorangehenden Kapiteln habe ich schon unterschiedliche Annahmen bezüglich der Obligatorik der Substantivkomplemente angesprochen. Ich finde es wichtig hervorzuheben, dass es in den obigen Arbeiten fast ausnahmslos keine längere Thematisierung dieser Frage gibt, ${ }^{118}$ sie beschränken sich im Allgemeinen auf eine kurze, nicht weiter erörterte Stellungnahme.

So stellt Teubert (1979:37) bei der Demonstration dessen, warum ,die Probe der Weglassbarkeit“ beim Substantiv problematisch ist, Folgendes fest: „Die Valenz der Substantive kennt obligatorische Ergänzungen nicht (... “). Auch in seinem HSK-Beitrag ist die gleiche - wenn auch differenzierter formulierte - Auffassung von Teubert (2003: 828) zu finden: „Es hat sich (...) bewährt, Obligatorik bzw. Fakultativität strikt im Hinblick auf syntaktische Wohlgeformtheit zu definieren. In diesem Sinne sind Nominalergänzungen prinzipiell fakultativ“، Ich bin jedoch - im Gegensatz zu dieser Argumentation - der Meinung, dass syntaktische und semantische Valenzaspekte nicht so streng voneinander getrennt werden dürfen. ${ }^{119}$

Auch Engel (2009b: 90f.) beschränkt sich im Zusammenhang mit der Obligatorikfrage der Substantivergänzungen auf eine kurze Stellungnahme, wonach das Nomen - mit Ausnahme des Determinativs, das er nämlich als Angabe betrachtet - über keine obligatorischen Satelliten verfügt. Einer ähnlichen Auffassung begegnen wir auch in Engels (2009a: 287) Grammatik, in der er Ergänzungen des Nomens grundsätzlich als fakultativ bezeichnet.

Wie ich oben in Kap. 2.4.3 schon thematisiert habe, sieht Eisenberg (2006: 268) einen der Beweisgründe gegen die Annahme einer Substantivvalenz sui generis darin gegeben, dass ihm zufolge „eine Unterscheidung zwischen fakultativen und obligatorischen Attributen nicht möglich ist". Ebenfalls in Kap. 2.4.3 habe ich angesprochen, dass Helbig / Buscha (2018: 528f.)

\footnotetext{
118 Wie sich unten in diesem Kapitel noch zeigen wird, kann als einzige Ausnahme davon Hölzners (2007) Monographie der Substantivvalenz betrachtet werden, in der es im theoretischen Teil ebenfalls keine eingehenden Ausführungen zu dieser Frage gibt, dafür aber wird sie im korpusanalytischen Teil durchgehend und vielseitig untersucht.

${ }^{119}$ So wird diese Frage u. a. auch in multidimensionalen Valenzmodellen, wie z. B. in dem der GDS gesehen (vgl. dazu oben Kap. 2.5.1 und 2.5.2).
} 
die Substantivvalenz ähnlich wie z. B. Eisenberg als eine sekundäre Erscheinung darzustellen suchen, und an einigen Punkten - in meinen Augen - vage Einschränkungen treffen, so auch in Bezug auf die Frage der Obligatorik von Substantivergänzungen. Sie geben nämlich an, „dass die Aktanten des Substantivs in der Regel fakultativ, nicht obligatorisch sind“ (Helbig / Buscha 2018: 529; meine Hervorhebung Á. S.-T.), gehen aber weder in Form von Beispielen, noch im Rahmen weiterer Ausführungen näher auf die Problematik ein.

Einen ähnlichen Standpunkt vertreten die Verfasser der GDS (1968ff.), die jedoch betreffend die Ausnahmen schon konkreter formulieren. Sie erörtern nämlich bei der Betrachtung der Frage, wie die für das Verb entwickelten Valenzrelationen und Tests beim Substantiv zur Geltung kommen können, die Problematik, dass es bei substantivischen Valenzträgern wenig Sinn macht, einen Reduktionstest als Unterscheidungsverfahren zu definieren: „Ein Reduktionstest im Bereich der Nominalphrasen zeigt lediglich, daß sich hier so gut wie keine obligatorischen Elemente finden“ (GDS 1970). Nach der Meinung der Verfasser gelten ausschließlich die „obligatorischen Zahlattribute in Maß- und Behälterkonstruktionen“120 als Ausnahmen von dieser Feststellung (GDS 1970).

Eine andere Grundauffassung als die obigen finden wir bei Sandberg (1979), dessen Arbeit in Bezug auf die Obligatorik der substantivischen Ergänzungen in der Fachliteratur oft thematisiert wird. Er geht nämlich davon aus, dass nicht-lexikalisierte ${ }^{121}$ Verbalsubstantive auch obligatorische Ergänzungen haben können, denn die von ihnen eröffneten Leerstellen entsprechen den Leerstellen beim Basisverb (vgl. z. B. Sandberg 1979: 5; auch 25).

Sandberg (1979) beschäftigt sich auch mit den Realisierungsmöglichkeiten der (obligatorischen) Ergänzungen. Er vertritt dabei die Meinung, dass der Kontext ebenfalls in die Analyse miteinbezogen werden soll. Im Zusammenhang damit erklärt er, dass die von ihm untersuchten Ergänzungen - d. h. Agens und Patiens - nicht nur als Genitivattribute oder Possessivpronomina ausgedrückt werden können (vgl. Sandberg 1979: 6f). ${ }^{122}$ Es gibt nämlich Fälle, in denen die Ergänzung zwar in demselben Satz wie das Substantiv, aber durch ein Partnerwort, d. h. nicht innerhalb der Nominalphrase, realisiert wird. ${ }^{123}$ Darüber hinaus kann es auch vorkommen, dass die Ergänzung erst im vorangehenden Kontext realisiert wird. ${ }^{124}$ Auf

\footnotetext{
${ }^{120}$ Wie z. B. das Zahlattribut in drei Glas Honig. Zur detaillierten Beschreibung solcher Konstruktionen vgl. die GDS (1979ff.).

${ }^{121}$ Sandberg (1979: 5) nennt die nicht-lexikalisierten Verbalsubstantive auch reverbalisierbare Verbalsubstantive.

${ }^{122}$ Bei Sandberg (1979) werden die Realisierungen von Agens und Patiens getrennt untersucht, wobei auch einige kleinere Unterschiede festgestellt werden, auf die hier jedoch nicht eingegangen wird.

${ }^{123}$ Ein Beispiel dafür an einer späteren Stelle bei Sandberg (1979: 29f.; meine Hervorhebungen Á. S.-T.) ist der folgende Satz: Motor nach dem Anspringen etwa 3-5 Sekunden laufen lassen.

${ }^{124}$ Als Beispiel dafür kann von einer späteren Stelle bei Sandberg (1979: 49; meine Hervorhebungen Á. S.-T.) Folgendes dargestellt werden: Hast du schon die Bilder vom Urlaub gezeigt? Ich bin ja gerade beim Zeigen.
} 
Grund der Art und Weise der Realisierung der obligatorischen Ergänzungen unterscheidet Sandberg (1979: 52f.) zwischen Direktobligatorium und Fernobligatorium. Dabei meint das erstere die Realisierungsnotwendigkeit innerhalb der Nominalphrase des Bezugssubstantivs in Form eines Genitivattributs, das zweitere dagegen die Realisierungsnotwendigkeit im vorhergehenden Kontext. Wie auch bei Helbig (1992: 115f.) angesprochen wird, steckt hinter dem Ausdruck des Direktobligatoriums eigentlich das Wesen der obligatorischen Ergänzungen, die demnach also auch bei einigen Substantiven vorhanden sein sollen. Ich bin der Meinung, dass die von Sandberg (1979) Direktobligatorium genannte obligatorische Ergänzung sowohl semantisch als auch morphologisch-syntaktisch als obligatorische Ergänzung des jeweiligen Substantivs betrachtet werden kann, während das sog. Fernobligatorium nur als semantisch obligatorisch zu bewerten ist, deren Realisierung aber in ihren morphologischen Eigenschaften vom „Bezugssubstantiv“ (weithegend) unabhängig ist.

Sommerfeldt / Schreiber (1977: 18) sind der Ansicht, dass Obligatorik der valenzbedingten Dependentien unterschiedlich fassbar ist. Wenn sie syntaktisch $\mathrm{zu}$ verstehen ist, können Substantive - ihnen zufolge - ,fast nur“ fakultative Komplemente haben, wobei sie nicht näher darauf eingehen, welche Substantive die Ausnahmen darstellen und über syntaktisch obligatorische Aktanten verfügen. Dies ist für sie jedoch auch weniger wichtig, da sie dafür plädieren, Obligatorik anders $\mathrm{zu}$ interpretieren. Ihres Erachtens sollten nämlich alle Komplemente als obligatorisch bezeichnet werden, durch deren Weglassung die Bedeutung(svariante) des jeweiligen Valenzträgers beeinflusst wird.

Wie bereits in Kap. 2.4.3 erörtert, scheint Wolf (1982: 55ff.) auch bei valenten Substantiven davon auszugehen, dass sie nicht nur sinn-, sondern sogar syntaktisch notwendige Adjunkte haben können, wodurch in seinem Ansatz auch beim Substantiv obligatorische Komplemente etabliert werden.

Die Idee von obligatorischen Substantivkomplementen lehnt auch Hölzner (2007: 154) theoretisch nicht $a b$, und fokussiert in seinen Korpusanalysen u. a. auf diese Frage. Dabei überprüft er die Valenzeigenschaften von mehreren Substantiven, die als Beispiele in der Fachliteratur zur Untermauerung bestimmter Argumentrealisierungseigenschaften gebracht wurden, darunter auch welche von Sandberg (1979) zur Demonstration der Obligatorik von Ergänzungen deverbaler Substantive (vgl. Hölzner 2007: 169ff., v. a. 196ff.). Eine wesentliche Konklusion seiner korpusanalytischen Untersuchungen ist, dass die Argumentrealisierungen von Substantiven so große Selbstständigkeit zeigen, dass weder Ansätze, wie der Nominalisierungs- oder der Stützverbgefügeansatz, noch introspektive Annahmen von Untersuchenden geeignet sind, sie zuverlässig zu prognostizieren (vgl. Hölzner 2007: 304). 
In Bezug auf die Obligatorik von Substantivergänzungen gibt es - meines Erachtens - zwei wichtige Folgerungen bei ihm. Einerseits vertritt er - auf Grund der Ergebnisse seiner Korpusanalysen - die Auffassung, dass ,[e]tliche Realisierungswerte bestimmter Argumente [...] sehr hoch [sind], und zwar in der Tat ähnlich hoch wie die Realisierungswerte von obligatorischen Ergänzungen bei Verben“ (Hölzner 2007: 309). Andererseits rät Hölzner (2007: 309ff., Zitat von 311) zum Umdenken der Frage der Obligatorik von Substantivargumenten, da ,... Substantive ihre einzelnen Argumentstellen in unterschiedlichem Maße ins Spiel zu bringen und nahezu ausnahmslos eine bestimmte Argumentstelle zu fokussieren [scheinen]" und diese Fokussierung die Wahrscheinlichkeit der intraphrastischen Realisierung der jeweiligen Argumente erhöht.

Ich halte es für wichtig an dieser Stelle auch die Arbeit von Bassola / Bernáth (1998) zu behandeln, weil sie sich darin - scheinbar genauso wie Sandberg (1979) - ebenfalls mit deverbalen Substantiven und ihren Valenzrealisierungseigenschaften beschäftigen. Bassola / Bernáth (1998: 173) definieren in diesem Artikel „deverbal im weiteren Sinne“, sie betrachten dementsprechend alle Substantive, die „mit Verben verwandt sind“. Diese „Verwandtschaft“ besteht entweder darin, dass sie aus Verben gebildet sind oder dass „Verben aus ihnen gebildet werden können“. Die Verfasser haben auch die Frage im Auge, was von der Valenzstruktur der untersuchten deutschen und ungarischen deverbalen Substantive im Satz, im Text immanent oder nicht einmal immanent im Text ausgedrückt wird.

Bezüglich des Problemkreises der Obligatorik von Substantivergänzungen fassen Bassola / Bernáth (1998: 195) ihre Ergebnisse wie folgt zusammen: „Die Untersuchungen haben (...) gezeigt, daß die Ergänzungen des Substantivs nicht obligatorisch sind; oft sind sie dem Text auch immanent nicht zu entnehmen“. Diese Annahme scheint mit der oben dargestellten Auffassung von Sandberg (1979) in Widerspruch zu stehen. Dies ist aber - wie ich es sehe - nicht der Fall. Vielmehr bin ich der Meinung, dass - obzwar Bassola / Bernáth (1998) den Begriff deverbal im weiteren Sinne verstehen - der von den Verfassern betrachtete Untersuchungsrahmen Substantive, bei denen Sandberg (1979) obligatorische Ergänzungen annimmt, dennoch nicht erfasst. All die präsentierten authentischen Beispiele, die von Verben abgeleitete Substantive darstellen, sind nämlich - meiner Ansicht nach - lexikalisierte Ableitungen, die auch Sandberg (1979) zufolge nicht über obligatorische Ergänzungen verfügen.

Wie schon bei der Darstellung der Bewertung der Obligatorikfrage in der GDS oben angedeutet wurde, kann auch bei nicht abgeleiteten Substantiven über die Obligatheit der Substantivkomplemente gesprochen werden. Dort wurde das Zahlattribut bei Maß- und 
Behälterkonstruktionen als obligatorisch bezeichnet. Auch Tamássy-Bíró (1998b) weist darauf hin, dass Zahlattribute in Verbindung mit Maßausdrücken oder Maßeinheiten als obligatorische Valenzpartner betrachtet werden. Darüber hinaus werden bei Tamássy-Bíró (v. a. 1997 und 1998a; aber auch 1998b) ${ }^{125}$ „einige besondere ungarische Substantivsubklassen“ behandelt, die über „nichteliminierbare morphologische Ergänzungen“ verfügen. Dabei ist sie bemüht zu zeigen, „daß es sich um Substantivsubklassen handelt, deren Entsprechungen auch in der deutschen Valenzliteratur als valent (...) ausgewiesen wurden“ (Tamássy-Bíró 1998a: 318). Im Zusammenhang mit dem Begriff der morphologischen Ergänzungen weist Tamássy-Bíró (1998a: 319ff.) darauf hin, dass die Valenzrealisierung auf zwei Ebenen beschrieben werden kann. Mikrovalenz bedeutet dabei die Realisierung der Valenzpotenz auf morphologischer Ebene, mit Makrovalenz wird die Realisierung der Valenzpotenz auf syntaktischer Ebene bezeichnet. ${ }^{126}$ Die Besonderheit der von Tamássy-Bíró behandelten ungarischen Substantivsubklassen liegt darin, dass ihre Vertreter über keine „absolute Form“ verfügen, weil das ungarische Possessivum -(j)a, -(j)e schon Teil ihrer Grundform geworden ist. Damit weisen sie „einen nicht eliminierbaren Erstaktanten auf Mikroebene“ auf (Tamássy-Bíró 1998a: 324).

Hier geht es nicht darum, alle Substantivsubklassen einzeln darzustellen, vielmehr sollen die aus der Sicht meiner Arbeit wesentlichsten Gedanken von Tamássy-Bíró erörtert bzw. eventuelle Problemfragen behandelt werden. Bei den beschriebenen Substantivsubklassen gibt es neben verbalen Ableitungen auch solche, die nicht aus abgeleiteten Substantiven bestehen. Unter ihnen bilden relationale Verwandtschaftsbezeichnungen, wie z. B. Vater (apa), Mutter (anya), Tochter (lány $\underline{a}^{127}$ ) eine der Gruppen. Wie auch Tamássy-Bíró (1998a: 331) die Aufmerksamkeit darauf lenkt, kann „der obligatorische Mikropossessor“ nicht bei allen dieser Substantive erwiesen werden. So ist z. B. beim Substantiv apa (Vater) in der Grundform des Wortes das Possessivsuffix nicht präsent, während es bei fia (Sohn sein/ihr) schon in der Grundform des Wortes realisiert ist. Dementsprechend kann - meines Erachtens - bei den beiden Typen nicht in gleichem Maße und in gleicher Weise über Obligatorik der Ergänzung gesprochen werden. Ich glaube deswegen, dass es bezüglich des Ungarischen sinnvoller wäre, eine graduelle Unterscheidung unter diesen Typen vorzunehmen. Bei den deutschen

\footnotetext{
${ }^{125}$ In Zusammenhang mit Tamássy-Bíró (1997 und 1998a) soll hervorgehoben werden, dass die beiden Artikel eigentlich dasselbe Thema umfassen, aber in Tamássy-Bíró (1998a) die ungarischen und deutschen Substantive von Anfang an parallel behandelt werden. Deswegen nehme ich diesen Artikel als Wegweiser bei der Darstellung ihrer wichtigsten Feststellungen.

${ }^{126} \mathrm{Zu}$ den Begriffen von Mikro- und Makrovalenz vgl. z. B. László (1988) und Ágel (2000: 220ff.).

${ }^{127}$ Bei den ungarischen Ausdrücken markiere ich (auch im Folgenden) das realisierte Possessivsuffix durch Unterstreichung.
} 
Entsprechungen tauchen - meiner Ansicht nach - solche Unterschiede nicht auf, was eine solche Differenzierung bei ihnen überflüssig erscheinen lässt.

Ebenfalls nicht abgeleitete Substantive gehören zu der Substantivsubklasse, deren Vertreter ein Teil-Ganzes-Verhältnis ausdrücken (vgl. Tamássy-Bíró 1998a: 332ff.). Bei diesen Substantiven kommt das Possessivum im Ungarischen wiederum schon in der Grundform des Wortes vor. Zu dieser Gruppe gehören z. B. széle (Rand sein/ihr), eleje (Vorderteil sein/ihr). ${ }^{128}$

Bei den Verbalsubstantiven, die zu den von Tamássy-Bíró behandelten Substantivsubklassen gehören, stellt sie fest, dass durch das mikrovalenziell realisierte ungarische Possessivsuffix entweder das Agens oder der Träger eines Vorgangs bezeichnet wird (Tamássy-Bíró 1998a: 335). ${ }^{129}$ Während so bei diesen Verbalsubstantiven weniger die Frage aufgeworfen werden kann, ob sie tatsächlich Ergänzungen realisieren, merkt TamássyBíró (1998a: 331) bezüglich der oben schon angesprochenen nicht-abgeleiteten Substantive (wie z. B. lánya - Tochter ihre/seine) Folgendes an: „Nach der Auffassung von U. Engel würden diese Genitivattribute in die Klasse Genitivus possessivus gehören und somit nicht als Ergänzung, sondern als Angabe zum Nomen klassifiziert“. ${ }^{130}$

Bei ihren zusammenfassenden Gedanken stellt Tamássy-Bíró (1998a: 336) fest, dass alle behandelten ungarischen Substantivsubklassen deutschen Substantivgruppen entsprechen, „denen die Valenzliteratur schon von Anfang an das Prädikat ,valent' zugeschrieben hat“. Die Entsprechungen zwischen den ungarischen Substantiven und ihren deutschen Äquivalenten erklärt sie mit der Valenzpotenz der einzelnen Substantive, „die sich in den beiden Sprachen unterschiedlich realisiert“ (Tamássy-Bíró 1998a: 336). Diesbezüglich vertrete ich eine teilweise andere Auffassung, wonach man auch bei der Valenzpotenz dieser Ausdrücke Differenzen annehmen könnte, die sich auf die morphosyntaktischen Relationen beziehen. So gehe ich davon aus, dass es eine semantische Seite der Valenz gibt, die bei äquivalenten Ausdrücken universeller Geltung ist, zu der aber entsprechend den einzelsprachlichen Besonderheiten andere morphosyntaktische Valenzpotenzen und Valenzrealisierungen gehören können.

Nach dieser Darstellung unterschiedlicher Ansätze zur Beurteilung der Obligatorik von Substantivkomplementen verbleibe ich auch im nächsten Unterkapitel immer noch bei der

\footnotetext{
${ }^{128}$ Beispiele von Tamássy-Bíró (1998a: 332ff.).

129 So drückt z. B. in felkelte (Aufstehen sein/ihr) das Possessivsuffix das Agens aus (vgl. Tamássy-Bíró 1998a: 335).

${ }^{130}$ An dieser Stelle verweise ich auf meine Argumentation bezüglich der Beurteilung des Komplementstatus von Genitiv- und Präpositionalattributen in Unterkapitel 2.5.2, wo ich dafür plädiert habe, dass auch diese - oft als nicht-subklassenspezifisch und damit als Supplemente eingestufte - Attribute ggf. als Komplemente zu klassifizieren sind.
} 
Frage der Substantivkomplemente. Dabei stelle ich die Problematik in den Mittelpunkt, wie sie in verschiedenen Arbeiten bezeichnet bzw. klassifiziert werden.

\subsubsection{Zur Nomenklatur der Substantivkomplemente in unterschiedlichen linguistischen Arbeiten}

Es gehört schon zur Problemstellung, dass zwar in der Überschrift des Kapitels Substantivkomplemente angesprochen werden, diese Erweiterungen des Substantivs in vielen der untersuchten Arbeiten jedoch gar nicht Komplemente genannt werden. Diese, in der Valenztheorie allgemein herrschende, terminologische Vielfalt habe ich bereits in Kap. 2.2 angesprochen. An dieser Stelle ist in erster Linie von Belang, wie viele und welche Subklassen der valenzbedingten Substantiverweiterungen in den einzelnen Ansätzen beschrieben werden und in welchem Maße die Bezeichnungen der einzelnen Klassen Hinweise auf die jeweilige theoretische Grundlegung ihrer Klassifizierung bieten.

Bei der Beschreibung der Aktanten bestimmen Sommerfeldt / Schreiber (1977: 37f.) die morphologische Form und die „[s]emantische Beschaffenheit der Aktanten“. Das heißt, dass bei ihnen keine Aktantenklassen an sich erkannt werden können, und somit nur von der Nomenklatur der Attributarten, die Aktanten realisieren, geredet werden kann. Wie den Bezeichnungen Substantive im Genitiv, präpositionale Substantive, Possessivpronomina, relative Adjektive, Infinitivgruppen und Nebensätze entnommen werden kann, ist diese Nomenklatur eindeutig formal, morphologisch-syntaktisch motiviert.

Teubert (1979: 81ff.) bestimmt in seiner Valenzmonographie 17 „denominale ${ }^{131}$ Ergänzungsklassen“, die er sowohl semantisch als auch morphologisch-syntaktisch charakterisiert und unterschiedlichen Klassen der Bezugssubstantive zuordnet. Wie bereits in Kap. 2.5.2 erörtert, hat Helbig (1992: 120; meine Hervorhebungen Á. S.-T.) in Bezug auf diese Ergänzungsklassen festgestellt, dass sie ,ein Amalgam von Kasusetiketten ${ }^{132}$ (z. B. Agentiv, Objektiv), von semantisch-denotativen Klassen (z. B. Personen-, Stoff-, Sachergänzung) und von Satzgliedumschreibungen (z. B. Gleichsetzung)“ darstellen. Dies kann - meiner Meinung nach - teilweise dadurch begründet werden, dass Teubert (1979) zur Unterscheidung von Ergänzungen von Angaben sowohl logisch-semantische, als auch formale Aspekte betrachtet (zur Beschreibung davon vgl. Kap. 2.5.2). Ferner ist von Belang, dass er bei den einzelnen

\footnotetext{
${ }^{131}$ D. i. in Teuberts (1979) Terminologie vom Substantiv abhängige.

132 Dabei halte ich es für sehr wichtig zu betonen, dass Kasus hier nicht im Sinne von morphologischem Kasus, sondern in dem von Tiefenkasus verwendet wird.
} 
Ergänzungsklassen ihre „Aktualisierungsarten“ (d. h. Realisierungsformen) zusätzlich aufführt und (dementsprechend) darum bemüht $\mathrm{zu}$ sein scheint, in den Bezeichnungen der Ergänzungsklassen keine formalen Aspekte zur Geltung kommen zu lassen.

Was die Charakterisierung der Komplemente von Substantiven in der GDS (1968ff.) betrifft, fällt ins Auge, dass dort deutlich weniger Komplementklassen festgelegt werden und dass ihr System auch wesentlich weniger leicht überschaubar dargestellt wird als im verbalen Bereich. ${ }^{133}$ Insgesamt können 4 große Komplementklassen der Substantive erkannt werden, deren Bezeichnungen formale Merkmale widerspiegeln: Genitivphrasen als Komplemente, Präpositionalphrasen als Nomenkomplemente, Nebensätze als Nomenkomplemente und Numerativkonstruktionen.

Engel (2009a: 289) teilt Satelliten des substantivischen Kopfes der Nominalphrase erstens aufgrund ihrer Stellung in zwei große Gruppen ein, und zwar in Satelliten, die im linken Feld vorkommen und in Satelliten des rechten Feldes. Bei der Beschreibung der beiden Felder werden Ergänzungen und Angaben nicht gesondert aufgelistet, sondern bei jedem Satelliten wird erörtert, ob er als Angabe oder als Ergänzung einzustufen ist. Beim Überblick der Satelliten des linken Feldes können in Engels Klassifizierung der Genitivus subiectivus, der Genitivus obiectivus, das Nomen invarians und das Nomen varians ${ }^{134}$ als Ergänzungen herausgefiltert werden. Unter den Satelliten des rechten Feldes bezeichnet er präpositive Attribute, direktive Attribute, expansive Attribute, nominale Attribute und verbative Attribute als Ergänzungen und fügt dieser Gruppe noch Relativsätze und Genitivattribute ${ }^{135}$ hinzu, da sie sowohl Ergänzungen als auch Angaben realisieren können (vgl. Engel 2009a: 293). Wenn wir die als Ergänzungen bezeichneten Satelliten in den beiden Feldern zusammenrechnen, ergibt das insgesamt 13 Ergänzung(sklass)en. Ein Teil der Bezeichnungen ist eindeutig morphologisch motiviert, wobei dies bei einigen - in meinen Augen - nicht so leicht zu

\footnotetext{
${ }^{133}$ Bei der Beschreibung der Valenz spielen in der GDS (1030ff.) - entsprechend ihrem multidimensionalen Valenzkonzept - bei der Bestimmung der Komplemente sowohl Form- als auch Bedeutungsrelationen eine Rolle. Die Bezeichnungen der verbalen Komplementklassen, von denen es insgesamt 11 in der GDS gibt, haben die Autoren so gewählt, damit sie „als Merkhilfen genutzt werden können“ (GDS 1073). Dabei scheinen in einigen Fällen ihrer Benennung morphologische (vgl. Akkusativkomplement, Dativkomplement, Genitivkomplement, Präpositivkomplement), in anderen syntaktische (vgl. Subjekt, Prädikativkomplement, AcI-Komplement, Verbativkomplement), in wiederum anderen semantische Aspekte (vgl. Situativkomplement, Direktivkomplement, Dilativkomplement) ausschlaggebend gewesen zu sein.

${ }^{134} \mathrm{Zu}$ diesen Termini vgl. Engels (2009a: 292, aber v. a. 449ff.) Kritik an der Verwendung des Terminus engere Apposition.

${ }^{135}$ Bei der Charakterisierung der Genitivattribute im rechten Feld teilt Engel (2009a: 294ff.) sie auf semantischer Grundlage in Subklassen ein, von denen - seines Erachtens - der Genitivus subiectivus, der Genitivus obiectivus und der Genitivus explicativus als Ergänzungen zu bezeichnen sind.
} 
erkennen ist (z. B. nominale Attribute ${ }^{136}$ ) wie bei anderen (z. B. präpositive Attribute). Darüber hinaus gibt es Benennungen, die rein semantisch fundiert sind, wie z. B. direktive Attribute oder expansive Attribute. Schließlich sind in Kennzeichnungen wie z. B. Genitivus subiectivus oder Genitivus obiectivus morphologische und zugleich auch semantische Merkmale bestimmend.

Hölzner (2007: 163ff., v. a. 167ff.) unterscheidet zwischen intra- und transphrastischer Argumentrealisierung ${ }^{137}$, wobei bei ihm Komplementklassen als solche nicht definiert werden. Dafür benennt er bei den intraphrastischen Argumentrealisierungen die einzelnen Gruppen nach ihrer Realisierungsform. So finden sich unter den intraphrastischen Argumentrealisierungen folgende, morphologisch-syntaktisch motivierte Bezeichnungen: Genitivattribut, Präpositionalattribut, Attributsatz, Adjektivattribut, Kompositum und Pronomen. Bei den transphrastischen Argumentrealisierungen bestimmt er abhängig davon, auf welcher Ebene die transphrastische Realisierung stattfindet, insgesamt zwei Gruppen: Partnerwort im Satz und Kontextrealisierung.

Helbig / Buscha (2018: 528ff.) bieten keine ausführliche Auflistung aller Substantivaktanten und somit kann auch bei ihnen von keiner expliziten Nomenklatur der Aktanten gesprochen werden. Es gibt jedoch bei ihnen Beispiele für Satzmodelle mit valenten Substantiven, in denen Substantive mit unterschiedlicher Anzahl an realisierten Aktanten präsentiert werden. Diesen Beispielen ist zu entnehmen, dass sie die einzelnen Aktanten - ähnlich wie Hölzner (2007) die intraphrastischen Argumentrealisierungen - eindeutig aufgrund ihrer Realisierungsform bestimmen (vgl. z. B. Substantiv im Genitiv, Präposition + Substantiv) (vgl. die Abkürzungen bei den Satzmodellen bei Helbig / Buscha 2018: 529ff., bzw. die Liste der entsprechenden Abkürzungen auf Seite 521f.).

Da Eisenberg (2006: 266ff.) - wie bereits in Kap. 2.4.3 erörtert - generell davon ausgeht, dass Attribute als Modifikatoren keine Komplemente darstellen, kann in seiner Grammatik überhaupt keine Klassifizierung der Nomenkomplemente erwartet werden. Bezüglich der Beschreibung der Attribute ist festzustellen, dass ihre Bezeichnungen bei Eisenberg (2006: 235ff.) morphologisch-syntaktisch motiviert sind. Erstens unterscheidet er dabei aufgrund der Wortartenzugehörigkeit (des Kopfs) des jeweiligen Attributs - adjektivische Attribute, substantivische Attribute, Präpositionalattribute und Relativpronomina bzw.

\footnotetext{
${ }^{136}$ Diese definiert Engel (2009a: 299) als Attribute, die bei Vorgangs- oder Tätigkeitsbezeichnungen vorkommen und „entweder einen Ausführenden - ,Agens“ - oder eine Subkategorie angeben“. Als wichtiges formales Merkmal gilt für diese Attribute, dass sie mit als ans Substantiv angeschlossen werden: z. B. Arbeit als Obermelker.

${ }^{137}$ Ferner führt er bei der Darstellung seiner Analyseergebnisse bei jedem Substantiv die Anzahl „,indefiniter Auslassungen", aber diese Gruppe spielt aus der Sicht der Untersuchung der Nomenklatur der Komplemente keine Rolle und wird deswegen hier ausgegrenzt (vgl. Hölzner 2007: 163ff.).
} 
Relativsätze. Des Weiteren werden unter den substantivischen Attributen - auf formaler Grundlage - Genitivattribute und Appositionen auseinandergehalten.

Ich bin der Ansicht, dass die Klassifizierung der Nomenkomplemente in der Fachliteratur sehr vielfältig ausfällt, wobei betreffend die Bezeichnung von Substantivkomplementen - wie auch den obigen exemplarischen Darstellungen zu entnehmen ist ${ }^{138}$ - generell vier Ansätze bestimmt werden können:

1) Es werden keine Komplementklassen als solche bestimmt, sondern die einzelnen Realisierungsformen der Argumente stehen stellvertretend für sie (vgl. z. B. Sommerfeldt / Schreiber 1977; die intraphrastischen Argumentrealisierungen bei Hölzner 2007; Helbig / Buscha 2018).

2) Eine unterschiedliche Anzahl von Komplementklassen wird bestimmt, deren Bezeichnungen formal, morphologisch-syntaktisch motiviert sind (vgl. die Komplementklassen der Substantive in der GDS).

3) Eine unterschiedliche Anzahl von Komplementklassen wird bestimmt, deren Bezeichnungen eindeutig nicht formal, dabei aber durch keinen einheitlichen Gesichtspunkt motiviert sind (vgl. z. B. Teubert 1979).

4) Eine unterschiedliche Anzahl von Komplementklassen wird bestimmt, deren Bezeichnungen z. T. formal, morphologisch(-syntaktisch), z. T. logisch-semantisch und z. T. morphologisch und zugleich semantisch motiviert sind (vgl. Engel 2009).

Nach der kritischen Darstellung unterschiedlicher Ansätze und Theorien der Fachliteratur in den Unterkapiteln von 2.5 fasse ich im nächsten Kapitel mein Konzept der Unterscheidung von Substantivkomplementen und -supplementen zusammen.

\subsection{Grundlagen des eigenen multidimensionalen Substantivvalenzkonzepts}

Die hier $\mathrm{zu}$ erörternden Gedanken entwickelten sich aus meiner kritischen Auseinandersetzung mit der Fachliteratur und den bisherigen korpusbasierten Analysen zur Valenzstruktur von Substantiven, die ich durchgeführt hatte. Ich gehe jedoch davon aus, dass ich an diesem Punkt nicht mehr als ein Grundgerüst meines Substantivvalenzkonzepts darlegen kann, das durch die Auswertung weiterer korpusanalytischer Ergebnisse allmählich verfeinert werden soll und wird.

\footnotetext{
${ }^{138}$ Eisenbergs (2006) Beschreibung der Attribute kann - in meinen Augen - keinem dieser Ansätze zugeordnet werden, da er generell davon ausgeht, dass Attribute nicht als Komplemente betrachtet werden können.
} 


\subsubsection{Logisch-semantische und formale Valenzbeziehungen}

Zur Darstellung der Substantivvalenz als ein System sui generis, und noch genauer zur Beschreibung der Valenzeigenschaften konkreter Substantive halte ich unbedingt ein multidimensionales Modell für nötig, in dem ich einerseits logisch-semantische, andererseits formale Relationen voraussetze. Darüber hinaus vertrete ich die Auffassung, dass die Klassifizierung von Attributen nicht dichotomisch, sondern graduell angelegt sein sollte, wobei - von Gruppen typischer bis zu denen peripherer Vertreter - mehrere Unterklassen von Komplementen und Supplementen anzunehmen sind.

Was die logisch-semantische Ebene der Substantivvalenz betrifft, gehe ich ähnlich wie die Verfasser der GDS (1969f.) davon aus, dass die Beziehung der Argumentselektion unentbehrlich ist, zu der ergänzend auch noch die Sortenselektion betrachtet werden kann. Ich stimme darüber hinaus mit der GDS (1969) auch darin überein, dass die „,semantischen Überlegungen“ beim Substantiv für Entscheidungen im Bereich der Syntax von großer Bedeutung sind. Das habe ich oben gezeigt, indem ich erörtert habe, dass z. B. die Genitiv- und Präpositionalattribute rein syntaktisch betrachtet zwar nicht subklassenspezifisch sind, mit Einschaltung der semantischen Ebene aber schon solche unter ihnen ermittelt werden können.

An dieser Stelle halte ich noch für wichtig und treffend eine Art Definition der Substantivvalenz von Tamássy-Bíró (1998b: 157) zu zitieren, in der ein wichtiger Aspekt der Problematik - meines Erachtens - sehr anschaulich ausgedrückt wird: „Die Substantivvalenz (...) ist eher durch eine nur abstrakte Formel beschreibbar, die aus der Semantik von Sb. [Substantiv; Á. S.-T.] ,herauszuanalysieren' ist und deren syntaktische Konsequenzen keinen festen Charakter haben“".

Als entscheidendes Unterscheidungskriterium zwischen Komplementen und Supplementen nehme ich ähnlich wie die Verfasser der GDS das Vorhandensein von Argumentselektion an, aber ich vertrete - im Gegensatz zur Betrachtung der Frage in der GDS (1974) - die Auffassung, dass nicht nur stark argumentselegierte Attribute als Komplemente zu klassifizieren sind, sondern alle, die beim betreffenden Bezugssubstantiv überhaupt als argumentselegiert gelten. Ähnlich sieht Kubczak (2006) die Frage, wenn sie in ihrem Artikel dafür argumentiert, dass die „Grenze von ,Komplement” bei der Valenz des Substantivs“ „verschoben“ werden sollte, damit nicht nur stark argumentselegierte Attribute als Komplemente betrachtet werden können. Der unterschiedliche Grad der Argumentselektion spielt - in meinen Augen - bei der Einordnung der Komplemente in Unterklassen eine Rolle, indem von zentralen bis zu peripheren mehrere Klassen der Komplemente bestimmt werden. Dabei ist starke Argumentselektion für Klassen 
der zum Zentrum oder in dessen Nähe gehörenden Gruppen charakteristisch (zu meinem Konzept zur Klassifizierung der Substantivkomplemente vgl. Kap. unten 2.6.2).

An dieser Stelle halte ich es für besonders wichtig, auf das Problem hinzuweisen, dass der Argumentbegriff in der Fachliteratur z. T. uneinheitlich verwendet wird (vgl. dazu z. B. Uzonyi 2019: 273ff.), wobei ich in Kap. 2.5.1 mit Jacobs' (2003) Interpretation von Argument sogar ein „Extrembeispiel“ thematisiert habe. Ohne hier ins Detail zu gehen, halte ich fest, dass ich den Terminus im logisch-semantischen Sinne gebrauche und die Leerstellen darunter verstehe, die von einem Prädikat bzw. einer Funktion eröffnet werden (vgl. Bußmann 2008: 56). Die Beziehung der Argumentselektion wird in der GDS (1038f.) im Rahmen der Beschreibung der Verbvalenz als „Relation der Sachverhaltsbeteiligung eingeführt“, zu deren Ermittlung der Folgerungstest dient (vgl. GDS 1046ff.). Daraus folgt für mich, dass auch Argumente des Substantivs mit Hilfe des Folgerungstests bestimmt werden können.

Im Zusammenhang mit dem Kriterium der Argumentselektion halte ich noch für besonders wichtig, auf Erkenntnisse meiner Korpusanalysen hinzuweisen, die unten (v. a.) in Kap. 4.2.1.1 detaillierter erklärt werden: Sie führten mich zu der Ansicht, dass die Klasse der Argumente bei bestimmten valenten Substantiven um ein sog. Quasiargument ( $\left.A^{\prime}\right)$ erweitert werden sollte. Ein Beispiel für die Realisierung eines Quasiarguments stellt in meinen Augen in der Nominalphrase der Ausdruck ,tiefere Lagen“ die Apposition ,tiefere Lagen“ (vgl. Beisp. 11) in Kap. 4.2.1.2) dar. Sie bringt nämlich nicht etwas Sachverhaltbeteiligtes zum Ausdruck, sondern steht mit dem Bezugssubstantiv im Verhältnis einer Quasigleichsetzung und referiert somit auf dasselbe wie das Bezugssubstantiv. Wie meinen Erörterungen in Kap. 2.6.2 zu entnehmen sein wird, nehme ich im Falle von Komplementkandidaten, die Quasiargumente realisieren, eine abgeschwächte Argumentselektion vor, was ich durch die Klammersetzung (+) kennzeichne.

Da mit Sortenselektion gemeint ist, dass der Valenzträger sortale Merkmale des Arguments bestimmt, ist die Untersuchung dieser Beziehung nur bei Attributen sinnvoll, die aufgrund des Folgerungstests als argumentselegiert gelten. Ist ein Attribut sortenselegiert, soll das als ein weiterer Komplement-Fürsprecher betrachtet werden und bei der Einteilung eines Attributs in die Subklassen der Komplemente eine Rolle spielen.

Zwar spielt in meinem multidimensionalen Konzept zur Beschreibung von Substantivkomplementen und -supplementen die logisch-semantische Relation der Argumentselektion die primäre Rolle, nicht unwesentlich sind jedoch Relationen der Formseite, wobei ich aber diesbezüglich wesentlich mehr Problemfragen annehme als betreffend die ebenfalls nicht als eindeutig zu bezeichnende - logisch-semantische Ebene. 
In meinem multidimensionalen Konzept gehe ich vorerst von den formalen Relationen der Obligatorik und der Rektion aus. Wie ich in Kap. 2.5.3 schon thematisiert habe, gehen die meisten Autoren davon aus, dass Substantivkomplemente fakultativ sind. Trotz alledem finde ich die Grundidee von Obligatorik auch im substantivischen Bereich absolut berechtigt und vertrete die Ansicht, dass das Vorhandensein der Relation der Obligatorik eine argumentselegierte Konstituente dem Zentrum der Komplemente zuordnen lässt. Dabei bewerte ich die - in Kap. 2.5.3 bereits angesprochenen - Ansichten und Beispiele der GDS und Tamássy-Bírós als besonders plausibel. Ferner leuchtet mir Hölzners - ebenfalls im vorangehenden Kapitel erörterter - Vorschlag ein, Obligatorik in Bezug auf die Substantivvalenz eher als eine Art Fokussierung neu zu interpretieren.

Im Zusammenhang mit einer Neuinterpretation von Obligatorik im substantivischen Bereich halte ich noch für wichtig, auf Wolfs (1982) in Kap. 2.4.3 bereits thematisierten Beispiele mit Relationalia hinzuweisen, die obligatorische Substantivkomplemente darzustellen scheinen. Zwar bin ich mit Wolf in der Hinsicht nicht einverstanden, dass all die Beispiele mit Weglassung des Komplements der jeweiligen Relationalia ungrammatisch seien, ${ }^{139}$ aber ich bin der Ansicht, dass (u. a.) bestimmte Relationalia tatsächlich überwiegend mit einem Komplement zusammen realisiert werden. Dementsprechend könnte man für solche Komplemente einen Schwellenwert festlegen und diejenigen als obligatorisch betrachten, deren Vorkommenshäufigkeit in einer korpusbasierten Analyse diesen Schwellenwert erreicht oder überschreitet. Zur Bestimmung dessen, bei welchem Realisierungsanteil dieser Schwellenwert liegen sollte, finde ich nützlich, mit zahlreichen Substantiven Korpusanalysen durchzuführen, die potenziell über „obligatorische“ Komplemente verfügen, und eine Art Durchschnittswert der Gebrauchsfrequenzen der entsprechenden Komplementkandidaten zu errechnen.

Im Falle von Präpositionalattributen halte ich - ähnlich dem Konzept der GDS (1035ff.) bei der Klassifizierung der Substantivkomplemente und -supplemente noch eine bzw. zwei formale Relationen für bestimmend. Die bei allen Präpositionalattributen zu untersuchende Beziehung ist die Konstanz, womit gemeint ist, ob das jeweilige Präpositionalattribut in der gegebenen Bedeutung nur durch eine Präposition ans Bezugssubstantiv angeschlossen werden kann (+KONST), oder ob es alternativ verwendbare Präpositionen dafür gibt. Bei Präpositionalattributen, die durch Wechselpräpositionen mit ihrem Bezugssubstantiv verbunden sind, ist noch die Relation von Kasustransfer $\mathrm{zu}$ beschreiben, d. h. ob im Präpositionalattribut sowohl der Akkusativ als auch der Dativ zu gebrauchen sind, oder eben

${ }^{139}$ So finde ich z. B. den Satz Der Redner bekräftigte den guten Zweck. im Gegensatz zu *Ihr mißfällt die Art. gar nicht ungrammatisch (Beispiele von Wolf 1982: 56). 
nur einer der beiden (+KASUSTR). Bei der Klassifizierung der Präpositionalattribute sehe ich + KONST und +KASUSTR als Komplement-Fürsprecher an.

Im Folgenden gehe ich auf wichtige Aspekte der Beschreibung von formalen Beziehungen und auf die mit ihnen verbundenen Probleme näher ein, wobei ich zuerst das Merkmal der Subklassenspezifik anspreche, das es in der Form in meinem Konzept zwar nicht gibt, das aber in der Fachliteratur sehr häufig eine (überaus) wichtige Rolle spielt. Wie schon erörtert, führt dieses Kriterium und dessen zu enge (einseitig auf formale Aspekte beschränkte) Definition oft dazu, dass bestimmte Attributsklassen - wegen ihrer (scheinbar) nicht-subklassenspezifischen Art - von vornherein als Supplemente abgetan werden (vgl. oben v. a. Kap. 2.5.1 aber auch 2.5.2). Einer solchen Einstellung kann ich weitestgehend nicht zustimmen. Für mich ist jedes Attribut, das ein Argument des Bezugssubstantivs realisiert, zumindest ein peripheres Komplement.

Als Beispiele für - aus den obigen Gründen - von vielen automatisch als Supplemente eingestufte Attributsklassen können das attributive Adjektiv, das Possessivpronomen bzw. teilweise die Genitiv- und Präpositionalattribute betrachtet werden (vgl. dazu auch Kap. 2.5.2 oben). In diesem Zusammenhang halte ich für wichtig, den Beitrag von Bassola / Bernáth (1998: 193) anzusprechen, die Folgendes hervorheben: „Die Untersuchungen haben ergeben, daß Ergänzungen von Substantiven auch durch Aktualisierungsformen zum Ausdruck gebracht werden können, die in einschlägigen Werken nicht behandelt werden. Als eine solche Form ist das Adjektiv zu nennen ..." Als Beispiele für attributive Adjektive, die - ihres Erachtens - als Ergänzungen zu klassifizieren sind, führen sie folgende Beispiele auf:

die Hilfe Japans = die japanische Hilfe;

die Gespräche zwischen Deutschland und Japan = deutsch-japanische Gespräche (Bassola / Bernáth 1998: 194).

Von diesen Beispielen betrachte ich das Adjektivattribut in japanische Hilfe im Vergleich zum Adjektivattribut im anderen Ausdruck als peripherer, weil es im Gegensatz zu der adjektivischen Konstituente in deutsch-japanische Gespräche eine mehrdeutige Konstruktion ergibt. So ist japanische in der obigen Nominalphrase sowohl agentiv als auch benefaktiv zu verstehen, was - meines Erachtens - ein Beweis für eine schwächere Argumentselektion darstellt.

Bassola / Bernáth (1998: 194) behandeln noch das Kompositum als Realisierungsform, die auch Ergänzungen repräsentieren kann aber in der Fachliteratur nicht beachtet wird. Zu den wenigen Autoren gehört auch Hölzner (2007: v. a. 304), der sowohl attributive Adjektive als auch Erstglieder von Komposita durchaus als Ergänzungen valenter Substantive betrachtet. Aus 
eben diesem Grund halte ich für überraschend und nur für eine fehlerhafte Formulierung, was in seinen zusammenfassenden Gedanken in Bezug auf Erstglieder von Komposita als potenzielle Argumentrealisierungen zu lesen ist:

Die Realisierung von Argumenten als Erstglied eines Determinativkompositums mit dem entsprechenden Bezugssubstantiv als Grundwort erweist sich nach den empirischen Analysen sowohl qualitativ als auch quantitativ als Besonderheit. Denn zum einen scheint es so gut wie keine semantische Rolle zu geben, für die vom System her keine Möglichkeit besteht, als Erstglied eines Kompositums realisiert zu werden. Zum anderen wird im realen Sprachgebrauch von dieser multifunktionalen Einsetzbarkeit der Kompositumsvariante überraschend häufig Gebrauch gemacht. $[\ldots]$

Dass die Kompositumsvariante in bisherigen Valenzdarstellungen (wenn sie dort überhaupt aufgeführt wird) eine absolute Nebenrolle spielt, ist angesichts der erhobenen Daten nicht nachvollziehbar; [...] (Hölzner 2007: 307f.; meine Hervorhebungen Á. S.-T.)

Sowohl die Ergebnisse seiner Korpusanalysen als auch die hier zitierte Beweisführung von Hölzner deuten - in meinen Augen - darauf hin, dass er - im Gegensatz zu seinem obigen Wortlaut - die Auffassung vertritt, dass Erstglieder von Determinativkomposita als Argumentrealisierungen von valenten Substantiven keine Besonderheit darstellen.

Mit Erstgliedern von Komposita habe ich schon eine potenzielle Komplementrealisierung in mein Konzept integriert, die wegen der „ungewöhnlichen“ Realisierungsebene (da es nicht intraphrastisch ${ }^{140}$ ist) oft ausgegrenzt aber in meinem Konzept als mit den intraphrastischen Realisierungsformen gleichberechtigte Realisierungsform betrachtet wird. Aus ähnlichem Grund werden die von Hölzner (2007) als transphrastisch bezeichneten Realisierungen innerhalb des betreffenden Satzes als Partnerwort oder sogar außerhalb des Satzes - nicht beachtet. ${ }^{141}$ Da ich Argumenthaftigkeit als Kriterium für die Zuweisung des Komplementstatus

${ }^{140}$ Zu den Begriffen intra- bzw. transphrastisch vgl. Hölzner (2007: v. a. 154 und 165).

${ }^{141}$ An dieser Stelle führe ich Beispiele mit solchen Komplementen aus einem früheren Beitrag von mir auf (vgl. Sántáné-Túri 2014), in dem ich Argumentrealisierungen des valenten Substantivs Geschenk auf allen, in mein Konzept integrierten Ebenen untersucht habe. Dabei habe ich exemplarisch gezeigt, dass Komplemente von Substantiven nicht nur innerhalb der jeweiligen Nominalphrase oder davon abhängiger Nebensätze, sondern auch als Erstglieder von Komposita unterhalb der Phrasenebene; in Form von Partnerwörtern zwar außerhalb der Nominalphrase, aber noch im betreffenden Satz und ferner auch außerhalb des jeweiligen Satzes, auf Textebene realisiert werden. (Die Abkürzungen A1, A2 und A3 stehen für Argumente von Geschenk, wobei ich folgende Argumente von Geschenk annehme: ein Geschenk von jmdm (A1) an jmdn (A2) aus einem Anlass (A3).)

Beisp. a)

Vereine-(A2), die ihr(A2) DFB-(A1)Geschenk noch nicht abgeholt haben, bekommen eine weitere Gelegenheit dazu: Morgen Vormittag (10 bis 12 Uhr) werden sie beim TV 1860 Jahn-Schweinau (Daimlerstraße 71) ausgeteilt. (Nürnberger Zeitung, 12.10.2007)

In diesem Satz ist A1 von Geschenk als Erstglied eines Kompositums mit dem betreffenden Substantiv als Bestimmungsglied realisiert ( $D F B-)$, während A2 sowohl innerhalb der NP (ihr) als auch außerhalb der NP, aber innerhalb des betreffenden Satzes (Vereine) erscheint. (Beispiel von Sántáné-Túri 2014: 402f.)

Beisp. b) 
für ausreichend halte, müssen streng genommen auch Argumentrealisierungen eines Substantivs außerhalb des jeweiligen Satzes, in dem das betreffende valente Substantiv als Valenzträger vorkommt, als Komplemente gelten können. In einem früheren Beitrag (vgl. Sántáné-Túri 2014) habe ich auch diesbezüglich Korpusbelege analysiert und gezeigt, wie

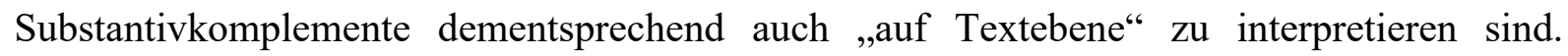
Tatsächlich entziehen sich die morphologisch-syntaktischen Merkmale dieser Argumentrealisierungen des Rektionseinflusses des Bezugssubstantivs. Dennoch halte ich es abhängig von den Zielsetzungen der jeweiligen Untersuchung der Substantivvalenz - für sinnvoll auch sie zu beachten. Hölzner (2007: 303) plädiert - auf Grund der Ergebnisse seiner Korpusanalysen - sogar dafür, dass im nominalen Bereich eben die transphrastische Realisierung die typische ist.

Zwar halte ich solche Argumentrealisierungen besonders aus Sicht der Untersuchung des Beitrags von valenten Substantiven zur Stiftung von Textkohärenz für wichtig, aber ich werde in meiner vorliegenden Arbeit die Analyse der Komplemente auf die Satzebene als höchste beachtete Realisierungsebene eingrenzen. Der wichtigste Grund dafür liegt darin, dass ich, neben dem primären Fokus auf der Beschreibung der Substantivvalenz, als sekundären Untersuchungsaspekt den Vergleich des Valenzverhaltens von valenten Substantiven in und außerhalb von SVG vor Augen halten will. Dabei spielen in der Beschreibung formale Merkmale eine wichtige Rolle. Deswegen befasse ich mich vorerst nur mit Komplementrealisierungen, bei denen neben der logisch-semantischen Dimension auch die Möglichkeit von formalen Relationen gegeben ist.

Nach der Darstellung der einzelnen Valenzrelationen in meinem multidimensionalen Konzept der Substantivvalenz beschreibe ich im nächsten Unterkapitel kurz ihre Funktion und Anwendung bei der Bestimmung der Substantivkomplemente.

\subsubsection{Zum Bestimmungsverfahren und zur Klassifizierung der Substantivkomplemente}

Die in Kap. 2.6.1 erörterten logisch-semantischen und formalen Valenzrelationen sollen in einem Filterverfahren in einer geordneten Reihenfolge der Beurteilung des Komplementstatus

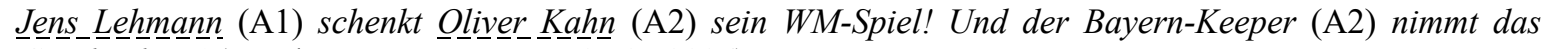
Geschenk an! (Hamburger Morgenpost, 07.07.2006)

Hier sind keine Komplemente innerhalb der jeweiligen Nominalphrase zu finden. A2 wird sowohl auf Textebene (Oliver Kahn), als auch innerhalb des betreffenden Satzes aber außerhalb der Nominalphrase (der Bayern-Keeper) ausgedrückt, während A1 nur auf Textebene, d. h. außerhalb des gegebenen Satzes (Jens Lehmann) vorkommt. (Beispiel von Sántáné-Túri 2014: 405f.) 
eines Komplementkandidaten dienen und ferner zur Bestimmung von Komplementklassen führen. Dabei ist die Ermittlung der Argumentselektion mithilfe eines Folgerungstests von primärer Wichtigkeit, da alle Komplementkandidaten, die als argumentselegiert gelten, mindestens der Klasse der peripheren Komplemente zugeordnet werden. Abhängig von der Realisierungsform des jeweiligen Komplementkandidaten werden nicht alle formalen Relationen überprüft, da - wie in Kap. 2.6.1 bereits thematisiert - Kasustransfer und Konstanz nur bei Präpositionalattributen ihre Relevanz haben.

Bei den Korpusanalysen in Kap. 4 wird es mir vorerst darum gehen, in den Belegen alle Attribute herauszufiltern, die aufgrund der Relation der Argumentselektion als Komplemente gelten, sie systematisch den einzelnen Argumenten zuzuordnen und zusätzlich durch ihre Realisierungsformen zu klassifizieren. Dementsprechend etabliere ich (vorerst) keine Nomenklatur der Substantivkomplemente in meinem Konzept, sondern identifiziere die einzelnen Komplementtypen dadurch, welches Argument sie durch welche Attributsklasse, d. h. Realisierungsform, zum Ausdruck bringen.

Die Ergebnisse der korpusbasierten Untersuchung sollen dabei behilflich sein, einen Einblick darin gewinnen zu können, welchen Einfluss die einzelnen formalen Relationen darauf haben, ob die einzelnen Komplemente als typisch oder eher als weniger typisch gelten. ${ }^{142}$ So bin ich der Ansicht, dass erst nach ausführlichen Korpusanalysen bestimmt werden kann, wie genau Zentrum, Peripherie und weitere Übergänge der Komplementklassen zu definieren sind. Ich gehe jedoch davon aus, dass mindestens drei „Eckpunkte“ nach diesem Gesichtspunkt zu bestimmen sind, und zwar die von Zentrum, Peripherie und absoluter Peripherie. Ferner sollte man mit Übergängen zwischen Zentrum und Peripherie rechnen, aber ob es eine oder eben zwei Klassen sein sollen, kann - meines Erachtens - nicht im Voraus festgelegt werden. Um das zu entscheiden, sollte man einen klaren Überblick davon haben, wie divers das Bild der unterschiedlichen Komplemente ist, wenn sie durch die einzelnen Valenzrelationen beschrieben werden. Vorerst will ich deswegen nur festhalten, dass ein Komplementkandidat desto mehr zum Zentrum der Komplemente gerechnet werden sollte, durch je mehr und je stärkere Komplementfürsprecher er charakterisiert werden kann. Außerdem würde ich stark argumentselegierte Komplemente, die obligatorisch sind, automatisch zum Zentrum rechnen. Wie in Kap. 2.6.1 bereits angesprochen, gehören - in meinem Konzept - Komplemente, die

\footnotetext{
${ }^{142}$ Dabei vertrete ich die Auffassung, dass dies nicht generell bestimmt werden kann, sondern von verschiedenen Faktoren, darunter vor allem von der Semantik der jeweiligen Substantive, abhängig ist. Das hat jedoch zur Folge, dass bei der Bestimmung der einzelnen Komplementklassen davon auszugehen ist, dass einzelne, z. B. semantisch definierte Klassen der Substantive auch in dieser Hinsicht ein unterschiedliches Bild zeigen werden.
} 
Quasiargumente realisieren, zu den absolut peripheren Komplementen, solange sie über keine weiteren Merkmale verfügen, die als Komplement-Fürsprecher gelten.

Entsprechend diesen Überlegungen fasse ich hier zwar die einzelnen KomplementFürsprecher in meinem Konzept tabellarisch zusammen, aber ich lege nicht genau fest, welche und wie viele Klassen der Komplemente nach der Unterscheidung Zentrum vs. Peripherie zu bestimmen sind. Nehme ich unterschiedliche Stufen der Stärke einer Valenzrelation an, signalisiere ich das durch die Anzahl der Pluszeichen.

\begin{tabular}{|c|c|c|c|c|}
\hline \multirow{4}{*}{ 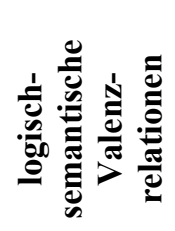 } & \multirow{3}{*}{\multicolumn{2}{|c|}{ Argumentselektion }} & stark & ++ \\
\hline & & & schwach & + \\
\hline & & & Quasiargument & $(+)$ \\
\hline & \multicolumn{2}{|c|}{ Sortenselektion } & \multicolumn{2}{|l|}{+} \\
\hline \multirow{5}{*}{ 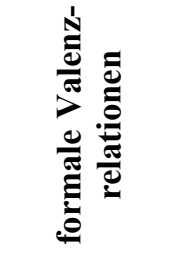 } & \multicolumn{2}{|c|}{ Obligatorik } & \multicolumn{2}{|l|}{+} \\
\hline & \multirow{2}{*}{\multicolumn{2}{|c|}{ Rektion }} & stark & ++ \\
\hline & & & schwach & + \\
\hline & \multirow{2}{*}{$\begin{array}{c}\text { nur bei } \\
\text { Präpattr-en }\end{array}$} & Konstanz & \multicolumn{2}{|l|}{+} \\
\hline & & Kasustransfer & \multicolumn{2}{|l|}{+} \\
\hline
\end{tabular}

Tab. 1: Komplement-Fürsprecher

Nach der Behandlung diverser Fragen aus dem Bereich der Valenz- und Substantivvalenztheorie verweile ich auch im nächsten Kapitel noch bei grundlegend theoretischen Überlegungen, und zwar in Bezug auf sog. Funktions- und/oder Stützverbgefüge, die später im Rahmen der korpusbasierten Untersuchung von ausgewählten Substantiven in Kap. 4 eine wichtige Rolle spielen werden. Auf das hier dargestellte multidimensionale Konzept zur Beschreibung von Substantivkomplementen werde ich ebenfalls in Kap. 4 zurückgreifen, wobei die eruierten Komplementrealisierungsformen eine Rückwirkung auf den theoretischen Rahmen haben und zur oben erwähnten Präzisierung der Bestimmung der Formrelationen beitragen sollen. 


\section{FVG / SVG im Rahmen der Untersuchung der Substantivvalenz}

Wie in Kap. 1 bereits erklärt, fokussiert sich meine Arbeit auf die Untersuchung der Substantivvalenz als ein System sui generis, wobei ich die Analyse und quantitative Auswertung von authentischen Korpusbelegen als unentbehrlich erachte. Dabei begegnet man aber valenten Substantiven oft in mehr oder weniger festen Verbindungen mit Verben, die in der Fachliteratur meistens mit den Termini $F V G$ oder $S V G$ bezeichnet werden. Deswegen halte ich es für wichtig und hoffentlich - zumindest als Vorarbeit für eine spätere, diese Konstruktionen in den Mittelpunkt stellende Studie - auch nutzbringend, bei meinen Korpusrecherchen solche Gefüge nicht auszugrenzen, sondern miteinzubeziehen, denn so können trotz oder sogar dank der Fokussierung auf die Beschreibung der Valenz der Substantive wichtige und vielleicht sogar ganz neue Erkenntnisse über das Verhalten bzw. die (Valenz)Eigenschaften dieser Verbindungen gewonnen werden, da die zur Zeit vorhandenen, nicht besonders zahlreichen korpusbasierten Untersuchungen $\mathrm{zu}$ FVG/SVG fast ausschließlich aus der Perspektive der Verbvalenz durchgeführt wurden (vgl. dazu detaillierter Kap. 3.2).

Damit ich Substantive in ihren Valenzeigenschaften auch in solchen Gefügen untersuchen kann, ist es wichtig, bestimmen zu können, was ich zu diesen Verbindungen rechne. Als Ausgangspunkt dafür biete ich in Kap. 3.1 einen kurzen Einblick in die vielfältige und zugleich auch sehr konfuse Diskussion der Forschung über FVG bzw. SVG, wobei ersichtlich werden soll, dass weder im Falle von FVG noch in dem von SVG eindeutig gesagt werden kann, was konkret unter diesen Begriffen zu verstehen ist und ob sie dementsprechend als synonyme oder nur als verwandte Bezeichnungen zu interpretieren sind. In Kap. 3.2 will ich einen Überblick darüber geben, wie diese Konstruktionen in der Fachliteratur in ihren Valenzeigenschaften beschrieben werden. Ein Thema, das mindestens genauso viele Fragen aufwirft wie die ontologische bzw. terminologische Charakterisierung dieser Verbindungen.

Nach der Erörterung unterschiedlicher Ansätze über FVG bzw. SVG fasse ich in Kap. 3.3 meinen eigenen Standpunkt zusammen. Dabei begründe ich meinen Terminusgebrauch und formuliere eine Art Arbeitsdefinition zur Ermittlung der betreffenden Konstruktionen im Rahmen meiner Korpusanalysen. Dabei will ich jedoch keinesfalls eine neue Definition oder neue Abgrenzungskriterien für diese Konstruktionen bestimmen, sondern stimme vielmehr

Kamber (2008: 13) zu, der im Zusammenhang mit den zahlreichen Abgrenzungs- und Definitionsversuchen von FVG - in Anlehnung an Van Pottelberge (2001: 3) - feststellt, dass sich die Forschung damit „,wirklich in eine Sackgasse hineinmanövriert“ hat. Vielmehr bemühe 
ich mich darum, einen möglichst flexiblen theoretischen Rahmen für meine Korpusanalysen zu schaffen, bei denen jedoch - wie ich oben bereits erklärt habe - diese Konstruktionen nicht im Mittelpunkt stehen, sondern nur als Teil einer möglichst umfassenden Analyse der Valenzeigenschaften von Substantiven mitberücksichtigt bzw. untersucht werden.

\subsection{FVG / SVG - Ein ontologisches bzw. terminologisches Problem aus der Perspektive} der Fachliteratur

FVG gelangten zuerst durch sprachpflegerische Kritik an der Verwendung von solchen Konstruktionen in den Fokus der Sprachwissenschaft. So entstand auch die Schrift von Polenz (1963) Funktionsverben im heutigen Deutsch. Sprache in der rationalisierten Welt als linguistische Reaktion darauf, dass „,der Direktor eines deutschen Universitätsinstituts von seinem Ministerium einen Antrag zurückgeschickt [erhielt]“, und zwar mit der Forderung, diesen auf der Grundlage der beigelegten „Broschüre ,Fingerzeig für die Gesetzes- und Amtssprache' $[\ldots]$, die von der Gesellschaft für deutsche Sprache herausgegeben und vom Bundesminister des Innern mit einem mahnenden Geleitwort versehen worden ist", sprachlich umzuarbeiten (Polenz 1963: 5). Hier will ich auf Fragen des Verhältnisses von Sprachpflege, Sprachkritik und Sprachwissenschaft, die Polenz (1963: 5ff.) erörtert, nicht eingehen, und auch die detaillierte, z. T. sogar sprachgeschichtlich fundierte Darstellung der Beschaffenheit und Verwendung dieser Konstruktionen soll nur verkürzt wiedergegeben werden. Diese Arbeit von Polenz (1963) ist aus der Sicht der Fragestellung dieses Kapitels jedoch von besonderer Bedeutung, weil der Terminus Funktionsverb durch sie in der germanistischen Linguistik etabliert wurde (vgl. Helbig 1979: 273) und die Definition des Begriffs bis heute noch großen Einfluss auf die Forschung dieses Gebiets hat. Deswegen konzentriere ich mich hier auf die Darstellung der Charakteristika von Funktionsverben, die diesem Beitrag entnommen werden können.

Polenz (1963: 12ff.) plädiert dafür, Verbindungen, in denen das Verbalabstraktum, d. h. das substantivische Element des FVG, akkusativisch ans Verb gebunden ist, von solchen mit einem präpositional ans Verb angeschlossenen Substantiv zu unterscheiden, da sie nicht nur formal, sondern auch in ihrer Funktion deutliche Unterschiede aufweisen sollen: In letzteren sei die Verbindung zwischen Verb und Nomen nämlich enger, außerdem verändere das Funktionsverb in diesen Konstruktionen auch die Vorgangsart ${ }^{143}$. Er nennt die ganze Gruppe dieser

\footnotetext{
${ }^{143}$ Als Beispiel für die unterschiedliche Funktion der beiden Typen der FVG bringt Polenz (1963: 13) das Vollverb über etw. entscheiden, mit dem eine Entscheidung über etw. treffen / fällen als FVG mit einem akkusativisch ans
} 
Verbindungen „Funktionsverbformeln“ und fokussiert in seiner Arbeit auf die Subklasse, in denen „Funktionsverben mit Präpositionen“ (Polenz 1963: 12) vorkommen.

Funktionsverben charakterisiert er als „Bewegungsverben mit ursprünglich räumlichkonkreter Bedeutung, die zusammen mit den richtungsbezeichnenden Präpositionen $z u$ und in die Vorgangsart in bestimmter Weise differenzieren“ (Polenz 1963: 13; Hervorhebungen von Polenz). Diese Verben sind ihm zufolge nicht mehr als Vollverben anzusehen, sondern mit Hilfsverben vergleichbar und ihre Verwendung ist mit der Tendenz in der deutschen Sprache zum analytischen Sprachbau und damit mit der seit althochdeutscher Zeit belegbaren „ständige[n] Zunahme von Hilfsverbumschreibungen“ gut vereinbar (Polenz 1963: 13f.). Ferner sieht er sowohl Hilfsverben als auch Funktionsverben, ,als Leitglieder von Vorgangsgefügen“ (Polenz 1963: 26) an. Während jedoch Hilfsverben einen Infinitiv als „Nennglied im Vorgangsgefüge“ haben sollen (z. B. ich will sprechen, ich wünsche zu sprechen $)^{144}$, seien die Nennglieder von Funktionsverben Verbalabstrakta. Betreffend die Funktionsverben, die mit Präpositionen zusammen realisiert werden, ${ }^{145}$ erklärt Polenz (1963:27), dass sie in ihrer Vollverbverwendung ,konkrete Bewegungen oder Zustände bezeichnen“, aber als Funktionsverben, d. h. als Leitglieder von Vorgangsgefügen mit Verbalabstrakta als Nennglieder, sich ihre konkret-räumliche Bedeutung in ,zeitliche Bewegungs- oder Zustandsbezeichnungen“" verwandle.

Aufbauend auf der obigen Beschreibung von Funktionsverben von Polenz (1963) verwendet und definiert Engelen (1968) zum ersten Mal den Terminus FVG. Es ist jedoch wichtig hervorzuheben, dass er nur solche Konstruktionen als FVG bezeichnet, in denen das Nomen,

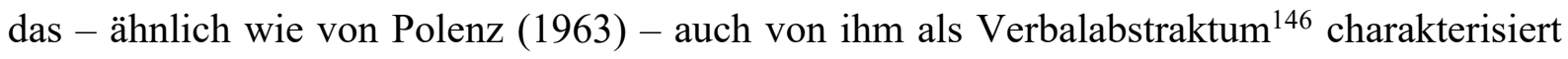
wird, präpositional ans Funktionsverb angeschlossen ist (vgl. Engelen 1968: 289 und 292). In seinem Artikel formuliert Engelen (1968: 289ff.) zuerst Kriterien, mit deren Hilfe FVG bestimmt bzw. von anderen Konstruktionen abgegrenzt werden können. Diese erörtere ich hier

Funktionsverb angeschlossenen Substantiv verbunden werden kann, bei dem Polenz zufolge ,der Vorgangsbegriff ,entscheiden " gegenüber der rein verbalen Stilvariante [...] nicht differenziert [ist]“". Im Gegensatz dazu betrachtet er beim FVG etwas zur Entscheidung bringen, in dem ein mit dem Funktionsverb durch eine Präposition verbundene Substantiv realisiert wird, die Funktion des FVG in Bezug auf den Ausdruck der Vorgangsart komplett anders, da sei nämlich ,der Vorgangsbegriff selbst, der sich semantisch von der rein verbalen Formulierung ich entscheide über etwas unterscheidet" (Polenz 1963: 13; Hervorhebung von Polenz).

${ }^{144}$ Beispiele von Polenz (1963: 26). Es sei an dieser Stelle dahingestellt, dass ich die Festlegung der Nennglieder von Hilfsverben auf Infinitive (statt auf infinite Verbformen) bei einer herkömmlichen Deutung des Terminus Hilfsverb sehr problematisch finde.

${ }_{145}$ Polenz (1963: 27) gibt folgende Liste dieser Funktionsverben an: bringen, stellen, setzen, gehen, nehmen, ziehen, kommen, gelangen, geraten, stehen.

${ }^{146}$ Als Ausnahmen davon betrachtet Engelen (1968: 292) die Gruppe der volllexikalisierten FVG, die aber - seiner Ansicht nach - eventuell gar nicht als FVG bezeichnet werden sollten (vgl. dazu die Zusammenfassung von Engelens Gruppierung der FVG nach ihrer Transparenz und ihrem Lexikalisierungsgrad). 
nicht detailliert, sondern bemühe mich eher darum, einen kurzen, zusammenfassenden Überblick über sie zu bieten.

Was die semantische Struktur von FVG betrifft, betrachtet Engelen (1968: 289f.) das nominale Element des FVG als Hauptträger der Bedeutung, während das Funktionsverb innerhalb der Konstruktion seine Vollverbbedeutung verliert und ,im großen und ganzen nur eine grammatische Funktion" ausübt, wobei er auch darauf hinweist, dass diese Charakterisierung der Funktionsverben in der Hinsicht verfeinert werden soll, dass sie öfter doch nicht ganz entsemantisiert und als bloße Funktionswörter anzusehen sind, weil sie ,auch eine vage inhaltliche Komponente enthalten“(Engelen 1968: 290).

Die Eigenschaften des nominalen Elements, dass es nicht unmittelbar erfragbar und nicht pronominalisierbar ist, die in der Fachliteratur bis heute noch immer wieder als definitorische Merkmale der FVG aufgegriffen werden, fasst er als notwendige, aber nicht ausreichende Kriterien der Definition von FVG auf. Ebenfalls eine wiederkehrende Frage der Beschreibung von FVG ist die Attribuierbarkeit ihres nominalen Teils. Diesbezüglich unterscheidet Engelen (1968: 292), abhängig von ihrer semantischen Transparenz und - in engem Zusammenhang damit - von ihrem Lexikalisierungsgrad drei Gruppen der FVG. Zur ersten Gruppe zählt er nicht-lexikalisierte FVG mit einer kompositionellen Bedeutung, deren substantivisches Element „ziemlich leicht“ attribuiert werden kann. ${ }^{147} \mathrm{Zu}$ den beiden anderen Gruppen gehören schon lexikalisierte FVG mit einer durchschaubaren, aber nicht ganz aus den Bedeutungen ihrer Konstituenten erschließbarer Bedeutung ${ }^{148}$ bzw. volllexikalisierte FVG, die semantisch nicht mehr auf der Grundlage ihrer Konstituenten interpretierbar sind ${ }^{149}$. Für diese beiden Subklassen der FVG ist nach seiner Auffassung die Nicht-Attribuierbarkeit des nominalen Teils charakteristisch, wobei er auch die Möglichkeit dessen in Betracht zieht, dass die volllexikalisierten FVG gar nicht mehr zu den FVG gerechnet, sondern als „lexikalisierte komplexe Prädikate“ bezeichnet werden sollten. Hier halte ich noch für wichtig hervorzuheben, dass er zwar darauf nicht gesondert eingeht, aber seiner Beschreibung der unterschiedlichen Abgrenzungskriterien der FVG an mehreren Stellen zu entnehmen ist, dass Engelen (1968: 292 und 294) FVG als Prädikate charakterisiert, was ebenfalls bis heute in der Forschung

\footnotetext{
${ }^{147}$ Als Beispiele von solchen FVG finden wir bei Engelen (1968: 292) u. a. in Not geraten oder zur Verfügung stellen.

${ }^{148} \mathrm{Zu}$ dieser Gruppe gehören Engelen (1968: 292) zufolge z. B. in Frage kommen/stellen und in bezug [sic] setzen.

${ }^{149}$ Als Beispiele für solche volllexikalisierte (FVG-)Konstruktionen führt Engelen (1968: 292) in Harnisch setzen, instand setzen, zuwege bringen auf.
} 
überwiegend der Fall ist, aber - wie ich unten noch thematisieren werde - keinesfalls als unumstritten gelten kann. ${ }^{150}$

Das Kriterium der Paraphrasierbarkeit von FVG durch ein Vollverb oder ein Adjektiv in Verbindung mit einem Kopulaverb kennzeichnet Engelen (1968: 291) als distinktives, aber nicht notwendiges Merkmal von FVG, da es von vielen FVG nicht erfüllt wird. Aus der Sicht der Beschreibung von FVG in der Grammatik findet er zwei ihrer Eigenschaften wichtig: Einerseits gibt es begrenzt viele Funktionsverben und noch weniger Präpositionen, die mit diesen Funktionsverben in Verbindung treten können, andererseits bilden Funktionsverben und Präpositionen Reihen von FVG (vgl. Engelen 1968: 293).

Im Zusammenhang mit der Gruppe der Funktionsverben soll noch betont werden, dass Engelen (1968: 289) zwar explizit darauf hinweist, dass er sich in seiner Definition von FVG nach Polenz (1963) richtet, seine Beispiele für Funktionsverben jedoch - meiner Ansicht nach - nicht ganz mit der Auffassung von Polenz vereinbart werden können. So sind bei Engelen (1968: 293ff.) unter den Funktionsverben u. a. sowohl haben, als auch bleiben und sein anzutreffen, ${ }^{151}$ die - im Gegensatz zur Funktionsverbinterpretation von Polenz (1963: 13) keine „Bewegungsverben mit ursprünglich räumlich-konkreter Bedeutung“ darstellen.

An diesem Punkt halte ich es noch für interessant und wichtig, vor der weiteren Thematisierung von Engelens (1968) Beitrag, das Problem anzusprechen, dass die Interpretation des Begriffs Funktionsverb in der Fachliteratur - meiner Ansicht nach mindestens so vielfältig ist, wie die von FVG. ${ }^{152}$ Zwar wird diese Frage unten bei der Darstellung der unterschiedlichen FVG (/ SVG)-Definitionen implizit, oder an einigen Stellen auch explizit noch mehrmals aufgegriffen, aber ich will hier auf die Deutung des Begriffs bei Lehmann (1983) hinweisen, der sich mit „einigen Problemen bei der Beschreibung von Funktionsverbgefügen“ befasst. Zwar wird sein Beitrag aus der Sicht meiner Arbeit eher bei der Charakterisierung der Valenzeigenschaften von FVG (vgl. Kap. 3.2) von Belang sein, aber ich finde auch seinen Versuch, Funktionsverben zu definieren, aufschlussreich, da darin gleichzeitig auch die Tatsache klar wird, dass ihre Abgrenzung gar nicht eindeutig und in konkreten Beispielen häufig von der subjektiven Einschätzung des Untersuchenden abhängig ist.

\footnotetext{
150 Vgl. dazu unten v. a. meine Zusammenfassung der Bedenken von Van Pottelberge (2001) in diesem Zusammenhang oder meine Begründung dafür, warum ich FVG nicht (mehr) als (komplexe) Prädikate betrachte. 151 Über die Einschätzung des Funktionsverbstatus von haben und sein sind Forscher von FVG auch heute noch geteilter Meinung (vgl. dazu in diesem Kapitel z. B. Eisenberg 2006 oder Heine 2006).

${ }^{152}$ Eine andere Meinung vertritt in diesem Zusammenhang zum Beispiel Eisenberg (2006: 309), der den Status des Begriffs $F V G$ als „viel unklarer“ einschätzt als den von Funktionsverb.
} 
Er geht davon aus, dass FVG als Prädikatsausdrücke zu betrachten sind, in denen das substantivische und verbale Element eine semantische Einheit bilden, wobei die ,inhaltliche Benennung“ vom Nomen realisiert wird, während das Funktionsverb seine „spezifische eigene

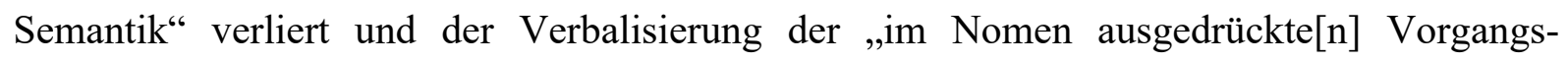
/Zustandsbezeichnung“ dient. Dabei könne das Funktionsverb jedoch ,allgemeine semantische Merkmale wie Aktionalität (ins Gerede kommen) und Kausativität (zum Reden bringen) in das Gefüge einbringen, die es aufgrund seiner Zugehörigkeit zur Klasse der Verben besitzt“" (Lehmann 1983: 43, Hervorhebungen von Lehmann).

Er zeigt jedoch, dass seines Erachtens auch eine solche aus der Sicht der Abgrenzung von FVG zu freien Verbindungen besonders vielversprechende, semantische Beschreibung von FVG an bestimmten Punkten an ihre Grenzen stößt:

Gerade das Verb durchführen zeigt aber deutlich, daß auch inhaltsschwache Verben einmal als Vollverben (eine Meinungsumfrage durchführen) aufscheinen können (Realisierung des spezifischen Sembestands), in anderen Fällen (eine Messung durchführen) ihren spezifischen Sembestand nicht realisieren, also als Funktionsverben auftreten. (Lehmann 1983: 46; Hervorhebungen von Lehmann)

Dies führt dann Lehmann (1983:46) zufolge dazu, dass es Fälle gibt, in denen die Zugehörigkeit des jeweiligen verbalen Elements der Konstruktion zu den Voll- oder Funktionsverben nicht eindeutig bestimmt werden kann und der Untersuchende auf seine „Intuition“ angewiesen ist. Dies stellt - meines Erachtens - ein Problem bei der Analyse der FVG dar, das - egal wie viele Konstruktionen wie gründlich untersucht werden höchstwahrscheinlich nie ganz behoben werden kann.

Nach diesem kurzen Exkurs über die Definitionsproblematik von Funktionsverben kehre ich zu Engelens (1968) Artikel zurück. Neben der Bestimmung der Abgrenzungskriterien von FVG ist die zweite Zielsetzung von Engelen (1968: 293ff.), in seinem Beitrag die einzelnen „Funktionsverbreihen“ zu beschreiben. Dabei ist - meines Erachtens - von Belang, dass er bei der Zusammenstellung der Listen der einzelnen Funktionsverbreihen und bei deren semantischen Klassifizierung bzw. bei der Beschreibung ihrer Gebrauchstendenzen und Produktivitätseigenschaften nur an wenigen Punkten für wichtig gehalten hat, Korpusanalysen durchzuführen. Stattdessen ging er bei der Zusammenstellung seiner FVG-Listen von FVGBeschreibungen von Wörterbüchern und der eigenen Intuition aus. Generell hält er bei der Untersuchung von FVG Korpusanalysen aus zweierlei Sicht problematisch: Einerseits sollen FVG „,relativ selten“ realisiert werden, was dazu führt, dass ,[m]an [...] ein riesiges Corpus 
durchgehen [müßte], um eine hinreichende Zahl von Belegen zu finden“. ${ }^{153}$ Andererseits hat er an Korpora auszusetzen, dass sie - seiner Meinung nach - häufig „Ad-hoc-Bildungen“ enthalten, was zur Folge hat, dass man sie ,wieder auf Grund des eigenen Sprachgefühls oder auf Grund von Informantenbefragungen - ausschließen müßte“ (Engelen 1968: 294).

Engelen (1968: 293ff.) teilt FVG nach den Merkmalen perfektiv/durativ und intransitiv / transitiv in Subklassen ein. Genauso wie Polenz (1963: 12ff.), führt auch er FVG auf, in denen die Präpositionen in und $z u$ vorkommen, da sie bei weitem die am häufigsten realisierten präpositionalen Elemente in FVG sind. Dabei gibt es bei Engelen (1968: 298) jedoch auch einige wenige Beispiele für die Verwendung von außer, die eine Reihe darstellen, die er als eine Variante einer anderen Reihe mit in betrachtet und als sehr unproduktiv bezeichnet. Bezüglich der von Engelen aufgeführten FVG ist noch hervorzuheben, dass sie erkennbar mehr und - wie ich oben schon angedeutet habe $-z$. T. auch mehr unterschiedliche Funktionsverben enthalten, als die Beispiele von Polenz.

Im Vergleich zu den beiden, oben referierten Arbeiten von Polenz (1963) und Engelen (1968), die zu den ersten grundlegenden Arbeiten gerechnet werden müssen, durch die die linguistische Untersuchung von FVG in die Wege geleitet wurde, geht Helbig 1979 bereits von einer wesentlich breiter gefassten Gruppe der FVG aus, wobei er auch das Problem anspricht, dass sich die Forschung nicht einig darin ist, welche Konstruktionen überhaupt zu den FVG gerechnet werden sollen (vgl. Helbig 1979: 275). Auf der Grundlage morphologischer Kriterien unterscheidet er fünf Gruppen der FVG, mit einer weiteren Differenzierung bei der fünften in zwei Subklassen. Seiner Definition zufolge bestehen FVG aus einem Funktionsverb und einem Substantiv, das sowohl präpositional als auch akkusativisch oder sogar in einem der weiteren reinen Kasus ans Funktionsverb angeschlossen werden kann.

Bei der Systematisierung der $-\mathrm{zu}$ der Zeit in der Fachliteratur thematisierten operationellen Kriterien, die zur Abgrenzung der FVG von freien Wortverbindungen dienen sollen, beschreibt er insgesamt 16 Kriterien (vgl. Helbig 1979: 276 f.), wobei er jedoch gleich feststellt, dass ,sich die einzelnen FVG bei der Anwendung der verschiedenen Kriterien unterschiedlich verhalten“ (Helbig 1979: 279). Dies erklärt er mit dem unterschiedlichen Lexikalisierungsgrad der FVG und geht davon aus, dass die untersuchten Klassifizierungskriterien nur auf die lexikalisierten FVG ,ganz oder fast ganz

${ }^{153}$ Dieses Argument von Engelen (1968: 294) kann zu seiner Zeit, als noch keine digitalisierten Korpora und keine Suchmaschinen für die Durchsuchung oder sogar Analyse von solchen Korpora zur Verfügung standen, deutlich besser vertretbar gewesen sein als es heute ist. Es sei hier jedoch dahingestellt, wieweit solche Beschreibungen von (möglichen) Konstruktionen imstande sind, den tatsächlichen Sprachgebrauch adäquat zu schildern. 
zutreffen“ (Helbig 1979: 283; meine Hervorhebung, Á. S.-T.). ${ }^{154}$ Hier sollen nicht alle Kriterien detailliert erörtert werden, vielmehr geht es mir darum, durch die zusammenfassende Darstellung seiner Ansichten die Stelle von Helbigs (1979) Beitrag in der linguistischen Diskussion über FVG zu umreißen.

Helbig (1979: 283) unterscheidet bei der Klassifizierung von FVG zwischen zentralen und zur Peripherie gehörenden FVG. Zur ersteren Gruppe rechnet er diejenigen Konstruktionen, in denen das Nomen entweder im Akkusativ oder präpositional ans Funktionsverb angeschlossen ist. Im Zusammenhang mit diesen zentralen Subklassen merkt er an, dass auch ihre Vertreter nicht ausnahmslos alle operationellen Kriterien erfüllen. Obwohl - seiner Meinung nach - vor allem FVG mit akkusativischem Nomen nicht allen Kriterien entsprechen, klassifiziert er beide Subklassen als zentral (vgl. Helbig 1979:275), wobei FVG mit einem präpositional angeschlossenen Nomen ,als innerste Schicht“ zu betrachten sind. Zur Peripherie zählen ihm zufolge FVG, in denen das Nomen im Nominativ realisiert ${ }^{155}$ oder im Genitiv mit sein als Funktionsverb ${ }^{156}$ verbunden wird.

Die Substantive in FVG sind auch seiner Auffassung nach Verbal- oder Adjektivabstrakta, die die Hauptbedeutung des FVG tragen, während die Funktionsverben und - bei präpositional mit dem Funktionsverb verbundenen Substantiven - auch die Präpositionen in ihrer Semantik reduziert sind. Die Frage der Entsemantisierung von Funktionsverben betrachtet er als eines der Beispiele für Kriterien der Beschreibung von FVG, die in der Forschung umstritten sind. Dabei plädiert er dafür, Funktionsverben semantisch nicht als völlig leer anzusehen, da sie „nicht nur Träger von morphosyntaktischen Funktionen, sondern auch Träger von semantischen Funktionen sehr allgemeiner Art“ sind, die ,zumeist den Aktionsarten zugeschrieben werden“ (Helbig 1979: 274). Es sei hier nur am Rande angemerkt, dass er dabei auch kausativ zu den Aktionsarten rechnet, (vgl. Helbig 1979: 281) wodurch er jedoch (ungewollt) eine weitere in der (späteren) Forschung ebenfalls nicht einheitlich gesehene Frage in die Diskussion miteinbezieht. ${ }^{157}$

Unter den operationellen Kriterien zur Bestimmung der FVG, die er dem Überblick der zeitgenössischen Forschung entnimmt, befasst er sich neben den oben - bei Polenz (1963) bzw.

\footnotetext{
154 Vgl. dazu v. a. Van Pottelberges Meinung (2001: 4), der diese Art der Unterscheidung von FVG-Klassen deswegen weniger zufrieden stellend findet, weil - seiner Ansicht nach - dabei nur die ,unterschiedliche Festigkeit" der einzelnen FVG-Konstruktionen eine Rolle spielt, wobei diese aber nicht weiter von Phraseologismen auf der einen und freien syntaktischen Verbindungen auf der anderen Seite abgegrenzt werden können.

${ }^{155}$ Ein Beispiel dafür von Helbig (1979: 275) ist: Zwischen den Delegierten besteht keine Übereinstimmung.

${ }^{156}$ Dafür führt Helbig (1979: 275) folgendes Beispiel an: Wir sind der Meinung, daß er kommt.

157 Vgl. dazu z. B. Lehmann (1983) oder Van Pottelberge (2001), deren Ansicht nach kausativ nicht zu den Aktionsarten gerechnet werden kann.
} 
Engelen (1968) - schon angesprochenen Merkmalen, wie Abstrakta als substantivische Elemente von FVG, Reihenbildung, eingeschränkte oder gar ausgeschlossene Attribuierbarkeit des Substantivs im FVG, fester Artikelgebrauch und aufgehobene Numerusopposition beim substantivischen Element, Paraphrasierbarkeit von FVG durch Vollverben oder Verbindungen von Adjektiven mit Kopulaverben, auch mit Fragen der Negation und der Valenzeigenschaften von FVG, von denen die letztere unten in Kap. 3.2 eingehender thematisiert wird.

In seiner Beschreibung der FVG grenzt Eisenberg (2006: 309ff.) den Kernbereich der FVG auf Konstruktionen aus einem Funktionsverb und einer Präpositionalgruppe ein und befasst sich ausschließlich mit solchen Gefügen. Die Gruppe der aus der Behandlung ausgeschlossenen Verbindungen beschreibt er folgendermaßen:

Nicht behandelt werden die manchmal zu den FVG gerechneten [...] Fügungen mit Kopulaverben (in Aufregung sein), mit haben + PrGr [Präpositionalgruppe; Á. S.-T.] (zur Verfügung haben), sowie Konstruktionen mit rausgebleichter` Bedeutung, die ein Nominal im Akk [Akkusativ; Á. S.-T.] (Kenntnis bekommen) oder Dat [Dativ; Á. S-T.] (einem Irrtum unterliegen) regieren. (Eisenberg 2006: 310; Hervorhebungen von Eisenberg).

Die Nicht-Behandlung dieser (FVG-)Subklassen begründet er damit, dass sie kein „Funktionsverb im engeren Sinne“ enthielten.

Bei Eisenberg (2006: 309ff.) werden also nur Konstruktionen mit einem präpositional angeschlossenen Substantiv unter den (zum Kernbereich gehörenden) FVG untersucht, wobei sein und haben von den Funktionsverben ausgeklammert werden ${ }^{158}$. Seine, im obigen Zitat anzutreffende Formulierung „Konstruktionen mit >ausgebleichter« Bedeutung“ finde ich jedoch ungenau, da mit ,ausgebleicht“ wahrscheinlich nur die Bedeutung des betreffenden Funktionsverbs - und nicht die der Gesamtkonstruktion - gemeint sein soll.

Eisenberg (2006: 310; Hervorhebung von Eisenberg) ist mit Van Pottelberge (2001) in der Hinsicht einverstanden, dass FVG mit anderen verbonominalen Konstruktionen Ähnlichkeiten aufweisen, „die ebenfalls ein semantisch >ausgebleichtes` oder sonstwie merkmalarmes Verb (engl. light verb) ${ }^{159}$ und nominale Elemente der einen oder anderen Art enthalten“. Dementsprechend vertritt er auch die Auffassung, dass FVG kein ,ganz besondere[r] Status unter den $\gg$ Nominalprädikaten $<{ }^{160}$ zuzuweisen“ “ ist.

\footnotetext{
${ }^{158}$ Vgl. dazu auch Heine (2006: 48).

159 Aus dieser Erklärung von Eisenberg (2006: 310) ist darauf zu schließen, dass - seiner Ansicht nach Funktionsverben als (eine Art) light verb zu klassifizieren seien.

${ }^{160}$ Das heißt jedoch zugleich, dass Eisenberg (2006) - trotz Van Pottelberges (2001) Bedenken im Zusammenhang mit der Verwendung des Begriffs (komplexes) Prädikat (vgl. die Darstellung dieses Problems unten) - an der in der Fachliteratur häufig vorkommenden Bezeichnung von FVG als (Nominal-)Prädikate festhält (vgl. ferner noch mein Vorschlag unten, FVG als komplexe Satzkonstituenten zu charakterisieren).
} 
Zwar sind Funktionsverben - Eisenberg (2006: 309ff.) zufolge - relativ eindeutig zu definieren, während das auf den Begriff $F V G$ nicht zutreffen soll, er gibt jedoch keine explizite Definition des Terminus Funktionsverb an, sondern verweist auf Polenz (1963) bzw. zählt die häufigsten Funktionsverben ${ }^{161}$ auf. Während Polenz (1963: 12) über „Funktionsverben mit Präpositionen“" spricht, erklärt Eisenberg (2006: 311) die Relationen in FVG anders und sieht die Präposition nicht ans Verb, sondern ,an das Nominal der PrGr [Präpositionalgruppe; Á. S.-T.)“ gebunden. Dabei stellt er fest, dass in über $90 \%$ der (von ihm untersuchten) FVG entweder in oder $z u$ als Präposition vorkommt. Während die Menge der potenziellen Präpositionen in FVG so deutlich einzuschränken ist, ist es besonders problematisch, die Substantive zu charakterisieren, die in FVG vorkommen können - dementsprechend beschränkt er sich darauf, bestimmte morphosyntaktische Merkmale von FVG und darin vorkommenden substantivischen Gliedern zu beschreiben, die hier jedoch nicht eingehender thematisiert werden, da sie im Vergleich zu den bisher dargestellten Charakteristika von FVG keine neuen darstellen (vgl. Eisenberg 2006: 312ff.).

Betreffend die Struktur von FVG vertritt Eisenberg (2006: 314), genauso wie Van Pottelberge (2001), die Auffassung, dass diese zwar Einschränkungen aufweisen, aber den grammatischen Regularitäten entsprechend. Das untermauert die obige These, derzufolge die FVG nicht als besondere Gruppe der Nominalprädikate zu klassifizieren sein sollen. Im Gegensatz zu Helbig (1979) geht Eisenberg ferner davon aus, dass es wohl Beispiele für lexikalisierte FVG gibt, die Lexikalisierung von FVG aber nicht als allgemeine Tendenz angesehen werden kann.

Etwas mehr als dreißig Jahre später, als der oben thematisierte, die Untersuchung der FVG bis heute noch deutlich prägende Beitrag von Helbig (1979) ${ }^{162}$ veröffentlich wurde, fasst Bruker (2013: 17 ff.) bereits 27 Kriterien und Testverfahren zusammen, die in der Fachliteratur wiederkehrend zur Bestimmung der FVG verwendet werden. Gleich im Anschluss an die ausführliche Liste ist aber auch bei ihr die folgende - meines Erachtens sehr wichtige Bemerkung zu finden: ,Zu fast allen dieser Kriterien gibt es jedoch Ausnahmen, so daß sie nur als Anhaltspunkte für Abgrenzungsüberlegungen dienen können. Darauf wird auch von den meisten Autoren ausdrücklich hingewiesen.“ (Bruker 2013: 19)

\footnotetext{
161 Und zwar nur solche, die in FVG mit einem präpositional angeschlossenen Substantiv vorkommen, da Eisenberg - wie oben schon erklärt - nur solche Konstruktionen zum Kernbereich der FVG rechnet und zudem ausschließlich diese Gruppe der FVG in seiner Grammatik beschreibt.

162 Bezüglich der Arbeiten von Helbig (1979 und 1984) stellt Van Pottelberge in seiner im Jahre 2001 erschienenen Monographie fest, dass seit ihrer Erscheinung der Frage der Abgrenzungsmöglichkeiten der FVG ,nichts Wesentliches mehr hinzugefügt worden [ist]“ (Van Pottelberge 2001: 3). Diese Einschätzung der Wichtigkeit von Helbigs Beiträgen finde ich auch heute noch zutreffend.
} 
In ihrer eigenen FVG-Definition geht Bruker (2013: 28 f.) davon aus, dass FVG aus einem nominalen und einem verbalen Teil zusammengesetzte Konstruktionen sind, die die Funktion eines Prädikats haben. Beim nominalen Teil hält sie alle Kasus einschließlich des Präpositionalkasus für mögliche Realisierungsformen. Im Zusammenhang mit dem verbalen Element betont sie, dass es entsemantisiert und ,auf eine grammatikalische Funktion, nämlich Darstellung von Tempus, Numerus, Modus, Person und Genus verbi [reduziert ist]“". Bezüglich des nominalen Elements spricht sie von Verbalabstrakta und sieht die Paraphrasierbarkeit des FVG durch das entsprechende Basisverb ${ }^{163}$,[h]äufig, aber durchaus nicht immer“" gegeben. Als weitere Merkmale der FVG nennt sie noch Charakteristika der morphologischen Fixiertheit. Schließlich hält sie fest, dass nicht regelmäßig bestimmt werden kann, welches Verb mit welchem Nomen ein FVG bildet, wodurch FVG sowohl im Fremdsprachenunterricht als auch bei der maschinellen Übersetzung ${ }^{164}$ besondere Herausforderungen darstellen.

In Bezug auf die Abgrenzbarkeit der FVG hebt Bruker (2013:17) auch den von Krenn (2004) speziell fürs Deutsche konzipierten Entscheidungsbaum hervor, geht jedoch nicht darauf ein, dass dieses Verfahren von Krenn (2004: 8 f.) nur für solche Konstruktionen einsetzbar ist, in denen das vermeintliche Funktionsverb mit einem Nomen im Präpositionalkasus verbunden ist. Das heißt aber, dass viele der potenziellen FVG, und zwar diejenigen, in denen der nominale Bestandteil nicht präpositional an den verbalen Teil angeschlossen ist, diesen Test gar nicht durchlaufen können. Im Zusammenhang mit diesem Modell halte ich es für wichtig anzusprechen, dass Krenn (2004: 9, Abb. 1) als englische Entsprechung für den deutschen Terminus Funktionsverb den Ausdruck support verb verwendet. Auch Bruker (2013: 19f.) betrachtet den englischen Terminus support verb construction als eines der englischen Äquivalente für $F V G$. Das kann durchaus auch damit erklärt werden, dass sie im Deutschen unter anderem $S V G$ als Synonym von $F V G$ ansieht. Ferner wird von ihr auch der Terminus light verb construction (im Folgenden $L V C$ ) mit dem deutschen $F V G$ gleichgesetzt (vgl. Bruker 2013: 19 f.). So scheinen bei ihr $F V G$, SVG und $L V C$ ein und dasselbe, wenn auch vielfältige Phänomene zu bezeichnen (zu den LVC vgl. unten noch Van Pottelberge 2001). Dadurch wird auch das am Anfang von Kap. 3 angedeutete Problem ersichtlich, wonach nicht nur der Terminus $F V G$ uneinheitlich definiert wird, sondern auch dessen Verhältnis zum in der

\footnotetext{
${ }^{163}$ Damit meint Bruker (2013: 28) das Vollverb, aus dem das nominale Element des FVG abgeleitet wurde.

${ }^{164}$ Diese Bemerkung von Bruker sollte - meiner Ansicht nach - dahingehend erweitert werden, dass diese sprachlichen Konstruktionen generell bei der maschinellen Sprachverarbeitung problematisch handzuhaben sind (vgl. dazu u. a. Langer 2004, Fazly / Stevenson 2005).
} 
Fachliteratur ebenfalls kontrovers behandelten SVG-Begriff nicht eindeutig ist. Im Folgenden wende ich mich auch diesem Thema zu.

Zwar weist auch Storrer (2007: 164) in ihrem Beitrag Corpus-based investigations on German support verb constructions darauf hin, dass u. a. die Termini support verb construction und function verb construction in der Fachliteratur z. T. als Bezeichnung für ein und dieselbe besondere Gruppe von komplexen Prädikaten verwendet werden, aber sie unterschiedet zwei Richtungen der Forschung voneinander (vgl. Storrer 2007: 168ff.). Die eine nennt sie SVC line und verbindet diese mit der französischen Linguistik, der maschinellen Übersetzung und der Computerlinguistik. Die andere Richtung, die sie als FVC line bezeichnet, leitet sie aus der deutschen Linguistik her. Den wichtigsten Unterschied zwischen den beiden Forschungsrichtungen sieht sie darin, dass sie unterschiedliche Typen dieser komplexen Prädikate als prototypisch betrachten. Während Vertreter der SVC line die Konstruktionen als prototypisch ansehen, in denen sich das nominale Element in der Position des direkten Objekts des verbalen Bestandteils befindet, ${ }^{165}$ geht man der FVC line zufolge davon aus, dass in prototypischen Fällen das Nomen präpositional ans verbale Element angeschlossen ${ }^{166}$ ist. Das heißt also, dass dementsprechend FVG und SVG - zumindest morphologisch gesehen - durch den unterschiedlichen prototypischen Kasus des nominalen Bestandteils voneinander abgegrenzt werden könnten. Die Idee, nach der in prototypischen FVG das Nomen durch eine Präposition mit dem verbalen Bestandteil verbunden ist und in prototypischen SVG im reinen Akkusativ steht, kann jedoch mit vielen anderen FVG- und SVG-Ansätzen nicht vereinbart werden. Dafür reicht vorerst nur der Hinweis u. a. auf die oben genannten FVGBeschreibungen von Helbig (1979), Lehmann (1983) oder Bruker (2013).

Langer (2004: 171 ff.) vertritt ebenfalls die Auffassung, dass die Termini FVG und SVG miteinander verwandt sind, betont aber ferner, dass sie nicht miteinander gleichgesetzt werden sollten. Während er bezüglich des in der deutschen Linguistik entstandenen Terminus $F V G$ auf die Uneinheitlichkeit der Definitionen hinweist, soll der - in erster Linie in der Romanistik erarbeitete - Terminus $S V G$ seiner Meinung nach „quite well defined“ sein.

Er charakterisiert SVG als semi-kompositionelle Verbindungen eines prädikativen Nomens (engl. predicative noun) und eines Stützverbes (engl. support verb), in denen die Argumente des Nomens als syntaktische Aktanten (Komplemente) des Stützverbs realisiert werden. In prototypischen SVG betrachtet er die Nomina als Prädikate innerhalb der Konstruktion und

\footnotetext{
${ }^{165}$ Ein Beispiel von Storrer (2007: 165; meine Hervorhebung, Á. S.-T.) dafür ist: Peter trifft eine Entscheidung.

166 Wie im folgenden Beispiel von Storrer (2007: 165; meine Hervorhebung, Á. S.-T.): Peter tritt in Verbindung mit dem Minister.
} 
schreibt dem Stützverb überwiegend syntaktische Relevanz zu. Zur inneren Morphologie der SVG stellt er Folgendes fest: „The predicative noun is realised as head of a noun phrase in a syntactic slot provided by the support verb; in many cases but not always this is the direct object position“ (Langer 2004: 172; meine Hervorhebung, Á. S.-T.). Aus semantischer Sicht hält er außerdem eine Art Entsemantisierung des Stützverbs für ein weiteres Charakteristikum der SVG. Während in der Erklärung von Langer in prototypischen SVG das Nomen meistens, aber nicht ausschließlich in der Position des direkten Objekts des Stützverbs realisiert wird, schränkt er FVG eindeutig auf ein formales Muster ein: „A related kind of constructions are the ones that have been in the main focus of German research on semi-compositional verb-noun constructions. They consist of a predicative noun embedded in a prepositional phrase, again

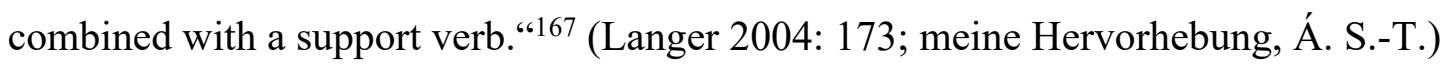

Ähnlich wie bei den SVG würden auch bei den FVG die syntaktischen Aktanten des verbalen Bestandteils nicht vom Verb semantisch subkategorisiert, sondern gehörten bedeutungsmäßig zum nominalen Element. Im Gegensatz zu den - seiner Meinung nach - gut abgrenzbaren SVG findet er die weitere, v. a. semantische Charakterisierung der FVG problematisch:

This type of construction is more difficult to describe semantically, allows much less generalizations and especially does not easily allow the construction of semantic equivalence classes between verbal and nominal predicates as in the case of prototypical support verb constructions. (Langer 2004: 173)

Obwohl er in seinem Artikel einen ausführlichen Überblick der bis dahin in der Forschung verwendeten Testverfahren zur Abgrenzung der SVG gibt, von denen einige seiner Ansicht nach auch zuverlässig zu sein scheinen, stellt Langer (2004: 182 f.) schließlich fest, dass eigentlich keiner dieser Tests den ganzen Bereich der SVG ausnahmslos abdecken kann. Außerdem sind diese Verfahren nicht automatisierbar, was die maschinelle Verarbeitung bzw. Identifizierung der SVG sehr problematisch macht.

In seiner Monographie Verbonominale Konstruktionen, Funktionsverbgefüge: vom Sinn und Unsinn eines Untersuchungsgegenstandes betrachtet Van Pottelberge (2001) die Frage, wie Funktionsverbgefüge von Nicht-Funktionsverbgefügen unterschieden werden können, wobei er auch die vorangehende Forschung akribisch und kritisch unter die Lupe nimmt. Seine ausführliche und umfangreiche Arbeit kann hier nicht in all ihren Einzelheiten detailliert dargestellt werden, vielmehr kommt es darauf an, Gedanken hervorzuheben, die die

\footnotetext{
${ }^{167}$ Als Beispiel für ein deutsches FVG führt Langer (2004: 173) den Ausdruck in Blüte stehen an.
} 
Aufmerksamkeit auf - meines Erachtens - wichtige problematische Thesen der Forschung lenken.

Ähnlich wie viele andere vertritt Van Pottelberge (2001:1) die Auffassung, dass im Deutschen generell der Terminus $F V G$, im Französischen dagegen $S V G$ (genauer ,construction à verbe support ${ }^{*}$ ) verwendet wird. Außerdem weist er darauf hin, dass es für die betreffenden, in vielen Sprachen üblichen Konstruktionen keine „,internationale Bezeichnung“ gibt. Dem Problem des „fehlenden Einheitsbegriffs“ im Englischen widmet er sogar ein eigenes Unterkapitel (vgl. Van Pottelberge 2001: 66ff.), in dem er feststellt, dass „,[k]ein einziger Terminus für Verben in verbonominalen Konstruktionen in der englischsprachigen Forschung so verbreitet wie light verb [ist]“, wobei er jedoch auch darauf hinweist, dass der Begriff, ,heute mit verschiedenen Inhalten vor[kommt]“ (Van Pottelberge 2001: 78 und 77). ${ }^{168}$

Van Pottelberge (2001: 2ff.) stellt auch klar, dass der Terminus FVG keinesfalls einheitlich verwendet wird. Bei der Abgrenzung der zur Klasse der FVG gehörenden verbonominalen Konstruktionen werden meist formale und semantische Kriterien zugleich herangezogen, weil sie als „ein systematisches Muster oder Verfahren“ betrachtet werden, was dazu führt, dass ihre Beschreibung als Aufgabe der Grammatik angesehen wird (Van Pottelberge 2001: 6). Als gemeinsamen Nenner der zahlreichen und z. T. deutlich auseinandergehenden FVGKlassifikationen sieht er das formale Kriterium, nach dem in einem FVG ein Verb und ein Substantiv miteinander verbunden werden, und das Substantiv „meist in Form eines Akkusativobjekts oder eines Präpositionalobjekts" realisiert wird (Van Pottelberge 2001: 15), wobei ich jedoch die Formulierung Akkusativ-oder Präpositionalobjekt in Bezug auf diese Konstruktionen etwas unglücklich finde, da es in diesen Verbindungen überhaupt nicht als unumstritten gilt, dass das nominale Element als Objekt des Funktionsverbs betrachtet werden kann. ${ }^{169}$

Die in der Fachliteratur vorfindlichen Kriterien, die zur Unterscheidung der FVG von anderen verbonominalen Konstruktionen verwendet werden, betrachtet Van Pottelberge aus mehrerlei Sicht kritisch. Ohne auf jedes Detail eingehen zu können, stehe hier in diesem Zusammenhang zuerst eine summarische Feststellung von Van Pottelberge (2001: 3; meine

\footnotetext{
168 Neben light verb betrachtet Van Pottelberge (2001: 78ff.) nur composite predicates als einen Terminus, der „mehr als gelegentlich benutzt wird“. Er geht noch auf einige weitere Termini ein, die zur Bezeichnung dieses Phänomens verwendet werden; er erklärt jedoch, dass sie „einmalig oder nicht konsequent mit demselben Inhalt vor[kommen]“ (Van Pottelberge 2001: 79). Dabei ist darauf hinzuweisen ist, dass er an anderen Stellen - ganz berechtigt - auch die uneinheitliche Verwendung der weiter verbreiteten, geläufigen light verb und composite predicates demonstriert.

${ }^{169}$ Vgl. dazu z. B. Storrers (2007) oder Langers (2004) Formulierung, die betreffend das nominale Element in diesen Verbindungen nicht über Objekte, sondern über ein Element in Objektposition reden. Vgl. ferner in Kap. 3.2 die Überlegungen zur internen und externen Valenz von solchen Konstruktionen.
} 
Hervorhebung, Á. S-T.): „Die Leichtigkeit, mit der Definitionsprobleme gegenwärtig unter den Teppich gekehrt werden, hängt wohl mit den gescheiterten Abgrenzungsversuchen in der Vergangenheit zusammen.“

Als Hauptfragestellung von Van Pottelberges (2001: 6) Arbeit gilt, ob sich FVG überhaupt konsequent durch semantische und syntaktische Merkmale charakterisieren lassen und so als „eine eigene Klasse oder eine Kategorie (ein sprachliches Zeichen mit einer Form und einer Bedeutung)“ betrachtet werden können. Zur Beantwortung dieser Frage setzt er sich kritisch mit früheren Klassifikationsversuchen auseinander, und kommt letztendlich zur Schlussfolgerung, dass die verbonominalen Konstruktionen, die in der Forschung bis dahin gemeinhin als FVG oder mit einem der verwandten Termini bezeichneten wurden, keine eigene Klasse bilden. Entsprechend der Heterogenität des Untersuchungsgegenstandes vertritt Van Pottelberge (2001: 10) darüber hinaus die Auffassung, dass ,es eine schlüssige Abgrenzung der verbonominalen Konstruktionen nicht geben kann“.

Eine weitere interessante Frage, die von Van Pottelberge (2001: 18ff.) thematisiert wird, ist die Bezeichnung von FVG als „komplexe Prädikate“, wofür es zahlreiche Beispiele in der Fachliteratur gibt, wie auch aus den Darstellungen in diesem Unterkapitel ersichtlich werden kann. Diese Bezeichnung findet Van Pottelberge jedoch problematisch, da sie ebenfalls uneinheitlich gebraucht wird. Dabei zeigt er, dass die Verwendung dieses Begriffs von Partikelund Präfixverben über analytische Verbformen bis zur Verbindung eines Verbs mit einem Substantiv oder sogar Adjektiv reicht. Van Pottelberge (2001: 20) lenkt die Aufmerksamkeit außerdem auch darauf, dass es überhaupt problematisch ist, ob mit Prädikat ein grammatisches oder ein logisch-semantisches Phänomen gemeint ist.

Da seiner Ansicht nach die Bezeichnung der FVG als (komplexe) Prädikate auf Grund des uneinheitlichen Gebrauchs Probleme aufwirft, plädiert Van Pottelberge (2001: 31ff.) in Anlehnung an Fourquets Konnexionsmodell dafür, FVG als primäre Konnexionen zu betrachten. Unter primärer Konnexion ist zu verstehen, dass ein Glied des Satzes sich zuerst mit dem Verb als Kernteil des Satzes verbindet, wodurch ein Komplex entsteht. Die weiteren Glieder des Satzes knüpfen sich nicht einfach ans Verb, sondern an diesen Komplex an. ${ }^{170}$

\footnotetext{
${ }^{170}$ Zwar weist Van Pottelberge (2001: 33 und 37) darauf hin, dass Forquets Konnexionsmodell ,,vielmehr eine theoretische Skizze als ein fertig ausgearbeitetes Analysemodell [ist]“, das zudem ,in der deutschen Grammatikbeschreibung weitgehend unbekannt geblieben [ist]", findet er einen solchen Beschreibungsrahmen in der Hinsicht besonders vorteilhaft, dass dadurch nicht nur Verb-Substantiv-Verbindungen sondern auch VerbAdjektiv-Verbindungen bzw. die Verbindung von Kopulaverben mit den entsprechenden Prädikativen ,auf einer gemeinsamen Ebene" behandelt werden können.
} 
Ein großes Verdienst von Van Pottelberge (2001) ist - in meinen Augen -, dass seine Arbeit nicht nur theoretisch ausgerichtet ist, sondern in ihrem zweiten Teil auch auf empirischen Korpusanalysen beruht. Nach der Beweisführung im ersten Teil, dass FVG nicht als gesonderte Klasse betrachtet werden sollten, will er im zweiten Hauptteil seiner Monographie durch die Analyse von verbonominalen Konstruktionen mit den Verben versetzen und bringen zeigen, wie solche Verbindungen zu beschreiben sind. Im Vergleich zu vorangehenden Abhandlungen sieht er, neben der nicht nur synchronisch, sondern auch diachronisch ausgerichteten Untersuchung, seine Vorgehensweise als wichtige Neuerung an, wobei „erstmals ausgewählte Valenzmuster der Verben bringen und versetzen exhaustiv beschrieben werden und nicht nur diejenigen Verbindungen, die mit einem Nomen actionis oder Abstraktum gebildet sind (...)“ (Van Pottelberge 2001: 9; Unterstreichungen von mir, Á. S.-T.). Im Zusammenhang mit seinen Beleganalysen halte ich es jedoch für wichtig hervorzuheben, dass er auf eine „statistische Korpusauswertung verzichtet“, da er eher darum bemüht ist, „das Mögliche [zu] zeigen“(Van Pottelberge 2001: 10).

Für die vorliegende Darstellung des FVG-Problems finde ich die Arbeit von Heine (2006) unter dem Titel Funktionsverbgefüge in System, Text und korpusbasierter (Lerner-)Lexikographie aus zweierlei Sicht bedeutend. Einerseits geht sie zur Definition ihres FVG-Begriffs von einer ausführlichen, vergleichenden Analyse der FVG-Beschreibung von neun Grammatiken aus, andererseits ist sie auf die korpusbasierte Untersuchung von FVG fokussiert. ${ }^{171}$ Ohne hier auf ihre Grammatikanalysen detailliert eingehen zu können, sollen hier lediglich Heines (2006: 44ff., v. a. 49) wichtigste Ergebnisse und ihre daraus resultierende FVG-Definition erörtert werden.

Auch Heine (2006: 17) weist schon am Anfang ihrer Untersuchung darauf hin, dass in der Forschung für die Beschreibung der „Wesens- und Abgrenzungsmerkmale“ von FVG ,eine große Divergenz" charakteristisch ist. Nach der eingehenden Behandlung des FVG-Bildes der von ihr recherchierten Grammatiken gelangt sie zu 24 Kriterien, die in den Arbeiten zur Definition von FVG verwendet werden, wobei sie ebenfalls das Problem andeutet, dass zur

\footnotetext{
171 Der Grund, warum Heine (2006) in der Vorphase der Formulierung ihrer eigenen FVG-Definition vorerst (ausschließlich) Grammatiken unter die Lupe nimmt, liegt darin, dass sie sich mit FVG aus der Perspektive des Deutsch als Fremdsprache-Unterrichts auseinandersetzt und ,[d]en Grammatiken [...] neben den Wörterbüchern die wohl größte Bedeutung als Nachschlagewerke für Fremdsprachenlerner, -studenten und -lehrer zu[kommt] [...]“ (Heine 2006: 18). Es soll hier noch angemerkt werden, dass sie sich auch mit lexikographischen Fragestellungen zur Untersuchung der FVG befasst, aber die spielen für meine Arbeit nur eine geringe Rolle und beeinflussen auch die Definition von Heine (2006) nicht, deswegen werden sie hier ausgegrenzt.

Ferner soll noch betont werden, dass Heine (2006: 50ff.) auch zahlreiche weitere Arbeiten aus der Fachliteratur recherchiert, die keine Grammatiken sind. Diese nimmt sie jedoch nicht als Grundlage für die Erarbeitung ihrer Arbeitsdefinition von $F V G$, sondern braucht sie bei den Versuchen, FVG von anderen verbonominalen Konstruktionen abzugrenzen.
} 
zusammenfassenden Darstellung ${ }^{172}$ der in der Fachliteratur beschriebenen Merkmale von FVG „Vereinfachungen vorgenommen und gegebenenfalls verschiedene Perspektiven und vor allem Termini der Autoren einander angepasst werden [mussten]" (Heine 2006: 44; meine Hervorhebung, Á. S.-T.)

Außerdem stellt Heine (2006: 44f.) auch klar, dass es in der Fachliteratur öfter Beispiele dafür gibt, dass bestimmte Definitionsmerkmale in ihrer Gültigkeit eingeschränkt werden, ${ }^{173}$ was für sie bedeutet, dass diese nicht auf den Kernbereich der FVG zutreffen und dementsprechend bei der Grundlegung ihrer Definition außer Acht gelassen werden. So spielen für sie die Frage der Festigkeit des Artikelgebrauchs, die der Negation und die der Attribuierbarkeit des Substantivs in FVG bei der Etablierung ihres FVG-Begriffs von vornherein keine Rolle. Ferner bestimmt sie sechs Wesensmerkmale der FVG, die in der Fachliteratur kontrovers behandelt werden, wobei der Weg zur Formulierung ihrer eigenen FVG-Definition schrittweise über ihre Stellungnahmen zu diesen Fragen führt, was im Folgenden kurz zusammengefasst wird.

Als erstes greift sie die Problematik der Struktur dieser Konstruktionen auf, womit gemeint ist, in welcher Form Verb und Nomen in FVG miteinander verbunden sind, d. h., ob nur präpositional angeschlossene Substantive oder aber auch Substantive im Akkusativ, oder wofür wesentlich seltener in der Fachliteratur argumentiert wird, Substantive ohne Kasuseinschränkung als nominales Glied von FVG anzunehmen sind. Heine (2006: 46) entscheidet sich für die Anerkennung von präpositional und akkusativisch mit dem verbalen Glied verbundenen Substantiven in FVG, wobei sie der Meinung ist, dass noch zu überprüfen ist, ob durch diese Strukturmerkmale tatsächlich sämtliche FVG abgedeckt werden können.

Dabei schließt sie jedoch schon auf theoretischer Grundlage die Möglichkeit dessen aus, dass Substantive im Genitiv oder im Nominativ als Teil von FVG betrachtet werden können. Das begründet sie einerseits damit, dass die wenigen Verben, die im Gegenwartsdeutschen den Genitiv regieren nicht als Funktionsverben in Frage kommen, „denn keines dieser Verben kann als (hochgradig) polysem oder als eine sehr allgemeine Bedeutung tragend eingestuft werden“ (Heine 2006: 46), was sie jedoch - der am weitesten verbreiteten Auffassung in der Fachliteratur entsprechend - als grundlegendes Merkmal von Funktionsverben betrachtet. ${ }^{174}$

\footnotetext{
${ }^{172}$ Das erfolgt in Heines Arbeit in Form einer Tabelle (vgl. Heine 2006: 43f., Tab. 10).

173 Dieses Problem wird auch in meiner Arbeit eindeutig, vgl. dazu z. B. (oben in diesem Unterkapitel) die Darstellung von Helbigs (1979) FVG-Kriterien oder Brukers (2013) Beschreibung der Forschungsgeschichte bzw. der Definitionsproblematik.

${ }^{174}$ Vgl. dazu z. B. (oben in diesem Unterkapitel) die Schilderung von Lehmanns (1983) Charakterisierung von Funktionsverben.
} 
Andererseits grenzt sie Konstruktionen wie die Untersuchung erfolgt ${ }^{175}$, in denen das Substantiv im Nominativ steht, aus, „da das Substantiv im Nominativ das Subjekt des Satzes wäre und damit nicht gleichzeitig lexikalischer Prädikatsteil (eines Funktionsverbgefüges) sein kann“ (Heine 2006: 46). Ebenfalls auf theoretischer Grundlage, aber weniger entschlossen, schließt sie Konstruktionen zumindest vom Kernbereich ${ }^{176}$ der FVG aus, in denen das Substantiv im Dativ realisiert wird. Das erklärt sie wiederum durch die Charakterisierung von Funktionsverben als „semantisch entleert“ oder „,in einem hohen Maße polysem“ (Heine 2006: 46f.), was im Falle der Verben unterziehen und unterliegen allerdings nicht gelte, da sie ihren Recherchen zufolge in der Fachliteratur als (potenzielle,) den Dativ regierende Funktionsverben betrachtet werden.

In Bezug auf die semantischen Merkmale des verbalen Glieds von FVG vertritt Heine (2006: 49) - wie es aus den obigen Ausführungen z. T. schon deutlich wurde - die Auffassung, dass das Verb „,von sehr allgemeiner Bedeutung und Träger semantischer Merkmale hinsichtlich Phase/Aktion/Perspektive [...] [ist]“. Es ist wichtig hervorzuheben, dass die beiden Verben haben und sein von Heine (2006: 48) ${ }^{177}$,auf Grund ihrer ,Sonderstellung a aus der Gruppe der Funktionsverben ausgeschlossen [werden]“. Im Substantiv sieht sie den Vermittler der „Hauptbedeutung“. Ferner drückt das Substantiv eine „Handlung“, ein „Ergebnis“, einen „Vorgang“ oder einen „Zustand“ aus, und in FVG (des Kernbereichs) ist es - Heines Ansicht nach - nicht in übertragener Bedeutung zu interpretieren und stellt keine unikale Komponente dar.

Aus der Sicht meiner auf die Substantivvalenz fokussierten Arbeit und der in Kap. $3.2 \mathrm{zu}$ thematisierenden problematischen Betrachtung der Valenzverhältnisse in und von FVG sind noch weitere bestimmte Merkmale, mit deren Hilfe Heine (2006: 47ff.) die Substantive in FVG weiter charakterisiert, von großer Bedeutung. Zuerst erklärt sie, dass sie die von vielen Autoren festgelegte Abstraktheit als Charakteristikum der Substantive in FVG problematisch findet, da viele dieser Substantive mehrere Lesarten haben, unter denen häufig auch die Interpretation als Konkretum vorkommt. Außerdem ist sie der Meinung, dass nicht allgemein behauptet werden

\footnotetext{
175 Beispiel von Heine (2006: 46).

${ }^{176}$ Im Sinne des Prototypenansatzes geht Heine (2006: 241) von einem Kontinuum der FVG aus, wobei die prototypischen Vertreter der Gruppe den Kernbereich der FVG ausmachen, während weniger typische Mitglieder der Gruppe zur Peripherie gerechnet werden sollen bzw. „sich sowohl den Funktionsverbgefügen als auch den Kollokationen bzw. verbalen Phraseolexemen zuordnen lassen, da sie jeweils nur einen Teil der Kriterien erfüllen“.

177 In diesem Zusammenhang weist Heine (2006: 48) auf die zweite Auflage von Eisenbergs Grundriß der deutschen Grammatik. Bd. 2: Der Satz hin. In meiner Arbeit wird Eisenbergs FVG-Beschreibung aufgrund der dritten, durchgesehenen Auflage dieser Grammatik (Eisenberg 2006) referiert, in der allerdings haben und sein genauso von der Gruppe der Funktionsverben ausgeschlossen werden (vgl. oben in diesem Unterkapitel).
} 
kann, dass nur deverbale und deadjektivische Substantive in FVG vorkommen können, womit ich absolut einverstanden bin, ihre Begründung jedoch finde ich nicht besonders zutreffend. Sie führt nämlich als Erklärung auf, dass ,,mittlerweile eine Reihe von substantivischen Fremd- und Lehnwörtern in die deutsche Sprache Einzug gehalten haben, mit denen nicht immer auch das entsprechende Verb oder Adjektiv entlehnt worden ist“" (Heine 2006: 47). Dennoch bezeichnet sie deverbale und deadjektivische Substantive für die Untersuchung von FVG ,als besonders interessant“ (Heine 2006: 47), da ihr zufolge „Valenz und Deverbalität/-adjektivität in einem direkten Verhältnis zueinander stehen“ (Heine 2006: 48). ${ }^{178}$ Schließlich gibt sie auch in ihrer FVG-Definition als Charakteristikum des Substantivs in einem FVG an, dass es valent ist (vgl. Heine 2006: 49).

Als Teil ihrer FVG-Definition erklärt Heine (2006: 49f.) ferner, dass „Funktionsverb und Nominalgruppe [...] eine derart enge Verbindung ein[gehen], dass sie gemeinsam Satzgliedcharakter haben und die Nominalgruppe weder pronominalisierbar noch erfragbar ist". Als weitere Folge der Einheit des verbalen und nominalen Glieds betrachtet sie, dass FVG, in denen das Substantiv im Akkusativ steht, nicht passivierbar sind.

Die Paraphrasierbarkeit von FVG ,durch ein mit dem Nomen stammverwandtes Vollverb (oder Adjektiv + Kopula)“ (Heine 2006: 45), die ein umstrittenes Kennzeichen von FVG in der Fachliteratur darstellt, grenzt Heine (2006: 47) als definitorische Eigenschaft aus, da sie - ihres Erachtens - einerseits nicht bei jedem FVG gegeben ist, andererseits jedoch auch bei anderen verbonominalen Konstruktionen - wie z. B. bei Kollokationen oder freien Verbindungen vorzufinden ist.

Ich halte es noch für wichtig anzumerken, dass sich Heine (2006: 76ff.) in einem „Exkurs“ auch mit „,[e]in[em] Versuch, die Existenz von Funktionsverbgefügen zu widerlegen“ befasst, indem sie sich ausführlich mit Van Pottelberges (2001) oben thematisierter Monographie auseinandersetzt. Ihrer Darstellung ist an mehreren Punkten eine eindeutige Kritik an Van Pottelberges Annahmen zu entnehmen, ich will an dieser Stelle freilich nur die wichtigsten Aspekte von Heines (2006: 76) „(Haupt-)Kritikpunkt“ erörtern. Sie vermisst nämlich bei Van

\footnotetext{
178 Besonders problematisch finde ich Heines (2006: 47f.) Ausführungen, die der obigen Schlussfolgerung vorangehen und in denen sie sich auf die Arbeiten von Bassola (1998) und Kubczak/Schumacher (1998) beruft, da ich der Ansicht bin, dass aus den beiden zitierten Schriften nur voreilig die Konklusion gezogen werden kann, wonach zwischen „Deverbalität/-adjektivität“ und Valenz ein „direktes“, d. h. für mich einander gegenseitig voraussetzendes, Verhältnis bestehe.

Außerdem vertrete ich, wie ich es schon ausführlich thematisiert habe, die Auffassung, dass Substantive, darunter auch nicht-abgeleitete generell über Valenz verfügen können (vgl. v. a. Kap. 2.4.2, aber auch 2.6). Dementsprechend werden im korpusanalytischen Teil meiner Arbeit u. a. nicht-abgeleitete Substantive untersucht, die zudem nicht als Fremd- oder Lehnwörter betrachtet werden können und aus der Analyse soll hervorgehen, dass auch die in solchen Verb-Substantiv-Verbindungen vorkommen können.
} 
Pottelberge (2001) die Beschreibung von Kollokationen und die Klarstellung des Verhältnisses von Kollokationen und FVG zueinander. Ferner ist sie der Meinung, dass Van Pottelberges Phraseologismus-Begriff nicht der gängigen Interpretation in der Fachliteratur entspricht und er auch demzufolge Konstruktionen wie $j m d m$ etw $w_{A}$ versetzen und jmdn/ etw $w_{A}$ in etw versetzen $^{179}$ statt als Kollokationen falsch als freie Verbindungen klassifiziert (vgl. Heine 2006: 77).

Heine (2006: 83) führt diesen Gedanken weiter aus, indem sie die potenzielle Zuordnung der von Van Pottelberge (2001) untersuchten Konstruktionen mit bringen und versetzen zu den FVG aus der Perspektive ihrer eigenen FVG-Definition abwägt. Dabei findet sie die Möglichkeit der Klassifizierung der betreffenden Verbindungen mit dem Verb versetzen aus mehrerlei Sicht von vornherein problematisch. Einerseits verfügt dieses Verb in den gegebenen Konstruktionen nicht über eine allgemeine Bedeutung und entspricht dementsprechend ihrer Definition von Funktionsverben nicht; außerdem findet sie auch die Substantive, die sich potentiell mit dem Verb in den oben zitierten, von Van Pottelberge untersuchten Valenzmustern verbinden können, auch nicht ihrer FVG-Definition entsprechend. Darüber hinaus sieht sie sowohl durch Korpusanalysen als auch durch Van Pottelberges (2001: 262 bzw. 268) eigene Erklärung, wonach sich bestimmte Substantive auf Grund von „Selektion“ bzw. „Affinität“ mit versetzen in den gegebenen Valenzmustern verbinden, bewiesen, dass diese Konstruktionen weder als FVG noch als freie Verbindungen, sondern als Kollokationen zu klassifizieren seien.

Am Ende meiner Abhandlungen über das Problem der terminologischen und ontologischen Vielfalt des Phänomens FVG / SVG kehre ich nochmals zu der bereits am Anfang von Kap. 3 zitierten Monographie von Kamber (2008) zurück, da ich Kambers Vorgehensweise bezüglich der Beschreibung der $\mathrm{FVG}^{180}$ aus mehreren Gründen vorbildlich finde. Er geht nämlich ebenfalls von der Annahme aus, dass das entsprechende sprachliche Phänomen so weit gefasst und vielfältig ist, dass FVG nicht als eine homogene Gruppe betrachtet werden können (vgl. z. B. Kamber 2008: 9). Zwar ordnet auch Kamber (2008: 13f.) FVG auf einem Kontinuum zwischen semantisch kompositionellen, „regulären spezifischen Wortverbindungen“ und Idiomen als nicht idiomatische Phraseologismen an, weist aber zugleich darauf hin, dass

\footnotetext{
179 Wie ich in meiner zusammenfassenden Darstellung von Van Pottelberges (2001) Monographie schon angesprochen habe, befasst sich Van Pottelberge im praktischen, korpusanalytisch angelegten zweiten Hauptteil seiner Arbeit mit der Untersuchung von ausgewählten Valenzmustern der Verben bringen und versetzen (vgl. oben in diesem Unterkapitel), zu denen die obigen, von Heine (2006: 77ff.) kritisierten Beispiele gehören.

${ }^{180}$ Es sei hier kurz angemerkt, dass Kamber (2008: 34) an einer Stelle die beiden Termini Funktionsverb und Stützverb als Entsprechungen voneinander verwendet, wobei er jedoch darauf hinweist, dass die beiden in der Fachliteratur nur teilweise als Synonyme, teilweise jedoch als Termini unterschiedlicher Extension interpretiert werden. Er entscheidet sich in seiner Monographie für den Terminus Funktionsverb und bezeichnet dementsprechend die betreffenden Konstruktionen als FVG.
} 
zwischen diesen drei Gruppen mit keinen scharfen Grenzen zu rechnen ist. Das führt jedoch auch seiner Ansicht nach - dazu, dass das Problem der Abgrenzung der FVG kaum endgültig gelöst werden kann. Das hat wiederum zur Folge, dass „[v]ieles [...] hier im Ermessensspielraum des Sprachwissenschaftlers [bleibt]“ (Kamber 2008: 15), was ihn jedoch keinesfalls dazu veranlasst, FVG als Untersuchungsgegenstand zu verwerfen; vielmehr plädiert er für „einen möglichst flexiblen theoretischen Rahmen“ (Kamber 2008: 20) für deren Untersuchung. Dabei bedient sich Kamber (2008: 20 ff.) des Ansatzes der Prototypensemantik und entwirft zur Klassifizierung der FVG „,das Modell der umrahmten Schnittmengen“.

Ohne dieses Modell hier detailliert behandeln zu können, soll es dennoch in seinen Grundzügen charakterisiert werden. Kamber nimmt ein einziges Basiskriterium, dessen Erfüllung schon dazu ausreicht, einen FVG-Kandidaten tatsächlich als FVG einzustufen. Dabei wird untersucht, ob das Verb in der jeweiligen Konstruktion als Funktionsverb verwendet wird. ${ }^{181}$ Dieses Kriterium ist zwar „ein ,weiches“ semantisches Kriterium“, das eine „gewisse Subjektivität" (Kamber 2008: 30) zulässt; nichtsdestotrotz finde ich die Idee richtig, ein solches semantisches Merkmal als nicht einziges, jedoch hinreichendes Kriterium für die Zuordnung zur Klasse der FVG zu betrachten. Neben diesem Basiskriterium arbeitet das Modell mit drei Subklassifizierungskriterien, durch die überprüft wird, ob der nominale Bestandteil ein Verbalabstraktum ist, ob der verbale Teil durch ein Bewegungs- oder Zustandsverb realisiert wird und ob das Nomen präpositional mit dem verbalen Teil verbunden ist (im Einzelnen dazu vgl. ausführlich Kamber 2008: 25ff.). Prototypische FVG erfüllen nach Kamber neben dem Basiskriterium alle drei Subkategorisierungskriterien, weniger typische nur zwei oder eins davon und zur Peripherie der Gruppe gehörende FVG entsprechen nur dem Basiskriterium.

Als großen Vorteil von Kambers Vorgehensweise bewerte ich neben dem flexiblen theoretischen Rahmen auch die Tatsache, dass er von möglichst wenig Vorannahmen ausgehend auf der Grundlage einer detaillierten Korpusanalyse weitere Informationen über das Verhalten und die Eigenschaften der FVG gewinnt (vgl. dazu v. a. Kamber 2008: 43ff.). Außerdem ist für jemanden, der - wie auch ich - die sui generis-Auffassung der Substantivvalenz vertritt, besonders attraktiv, dass in Kambers Modell nicht nur Konstruktionen zum Untersuchungsgegenstand gerechnet werden, in denen das nominale

${ }^{181}$ In diesem Zusammenhang soll erwähnt werden, dass ich Kambers (2008: 23ff.) Präzisierung des Begriffs Funktionsverb sehr begrüße, bei der dieses in Verbindung mit dem nominalen Element immer in der konkreten Konstruktion untersucht und nicht bloß als entsemantisiertes, mit (eher) nur grammatischen Funktionen bekleidetes Element betrachtet wird. 
Element ein Verbalabstraktum ist. D. h., dass auch Kandidaten mit nicht-abgeleitetem nominalen Bestandteil als FVG in Frage kommen.

Nachdem ich einen Überblick davon zu geben versuchte, wie unterschiedlich die Begriffe FVG bzw. SVG in der Forschung interpretiert und ggf. miteinander in Verbindung gesetzt werden, wende ich mich im folgenden Unterkapitel der Frage zu, wie die Valenzverhältnisse in und von diesen Konstruktionen in der Fachliteratur dargestellt werden.

\subsection{Valenzbeschreibungen von FVG / SVG}

Die Interpretation der Valenzverhältnisse und -eigenschaften von FVG / SVG wird in der Forschung mindestens so häufig und vielfältig thematisiert wie die Bestimmung der Begriffe selbst. Bei der Darstellung der Valenzbeschreibung dieser Konstruktionen kann man - meines Erachtens - einer weiteren Schwierigkeit begegnen, und zwar der, dass nicht nur die Termini $F V G / S V G$ in der Forschung problematisch sind, sondern die Zuordnung der damit bezeichneten Phänomene zum Bereich der Phraseologie ebenfalls nicht unumstritten ist. Abhängig davon, ob einem Ansatz ein weiter oder aber ein enger Phrasem- bzw. PhraseologieBegriff zugrunde liegt, können Phänomene wie FVG/SVG nämlich in den jeweiligen Abhandlungen ausgegrenzt oder mitberücksichtigt werden. Dementsprechend werden hier nur solche phraseologischen Arbeiten rezipiert, die sich entweder explizit mit FVG / SVG befassen oder in deren Phraseologiedeutung auch FVG / SVG eindeutig miteinbegriffen sind.

Helbig (1979: 277) vertritt die Auffassung, dass sich das Funktionsverb sowohl semantisch als auch in seinen quantitativen und qualitativen Valenzeigenschaften vom ursprünglichen Vollverb unterscheidet. Er betrachtet das Substantiv im FVG als semantischen Schwerpunkt der Konstruktion und auch als „Hauptvalenzträger im Satz“. In diesem Zusammenhang stellt er Folgendes fest: „Weitere Objekte und Adverbialien im Satz hängen nicht direkt vom FV

[Funktionsverb, Á. S-T.], sondern vom SF [Substantiv im Funktionsverbgefüge, Á. S-T.] und erst über dieses SF von der Gesamtheit des FVG ab“.

Obwohl er „,von weiteren Objekten“ spricht, was darauf hindeuten könnte, dass er das Substantiv im FVG als Objekt einstuft, hebt er an einer späteren Stelle hervor, dass das Substantiv im FVG weder als Objekt noch als Adverbialbestimmung betrachtet werden kann, da es Teil des Prädikats sei (vgl. Helbig 1979: 282). Diese Feststellung schränkt er zuerst auf die FVG ein, die zum „Zentrum der FVG“ gehören und damit bei ihm - wie ich in Kap. 3.1 schon thematisiert habe - diejenigen Konstruktionen sind, in denen das Substantiv akkusativisch oder präpositional ans Verb gebunden ist. Außerdem soll dies noch für FVG 
gelten, in denen sein oder bleiben mit einem Substantiv im Genitiv verbunden ist. Bei FVG mit einem substantivischen Teil im Nominativ muss jedoch das Substantiv - seiner Ansicht nach als syntaktisches Subjekt des Satzes eingestuft werden ${ }^{182}$.

Helbig (1979: 282f.) geht auch auf die problematische Frage ein, wie bei zum Zentrum gehörenden FVG der Satzgliedstatus von präpositional ans Substantiv im FVG angeschlossenen Elementen beurteilt werden soll, wie z. B. in: „Er geriet in Abhängigkeit von $\underline{\mathrm{ihm}}$ “. ${ }^{183}$ Diese Frage wird in der Fachliteratur auch heute noch kontrovers beantwortet, ähnlich wie von Helbig (1979: 283) zusammengefasst: „Teilweise wird von einer ,Zwitterstellung“ zwischen Satzglied und Attribut gesprochen, teilweise aber auch eindeutig eine Entscheidung gegen das Attribut und für das Satzglied getroffen“. Da diese Erweiterungen - seines Erachtens - vom Substantiv im FVG abhängen, das Teil des Prädikats ist, können sie als vom Prädikat abhängige Elemente, d. h. als Satzglieder angesehen werden. Für ihre Klassifizierung als Attribute spricht Helbig (1979: 283) zufolge, dass „die entsprechenden SF ähnliche Kombinationsmöglichkeiten [haben] auch ohne FV, also außerhalb von FVG“.

Diesen Gedanken finden wir auch bei Teubert (2003: 829), der erklärt, dass die Valenz sowohl abgeleiteter als auch nicht-abgeleiteter Substantive auf FVG zurückgeführt werden kann, da sie auch außerhalb von Funktionsverbgefügen dieselben Komplementrealisierungen aufweisen. In Bezug auf deverbale valente Substantive findet sich eine weitere interessante Feststellung bei ihm, wonach „fast immer, wenn sich die Valenz eines Substantivs nicht von der des Verbs ableiten lässt, es ein Funktionsverb- oder Stützverbgefüge gibt, das dieselben Valenzeigenschaften zeigt wie das Substantiv“".

Teubert (2003: 827) erklärt bei der Thematisierung der drei Beschreibungsansätze der Substantivvalenz den SVG-Ansatz so, dass dieser ,die Valenz der Substantive aus der Valenz von Stützverbkonstruktionen mit diesem Substantiv herleitet“" (vgl. dazu oben Kap. 2.4.1). Er weist darauf hin, dass dadurch auch in diesem Ansatz, ähnlich wie im Nominalisierungsansatz, bei dem nur (aus Verben) abgeleitete Substantive betrachtet werden (und zwar so als ließe sich ihre Valenz regelhaft aus der Valenz der entsprechenden Basiswörter ableiten), „das Primat der verbalen Prädikation im Prinzip aufrechterhalten [wird], ohne dass die valenten Substantive von entsprechenden Verben abgeleitet sein müssten“.

182 Dafür führt Helbig (1979: 275, Helbigs Hervorhebung) folgendes Beispiel an: Zwischen den Delegierten besteht keine Übereinstimmung. Zur Problematik der im Nominativ realisierten substantivischen Elemente in potenziellen FVG / SVG vgl. unten noch in diesem Unterkapitel v. a. Heine (2006), Bassola (2009) und meinen Standpunkt in Kap. 3.3.

${ }^{183}$ Helbigs (1979: 283) Hervorhebung. 
Sommerfeldt (1980) untersucht FVG in ihren Valenzeigenschaften, die eine verbale Entsprechung haben, wobei er den Terminus FVG im Sinne von Helbig (1979) interpretiert. An dieser Stelle ist es wichtig zu betonen, dass bei der Charakterisierung der Valenzeigenschaften Sommerfeldt einerseits den Vergleich eines FVG und des entsprechenden Basisverbs vor Augen hat, andererseits die Rolle des FV und des Substantivs bei der Bestimmung der Valenz des FVG unter die Lupe nimmt, aber auch an diesem Punkt einem Vergleich mit dem entsprechenden Basisverbs sehr großen Raum gewährt. ${ }^{184}$

Als Basisverb wird bei ihm das Verb bezeichnet, aus dem das Substantiv des FVG abzuleiten ist. Entsprechend seiner Deutung des Begriffs $F V G$ in Anlehnung an Helbig (1979) betrachtet er ausschließlich solche verbonominalen Konstruktionen, in denen ein deverbales Substantiv vorkommt. ${ }^{185}$ Durch die Analyse der syntaktischen Valenz in einzelnen Gruppen von Beispielen, die er anhand des Satzmusters des Basisverbs bestimmt, zeigt Sommerfeldt (1980: 294f.), dass es diverse morphosyntaktische Abweichungen in der Valenz des FVG und des entsprechenden Basisverbs gibt. In Bezug auf die quantitative Valenz von FVG weist er auf die generelle Übereinstimmung zwischen dem Basisverb und dem FVG hin:

Lediglich bei einigen Fügungen, denen dreiwertige Verben zugrunde liegen, tritt der (fakultative) Aktant des zweiten Partners in der Regel nicht auf:

Der Redner bewies den Zuhörern die Richtigkeit der Maßnahme.

Der Redner stellte die Richtigkeit der Maßnahme unter Beweis.

(Sommerfeldt 1980: 295)

Die qualitativen Unterschiede betreffen - ihm zufolge - einerseits die Obligatheit von Komplementen ${ }^{186}$ im Vergleich des Basisverbs und des FVG, aber noch deutlicher kommen sie bei der syntaktischen Valenz zum Vorschein, was Sommerfeldt (1980: 296) teilweise damit erklärt, dass ,in der Fügung die syntaktische Valenz des Basisverbs und des ihm entsprechenden Substantivs zusammentreffen. Außerdem spielt die Valenz des Funktionsverbs eine Rolle.“" ${ }^{187}$

\footnotetext{
184 Sommerfeldt (1980: 296f.) untersucht auch einen dritten Aspekt, nämlich den „Unterschied zwischen passivischen Funktionsverbfügungen und dem Passiv der Basisverben“. Diese Fragestellung ist jedoch aus der Sicht meiner Arbeit von marginaler Bedeutung, da es sich nur auf ein bestimmtes Valenzmerkmal von deverbalen Substantiven in FVG konzentriert, und wird hier deswegen ausgegrenzt.

${ }^{185}$ Wie in Kap. 3.1 schon angesprochen wurde, geht Helbig (1979: 274) beim substantivischen Element von FVG neben Verbal- auch von Adjektivabstrakta aus, letztere werden jedoch in der Untersuchung von Sommerfeldt (1980) nicht beachtet.

${ }^{186}$ Sommerfeldt (1980: 295) verwendet dafür den Terminus Aktanten, meint damit aber dasselbe, was in meiner Arbeit als Komplemente bezeichnet wird.

${ }^{187}$ Im Hinblick auf den Hauptuntersuchungsgegenstand meiner Arbeit halte ich es für wichtig anzumerken, dass der obigen Feststellung von Sommerfeldt (1980: 295f.) implizit der Gedanke zu entnehmen ist, dass die Valenz auch bei deverbalen Substantiven etwas Eigenständiges darstellt.
} 
Was die Funktion des Funktionsverbs im Hinblick auf die Valenz der Gesamtkonstruktion betrifft, ist Sommerfeldt (1980: 295f.) der Ansicht, dass sich die Valenz von FVG häufig von der des Funktionsverbs unterscheidet. Außerdem findet er in Bezug auf den Einfluss des Funktionsverbs auf die Valenz des FVG den Faktor ausschlaggebend, in welchem Maße es seine semantische Selbstständigkeit beibehält. Dementsprechend wird - seiner Meinung nach - die Valenz des FVG, je mehr das Funktionsverb semantisch verblasst ist, desto mehr von der Valenz des substantivischen Elements des FVG bestimmt. Aus der Sicht des Untersuchungsgegenstandes der vorliegenden Arbeit ist Sommerfeldts (1980:296) Feststellung von besonderem Interesse, wonach bei bestimmten FVG, vor allem bei FVG, in denen die Funktionsverben ,über eine sehr allgemeine Bedeutung [verfügen]“, „die Valenz der Fügung wesentlich von der Valenz des Substantivs determiniert wird“.

Eine deutlich andere Interpretation ist z. B. bei Lehmann (1983: 42ff.) zu finden, der FVG als analytische Prädikate betrachtet, wobei er semantisch dem nominalen Element ein wesentlich größeres Gewicht zuschreibt, während er jedoch syntaktisch das verbale Element als absolut dominierend ansieht. Als wichtigen Unterschied zwischen Hilfs- und Funktionsverben führt er nämlich die Eigenschaft von Funktionsverben an, dass sie ,auf der syntaktischen Ebene“ „satzkonstituierend“ seien (Lehmann 1983: 42). Dementsprechend geht er davon aus, dass „der Stellenplan des Satzes vom Funktionsverb festgelegt“ werde (Lehmann 1983: 43). Seiner Ansicht nach können nämlich syntaktisch gesehen von der Ganzheit des FVG nur solche Leerstellen eröffnet werden, die im Stellenplan des Funktionsverbs verankert sind, wobei das Funktionsverb in seiner syntaktischen Valenz überhaupt nicht von seiner Vollverbvariante abweicht. Was die syntaktische Valenz der Vollverbvariante und die des Funktionsverbs bzw. des FVG betrifft, hält er Differenzen nur in der Obligatheit der Komplemente für möglich, weil in dieser Hinsicht das FVG als Einheit einen Einfluss auf die Realisierung der Argumente habe. Das sieht er auch als Folge dessen an, dass eine Leerstelle des Funktionsverbs automatisch vom nominalen Element besetzt sei (vgl. Lehmann 1983: 45).

Torzova (1983: 284) nimmt in ihrem Beitrag Zur Valenz der Phraseologismen u. a. auf Sommerfeldt ${ }^{188}$ (1980) insofern Bezug, als sie die Angemessenheit der von ihr gewählten

\footnotetext{
${ }^{188}$ Eine Parallele liegt - meines Erachtens - in Sommerfeldts (1980) Vorgehensweise, die Valenz der FVG mit Entsprechungen zu vergleichen, die er „Basisverben“ nennt, und Torzovas Verfahren der Kontrastierung der Valenz von Phraseologismen mit den von ihr „synonymisch“ genannten verbalen Entsprechungen. Ich halte es jedoch für wichtig, darauf hinzuweisen, dass ich Torzovas Bezugnahme auf Sommerfeldt (1980) zur Untermauerung der Adäquatheit der von ihr gewählten Untersuchungsverfahren aus der Sicht weniger begründet finde, dass in ihrem Beitrag durchgehend Vergleiche mit den „homonymischen“ Verben durchgeführt werden, während die sog. „synonymischen“ Entsprechungen nur in ganz wenigen Beispielen als Kontrastpartner herangezogen - oder zumindest im Beitrag präsentiert - werden.
} 
Untersuchungsmethode der Valenzeigenschaften von verbalen Phraseologismen unter Beweis stellt. Obwohl sie sich - im Gegensatz zu Sommerfeldt (1980) - nicht direkt mit FVG, die sie als „eine begrenzte an der Peripherie der Phraseologie liegende Gruppe von sprachlichen Einheiten“ bezeichnet, befasst, soll hier auf ihre Analyse aus zweierlei Gründen eingegangen werden. Einerseits geht sie von einer weiten Phraseologieauffassung aus, wodurch ihre Thesen auch für FVG / SVG gelten sollen, andererseits findet ihre Arbeit in der späteren Forschung einen großen Widerhall.

Torzova (1983: 283) schließt sich der Charakterisierung von Phraseologismen als „ganzheitliche Spracheinheiten“ an, die ,ihnen eigene strukturelle Bindefähigkeiten besitzen“. Sie legt fest, dass Phraseologismen abhängig von ihrem Phraseologisierungsgrad auch in ihren Valenzmerkmalen Unterschiede aufweisen. Sie teilt zuerst die deutschen verbalen Phraseologismen aus ihrem Korpus nach der Anzahl ihrer obligatorischen und fakultativen Komplemente ${ }^{189}$ in Gruppen ein, woraus ersichtlich wird, dass sie ein bis drei Komplemente aufweisen. In ihrer Analyse vergleicht sie die Valenz- und Distributionseigenschaften „der verbalen Phraseologismen und der mit ihren Verbkomponenten genetisch verbundenen Lexemen“, die sie „unter Vorbehalt“ „'homonymische“ Verben“ (Torzova 1983: 283) nennt. Außer mit ihren „homonymischen“ Verbentsprechungen kontrastiert sie Phraseologismen in ihrem Valenzverhalten auch mit ihren „lexikalischen Identifikatoren“, die sie ebenfalls „unter Vorbehalt“ als „'synonymische“ Verben“ (Torzova 1983: 284) bezeichnet (zur kritischen Darstellung und zugleich zur Verwendung dieser Methoden vgl. unten in diesem Unterkapitel Stumpf 2015a: 233; 2015b: 11).

Rein quantitativ gesehen, kann Torzova (1983: 284) zufolge bei der Untersuchung der Valenz der Phraseologismen und der entsprechenden ,homonymischen“ Verben die Rolle des verbalen Elements bei der Vorbestimmung der Valenz des Phraseologismus nicht klar charakterisiert werden. So verfügen in ihrem Korpus 62\% der Phraseologismen über eine Valenzstelle weniger als ihre ,homonymischen“ Verbentsprechungen, bei $22 \%$ der Phraseologismen stimmen die quantitativen Valenzmerkmale der Phraseologismen mit der der „homonymischen“ Verben überein, bei 10\% der Phraseologismen gibt es 1-2 Komplemente mehr, bei $6 \%$ der Phraseologismen jedoch 2 Komplemente weniger als bei ihren „homonymischen“"Entsprechungen.

Bei der Analyse der Valenzeigenschaften von Phraseologismen gibt Torzova (1983: 284ff.) den qualitativen Eigenschaften gegenüber den quantitativen den Vorrang. Ihre Feststellungen

\footnotetext{
${ }^{189}$ Zwar findet sich bei Torzova (1983: 283ff.) der Terminus Mitspieler, damit wird jedoch dasselbe bezeichnet,
} wofür in meiner Arbeit der Terminus Komplemente steht. 
in Bezug auf die qualitative Valenz von Phraseologismen können hier nur kurz zusammengefasst werden. Abhängig vom Phraseologisierungsgrad der jeweiligen Konstruktion sieht sie die qualitative Valenz des Phraseologismus entweder durch das entsprechende „homonymische“ oder das jeweilige „synonymische“ Verb vorbestimmt. Je mehr eine Konstruktion als phraseologisiert gilt, desto weniger Ähnlichkeiten sollen nämlich in den Valenzeigenschaften des Phraseologismus mit dem entsprechenden „homonymischen“ Verb zu entdecken sein und immer mehr sollten seine Valenzmerkmale mit denen des „synonymischen“"Verbs korrelieren.

Im Zusammenhang mit der Beschreibung der Valenz von verbalen Phraseologismen baut Hessky (1988) stark auf Torzovas (1983) Arbeit auf und führt sie an mehreren Punkten weiter, indem sie die externe Valenz eines Phraseologismus sowohl quantitativ als auch qualitativ mit dem entsprechenden „homonymischen“ Verb vergleicht und dabei auch die interne Valenz des Phraseologismus beachtet. Obwohl - meines Erachtens - all die Beispiele, die Hessky (1988) behandelt, idiomatische Phraseme sind, die zum engeren Phraseologiebegriff gehören, kann davon ausgegangen werden, dass sie eine weite Phraseologieauffassung vertritt, und dementsprechend lassen sich ihre Feststellungen verallgemeinern bzw. auch auf nichtidiomatische Phraseme, wie z. B. FVG / SVG ausweiten. Dafür sprechen - in meinen Augen folgende Argumente: Erstens verwendet Hessky (1988) in ihrem Beitrag in Bezug auf den Untersuchungsgegenstand durchgehend den Oberbegriff verbale Phraseologismen, den sie zwar nicht weiter erörtert, aber wenn sie das Verhältnis der Valenzforschung zur Phraseologie bis zu den Achtzigern des zwanzigsten Jahrhunderts charakterisiert, weist sie nicht nur darauf hin, dass Phraseme valenziell sehr wenig untersucht wurden, sondern macht - in Anlehnung an die Valenzbibliographie von Schumacher (1987) - auch deutlich, dass die wenigen diesbezüglichen Beschreibungen überwiegend auf FVG fokussierten. Dies kennzeichnet implizit, dass sie auch FVG durchaus zur Phraseologie rechnet (vgl. Hessky 1988: 139). Zweitens stellt sie in ihrer, ein Jahr vor diesem Beitrag publizierten und der kontrastiven Untersuchung von deutschen und ungarischen Phraseologismen gewidmeten Monographie Phraseologie. Linguistische Grundfragen und kontrastives Modell deutsch $\rightarrow$ ungarisch klar, dass sie eine weite Phraseologieauffassung vertritt, wonach das Spektrum der Phraseologie durch zwei Kriterien bestimmt werden könne, nämlich Vorgeformtheit / Reproduzierbarkeit und Idiomatizität, von denen jedoch das erstere ausreiche, um eine Wortgruppe zum Bereich der Phraseologie gehörend zu klassifizieren (vgl. Hessky 1987: 13ff; v. a. 19f. und 22). Dementsprechend sind Hessky (1987: 19 bzw. 80ff.) zufolge auch FVG zur Phraseologie zu rechnen, die sie Streckformen des Verbs nennt. 
Obwohl Hessky (1988: 141ff.) die Unterscheidung zwischen „konstruktionsexterner“ und „konstruktionsinterner“ Valenz aus unterschiedlichen Gründen problematisch findet, geht sie vorerst doch von der Annahme und Untersuchung der beiden Phänomene bei Phraseologismen aus. Zur konstruktionsexternen Valenz rechnet sie die „Satzbildungsfähigkeit“ dieser Konstruktionen, wobei - ihres Erachtens - „stabile, nichtsubstituierbare Konstituenten der Verbindung nicht als ausgefüllte Leerstellen des phraseologisch gebundenen Verbs anzusehen sind“ und daraus folgt auch, dass für sie nicht das Funktionsverb selbst, sondern ,der Phraseologismus als integrale Einheit“ als Valenzträger anzusehen ist (vgl. Hessky 1988: 142, Hesskys Hervorhebung). Im Rahmen der Untersuchung der konstruktionsinternen Valenz hat sie - wie teilweise Torzova (1983) auch - den Vergleich der Valenzeigenschaften des Funktionsverbs innerhalb des Phraseologismus mit denen in seiner freien Verwendung im Fokus, wobei sie klarstellt, dass diese Vorgehensweise mit der oben erklärten Betrachtung von verbalen Phraseologismen als ,integrale Einheiten“ und damit als komplexe Valenzträger im Widerspruch steht. Nichtdestotrotz findet sie wichtig, die Binnenstruktur von verbalen Phraseologismen zu beschreiben, wobei sie vom Funktionsverb als Valenzträger ausgeht, auch wenn diese Vorgehensweise, „dem Wesen eines phraseologischen Sprachzeichens [widerspricht]“ (Hessky 1988: 142). Durch diese Art der Untersuchung der internen Valenz von Phraseologismen könne man einerseits den Phraseologisierungsprozess besser verstehen bzw. charakterisieren, andererseits die Eigenschaften von phraseologischen Verbindungen mit denen von freien Syntagmen vergleichen. ${ }^{190}$

Hier kommt es nicht darauf an, die einzelnen von Hessky (1988: 144ff.) beschriebenen, durch den quantitativen und qualitativen Vergleich der Valenz des FVG und der des entsprechenden „homonymischen“ Verbs entstandenen Klassen von Phraseologismen detailliert darzustellen, vielmehr sollen ihre wichtigsten Folgerungen zusammengefasst werden. Einerseits begegnet man bei ihr einem ähnlichen Gedanken wie bei Schumacher (1980: 295f.), wonach zwischen dem valenziellen und dem semantischen Einfluss des verbalen Phrasemelements auf die Valenzstruktur und Bedeutung der Gesamtkonstruktion Parallelitäten zu entdecken seien: „In dem Maße, wie die Bedeutung des isolierten Verbs durch die komplexe phraseologische Bedeutung überlagert wird, büßt das Verb auch seine ursprüngliche

\footnotetext{
${ }^{190}$ In diesem Zusammenhang finde ich wichtig, auf Van Pottelberges (2001) Auffassung hinzuweisen, der zufolge Konstruktionen, die in der Fachliteratur als FVG klassifiziert werden, in ihren Eigenschaften oft nicht von freien Syntagmen zu unterscheiden und dementsprechend nicht als selbstständige Klasse zu beschreiben seien. Ferner ist der Kontrast mit Van Pottelberge auch in der Hinsicht interessant, wie er die Rolle der verbalen und nominalen Konstituente von FVG bei der Bestimmung der Valenzeigenschaften der FVG betrachtet. (vgl. unten in diesem Unterkapitel)
} 
syntaktisch-strukturelle Dominanz ein“ (Hessky 1988: 146). Demzufolge sei die Rolle des verbalen Elements bei der Bestimmung der valenziellen Eigenschaften eines Phraseologismus von dessen semantischer Dominanz bzw. Selbstständigkeit und dadurch vom Phraseologisierungsgrad der Gesamtkonstruktion abhängig. Andererseits vertritt Hessky (1988: 148) die Auffassung, dass „,[es] [a]ngesichts der Spezifik phraseologischer Sprachzeichen [...] nicht angemessen ist, den gängigen Begriff der Verbvalenz mechanisch auf sie zu übertragen“.

Die letztere Feststellung von Hessky bietet sich - meines Erachtens - als perfekten Anknüpfungspunkt für die Darstellung von Ágels (2004) Überlegungen, der in seinem Beitrag von „Minimalkriterien der Phraseologizität (Polylexikalität und Fixiertheit)“ (Ágel 2004: 66) ausgeht und dadurch eine weite Phraseologieauffassung vertritt, in die u. a. auch FVG explizit miteinbezogen werden. Wie schon der Titel seines Artikels verrät, stellen für ihn Phraseologismen den „(valenz)syntaktischen Normalfall“ dar. Dementsprechend lenkt er die Aufmerksamkeit auf Defizite der Valenztheorie in Bezug auf die Untersuchung von Phrasemen (vgl. Ágel 2004: 66f.), wobei sein Ziel ist, zu zeigen, wie Phraseme in eine Valenztheorie integriert werden können (vgl. dazu auch Ágel (2003) über Wort- und Ausdrucksvalenzträger). Ferner will er als wichtigen Schritt dieser Integration eine „,integrative Valenzträgertheorie“ und eine ,integrative Aktantenpotenzialtheorie entwickeln (vgl. Ágel 2004: 71ff. bzw. 80ff.). ${ }^{191}$

Auch Ágel (2004: 71ff.) befasst sich mit der Unterscheidung zwischen interner und externer Valenz bei Phraseologismen, wobei er zugleich auf die problematische Art des Begriffs „interne Valenz" hinweist. Im Zusammenhang mit der internen und der externen Valenz hebt er zweierlei hervor: Einerseits, dass die externe Valenz von der internen in der Hinsicht abhängt, dass die intern schon vorkommenden Realisierungsformen extern nicht mehr realisiert werden können, ${ }^{192}$ andererseits vertritt er auch die Auffassung, dass die Grenze zwischen interner und externer Valenz fließend ist (vgl. Ágel 2004: 72).

Er weist ferner auf ein - seines Erachtens - ,gewaltige[s] Theoriedefizit“ (Ágel 2004: 72) hin, wonach als wichtiges Merkmal von Phraseologismen ihre Festigkeit zu betrachten ist, wobei sie allerdings häufig eine deutliche Variabilität aufweisen. Dieser Gedanke setzt sich auch in seinem Stufenmodell der Formvariabilität der Valenzträger durch (vgl. Ágel

\footnotetext{
${ }^{191}$ Diese integrativen Schritte haben auch für Ágels (2000) frühere Valenztheorie Relevanz und führen eigentlich zu ihrer Modifizierung, worauf hier jedoch nicht näher eingegangen werden kann.

${ }^{192}$ Wie unten in diesem Unterkapitel noch erklärt wird, spielt die Erkenntnis dieses Zusammenhangs auch bei Stumpf (2015a und 2015b) eine wichtige Rolle.
} 
2004: 73ff.), in dem - auf vier Stufen von 0-Variabilität bis zur hohen Variabilität - der stufenweise Übergang von geschlossenen bis zu offenen Valenzträgern präsentiert wird. Hier kommt es nicht darauf an, dieses Modell detailliert darzustellen, vielmehr fokussiere ich auf die Aspekte des Ansatzes, die sich auf FVG beziehen (lassen).

Ágel (2004: 73ff.) zufolge gehören FVG der Stufe der mittleren Formvariabilität an und können außerdem noch durch eine, „prinzipiell uneingeschränkte“ Bedeutungsvariation charakterisiert werden. Valenzträger, die eine mittlere Formvariabilität aufweisen, bezeichnet er als „offene VT [Valenzträger, Á. S.-T.], d. h. [...] VT mit einem Slot “. Unter Slot ${ }^{193}$ versteht er den „Bestandteil des VT, der obligatorisch aufzufüllen ist, damit ein VT als VT überhaupt funktionstüchtig wird. Im Unterschied zu Leerstellen gestalten Slots die Binnenstruktur von PH [Phraseologismen Á. S.-T.] mit.“ (Ágel 2004: 75; Ágels Hervorhebungen) Durch diese Überlegungen wird auch der Begriff der internen Valenz, den Ágel (2004: 71ff.) - wie oben schon angesprochen - deutlich kritisiert, relativiert, da Slots nicht als valenzbedingte Leerstellen betrachtet werden können und dementsprechend phrasemintern auch nicht von Valenz zu sprechen sei. Wie ich unten noch detaillierter ausführen werde, vertritt diesbezüglich auch Stumpf (2015a und 2015b) dieselbe Auffassung und schlägt vor, bei Phrasemen statt des Begriffs interne Valenz den von ,intern fixierten Komponenten“ zu verwenden (vgl. dazu unten in diesem Unterkapitel).

In Anlehnung an Welke (1994; 2002: 103ff.) geht Ágel (2004: 76ff.) bei jedem Valenzträger von einer Aktantenhierarchie aus, „die die logisch-pragmatische Rangfolge der Beteiligtenrollen (=Argumente) widerspiegelt“. Dies ist v. a. bei polylexikalischen Valenzträgern besonders wichtig, damit man die Binnenstruktur von Phraseologismen bestimmt und dadurch auch ihre externen Valenzverhältnisse besser erklären kann. Was die Grundgedanken betrifft, lässt sich die Annahme einer Aktantenhierarchie dieser Art - meines Erachtens - gut mit Fourquets Konzept der „primären Konnexion“ verbinden, für dessen Anwendbarkeit zur Beschreibung der (inneren) Valenzverhältnisse von FVG auch Van Pottelberge (2001: 31ff.) plädiert (vgl. Kap. 3.1 und auch unten in diesem Unterkapitel).

Helbigs (1979: 277) oben angesprochene These, nach der das Funktionsverb im Vergleich zum Vollverb seine lexikalische Bedeutung verliere und auch seine Valenz ändere, wird von Stein (1996: 192f.) kritisiert. Seiner Ansicht nach geht es bei diesen Verb-NomenVerbindungen um kontextbedingte Selektion: Von den Lesarten des betreffenden Verbs selektiert das prädikative Substantiv ,genau eine [...] und benutzt die Argumentstruktur dieser

\footnotetext{
${ }^{193}$ Ágel nimmt fünf Typen von Slots an. Zu deren Erörterung vgl. Ágel (2004: 75).
} 
Lesart für seine eigenen Argumente“ (Stein 1996: 200). ${ }^{194}$ Das bedeute ferner, dass (die) Argumente des Substantivs und des Verbs unifizieren. Er betrachtet nicht nur das prädikative Substantiv, sondern die ganze Verb-Nomen-Verbindung als Valenzträger und sieht bewiesen, dass bei der Untersuchung der Substantivvalenz nicht nur die isolierte Realisierung der Substantive beachtet werden soll, ,da auch latente Argumente aus dem zugrunde liegenden Verb in Verb-Substantiv-Verbindungen relevant werden können“195 (Stein 1996: 200).

Ähnlich wie von Stein wird auch von Storrer (2006: 279) die Argumentstruktur von Nominalisierungsverbgefügen semantisch mit Argumenttransfer, syntaktisch mit „Amalgamierung der Valenzmuster“ der verbalen und nominalen Komponenten erklärt. In ihrer Terminologie richtet sich Storrer (2006) nach Polenz (1987) und nach der GDS, trifft aber weitere Verfeinerungen. Dementsprechend ist bei $\mathrm{ihr}$ Nominalisierungsverbgefüge ein Oberbegriff für eine heterogene Gruppe von Verb-Nomen-Konstruktionen, die als Prädikatsausdruck dienen und aus einem Nominalisierungsverb und einem deverbalen oder deadjektivischen Substantiv bestehen. Eine Teilmenge der Nominalisierungsverbgefüge stellen die FVG dar, in denen das Funktionsverb über ,eine systematisch beschreibbare Eigenbedeutung“"verfüge und dadurch eine zusätzliche prädikative Funktion habe (vgl. Storrer 2006: 276 bzw. Polenz 1987:170). Für „Nominalisierungsverbgefüge, die keine Funktionsverbgefüge sind“, verwendet Storrer (2006: 277f.) den Terminus Streckverbgefüge. Sie spricht sich dafür aus, dass den nominalen Komponenten der Nominalisierungsverbkonstruktionen in FVG ein anderer Status zugeschrieben werden soll als in Streckverbgefügen (vgl. Storrer 2006: 278ff., 292f.). Während sie nominale Komponenten in FVG - in Einklang mit der GDS - als Prädikativkomplemente betrachtet, schreibt sie den nominalen Komponenten in Streckverbgefügen - auch wenn sie nicht prototypisch erfragbar und anaphorisierbar sind - semantisch einen Argumentstatus zu und bezeichnet sie auf der syntaktisch-strukturellen Ebene als Termkomplemente.

Wie in Kap. 3.1 bereits erklärt, weist auch Langer (2004: 171ff.), ähnlich wie Storrer (2006: 275ff. und 2007: v. a. 168ff.), die Termini $S V G$ und FVG unterschiedlichen Diskussionslinien der Linguistik zu, die verschiedene Typen der Verb-Nomen-Verbindungen als prototypisch betrachten. Im Zusammenhang mit den Valenzeigenschaften der SVG, in

\footnotetext{
${ }^{194}$ An einer anderen Stelle relativiert Stein (1998: 224) diese Feststellung und hebt hervor, dass es nicht berechtigt sei, einseitig diese selektierende Rolle des Substantivs als primär zu betrachten, da ,auch das Verb einen beträchtlichen Einfluß auf die Substantivbedeutung haben kann“".

${ }^{195}$ Hier ist es wichtig, darauf hinzuweisen, dass von Stein (1996) nur Verb-Substantiv-Verbindungen beachtet werden, in denen ein deverbales Substantiv das nominale Element darstellt.
} 
denen - seiner Ansicht nach - das Substantiv meistens im Akkusativ steht, finden wir bei ihm folgende Erklärung:

The fundamental idea of a SVC [support verb construction, Á. S-T.] is the realization of the arguments (i.e. the semantic actants) of the predicative noun as syntactic actants of the support verb. In prototypical support verb constructions, the verb does not semantically subcategorize any of its syntactic complements. This means that the noun is the predicate of the construction, the verb has mainly syntactic relevancy. (...) the verb determines which nominal arguments receive which syntactic slot. (Langer 2004: 172, meine Hervorhebungen, Á. S-T.)

Das legt nahe, dass Komplemente eines SVG semantisch gesehen als Argumente des valenten Substantivs betrachtet werden können, ihre formale Realisierung jedoch vom Stützverb (mit)bestimmt wird.

SVG sind für Bassola (2009: 84) Verbindungen prädikativer, d. h. valenter Substantive, mit Nominalverben ${ }^{196}$, die er als Stützverben bezeichnet, durch die die valenten Substantive ihre Komplemente ,auf der Satzebene zum Satzkomplement machen [können]“. Primär geht er davon aus, dass diese Satzkomplemente vom valenten Substantiv abhängen (vgl. Bassola 2009: 80 bzw. 81 Tab. 2; 2015: 16). Die Zugehörigkeit dieser Komplemente zum verbalen oder nominalen Teil ist jedoch häufig nicht eindeutig zu beurteilen. So ist Bassolas (2009: 86) Ansicht nach bei den SVG, in denen das verbale Element und das Substantiv in ihrer Rektion übereinstimmen, problematisch $\mathrm{zu}$ entscheiden, $\mathrm{zu}$ welchem der beiden Elemente die Komplemente gehören. So könne in (Det.) $)^{197}$ Krieg gewinnen gegen + Akk. das Präpositivkomplement gegen $+A k k$. sowohl dem Verb als auch dem Substantiv als Regens zugeordnet werden, während es in (Det.) Krieg führen gegen + Akk. eindeutig nur zum Substantiv gehören könne. ${ }^{198}$ Als besonderen Problemfall betrachtet er die Verbindung von valenten Substantiven mit Stützverben, in der das Substantiv im Nominativ steht und somit zum Subjekt des Satzes wird, wie z. B. in Diskussionen über + Akk. finden statt. ${ }^{199}$ So ist das Substantiv nicht mehr Teil des Prädikats, sondern selbst ein Satzglied. Im Satz bzw. auch innerhalb des SVG soll Bassola (2015: 26) zufolge eine gestufte Abhängigkeit angenommen werden, wobei das valente Substantiv vom Stützverb, die Komplemente des Substantivs vom SVG und innerhalb dessen vom Substantiv abhängen.

Gerade auf Grund dieser Eigenschaften und wegen der Problemfälle werden im Deutschungarischen Wörterbuch zur Substantivvalenz (Bassola et al. 2003 und 2012) SVG zur

\footnotetext{
${ }^{196}$ Den Terminus verwendet Bassola (2009) im Sinne von Polenz (1988).

197 d. h. Determinans

198 Die Beispiele wurden vereinfacht Bassola (2009: 86) entnommen.

${ }^{199}$ Das Beispiel stammt aus einem Korpusbeleg von Bassola (2009: 86), wurde aber nur in seiner Grundstruktur übernommen.
} 
Bestimmung der Argumente der valenten Substantive verwendet, bei der Bestimmung der Realisierungsformen der Komplemente aber ausgegrenzt. In diesem Zusammenhang halte ich es noch für wichtig, auf das PROCOPE-Projekt ${ }^{200}$ hinzuweisen, in dessen Rahmen Mitarbeiter des Instituts für deutsche Sprache und des Laboratoire d'Automatique Documentaire et Linguistique mit dem Ziel zusammengearbeitet haben, „eine neue Basis für zweisprachige syntagmatische Wörterbücher zu erarbeiten“ (Bresson / Kubczak 1998: 7). Die Arbeit an der Erstellung deutsch-französischer Lemmata zur Nominalsyntax hat nämlich bezüglich der SVG ergeben, dass es im Deutschen ,nicht immer möglich [war], die Form und die Anzahl der Argumente innerhalb der Nominalgruppe über das Modell der Weglassung des Stützverbs zu ermitteln“, wobei jedoch die Anzahl und Rolle der potenziellen Argumente des Substantivs so bestimmt werden konnten (Kubczak / Costantino 1998: 19).

Die Frage der Valenzverhältnisse in bzw. von FVG wird in der Forschung auch Van Pottelberge (2001: 37ff.) zufolge sehr kontrovers behandelt. Entsprechend seinem Vorschlag, das FVG als primäre Konnexion zu betrachten, findet er es absolut richtig, wenn zwischen dem FV und dem nominalen Element eine engere Verbindung angenommen wird, er lehnt jedoch Ansätze ab, in denen das FVG als oberstes Regens des Satzes betrachtet und somit „kategorial zum Verb erhoben wird“ (Van Pottelberge 2001: 37). ${ }^{201}$ Außerdem hält er Annahmen der Forschung, nach denen ein FVG als lexikalische Einheit besondere Valenzeigenschaften habe, für unhaltbar. Er ist ferner bemüht, auch unterschiedliche Thesen zu widerlegen, denen zufolge die Valenz des FVG von der des Funktionsverbs quantitativ und/oder qualitativ abweichen würde. So kommt er im ersten, theoretisch ausgerichteten Hauptteil seiner Monographie, in dem er unterschiedliche Forschungspositionen kritisch unter die Lupe nimmt, immer wieder zur Feststellung, dass die Valenz eines FVG von der Valenz des darin vorkommenden Verbs bestimmt wird (vgl. z. B. Van Pottelberge 2001: 8, 44, 48). Er schwächt diese Behauptung meines Erachtens absolut berechtigt - etwas ab, indem er (zumindest) bei der Erörterung der Valenzeigenschaften von FVG mit präpositionalen Erweiterungen auch den Einfluss des nominalen Teils auf die syntaktische Valenz des FVG einräumt:

Es gibt in der deutschen Sprache kein Funktionsverbgefüge, das eine Präpositionalphrase aufweist, die nicht aufgrund des Satzbauplans des Funktionsverbs oder aufgrund der Verwendungsmöglichkeiten (das heißt der Valenz) des Substantivs zu erklären wäre, ausgenommen in lexikalisierten Verb-Substantiv-Verbindungen, wo Substantive und/oder

\footnotetext{
${ }^{200}$ Die Forschungsergebnisse dieses Projekts durften von Mitarbeitern des Deutsch-ungarischen Wörterbuches zur Substantivvalenz (Bassola et al. 2003 und 2012) ins Konzept des Wörterbuches integriert werden.

${ }^{201}$ Vgl. z. B. Van Pottelberges (2001: 37ff.) Kritik an Heringer 1968.
} 
Verben nicht mehr mit ihren freien Verwendungen vergleichbar sind (z. B. mit jmdm. ins Gericht gehen) oder das Substantiv sogar unikal ist (z. B. nach jmdm./etw. Ausschau halten). (Van Pottelberge 2001: 55; Unterstreichung von mir, Á. S.-T.).

Van Pottelberge (2001: 45 und 55) ist mit Lehmann (1983: 43) in der Hinsicht jedoch absolut einverstanden, dass „der Stellenplan des Satzes vom Funktionsverb festgelegt [wird]“. Er ist dabei ferner - im Einklang mit Lehmann - der Ansicht, dass ein Verb in seiner Funktions- und Vollverbvariante keine valenz-syntaktischen Unterschiede aufweise.

Was die Valenzverhältnisse von FVG betrifft, befasst sich Van Pottelberge (2001: 55ff.) auch mit der Frage einer Funktionsverteilung in FVG, wonach die syntaktische Valenz vom Funktionsverb und die semantische vom Substantiv des FVG bestimmt ist, welche These wenn auch unterschiedlich deutlich ausgeprägt - häufig in der Fachliteratur vorkommt. Zwar ist er selbst der Auffassung, dass das Substantiv in FVG wesentlich bei der semantischen Selektion der Argumente ist, er sieht darin aber kein Merkmal, das nur für FVG charakteristisch wäre und so zu ihrer Abgrenzung als eigene Klasse dienen könnte.

Heine (2006), die FVG mit präpositional oder akkusativisch ans Funktionsverb angeschlossenen Substantiven betrachtet, folgert aus der engen Verbindung von Funktionsverb und Substantiv darauf, dass sie einen komplexen Prädikatsausdruck darstellen und dementsprechend gemeinsam als Valenzträger fungieren (Heine 2006: 50ff. v. a. 52f.). Ferner stellt Heine im Zusammenhang mit der Valenzbeschreibung von FVG fest, dass das Substantiv in FVG - im Gegensatz zu Substantiven in Kollokationen - keine Leerstelle füllt, da es Prädikatsteil ist.

Stumpf (2015a) vertritt sowohl in seiner Monographie Formelhafte (Ir-)Regularitäten. Korpuslinguistische Befunde und sprachtheoretische Überlegungen als auch in seinem in demselben Jahr, aber etwas später erschienen Artikel Phraseologie und Valenztheorie. Status quo, Forschungsprobleme und (korpusanalytische) Perspektiven (Stumpf 2015b) eine weite Phraseologieauffassung, darüber hinaus widmet er in diesem Beitrag der Frage der Valenz von FVG sogar einen eigenen Punkt (vgl. Stumpf 2015b: 17ff.). Dabei ist jedoch ganz wichtig hervorzuheben, dass er in seinem Artikel FVG lediglich zu den nicht-idiomatischen Phrasemen rechnet, aber den Begriff $F V G$ überhaupt nicht definiert, was sich vielleicht damit erklären lässt, dass er in diesem Zusammenhang eine Art linguistischen Common Sense annimmt, was aber in Anbetracht der Widersprüchlichkeit des Terminusgebrauchs - meines Erachtens nicht begründet ist.

Zwar stehen FVG in seiner oben genannten Monographie an keiner Stelle so im Fokus wie in seinem Beitrag, aber wir können seiner Übersicht 2-10, die die Untersuchungsgegenstände 
einer weit gefassten Phraseologie darstellt, eine Definition von FVG entnehmen. Dort werden FVG nämlich als „Zusammensetzungen aus einem nominalisierten Verb und semantisch ,leeren“ Verben, mit denen die Aktionsart differenziert wird“" charakterisiert (Stumpf 2015a: 49).

In Bezug auf die Beschreibung der Valenz (verbaler) Phraseme findet Stumpf (2015a: 219ff., 2015b) eine Unterscheidung in „,interne“ und „externe“ Valenz wichtig. Ähnlich wie z. B. Ágel (2004) weist er allerdings darauf hin, dass der Terminus interne Valenz etwas kontrovers ist, da mit Valenz die Fähigkeit gemeint werden soll, Leerstellen zu eröffnen, die durch verschiedene Elemente des Satzes besetzt werden können bzw. müssen, wobei interne Valenzstellen jedoch automatisch durch Elemente des Phrasems fest besetzt sind. Dementsprechend verzichtet er auf die Verwendung dieses Terminus und schlägt dafür den von „intern fixierten Komponenten“ vor, mit dem also Elemente eines Phrasems gemeint sind, die vom verbalen Teil eröffnete Leerstellen meistens in einer festgesetzten, nicht substituierbaren Form ausfüllen und zur Nennform des Phrasems gehören (vgl. Stumpf 2015a: 220; 2015b: 6ff.). Ihm zufolge bilden das verbale Element und die intern fixierten Komponenten eines Phrasems gemeinsam „den komplexen phraseologischen Verbalträger“, wobei ich anstelle von Verbalträger den Terminus Valenzträger auch in diesem Kontext wesentlich treffender finde (vgl. Stumpf 2015a: 220; 2015b: 7). Durch die Umbenennung der internen Valenz(stellen) erübrigt sich jedoch für ihn im Grunde genommen die zusätzliche Markierung der weiteren Leerstellen des Phrasems als zur externen Valenz gehörend, dementsprechend begegnen wir bei Stumpf (2015a) dem etwas modifizierten Terminus (externer) Valenz, ${ }^{202}$ der jedoch in seinem wenig später erschienen Beitrag wiederum durch den „herkömmlichen“ Terminus externe Valenz aufgelöst wird (vgl. Stumpf 2015b).

Was das Verhältnis von intern fixierten Komponenten und externer Valenz anbelangt, vertritt Stumpf (2015a: 221f., 2015b: 15ff.) die Auffassung, dass die intern fixierten Komponenten zwar kaum Einfluss auf die quantitative und selektionale Valenz des Phrasems haben, ${ }^{203}$ wohl aber auf die qualitative. Die syntaktische Realisierung der externen Valenz hängt nämlich auch davon ab, welche Kasusformen durch die intern fixierten Komponenten schon belegt und dadurch für die externe Valenz des Phrasems blockiert sind. ${ }^{204}$ Außerdem hebt er hervor, dass die Zuordnung der einzelnen Konstituenten zu den intern fixierten Komponenten bzw. zur externen Valenz nicht immer problemlos verläuft, „da es auch

\footnotetext{
${ }^{202}$ Vgl. Anm. 184 bei Stumpf (2015a).

${ }^{203}$ Vgl. dazu auch Hessky (1988: 146), die dieselbe Meinung vertritt.

${ }^{204}$ Vgl. auch Kolde 1979: 82.
} 
Übergangsfälle zwischen variablen und stabilen Elementen von verbalen Idiomen gibt" (Stumpf 2015a: 221). Als Beispiel für einen solchen Problemfall betrachtet er das adverbial verwendete Adjektiv hart in jmd. geht mit jmdm. (hart) ins Gericht. Da die Modalerweiterung in diesem Phrasem außer hart auch durch andere, semantisch verwandte Komponenten realisiert werden kann, könnte es - seiner Meinung nach - einerseits als (variable) externe Valenz betrachtet werden, andererseits sieht er es aufgrund seiner korpusanalytischen Erkenntnisse, die eine hohe Vorkommenshäufigkeit von hart belegen, jedoch eher als intern fixierte Komponente des Phrasems an.

Im Zusammenhang mit dem Problem der Unterscheidung zwischen intern fixierten Komponenten und externer Valenz spricht er Ágels (2004) Stufenmodell an, als dessen großen Vorteil er seine nicht-dichotomische Art der Differenzierung bewertet. Er macht freilich zugleich deutlich, dass durch die fließenden Grenzen zwischen den einzelnen Variationsstufen die Zuordnung einzelner Komponenten zu den intern fixierten Komponenten oder zur externen Valenz problematisch bzw. subjektiv wird, wobei auch ausführliche Korpusanalysen keine eindeutige Lösung bieten können (vgl. v. a. Stumpf 2015b: 16f.).

Bei der externen Valenz hält er es für wichtig, zwischen quantitativer und qualitativer Valenz zu unterscheiden. In Bezug auf die quantitative Valenz betont er, dass im Falle von verbalen Idiomen keinesfalls angenommen werden kann, dass durch die Realisierung der intern fixierten Komponenten die quantitative externe Valenz automatisch reduziert wäre (vgl. Stumpf 2015b: 10). Er ist mit Hessky (1988: 144ff.) in der Hinsicht einverstanden, dass die externe Valenz des Phrasems durch die Valenz der nicht-phraseologisch realisierten Entsprechung der verbalen Phrasemkomponente nur zum Teil bestimmt wird, und je weniger das der Fall ist, desto mehr büßt sie ihre Semantik innerhalb des Phrasems ein.

Bezüglich des Vergleichs zwischen der externen Valenz von verbalen Idiomen und der Valenz des „formativisch identischen“, 205 nicht-phraseologisch verwendeten Verbs, zeigt Stumpf (2015a: 233; 2015b: 11), wie problematisch dieser ist. Das betreffende Verb verfügt nämlich in seiner außerphraseologischen Verwendung über mehrere Valenzstrukturen, von denen nicht immer eindeutig entschieden werden kann, welche als Entsprechung des Verbs innerhalb des Idioms betrachtet werden soll. In seinem Beitrag geht er auch auf einen anderen Vergleich ein, und zwar auf den zwischen der Valenz des verbalen Idioms und einem verbalen Synonym des ganzen Idioms, der - neben dem obigen Vergleich - z. B. auch von Torzova

\footnotetext{
${ }^{205}$ Mit der Bezeichnung formativisch identisch in Bezug auf die außerphraseologisch verwendete Entsprechung des verbalen Elements eines Phrasems meint Stumpf (2015b: 10ff.) dasselbe, wofür bei Torzova (1983) ,homonymisch“ steht (vgl. oben in diesem Unterkapitel).
} 
(1983) unternommen wird. Diese Kontrastierung findet er jedoch noch prekärer, da es im Falle eines Idioms - durch die Vagheit der Bedeutungsumschreibungen - „teilweise mehrere (mögliche) ,synonyme“ Verben geben kann“ (vgl. Stumpf 2015b: 11).

Trotz der Probleme, die mit dem Vergleich der externen Valenz von verbalen Phrasemen und der Valenz des jeweiligen verbalen Elements in „freier“, d. h. nicht-phraseologischer Verwendung verbunden sind, ist er für Stumpf (2015a: 222ff. und 2015b) von großer Bedeutung, da er Valenz(ir)regularitäten auf der Grundlage eines solchen Vergleichs bestimmt. Unter Valenz(ir)regularität versteht er nämlich das Phänomen, wenn verbale Phraseme in ihrer externen und/oder internen qualitativen Valenz von der Valenz des jeweiligen außerphraseologisch gebrauchten Verbs abweichen, d. h. solche Ergänzungsklassen aufweisen, „die in freier Verwendung nicht der Rektion des Verblexems unterliegen“ (Stumpf 2015a: 223). Valenz(ir)regularitäten betrachtet er als eine Form phraseologischer und damit formelhafter (Ir-)Regularitäten, die charakteristisch für Phraseme sind. Ferner ist er der Meinung, dass besonders diese Valenzphänomene durch ihre Häufigkeit und oft gar nicht irreguläre Art auch nicht mit einem etwas Negatives konnotierenden Begriff der Irregularität bezeichnet werden sollten. Damit erklärt sich die Klammerung in seinem Terminusgebrauch formelhafte (Ir-)Regularitäten bzw. Valenz(ir)regularitäten (vgl. Stumpf 2015a: 233; 2015b: 26f.).

Ich bin mit Stumpf (2015a; 2015b) in der Hinsicht absolut einverstanden, dass er sehr großen Wert auf Korpusanalysen legt, mit deren Hilfe man zuverlässig(er)e Informationen über den Gebrauch von Phrasemen und darunter auch von FVG erhalten kann. Die Auswertung von korpusanalytischen Daten können u. a. auch dazu dienen, Entscheidungen über die Valenzverhältnisse in bzw. von Phrasemen zu treffen. Dabei beachtet er sowohl morphologisch-syntaktische als auch semantische Merkmale einzelner Elemente und deren Frequenz in den Belegen.

FVG stehen - Stumpf (2015b:5) zufolge - ,stellvertretend für eine weite Phraseologieauffassung“ und dementsprechend können sie - ähnlich wie Idiome - in ihren Valenzeigenschaften charakterisiert werden. Stumpf (2015b: 18f.) schließt sich dem Ansatz an, wonach in FVG das verbale und das substantivische Element gemeinsam als Valenzträger zu verstehen sind. Er betont die Wichtigkeit von Korpusanalysen auch bei der Untersuchung von FVG und verweist auf die korpusbasierten Arbeiten von Heine (2006 und 2008).

Im Anschluss an den Überblick unterschiedlicher Ansätze der Forschung über das Wesen und die Valenzinterpretation von FVG / SVG erörtere ich im nächsten Unterkapitel meine Einstellung zur Frage der terminologischen und ontologischen Vielfalt der Begriffe in diesem 
Bereich und erkläre die Stellung der Untersuchung der betreffenden Gefüge im Rahmen meiner Arbeit.

\subsection{Eigener Standpunkt}

Nach dem Überblick diverser Ansätze zur Beschreibung von FVG, SVG bzw. ihrem Verhältnis zueinander können die Uneinheitlichkeit des Terminusgebrauchs und die Vielfalt der Definitionsmerkmale der betreffenden Verb-Nomen-Verbindungen - meines Erachtens in erster Linie mit der Heterogenität des Untersuchungsgegenstandes erklärt werden (vgl. dazu auch Kamber 2008). Es gibt zwischen den Theorien zur Beschreibung dieser Gefüge in mehreren Punkten z. T. wesentliche Unterschiede. So werden semantischer und syntaktischer Status des Substantivs innerhalb der Verbindung unterschiedlich bestimmt. Es herrscht auch darüber kein Konsens, ob die Gesamtkonstruktion oder ihre Elemente separat bzw. verteilt als Valenzträger anzusehen sind. Außerdem ist auch noch die Frage von Belang, ob nur abgeleitete, und darüber hinaus, nur deverbale Substantive als nominales Element angenommen, oder - was nur ganz selten überlegt wird - auch Konstruktionen mit nicht-abgeleiteten Substantiven in die Analyse miteinbezogen werden sollen.

In meiner Arbeit verbleibe ich aus mehrerlei Gründen vorerst beim Terminus $S V G$. Einerseits ist sein Gebrauch im Sinne der Tradition des Deutsch-ungarischen Wörterbuches zur Substantivvalenz (Bassola et al. 2003 und 2012; vgl. dazu u. a. Bassola/László 1996: 34 ff.)., das ich sowohl aus der Sicht der ungarischen Auslandsgermanistik als auch aus der der (kontrastiven) Substantivvalenzforschung als sehr bedeutend einschätze, u. a. auch deswegen, weil sein Grundkonzept in einer langen, gut durchdachten und ausführlich dokumentierten Vorbereitungsphase erarbeitet wurde (vgl. u. a. Bassola/László/Tamássy Bíró 2005). Andererseits konzentriert sich die SVG-Diskussionslinie auf das prädikative Nomen in der Verb-Nomen-Konstruktion und untersucht, wie „die Argumente des prädikativen Nomens in die gesamte Konstruktion integriert werden“ (Storrer 2006: 276f.), was mit meiner Interpretation der Substantivvalenz als ein System sui generis gut vereinbart werden kann.

Da ich jedoch die Auffassung vertrete, dass mit dem Terminus $S V G$ eine heterogene Gruppe von Verb-Nomen-Konstruktionen abgedeckt werden soll, fasse ich ihn wesentlich weiter als er generell definiert wird. Dementsprechend gehe ich davon aus, dass SVG komplexe Satzkonstituenten sind, die aus einem nominalen und einem verbalen Teil bestehen. ${ }^{206}$ Dabei

\footnotetext{
${ }^{206}$ Ersteres bezeichne ich als Substantiv im SVG und letzteres als Stützverb.
} 
verstehe ich unter ,Konstituente eines sprachlichen Ausdrucks s [...] jede Einheit in s, die unmittelbar oder mittelbar Bestandteil von s ist" ${ }^{“ 207}$. Bezüglich des Substantivs im SVG bin ich der Meinung, dass nicht nur akkusativisch und eventuell noch präpositional angeschlossene Substantive berücksichtigt werden sollen, sondern auch Substantive in den weiteren Kasus, auch wenn solche Verbindungen wesentlich seltener sind und zum Teil in ihren semantischen und syntaktischen Eigenschaften Besonderheiten aufweisen. ${ }^{208}$ Entsprechend meiner Interpretation der Substantivvalenz als selbstständigem Phänomen halte ich auch nicht abgeleitete Substantive in SVG für möglich.

In meiner Charakterisierung der SVG als komplexe Satzkonstituenten verzichte ich bewusst auf die Bezeichnung komplexe Prädikate, da ich teilweise mit Van Pottelberges (2001: 18ff.) Kritik an der uneinheitlichen Interpretation des Begriffs einverstanden bin, andererseits will ich auch nominativisch mit dem Stützverb verbundene Substantive aus meinen Untersuchungen nicht ausschließen, die jedoch als Subjekt des Satzes nicht als Teile eines komplexen Prädikats betrachtet werden können.

Wie schon an anderer Stelle darauf hingewiesen, wird die Untersuchung von SVG - meines Erachtens - dadurch erschwert, dass ,bis jetzt nicht klar definiert werden [konnte] - vielleicht, weil es auch nicht klar definiert werden kann -, welche Verben als Stützverben gelten“ (Sántáné-Túri 2016: 89). Die Frage, in welchem Maße das Verb in der jeweiligen Konstruktion seine Semantik ändert ${ }^{209}$ und ob ggf. auch die Bedeutung des nominalen Bestandteils modifiziert wird, kann - meiner Ansicht nach - (im Voraus) nicht bestimmt werden. Nur soviel scheint mir - in dieser Hinsicht - klar feststellbar zu sein, dass diese Konstruktionen semi-kompositionell sind.

Behauptungen über die Valenzeigenschaften und weitere, z. T. kontrovers diskutierte Charakteristika dieser Gefüge, wie z. B. Festigkeit des Artikelgebrauchs vor dem Substantiv des SVG, Numeruskonstanz oder eingeschränkte bzw. nicht mögliche Attribuierbarkeit des Substantivs im SVG will ich erst nach ausführlichen Korpusanalysen machen. Im Rahmen der korpusbasierten Beschreibung der ausgewählten Substantive in meiner Arbeit werden die Valenzeigenschaften der SVG, die in den Belegen mit den betreffenden Substantiven ermittelt werden, mituntersucht, wobei die weiteren Wesensmerkmale vorerst nur beispielhaft, am

\footnotetext{
207 Konstituente. In: grammis. Grammatisches Informationssystem. https:/grammis.idsmannheim.de/terminologie/781 (zuletzt gesehen am 31. 5. 2020).

${ }^{208}$ Vgl. dazu u. a. die FVG-Klassifizierung von Helbig (1979: 275ff.), Storrers (2006: 275ff., bzw. 2007 , v. a. Punkt 168ff.) Erklärung zu prototypischen SVG oder die Bemerkung von Bassola (2009: 86 oder 2015: 25ff.) im Zusammenhang mit SVG, in denen das Substantiv nicht im Akkusativ steht.

${ }^{209}$ Es sei vorerst dahingestellt, ob es nur Entsemantisierung bedeuten kann, wie es meistens in der Fachliteratur angenommen wird.
} 
Rande der Hauptuntersuchungen berücksichtigt werden können. Dabei ist nochmals hervorzuheben, dass den Ausgangspunkt meiner Analysen - dem Hauptinteresse meiner Dissertation entsprechend - jedoch nicht (Stütz)verben, sondern valente Substantive bilden, die in ihren Valenzeigenschaften möglichst exhaustiv beschrieben werden sollen. Dabei halte ich - im Gegensatz zu Van Pottelberge (2001) - auch eine quantitative Auswertung der Ergebnisse der Korpusanalyse für wichtig, damit die Untersuchung nicht nur für die Darstellung „des Möglichen“, sondern auch für die des Üblichen Anhaltspunkte bietet. 


\section{Korpusbasierte Untersuchung der Valenz ausgewählter Substantive}

\subsection{Das Analyseverfahren}

\subsubsection{Fragestellungen und Vorgehensweise}

Wie bereits in Kap. 3.3 angesprochen, wende ich mich - nach den theoretischen Grundlegungen in Kap. 2 und 3 - in diesem Kapitel der korpusbasierten Untersuchung von sechs ausgewählten Substantiven zu. Dabei entnehme ich dem Deutschen Referenzkorpus (DeReKo) mit Hilfe von COSMAS II ${ }_{w e b}$, dem Online-Korpusrecherche- und Analysesystem des Leibniz-IDS Mannheim, Belege, indem ich ,alle öffentlichen Korpora des Archivs W (mit Neuaquisitionen ohne Wikipedia)“ mit Suchanfragen durchsuche, die immer aus dem Grundformoperator \& und dem jeweiligen Substantiv bestehen. Bei den Recherchen sind in den Optionen zur Lemmatisierung Flexionsformen und Komposita aktiviert. Dies hat zum Ziel, dass unter den Treffern auch Komposita zu erwarten sind, die als Bestandteil das jeweilige Substantiv enthalten. Die so ermittelten Treffer werden immer durch Zufallsauswahl auf 1000 reduziert, ${ }^{210}$ von denen in den einzelnen Unterkapiteln von 4.2.1 - 4.2.3 vorerst die ersten 100 relevanten Treffer untersucht und ausgewertet werden. Dabei können bestimmte Belege nicht in die Analyse miteinbezogen werden. Es ist nämlich darauf hinzuweisen, dass man mit dieser Suchanfrage und diesen Sucheinstellungen z. B. auch solche Belege mit Komposita erhält, in denen das betreffende Substantiv nicht als Leitglied realisiert wird und die deswegen automatisch aus der Analyse ausgegrenzt werden (vgl. z. B. Übersetzungshilfe, Ausdrucksmöglichkeiten). Ferner bleiben auch Belege unbeachtet, in denen die untersuchten Substantive als Teil fester phraseologischer Einheiten vorkommen wie z. B. in der Tat oder keine Ursache, da solche Verwendungen über das Valenzverhalten der jeweiligen Substantive keine relevanten Informationen bieten.

Der Beschreibung der Valenzeigenschaften der einzelnen Substantive liegen folgende Fragstellungen zugrunde:

a) Welche (valenten) Bedeutungen hat das jeweilige Substantiv?

b) Welche Argumente hat das Substantiv (bedeutungsabhängig)?

\footnotetext{
210 Die relativ hohe Anzahl der gespeicherten Treffer im Vergleich zur Anzahl der (im ersten Schritt) hier analysierten und ausgewerteten Belege lässt sich einerseits dadurch begründen, dass bei jedem Substantiv mehr als 100 Belege untersucht werden müssen, um tatsächlich 100 relevante Belege aussortieren zu können. Andererseits tauchen - aufgrund der Erfahrungen früherer eigener korpusbasierten Analysen zum Valenzverhalten ausgewählter Substantive (vgl. Sántáné-Túri) - immer Fragen im Zusammenhang mit bestimmten Argumentrealisierungen bzw. Verwendungsweisen auf, die gezielt noch untersucht werden sein sollten, wofür so ein entsprechender Vorrat am weiteren Belegmaterial gesichert wird.
} 
c) In welcher Form und in welcher Kombination werden Argumente des Substantivs realisiert?

d) Mit welchen Stützverben verbindet sich (bedeutungsabhängig) das Substantiv?

e) Gibt es Unterschiede in der Argumentrealisierung des Substantivs abhängig davon, ob es innerhalb eines SVG oder ohne Stützverb vorkommt?

Durch die Auswertung der korpusanalytischen Ergebnisse will ich in einem ersten Schritt über das Valenzverhalten dieser Substantive im Lichte des Sprachgebrauchs ein möglichst vielfältiges und authentisches Bild geben. In einem nächsten Schritt führt der Weg zur Beantwortung der aus der Sicht meiner Arbeit primär wichtigen Frage, wieweit die Substantivvalenz als ein in ihren Eigenschaften eigenständiges, selbstständiges Phänomen bezeichnet werden kann. Dieser Zielsetzung ist natürlich auch die Auswahl der untersuchten Substantive untergeordnet, die ich in Kap. 4.1.2 kurz erörtere.

\subsubsection{Zur Auswahl der untersuchten Substantive}

Im vorliegenden korpusanalytischen Kapitel meiner Arbeit untersuche ich je zwei, d. h. insgesamt sechs, auf Grund ihrer Beschreibung im DHW bzw. Kluge, deverbale, deadjektivische und nicht-abgeleitete Substantive. Ich halte es aus mehrerlei Sicht für wichtig, sowohl abgeleitete als auch nicht-abgeleitete Substantive in die Valenzbeschreibung miteinzubeziehen. Einerseits will ich nämlich herausfinden, welche Ähnlichkeiten und / oder Unterschiede zwischen diesen Gruppen von Substantiven auf dieser Grundlage entdeckt werden können. Die Frage besteht für mich dabei auch darin, ob die (quantitative) Auswertung der Belegdaten und deren Vergleich bezüglich der deverbalen, deadjektivischen und nichtabgeleiteten Substantive gute Beweise für meinen theoretischen Ausgangspunkt bietet, wonach die Substantivvalenz als ein System sui generis zu interpretieren ist. Dabei finde ich einerseits wichtig, zu untermauern, dass auch nicht-abgeleitete Substantive Valenz haben. Andererseits ist $\mathrm{zu}$ untersuchen, ob Vertreter der, von den meisten als generell nicht valenzfähig bezeichneten, nicht-abgeleiteten Substantive ähnliche oder aber andere Besonderheiten in ihren Valenzeigenschaften aufweisen, als die von mehreren als potenziell valent angenommenen deverbalen und deadjektivischen Substantive.

Wie schon früher erwähnt (vgl. v. a. Kap. 3.3 und 4.1.1) will ich auch dafür ein Auge haben, ob die untersuchten Substantive in den Belegen als Substantive im SVG ${ }^{211}$ oder eben nicht als

\footnotetext{
${ }^{211}$ Solche Belege bezeichne ich im Folgenden als $S V G$-Belege.
} 
nominaler Teil eines $\mathrm{SVG}^{212}$ vorkommen. Dafür gibt es zwei Hauptgründe: Erstens will ich (bedeutungsabhängig) das Valenzverhalten der Substantive in ihren SVG-Belegen mit den ermittelten Komplementstrukturen in ihren Nicht-SVG-Belegen einander gegenüberstellen, um beurteilen zu können oder zumindest Anhaltspunkte dafür zu finden, wieweit sich die Valenzeigenschaften der Substantive in den Nicht-SVG-Belegen abweichend von den Valenzeigenschaften der SVG-Belege beschreiben lassen und wieweit diese dadurch die Selbstständigkeit der Substantivvalenz unterstützen. Zweitens wurden SVG (zu meiner Deutung des Terminus vgl. Kap. 3.3) in der Fachliteratur bis jetzt fast ausschließlich ausgehend von ihren verbalen Elementen untersucht und beschrieben (vgl. z. B. Van Pottelberge 2001; Kamber 2008). Im Gegensatz dazu verfolge ich die genau umgekehrte Herangehensweise.

Bei der Auswahl der konkreten deverbalen, deadjektivischen und nicht-abgeleiteten Substantive haben mich zwei Gesichtspunkte geleitet. Einerseits spielten meine Annahmen über die Argumentstruktur bzw. die potenzielle Komplementstruktur der Substantive eine Rolle. So fiel meine Wahl auf das deverbale Substantiv Übersetzung, über dessen Argumentstruktur ich angenommen habe, dass sie komplex ist. Das ebenfalls deverbale Nomen Verspätung habe ich deswegen interessant gefunden, weil ich herausfinden wollte, wie eines seiner Argumente, das die Zeitdauer ausdrückt, um die sich jemand verspätet, sprachlich realisiert wird, ob da z. B. ähnliche Phänomene zu belegen sind, wie im Falle von Maß- und Behälterkonstruktionen. Unter den nicht-abgeleiteten Substantiven habe ich Ursache als eine Art Gegenpol zu Übersetzung ausgesucht, weil ich davon ausging, dass es eine einfache Argumentstruktur hat, bei der es interessant zu beobachten wäre, ob sie sich auch in der Konstanz der Komplementstruktur widerspiegelt. Andererseits war die Zusammenstellung der $\mathrm{zu}$ untersuchenden Substantive dadurch beeinflusst, was meine Vorannahmen über ihre möglichen SVG-Realisierungen waren. So habe ich Ausdruck (deverbal), Freiheit (deadjektivisch) und Problem (nicht-abgeleitet) in meine Analysen miteinbezogen, weil ich vermutet habe, dass sie öfter in SVG bzw. in vielfältigen SVG realisiert werden. Wie es sich aus den Beschreibungen der Analyse (vgl. v. a. Kap. 4.2) herausstellen wird, haben sich meine Vorannahmen zwar überwiegend bestätigt, aber einige mussten auf Grund der korpusbasierten Resultate modifiziert werden.

Vor der Präsentation der konkreten Ergebnisse meiner korpusanalytischen Untersuchungen fasse ich im nächsten Kapitel wichtige Aspekte der Auswertung und Präsentation der Belegdaten in meiner Arbeit kurz zusammen.

\footnotetext{
${ }^{212}$ Solche Belege nenne ich im Folgenden Nicht-SVG-Belege.
} 


\subsubsection{Zur Auswertung und Darstellung der korpusanalytischen Daten und Ergebnisse}

Die Grundlage für die Beschreibung der Valenzeigenschaften der ausgewählten Substantive stellt die Analyse von sechsmal hundert Belegen aus DeReKo dar. Dabei wird bei jedem Beleg angegeben,

- welche Bedeutung(svariante) des untersuchten Substantivs darin vorkommt, wenn das Substantiv nicht nur in einer Bedeutung(svariante) identifiziert werden konnte;

- wie viele Komplemente es enthält;

- in welcher Form kommen die Komplemente vor;

- welche Argumente durch die identifizierten Komplemente realisiert werden;

- ob das Substantiv im Beleg in einem SVG erscheint.

Im Laufe des Analyseprozesses mache ich zuerst bei jedem Substantiv Vorannahmen darüber, welche Bedeutung(svariant)en des Substantivs valent sein können und welche Argumentstrukturen zu denen gehören, wobei bei der (Vor)bestimmung der Argumente die Ermittlung der Sachverhaltsbeteiligung und damit der Folgerungstest eine ausschlaggebende Rolle spielt. Generell werden die Argumentstrukturen entweder mithilfe der entsprechenden valenten Verben bzw. Adjektive ${ }^{213}$ oder aber SVG (ähnlicher) Konstruktionen ${ }^{214}$ dargestellt. Es ist jedoch wichtig zu betonen, dass diese Vorannahmen nur als Ausgangspunkt dienen und immer durch Erkenntnisse der Korpusanalyse verifiziert oder zumindest modifiziert werden. An bestimmten Stellen werden die eigenen korpusbasierten Ergebnisse in Bezug auf die valenten Bedeutungen und ihren Argumentstrukturen mit den relevanten Angaben im DUW abgeglichen.

Bei der Angabe der Zahl und Form der Komplementrealisierungen wird zwischen zwei Gruppen der Realisierungsformen unterschieden, was besonders wegen der Untersuchung der Valenzeigenschaften von SVG-Belegen nötig ist. So werden zuerst immer die Komplemente ermittelt, die rein morphologisch-syntaktisch gesehen direkt vom Substantiv abhängig sein können, und im Beleg durch Unterstreichung hervorgehoben. ${ }^{215}$ Wenn der Beleg darüber hinaus noch weitere Argumentrealisierungen enthält, die aus formalen Gründen nicht direkt

\footnotetext{
${ }^{213}$ vgl. z. B.jd. (A1) übersetzt etw. (A2) aus etw. (A3) in etw. (A4) (in Kap. 4.2.1.1) oder etw. (A1) ist frei (in Kap. 4.2.2)

${ }^{214}$ vgl. z. B.jd. (A1) hat die Freiheit etw. zu tun (A2) (in Kap. 4.2.2)

${ }^{215} \mathrm{Zu}$ dieser Gruppe der Realisierungsformen gehören in meinem Konzept Bestimmungsglieder von Komposita, Genitivattribute, Präpositionalattribute, attributive Adjektive, (Possessiv- und Relativ)pronomina, Appositionen, Pronominaladverbien und bestimmte Nebensätze.
} 
vom Substantiv abhängig sein können (wie z. B. das Subjekt), wird ihre Zahl durch „,+“ der Anzahl der anderen Komplemente hinzugefügt und die jeweiligen Komplemente im Beleg durch Strichlinien von den übrigen unterschieden und markiert. Die Aufführung der Realisierungsformen und der Argumente folgt nicht automatisch der Reihenfolge des Vorkommens der Komplemente, sondern - gerade wie bei der Angabe ihrer Anzahl - zuerst werden immer die Informationen bezüglich der ersten Gruppe der Komplemente aufgeführt und im Anschluss darauf folgen die Angaben zur zweiten Gruppe. Zur Verdeutlichung stehe hier ein Beispiel mit Ausdruck:

$\{\{1+2 ;$ mit + Dat.; Subj.; Akk.; A3; A1; A2 $\}\} \quad\{\{\text { SVG; zum A. bringen }\}\}^{216}$ Mit ihrem Einsatz möchte die Pastoralassistentin 217 ihre Solidarität mit den zum Ausdruck bringen und selbst einen Beitrag leisten, dass die Welt ein wenig gerechter wird.

(St. Galler Tagblatt, 16.02.1999)

In Beisp. 1) bedeutet 1+2, dass ein Komplement ermittelt werden konnte, das formal gesehen direkt vom Substantiv Ausdruck abhängig sein kann und zwei weitere, die formal gesehen nicht als direkte Dependentien des Substantivs behandelt werden können. Die drei Realisierungsformen und drei Argumente werden diesem 1+2 System entsprechend aufgeführt, d. h., dass mit + Dat. als potenziell direkt vom Substantiv abhängiges Komplement A3 realisiert, während A1 in Form des Subjekts und A2 im Akkusativ (hier als Akkusativobjekt des Satzes) vorkommen. Die zweite Gruppe der Informationen folgt wiederum in Klammern und drückt aus, dass es sich um einen SVG-Beleg mit zum Ausdruck bringen handelt. Die Elemente des SVG sind außer Ausdruck, der als Bezugssubstantiv immer fett markiert wird, im Beleg kursiv gesetzt.

In den einzelnen Unterkapiteln werden nur bestimmte Belege, die der Veranschaulichung der eruierten Komplementstrukturen oder der Darstellung interessanter Probleme dienen, dargeboten, während alle 600 analysierten Belege in ihrer Form unverändert, aber mit den eigenen Analysedaten ergänzt, in Kap. 8, im Anhang, zur Verfügung gestellt werden. ${ }^{218}$ Alle in

\footnotetext{
${ }^{216}$ Die Form der Klammern signalisiert zu welcher Bedeutung von Ausdruck der jeweilige Beleg gehört.

${ }^{217}$ Damit die Strichlinien bei den zwei nebeneinanderstehenden Komplementen nicht zusammenfließen, wurde das eine Komplement durch kürzere Strichlinien markiert. Diese Markierung hat aus der Sicht der Analyse keine zusätzliche Bedeutung, sie dient ausschließlich der visuell besseren Abgrenzung der Komplemente.

${ }^{218}$ Es muss hervorgehoben werden, dass in jedem Beleg das jeweilige valente Substantiv nur einmal, und zwar in seiner durch Fettdruck hervorgehobenen Realisierung beachtet werden darf, auch wenn es Belege gibt, in denen das jeweilige Substantiv mehrmals vorkommt. Der Grund dafür liegt darin, dass diese Hervorhebungen im Laufe der DeReKo-Recherche vom COSMAS, dem Analyse- und Recherchesystem, gemacht werden und die in einem Beleg nicht hervorgehobenen Realisierungen des betreffenden Substantivs unter den späteren Belegen vorkommen oder zumindest vorkommen können. (Bei einer Reduzierung von mehreren Zehntausenden oder von noch mehr Belegen auf 1000 Treffer ist natürlich nicht sicher, dass die jeweiligen Belege tatsächlich unter diesen 1000 Treffern vorkommen. Damit man aber nicht Gefahr läuft, ein und denselben Beleg mehrmals zu analysieren, sollte den von COSMAS gemachten Hervorhebungen gefolgt werden.)
} 
Bezug auf die obigen Aspekte ermittelten Daten werden in der gerade erklärten Form bei jedem Substantiv tabellarisch bereitgestellt (vgl. Tab. 2-11). In den Tabellen wird zusätzlich die Anzahl der mit den betreffenden Komplementstrukturen ermittelten Belege angegeben, damit so auch ein quantitativer Vergleich der Daten ermöglicht wird.

Nach diesen einleitenden Gedanken zu den Grundlagen meiner Untersuchung stelle ich im nächsten Unterkapitel die wichtigsten Ergebnisse meiner Analysen in Bezug auf die einzelnen Substantive dar.

\subsection{Die untersuchten Substantive im Korpus}

\subsubsection{Die deverbalen Substantive}

\subsubsection{Das Substantiv Übersetzung}

Unter den analysierten ersten 100 Treffern für Übersetzung konnten zwei Hauptbedeutungen bestimmt werden, mit zwei Bedeutungsvarianten in der einen Hauptbedeutung. Dieses Bild deckt sich ganz mit der Darstellung im DUW. Dementsprechend sind folgende Bedeutung(svariant)en von Übersetzung zu unterscheiden:219
Ia) das Übersetzen
Ib) übersetzter Text
II) (Technik) Verhältnis der Drehzahlen zweier über ein Getriebe gekoppelter Wellen; Stufe der mechanischen Bewegungsübertragung.

Wie Tab. 2 zu entnehmen ist, überwiegen die Belege für Bedeutung Ib) (70,00\%), wie in Beisp. 2). Beleg (3) und (4) stellen je ein Beispiel für Bed. Ia) und Bed. II) dar, von denen letztere mit 4,00\% einen Bruchteil der Belege ausmacht.

(2) Das Lied war eine Übersetzung eines Songs des zeitgenössischen englischen Liedermachers Leon Rosselsen. ${ }^{220}$ (St. Galler Tagblatt, 05.06.2008, S. 46)

(3) Hofmann hat sich stets konsequent einer Nachbildung der gegenständlichen Welt verweigert und in der "Übersetzung des Seelischen in die Form" einen Weg gefunden, der auch für seine Schüler richtungweisend geworden ist. (Salzburger Nachrichten, 18.08.1999.)

(4) Er hatte eine viel zu harte Übersetzung gewählt, die er schon nach wenigen Metern nicht mehr treten konnte. (Kleine Zeitung, 08.06.2000)

\footnotetext{
${ }^{219}$ Die einzelnen Stichworte werden hier dem DUW ohne Änderung entnommen.

${ }^{220}$ Die Hervorhebungen in den DeReKo-Belegen sind - wenn nicht anders markiert - meine Hervorhebungen, Á. S-T. Durch Fettdruck wird das jeweilige untersuchte Substantiv markiert, Elemente eines SVG mit ihm werden durch Kursivsetzung hervorgehoben und Unterstreichung kennzeichnet Argumentrealisierungen des Substantivs, wobei Realisierungsformen, die aus rein morphologisch-syntaktischen Gründen nicht direkt vom Substantiv abhängig sein können (wie z. B. das Subjekt) durch Strichlinien von den weiteren Argumentrealisierungen unterschieden werden.
} 


\begin{tabular}{|c|c|c|c|}
\hline & Bed. Ia) & Bed. Ib) & Bed. II \\
\hline -SVG & 26 & 70 & 4 \\
\hline +SVG & 0 & 0 & 0 \\
\hline
\end{tabular}

Tab. 2: Anzahl der SVG- und Nicht-SVG-Belege für Übersetzung nach Bedeutungsvarianten klassifiziert

Unter den ausgewerteten 100 Treffern finden sich keine SVG-Belege, womit aber im Voraus schon gerechnet werden konnte, aber wie in Kap. 4.1.2 bereits angesprochen, war der Grund für die Auswahl dieses Substantivs meine Annahme, dass seine Argumentstruktur, zumindest in den Bedeutungen Ia) und $\mathrm{Ib}$ ) sehr komplex ist. Wie auch in Tab. 3 und 4 gezeigt wird, hat sich diese Vermutung bestätigt, indem in der ersten Hauptbedeutung von A1 bis A4 vier Argumente ermittelt werden konnten, wobei ihre Funktion in der folgenden Grundstruktur erläutert werden kann: jd. (A1) übersetzt etw. (A2) aus etw. (A3) in etw. (A4).

\begin{tabular}{|c|c|c|c|c|}
\hline & Kreal. quant. & Kreal. qual. & Areal. & Anzahl der Belege \\
\hline \multirow{11}{*}{ Bed Ia) } & 0 & keine Real. & kein $\mathrm{A}$ & 8 \\
\hline & 1 & von + Dat & $\mathrm{A} 2$ & 3 \\
\hline & 1 & in + Akk & A4 & 2 \\
\hline & 1 & in $+\mathrm{Akk}$ & A3 & 1 \\
\hline & 1 & Posspr & A1 & 1 \\
\hline & 1 & attr. Adj & A1 & 1 \\
\hline & 1 & Gen & A2 & 4 \\
\hline & 2 & \begin{tabular}{|l} 
Gen \\
in + Akk
\end{tabular} & $\begin{array}{l}\text { A2 } \\
\text { A4 } \\
\end{array}$ & 1 \\
\hline & 2 & \begin{tabular}{|l|} 
Gen \\
in + Akk \\
\end{tabular} & $\begin{array}{l}\text { A2 } \\
\text { A3 } \\
\end{array}$ & 3 \\
\hline & 2 & \begin{tabular}{|l|} 
attr. Adj \\
von + Dat \\
\end{tabular} & $\begin{array}{l}\text { A1 } \\
\text { A2 } \\
\end{array}$ & 1 \\
\hline & 2 & $\begin{array}{l}\text { Demonstrpr. im Gen } \\
\text { in + Akk }\end{array}$ & \begin{tabular}{|l|} 
A2 \\
A4 \\
\end{tabular} & 1 \\
\hline \multirow{2}{*}{ Bed.II) } & 0 & keine Real. & kein $\mathrm{A}$ & 3 \\
\hline & 1 & attr. Adj. & A1 & 1 \\
\hline
\end{tabular}

Tab. 3: Zahlenmäßige Verteilung der Belege für Übersetzung Bed. Ia) und II), nach Komplement- und Argumentstrukturen klassifiziert

Tab. 3 und 4 sind auch noch weitere wichtige Informationen bezüglich der Argumentstruktur und deren Realisierung durch Komplemente zu entnehmen. Zuerst gehe ich dabei auf die Frage ein, dass unter den Argumenten neben den oben bereits erwähnten A1 - A4 noch ein A`zu entdecken ist. Um die Funktion von A` erläutern zu können, mache ich hier einen kurzen Exkurs 
und stelle einen Schritt des gesamten Analyseverfahrens dar, denn dieser hat eine entscheidende Rolle in der Bestimmung von $\mathrm{A}^{\text {‘ gespielt. }}$

\begin{tabular}{|c|c|c|c|}
\hline Kreal. quant. & Kreal. qual. & Areal. & Anzahl der Belege \\
\hline 0 & keine Real. & kein $\mathrm{A}$ & 16 \\
\hline 1 & Posspr. & $\mathrm{A} 2$ & 1 \\
\hline 1 & Posspr. & A1 & 1 \\
\hline 1 & Gen. & A2 & 6 \\
\hline 1 & Gen. & A1 & 2 \\
\hline 1 & sächs. Gen. & A1 & 3 \\
\hline 1 & von + Dat & A1 & 4 \\
\hline 1 & attr. Adj & A4 & 8 \\
\hline 1 & Demonstrativpr. im Gen. & A2 & 1 \\
\hline 1 & in + Akk. & A4 & 1 \\
\hline 1 & Komp. & A2 & 1 \\
\hline 1 & für + nicht dekl. & A2 & 1 \\
\hline 1 & Apposition & $\mathrm{A}^{\prime}$ & 1 \\
\hline 1 & Relativpr. im Gen. & A2 & 1 \\
\hline 1 & für + Akk. & $\mathrm{A} 2$ & 1 \\
\hline 2 & $\begin{array}{l}\text { aus + Dat. } \\
\text { von + Dat. }\end{array}$ & $\begin{array}{l}\text { A3 } \\
\text { A1 }\end{array}$ & 2 \\
\hline 2 & $\begin{array}{l}\text { Gen. } \\
\text { Komp. }\end{array}$ & $\begin{array}{l}\text { A1 } \\
\text { A2 }\end{array}$ & 1 \\
\hline 2 & $\begin{array}{l}\text { attr. Adj. } \\
\text { Gen. }\end{array}$ & $\begin{array}{l}\text { A4 } \\
\text { A2 }\end{array}$ & 6 \\
\hline 2 & $\begin{array}{l}\text { Posspr. } \\
\text { Apposition }\end{array}$ & $\begin{array}{l}\text { A2 } \\
\text { A }^{6}\end{array}$ & 1 \\
\hline 2 & $\begin{array}{l}\text { Gen. } \\
\text { in }+ \text { Akk. }\end{array}$ & $\begin{array}{l}\text { A2 } \\
\text { A4 }\end{array}$ & 1 \\
\hline 2 & $\begin{array}{l}\text { attr. Adj. } \\
\text { von + Dat. }\end{array}$ & $\begin{array}{l}\text { A4 } \\
\text { A1 }\end{array}$ & 1 \\
\hline 2 & $\begin{array}{l}\text { attr. Adj. } \\
\text { von + Dat. }\end{array}$ & $\begin{array}{l}\text { A1 } \\
\text { A2 }\end{array}$ & 1 \\
\hline 2 & $\begin{array}{l}\text { sächs. Gen. } \\
\text { als }\end{array}$ & $\begin{array}{l}\text { A1 } \\
\text { A }^{\prime}\end{array}$ & 1 \\
\hline 2 & $\begin{array}{l}\text { für + Akk. } \\
\text { Apposition }\end{array}$ & $\begin{array}{l}\text { A2 } \\
\text { A }^{\prime}\end{array}$ & 1 \\
\hline 2 & $\begin{array}{l}\text { sächs. Gen. } \\
\text { Gen. }\end{array}$ & $\begin{array}{l}\text { A1 } \\
\text { A2 }\end{array}$ & 1 \\
\hline 2 & $\begin{array}{l}\text { sächs. Gen. } \\
\text { von + Dat. }\end{array}$ & $\begin{array}{l}\text { A1 } \\
\text { A2 }\end{array}$ & 1 \\
\hline 2 & $\begin{array}{l}\text { als } \\
\text { attr. Adj. }\end{array}$ & $\begin{array}{l}\mathrm{A}^{\prime} \\
\mathrm{A} 4\end{array}$ & 1 \\
\hline 2 & $\begin{array}{l}\text { attr. Adj. } \\
\text { für + Akk. }\end{array}$ & $\begin{array}{l}\text { A4 } \\
\text { A2 }\end{array}$ & 1 \\
\hline 3 & $\begin{array}{l}\text { attr. Adj. } \\
\text { Apposition } \\
\text { von + Dat. }\end{array}$ & $\begin{array}{c}\text { A4 } \\
\text { A }^{\prime} \\
\text { A1 }\end{array}$ & 1 \\
\hline
\end{tabular}




\begin{tabular}{|c|l|c|c|}
\hline Kreal. quant. & \multicolumn{1}{|c|}{ Kreal. qual. } & Areal. & Anzahl der Belege \\
\hline \multirow{3}{*}{3} & Posspr. & A1 & \\
& von + Dat. & A2 & 1 \\
& als & A $^{\prime}$ & \\
\hline \multirow{3}{*}{3} & unmarkierte Gleichsetzung & A' & \\
& attr. Adj. & A4 & 1 \\
& von + Dat. & A2 & \\
\hline
\end{tabular}

Tab. 4: Zahlenmäßige Verteilung der Belege für Übersetzung Bed. Ib), nach Komplement- und Argumentstrukturen klassifiziert

Dabei gehe ich von einem Beispiel (5) von Bed. Ib) aus, in dem A4 durch Pfälzische, A1 durch von Bastiane Franke aus Fischbach und A“ in Form einer Apposition durch „Waarde uf de Godot" realisiert wird:

(5) Anerkennungspreise von jeweils 1000 Mark erhalten diesmal die Pfälzische Übersetzung "Waarde uf de Godot" von Bastiane Franke aus Fischbach, die Komödie "Arwettplätz vun A bis Z" des Zweibrückers Horst Scherer sowie das Volksstück "Herrgott von Bockshiwwel" von Vorjahrespreisträger Heinrich Kraus. (Mannheimer Morgen, 25.03.1998)

Ursprünglich war A' in die Darstellung der Argumente nicht involviert, als ich aber auch bei der Analyse weiterer Substantive (vgl. v. a. Kap. 4.2.1.2; 4.2.3.1, aber auch 4.2.3.2) mehrere Beispiele gefunden habe, in denen $A^{`}$ zu entdecken ist, habe ich mein Konzept geändert und dementsprechend frühere Belege neuanalysiert. Wie ich es auch bei den übrigen betroffenen Substantiven ansprechen werde, habe ich A' als Argument problematisch gefunden und ich nehme es immer noch nicht als vollwertiges Argument an. Der Grund dafür liegt darin, dass in meinen Augen - $A^{\text {‘ }}$ zum jeweiligen Bezugswort (hier zu Übersetzung) in einem Quasigleichsetzungsverhältnis steht. Wie ich in Kap. 2.6.1 erklärt habe, gehe ich bei der Bestimmung der Argumenthaftigkeit von der Interpretation von Argument in logischsemantischem Sinne aus und bezeichne dementsprechend Leerstellen damit, die von einem Prädikat bzw. einer Funktion eröffnet werden. Da ich jedoch hier eine Quasigleichsetzung von

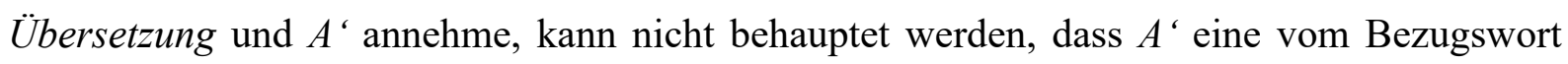
Übersetzung eröffnete Leerstelle besetzen würde und somit kann es auch nicht als typisches Argument betrachtet werden. Dadurch ist auch zu erklären, warum A' nicht, wie sonst in meinen Strukturdarstellungen im Falle von Argumenten üblich, durchnummeriert, sondern mit einem Apostroph markiert ist.

Bezüglich der Realisierungsform Apposition, deren problematische Einstufung als Komplement ich in Kap. 2.4.2 bereits angesprochen habe, bin ich zu der Feststellung gekommen, dass Appositionen, die mit der Formel $x$ stellt ein y dar umzuschreiben sind, wobei $x$ für die appositive Konstituente und $y$ für das Bezugswort steht, Realisierungen von 
Quasiargumenten darstellen. Das gilt, wie gerade ausgeführt, auch für die Apposition in die (...) Übersetzung "Waarde uf de Godot" aus Beisp. 5), da ihre Bedeutung als "Waarde uf de Godot" stellt eine Übersetzung dar paraphrasiert werden kann.

Im Zusammenhang mit der Klassifizierung von Konstituenten, die A‘ zum Ausdruck bringen, habe ich folgendes Konzept entwickelt: Da Komplementkandidaten, die A`sprachlich (unterschiedlich) realisieren, das - in meinem Modell - primäre Kriterium der Argumenthaftigkeit mit der Beschränkung erfüllen, dass sie ein Quasiargument zu realisieren scheinen, bin ich zu der Entscheidung gekommen, sie als absolut periphere oder als periphere Komplemente zu klassifizieren (vgl. Kap. 2.6.2). Wie ich in Kap. 2.6.1 erklärt habe, spielen formale, morphologisch-syntaktische Aspekte in meinem Modell eine wichtige Rolle dabei, Komplemente und Supplemente in zentrale und periphere Subklassen einzuteilen. Das trifft auch auf die A'-Kandidaten voll zu. Wenn nämlich diese Quasiargumente auf Grund der Belege nur durch Appositionen realisiert werden, bei denen kaum Komplement-Fürsprecher unter den formalen Valenzrelationen auszumachen sind, betrachte ich ihre Realisierungen als absolut periphere Komplemente. Wenn es jedoch bei einem Valenzträger neben Appositionen auch weitere Realisierungsformen des Quasiarguments gibt, bei denen auch formale KomplementFürsprecher ermittelt werden können, gehe ich davon aus, dass diese Komplemente als weniger peripher einzustufen sind. Da im Falle von Übersetzung die Ausdrucksmöglichkeiten von A` auf Grund der Belege - nicht nur auf die Apposition begrenzt sind (vgl. z. B. als „,Zurück-SitzFahren " in 5)), ordne ich diese Komplementkandidaten nicht den absolut peripheren, sondern den peripheren Vertretern der Komplemente zu.

(6) Nur bei seiner Übersetzung von "Back Seat Driving" als "Zurück-Sitz-Fahren" runzeln die Zuschauer dann doch die Stirn. (Mannheimer Morgen, 30.08.1999)

Nach diesem Exkurs zur Frage der von mir als Quasiargumente bezeichneten A'-Positionen und zur Klassifizierung der in diesen Positionen realisierten Konstituenten als Komplemente, halte ich für wichtig, noch einige interessante Aspekte der Argumentrealisierungen in den Belegen mit Übersetzung hervorzuheben. Wie ich in Kap. 2.6.1 bereits thematisiert habe, werden attributive Adjektive sehr oft als potenzielle Realisierungsform von Komplementen ausgeschlossen, womit ich jedoch absolut nicht einverstanden bin. Unter den Belegen mit Übersetzung kommen in Bed. 1b) 20-mal attributive Adjektive vor, was 28,57\% der Gesamtbelegzahl in Bed. 1b) ausmacht. (vgl. Tab. 4). Diese Komplemente realisieren mit einer einzigen Ausnahme immer A4 (vgl. Beisp. 7)). Diese Ausnahme stellt Beisp. 8) dar, in dem das attributive Adjektiv amtlich A1 zugeordnet werden kann. 
(7) In diesen Tagen erscheint die deutsche Übersetzung seines Werkes «Die Kugel und das Opium» über die Massaker auf dem Platz des Himmlischen Friedens 1989. (St. Galler Tagblatt, 13.10.2012)

(8) Obwohl die amtliche Übersetzung von Schreibers streng geheimer Aussage in Toronto erst in "etwa ein bis zwei Monaten" in Bern erwartet wird, ist klar, was jetzt kommt. (NEWS, 07.03.2002, S. 16)

Zwar gab es unter den ersten 100 relevanten Belegen für Übersetzung nur zwei, in denen es als Leitglied eines Kompositums vorkam und das Erstglied - als Realisierung von A2 - als Komplement eingestuft werden konnte ${ }^{221}$ (vgl. Tab. 4 bzw. z. B. 9)), aber ich vermute, dass die Zahl solcher Beispiele bei einer größeren Gesamtbelegzahl auch deutlich höher ausfallen würde. Eine Vermutung, die jedoch später noch zu überprüfen bleibt.

(9) Sie wird von ihrem sprachschöpferischen Einfluss auf die deutsche Sprache her mit Martin Luthers Bibelübersetzung verglichen. (Nordkurier, 04.06.2012)

Schließlich ist in Beisp. 10) ein weiteres Charakteristikum für die Stellung von Substantivkomplementen zu erkennen, wofür es noch andere Belege unter den ersten 100 Treffern für Übersetzung gibt. Die Komplemente des Substantivs können sich im Satz von ihrem Bezugssubstantiv entfernen (vgl. auch Bassola 2014: 22), so wie es in 10) mit Für ihren Namen der Fall ist, der am Satzanfang, vor dem finiten Verb in markierter Stellung realisiert wird und dadurch sogar zusätzlichen Akzent trägt.

(10) Für ihren Namen haben die neun Tänzerinnen der Gruppe eine sehr freie Übersetzung gefunden. (Nordkurier, 06.08.2002)

\subsubsection{Das Substantiv Ausdruck}

Unter den ersten 100 Treffern für Ausdruck kam das Substantiv 99-mal in den Bedeutungen Ausdrucksweise bzw. Wort, Bezeichnung vor, während es einen einzigen Beleg für die Bedeutung vom Drucker ausgedruckter Output gab. Da letztere Bedeutung - auch dem DUW zufolge - nicht demselben Wort zugeordnet werden kann wie die beiden anderen, sondern einem homonymen Substantiv, wurde der einzige Beleg mit der Bedeutung Output ausgegrenzt und durch den nächstfolgenden Beleg für eine der beiden anderen Bedeutungen ersetzt.

\footnotetext{
${ }^{221}$ Zwar kam Übersetzung z. B. auch in einem Kompositum wie Neü̈bersetzung vor, aber es ist wichtig darauf hinzuweisen, dass in solchen Komposita das Erstglied kein Argument von Übersetzung und somit kein Komplement realisiert.
} 
So konnten auf der Grundlage der untersuchten Belege zwei Bedeutungen und zwei Argumentstrukturen von Ausdruck ermittelt werden, die folgendermaßen darzustellen sind:

I) Ausdrucksweise - jd. (A1) drückt etw. (A2) irgendwie (A3) aus

II) Wort, Bezeichnung - etw. (A') stellt einen Ausdruck für etw. (A2) dar

Wie den obigen Strukturdarstellungen zu entnehmen ist, ist in Bed. II) auch hier das in Kap. 4.2.1.1 schon thematisierte Quasiargument $\mathrm{A}^{`} \mathrm{zu}$ entdecken, das in sechs Belegen in Form einer Apposition realisiert wird (vgl. Tab. 6). So entspricht in 11) «tiefere Lagen» A', während für den Bereich zwischen 600 und 1000 Meter A2 zugeordnet werden kann.

... und so habe er eines Tages einem Meteorologen in Zürich vorgeschlagen, den Ausdruck «tiefere Lagen» für den Bereich zwischen 600 und 1000 Meter einzuführen - mit Erfolg. (St. Galler Tagblatt, 29.12.2012)

\begin{tabular}{|c|c|c|}
\hline & Bed. I) & Bed. II) \\
\hline -SVG & 33 & 9 \\
\hline +SVG & 58 & 0 \\
\hline
\end{tabular}

Tab. 5: Anzahl der SVG- und Nicht-SVG-Belege für Ausdruck nach Bedeutungen

Wie erwartet, kommt Ausdruck in Bed. I) häufig als Substantiv im SVG vor, 63,74\% der Belege für Bed. I) machen SVG-Belege aus. In Bezug auf die SVG mit Ausdruck ergaben sich mehrere Fragen, die jedoch alle zum Problemkreis gerechnet werden können, wo die Grenze der Gruppe der Stützverben liegt. Auf der Grundlage der Analyse der Belege für Ausdruck bin ich im Zusammenhang mit der in der Forschung umstrittenen Zuordnung von sein zu den Stützverben (vgl. Kap. 3.1) zu der Entscheidung gekommen, dass ich sein - im Gegensatz z. B. zum ebenfalls uneinheitlich beurteilten haben - nicht als Stützverb betrachte, da es nur der Funktion der Gleichsetzung zu dienen scheint.

\begin{tabular}{|c|c|c|c|c|c|c|}
\hline $\begin{array}{l}\underset{ٌ}{2} \\
\stackrel{\square}{2}\end{array}$ & & Kreal. quant. & Kreal. qual. & Areal. & SVG-Strukturen & $\begin{array}{c}\text { Anzahl } \\
\text { der } \\
\text { Belege }\end{array}$ \\
\hline \multirow{7}{*}{ I) } & \multirow{9}{*}{$\sum_{a}^{i n}$} & 0 & keine Real. & kein $\mathrm{A}$ & & 6 \\
\hline & & 1 & davon + Nebensatz & $\mathrm{A} 2$ & & 1 \\
\hline & & 1 & Gen. & $\mathrm{A} 2$ & & 20 \\
\hline & & 1 & dass-Satz & $\mathrm{A} 2$ & & 1 \\
\hline & & 1 & von + Dat. & $\mathrm{A} 2$ & & 3 \\
\hline & & 1 & Posspr. & $\mathrm{A} 2$ & & 1 \\
\hline & & 1 & Posspr. & A1 & & 1 \\
\hline \multirow{2}{*}{ II) } & & 1 & Apposition & $\mathrm{A}^{\prime}$ & & 5 \\
\hline & & 1 & für + Akk. & $\mathrm{A} 2$ & & 1 \\
\hline
\end{tabular}




\begin{tabular}{|c|c|c|c|c|c|c|}
\hline$\underset{\mathscr{Q}}{\mathscr{Q}}$ & & Kreal. quant. & Kreal. qual. & Areal. & SVG-Strukturen & $\begin{array}{l}\text { Anzahl } \\
\text { der } \\
\text { Belege }\end{array}$ \\
\hline & & 2 & $\begin{array}{l}\text { Apposition } \\
\text { für }+ \text { Akk. }\end{array}$ & $\begin{array}{l}\mathrm{A}^{\prime} \\
\mathrm{A} 2\end{array}$ & & 1 \\
\hline \multirow{18}{*}{ I) } & \multirow{18}{*}{$\sum_{a}^{+}$} & $0+1$ & $\begin{array}{l}\text { keine Real. } \\
\text { Subj. }\end{array}$ & $\begin{array}{c}\text { kein Argument } \\
\text { A2 }\end{array}$ & $\begin{array}{c}\text { zum A. kommen } \\
\text { zum A. bringen + PASSIV }\end{array}$ & 6 \\
\hline & & $0+1$ & $\begin{array}{l}\text { keine Real. } \\
\text { dass-Subjektsatz }\end{array}$ & $\begin{array}{l}\text { kein Argument } \\
\text { A2 }\end{array}$ & zum A. kommen & 1 \\
\hline & & $0+1$ & $\begin{array}{l}\text { keine Real. } \\
\text { Akk. }\end{array}$ & $\begin{array}{l}\text { kein Argument } \\
\text { A2 }\end{array}$ & zum A. bringen & 2 \\
\hline & & $0+2$ & $\begin{array}{l}\text { keine Real. } \\
\text { Subj. } \\
\text { Akk. }\end{array}$ & $\begin{array}{c}\text { kein Argument } \\
\text { A1 } \\
\text { A2 }\end{array}$ & zum A. bringen & 13 \\
\hline & & $0+2$ & $\begin{array}{l}\text { keine Real. } \\
\text { Subj. } \\
\text { dass-Objektsatz }\end{array}$ & $\begin{array}{c}\text { kein Argument } \\
\text { A1 } \\
\text { A2 }\end{array}$ & zum A. bringen & 3 \\
\hline & & $0+2$ & $\begin{array}{l}\text { keine Real. } \\
\text { Subj. } \\
\text { Dat. }\end{array}$ & $\begin{array}{c}\text { kein Argument } \\
\text { A1 } \\
\text { A2 }\end{array}$ & $\begin{array}{l}\text { Dat. A. verschaffen } \\
\text { Dat. A. geben } \\
\text { Dat. A. verleihen }\end{array}$ & 7 \\
\hline & & $0+3$ & $\begin{array}{l}\text { keine Real. } \\
\text { Akk. } \\
\text { Subj. } \\
\text { Pronadv. damit }\end{array}$ & $\begin{array}{c}\text { kein Argument } \\
\text { A2 } \\
\text { A1 } \\
\text { A3 } \\
\end{array}$ & zum A. bringen & 1 \\
\hline & & $1+1$ & $\begin{array}{l}\text { Pronadv. damit } \\
\text { dass-Subjektsatz }\end{array}$ & $\begin{array}{l}\mathrm{A} 3 \\
\mathrm{~A} 2\end{array}$ & zum A. bringen + Passiv & 1 \\
\hline & & $1+1$ & $\begin{array}{l}\text { Pronadv. damit } \\
\text { Subj. }\end{array}$ & $\begin{array}{l}\mathrm{A} 3 \\
\mathrm{~A} 2\end{array}$ & zum A. bringen + Passiv & 1 \\
\hline & & $1+1$ & $\begin{array}{l}\text { mit + Dat. } \\
\text { Subj. }\end{array}$ & $\begin{array}{l}\mathrm{A} 3 \\
\mathrm{~A} 2\end{array}$ & zum A. kommen & 1 \\
\hline & & $1+1$ & $\begin{array}{l}\text { mit + Dat. } \\
\text { dass-Subjektsatz }\end{array}$ & $\begin{array}{l}\mathrm{A} 3 \\
\mathrm{~A} 2\end{array}$ & zum A. bringen + Passiv & 1 \\
\hline & & $1+1$ & $\begin{array}{l}\text { in + Dat. } \\
\text { Subj. }\end{array}$ & $\begin{array}{l}\mathrm{A} 3 \\
\mathrm{~A} 2 \\
\end{array}$ & $\begin{array}{l}\text { zum A. gelangen } \\
\text { zum A. kommen }\end{array}$ & 3 \\
\hline & & $1+1$ & $\begin{array}{l}\text { durch + Akk. } \\
\text { Subj. }\end{array}$ & $\begin{array}{l}\text { A3 } \\
\text { A2 }\end{array}$ & zum A. kommen & 1 \\
\hline & & $1+2$ & $\begin{array}{l}\text { mit + Dat. } \\
\text { Subj. } \\
\text { Dat. }\end{array}$ & $\begin{array}{l}\mathrm{A} 3 \\
\mathrm{~A} 1 \\
\mathrm{~A} 2\end{array}$ & $\begin{array}{l}\text { Dat. A. geben } \\
\text { Dat. A. verleihen }\end{array}$ & 2 \\
\hline & & $1+2$ & $\begin{array}{l}\text { mit + Dat. } \\
\text { Subj. } \\
\text { Akk. }\end{array}$ & $\begin{array}{l}\mathrm{A} 3 \\
\mathrm{~A} 1 \\
\mathrm{~A} 2\end{array}$ & zum A. bringen & 6 \\
\hline & & $1+2$ & $\begin{array}{l}\text { attr. Adj. } \\
\text { Subj. } \\
\text { Dat }\end{array}$ & $\begin{array}{l}\mathrm{A} 3 \\
\mathrm{~A} 1 \\
\mathrm{~A} 2\end{array}$ & Dat. A. verleihen & 1 \\
\hline & & $1+2$ & $\begin{array}{l}\text { in + Dat. } \\
\text { Subj. } \\
\text { Akk. }\end{array}$ & $\begin{array}{l}\mathrm{A} 3 \\
\mathrm{~A} 1 \\
\mathrm{~A} 2\end{array}$ & zum A. bringen & 1 \\
\hline & & $1+2$ & $\begin{array}{l}\text { durch + Akk. } \\
\text { Subj. } \\
\text { Akk. }\end{array}$ & $\begin{array}{l}\mathrm{A} 3 \\
\mathrm{~A} 1 \\
\mathrm{~A} 2\end{array}$ & zum A. bringen & 1 \\
\hline
\end{tabular}




\begin{tabular}{|c|c|c|c|c|c|}
\hline 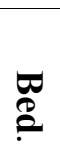 & Kreal. quant. & Kreal. qual. & Areal. & SVG-Strukturen & $\begin{array}{c}\text { Anzahl } \\
\text { der } \\
\text { Belege }\end{array}$ \\
\hline & $2+1$ & $\begin{array}{l}\text { Posspr. } \\
\text { attr. Adj. } \\
\text { Subj. }\end{array}$ & $\begin{array}{l}\mathrm{A} 2 \\
\mathrm{~A} 3 \\
\mathrm{~A} 2 \\
\end{array}$ & Poss. Ausdruck erhalten & 1 \\
\hline & $2+1$ & $\begin{array}{l}\text { Posspr. } \\
\text { mit + Dat. } \\
\text { Subj. }\end{array}$ & $\begin{array}{l}\text { A2 } \\
\text { A3 } \\
\text { A2 }\end{array}$ & Poss. A. finden & 1 \\
\hline & $2+1$ & $\begin{array}{l}\text { Posspr. } \\
\text { in + Dat. } \\
\text { Subj. }\end{array}$ & $\begin{array}{l}\text { A2 } \\
\text { A3 } \\
\text { A2 }\end{array}$ & Poss. A. finden & 4 \\
\hline
\end{tabular}

Tab. 6: Zahlenmäßige Verteilung der SVG- und Nicht-SVG-Belege für Ausdruck, nach Bedeutungen bzw. Komplement- und Argumentstrukturen klassifiziert

Unter den Belegen bin ich vielen Beispielen begegnet, die sich - meines Erachtens - an der Grenze der Stützverben befinden und von vielen nicht mehr zu den Stützverben gerechnet würden, da sie semantisch nicht entleert sind (vgl. auch dazu Kap. 3.1). Da ich jedoch die Auffassung vertrete, dass SVG in ihrer Bedeutung nicht dadurch zu charakterisieren sind, dass das Stützverb seine Semantik weitgehend einbüßt, sondern vielmehr durch die semikompositionelle Art der Verbindung, habe ich Konstruktionen wie z. B. in 12) und 13) als SVG eingestuft (zu meinem Standpunkt über SVG vgl. Kap. 3.3, zur ausführlichen Auflistung der ermittelten SVG mit Ausdruck vgl. Tab. 6).

(12) Im «Incarnatus» erhält jeder_Gedanke seinen eigenen musikalischen Ausdruck. (St. Galler Tagblatt, 17.01.2001)

(13) In der Beton-Burg Stadthalle präsentierte der „Urvater der deutschen Rockmusik“ gestern Abend

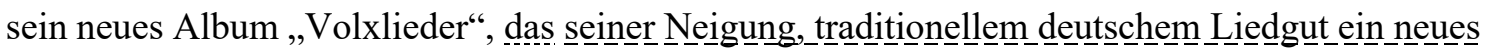

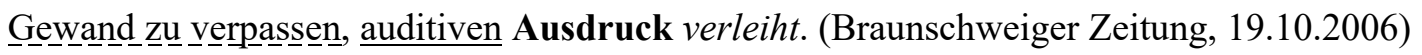

Beisp. 12) und 13) weisen attributive Adjektive (musikalischen und auditiven) als Realisierungen von A3 in Bed. I) auf, die somit - meiner Ansicht nach - als Komplementrealisierungen zu klassifizieren sind. Zudem sind sie auch deswegen von Belang, weil sie Attribute des Substantivs im SVG darstellen, wobei die nominalen Teile von SVG den meisten Autoren zufolge - nur sehr eingeschränkt attribuierbar sein sollen.

Beisp. 12) zeigt ein weiteres interessantes Phänomen, indem darin ein Argument (A2) sowohl auf Satzebene (durch das Subjekt jeder Gedanke) als auch innerhalb der Nominalphrase (durch das Possessivpronomen seinen) realisiert wird. Wie Tab. 6 zu entnehmen ist, kann eine 
solche doppelte Argumentrealisierung nicht als Ausnahme betrachtet werden, da es fünf weitere Belege mit einer doppelten Realisierung von A2 gibt. ${ }^{222}$

Wenn wir die Komplementstrukturen der SVG- und Nicht-SVG-Belege in Bed. I) miteinander vergleichen, kann festgestellt werden, dass es nur wenige Übereinstimmungen in den Realisierungsformen gibt. Während A3 sowohl in SVG- als auch in Nicht-SVG-Belegen oft in Form von mit + Dat. (oder entsprechenden Formen, wie z. B. durch ein Pronominaladverb mit mit) vorkommt, können im Falle der Komplemente, die A1 und A2 realisieren, kaum Parallelen zwischen SVG- und Nicht-SVG-Realisierungen entdeckt werden. Dies spricht meines Ermessens - dafür, dass zwar SVG dazu geeignet sein können, die Argumentstrukturen der Substantive aufzudecken, aber zur Ermittlung der Realisierungsformen der jeweiligen Komplemente sie nur sehr eingeschränkt verwendet werden können (vgl. dazu auch die Vorgehensweise von Bassola et al. (2003 und 2012) bei der Ermittlung der Valenzstruktur von valenten Substantiven; bzw. Bresson / Kubczak 1998: 7).

\subsubsection{Das Substantiv Verspätung}

Verspätung konnte in einer Bedeutung ohne Bedeutungsvariante ${ }^{223}$ mit der folgenden Argumentstruktur ermittelt werden: jd. / etw. (A1) verspätet sich (um) ... (A2). Mit 73\% überwiegen die Belege, in denen ein Komplement vorkommt, wobei in 91,78\% dieser Fälle A2 durch das jeweilige Komplement realisiert wird, was deswegen von großer Bedeutung ist, weil Verspätung in erster Linie aus dem Grund in die Untersuchung miteinbezogen wurde, weil ich herausfinden wollte, wie oft und in welcher Form A2 in Konstruktionen mit Verspätung versprachlicht wird. Ich finde diese Frage interessant, weil im Zusammenhang mit dem Problem der Obligatorik von Substantivkomplementen von den wenigen Autoren, die obligatorische Komplemente beim Substantiv für möglich halten, die meisten nur sog. Maßund Behälterkonstruktionen als Vertreter dieser Gruppe annehmen (vgl. Kap. 2.5.3). Da Verspätung keine Maß- oder Mengenangabe ist, ging ich nie davon aus, dass dieses Substantiv diesem Problemkreis zugeordnet werden könnte, aber eine Art Analogie habe ich deswegen vermutet, weil durch die Realisierung von A2 eben das Maß an Verspätung zum Ausdruck gebracht wird.

\footnotetext{
${ }^{222}$ Somit liegt der Anteil der doppelten Realisierung von A2 bezogen auf die Gesamtzahl der Belege, in denen mindestens zwei Komplemente vorkommen, bei $14 \%$.

${ }^{223}$ Dies entspricht auch der Bedeutungsbeschreibung im DUW.
} 


\begin{tabular}{|c|c|c|c|c|c|}
\hline & $\begin{array}{l}\text { Kreal. } \\
\text { quant. }\end{array}$ & Kreal. qual. & Areal. & SVG-Strukturen & $\begin{array}{c}\text { Anzahl der } \\
\text { Belege }\end{array}$ \\
\hline \multirow{7}{*}{$\sum_{a}^{i}$} & 0 & keine Real. & kein $\mathrm{A}$ & & 21 \\
\hline & 1 & Gen. & A1 & & 3 \\
\hline & 1 & Komp. & $\mathrm{A} 1$ & & 1 \\
\hline & 1 & Messwert & $\mathrm{A} 2$ & & 48 \\
\hline & 1 & Indefpr. & $\mathrm{A} 2$ & & 7 \\
\hline & 1 & attr. Adj. & $\mathrm{A} 2$ & & 9 \\
\hline & 1 & eine ... von & $\mathrm{A} 2$ & & 2 \\
\hline \multirow{3}{*}{$\stackrel{+}{\stackrel{+}{\infty}}$} & $0+1$ & $\begin{array}{l}\text { keine Real. } \\
\text { Subj. }\end{array}$ & $\begin{array}{c}\text { kein Arg } \\
\text { A1 }\end{array}$ & V. haben & 3 \\
\hline & $0+1$ & $\begin{array}{l}\text { keine Real. } \\
\text { Subj. }\end{array}$ & $\begin{array}{c}\text { kein Arg } \\
\text { A2 }\end{array}$ & V. haben & 1 \\
\hline & $1+1$ & $\begin{array}{l}\text { Messwert } \\
\text { Subj. }\end{array}$ & $\begin{array}{l}\text { A2 } \\
\text { A1 }\end{array}$ & V. haben & 5 \\
\hline
\end{tabular}

Tab. 7: Zahlenmäßige Verteilung der SVG- und Nicht-SVG-Belege für Verspätung, nach Komplement- und Argumentstrukturen klassifiziert

Wie Tab. $7 \mathrm{zu}$ entnehmen ist, ist für die Komplemente, die A2 in den oben genannten Belegen realisieren, eine große formale Vielfalt charakteristisch. So kommen neben zwei Realisierungen durch die Konstruktion eine ... von und einer Realisierung auf Satzebene durch das Subjekt des Satzes, mehrmals Indefinitpronomina (vgl. z. B. 14)) und attributive Adjektive (vgl. z. B. 15)) vor. Mit einem Anteil von 73,61\% überwiegt jedoch eindeutig die Realisierungsform, die ich in Anlehnung an die GDS (1985) ${ }^{224}$ - als Messwert bezeichne, worunter ich hier die Verbindung einer Zeiteinheit mit einem Zahladjektiv verstehe (vgl. 16) und 17)).

(14) Denn gestartet wurde der Umzug mit einiger Verspätung und auch dann wollte er zunächst nicht so recht in Gang kommen. (St. Galler Tagblatt, 01.03.2010)

(15) In einer schwachen Viertelstunde war der Schaden wieder so weit repariert, dass der Zug mit einer kleinen Verspätung die Fahrt fortsetzen konnte. (St. Galler Tagblatt, 05.09.2007)

(16) Dafür kommt der vergrößerte Jumbo-Jet 747-8 erst mit eineinhalb Jahren Verspätung auf den Markt und der - einstige - Hoffnungsträger B 787 nach sieben Verspätungsrunden frühestens im dritten Quartal. (FOCUS, 31.01.2011)

(17) Bei Flügen bis zu 1500 Kilometern haben Fluggäste ab zwei Stunden Verspätung Anspruch auf freie Telefonate, Getränke, Mahlzeiten, gegebenenfalls auch Hotel-Übernachtungen. Bei Strecken bis zu 3500 Kilometern gilt dies nach drei Stunden Verspätung, ab 3500 Kilometern nach vier Stunden. (Hamburger Morgenpost, 07.09.2012)

\footnotetext{
${ }^{224}$ In der GDS (1985) wird Messwert als Verbindung eines Zahladjektivs mit einem Maß definiert.
} 
Unter den ersten 100 relevanten Treffern konnte ein Beleg ermittelt werden, in dem Verspätung als Leitglied eines Kompositums vorkommt, dessen Bestimmungsglied ein Komplement realisiert. In Beisp. 18) bringt nämlich das Erstglied Zug A1 zum Ausdruck.

(18) Bahnreisende bekommen bei Zugverspätungen ab einer Stunde künftig einen Teil des Fahrpreises zurück. (Braunschweiger Zeitung, 24.04.2009)

SVG-Belege machen mit 8\% einen relativ kleinen Anteil der Belege aus und kommen ausnahmslos mit haben als Stützverb vor. A1 wird insgesamt in 12\% der Belege realisiert, in SVG-Belegen wird es durch das Subjekt des Satzes ausgedrückt, während es in Nicht-SVGBelegen - mit der einzigen oben erwähnten Ausnahme (vgl. 18) - immer in Form eines Genitivattributs realisiert wird (vgl. dazu 19) bzw. Tab. 6).

(19) Die Journalistin Trautl Brandstaller sucht als Herausgeberin des Bandes Zuflucht bei Robert Musil, der in seinem "Mann ohne Eigenschaften" die chronische Verspätung Österreichs in allen Lebensbereichen beschrieben hat. (Tiroler Tageszeitung, 17.12.1996)

\subsubsection{Das deadjektivische Substantiv Freiheit}

Nach der Beschreibung des DUW verfügt das Substantiv Freiheit über drei Bedeutungsvarianten, die wie folgt umrissen werden können: ${ }^{225}$

I) Zustand, in dem man unabhängig, ungebunden ist

II) das Nicht-gefangen-Sein

III) Recht, etw. zu tun

\begin{tabular}{|c|c|c|c|}
\hline & Bed. I) & Bed. II) & Bed. III) \\
\hline -SVG & 72 & 12 & 9 \\
\hline +SVG & 1 & 0 & 6 \\
\hline
\end{tabular}

Tab. 8: Anzahl der SVG- und Nicht-SVG-Belege für Freiheit nach Bedeutungen klassifiziert

Im Korpus gehören 73,00\% der ersten 100 Belege für Freiheit zu Bed. I), während Bed. II) $12 \%$ und Bed. III) 15,00\% der analysierten Belege ausmachen. Auf Grund der ermittelten Daten können folgende Argumentstrukturen mit den drei Bedeutungen verbunden werden:

I) etw. (A1) ist frei, ungebunden

II) jd. (A1) ist in Freiheit

III) $j d$. (A1) hat die Freiheit etw. zu tun (A2)

${ }^{225}$ Die hier dargelegten Bedeutungsparaphrasen basieren auf Stichwörtern der Bedeutungsbeschreibung im DUW. 
Die Unterscheidung zwischen den Bedeutungen I) und III) hat sich in bestimmten Belegen problematisch erwiesen. Während Beisp. 20) für Bed. I) steht, lässt sich Freiheit in Beisp. 21) auf Grund des vorangehenden Satzes und der Verwendung des Demonstrativpronomens als Artikelwort - meines Erachtens - Bed. III) zuordnen, in dem darin ein bestimmtes Recht, etwas $\mathrm{zu}$ tun, zum Ausdruck gebracht wird. Weniger eindeutig scheint mir dagegen $\mathrm{u}$. a. die Bestimmung dessen, zu welcher Bedeutung Beisp. 22) oder 23) gerechnet werden sollen. Da ich davon ausgegangen bin, dass diese Freiheiten auf Rechte hinweisen, die einem entweder etwas ermöglichen oder eben dadurch verantwortlich für etwas machen, habe ich beide in die Gruppe von Bed. III) eingeordnet.

(20) Der künstlerische Leiter des Sandskulpturen-Festivals in Rorschach, Urs Koller, hat das diesjährige Motto in einem Gedichtband des deutschen Schriftstellers Hans Magnus Enzensberger entdeckt. Dass es immer einen gebe, der grösser oder kleiner ist als du, sei ein universelles Thema, das die Menschen tagtäglich bewege. «Es lässt den_K Künstlern sehr viel Freiheit.» (St. Galler Tagblatt, 05.06.2013)

(21) $\mathrm{Ab}$ 19. Februar 1999 dürfen 75 Industriefirmen (ab 40 Mill. kWh Jahresbedarf) ihre Stromlieferanten EU-weit frei wählen. Diese Freiheit droht aber an prohibitiven Durchleitungstarifen zu scheitern: Sie sind im Extremfall doppelt so hoch wie in Bayern. (Salzburger Nachrichten, 30.12.1998)

(22) „Wir wissen, dass einige Pädagogen unter dem Mantel der Freiheit ihre thematischen Steckenpferde im Unterricht bis zur Pensionierung ritten“, räumt der GEW-Landesvorsitzende Eberhard Brandt zwar Mängel des alten Systems ein. (Braunschweiger Zeitung, 20.04.2006)

(23) Freiheit heißt Verantwortung. (Hamburger Morgenpost, 11.06.2007)

Meine Annahme in Bezug auf die große Häufigkeit von SVG-Belegen mit Freiheit hat sich nur in Bed. III) bewahrheitet, in der SVG-Belege mit 40,00\% tatsächlich einen großen Anteil vertreten. Dagegen habe ich in Bed. II) keinen, in Bed. I) lediglich einen einzigen der insgesamt 73 Belege als SVG-Belege eingestuft. Im Zusammenhang mit den ermittelten SVG-Belegen halte ich für besonders wichtig hervorzuheben, dass sie bezüglich des Artikelgebrauchs vor dem nominalen Teil große Varianz bzw. Flexibilität aufweisen. So kommt das Substantiv im SVG jm. ... Freiheit lassen z. B. in 20) mit dem erweiterten Indefinitpronomen sehr viel, in 24) mit dem bestimmten Artikel und in 25) mit der Maßangabe ein Stück zusammen vor.

(24) «Wir lassen ja dem Bauherrn die Freiheit, wem er die Aufträge vergeben will. (St. Galler Tagblatt, [Tageszeitung], 28.05.1999)

(25) Dies weil es mir ein Stück Freiheit lässt, spontan doch eine andere Strecke zu fahren oder gegen einen Aufpreis die Klasse zu wechseln. (St. Galler Tagblatt, 21.06.2011) 


\begin{tabular}{|c|c|c|c|c|c|c|}
\hline$\stackrel{\varpi ్}{\mathscr{0}}$ & & $\begin{array}{l}\text { Kreal. } \\
\text { quant. }\end{array}$ & Kreal. qual. & Areal. & SVG-Strukturen & $\begin{array}{c}\text { Anzahl der } \\
\text { Belege }\end{array}$ \\
\hline \multirow{6}{*}{ I) } & \multirow{12}{*}{$\sum_{a}^{1}$} & 0 & keine Real. & $\operatorname{kein} \mathrm{A}$ & & 32 \\
\hline & & 1 & attr. Adj. & A1 & & 10 \\
\hline & & 1 & Gen. & A1 & & 12 \\
\hline & & 1 & Komp. & A1 & & 10 \\
\hline & & 1 & für + Akk. & A1 & & 3 \\
\hline & & 1 & Posspr. & A1 & & 5 \\
\hline \multirow{2}{*}{ II) } & & 0 & keine Real. & kein $\mathrm{A}$ & & 11 \\
\hline & & 1 & Posspr. & A1 & & 1 \\
\hline \multirow{4}{*}{ III) } & & 0 & keine Real. & $\operatorname{kein} \mathrm{A}$ & & 5 \\
\hline & & 1 & Komp. & $\mathrm{A} 2$ & & 1 \\
\hline & & 1 & Infsatz. & $\mathrm{A} 2$ & & 2 \\
\hline & & 2 & $\begin{array}{l}\text { Komp. } \\
\text { Gen. }\end{array}$ & $\begin{array}{l}\mathrm{A} 2 \\
\mathrm{~A} 1 \\
\end{array}$ & & 1 \\
\hline I) & \multirow{7}{*}{$\stackrel{+}{i s}$} & $1+1$ & $\begin{array}{l}\text { Posspr. } \\
\text { Dat. }\end{array}$ & $\begin{array}{l}\text { A1 } \\
\text { A1 }\end{array}$ & Dat. F. lassen & 1 \\
\hline \multirow{6}{*}{ III) } & & $0+1$ & $\begin{array}{l}\text { keine Real. } \\
\text { Subj. }\end{array}$ & $\begin{array}{c}\text { kein Arg } \\
\text { A1 }\end{array}$ & F. haben & 1 \\
\hline & & $0+1$ & $\begin{array}{l}\text { keine Real. } \\
\text { Dat. }\end{array}$ & $\begin{array}{c}\text { kein Argument } \\
\text { A1 }\end{array}$ & Dat. F. lassen & 1 \\
\hline & & $1+1$ & $\begin{array}{l}\text { NS } \\
\text { Dat. }\end{array}$ & $\begin{array}{l}\mathrm{A} 2 \\
\mathrm{~A} 1 \\
\end{array}$ & Dat. F. lassen & 1 \\
\hline & & $1+1$ & $\begin{array}{l}\text { Infsatz } \\
\text { Dat. }\end{array}$ & $\begin{array}{l}\text { A2 } \\
\text { A1 }\end{array}$ & Dat. F. lassen & 1 \\
\hline & & $1+1$ & $\begin{array}{l}\text { Inf(satz) } \\
\text { Subj. }\end{array}$ & $\begin{array}{l}\mathrm{A} 2 \\
\mathrm{~A} 1\end{array}$ & F. haben & 1 \\
\hline & & $1+1$ & $\begin{array}{l}\text { Gen. } \\
\text { Dat. }\end{array}$ & $\begin{array}{l}\text { A2 } \\
\text { A1 }\end{array}$ & Dat. bleibt die F. & 1 \\
\hline
\end{tabular}

Tab. 9: Zahlenmäßige Verteilung der SVG- und Nicht-SVG-Belege für Freiheit, nach Bedeutungen bzw.

Komplement- und Argumentstrukturen klassifiziert

Was die Realisierungsformen der Komplemente betrifft, sind die Belege für Bed. I) besonders aus der Sicht von Belang, dass sie bei der Realisierung von A1 von einer deutlichen Vielfalt zeugen (vgl. Tab. 9). In Bed. I) gibt es ausschließlich Belege für die Realisierung von A1, das in $24,39 \%$ der Belege in Form eines attributiven Adjektivs, ebenfalls zu 24,39\% durch das Bestimmungsglied eines Kompositums, in 29,27\% der Fälle durch ein Genitivattribut, in 7,32\% der Belege durch ein Präpositionalattribut mit für $+A k k$. und in 12,20\% der Beispiele durch ein Possessivpronomen ausgedrückt wird. Darüber hinaus gibt es auch einen Beleg (2,44\%) für die doppelte Realisierung von A1. In 26) erscheint es nämlich auf der Phrasenebene in Form eines Possessivpronomens (seine) und auf der Satzebene im Dativobjekt des Satzes (Hongkong).

(26) China lässt ㅌongkong - mit Abstrichen - tatsächlich seine Freiheit. (Salzburger Nachrichten, 17.12.1999) 
Wie auch der obigen Aufzählung zu entnehmen ist, machen Bestimmungsglieder von Komposita einen wesentlichen Anteil der Komplementrealisierungen in Bed I) aus. Im Zusammenhang mit den entsprechenden Belegen ist noch hervorzuheben, dass in Bezug auf die betreffenden Bestimmungsglieder eine große Varianz festgestellt werden kann. Zwar scheinen Bestimmungsglieder wie Presse oder Wahl (vgl. 27) und 28)) deutlich häufiger vorzukommen, aber daneben werden unterschiedlichste Wörter zur Füllung der entsprechenden Position gewählt, wie z. B. Bein in 29) bzw. Lehr- und Methoden in 30).

(27) Weil es um Pressefreiheit geht, könnte das Verfahren bis zum Bundesverfassungsgericht gehen. (Berliner Morgenpost, 24.05.1998)

(28) Der Kanton hat die Schulpflicht nun auf den Kindergarten ausgedehnt. Fällt damit die Wahlfreiheit weg? (St. Galler Tagblatt, 22.02.2008)

(29) Kein hektisches Kartenlesen und auf die richtige Autobahnabfahrt warten, genügend Beinfreiheit, um sich auch ausstrecken zu können, und ein deutschsprachiger Reiseleiter, der einem immer darauf hinweist, wo man gerade ist, und was es hier zu sehen gibt. (Kleine Zeitung, 11.03.1998)

(30) Gerade weil in den Mittelschulen die Lehr- und Methodenfreiheit gross [sic] sei, brauche es entsprechende Kontrollen. (St. Galler Tagblatt, 30.05.2011)

Während das Bestimmungsglied eines Kompositums in Belegen für Bed. I) A1 zum Ausdruck bringt, ist diese Realisierungsform im Lichte der ermittelten Belege für Bed. III) in der Verwendung von Freiheit mit A2 verbunden. So ist in Beisp. 31) das Genitivattribut des öffentlichen Auftraggebers als Realisierung von A1 zu betrachten, während Entscheidung als Teil des Kompositums für A2 steht.

(31) Der tiefere Wert öffne den Marktzutritt für mehr Firmen, begründen die Verbände die Reduktion. Sie enge im Gegenzug aber die Entscheidungsfreiheit des öffentlichen Auftraggebers ein. (St. Galler Tagblatt, 26.01.2000)

Bezüglich der Realisierungsformen der Komplemente in Bed. III) lässt sich außerdem feststellen, dass sich die morphologisch-syntaktischen Eigenschaften der Komplemente in Nicht-SVG-Belegen aus denen der Komplemente in SVG-Belegen nicht ableiten lassen (vgl. Tab. 9). Hier wird wiederum deutlich, dass SVG zwar dazu geeignet sind bzw. sein können, Informationen über die Argumentstrukturen der in ihnen enthaltenen Substantive zu liefern, aber die Komplementrealisierungsformen des betreffenden Substantivs können in Nicht-SVGVerwendungen auf Grund der morpho-syntaktischen Valenzeigenschaften des SVG nur sehr eingeschränkt vorausgesagt werden (vgl. auch Kap. 3.2 und 4.2.1.2). 


\subsubsection{Die nicht-abgeleiteten Substantive}

\subsubsection{Das Substantiv Problem}

Auf Grundlage der ersten 100 Belege für Problem konnte eine Bedeutung des Substantivs ermittelt werden, aber $\mathrm{zu}$ dieser einen Bedeutung gehören zwei miteinander nicht kombinierbare Argumentstrukturen, die folgendermaßen angegeben werden können:

Ia) jd. (A1) hat ein Problem mit etw. (A2)

Ib) etw. (A') stellt ein Problem dar

Wie der Darstellung der Argumentstrukturen entnommen werden kann, erscheint auch hier wie in Kap. 4.2.1.1, 4.2.1.2 und 4.2.3.2 - A' in einer der Strukturen, wobei ich jedoch beim A von Problem einen leichten Unterschied im Vergleich zum A` von Ausdruck oder Übersetzung

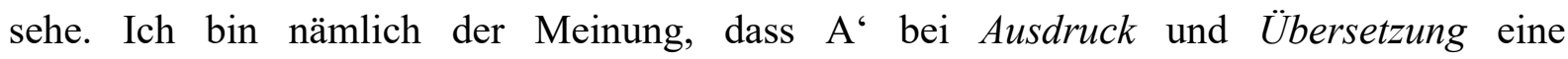
Quasigleichsetzung ausdrückt und es keine (andere) vom Bezugssubstantiv eröffnete Leerstelle gibt, die logisch-semantisch gesehen von Realisierungen von A' besetzt sein könnte. Dafür liegt bei Problem - meines Erachtens - ebenfalls eine Quasigleichsetzung vor, wobei jedoch eine Verbindung zu einem der weiteren Argumente, nämlich zu A2, hergestellt werden kann. Aus diesem Grund habe ich auch überlegt, in der Strukturdarstellung Ib) nicht A', sondern A2 anzugeben, aber schließlich habe ich diese Unterscheidung beibehalten, da A1 und A2 in ihrer Funktion nicht identisch sind. Trotzdem ist A' hier vielleicht auch nicht ganz angebracht, da sich dieses Argument von Problem auf einer Zwischenstufe zwischen einem Quasiargument A' und einem echten Argument befindet. Diese Frage soll in der nahen Zukunft durch weitere Beleganalysen noch weiter untersucht werden, bis dahin verbleibe ich bei der Verwendung von A' auch für das betreffende Argument in der Struktur Ib) von Problem.

Ferner sehe ich den Eindruck, dass es sich bei A ‘ von Problem um einen Übergang zwischen einem Quasiargument und einem echten Argument handle, nicht zuletzt dadurch unterstützt, dass sich der Unterschied auch in morphologisch-syntaktischen Aspekten der Komplementrealisierung widerzuspiegeln scheint. Während das A' von Ausdruck nur in Form von Appositionen, das von Übersetzung neben der Apposition noch durch eine Verbindung mit als realisiert werden kann (vgl. Tab. 6 und Tab. 4), gibt es A'-Realisierungen im Falle von Problem nicht nur in Form einer unmarkierten Apposition wie in 32), die sogar hier am seltensten als Realisierungsform vorkommt, sondern in vielerlei anderen Formen, wie z. B. durch ein Genitivattribut (vgl. z. B. 33)) oder durch einen dass-Satz (vgl. z. B. 34)) (vgl. dazu auch Tab. 10). 
(32) In der Selbsthilfegruppe Al-Anon sind alle willkommen, welche das Problem Alkoholismus in ihrer Familie oder im Freundeskreis haben. (St. Galler Tagblatt, 24.02.1999)

(33) Beim zentralen Problem der Arbeitslosigkeit rangen alle Wahlkämpfer um die richtigen Worte. (Tiroler Tageszeitung, 26.09.1998)

(34) «Erstens hatten wir zu Beginn das Problem, dass mehrere Spieler wegen Auslandaufenthaltes oder Rekrutenschule abwesend waren. (St. Galler Tagblatt, 07.01.2012)

\begin{tabular}{|c|c|c|c|c|c|}
\hline & $\begin{array}{l}\text { Kreal. } \\
\text { quant. }\end{array}$ & Kreal. qual. & Areal. & SVG-Strukturen & $\begin{array}{c}\text { Anzahl der } \\
\text { Belege }\end{array}$ \\
\hline \multirow{11}{*}{$\sum_{a}^{1}$} & 0 & keine Real. & kein $\mathrm{A}$ & & 61 \\
\hline & 1 & Gen. & $\mathrm{A}^{\prime}$ & & 5 \\
\hline & 1 & Gen. & A1 & & 1 \\
\hline & 1 & für + Akk. & A1 & & 5 \\
\hline & 1 & mit + Dat. & $\mathrm{A} 2$ & & 1 \\
\hline & 1 & attr. Adj. & $\mathrm{A} 2$ & & 2 \\
\hline & 1 & attr. Adj. & A1 & & 2 \\
\hline & 1 & Komp. & $\mathrm{A} 2$ & & 7 \\
\hline & 1 & Inf.-Satz & $\mathrm{A}^{\prime}$ & & 1 \\
\hline & 1 & dass-Satz & $\mathrm{A}^{\prime}$ & & 1 \\
\hline & $1+1$ & $\begin{array}{l}\text { mit - Pronadv. } \\
\text { Subj. }\end{array}$ & $\begin{array}{l}\mathrm{A} 2 \\
\mathrm{~A} 1 \\
\end{array}$ & & 1 \\
\hline \multirow{12}{*}{$\stackrel{+}{\infty}_{a}^{+}$} & $0+1$ & $\begin{array}{l}\text { keine Real. } \\
\text { Subj. }\end{array}$ & $\begin{array}{c}\text { kein } \mathrm{A} \\
\mathrm{A} 1\end{array}$ & P. haben & 2 \\
\hline & $0+1$ & $\begin{array}{l}\text { keine Real. } \\
\text { Subj. }\end{array}$ & $\begin{array}{c}\text { kein } \mathrm{A} \\
\mathrm{A} 1\end{array}$ & P. haben & 1 \\
\hline & $0+2$ & $\begin{array}{l}\text { keine Real. } \\
\text { Dat. } \\
\text { Subj. }\end{array}$ & $\begin{array}{c}\text { kein } \mathrm{A} \\
\mathrm{A} 1 \\
\mathrm{~A}^{\prime}\end{array}$ & Dat. P. bereiten & 1 \\
\hline & $1+1$ & $\begin{array}{l}\text { Apposition } \\
\text { Subj. }\end{array}$ & $\begin{array}{l}\mathrm{A}^{\prime} \\
\mathrm{A} 1\end{array}$ & P. haben & 1 \\
\hline & $1+1$ & $\begin{array}{l}\text { Infsatz } \\
\text { Subj. }\end{array}$ & $\begin{array}{l}\text { A2 } \\
\text { A1 }\end{array}$ & P. haben & 1 \\
\hline & $1+1$ & $\begin{array}{l}\text { dass-Satz } \\
\text { Subj. }\end{array}$ & $\begin{array}{l}\mathrm{A}^{\prime} \\
\mathrm{A} 1\end{array}$ & P. haben & 1 \\
\hline & $1+1$ & $\begin{array}{l}\text { Komp.; } \\
\text { Subj. }\end{array}$ & $\begin{array}{l}\text { A2 } \\
\text { A1 }\end{array}$ & P. haben & 1 \\
\hline & $1+1$ & $\begin{array}{l}\text { bei + Dat. } \\
\text { Subj. }\end{array}$ & $\begin{array}{l}\text { A2 } \\
\text { A1 }\end{array}$ & P. haben & 1 \\
\hline & $1+1$ & $\begin{array}{l}\text { attr. Adj. } \\
\text { Subj. }\end{array}$ & $\begin{array}{l}\text { A2 } \\
\text { A1 }\end{array}$ & P. haben & 1 \\
\hline & $1+1$ & $\begin{array}{l}\text { in + Dat. } \\
\text { Subj. }\end{array}$ & $\begin{array}{l}\mathrm{A} 2 \\
\mathrm{~A} 1\end{array}$ & P. haben & 1 \\
\hline & $1+1$ & $\begin{array}{l}\text { mit + einander } \\
\text { Subj. }\end{array}$ & $\begin{array}{l}\text { A2 } \\
\text { A1 }\end{array}$ & P. haben & 1 \\
\hline & $1+1$ & $\begin{array}{l}\text { mit + Dat. } \\
\text { Subj. }\end{array}$ & $\begin{array}{l}\mathrm{A} 2 \\
\mathrm{~A} 1\end{array}$ & P. haben & 1 \\
\hline
\end{tabular}

Tab. 10: Zahlenmäßige Verteilung der SVG- und Nicht-SVG-Belege für Problem, nach Komplement- und Argumentstrukturen klassifiziert 
Je mehr Komplement-Fürsprecher die Realisierungen des Quasiarguments $A$ ' neben der Quasiargumentselegiertheit aufweisen, desto weniger sind sie - meinem multidimensionalen Konzept zufolge - zu den absolut peripheren Komplementen zu rechnen. Daraus folgt für mich, dass die nicht-appositiven A'-Realisierungen von Problem als weniger periphere Komplemente zu betrachten sind als die Komplemente von Ausdruck oder Übersetzung, die ebenfalls A realisieren. Dabei bin ich der Ansicht, dass von diesen Beispielen - aus den oben genannten formalen Gründen - die A' realisierenden Komplemente von Ausdruck am meisten zur Peripherie der Komplemente gehören.

Unter den ersten 100 Treffern für Problem wurden 13 als SVG-Belege klassifiziert. Diese werden überwiegend mit haben als Stützverb formuliert (vgl. Tab. 10). Es ist wichtig hervorzuheben, dass auch diese SVG, wie die meisten, die im Rahmen der Untersuchung der in der vorliegenden Arbeit analysierten 600 Belege ermittelt wurden, große Flexibilität in Bezug auf den Artikelgebrauch vor dem Substantiv im SVG aufweisen und auch Beispiele für die Attribuierung des Substantivs zeigen. So wird das SVG ... Problem haben in 32) und 34) mit dem bestimmten Artikel, in 35) mit dem unbestimmten Artikel, in 36) mit dem negierenden Artikelwort kein und in 37) mit dem unbestimmten Artikel und attribuiert verwendet. Noch interessanter ist Beisp. 38), in dem das Substantiv im SVG - ähnlich wie in 37) - durch ein attributives Adjektiv ergänzt ist, aber darüber hinaus das valente Substantiv als Substantiv im SVG in einem Kompositum vorkommt und zusätzlich durch das Erstglied modifiziert wird.

(35) Im defensiven Mittelfeld hat S04 ein Problem. (Braunschweiger Zeitung, 13.11.2010)

(36) Lichtentenstein dagegen hat mit der Unterzeichnung kein Problem, nachdem das Umsteuern in Richtung auf einen transparenten Finanzsektor lange beschlossene Sache ist. (Luxemburger Tageblatt, 30.10.2014)

(37) Aber der eher tre des Politischen geschnitzt. (Berliner Morgenpost, 10.05.1999)

(38) Und doch gibt es keinen Grund zur Entwarnung, denn Luxemburg hat ein handfestes Bildungsproblem. (Luxemburger Tageblatt, 11.03.2008)

\subsubsection{Das Substantiv Ursache}

Ursache wurde in erster Linie deswegen in die Analyse miteinbezogen, weil ich davon ausging, dass es über die einfache Argumentstruktur etw. (A1) hat eine Ursache verfügt, in der es ein einziges Argument gibt, und ich wollte untersuchen, wie oft und in welcher Form dieses 
Argument realisiert wird. Auf Grund der Belegdaten und der daraus resultierenden Modifizierung meines Konzepts bezüglich der Bestimmung der Argumente, musste meine Ausgangshypothese betreffend die Argumentstruktur von Ursache so geändert werden, dass auch im Falle dieses Substantivs mit dem Quasiargument A`gerechnet werden muss.

\begin{tabular}{|c|c|c|c|c|c|}
\hline & $\begin{array}{l}\text { Kreal. } \\
\text { quant. }\end{array}$ & Kreal. qual. & Areal. & SVG-Strukturen & $\begin{array}{c}\text { Anzahl der } \\
\text { Belege }\end{array}$ \\
\hline \multirow{6}{*}{$\stackrel{i}{\infty}$} & 0 & keine Real. & kein $\mathrm{A}$ & & 22 \\
\hline & 1 & Gen. & A1 & & 30 \\
\hline & 1 & Komp. & A1 & & 9 \\
\hline & 1 & für - Pronadv. & $\mathrm{A} 1$ & & 6 \\
\hline & 1 & für + Akk. & $\mathrm{A} 1$ & & 27 \\
\hline & 1 & von + Dat. & A1 & & 2 \\
\hline \multirow{4}{*}{$\stackrel{+}{\infty}$} & $0+1$ & $\begin{array}{l}\text { keine Real. } \\
\text { Subj. }\end{array}$ & $\begin{array}{c}\text { kein Arg } \\
\text { A1 }\end{array}$ & U. haben & 1 \\
\hline & $1+1$ & $\begin{array}{l}\text { Posspr. } \\
\text { dass-Subjsatz }\end{array}$ & $\begin{array}{l}\text { A1 } \\
\text { A1 }\end{array}$ & U. haben & 1 \\
\hline & $1+1$ & $\begin{array}{l}\text { Posspr. } \\
\text { Subj. }\end{array}$ & $\begin{array}{l}\text { A1 } \\
\text { A1 }\end{array}$ & Poss. U. haben & 1 \\
\hline & $1+2$ & $\begin{array}{l}\text { Posspr. } \\
\text { Subj. } \\
\text { in + Dat. }\end{array}$ & $\begin{array}{l}\text { A1 } \\
\text { A1 } \\
\text { A }^{\prime}\end{array}$ & Poss. U. finden & 1 \\
\hline
\end{tabular}

Tab. 11: Zahlenmäßige Verteilung der SVG- und Nicht-SVG-Belege für Ursache, nach Komplement- und Argumentstrukturen klassifiziert

Unter den ersten 100 analysierten Treffern ${ }^{226}$ konnte ein Beleg für die Realisierung von $A^{6}$ ermittelt werden, der zugleich der einzige Beleg für das SVG mit der Struktur Poss. U. finden ist (vgl. 39)). Darin erscheint $A^{`}$ - neben der doppelten Realisierung von A1 durch ein Possessivpronomen (ihre) und durch das Subjekt des Satzes (Viele Defizite der jetzigen Situation) - auf Satzebene, in Form der Präpositionalphrase in + Dat..$^{227}$

\footnotetext{
${ }^{226}$ Wie bereits in Kap. 4.1.1 darauf hingewiesen wurde, fallen die ersten 100 analysierten Belege bei keinem der analysierten Substantive mit den ersten 100 Treffern für das jeweilige Substantiv zusammen, da bestimmte Belege ausgelassen werden müssen, v. a. deswegen, weil das untersuchte Substantiv in ihnen in phraseologischen Einheiten vorkommt, in denen das Valenzverhalten des Substantivs nicht zuverlässig untersucht werden kann. Bei Ursache war die Anzahl der aus diesen Gründen übersprungenen Belege (z. B. aus ungeklärter Ursache; kleine Ursache, ... große Wirkung; keine Ursache - als Antwort auf eine Dankesbezeigung) bei weitem am höchsten, was zwar keinen direkten Einfluss auf die Analyseergebnisse hat, jedoch informativ in Bezug auf die Gebrauchstendenzen von Ursache ist.

${ }^{227}$ Zwar reichen die bisher durchgeführten Analysen an 100 Belegen für Ursache, darunter an einem einzigen mit dem SVG Poss. U. finden, bei weitem nicht dafür aus, in Bezug auf die Valenzeigenschaften dieses SVG Folgerungen abzuleiten, aber ich halte es für wahrscheinlich, dass die Realisierungsform in + Dat. nicht durch das Substantiv Ursache (alleine) bestimmt sein kann.
} 
(39) Viele_ Defizite der_ jetzigen Situation finden ihre Ursache in der_ Überbetonung fachlich

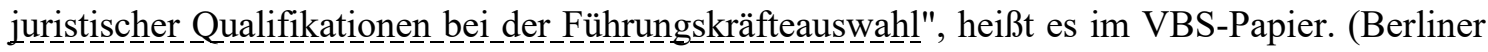
Morgenpost, 12.07.1999)

Wie Tab. 11 zu entnehmen ist, sind SVG-Belege mit insgesamt 4,00\% in sehr geringem Anteil vertreten. Das anfangs von mir als einziges Argument von Ursache angenommene A1 wird in 78\% der Belege realisiert und wird davon zu 42,30\% durch ein Präpositionalattribut mit für $+A k k$. oder ein Pronominaladverb mit für (vgl. z. B. 40)) und zu 38,46\% durch ein Genitivattribut (vgl. z. B. 41) bzw. 45)-46)) versprachlicht. Außerdem machen 11,54\% der Realisierungsformen Bestimmungsglieder von Komposita aus, deren lexikalische Füllung eine große Varianz aufweist (vgl. z. B. 42)-44)).

(40) Die Ursache für die unterschiedliche Schmerzempfindlichkeit dagegen liege vermutlich in den Genen: Experimente mit Mäusen hätten ergeben, daß das weibliche Hormon Östrogen und das männliche Testosteron für das Schmerzempfinden verantwortlich seien. (Mannheimer Morgen, 14.04.1998)

(41) Die Ursache des Unglücks waren kaputte Dichtungsringe an einer Feststoffrakete. (Luxemburger Tageblatt, 03.12.2013)

(42) Die Spezialdienste der Kantonspolizei haben die Ermittlungen zur Brandursache aufgenommen. (St. Galler Tagblatt, 20.01.2010)

(43) "Es wird nur nach absoluten Tempoüberschreitungen gestraft. Auf die Hauptunfallursache, die nicht angepasste Geschwindigkeit, wird nicht Rücksicht genommen." (Kleine Zeitung, 25.10.2000)

(44) Ein ärztliches Gutachten soll die genaue Verletzungsursache klären helfen. (Salzburger Nachrichten, 18.07.1995)

45) und 46) stellen zwei interessante Beispiele dafür dar, dass das Genitivkomplement von Ursache - wohlbemerkt in Nicht-SVG-Belegen - von dem Bezugssubstantiv entfernt erscheint. Dies wird in 45) dadurch ermöglicht, dass Ursache mit Wirkung, einem anderen valenten Substantiv, in koordinativer Verbindung steht und das als Genitivkomplement von Wirkung realisierte Komplement der Gewalt, somit auch an Ursache „weitergeleitet“" wird. In 46) zeigt sich - meines Erachtens - noch deutlicher die „Kraft der Substantivvalenz“: Das Genitivattribut, das von Ursache entfernt realisiert wird, gehört nicht einmal zum anderen, Ursache nebengeordneten Substantiv Lösungsansätze, sondern zum Nomen Bewältigung, das Teil des Präpositionalattributs von Lösungsansätze ist.

(45) Doch zwischen den Bildern, wenn er über Ursache und Wirkung der Gewalt räsoniert, Familienstrukturen erforscht und in seelische Abgründe abtaucht, dann erweist er sich als existenzialistischer Autorenfilmer reinsten Wassers. (Mannheimer Morgen, 12.10.2005) 
(46) Zwei zentrale strukturelle Defekte der deutschen Demokratie sind sowohl Ursache als auch Lösungsansätze zur Bewältigung der Situation: die mangelnde Wahrnehmung einer nachhaltigen Wachstumsperspektive und unzureichende Strukturen der Information und Mitbestimmung. (Mannheimer Morgen, 29.10.2011)

\subsection{Zusammenfassung der Ergebnisse der korpusbasierten Untersuchung der ausgewählten Substantive}

Nach der Darstellung und Auswertung der Ergebnisse der korpusbasierten Untersuchung der ausgewählten sechs Substantive in Kap. 4.2 fasse ich hier die wichtigsten Erkenntnisse dieser Analyse zusammen, wobei ich mich in erster Linie auf Aspekte fokussiere, die aus der Sicht der Charakterisierung der Substantivvalenz von Belang sind. Ferner hebe ich Merkmale hervor, die auf Grund der Belegdaten bezüglich der SVG-Konstruktionen festgestellt werden können.

Einerseits zeigen alle drei Gruppen (deverbale, deadjektivische und nicht-abgeleitete Substantive) Ähnlichkeiten in ihrem Valenzverhalten. Dies kann in den einzelnen Tabellen mit den Analysedaten zur Komplementrealisierung der Substantive auch überprüft werden. Zu diesen gemeinsamen Merkmalen gehört die oft $\mathrm{zu}$ beobachtende Vielfalt der Realisierungsformen der Komplemente, die in einer Bedeutung ein und dasselbe Argument realisieren. So kann z. B. A2 in Bed. Ib) von Übersetzung sowohl durch ein Genitivattribut, als auch durch Präpositionalphrasen mit für + Akk. oder von + Dat. oder aber auch durch ein Bestimmungsglied im Kompositum bzw. ein attributives Adjektiv zum Ausdruck gebracht werden (vgl. Tab. 4). Ähnlich bunt ist die Palette der Realisierungsformen z. B. für A1 von Freiheit, die von Genitivattributen über attributive Adjektive und Bestimmungsglieder von Komposita bis Präpositionalphrasen mit für + Akk. reicht (vgl. Tab. 9), um den Reichtum der Ausdrucksmöglichkeiten von A1 von Ursache (vgl. Tab. 11) gar nicht zu erwähnen.

In Bezug auf die Stellung der Komplemente der valenten Substantive weist auch die Fachliteratur darauf hin, dass in SVG-Konstruktionen die Komplemente sich auf Satzebene von ihren Bezugswörtern entfernen können (vgl. v. a. Bassola 2014), was auch in der vorliegenden Analyse eindeutig nachgewiesen werden konnte. Ferner wurde durch Belege für Ursache gezeigt, dass zu dieser Art der Disposition nicht einmal SVG-Konstruktionen nötig sind. Die kohäsive Kraft der Substantivvalenz ermöglicht es auch auf Phrasenebene, dass bei nebengeordneten Valenzträgern ihre Komplemente gemeinsam und dadurch von einem der valenten Köpfe entfernt realisiert werden (vgl. 45) und auch 46)). 
$\mathrm{Zu}$ besonders umstrittenen Realisierungsformen der Substantivkomplemente gehören attributive Adjektive und Bestimmungsglieder von Komposita. Die Analysen haben meine Annahme, wonach sie durchaus Komplemente realisieren können, jedoch eindeutig untermauert (vgl. dazu v. a. den Anteil von attr. Adj. in Tab. 4; den Anteil von Komp. in Tab 11 und den Anteil beider Realisierungsformen in Tab. 9 und 10).

Die Analyse hatte an mehreren Punkten auch Rückwirkungen auf meine theoretischen Ausgangpunkte sowohl in Bezug auf mein multidimensionales Konzept zur Beschreibung der Substantivkomplemente und -supplemente (vgl. Kap. 2.6) als auch auf meine „Arbeitsdefinition“ von SVG (vgl. Kap. 3.3). Das primär wichtige Kriterium der Argumenthaftigkeit erwies sich - meines Erachtens - als vertretbar bei der Unterscheidung von Komplementen und Supplementen. Die Belegdaten haben mich jedoch dazu bewogen, meinen Argumentbegriff $\mathrm{zu}$ erweitern, was in meinen Analysen zur Etablierung von $\mathrm{A}^{\text {‘ }}$ als Quasiargument führte (vgl. v.a. Kap. 4.2.1.1, 4.2.1.2 und 4.2.3.1). Anhand von Belegen von Ausdruck, Übersetzung und Problem konnte ich zeigen, dass A' einen stufenweisen Übergang von einem absolut peripheren zu einem mit einem echten Argument des jeweiligen Substantivs eng verwandten Quasiargument aufweist. Diese Annahme scheint auch mit formalen Aspekten der Komplementrealisierung, die in meinem Modell der Feineinteilung der Komplemente und Supplemente in zentrale bis periphere Subklassen dient, sehr schön im Einklang zu stehen (vgl. dazu v. a. Kap. 4.2.3.1).

In Kap. 3.1 habe ich gezeigt, dass die Gruppe der Stützverben in der Forschung uneinheitlich bestimmt wird. In Kap. 3.3 bin ich zu der Entscheidung gekommen, vorerst kein Verb als potenzielles Stützverb auszuschließen. Dies hat sich im Laufe der ersten Phase der korpusbasierten Analyse der sechs Substantive dahingehend geändert, dass ich in den Belegen Konstruktionen mit sein nicht als SVG betrachtet habe, weil es in den meisten Fällen nur Beispiele dafür gab, dass die jeweiligen valenten Substantive entweder als Subjekte oder als Gleichsetzungsnominative durch sein an eine entsprechende Konstituente im Satz angeschlossen wurden oder aber sein, mit einer Präpositionalphrase, mit dem jeweiligen valenten Substantiv verbunden, feste Einheiten bildete wie z. B. in Freiheit sein. Solche Belege gehören m. E. eindeutig zu den Nicht-SVG-Belegen. ${ }^{228}$

\footnotetext{
${ }^{228}$ Durch die Auswertung der ermittelten Belegdaten sollte überlegt werden, ob der Stützverbstatus von sein abhängig davon, ob es sich mit abgeleiteten oder nicht-abgeleiteten Substantiven im Nominativ verbindet, unterschiedlich beurteilt werden sollte. Diese Frage bleibt noch weiter zu untersuchen, wobei auch andere Konstruktionen auf ihren SVG-Status hin überprüft werden sollten, in denen valente Substantive nicht im Nominativ, sondern präpositional an sein angeschlossen werden, da ich vermute, dass es - im Gegensatz zu den Belegdaten, die durch die Analyse der hier beschriebenen 600 Treffer eruiert werden konnten - auch unter ihnen
} 
Betreffend die SVG finde ich die Ergebnisse der Korpusanalyse aus mehrerlei Sicht wichtig. Einerseits konnte bei jedem Substantiv, das in den Belegen überhaupt SVG-Konstruktionen einging, eindeutig gezeigt werden, dass es sowohl in Bezug auf den Artikelgebrauch vor dem jeweiligen Substantiv im SVG als auch auf die Attribuierbarkeit des Substantivs eine (relativ) große Flexibilität aufweist. Dies ist deswegen von Belang, weil in der Forschung im Zusammenhang mit diesen Merkmalen überwiegend gerade eine entgegengesetzte Auffassung vertreten wird. Andererseits haben die Ergebnisse bestätigt, dass SVG nur zur Eruierung der Argumentstruktur der in ihnen enthaltenen valenten Substantive geeignet sind und durch die Untersuchung ihrer Valenzeigenschaften über die potenziellen Realisierungsformen der Substantive nur sehr eingeschränkt Vorannahmen gemacht werden können. Das auch untermauert - meines Ermessens - eindeutig die Selbstständigkeit der Substantivvalenz, da entsprechend meiner multidimensionalen Betrachtung der Substantivvalenz, neben logischsemantischen auch formale, morphologisch-syntaktische Aspekte eine bedeutende Rolle in der Beschreibung der Substantivkomplemente bzw. -supplemente spielen.

Nach der Zusammenfassung der wichtigsten Ergebnisse der korpusbasierten Untersuchung der sechs ausgewählten Substantive überblicke ich im nächsten, abschließenden Kapitel meiner Arbeit die wichtigsten Erkenntnisse und Teilergebnisse der vorangehenden Kapitel und ordne sie in ein möglichst vollständiges Bild über das Problem der Selbstständigkeit der Substantivvalenz.

Kandidaten für SVG geben kann. Dies bedeutet also, dass die Frage über den Stützverbstatus von sein mindestens bis zur Auswertung weiterer korpusbasierten Daten für mich offenbleibt. 


\section{Schlussfolgerungen und Ausblick}

Da ich die wichtigsten Erkenntnisse und Teilergebnisse meiner Arbeit bereits in einzelnen Unterkapiteln detaillierter erörtert habe (vgl. Kap. 2.4.2; 2.6; 3.3; 4.3), will ich sie im vorliegenden Kapitel nicht einzeln Revue passieren lassen. Vielmehr geht es mir hier darum, sie bündig zu einem Ganzen zusammenzufassen und auch einen Ausblick in Bezug darauf zu bieten, welche offenen Fragen und Möglichkeiten der Vervollkommnung und Fortsetzung der vorliegenden Arbeit ich zurzeit sehe.

Meine theoretische Grundlegung war, dass die Substantivvalenz als ein System sui generis interpretierbar ist, was mehrerlei Folgen hat. Erstens ist anzunehmen, dass nicht nur abgeleitete Substantive über Valenz verfügen können, sondern auch nicht-abgeleitete. Zweitens zeigt zwar die Valenz der abgeleiteten Substantive Ähnlichkeiten mit der Valenz der jeweiligen Basiswörter, sie kann aber nicht regelhaft davon erklärt bzw. abgeleitet werden. Drittens gibt es Besonderheiten der Substantivvalenz, die nur ihr eigen sind.

Was den ersten und zweiten Punkt betrifft, konnte durch die korpusanalytischen Untersuchungen des vorangehenden Kapitels - meines Ermessens - überzeugend erwiesen werden, dass auch die nicht-abgeleiteten Substantive vielfältige Argumentrealisierungen aufweisen und sich ihre Komplemente in ihren formalen, morpho-syntaktischen Merkmalen nicht von denen der deverbalen und deadjektivischen Substantive unterscheiden. Die in Kap. 4 durchgeführte Analyse könnte zwar noch im Falle der abgeleiteten Substantive durch den systematischen Vergleich ihrer Valenzeigenschaften mit denen ihrer Basiswörter ergänzt werden, aber aus der Sicht der Frage nach der Selbstständigkeit der Substantivvalenz halte ich es für viel wichtiger, dass die Analysen im Valenzverhalten der Substantive Parallelitäten zwischen den drei Gruppen gezeigt haben, was - meines Erachtens - mit den oben erwähnten Besonderheiten der Substantivvalenz erklärt werden können. Die korpusanalytischen Untersuchungen haben auch bezüglich solcher Besonderheiten zahlreich Daten geliefert, von denen die Vielfältigkeit der Realisierungsformen der Komplemente und Möglichkeiten der doppelten Komplementrealisierung auf Phrasen- und Satzebene in SVG-Belegen zu den wichtigsten gehören.

Das in Kap. 2.6 entworfene multidimensionale Konzept zur Unterscheidung von Substantivkomplementen und -supplementen hat sich bei der korpusbasierten Analyse der sechs ausgesuchten Substantive als in die Praxis umsetzbar erwiesen und dessen Kriterium der Argumenthaftigkeit konnte durch die Etablierung eines Quasiarguments A' präzisiert werden. Wie in Kap. 4.3 schon erörtert, kann im Falle von A' auf der logisch-semantischen Seite ein 
stufenweiser Übergang von einem absolut peripheren Quasiargument zu einem weniger peripheren angenommen werden, das mit einem der jeweiligen echten Argumente des betreffenden Substantivs Ähnlichkeiten aufweist. Die Wichtigkeit der multidimensionalen Betrachtung der Substantivkomplemente und -supplemente sehe ich auch dadurch bestätigt, dass sich die gerade erwähnte skalare Art von $A^{6}$ auch in formalen Aspekten der Argumentrealisierung widerspiegelt.

Aus der Sicht der vorliegenden Arbeit betrachte ich als wichtigstes Resultat dessen, dass in die korpusbasierte Untersuchung der Substantive auch SVG-Belege miteinbezogen wurden, dass durch den (bedeutungsabhängigen) Vergleich der Komplementrealisierungen der Substantive in ihren SVG- und Nicht-SVG-Belegen - meiner Ansicht nach - überzeugend nachgewiesen werden konnte, dass die Realisierungsformen der Komplemente in Nicht-SVGVerwendungen aus denen der SVG-Belege nicht abzuleiten sind. Eine Tatsache, die - in meinen Augen - wiederum die Eigenständigkeit der Substantivvalenz untermauert. Im Zusammenhang mit der Auswertung der Belegdaten betreffend die SVG-Konstruktionen finde ich noch interessant, dass sich die ermittelten SVG in Bezug auf die Merkmale des Artikelgebrauchs vor dem Substantiv im SVG und auch bezüglich dessen Attribuierbarkeit in den meisten Fällen anders verhalten, als in der Fachliteratur generell angenommen (vgl. Kap. 4.2 und 4.3).

Schließlich muss hervorgehoben werden, dass die korpusbasierte Beschreibung der (Substantiv)valenz, wie auf dieses Problem oft auch in der Forschung hingewiesen wird, sehr zeitaufwendig ist. Ich finde es interessant und wichtig, in Zukunft eingehender zu untersuchen, wieweit der mühsame Arbeitsprozess der Eruierung der Valenzstrukturen von valenten Substantiven durch den Einsatz von automatisierten Korpusanalyseverfahren wie die Kookkurrenzanalyse in COSMAS II effektiver gemacht werden könnte. Dazu finde ich den ersten Grundstein in meiner Arbeit gelegt: Die hier praktizierte Methode könnte um das gerade erwähnte automatisierte Verfahren der Kookkurrenzanalyse ergänzt werden: Die schon ermittelten Analysedaten würden mit den so neu dazugewonnen verglichen, um herausfinden zu können, wie diese automatisierte Analysemethode am meisten zur Beschreibung der Substantivvalenz und darunter auch von SVG-Konstruktionen beitragen kann. Dies wäre auch deswegen von Belang, weil es ebenfalls zu meinen Zielsetzungen gehört, meine Analysen auf weitere abgeleitete und nicht-abgeleitete Substantive auszuweiten, wobei die Erhöhung der Anzahl der zu untersuchenden Belege die Optimierung des Analyseprozesses noch bedeutender macht. 


\section{Abkürzungsverzeichnis}

A - Argument

Akk. - Akkusativ

Areal. - Argumentrealisierung

Artikelw. - Artikelwort

attr. Adj. - attributives Adjektiv

Bed. - Bedeutung

Beisp. - Beispiel

Dat. - Dativ

dekl. - dekliniert

Demonstrativpr. - Demonstrativpronomen

etw. - etwas

FVG - Funktionsverbgefüge

Gen. - Genitiv

Indefpr. - Indefinitpronomen

Infsatz - Infinitivsatz

jd. - jemand

Komp. - Kompositum

Kreal. - Komplementrealisierung

Nom. - Nominativ

NS - Nebensatz

Objsatz - Objektsatz

Poss. - Possessiv

Posspr. - Possessivpronomen

Präpattr. - Präpositionalattribut

Pronadv. - Pronominaladverb

qual. - qualitativ

quant. - quantitativ

Real. - Realisierung

Relativpr. - Relativpronomen

sächs. Gen. - sächsischer Genitiv

Subj. - Subjekt

Subjsatz - Subjektsatz 
SV - Stützverb

SVG - Stützverbgefüge

Tab. - Tabelle 


\section{Literaturverzeichnis}

\subsection{Quellen}

DeReKo = Das Deutsche Referenzkorpus. Abgefragt über COSMAS II $\mathrm{web}_{\mathrm{we}}$. https://cosmas2.idsmannheim.de/cosmas2-web/

\subsection{Sekundärliteratur}

Askedal, John Ole (2003): Das Valenz- und Dependenzkonzept bei Lucien Tesnière. In: Ágel, Vilmos / Eichinger, Ludwig M. / Eroms, Hans-Werner / Hellwig, Peter et al. (Hrsg.): Dependenz und Valenz. 1. Halbband. Berlin/ New York: de Gruyter. (= Handbücher zur Sprach- und Kommunikationswissenschaft Bd. 25.1), 80-99.

Ágel, Vilmos (1988): Überlegungen zur Theorie und Methode der historisch-synchronen

Valenzsyntax und Valenzlexikographie. Mit einem Valenzlexikon $\mathrm{zu}$ den „Denkwürdigkeiten der Helene Kottanerin (1439-1440)“. Tübingen: Niemeyer. (= Lexicographica Series Maior 25).

Ágel, Vilmos (1996): Finites Substantiv. In: Zeitschrift für germanistische Linguistik 24 (1996), 16-57.

Ágel, Vilmos (2000): Valenztheorie. Tübingen: Narr. (Narr Studienbücher).

Ágel, Vilmos (2003): Wort- und Ausdrucksvalenz(träger). In: Cornell, Alan/Fischer,

Klaus / Roe, Ian F.: Valency in Practice / Valenz in der Praxis. Oxford u.a.: Lang. (= German Linguistic and Cultural Studies 10), 17-36.

Ágel, Vilmos (2004): Phraseologismus als (valenz)syntaktischer Normalfall. In: Steyer, Kathrin (Hrsg.): Wortverbindungen - mehr oder weniger fest. Berlin/New York: de Gruyter. (= Institut für Deutsche Sprache 2003), 65-86.

Ágel, Vilmos / Fischer, Klaus (2010): 50 Jahre Valenztheorie und Dependenzgrammatik. In Zeitschrift für germanistische Linguistik Jg. 38, Heft 2, 249-290.

Bassola, Peter (1998): Arbeitsbericht: Das Projekt „Erstellung eines deutsch-ungarischen Substantivvalenzwörterbuches“. In: Bresson, Daniel / Kubczak, Jacqueline (Hrsg.) (1998): Abstrakte Nomina. Vorarbeiten $\mathrm{zu}$ ihrer Erfassung in einem zweisprachigen syntagmatischen Wörterbuch. Tübingen: Narr. (= Studien zur deutschen Sprache 10), 121144.

Bassola, Péter (2009): Stellung der Komplemente des prädikativen Substantivs. In: Di Meola, Claudio / Gaeta, Livio / Hornung, Antonie / Rega, Lorenza (Hrsg.): Perspektiven Drei. 
Akten der 3. Tagung „Deutsche Sprachwissenschaft in Italien“. Rom, 14.-16. Februar 2008. Frankfurt/Main: Peter Lang, 79-89.

Bassola, Péter (2014): Nominale Satelliten an der Leine. Nominalphrasen-, Satz- und Textbereich. In: Bassola, Péter / Drewnowska-Vargáné, Ewa / Kispál, Tamás / Németh, János / Scheibl, György (Hrsg.) (2014): Zugänge zum Text. Frankfurt/Main: Peter Lang, (= Szegediner Schriften zur germanistischen Linguistik 3), 17-34.

Bassola, Péter (2015): Verben und valente Nomina. In: Dominguez Vazquez, Maria José / Eichinger, Ludwig M. (Hrsg.): Valenz im Fokus: Grammatische und lexikographische Studien. Festschrift für Jacqueline Kubczak. Mannheim: Institut für Deutsche Sprache, 1530. Online verfügbar unter: https://ids-pub.bsz-bw.de/files/3539/FestschriftKubczak_Text.pdf(zuletzt gesehen am 17.12.2019).

Bassola, Péter / Bernáth, Csilla (1998): Realisierung der Valenzstruktur von deutschen und ungarischen deverbalen Substantiven. In: Bassola, Péter (Hrsg.) (1998): Beiträge zur Nominalphrasensyntax. Szeged: JATE. (= Acta Germanica 6), 173-196.

Bassola et al. (2003) = Bassola, Péter / Hum, Rozália / Kubczak, Jacqueline / Tamássy Bíró, Magda (2003): Deutsch-ungarisches Wörterbuch zur Substantivvalenz. Bd. 1. Szeged: Grimm.

Bassola et al. (2012) = Bassola, Péter / Hum, Rozália / Kubczak, Jacqueline / Tamássy Bíró, Magda (2012): Deutsch-ungarisches Wörterbuch zur Substantivvalenz. Bd. 2. Szeged: Grimm.

Bassola, Peter / Kubczak, Jacqueline / László, Sarolta (2003): Zweisprachige Substantivvalenz in Theorie und Praxis. In: Speranţa, Stănescu (Hrsg.): Die Valenztheorie. Bestandsaufnahme und Perspektiven. Dokumentation einer wissenschaftlichen Tagung in Sibiu/Hermannstadt im Februar 2002. Frankfurt am Main: Peter Lang, 179-191.

Bassola, Péter / László, Sarolta (1996): Wörterbücher zur Substantivvalenz aus heutiger Sicht. In: Bassola, Péter / László, Sarolta / Tamássy Bíró Magda (2005): Theoretische und praktische Überlegungen zu einem kontrastiven Substantivvalenzwörterbuch. Szeged: Grimm, 26-61.

Bassola, Péter / László, Sarolta / Tamássy Bíró Magda (2005): Theoretische und praktische Überlegungen zu einem kontrastiven Substantivvalenzwörterbuch. Szeged: Grimm.

Blume, Kerstin (2000): Markierte Valenzen im Sprachvergleich. Lizensierungs- und Linkingbedingungen. Tübingen: Niemeyer. 
Bresson, Daniel / Kubczak, Jacqueline (Hrsg.) (1998): Abstrakte Nomina. Vorarbeiten zu ihrer Erfassung in einem zweisprachigen syntagmatischen Wörterbuch. Tübingen: Narr. (= Studien zur deutschen Sprache 10).

Bruker, Astrid (2013): Funktionsverbgefüge im Deutschen. Computerlexikographische Probleme und Lösungsansätze. Hamburg: Bachelor + Master Publishing.

Bußmann, Hadumod (Hrsg.) (2008): Lexikon der Sprachwissenschaft. Vierte, durchges. und bibl. erg. Auflage unter Mitarbeit von Hartmut Lauffer. Stuttgart: Kröner.

DHW $=$ Duden. Das Herkunftswörterbuch. 5., neu bearb. Aufl. Hrsg. von der Dudenredaktion. Berlin: Duden. 2014. (= Duden 7).

DUW = Duden: Deutsches Universalwörterbuch (2003). Hrsg. vom Wissenschaftlichen Rat der Dudenredaktion. 5., überarb. Aufl. Mannheim, Leipzig, Wien \& Zürich: Duden.

Dürr, Michael / Schlobinski, Peter (2006): Deskriptive Linguistik. Grundlagen und Methoden. Vandenhoeck \& Ruprecht.

Dürscheid, Christa (2000): Syntax. Wiesbaden: Westdeutscher Verlag.

Eisenberg, Peter (2006): Grundriss der deutschen Grammatik. Band 2: Der Satz. 3., durchges. Aufl. Stuttgart / Weimar: Metzler.

Engel, Ulrich (1996): Tesnière mißverstanden. In: Gréciano, Gertrud / Schumacher, Helmut (Hrsg.): Lucien Tesnière - Syntaxe structurale et opérationis mentales. Akten des deutschfranzösischen Kolloquiums anläßlich der 100. Wiederkehr seines Geburtstages, Straßbourg 1993. Tübingen: Niemeyer. (= Linguistische Arbeiten 348), 53-61.

Engel, Ulrich (2009a): Deutsche Grammatik. Neubearbeitung. 2. durchges. Aufl. München: Iudicium.

Engel, Ulrich (2009b): Syntax der deutschen Gegenwartssprache. 4., völlig neu bearb. Aufl. Berlin: Erich Schmidt. (= Grundlagen der Germanistik 22).

Engelen, Bernhard (1968): Zum System der Funktionsverbgefüge. In: Wirkendes Wort 18, 289-303.

Eroms, Hans-Werner (2000): Syntax der deutschen Sprache. Berlin / New York: de Gruyter. Fazly, Afsaneh / Stevenson, Suzanne (2005): Automatic Acquisition of Knowledge About Multiword Predicates. In: Proceedings of PACLIC 19, the 19th Asia-Pacific Conference on Language, Information and Computation. Online verfügbar unter: http://www.aclweb.org/anthology/Y05-1003 (zuletzt gesehen am 16. 1. 2020).

Fischer, Klaus (2001): Noch immer: Ergänzungen und Angaben. In: Sprachwissenschaft, Jg. 26, Heft 3, 239-268. 
GDS = Zifonun, Gisela / Hoffmann, Ludger / Strecker, Bruno (1997): Grammatik der deutschen Sprache. Bde. 1-3. Berlin / New York: de Gruyter. (Schriften des Instituts für Deutsche Sprache 7,1-7,3).

grammis. Grammatisches Informationssystem. Leibniz-Institut für deutsche Sprache. https://grammis.ids-mannheim.de (zuletzt gesehen am 31. 5. 2020).

Heine, Antje (2006): Funktionsverbgefüge in System, Text und korpusbasierter (Lerner-)Lexikographie. Frankfurt am Main: Peter Lang. (= Finnische Beiträge zur Germanistik 18).

Heine, Antje (2008): Funktionsverbgefüge richtig verstehen und verwenden. Frankfurt am Main: Peter Lang. (= Finnische Beiträge zur Germanistik 23).

Helbig, Gerhard (1979): Probleme der Beschreibung von Funktionsverbgefügen im Deutschen. In: Deutsch als Fremdsprache, Jg. 16 (1979), Heft 5, 273-285.

Helbig, Gerhard (1986): Zu umstrittenen Fragen der substantivischen Valenz. In: Deutsch als Fremdsprache. Jg. 23, Heft 4, (1986), 200-207.

Helbig, Gerhard (1992): Probleme der Valenz- und Kasustheorie. Tübingen: Niemeyer.

Helbig, Gerhard / Buscha, Joachim (2018): Deutsche Grammatik. Ein Handbuch für den Ausländerunterricht. Erstausgabe erschienen 2001 bei der Langenscheidt KG, München. Stuttgart: Klett.

Heringer, Hans-Jürgen (1968): Die Opposition von "kommen" und "bringen" als Funktionsverben. Untersuchungen zur grammatischen Wertigkeit und Aktionsart. Düsseldorf: Schwann. (= Sprache der Gegenwart 3).

Heringer, Hans-Jürgen (1986): The Verb and Its Semantic Power: Association as a Basis for Valence Theory. In: Journal of Semantics 4, 79-99

Hessky, Regina (1987): Phraseologie. Linguistische Grundfragen und kontrastives Modell deutsch $\rightarrow$ ungarisch. Tübingen: Niemeyer. (= Reihe Germanistische Linguistik 77).

Hessky, Regina (1988): Verbale Phraseologismen: valenzkonform oder nicht? In: Mrazović, Pavica/ Teubert, Wolfgang (Hrsg.): Valenzen im Kontrast. Ulrich Engel zum 60. Geburtstag. Heidelberg: Groos, 139-149.

Hölzner, Matthias (2007): Substantivvalenz. Korpusgestützte Untersuchungen zu Argumentrealisierungen deutscher Substantive. Tübingen: Niemeyer.

Jacobs, Joachim (1994): Kontra Valenz. Trier: WVT Wissenschaftlicher Verlag. (= Fokus; Bd. 12).

Jacobs, Joachim (2003): Die Problematik der Valenzebenen. In: Ágel, Vilmos / Eichinger, Ludwig M. / Eroms, Hans-Werner /Hellwig, Peter et al. (Hrsg.): Dependenz und Valenz. 1. 
Halbband. Berlin/New York: de Gruyter. (= Handbücher zur Sprach- und Kommunikationswissenschaft Bd. 25.1), 378-399.

Kamber, Alain (2008): Funktionsverbgefüge - empirisch. Eine korpusbasierte Untersuchung zu den nominalen Prädikaten des Deutschen. Tübingen: Niemeyer (= Reihe Germanistische Linguistik 281).

Kluge $=$ Kluge - Etymologisches Wörterbuch der deutschen Sprache. Bearb. von Elmar Seebold. 25., durchges. und erw. Aufl. Berlin / Boston: de Gruyter. 2011.

Kolde, Gottfried (1979): Zur Valenz fester verbaler Syntagmen. In: Löffler, Heinrich / Pestalozzi, Karl / Stern, Martin (Hrsg.): Standard und Dialekt. Studien zur gesprochenen und geschriebenen Gegenwartssprache. Festschrift für Heinz Rupp zum 60. Geburtstag. Bern: Francke, 73-87.

Krenn, Brigitte (2004): Manual zur Identifikation von Funktionsverbgefügen und figurativen Ausdrücken in PP-Verb-Listen. Online verfügbar unter: http://collocations.de/guidelines/Krenn2000-Guidelines.pdf (zuletzt gesehen am 15. 12. 2019).

Kubczak, Jacqueline (2006): Kleines Plädoyer für eine Verschiebung der Grenze von „Komplement“ bei der Valenz des Substantivs. In: Breindl, Eva / Gunkel, Lutz / Strecker, Bruno (Hrsg.): Grammatische Untersuchungen. Analysen und Reflexionen. Gisela Zifonun zum 60. Geburtstag. Tübingen: Narr. (= Studien zur deutschen Sprache Bd. 36), 97-109.

Kubczak, Jacqueline / Costantino, Sylvie (1998): Exemplarische Untersuchungen für ein syntagmatisches Wörterbuch. In: Bresson, Daniel / Kubczak, Jacqueline (Hrsg.) (1998): Abstrakte Nomina. Vorarbeiten $\mathrm{zu}$ ihrer Erfassung in einem zweisprachigen syntagmatischen Wörterbuch. Tübingen: Narr. (= Studien zur deutschen Sprache 10), 11119.

Kubczak, Jacqueline / Schumacher, Helmut (1998): Verbvalenz - Nominalvalenz. In: Bresson, Daniel / Kubczak, Jacqueline (Hrsg.) (1998): Abstrakte Nomina. Vorarbeiten zu ihrer Erfassung in einem zweisprachigen syntagmatischen Wörterbuch. Tübingen: Narr. (= Studien zur deutschen Sprache 10), 273-286.

Langer, Stefan (2004): A linguistic test battery for support verb constructions. In: Lingvisticae Investigationes 27, 171-184.

László, Sarolta (1988): Mikroebene. In: Mrazović, Pavica / Teubert, Wolfgang (Hrsg.) (1988):

Valenzen im Kontrast. Ulrich Engel zum 60. Geburtstag. Heidelberg: Groos, 218-233.

Lehmann, Sieghardt (1983): $\mathrm{Zu}$ einigen Problemen bei der Beschreibung von Funktionsverbgefügen. In: Zielsprache Deutsch 14, 42-46. 
Polenz, Peter von (1963): Funktionsverben im heutigen Deutsch. Sprache in der rationalisierten Welt. Düsseldorf: Pädagogischer Verlag Schwann.

Polenz, Peter von (1987): Funktionsverben, Funktionsverbgefüge und Verwandtes. Vorschläge zur satzsemantischen Lexikographie. In: Zeitschrift für Germanistische Linguistik 15, 169189.

Polenz, Peter von (1988): Deutsche Satzsemantik. Grundbegriffe des Zwischen-den-ZeilenLesens. 2., durchges. Aufl. Berlin / New York: de Gruyter. (= Sammlung Göschen 2226).

Sandberg, Bengt (1979): Zur Repräsentation, Besetzung und Funktion einiger zentraler Leerstellen bei Substantiven. Kungsbacka: Kungsbacka Tryckeri AB. (= Acta Universitatis Gothoburgensis. Göteborger Germanistische Forschungen 18).

Schierholz, Stefan (2004): Valenzvererbung? Präpositionalattributskonstruktionen und ihre Herleitung. In: Stănescu, Speranța (Hrsg.): Die Valenztheorie. Bestandsaufnahme und Perspektiven. Dokumentation einer wissenschaftlichen Tagung in Sibiu / Hermannstadt im Februar 2002. Frankfurt a. M.: Peter Lang, 79-96.

Schumacher, Helmut (1987): Valenzbibliographie. Mannheim: IDS.

Sommerfeldt, Karl-Ernst (1980): Zur Valenz von Funktionsverbfügungen. In: Deutsch als Fremdsprache, Jg. 17 (1980), Heft 5, 294-297.

Sommerfeldt, Karl-Ernst / Schreiber, Herbert (1977): Wörterbuch zur Valenz und Distribution der Substantive. Leipzig: VEB Bibliographisches Institut.

Stein, Achim (1996): Zur Valenz komplexer Prädikate am Beispiel deutscher und französischer Verb-Substantiv-Verbindungen. In: Gréciano, Gertrud / Schumacher, Helmut (Hrsg.): Lucien Tesnière - Syntaxe structurale et opérationis mentales. Akten des deutschfranzösischen Kolloquiums anläßlich der 100. Wiederkehr seines Geburtstages, Straßbourg 1993. Tübingen: Niemeyer. (= Linguistische Arbeiten 348), 191-201.

Stein, Achim (1998): Verb-Substantiv-Verbindungen mit mener, conduire, diriger und deutsche Entsprechungen. In: Bresson, Daniel/Kubczak, Jacqueline (Hrsg.): Abstrakte Nomina. Vorarbeiten $\mathrm{zu}$ ihrer Erfassung in einem zweisprachigen syntagmatischen Wörterbuch. Tübingen: Narr. (= Studien zur deutschen Sprache 10), 209-226.

Storrer, Angelika (2003): Ergänzungen und Angaben. In: Ágel, Vilmos / Eichinger, Ludwig M. / Eroms, Hans-Werner / Hellwig, Peter et al. (Hrsg.): Dependenz und Valenz. 1. Halbband. Berlin/New York: de Gruyter. (= Handbücher zur Sprach- und Kommunikationswissenschaft Bd. 25.1), 764-780.

Storrer, Angelika (2006): Zum Status der nominalen Komponenten in Nominalisierungsverbgefügen. In: Breindl, Eva / Lutz, Gunkel / Strecker, Bruno (Hrsg.): 
Grammatische Untersuchungen. Analysen und Reflexionen. Gisela Zifonun zum 60. Geburtstag. Tübingen: Narr. (= Studien zur deutschen Sprache 36), 275-293. Online verfügbar unter: https://ids-pub.bszbw.de/frontdoor/deliver/index/docId/7195/file/Storrer_Zum_Status_der_nominalen_Komp onenten_2006.pdf (zuletzt gesehen am 16. 1. 2020).

Storrer, Angelika (2007): Corpus-based investigations on German support verb constructions. In: Fellbaum, Christiane (Hrsg.): Idioms and Collocations. Corpus-based Linguistic and Lexicographic Studies. London: Continuum, 164-187.

Stumpf, Sören (2015a): Formelhafte (Ir-)Regularitäten. Korpuslinguistische Befunde und sprachtheoretische Überlegungen. Frankfurt: Peter Lang. (= Sprache - System und Tätigkeit, 67). Online verfügbar unter: https://www.peterlang.com/view/title/18735 (zuletzt gesehen am 8. 11. 2019).

Stumpf, Sören (2015b): Phraseologie und Valenztheorie. Status quo, Forschungsprobleme und (korpusanalytische) Perspektiven. In: Yearbook of Phraseology, 2015 (Volume 6, Issue 1), 3-34. Online verfügbar unter: https://www.degruyter.com/downloadpdf/j/yop.2015.6.issue1/phras-2015-0003/phras-2015-0003.pdf (zuletzt gesehen am 23. 11. 2019).

Tamássy-Bíró, Magda (1997): Obligatorische Ergänzungen in der Nominalphrase? Zur Valenzstruktur deutscher und ungarischer Substantive mit besonderem Hinblick auf die Subklasse des Typs eleje, alja. In: Papiere zur Linguistik, Nr. 57 (1997), Heft 2, 135-150.

Tamássy-Bíró, Magda (1998a): Valente Substantive mit Aktanten auf Mikroebene. Valente Substantivsubklassen im Deutschen und Ungarischen. In: Sprachwissenschaft, Bd. 23 (1998), Heft 3, 317-339.

Tamássy-Bíró, Magda (1998b): Einige Gedanken zur Valenz der ungarischen Substantive. In: Bassola, Péter (Hrsg.) (1998): Beiträge zur Nominalphrasensyntax. Szeged: JATE. (=Acta Germanica 6), 143-172.

Tesnière, Lucien (1980): Grundzüge der strukturalen Syntax. Herausgegeben und übersetzt von Ulrich Engel. Stuttgart: Klett-Cotta.

Teubert, Wolfgang (1979): Valenz des Substantivs. Attributive Ergänzungen und Angaben. Düsseldorf: Schwann. (= Sprache der Gegenwart Bd. 49).

Teubert, Wolfgang (2003): Substantivvalenz. In: Ágel, Vilmos/Eichinger, Ludwig M. / Eroms, Hans-Werner / Hellwig, Peter et al. (Hrsg.): Dependenz und Valenz. 1. Halbband. Berlin/New York: de Gruyter. (= Handbücher zur Sprach- und Kommunikationswissenschaft 25.1), 820-835. 
Torzova, Marina V. (1983): Zur Valenz der Phraseologismen. In: Deutsch als Fremdsprache, Jg. 20 (1983), Heft 5, 283-287.

Uzonyi, Pál (2004): Valenz kontra Subklassenspezifik. In: Czicza, Dániel / Hegedüs, Ildikó / Kappel, Péter et al. (Hrsg.): Wertigkeiten, Geschichten und Kontraste. Festschrift für Péter Bassola zum 60. Geburtstag. Szeged: Grimm, 233-237.

Uzonyi, Pál (2019): Zur höchsten Zahl der Aktanten von verbalen Valenzträgern. In: Drewnowska-Vargáné, Ewa / Kappel, Péter / Modrián-Horváth, Bernadett / Rauzs, Orsolya (Hrsg.): ,vnd der gieng treulich, weislich vnd mëndlich mit den sachen vmb“. Festschrift für Péter Bassola zum 75. Geburtstag. Szeged: Institut für Germanistik, 271-285.

Van Pottelberge, Jeroen (2001): Verbonominale Konstruktionen, Funktionsverbgefüge: vom Sinn und Unsinn eines Untersuchungsgegenstandes. Heidelberg: Winter.

Varnhorn, Beate (1986): Nochmals Ergänzungen und Angaben. Forschungskritik und ein neuer Vorschlag. In: Deutsche Sprache. Zeitschrift für Theorie, Praxis, Dokumentation. 14. Jahrgang (1986), 1-11.

Vater, Heinz (1978): Probleme der Verbvalenz. In: Leuvense Bijdragen 67, 267-308.

Welke, Klaus M.: (1988): Einführung in die Valenz- und Kasustheorie. Leipzig: Bibliographisches Institut.

Welke, Klaus (1994): Valenz und Satzmodelle. In: Thielmann, Werner / Welke, Klaus (Hrsg.): Valenztheorie - Werden und Wirkung. Wilhelm Bondzio zum 65. Geburtstag. Münster, 227-244. Online verfügbar unter: https://www.linguistik.huberlin.de/de/staff/h1457b23/satzmodelle (zuletzt gesehen am 8. 12. 2019).

Welke, Klaus (2002): Deutsche Syntax funktional. Perspektiviertheit syntaktischer Strukturen. Tübingen: Stauffenburg.

Wolf, Norbert Richard (1982): Probleme einer Valenzgrammatik des Deutschen. Innsbruck. (= Mitteilungen aus dem Institut für Sprachwissenschaft der Universität Innsbruck; 3). Online verfügbar unter: https://opus.bibliothek.uniwuerzburg.de/frontdoor/index/index/docId/3604 (zuletzt gesehen am 25. 4. 2020).

\subsection{Zitierte bzw. verwendete eigene Publikationen}

Sántáné-Túri, Ágnes (2014): Substantivvalenz auf Textebene. Vorüberlegungen zu einer empirischen Untersuchung. In: Bassola, Péter / Drewnowska-Vargáné, Ewa / Kispál, Tamás / Németh, János / Scheibl, György (Hrsg.) (2014): Zugänge zum Text. 
Frankfurt/Main: Peter Lang, (= Szegediner Schriften zur germanistischen Linguistik 3), $381-411$.

Sántáné-Túri, Ágnes (2016): Cosmas $\mathrm{II}_{\mathrm{web}}$ im Dienste der Substantivvalenzlexikographie. In: Argumentum. The peer-reviewed open access journal of the Doctoral School of Linguistics, University of Debrecen, 12, S. 87-99. Online verfügbar unter: http://argumentum.unideb.hu/2016-anyagok/sondernummer/05_santaneta.pdf （zuletzt gesehen am 18. 1.2020).

Sántáné-Túri, Ágnes (2017): Stützverbgefüge - die Arbeit am „Deutsch-ungarischen Wörterbuch zur Substantivvalenz" als Vermittler zwischen Theorie und Praxis. In: Jahrbuch der ungarischen Germanistik 2016, 173-183.

Sántáné-Túri, Ágnes (2019): Substantivvalenz in und außerhalb von Stützverbgefügen Überlegungen zu einer korpusbasierten Untersuchung. In: Drewnowska-Vargáné, Ewa / Kappel, Péter / Modrián-Horváth, Bernadett / Rauzs, Orsolya (Hrsg.): „vnd der gieng treulich, weislich vnd mëndlich mit den sachen vmb“. Festschrift für Péter Bassola zum 75. Geburtstag. Szeged: Institut für Germanistik, 234-255.

Túri Ágnes (2006): Streitfragen der Substantivvalenztheorie. Diplomarbeit, verteidigt an der Universität Szeged. (Manuskript). 


\section{Anhang}

\subsection{Belege für Übersetzung}

(C) Leibniz-Institut für Deutsche Sprache, Mannheim

COSMAS II-Server, C2API-Version 4.17.7 - 13.02.20

1) $\{\{0\}\}^{229}$

Serie «Zeugen der Vergangenheit» (V): Ein Buch aus dem 16. Jahrhundert

Unter den wertvollen Büchern, die im Bischofszeller Museum ausgestellt sind, befindet sich eine Koran-Übersetzung von Theodor Buchmann, genannt «Bibliander». Damit diese

Übersetzung gedruckt werden konnte, war sogar die Unterstützung Martin Luthers nötig.

* alex thalmann

Die durch rege Handelsbeziehungen mit St.Gallen und Konstanz recht wohlhabende Bevölkerung von Bischofszell war im 16. Jahrhundert den Idealen des jugendfrischen Humanismus sehr zugetan. (A97/AUG.17886 St. Galler Tagblatt, [Tageszeitung], 09.08.1997, Jg. 53. Originalressort: TB-BIZ (Abk.); * alex thalmann: «Bibliander» übersetzte den Koran, [Bericht])

2) $((2 ;$ Gen.; in + Akk.; A2; A4))

Ernst Kunz ist überzeugt: «Diese Kommentierung von Leistung und Verhalten der Schülerinnen und Schülern fördert die Zusammenarbeit von Schule und Elternhaus». Weiter könnten gemeinsame Ziele formuliert werden - sowie (wenn nötig) die Erziehungsberechtigten um entsprechende Unterstüzung angegangen werden. Damit Eltern mit mangelhaften Deutschkenntnissen nicht im Unklaren gelassen werden, ist eine Übersetzung der Beobachtungsbogen in alle «gängigen Fremdsprachen» vorgesehen. Ob bei den Gesprächen die Kinder beigezogen werden, bleibt noch offen. (A98/JUL.47937 St. Galler Tagblatt, [Tageszeitung], 18.07.1998, Jg. 54. Originalressort: TB-OT (Abk.); enrico berchtold: In der Unterstufe kein Zeugnis mehr, [Bericht])

\section{3) $\{\{2$; aus + Dat.; von + Dat.; A3; A1 $\}\}$}

Eine schwache Verteidigung, werden Sie jetzt vielleicht einwenden, welche Chance hat das Theater schon gegen Industrien. Gar keine, die Schönheit und die Wahrheit sind immer schwach und chancenlos, und doch verteidigen sie den Menschen nun schon seit über dreitausend Jahren erfolgreich.

Übersetzung aus dem Bosnischen von Barbara Sax (A99/JUL.50845 St. Galler Tagblatt, [Tageszeitung], 21.07.1999, Jg. 55. Originalressort: TB-KUL (Abk.); Loblied auf das Gesicht, [Bericht])

\section{4) $\{\{2$; Posspr.; Apposition; A2; A` $\}\}$}

liebe St.Galler

Genauer müsste ich - besonders am heutigen Gallustag - schreiben: Liebe heilige Gallerinnen und liebe heilige Galler. Wir haben uns so sehr an das Sankt gewöhnt, dass wir seine Übersetzung - heilig - kaum mehr beachten. Unser Kanton heisst also «Heiliges Gallen». Noch andere Kantone der Schweiz haben (vielen Kantonseinwohnern unbekannt) eine heilige Persönlichkeit zum Patron. (A99/OKT.72653 St. Galler Tagblatt, [Tageszeitung], 16.10.1999, Jg. 55. Originalressort: WV-EXT (Abk.); Liebe St.Gallerinnen, [Bericht])

\footnotetext{
${ }^{229}$ Die unterschiedlichen Klammern stehen für die einzelnen Bedeutungen von Übersetzung: ((Bed. Ia)); \{\{Bed. $\mathrm{Ib}\}\} ;[[$ Bed. II]].
} 
5) $[[0]]$

Organisiert wurde das Ferien(s)pass-Projekt von der öffentlichen Energieberatungsstelle Hinterthurgau/Aadorf. Die Kinder sollten einerseits Spass haben, andererseits aber lernen, wie man die Solarenergie nutzen kann. Sie bekamen lediglich einen Motor, den Antrieb mit der Übersetzung und die Solarzellen zur Verfügung gestellt. Fachliche Hilfe erhielten sie von den Leitern des Projektes. Herausgekommen sind die verschiedensten Vehikel: Von Fahrzeugen, die nur mit dem Nötigsten ausgerüstet waren, über solche, die auch ästhetischen Ansprüchen genügten, bis hin zum richtigen Rennauto. (A01/AUG.19706 St. Galler Tagblatt, [Tageszeitung], 03.08.2001, Jg. 57. Originalressort: TB-THG1 (Abk.); Hinterthurgauer Kinder bauten Solar-Modellautos, [Bericht])

6) $\{\{0\}\}$

Er war der Urgroßvater des Autors.

Bitter-ironische Anspielung

Hier setzt die nun vorliegende Neuübersetzung von Giò Waeckerlin Induni an, ihr liegt erstmals der in Italien vor zwei Jahren veröffentlichte vollständige Text zu Grunde. »Il Gattopardo« wird mit »Die Pardelkatze« übersetzt, ein Synonym für den Ozelot, eine kleine Raubkatze, die wegen ihres wunderbaren Fells fast ausgerottet wurde. Der Leopard ist dagegen ein mächtiges Tier. (NUN04/JUL.01128 Nürnberger Nachrichten, 12.07.2004; Vom Scheitern eines Standes - Lampedusas »Il Gattopardo« liegt in einer deutschen Neuübersetzung vor [Ausführliche Zitierung nicht verfügbar])

7) $\{\{1$; Gen.; A2 $\}\}$

Verbürgt ist es nicht, aber vielleicht begannen sie ja mit dem Lied «Meitli hürat nie!»: die Begeisterung für Liedtexte, die Forschertätigkeit nach Klangfarben und alten Musikinstrumenten, die Liebe zu fast oder schon längst vergessenen musikalischen Traditionen und die Ahnung, dass in dem Lied vom Meitli der Sprengsatz von Jahrhunderten lag. Die Gruppe hiess damals noch Zupfgyge und stand am Lenzburger Folkfestival auf der Bühne. Das Lied war eine Übersetzung eines Songs des zeitgenössischen englischen Liedermachers Leon Rosselsen. Urs Klauser am 5-String- Banjo, Sämi Forrer an Geige und Bass, Christof Koch und Hans Fässler - damals gerade in der RS - an der Gitarre. Dessen persönlicher Beitrag war der von ihm komponierte «Swiss Army Talking Blues»; die Leute jubelten, klatschten. (A08/JUN.01259 St. Galler Tagblatt, [Tageszeitung], 05.06.2008, Nr. 129, S. 46. - Sachgebiet: Regionales / Regionales, Originalressort: SG-Regionkultur; b.schmid: Lauter Pfeifen)

8) [[1; attr-es Adj.; A1]]

Die Töne streichen dem Halbrund der rückseitigen Wand nach, statt in den Raum hineinzuklingen.

Zahlreiche Mängel

Zudem handelt es sich um eine der letzten Orgeln mit elektrischer Übersetzung. Von den Tasten gibt es keine direkte, mechanische Verbindung zu den Pfeifen. Vielmehr betätigt der Tastendruck ein elektrisches Relais, das sich als störungsanfällig erweist. (A08/DEZ.07057 St. Galler Tagblatt, [Tageszeitung], 23.12.2008, Nr. 300, S. 33. - Sachgebiet: Regionales, Originalressort: GO-Gossau; r.bolt: Abschied vom faden Orgelsound)

9) $\{\{2$; Gen.; Komp.; A1; A2 $\}\}$

In Wandsbek arbeitete Voß nicht nur mit Claudius am Wandsbeker Boten, er begann auch mit der Übersetzung von Homers Epos Odyssee, in dem der griechische Dichter die Irrfahrten des 
Odysseus auf seiner Heimreise vom Krieg um Troja ins heimische Ithaka beschreibt.1778 wurde Johann Heinrich Voß Rektor der Lateinschule in Otterndorf, heute im niedersächsischen Landkreis Cuxhaven, dort wo die Elbe sich in einer Trichtermündung zur Nordsee öffnet. Hier schloss er die Übersetzung der Odyssee ab, die 1781 veröffentlicht wurde. Sie wird von ihrem sprachschöpferischen Einfluss auf die deutsche Sprache her mit Martin Luthers Bibelübersetzung verglichen. Doch Voß blieb nur wenige Jahre in Otterndorf: Es gab gesundheitliche Probleme. Das Grundwasser ist wegen der nahen Nordsee brackig. (NKU12/JUN.01260 Nordkurier, 04.06.2012, Von der Müritz an die Wandse)

10) $((1$; von + Dat.; A2))

Gleichzeitig passt die bereits einmal im Zürcher Kunstraum R22 gezeigte Arbeit ausgesprochen gut zum Countdown im Nextex, der mit Barbara Signer in die dritte Runde steigt.

Fulminanter Einstieg

Der bildhaften Übersetzung von «Traumbildern» forscht sie seit Beginn ihrer künstlerischen Tätigkeit nach. Diese Zeit ist noch jung; im Kunstmuseum zeigte sie anlässlich des Heimspiels 2006 ihre erste Arbeit im öffentlichen Raum. Es waren auf Video aufgenommene Bildsequenzen, welche sie aus Filmklassikern direkt vom Bildschirm aufnahm und neu zusammensetzte. (A10/MAR.04382 St. Galler Tagblatt, 13.03.2010, Nr. 60, S. 46. Sachgebiet: Regionales / Kultur, Originalressort: SG-st.galler kultur; a.stock: Zwischen Tag und Traum)

\section{1) $\{\{1$; Gen.; A2 $\}\}$}

Dass der Fahrer aber bereits auf der Piste blau ist, ist schon eher ungewöhnlich. Vor allem wenn er in diesem Zustand auch noch die Silbermedaille an der WM gewinnt.

Sehr erstaunt waren deshalb die englischsprachigen Journalisten in Garmisch-Partenkirchen, als sie in der Übersetzung des Pressebulletins folgende Aussagen von Christof Innerhofer, dem Zweiten der Super-Kombination, lasen: «After the downhill I was wondering how I could endure the slalom because I was so drunk.» Angetrunken den richtigen Weg durch die Slalomstangen zu finden, das ist natürlich eine grosse Herausforderung.

Der Südtiroler hatte tatsächlich davon gesprochen, nach der Abfahrt «blau» gewesen zu sein. Allerdings meinte er damit nicht, dass er seinen Sieg schon vor dem Start gefeiert hatte.

(A11/FEB.05624 St. Galler Tagblatt, 17.02.2011, Nr. 40, S. 21. - Sachgebiet: Sport, Originalressort: sport; t.werner: Après-Ski)

\section{2) $((0))$}

Doch die Serie hat in ihren Feinheiten trotzdem Witz und Charme. Besonders Teds bester Freund Barney, der in jeder Folge erneut versucht, seinen Kumpel zu verkuppeln, schmückt das Format mit Männerweisheiten wie: „Der Bart muss weg, er passt nicht zum Anzug.“ Dass der Kumpeltyp Ted nie einen Anzug trägt, entwickelt sich dabei zum Dauerwitz. „Suit up!“‘ lautet der kurze Spruch im Original - und offenbart damit ein Grundproblem der Sitcoms im deutschen Fernsehen: die Übersetzung. So sehr sich Übersetzer und Synchronsprecher auch bemühen, der Humor des Originals geht verloren. Gerade die kurzen Dauerbrenner zwischen Ted und Barney büßen im Deutschen an Tempo ein. (HAZ08/SEP.02588 Hannoversche Allgemeine, 13.09.2008, S. 7; \&\#8222;Friends\&\#8220; 2.0 [Ausführliche Zitierung nicht verfügbar])

13) $\{\{1$; sächs. Gen.; A1 $\}\}$ Andererseits weiß der Chronist genau, dass Eccu bei seinem letzten Skiausflug nicht nur „angetrunken“, sondern auch ,in selbstmitleidige Gedanken versunken“ ist, als er im Eis 
einbricht und ertrinkt. Und von solchen erzähllogischen Brüchen gibt's mehrere in diesem Roman, und das sind nicht die einzigen Schwachstellen: Viele Episoden, etwa über die diversen in Helsingfors gastierenden Musiker wirken in ihrer Breitwandschilderung ohne echten Zusammenhang. Speziell für deutsche Leser kommt hinzu, dass der Autor allerlei Kenntnisse in finnischer Geschichte, Politik, Geografie und Eigenart voraussetzt - auch ist

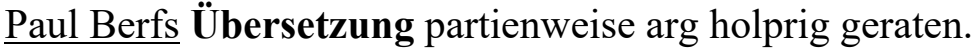

Dennoch: ein tiefgründiges, passioniertes Buch, das seinen Vorsatz, die Menschen jener Zeit lebendig in Erinnerung zu rufen, sehr wohl erfüllt. Und das bisweilen wirkt, als sei es ein Protokoll unserer Zeit. (HAZ08/NOV.04424 Hannoversche Allgemeine, 25.11.2008, S. 7; Die Helsinki-Saga [Ausführliche Zitierung nicht verfügbar])

14) $\{\{1$; Gen.; A2 $\}\}$

Im Juni findet ein Tag der offenen Tür statt.

Von Drogenelend erschüttert

Bis zu einem Dutzend ehemaliger Drogenabhängige werden ab Ende April nach ihrem Drogenentzug ins Pachtgebäude des Klosters Maria der Engel einziehen und maximal ein Jahr hier bleiben, um «sich wieder zu gewinnen», so die wörtliche Übersetzung der Rekuperation. «Die Fazenda ist eine Selbsthilfegruppe, keine Psychotherapie», macht Christian Heim klar. Darum zahlt keine Krankenkasse. (A12/APR.01473 St. Galler Tagblatt, 04.04.2012, Nr. 80, S. 33. - Sachgebiet: Regionales, Originalressort: TT-Aufschlag; h.kugler: Die Fazenda macht die Tore auf)

15) $\{\{2$; attr. Adj.; Gen.; A4; A2 $\}\}$

Friedenspreisträger Liao Yiwu hat sich für Fliehen entschieden: Er lebt seit einem Jahr in Deutschland im Exil. Sein früheres Leben mit Umerziehungsmassnahmen, Verfolgung und Gefängnis verarbeitet er in seinen Büchern. In diesen Tagen erscheint die deutsche Übersetzung seines Werkes «Die Kugel und das Opium» über die Massaker auf dem Platz des Himmlischen Friedens 1989. Alle seine Bücher sind in China verboten.

Die beiden Autoren haben somit auch die Wahl getroffen, ob sie in der Heimat publiziert werden oder nicht. (A12/OKT.05291 St. Galler Tagblatt, 13.10.2012, Nr. 240, S. 2. Originalressort: meinung; Die Wahl chinesischer Autoren: Schweigen oder Fliehen)

16) $\{\{1$; von + Dat.; A1 $\}\}$

Gewissermassen als Gegenleistung dafür, dass sich ein namenloser jüngerer Mann seine Biographie anhört und sie niederschreibt, unterweist Salagnon seinen Biographen in der Kunst des Beobachtens und des Zeichnens.

TV-Bilder vom Irak-Krieg der 90er-Jahre stehen zu Beginn von Jennis 750-Seiten-Roman. Wie stark Bilder vom Krieg auch sein können, wenn sie nur sprachlich vermittelt werden, zeigt dieses Buch, das vor einem Jahr den Prix Goncourt erhalten hat und in einer vortrefflichen Übersetzung von Uli Wittmann vorliegt.

Schlacht minuziös geschildert

Wenn französische Soldaten, Fremdenlegionäre und südvietnamesische Kämpfer schwer beladen durch die Sümpfe Vietnams waten oder verletzte Kameraden unter dem Feuer der gegnerischen kommunistischen Vietminh retten, wenn minuziös geschildert wird, wie Soldaten in Bodenfallen geraten, in denen sie von spitzen Bambusröhren aufgespiesst werden, dann muss man den Roman zur Seite legen, bevor man weiterlesen kann. (A13/JAN.11043 St. Galler Tagblatt, 30.01.2013, Nr. 24, S. 11. Originalressort: focus; BUCH DER WOCHE)

17) $\{\{2$; Gen.; in + Akk.; A2; A4 $\}\}$ 
Die erste findet am Donnerstag, 15. Dezember, um 18 Uhr, im Raabe-Haus, Leonhardstraße. 29 a, statt.

Anlässlich der aktuellen Übersetzung des Buches des Braunschweiger Schriftstellers Wilhelm Raabe in die japanische Sprache liest der Schauspieler und Regisseur Hans Jürgen Heinze aus dem Buch „Stopfkuchen“. Impressionen des japanischen Textes gibt Fujiko Spengler von der Deutsch-Japanischen Gesellschaft. Im Anschluss wird Stopfkuchen gereicht. Der Eintritt ist frei. (BRZ05/DEZ.13600 Braunschweiger Zeitung, 14.12.2005; Raabe auf Japanisch [Ausführliche Zitierung nicht verfügbar])

18) $\{\{0\}\}$

Seiner Meinung nach ist die Inschrift ein so genanntes Figurengedicht. Dessen Auflösung ergibt einen Namen: Lotharius - der lateinische Name Kaiser Lothars, derden Dom in Königslutter erbauen ließ.

Die spiegelverkehrte Inschrift über dem Jagdfries lautet: „Hoc opus eximum vario celamine mirum sc ...“ Die Übersetzung könnte lauten: „Dieses hervorragende Werk, durch mannigfaches Relief wunderbar, meißelte ..." Allerdings kann steht das Wort „,celamen“ auch für „Verbergung“ oder „Verschwiegenheit“. Deswegen kann die Inschrift auch übersetzt werden mit: „Dieses hervorragende Werk, durch mannigfaches Verbergen wunderbar, meißelte ..." Das wäre ein Hinweis auf eine verborgene Botschaft.

Für die Auflösung der Inschrift wendete Kuck das so genannte magische Schwindeschema an. Dabei wird die entspiegelte Inschrift über die gespiegelte geschrieben. (BRZ06/JUN.07443

Braunschweiger Zeitung, 15.06.2006; Versteckte Botschaften in der Inschrift am Kaiserdom [Ausführliche Zitierung nicht verfügbar])

\section{9) $((0))$}

Es gibt ja auch stärkere und schwächere Interviews, die ich geführt habe. Vielleicht habe ich schlecht gefragt, oder so. Es ist ja auch schwierig mit der Übersetzung, wenn ich etwa in der Ukraine bin. Ich spreche die Sprache nicht.

Generalisieren bedeutet, dass ich vereinfache und dadurch, dass ich vielleicht nicht mehr differenziere, die Wahrheit dessen, was ich sagen will, unter Umständen torpediere.

(BRZ06/DEZ.02023 Braunschweiger Zeitung, 05.12.2006; \&\#8222;Was man verdrängt, macht krank\&\#8220; [Ausführliche Zitierung nicht verfügbar])

\section{0) $\{\{1$; attr. Adj.; A4 $\}\}$}

Doch wer weiß schon was der Begriff wirklich bedeutet? Immerhin, die eifrigen Kirchgänger werden gemerkt haben, dass es der Name des ersten Sonntags nach Ostern ist -traditionell ein Konfirmationstermin.

Und die deutsche Übersetzung unterstreicht noch einmal das, was Jesus Christus uns durch sein Menschenleben, seinen Tod und seine Auferstehung erworben hat: „Quasi-modogeniti“ sind wir nämlich dadurch, das heißt: ,wie die neugeborenen Kinder“.

Mit Gottes Gnade, Erbarmen und Liebe dürfen wir alte Schuld hinter uns lassen und ein neues unbelastetes Leben anfangen. Wenn wir nurwollen. (BRZ08/MAR.14332 Braunschweiger Zeitung, 28.03.2008; [Ausführliche Zitierung nicht verfügbar])

21) $\{\{2$; attr. Adj.; Gen.; A4; A2 $\}\}$

An Häusern mit starkem Belcanto-Repertoire mag Mayr eine interessante Lücke schließen. So bringt die Staatsoper München in der nächsten Saison „Medea in Corinto“ heraus. Der CD fehlt leider die deutsche Übersetzung des Textes! 
Capucine Chiaudani als Fedra bei der Deutschen Erstaufführung in Braunschweig. Foto:

Christian Bort (BRZ09/MAI.01970 Braunschweiger Zeitung, 06.05.2009; Eine umstrittene Opern-Rarität [Ausführliche Zitierung nicht verfügbar])

22) $\{\{1$; Demonstrativpr. im Gen.; A2 $\}\}$

Für die Zensur der Post in der britischen Besatzungszone war nach dem Zweiten Weltkrieg eine Behörde in Peine zuständig. Ihre Existenz war fast vergessen, bis ein niederländisches Buch zum Thema auftauchte. Dessen Übersetzung liegt jetzt vor.

Drei Behörden für die Zensur jeglicher Post richteten die Briten in ihrer Besatzungszone ein: In Hamburg, Bonn, und eben in Peine. 2005 erschien ein Buch zum Thema in den Niederlanden; Gieselher Stoll hat nun die Passagen, die Peine betreffen, übersetzt.

(BRZ09/NOV.01749 Braunschweiger Zeitung, 05.11.2009; Briten ließen in Peine Briefe zensieren [Ausführliche Zitierung nicht verfügbar])

23) ((1; in + Akk.; A4))

EU: Künftig mit Dolmetscher vor Gericht

BRÜSSEL. Wer innerhalb der EU in die Mühlen der Justiz gerät, hat Anspruch auf

Übersetzung in die eigene Muttersprache. Das haben die EU-Justizminister bei ihrem Treffen in Luxemburg beschlossen. So soll Verdächtigen ein Dolmetscher für den Umgang mit Ermittlungs- und Justizbehörden gestellt werden. (BRZ10/OKT.04491 Braunschweiger Zeitung, 09.10.2010; EU: Künftig mit Dolmetscher vor Gericht [Ausführliche Zitierung nicht verfügbar])

24) ((1; Posspr.; A1))

Und da saß ich wie jeden Tag kurz vor Sonnenuntergang auf der halbrunden, mit Pandanus überdachten Veranda. Fünf oder sechs Nachbarskinder waren um mich herum versammelt, ich hatte Lollies verteilt und wir sangen immer abwechselnd Kinderlieder auf Maori und auf Deutsch. Ich hab keine Ahnung, was sie da sangen, und sie konnten umgekehrt trotz meiner Übersetzung nicht wirklich was mit einem Müller anfangen, noch dazu mit einem, der nicht wandert, und deshalb schlecht ist. Aber rätselhaftes waren sie von mir schon gewöhnt: am Vormittag war ich in der Schule gewesen und hatte in Hinanos Klasse ein bisschen was von Deutschland erzählt, von so merkwürdigen Dingen wie Schnee auf hohen Bergen, von Straßenbahnen, Kirchen, die tausend Jahre alt sind. Mit großen Augen hatten sie an meinen Lippen gehangen, sich totgelacht, als ich ihnen $\ddot{A}$, Ü und Ö vormachte. (BRZ11/SEP.01925 Braunschweiger Zeitung, 03.09.2011; Mataruas Vermächtnis [Ausführliche Zitierung nicht verfügbar])

25) $\{\{1$; in + Akk.; A4 $\}\}$

„Die Patientin hat ein deutliches Genu varum und eine medial und retropatellar betonte

Pangonarthrose links." Wer solche Sätze in seinem Arztbrief findet und kein Mediziner ist, ist nicht schlauer als vorher. Ärzte eignen sich während des Studiums erst die Fachbegriffe an, manch einer kann sie im Berufsalltag kaum noch ablegen.

Professor Karl-Dieter Heller, Chefarzt der Orthopädie im Braunschweiger HerzoginElisabeth-Hospital, hat für uns einen Arztbrief aufgesetzt - einmal in der medizinischen Fachsprache, dann in der Übersetzung ins Hochdeutsche. So eine Übersetzung ist vermutlich sehr viel seltener als die Diagnose, die am Ende auch wir verstehen: O-Beine, Kniegelenksverschleiß.

Arztbrief ist ein Austausch unter Kollegen (BRZ12/AUG.05405 Braunschweiger Zeitung, 08.08.2012; Aua, mein Knie \&\#8211; Was Ihr Arzt wirklich meint [Ausführliche Zitierung nicht verfügbar]) 
26) $\{\{1$; Gen.; A2 $\}\}$

Ihr Gegenüber, die Hauptrolle des Orlando, spielt Oliver Arno ("Les Miserables" im StadtTheater Klagenfurt und als Tod in "Elisabeth" in Belgien). Für Oliver Arno, der vornehmlich im Musiktheater zu Hause ist, ein sommerlicher Ausflug in die Theaterwelt.

Regie führt auch dieses Jahr Peter W. Hochegger, der wieder für die Übersetzung des Stückes verantwortlich zeichnet und die Feinheiten des englischen Humors bestens ins Deutsche übertragen hat. Hochegger über die Faszination Shakespeare: "Seine Werke sind für mich der Inbegriff all dessen, was Theater ausmacht. Im Mittelpunkt steht immer der einzelne Mensch im Konflikt zu seinem Umfeld - so klar und widersprüchlich wie das Leben selbst.

(BVZ09/JUL.06149 Burgenländische Volkszeitung, [Wochenzeitung], 22.07.2009, Nr. 30, Jg. 2009, S. 11. - Sachgebiet: Kultur, Originalressort: KULTUR)

27) ((1; attr. Adj.; A1))

ein internationales Symposium "Zeichen und System der Sprache" begann am Dienstag an der Technischen Hochschule "Otto von Guericke" in Magdeburg. die achttägige Fachberatung vereinigt 290 Wissenschaftler aus Europa, Asien und Amerika in der DDR.

Sprachwissenschaftler, Psychologen, Mathematiker und technische Experten beschäftigen sich mit der strukturellen Beschreibung der Sprache, der Bedeutung der Wörter und mit den Aufgaben der automatischen Übersetzung. Probleme der Sprachkybernetik, der Informationsverdichtung und des programmierten Unterrichts stehen ebenfalls auf dem Programm. (BZK/D64.00479 Neues Deutschland, [Tageszeitung], 10.09.1964, Jg. 19, Berliner Ausgabe, S. 4. Originalressort: FEUILLETON; Sprachkybernetik, [Feuilleton])

28) ((2; attr. Adj.; von + Dat.; A1; A2))

Zwar fördert die EG-Kommission schon seit Jahren Einzelprojekte der Sprachtechnik, bislang konnte jedoch kein umfassendes und ausgereiftes Übersetzungsverfahren entwickelt werden. Im Rahmen eines geplanten und auf fünf Jahre angelegten Förderprogramms versucht die EGKommission deshalb, die Kapazitäten zu bündeln. Im Vordergrund stehen dabei die maschinelle Übersetzung von Sprache in geschriebener und gesprochener Form sowie die integrierte Dokumentenerstellung und -verwaltung.

Eine Arbeitsgruppe unter Leitung von Professor Danzin hat den Mittelbedarf seitens der Kommission dafür auf rund 850 Millionen ECU veranschlagt. Forschungsgelder im Rahmen anderer Programme zum gleichen Thema sind dabei nicht berücksichtigt. (C93/JUN.02280 COMPUTER ZEITUNG, [Wochenzeitung], 17.06.1993, Nr. 24, Jg. 28, S. 16. Durchbruch bei Computerübersetzungen steht noch aus, [Bericht])

29) ((2; Demonstrpr. im Gen.; in + Akk.; A2; A4))

Als Anwendung schwebt den im texanischen Austin beheimateten Projektmachern zunächst ein allgemeiner Standard für formale Wissensrepräsentation vor. Mit ihm läßt sich Wissen aus unterschiedlichen Gebieten einfach zusammensetzen und austauschen.

Ein in Zukunft erfolgversprechender Bereich ist die automatische Erstellung von Bedienungsanleitungen und deren Übersetzung in verschiedene Sprachen. Um das gesamte kulturelle

Wissen, das einem normalen Erwachsenen zur Verfügung steht, zu erfassen, erstellten die Mitarbeiter des Cyc-Projekts "Mikrotheorien" zu einzelnen Themen. (C97/APR.01701

COMPUTER ZEITUNG, [Wochenzeitung], 10.04.1997, Nr. 15, Jg. 32, S. 28. Wissensbasiertes Datenbanksystem kümmert sich auch um die Banalitäten des Lebens, [Bericht]) 
30) $((0))$

Das Framework erleichtert die Anwendung der objektorientierten Middleware. Die gleiche Zielsetzung hat die Skriptsprache "Corba Scripting Language". Sie reduziert Komplexität, indem sie die Erstellung von Prozeduren zum Beispiel für Speicherreservierung, Übersetzung und Linking erlaubt. Die Integration von Internet-Anwendungen in Corba-Umgebungen erleichtert eine Brücke zwischen der Corba-eigenen Beschreibungssprache IDL und der Programmiersprache Java. (C98/OKT.04339 COMPUTER ZEITUNG, [Wochenzeitung], 01.10.1998, Nr. 40, Jg. 33, S. 16. - OMG erarbeitet ein Komponentenmodell, [Bericht])

31) $\{\{1$; Gen.; A2 $\}\}$

- Auf Deutsch erschienen bisher

die Storys "Kleines Mädchen mit komischen Haaren" (2001). Die Übersetzung des Megaromans "Infinite Jest" ist in Vorbereitung.

- Er lebt auf dem Land in Bloomington/ Illinois und unterrichtet Creative Writing an der Illinois State University. (FOC02/SEP.00475 FOCUS, 21.09.2002, S. 088-090, Sachgebiet: Kultur, Originalressort: KULT/Kultur; LITERATUR)

32) $\{\{0\}\}$

H. Shepard (Ill.), Verlag Kein \& Aber, 240 Seiten, 29,90 Euro

Maulwurf, Wasserratte, Kröte und Dachs sind die liebenswerten Helden in diesen weltberühmten Gutenachtgeschichten. Ein Kinderbuch-Klassiker in neuer Übersetzung. Ab 6 Xanthe (FOC05/FEB.00172 FOCUS, 14.02.2005, S. 064-064, Sachgebiet: Kultur, Originalressort: KULT/Kultur; Titel: DIE BESTEN 7 BÜCHER FÜR JUNGE LESER FEBRUAR)

33) $\{\{1$; attr. Adj.; A4 $\}\}$

Mehr als vierhunderttausend verkaufte Exemplare ihres erotischen Pilgerromans "Muschi denn zum Städtele hinaus". Chapeau! Und die chinesische Übersetzung kommt erst noch! Composing: FOCUS-Magazin

Fotos: face to face, action press (FOC08/APR.00355 FOCUS, 21.04.2008, S. 189-189, Sachgebiet: Mode, Originalressort: Modernes Leben; HIER SCHREIBT HARALD SCHMIDT)

34) $\{\{0\}\}$

Warum? Erstens gibt es, wie jeder anständige Dolmetscher und Übersetzer weiß, so etwas wie die perfekte Übersetzung nicht. Von Anfang an haben die ernsthaften, engagierten Übersetzer sich darüber wund diskutiert, wie die Feinheiten von Bedeutungen in den herausragenden Gedichten, Dramen und Romanen zu verstehen sind - von internationalen Abkommen ganz zu schweigen. (FOC13/JUL.00101 FOCUS, 08.07.2013, S. 110-111, Sachgebiet: Kultur, Originalressort: KULTUR UND LEBEN, MEDIEN; Für mich war es Liebe beim ersten Hören)

35) ((1; von + Dat.; A2))

Aber wer kennt sich schon zugleich in byzantinischen Erotica, apokryphen Fernsehserien und höherer Mathematik aus? Und wie soll man den verschlungenen Bandwurmsatz aufdröseln und neu zusammenknüpfen, mit dem David Foster Wallace die Fesselung eines verschnupften frankokanadischen Separatisten beschreibt? Bei der Übersetzung von „Infinite Jest“" („Unendlicher Spass“ erscheint, 1547 Seiten dick, am 24. August bei Kiepenheuer \& Witsch) 
hat der in Basel lebende Deutsche Ulrich Blumenbach, wie er kürzlich in einer Rede gestand, „Dinge gesehen, die mir selbst erfahrene Übersetzer kaum glauben würden“: Wörter, die kein Wörterbuch verzeichnet, verschachtelte Satzkonstruktionen, die Thomas Mann oder Proust wie „kurzatmige Asthmatiker“ aussehen lassen, Kapriolen und „lexikalische Vergewaltigungen“" einer irrwitzigen Spaßgesellschaft, neben denen selbst Thomas Pynchons Romane verblassen. Der gebürtige Hannoveraner Blumenbach, mit lebhaftem Temperament, einer gut sortierten Bibliothek und einem ,libidinösen Verhältnis zur Sprache“ gesegnet, hat die Herausforderung sportlich genommen und brillant bewältigt. Jetzt weiß er, dass Achondroplasie Zwergwuchs ist und ,gluteale Hyperadiposität“ ein vornehmeres Wort für Fettarsch, wi (HAZ09/AUG.02120 Hannoversche Allgemeine, 14.08.2009; Das göttliche Würstchen [Ausführliche Zitierung nicht verfügbar])

36) $\{\{1$; attr. Adj.; A4 $\}\}$

Das kinderlose Ehepaar Jonathan (Robert Wagner) und Jennifer Hart (Stefanie Powers) bezauberte in „Hart aber herzlich“ jedoch wegen der flapsigen Flirts. Jeder Kriminalfall, den die beiden Hobbydetektive actionreich in Bel Air lösten, war zugleich eine Frischzellenkur für ihre Liebe. Stets an ihrer Seite: Butler Max und Hund „Freeway“, aus dem in der deutschen Übersetzung ein biederer „Friedwart“ wurde. Die Serie basiert auf einer Idee des US-Bestsellerautors Sidney Sheldon. Von 1979 an wurden 118 Folgen gedreht, in den neunziger Jahren waren auch deutsche Schauspieler wie Katja Riemann und Ursula Karven zu Gast. (HAZ10/AUG.00773 Hannoversche Allgemeine, 14.08.2010; Seite an Seite [Ausführliche Zitierung nicht verfügbar])

37) $\{\{0\}\}$

Ein Überblick über moderne Bauweise

Der "Atlas der zeitgenössischen Architektur" zeigt die wichtigsten Bauwerke der Moderne in Text und Bild. Der spanische Architekturhistoriker Alex Sànchez Vidiella hat die Gebäude nach Kategorien wie öffentliche Gebäude, Städtebau oder Wohnhäuser nicht wie üblich nach Architekten geordnet. Ein informatives Kompendium, doch leider stört die hölzerne Übersetzung das Lesevergnügen. (HMP07/JUL.02761 Hamburger Morgenpost, 27.07.2007, S. 40; Ein Überblick über moderne Bauweise [Ausführliche Zitierung nicht verfügbar])

38) $\{\{2$; attr. Adj.; Gen; A4; A2 $\}\}$

Und nun ist plötzlich alles ein wenig anders. Denn Gri-sham ist unter die Kurzgeschichtenautoren gegangen. "Das Gesetz" lautet die deutsche Übersetzung des Titels der Sammlung, ein überdeutlicher Anklang an Megaseller wie "Die Firma", "Die Akte", "Die Jury" und "Der Klient".

Im Original heißt der Band "Ford County", ein fiktiver Landstrich in den Südstaaten der USA, in dem alle sieben Geschichten spielen. Es ist ein mal ernster, mal ironischer Blick, den der 55-Jährige auf seine Heimat wirft -auf die ganz normalen Menschen, die sich im Alltagswirrwarr behaupten müssen und dabei oft vergeblich gegen das eigene Schicksal anstrampeln. (HMP10/SEP.00935 Hamburger Morgenpost, 09.09.2010, S. 29; Deutschland-Premiere für den Thriller-Titanen John Grisham liest heute beim "Harbour Front Festival" [Ausführliche Zitierung nicht verfügbar])

39) $\{\{1$; Gen.; A1 $\}\}$

Dreh- und Angelpunkt ist Michael Maertens (aus der Hamburger Schauspieler-Dynastie). 
Er zieht als Alceste alle Register eines turbobetriebenen Rechthabers, der sich ständig selbst Steine in den Weg legt. Zweite Hauptfigur ist die tolle Übersetzung des verstorbenen Jürgen Gosch (mit Wolfgang Wiens), in der die Reime kunstvoll, aber wie geölt ineinandergreifen.

BU: Lässt sich nicht bedrängen: Menschenfeind Alceste (Michael Maertens, r., mit Thomas Loibl) hasst gewisse Umgangsformen. (HMP13/MAI.01461 Hamburger Morgenpost, 17.05.2013, S. 33; Keine Chance für Speichellecker Molieres "Menschenfeind" Umjubeltes Gastspiel im Thalia-Theater [Ausführliche Zitierung nicht verfügbar])

40) $\{\{1$; Posspr.; A1 $\}\}$

INNSBRUCK.

Theaterstücke ohne Inhalt und Aussage haben in der Ära Mentha/Hübsch am Tiroler Landestheater normalerweise nichts verloren. Donnerstag abend hat Georges Feydeaus Nulldrama "Herrenjagd", wie es Elfriede Jelinek in ihrer hiemit erstaufgeführten Übersetzung nennt, in den Kammerspielen doch etwas verloren: seine Unschuld. Feydeau deutete in "Monsieur chasse" 1892 zwar an, wie man Hasen jagt, macht sich im übrigen aber nicht einmal mehr die Mühe, die Motive des bürgerlichen Lustspiels aufzulisten. Nur das Gerüst bleibt, um den wahnwitzigen Mechanismus von Jagen und Gejagtsein in Gang zu bringen: Versteckspiel, Verwechslung, überraschende Begegnungen rund um der Herren angestrengte Seitensprünge. (I96/FEB.05470 Tiroler Tageszeitung, [Tageszeitung], 10.02.1996. - Sachgebiet: Kultur, Originalressort: Kultur; Das Nichts in den Aberwitz geschleudert)

\section{1) $[[0]]$}

Zwangsläufig, weil es nämlich an den erprobten Ausführungen dieser beiden Produktreihen ohne übertriebenen Aufwand kaum noch etwas zu verändern gab, beschränkten sich die Modifikationen hier hauptsächlich auf Maßnahmen, von denen die Sicherheit der Insassen ebenso profitiert wie die Umweltfreundlichkeit der Fahrzeuge bei der Produktion und im Betrieb. Schließlich nahmen im Zug der Modellpflege auch die Faktoren Wirtschaftlichkeit bzw. Fahrkomfort einen ähnlich hohen Stellenwert ein.

Dafür stehen beim 900er beispielsweise verbesserter Flankenschutz, hydraulische Betätigung der Kupplung, Bremsbeläge mit Verschleißwarner, geänderte Übersetzung des

Antriebsstrangs, die Verwendung wasserlöslicher Lacke bei der Farbgebung und ein erweitertes Zubehörprogramm. Der Saab 9000, der früher oder später durch den 9-5er abgelöst werden dürfte, wird vorerst einmal übers ganze Jahr 1997 hinweg in der Ausführung "Anniversary" mit Vierzylindertriebwerken in Leistungsstufen zwischen 130 und 225 PS zu haben sein.

Für den kleineren Bruder, den Saab 900, scheinen hingegen drei Antriebsaggregate jeweils kombiniert mit Fünfgangschalt- bzw. Automatikgetriebe im Programm auf. (I97/SEP.34777 Tiroler Tageszeitung, [Tageszeitung], 06.09.1997. - Sachgebiet: Auto / Motor, Originalressort: Motor; Saab-Palette weiter verfeinert)

42) ((1; Gen.; A2))

Wo reformiert Schlögl?

Mit der Übersetzung des bulgarischen Obduktionsberichts über den bei seiner Abschiebung aus Österreich umgekommenen Afrikaner Marcus Omofuma sind am Wochenende weitere Details durchgedrungen, die das Entsetzen über das Sterben dieses Mannes nicht geringer werden lassen. Und was tut die Disziplinarkommission des Innenministeriums, die gestern über die für das tödliche Knebeln verantwortlichen begleitenden Beamten zu befinden hatte? 
Sie suspendiert die Polizisten nicht. (I99/MAI.18844 Tiroler Tageszeitung, [Tageszeitung], 18.05.1999. - Sachgebiet: Politik, Originalressort: Innenpolitik; Wo reformiert Schlögl?)

43) $[[0]]$

Der Belgier "dribbelte" in damals neuem Streckenrekord von exakt 30:03 Minuten hinauf. Steinmayr wäre in den steilen Kehren fast vom Rad gekippt, keuchte mit 7:43 Minuten Rückstand ins Ziel. Er hatte eine viel zu harte Übersetzung gewählt, die er schon nach wenigen Metern nicht mehr treten konnte. In den Jahren darauf schrieb sich der Schweizer Beat Breu mit zehn Siegen und 27 Minuten ins Rekordbuch ein. Seit '96 gibt's das HornRennen nicht mehr. (K00/JUN.44195 Kleine Zeitung, [Tageszeitung], 08.06.2000. Sachgebiet: Sport, Originalressort: Sport; Die 7,5 Kilometer in)

44) $\{\{1$; attr. Adj.; A4 $\}\}$

Mittelpunkt.

Ein Getreidealtar mit liturgischen Geräten ist Blickfang und zugleich Mittelpunkt im Ausstellungsfoyer. Weiters: Eine uralte Bibel, ein Unikat - die älteste slowenische Übersetzung. Ebenfalls hinter Glas, Relikte aus dem Judentum, die Assoziationen zum Christentum widerspiegeln. Phänomenal: Schofar, ein im jüdischen Kult verwendetes Widderhorn, das z. B. zur Ankündigung des Sabbats geblasen wurde; Tefillin, ein Gebetsriemen der Juden, beim Morgengebet an Stirn und linkem Oberarm getragene Kapseln mit Schriftworten; Haggadah, eine belehrende Erzählung biblischer Passagen in der talmudischen Literatur. (K97/FEB.12148 Kleine Zeitung, [Tageszeitung], 16.02.1997. Originalressort: Völkermarkt; Bibelausstellung: Ausflug in religiöse Vergangenheit)

45) $\{\{0\}\}$

Für Regisseur Klaus Gmeiner ist die "zauberhafte, böse" Komödie durchaus aktuell, auch heute gäbe es die Gier, "Seitenblicke" auf die Hautevolee zu werfen. Angelika Ladurner spielt die Titelrolle, Peter Pikl steht diesmal selbst auf der Bühne. Die Übersetzung stammt - nicht belegbar, aber wahrscheinlich - von Alfred Polgar, dafür spreche, so Gmeiner, der meisterlich, glänzende Sprachwitz.

Aus dem Sattel in den Regiesessel wechselt Albert Tisal für seine Inszenierung von Molières Arzt wider Willen, womit man beim Handauf(-an)legen gelandet ist. Denn Sganarelle ist eigentlich Holzhac ker, soll aber Lucinde von ihrer Stummheit heilen. (K98/JUL.50643 Kleine Zeitung, [Tageszeitung], 01.07.1998. - Sachgebiet: Kultur, Originalressort: Kultur; Holzhacker und die Hautevolee)

46) $\{\{1$; Komp.; A2 $\}\}$

Klagenfurt. -

Mit Dextro- Energen-Traubenzucker bündelte er morgens Wissen und Kräfte. Dann legte Hannes Posch, 18, bei der heurigen Latein-Olympiade Mittwoch in Klagenfurt eine so exzellente Vergil-Übersetzung hin, daß er zum besten Lateinschüler Kärntens gekürt wurde. Der Maturant nahms' mit stoischer Ruhe - schließlich gilt er schon seit 1998 als drittbester Übersetzer dieser Sprache in ganz Österreich. Ein Opportunist? (K99/APR.27960 Kleine Zeitung, [Tageszeitung], 23.04.1999. Originalressort: Lokal; Der den Tag pflückte)

47) $\{\{0\}\}$

Das Stück zu regionalisieren, kam uns zu folkloristisch vor. Wir zielten auf die Reduktion zu einer kargen, nüchternen Sprache ab, wollten aber auch den Kampf mit der Sprache zeigen. Und so haben wir mit den Schauspielern gut eine Woche am Tisch gesessen, um die Übersetzung durchzusprechen. Im Jahr 2000 ist die Schaubühne am Lehniner Platz völlig 
leer. Es gibt dann nur noch einen Kasten mit einer mythischen Vergangenheit... Ostermeier:. .die Gott sei Dank ihre Glorie verloren hat. (L98/JUN.03251 Berliner Morgenpost, [Tageszeitung], 09.06.1998, S. 28. Originalressort: Text; BM: Rente allein macht nicht glücklich "Unser Theater will nicht schockieren": Thomas Ostermeier erhält heute für "Messer in Hennen" den Friedrich-Luft-Preis)

48) $\{\{1$; Gen.; A2 $\}\}$

Von Elke Rutschmann

Kikis Constantinou sitzt bequem in dem tiefen Ledersessel im Bibliotheksaal des Hotel Esplanade, zieht an seiner Zigarette und bläst versonnen Kringel in die Luft. Der Mann mit dem grauen Vollbart ist Hotelbesitzer aus Zypern und seit 25 Jahren Präsident und Mäzen von Anorthosis Famagusta. Der aufsteigende Zigarettenrauch passt zur Übersetzung von Anorthosis: Wie Phönix aus der Asche. Der heilige Vogel, mythologisches Symbol des sich erneuernden Lebens, ziert auch das Logo des 1911 gegründeten Vereins und steht gleichzeitig als Sinnbild für die Geschichte des zypriotischen Klubs. Mit Famagusta gibt es nämlich nur noch eine politische Verbindung. (L99/AUG.51325 Berliner Morgenpost, [Tageszeitung], 11.08.1999, S. 27. Originalressort: SPORT; Von Elke Rutschmann: Die verlorene Stadt beschwört Offensivgeist)

49) $\{\{2 ;$ attr. Adj.; von + Dat.; A4; A1 $\}\}$

die Texte der Kantate sind der Haggada entnommen, mit Ausnahme der seltsamsten, der Diskussion über weiße und schwarze Katzen, die zur Halacha gehört. es ist ein Geheimnis, wie ernst sie gemeint war. der Originaltext ist aramäisch; die italienische Version, nach der das Werk komponiert wurde, stammt von Fedele D'Amico, die deutsche Übersetzung von Rudolf Arneim".

Forum der Musik

Kommentare zu ausgewählten Schallplatten (LIM/LI1.00040 WDR-Hörfunkprogramm. Köln, Jan./März/Juli 1970)

50) ((2; Gen.; in + Akk.; A2; A3))

Vagina. Er zog Zwetschge immer noch vor. Eine Übersetzung des Bildes in die Wirklichkeit war nicht gelungen. Er hatte dann doch zu reden angefangen. Hoffend, daß dadurch eine Stimmung entstünde, die Rosis Verschlossenheit ein bißchen milderte. (WAM/SPB.00000 Walser, Martin: Ein springender Brunnen. Roman. - Frankfurt a.M.: Suhrkamp Verlag, 1998, 413 S. [S. 337])

51) $\{\{0\}\}$

Im Mittelpunkt von Leonards letztem auf Deutsch vorliegenden Werk "Raylan"(3) steht ein merkwürdiger Gesetzesvertreter, Raylan Givens, ein Top-Ermittler im US Marshals Service, ein ungewöhnlicher Cop, der schneller schießt als er denkt und sich in der trostlosen Einöde von Kentucky als unfreiwilliger Organspender wiederfindet, als er zwei ehemaligen DopeDealern, die auf menschliche Ersatzteile umgestiegen sind, auf die Schliche kommt. Auch in diesem Roman erweist sich Elmore Leonrad als der "King of Less", der alles Überflüssige weglässt, das der normale Leser sowieso schnell überblättern würde; der alles Ornamentale streicht, das den Krimi-Liebhaber von der Essenz der Geschichte entfernen würde; der hauptsächlich auf den Klang seiner temporeichen und spannenden Prosa mit herrlich farbenreichen Dialogen bedacht ist, auf dieses unaufdringliche Lässige, auf dieses stets mitreißende Swingende, dessen markanter Sound fast unmöglich in einer Übersetzung zur vollen Geltung gelangen kann. (LTB13/SEP.00297 Luxemburger Tageblatt, 05.09.2013, Auch seine Helden waren vor Pleiten, Pech und Pannen nicht gefeit) 
52) $((1 ;$ Gen.; A2))

(+1216): "Wer wüsste nicht, dass das eheliche Beilager niemals ohne die Glut der Unzucht, ohne Schmutz der Lust stattfindet, wodurch der empfangene Same beschmutzt und verderbt wird?"

Hieronymus ist der Vater der lateinischen Bibelübersetzung Vulgata. Papst Siricius $(+390)$ bezeichnete es als "Unzucht", ja "Verbrechen", wenn die Priester nach ihrer Weihe noch mit ihren Frauen verkehrten und Kinder zeugten, und verbot Hieronymus, bei der Übersetzung des Satzes von Paulus "Habe ich nicht das Recht, eine Ehefrau auf den Reisen mitzunehmen wie die übrigen Apostel und Petrus (1. Kor. 9,5)?" das lateinische Wort "uxor" (= Ehefrau) zu schreiben. Aus den Ehefrauen der Apostel werden in der lateinischen Übersetzung schließlich dienende Schwestern. (LTB14/NOV.01505 Luxemburger Tageblatt, 18.11.2014, ohne Titel)

53) $\{\{3$; attr. Adj.; Apposition; von + Dat.; A4; A’; A1 $\}\}$

Das teilte gestern die Gondrom-Universitätsbuchhandlung in Kaiserslautern mit, die den Preis zum zweiten Mal ausgeschrieben hatte. Insgesamt hatten sich mehr als 30 Autoren aus der Pfalz daran beteiligt.

Anerkennungspreise von jeweils 1000 Mark erhalten diesmal die Pfälzische Übersetzung "Waarde uf de Godot" von Bastiane Franke aus Fischbach, die Komödie "Arwettplätz vun A bis Z" des Zweibrückers Horst Scherer sowie das Volksstück "Herrgott von Bockshiwwel" von Vorjahrespreisträger Heinrich Kraus. Verliehen werden die Preise am 7. Mai abends im Foyer des Kaiserslauterer Pfalztheaters.

dpa (M98/MAR.26507 Mannheimer Morgen, [Tageszeitung], 25.03.1998, Jg. 53. Sachgebiet: Kultur, Originalressort: Feuilleton; Auszeichnung für Pfälzer Stücke, [Feuilleton])

54) $\{\{3$; Posspr.; von + Dat.; als; A1;A2; A‘ $\}\}$

Der quirlige Engländer Christopher Hawkins hat sich der Bilder aus dem Straßenverkehr bedient, um die Lektion anschaulich zu gestalten. Zwar erklärt er auf Englisch, doch damit jeder zumindest die Schlüsselbegriffe versteht hat er sie auf Deutsch parat: "Überholen, Straßenkreuzung, Notfallhalt, Umleitung." Nur bei seiner Übersetzung von "Back Seat Driving" als "Zurück-Sitz-Fahren" runzeln die Zuschauer dann doch die Stirn. Die Mienen glätten sich aber sofort, als er vorführt: "Du fährst mit deinem Mercedes durch die Gegend und deine entzückende Frau sagt: ,Stoooopp! Da kommt einer!" (M99/AUG.57549

Mannheimer Morgen, [Tageszeitung], 30.08.1999, Jg. 54. Originalressort: Lokal Mannheim; Spritziger Tanz-Unterricht mit Anleihen aus dem Straßenverkehr)

55) $\{\{1$; für + nicht dek1.; A2 $\}\}$

Etwa wenn, auch noch während der Predigt der Gemeindereferentin, die von einer Begegnung mit Gott in einem Menschen sprach, der Teufel bemerkte, es sei doch schon etwas, "wenn wir sagen können: Heute bin ich einem Menschen begegnet." Oder wenn Gott persönlich die Fürbitten kommentierte, die Klumb vortrug.

Alles in allem überwog aber der Spaß. Egal ob Klumb die Möglichkeiten des

Videotestamentes pries, als Prälat Stapelhoch den akademischen Vortrag zum Jubiläum hielt oder ihnen einen Crashkurs in pastoralem Englisch angedeihen ließ, bei dem sie unter anderem lernten, mit welchem Titel sie Pfarrer Haus in Englisch richtig ansprechen: "Spiritual bicycle" ist laut Klumb die einzig richtige Übersetzung für Geistlicher Rat. 
hh (M00/SEP.52325 Mannheimer Morgen, [Tageszeitung], 04.09.2000, Jg. 55. Originalressort: Lokal Viernheim; "Heiliger Bimbam" fordert wahre Lachsalven heraus)

56) $\{\{1$; sächs. Gen.; A1 $\}\}$

"Na gut, nachts hört ja eh keiner zu", diese Einschätzung allerdings, mit der sich die Konfirmanden Mut machten, erwies sich als falsch. Wie über den ganzen Samstagnachmittag und den Abend, so schauten und hörten auch in der Nacht immer wieder Besucher in der Kirche rein, nur für Minuten oder für Stunden. In Bibeln konnten sie den nach Martin Luthers Übersetzung aus der Altarbibel vorgetragenen Text mitverfolgen.

Dass man fürs Neue Testament rund einen Tag bräuchte, wusste das Marathon-Team. Dass am Ende aber nur vier Minuten vor 24-Stunden-Frist der Schlusssegen der Offenbarung erklang - mit einem so exaktem Timing hatte keiner gerechnet. (M01/JAN.07112

Mannheimer Morgen, [Tageszeitung], 30.01.2001, Jg. 56. Originalressort: RheinNeckar; Bibelmarathon endete vier Minuten vor 24 Stunden)

57) $\left\{\left\{1 ;\right.\right.$ Apposition; $\left.\left.A^{\prime}\right\}\right\}$

"Ich möchte die vertrauten Formen beibehalten, aber ihnen ein neues Innenleben geben", so der Künstler. Seine Formate beginnen in Postkartengröße.

Die "Postcans", ein Begriff, der sich zweideutig aus Postkarte und der Übersetzung "nach der Dose" zusammensetzt, greifen in die dritte Dimension. Durch Überlagerung der Fläche mit einer Wiederholung des aufgedruckten Motivs gewinnt das Werk Räumlichkeit. Eines seiner neueren Werke ist ein wandfüllendes Mosaik nach dem "Denker" von Rodin, den Menger in einen Engel verwandelt hat. (M01/OKT.80113 Mannheimer Morgen, [Tageszeitung], 23.10.2001, Jg. 56. Originalressort: Lokal Bürstadt / Biblis; Geleerte Dosen werden zum Kunstwerk)

58) $((0))$

Während der Woche finden Hausgruppen für Erwachsene und Jugendliche statt. Für weitere Infos schauen Sie mal im Internet unter www.HausdesHerrn.de

oder unter 0621/8 35-56 63. Power House, ein englischer "Music \& Message Event" (mit Übersetzung) findet um 18.30 Uhr im Trafohaus, Keplerstr. 22 (hinter Delta Park Hotel) statt. Thema: "A beautiful Mind". Gastredner ist Jerry Kragt, Berlin International Church. (M02/MAI.35708 Mannheimer Morgen, [Tageszeitung], 11.05.2002, Jg. 57)

59) $\{\{0\}\}$

"Wie kam Maria zum Kind?", Bericht vom 20.12. Bischöfin Margot Käßmann hat nur offen ausgesprochen, wovon viele Christen inzwischen fest überzeugt sind: Bei Zeugung und Geburt des Jeschua ben Josef ging es ganz "irdisch" zu. Alles, was die Evangelisten, die keine Augenzeugen der von ihnen geschilderten Ereignisse waren, etwa 70 Jahre später in griechischer Sprache schrieben, existiert nur noch in Abschriften von Abschriften mit Unstimmigkeiten und Übersetzungsfehlern. Kaum ein wissenschaftlich arbeitender Theologe bestreitet heute noch, dass die Legende von der Jungfrauengeburt durch spätere hellenistische Einflüsse und falsche Übersetzung entstanden ist. Dem Judentum, dem ursprünglichen Judenchristentum und auch dem Apostel Paulus war der Gedanke einer "Jungfrauengeburt" gänzlich fremd.

Im Gegensatz zu Professor Berger - für den nicht sein kann, was nicht sein darf - ist für die katholische Theologin Ranke-Heinemann die "Jungfrauengeburt" zweifelsfrei legendär; sie sagt dazu: "Die jungfräuliche Maria ist keine ursprünglich christliche Vorstellung, sie betrat gleichsam auf einem Umweg über die Heiden und die Heidenchristen das Glaubensgebäude 
des Christentums". (M03/JAN.02555 Mannheimer Morgen, [Tageszeitung], 14.01.2003, Jg. 58. Originalressort: Briefe an den Herausgeber; Es ging ganz "irdisch" zu, [Leserbrief])

60) $((0))$

Gleichzeitig begannen nach Ende der großen Abschlussveranstaltung um 18 Uhr bereits die Aufräumarbeiten, viele Gäste traten die teilweise sehr lange Heimreise an. Erstmals war am Samstag mit Kulturbürgermeister Dr. Peter Kurz (SPD) ein hochrangiger Vertreter der Stadt zu Besuch bei den Ahmadiyya, gestern folgte eine etwa 20-köpfige Delegation, der auch CDU-Fraktionsvorsitzender Dr. Sven Joachim Otto angehörte. Am Sonntagnachmittag lauschten etwa 400 deutsche Gäste einem 20-minütigen Vortrag des Kalifen in Übersetzung, eine Frage-und-Antwort-Runde gab Auskunft über Ziele und Grundsätze der Ahmadiyya. 3000 Helfer in 135 Teams hatten das Zusammensein im Namen Allahs generalstabsmäßig geplant und durchgezogen. "Wir sind froh, dass wir nach der Hitzewelle kamen", sagte ein Sprecher dankbar. (M03/AUG.55482 Mannheimer Morgen, [Tageszeitung], 25.08.2003, Jg. 58. Originalressort: Mannheim; Ahmadiyya räumen Mühlfeld)

61) $((0))$

"Sobibór" ist auch eine Reflexion über die Darstellbarkeit von Geschichte. Als "Fiktion des Realen" hat Lanzmann die schöpferische Qualität seiner Arbeit bezeichnet, die sich den üblichen Methoden musealer Vergangenheitsbewältigung bewusst entgegenstellt. Die "Verwaltung der Erinnerung" bekämpft er mit dem "lebendigen Wort", dessen Verdoppelung in der Übersetzung immer wieder Brüche erzeugt. Und mit Bildern, die weder illustrierend noch dramatisierend sein wollen. So entsteht eine Spannung zwischen historischen Fakten, erzählter Erinnerung und imaginierter Erfindung. (M03/SEP.59497

Mannheimer Morgen, [Tageszeitung], 10.09.2003, Jg. 58. - Sachgebiet: Kultur, Originalressort: Kultur (regional); Von unserem Mitarbeiter Wolfgang Nierlin: Handeln oder Sterben)

62) $\{\{1$; von + Dat.; A1 $\}\}$

Wenn man weiß, dass dieses merkwürdige Gespräch mit dem bulgarischen Kondukteur im Nachtexpress sich über Stunden hinzieht, obwohl Esti nur drei Worte Bulgarisch und der andere kein Ungarisch spricht, bekommt man eine Ahnung, von welcher Art der Budapester Urschelm ist. Dabei erreichen seine Interessiertheit am Leben, seine Zweifel, seine Unbedingtheit durchaus eine philosophische Dimension.

Ein Glücksfall ist die Übersetzung von Christina Viragh, deren Muttersprache Ungarisch, deren Literatursprache Deutsch ist. So geht nichts verloren von dem faszinierenden Satzbau in Kosztolányis Prosa.

Dezsö Kosztolányi: Ein Held seiner Zeit. (M04/AUG.57727 Mannheimer Morgen, [Tageszeitung], 24.08.2004, Jg. 59. - Sachgebiet: Kultur, Originalressort: Kultur; Von unserem Mitarbeiter Harald Loch: Budapester Urschelm, [Feuilleton])

63) $\{\{2$; aus + Dat.; von + Dat.; A3; A1 $\}\}$

59. Fortsetzung

Fortsetzung folgt Hotel Empire - Hongkong John Lanchester

Übersetzung aus dem Englischen von Matthias Fienbork (M04/DEZ.89584 Mannheimer Morgen, [Tageszeitung], 11.12.2004, Jg. 59. - Sachgebiet: Sport, Originalressort: Roman)

64) $\{\{0\}\}$ 
Begnadete Komponisten wie Guiseppe Verdi, Peter Tschaikowsky und Igor Stranwinski ließen sich ebenfalls von den erdumspannenden Worten inspirieren. Übrigens besteht heute Gelegenheit zu lauschen, wie "Paternoster" quer durch die Jahrhunderte klingt: In einer musikalischen Vesper, mit der heute der Caritasverband in der Jesuitenkirche sein Jubiläumsprogramm zum Hundertjährigen startet, gibt es ein Geburstagskonzert der besonderen Art. Mit historischen Vater-Unser-Vertonungen. Bekanntermaßen vermag die Musik mit einem Vorzug aufzutrumpfen: Ob es "Eise Papp am Himmel" (Luxemburgisch) oder "Heeregot, ii Foor, dear Di bes uunže Hemmel" (Helgoländisch) heißt, die "Übersetzung" nach Noten hört sich überall gleich an - in Minsk, Madrid oder Mannheim. wam übrigens... (M05/JAN.03150 Mannheimer Morgen, [Tageszeitung], 14.01.2005, Jg. 60. Originalressort: Lokal Mannheim für Hessen)

65) $\{\{1$; sächs. Gen; A1 $\}\}$

Dazwischen macht Manuel Diener als kleiner Titelheld ebenso gute Figur wie der Kinderchor "Doremi" der Mannheimer Musikschule, der den Knirps zunehmend doppelt, bis die Allmers in einem magischen, ernstlich rührenden Schlussbild umringt von singenden Geistern Klein Eyolfs sind.

Polanskis "Rosemary's Baby", eine Prise Nicolas Roegs' "Wenn die Gondeln Trauer tragen", ein Hauch Frankenstein und "Hui Buh": Metzen arbeitet filmisch, doch nach zwei pausenlosen Stunden ist nicht ganz sicher, ob er den Naturalismus mit den übernatürlichen Mittel der Natur auflöst oder über das selbst gesetzte Ziel hinausschießt. Mag der Horror manch einen erschrecken, eine pflegliche Behandlung von Emma Klingenfelds Übersetzung sowie gänzlicher Verzicht auf Improvisationen und Einschübe könnten versöhnlich stimmen. Weitere Aufführungen: 24. Oktober, 11., 12. und 23. November (Info und Karten unter 0621/1 680150 ).

Die Lage ist ernst: Michael Schmitter (Borgheim) und (M05/OKT.85183 Mannheimer Morgen, [Tageszeitung], 18.10.2005, Jg. 60. - Sachgebiet: Kultur, Originalressort: Kultur; Ralf-Carl Langhals: Die Untoten und ihr Schmerz auf dem Eiswürfel, [Feuilleton])

66) $\{\{0\}\}$

Dieses Werk, für über 2 Milliarden Menschen das Fundament ihres Glaubens, sei in mancher Hinsicht unter modernem Blickwinkel streitbar, sein Sinn sei zuweilen nicht direkt einsichtig. Grund genug, sich näher mit diesem wichtigen Zeugnis des Glaubens auseinanderzusetzen. Da jedoch jede Übersetzung bereits eine Interpretation des Übersetzers ist, scheint es gerade bei einem so zentralen Werk wie der Bibel sinnvoll, sich den Texten in ihrer Originalsprache anzunähern.

Zu diesem Zweck wird ein ökumenisches Bibelkolleg in der Auferstehungskirche angeboten. Am Anfang des Kurses soll eine Einführung in die Sprachen des Alten und Neuen Testamentes, Hebräisch und Altgriechisch, stehen. (M05/DEZ.99153 Mannheimer Morgen, [Tageszeitung], 01.12.2005, Jg. 60. Originalressort: Lokal Viernheim; Mit Heiliger Schrift auseinandersetzen)

67) $((1$; von + Dat.; A2))

Der Gast, "der 10000 Mal Liebe macht"

Bei der Übersetzung von Marken-Namen und Werbung lauern in China viele Fallstricke Von dpa-Korrespondent Frank Christiansen

Düsseldorf. (M06/JUL.59570 Mannheimer Morgen, [Tageszeitung], 29.07.2006, Jg. 61. Sachgebiet: Wirtschaft, Originalressort: Wirtschaft; Von dpa-Korrespondent Frank Christiansen: Der Gast, "der 10000 Mal Liebe macht") 
68) $\{\{1$; attr. Adj.; A4 $\}\}$

Birgit Pieper vom Christlichen Verein Junger Menschen (CVJM) Mannheim führte gemeinsam mit Ko-Moderatorin Miriam Frank durch das "Programm". Einen großen Teil des Abends nahm die Lobpreismusik ein: Die Band spielte moderne, rockige und meist englischsprachige Lieder. Zum Mitsingen wurden die Texte mit deutscher Übersetzung auf eine Leinwand projiziert.

Eine Theater-Einlage und eine Umfrage unter Feudenheimer Schülern, die als VideoEinspieler zu sehen war, veranschaulichten, um welche Fragen es an diesem Abend ging: Was brauche ich, um ein erfülltes Leben zu führen? Welche Rolle spielt Gott dabei?

(M07/FEB.03402 Mannheimer Morgen, [Tageszeitung], 14.02.2007, Jg. 62, Ausgabe Stadtteil Ost. - Sachgebiet: Lokales, Originalressort: Stadtteilausgabe Ost; Von unserer Mitarbeiterin Heidrun Hartelt: Kirche mal ganz anders)

69) ((1; in + Akk.; A4))

Elfjährige Pianistin überzeugt

Eine besondere Bereicherung dieser Ausstellungseröffnung waren Musikbeiträge am Piano von der erst elfjährigen Jutta Eberts, die in mehr als überzeugender Qualität gefühlvoll drei Stücke von Johann S. Bach, Rodion Schtschedrin und Claude Debussy zum Besten gab. Landrat Matthias Wilkes zeigt sich in seiner Ansprache von diesem jungen Talent begeistert: „Mit einer litauischen Mutter und einem Deutschen Vater zeigt Jutta, welch schönen Früchte aus einer Partnerschaft der beiden Länder wachsen können“, das findet auch das Publikum und lacht herzlich über diese Metapher, der zweite Lacher folgt wenig später - nach der Übersetzung ins Litauische.

Baaß und Klee begeistert

Auch Bürgermeister Matthias Baaß und Heinz Klee, Leiter der Friedrich-Fröbel-Schule, sind begeistert - vom Besuch der litauischen Delegation in Viernheim: „Das passiert nicht alle Tage, dass ein solcher Besuch den Weg in unsere Stadt findet", betont der Viernheimer Bürgermeister. (M08/JAN.06963 Mannheimer Morgen, [Tageszeitung], 28.01.2008, Jg. 63, Südhessen Morgen (Viernheim), S. 17. - Sachgebiet: Lokales, Originalressort: Viernheim; Künstler verbinden Kulturen, [Bericht [Bericht ; Nachrichten]])

70) $\{\{3$; unmarkierte Gleichsetzung; attr. Adj.; von + Dat.; A’; A4; A2 $\}\}$

Lellebollem-Fans sollten sich den Freitag, 26. September, rot im Kalender anstreichen: Dann beginnt von 17 bis 19 Uhr der Kartenvorverkauf für das neue Stück der Mundart-Schauspieler im Kadeheisl an der Beindstraße 8 (für Auswärtige: Nähe ev. Kirche). Weitere Vorverkaufstermine werden noch bekannt gemacht.

Der Titel des Stücks lautet „Hilfe, moi Alde kumme!““- die mundartliche Übersetzung von „Hilfe, meine Eltern ziehen bei mir ein!“”, ein Lustspiel in drei Akten aus der Feder von Erich

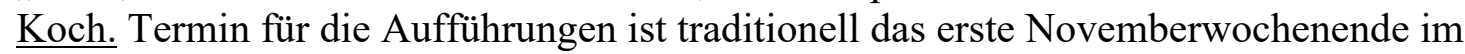
Bürgerhaus Heddesheim.

Die Premiere ist immer freitags, so dass es in diesem Jahr schon ab dem 31. Oktober (19.30 Uhr) losgeht. (M08/AUG.66108 Mannheimer Morgen, [Tageszeitung], 25.08.2008, Jg. 63, Rhein-Neckar, S. 14. - Sachgebiet: Regionales, Originalressort: Rhein-Neckar; Lellebollem: \&\#8222;Hilfe, moi Alde kumme\&\#8220; [Bericht [Bericht ; Nachrichten]])

71) $\{\{1$; von + Dat.; A1 $\}\}$ „Alles für das Feuer“" nennt sich das Kurzdrama, das dieses komplexe Thema ausgerechnet mit Hilfe eines Paars bewältigen will, dessen übermächtige Liebe alles Einzwängende sprengt. Die deutschsprachige Erstaufführung in Mannheims Kinder- und Jugendtheater 
„Schnawwl“ offenbart einige Stolperfallen bei dem Versuch, Kompliziertes zu vereinfachen. Die Übersetzung von Eva Maria Pieper schafft durch die Verwendung eines veralteten und eines zeitgemäßen Sprach-Stils zwei Zeitebenen. Teilweise rücken die Kunstfiguren weit zurück in die Vergangenheit, wenn Rittertugenden die Handlung regieren. Teilweise holt die Übertragung das Geschehen aber auch ganz nah an das Publikum heran, sobald das menschliche Dilemma erörtert wird. (M09/APR.32161 Mannheimer Morgen, [Tageszeitung], 27.04.2009, Jg. 64, Stadtausgabe, S. 31. - Sachgebiet: Kultur, Originalressort: Kultur; Von unserer Mitarbeiterin Monika Lanzendörfer: Wenn der Verstand einmal aussetzt, [Feuilleton])

72) $\{\{2$; sächs. Gen.; als; A1; A' $\}\}$

Ein trockener Humor stellt sich wie von selbst ein, wenn Wader Titel wie „Schön ist das Alter" singt und anschließend kundtut, dass er den Text vor 20 Jahren geschrieben hat. Man wird auf dieser Veranstaltung den Eindruck nicht los, dass alles etwas länger her zu sein scheint. Und so geht es mit poetischem Realismus weiter, wenn die berühmten Zeilen „Les feuilles mortes“ von Jacques Prévert in Waders eigener Übersetzung als „,Die welken Blätter“ ertönen. Oder sich Tom Paxtons „Last thing on my mind“ in ein „Ich werd” es überstehn" wandelt.

Hannes Wader, einst der sozial- und gesellschaftskritische Liedermacher und politische Widerstandssänger, ist alt geworden. (M10/APR.32819 Mannheimer Morgen, [Tageszeitung], 28.04.2010, Jg. 65, Stadtausgabe, S. 31. - Sachgebiet: Kultur, Originalressort: Kultur; Von unserer Mitarbeiterin Nora Abdel Rahman: Nichts bleibt, wie es war, [Feuilleton])

73) $\{\{0\}\}$

Interpols „Red Notice“

Die internationale Polizeiorganisation Interpol informiert ihre 188 Mitgliedsstaaten auf Antrag per „Red Notice“ regelmäßig über Verdächtige, die zur Fahndung ausgeschrieben sind. Bei Roten Benachrichtigungen - so die wörtliche Übersetzung - handelt es sich aber nicht um einen internationalen Haftbefehl. Interpol verbreitet lediglich Informationen zur Person - eine Art Steckbrief - sowie Angaben zum nationalen Haftbefehl. Die jeweiligen Mitgliedsstaaten müssen dann selber entscheiden, wie sie reagieren. (M10/DEZ.83972 Mannheimer Morgen, [Tageszeitung], 02.12.2010, Jg. 65, Stadtausgabe, S. 2. Sachgebiet: Politik, Originalressort: Politik; Interpols \&\#8222;Red Notice\&\#8220; [Bericht [Bericht ; Nachrichten]])

74) $\{\{1$; Relativpr. im Gen.; A2 $\}\}$

Allerdings nicht ohne Skepsis vor allem aus den Reihen jener, die nach Jahrzehnten einer fundamentalpolitischen Gegnerschaft einer solchen Zweckehe nicht recht über den Weg trauen wollen. Die alten Feindbilder scheinen noch lange nicht revidiert. Sogar KoalitionsBefürworter wie der energiepolitische Sprecher Ralf Löffler (Lindenfels) verleugneten in Lorsch nicht, dass es zum Teil erhebliche Vorbehalte gibt: „Ich misstraue diesen Leuten grundsätzlich." Löffler rechtfertigte ein schwarz-grünes Bündnis aber mit dem Anspruch der politischen Mitgestaltung: „Man muss mit ihnen koalieren, damit sie keinen Unsinn machen“, so Löffler über das grüne Ziel, die Reaktoren von Biblis ein für alle Mal vom Netz zu lassen: eine Forderung, die zwar im grünen Wahlprogramm steht, deren Übersetzung im Koalitionsvertrag einigen Mitgliedern aber zu schwammig formuliert ist. In dem 22-seitigen Papier ist von einer Energiewende und einem gleichzeitigen Ausbau erneuerbarer Energien die Rede. Von einem definitiven Aus für Biblis ist wörtlich nichts zu lesen. (M11/JUN.04740 Mannheimer Morgen, [Tageszeitung], 17.06.2011, Jg. 66, Südhessen Morgen 
(Lampertheim), S. 21. - Sachgebiet: Lokales, Originalressort: Keis Bergstrasse; Grüne mehrheitlich für schwarz-grünes Bündnis, [Bericht [Bericht ; Nachrichten]])

75) $\{\{1$; attr. Adj.; A4 $\}\}$

Mit den Spielerpässen gebe es keine Probleme. Trainingszeiten und Spielmodus bleiben, so sagt er, wie bisher. Für „Edingen Calcio“, der Name ist eine Hommage an den zur Heimat gewordenen Ortsteil „Edingen“ und eine Reminiszenz an die italienischen Wurzeln („Calcio“ heißt „Fußballsport“ in der deutschen Übersetzung), hat Fußballfan Fallico klare Ziele definiert: Möglichst schnell soll der Club von der C-Klasse in der Kreisliga in die A-Klasse aufsteigen. „Dann sehen wir weiter“, meint der knapp 50-jährige Geschäftsmann.

Wird sein neuer Verein die Konkurrenzsituation am Ort, den Kampf um Aktive und Zuschauer, noch verstärken? (M12/APR.06043 Mannheimer Morgen, [Tageszeitung], 21.04.2012, Jg. 67, Rhein-Neckar, S. 20. - Sachgebiet: Regionales, Originalressort: Rhein-Neckar; Von unserer Mitarbeiterin Nicoline Pilz: Bald fünf Fußballvereine in der Doppelgemeinde, [Bericht [Bericht ; Nachrichten]])

76) $((1 ;$ Gen; A2))

Auch von Landesseite habe das Projekt bislang keine Unterstützung erfahren, und das obwohl ,es als Konsequenz aus den Amokläufen in Winnenden und Wendlingen einen Landtagsbeschluss gab, der das Kultusministerium damit beauftragt hat, eine Konzeption zur flächendeckenden Einführung eines Konzeptes nach Dan Olweus über alle Schularten vorzulegen", sagt Jantzer.

Gespräche mit Olweus selbst verliefen Erfolg versprechender. „Er denkt darüber nach, einen Teil der Finanzierung, den die Übersetzung des Konzepts kosten würde, zu übernehmen“, erklärt Parzer. Sicher sei allerdings noch nichts. Umso wichtiger sei es, einen Geldgeber für das Projekt zu finden, um „endlich etwas gegen Mobbing zu tun.“ (M13/JAN.00344 Mannheimer Morgen, [Tageszeitung], 03.01.2013, Jg. 68, Stadtausgabe, S. 23. Sachgebiet: Lokales, Originalressort: Heidelberg; Von unserem Redaktionsmitglied Agnes Polewka: \&\#8222;Endlich etwas gegen Mobbing tun\&\#8220;, [Bericht [Bericht ; Nachrichten]])

77) $\{\{1$; Posspr.; A2 $\}\}$

Der Aufschwung beim maschinellen Übersetzen profitiert davon, dass sich zwei wissenschaftliche Lager einander annähern, die sich in der Vergangenheit heftige Grabenkämpfe lieferten. Da wäre die eine Seite - heute würde man sie die Google-Fraktion nennen -, die das Übersetzungsproblem mit schierer Rechenpower und ungeheuren Datenmengen bewerkstelligt. Deren Credo könnte lauten: Jeder Satz, oder zumindest ein ähnlicher Satz, steht schon irgendwo im Internet mit seiner Übersetzung. Datensammeln, Statistik und Wahrscheinlichkeitsrechnung reichen aus. Und wenn die Software den Satz „Wen brät die Hexe?" falsch übersetzt, liegt das in der geringen Textmenge an Märchen im Web. Ein Sprachverständnis setzt dieser Ansatz nicht voraus und kommt trotzdem zu verblüffenden Erfolgen, etwa bei Mehrdeutigkeiten. (M13/OKT.01239 Mannheimer Morgen, [Tageszeitung], 05.10.2013, Jg. 68, Stadtausgabe, S. 10. Originalressort: Forschung und Technik; Meist im Sinn der Verfasser, [Bericht ; Reportage])

78) $\{\{1$; von + Dat.; A1 $\}\}$

„Das Paket“ im Theater Felina

Neckarstadt. „Das Paket“, ein Theaterstück von Philippe Claudel in der Übersetzung von Rainer Escher, wird als deutschsprachige Erstaufführung produziert von der Gruppe Neues EnsemblE im Theater Felina (Holzbauerstraße 6) heute, Mittwoch, 12. und Freitag, 14. 
Februar, 20 Uhr, aufgeführt. Der Eintritt kostet zehn (ermäßigt fünf) Euro. (M14/FEB.03338

Mannheimer Morgen, [Tageszeitung], 12.02.2014, Jg. 69, Mannheim Mitte, S. 29. Sachgebiet: Lokales, Originalressort: Mannheim Mitte, [Bericht ; Nachrichten])

79) $\{\{2 ;$ für + Akk.; Apposition; A2; A` $\}\}$

ein Zufall brachte mich in den Ruf eines strategisch phantasiestarken "Militärschriftsteller" als nämlich, was mit dem Element des Stellungskrieges zusammenhing, lange Zeit gar nichts geschah, prophezeite ich die Somme-Schlacht, die psychologisch bedingt den gemeinsamen Einsatz französischer und britischer Divisionen fordern müßte. mein Rühmlein wuchs bei den Soldaten. nicht bei allen Zivilisten - mit dem Vorstand des örtlichen "Alldeutschen Verbandes", einem Studienrat, der im Unterricht für "ignavi et impavidi" die Übersetzung "Nau- und Flaumänner" vorschlug, machte ich einen Mordskrach, den ich bis ins Stuttgarter Ministerium trug. was von dieser Gruppe an Abstrusem vorgetragen wurde, ersah ich, als mir eine gedruckte Karte gezeigt wurde, in der ein "Korridor" die Gegend um Belfort mit Toulon verband, dort aber deutsche Kriegsschiffe stationiert wurden! das war ein Sonderstück aus dem Kapitel "Kriegsziele". (MK1/MHE.00000 Heuss, Theodor: Erinnerungen 19051933, [Memoiren]. Tübingen: Rainer Wunderlich Verlag Hermann Leins, 5. Auflage 1964 (1. Auflage 1963), 459 S. [S. 200])

80) $((0))$

Später werden die aus den vegetabilen Formen gewonnenen Objekte fotografiert und schließlich beginnt der Abstraktionsprozess durch die Übertragung in ein artfremdes Medium, z. B. Vlies, Textilien usw.

Der Künstler ist, wie es eine Studentin ausdrückt, "der Übersetzer der Natur". Künstler erkennen einander an der Art der "Übersetzung", die Form ist ihre Sprache der Verständigung. Ziel des Unterrichts ist nicht die Ideenfindung, sondern das Bewußtsein für das Tun und die Hingabe an jeden einzelnen Augenblick.

Jüngste Arbeiten zeigt Maria Blaisse in der Galerie 5020. (N00/AUG.38324 Salzburger Nachrichten, [Tageszeitung], 24.08.2000. - Sachgebiet: Kultur, Originalressort: FESTSPIELSOMMER 2000; Auch von der eigenen Natur lernen)

81) $\{\{2$; sächs. Gen; Gen.; A1; A2 $\}\}$

DARMSTADT (dpa).

Der Frankfurter Übersetzerin Eva Moldenhauer ist der mit umgerechnet rund $140.000 \mathrm{~S}$ dotierte Paul-Celan-Preis zuerkannt worden. Damit würdigt der Deutsche Literaturfonds Moldenhauers Übersetzung des Romans "Die Akazie" von dem Nobelpreisträger Claude Simon. (N91/JUN.01955 Salzburger Nachrichten, [Tageszeitung], 25.06.1991. - CelanÜbersetzerpreis für Eva Moldenhauer)

82) ((1; Gen.; A2))

Ein Instrumentarium soll Identitäten sichern o Die Unterstützung von Projekten mit einer Dimension, die dem europäischen Bewußtsein und einer europäischen Identität dienen, zum Beispiel die "Kulturstadt". o Unterstützung von Bildungsmaßnahmen, welche dem Austausch von Schülern, Lehrlingen und Studenten innerhalb der Mitgliedstaaten dienen, durch EGStipendien. o Förderung der europäischen Filmindustrie, eines einheitlichen hochauflösenden Fernsehsystems sowie ein Aktionsprogramm für die weitere Entwicklung der Telekommunikation. o Mit Rücksicht auf die neun Amtssprachen der EG - Deutsch, Französisch, Englisch, Niederländisch, Dänisch, Italienisch, Spanisch, Portugiesisch und Griechisch - wird die Übersetzung literarischer Werke sowohl durch Ausbildungsprogramme für Übersetzer als auch durch einen europäischen Übersetzerpreis gefördert. o Verhandlungen 
über die Angleichung der Urheberrechte. o Die Förderung des Buches in allen Facetten:

Preise und Stipendien für Schriftsteller und Übersetzer, Förderung von regionalen Verlagen sowie Gestaltungsvorschläge für den Buchhandel. (N92/SEP.32132 Salzburger

Nachrichten, [Tageszeitung], 02.09.1992. - Kulturerbe fördert das Verständnis)

83) $((0))$

Was die Besonderheit dieses Chors ausmacht, ist die nicht nur als "exotisch" zu definierende

- Magie eines expressiven, rauhen, ungeschminkten, offenen Klangs und einer aus bäuerlicher

Tradition stammenden ursprünglichen Farbe. Jede einzelne Stimme "erspielt" sich über ihre schier halsbrecherisch virtuose Individualität hinaus das Spektrum einer unverbraucht

archaischen Kraft, zeigt pure Vokal-Kunst aus einer reichen Gesangstradition, die sich auch in ihrem neuen "Kunst-Charakter" Ursprünglichkeit bewahrt.

Die Texte, die hier gesungen werden, brauchen nicht die wörtliche Übersetzung, sie erschließen sich im Kern ihrer Stimmung: Klagelieder, Volksepen, byzantinische Liturgik. Die Klangsprache orientiert sich hauptsächlich am reibenden Intervall der Sekund und an Mikrointervallen, die sich um einen Grundton ranken. So "transportiert" ein Chor auch musikalisch die Geschichte eines Volkes, die immer eine Geschichte von Leid und Tränen ist. (N93/MAI.16182 Salzburger Nachrichten, [Tageszeitung], 04.05.1993. - DIE AKTUELLE SCHALLPLATTE)

84) $\{\{1$; Gen.; A1 $\}\}$

Als ein "Grusical", wie es das Programmheft vorgibt, war mir die Story zu wenig furchterregend, als Parodie auf Horrorfilme zu wenig bissig, um als Märchen für Erwachsene zu gelten, zu vordergründig. Eine harmlose Nichtigkeit liegt also vor, ein stoffliches Leichtgewicht, das sich mit den großen Würfen des Genres nicht messen kann.

Dem mageren Plot und der flachen Übersetzung Michael Kunzes zum Trotz gelingt dem Linzer Musical-Team (Inszenierung: Wilfried Steiner, Ausstattung: Brigitte Erdmann, Choreographie: Antony Taylor) dennoch eine respektable, in Details liebevoll gearbeitete und strekenweise sogar recht komische Umsetzung. Pluspunkte setzt auch die Band, die unter der Leitung von Michael Cook die musikalischen Zügel dezent, aber straff in der Hand hat. Einfache Liednummern in der Machart der frühen sechziger Jahre sind zu hören, Balladen im Happy-Sound und ein paar "Schmachtfetzen", bei denen die Akteure förmlich bis zu den Knöcheln im Schmalz stehen. (N93/OKT.38363 Salzburger Nachrichten, [Tageszeitung], 20.10.1993. - Ein stoffliches Leichtgewicht zur "gruseligen" Unterhaltung)

85) $\{\{2$; sächs. Gen; von + Dat.; A1; A2 $\}\}$

Noch heute existiert in Argentinien der linke kulturpolitische Verband deutschsprachiger Einwanderer "Vorwärts"; Bauer, Mitglied der argentinischen KP, bekleidet das Amt des Vizepräsidenten.

Als Reverenz an seine zweite spanischsprachige Heimat hat Alfred Bauer seinen Vornamen hispanisiert: "Alfredo", und als Alfredo Bauer hat er in Argentinien einen bekannten Namen als Theater- und Romanschriftsteller, als Übersetzer und als Autor wissenschaftlicher Werke. Vor drei Jahren, zum Kolumbus-Jubiläum, führte eine Bühne in Buenos Aires Bauers

Übersetzung von Jura Soyfers "Broadway-Melodie 1492" auf, die erste Soyfer-Premiere überhaupt in einem lateinamerikanischen Land. Im Geiste Soyfers verfaßt ist eines von Bauers eigenen Stücken, "Des Teufels Wettermacher", das den Grundgedanken aus "Der Lechner Edi schaut ins Paradies" variiert.

Die größte Auflage aller seiner Bücher hat aber Bauers Stefan-Zweig-Roman "Der Mann von gestern und die Welt" (auf deutsch in der Edition Atelier, Wien 1993, erschienen) erlebt, und für diesen Wurf ist der gebürtige Wiener mit dem argentinischen Staatspreis für Literatur 
ausgezeichnet worden (als einziger Träger dieses Preises übrigens schon zum zweiten Mal).

(N95/MAI.19839 Salzburger Nachrichten, [Tageszeitung], 27.05.1995. - Dem NS-Regime entkommen Von Harald Steiner Österreichische)

86) $\{\{0\}\}$

Dort wurden sie ins Italienische übersetzt. Obwohl durch die Übersetzungsmühle gedreht, darf gesagt werden, daß sie nichts von ihrer Qualität verloren hatten. Die Gedichte wurden von den deutschsprachigen Autoren in ihrer Sprache vorgetragen, dann wurde die

Übersetzung gelesen.

Von solchem Applaus kann ein Autor hier nur träumen

Aus Klagenfurt war Ingram Hartinger zu Gast. (N96/JUL.30973 Salzburger Nachrichten, [Tageszeitung], 27.07.1996. - Mit Musik und Poesie Politik machen)

87) $\{\{0\}\}$

Der in London lebende Autor entwirft in "Des Mauren letzter Seufzer" ein Panoptikum seines Geburtslandes Indien im 20. Jahrhundert. Anhand der persönlichen Geschichte einer Gewürzhändlerdynastie schildert Rushdie mit meisterhafter Fabulierkunst die Veränderungen und den Werteverlust dieser Gesellschaft. Es spricht für die Qualität des Textes, daß man ihn zweimal hintereinander - eben im Original und in der Übersetzung - anhören kann, ohne daß einem auch nur eine Sekunde langweilig wird. Rushdie ist ein sehr traditioneller Erzähler, aber einer, der ein sehr hohes Niveau erreicht hat.

Helmut Schneider (N96/OKT.45348 Salzburger Nachrichten, [Tageszeitung], 31.10.1996. - Burgtheater wurde für Salman Rushdie zur "Festung")

88) $\{\{2$; attr. Adj.; Gen; A4; A2 $\}\}$

Die Branche "träumt" davon, daß sich in Deutschland ein ähnliches Leseverhalten entwickelt wie in Frankreich. Dort seien Bildgeschichten "ein ernsthaftes Medium der populären Literatur".

Als richtungsweisend für Comics mit historischen Themen nannte die Wissenschaftlerin unter anderem Goethes "Faust" oder die umstrittene Verarbeitung schmerzlicher Erlebnisse aus der Hitlerdiktatur, wie die deutsche Übersetzung der "Maus" des US-Amerikaners Art Spiegelmann. Auch "Der Wind in den Weiden" (1998) von Michael Plessix sei ein Stoff aus Fantasy und Märchenhaftem für Kinder, Jugendliche und Erwachsene gleichermaßen.

Überhaupt seien Comics nicht mehr so leicht bestimmten Altersgruppen zuzuordnen wie früher. (N99/FEB.04635 Salzburger Nachrichten, [Tageszeitung], 04.02.1999. Sachgebiet: Kultur, Originalressort: Kultur; Der Comic wird anspruchsvoller)

89) ((2; Gen.; in + Akk.; A2; A3))

Immer wieder sind es Farbakkorde, reich orchestriert und hart gegeneinander gesetzt, die scheinbar unvereinbare Entwicklungen zu einer Synthese verweben. Der Eigenwert der Farbe erfährt in seinen Bildern eine sublime Steigerung.

Hofmann hat sich stets konsequent einer Nachbildung der gegenständlichen Welt verweigert und in der "Übersetzung des Seelischen in die Form" einen Weg gefunden, der auch für seine Schüler richtungweisend geworden ist.

Wolfgang Richter (N99/AUG.34391 Salzburger Nachrichten, [Tageszeitung], 18.08.1999. - Sachgebiet: Kultur, Originalressort: FESTSPIELSOMMER 1999; Ölmalerei von mitreißender Kraft)

90) $\{\{2$; attr. Adj.; von + Dat.; A1;A2 $\}\}$ 
des zuständigen Berner Amtsgerichts, bestätigt NEWS, dass Kanzler Schüssel bereits als Zeuge beantragt ist. Der Schweizer Justiz macht diese Tatsache mittlerweile schweres Kopfzerbrechen. Obwohl die amtliche Übersetzung von Schreibers streng geheimer Aussage in Toronto erst in "etwa ein bis zwei Monaten" in Bern erwartet wird, ist klar, was jetzt kommt. Studiger: "Wir haben umfangreiche Vorprüfungen betreffend des beantragten Zeugen Wolfgang Schüssel durchgeführt. Es ist rechtlich wohl ausgeschlossen, einen ausländischen Bundeskanzler zu einer Zeugenaussage zu zwingen, die er nicht tätigen will.

(NEW02/MAR.00007 NEWS, 07.03.2002, S. 16, Sachgebiet: Politik, Originalressort: POL Politik; Zeuge Schüssel)

91) $\{\{2$; attr. Adj.; Gen.; A4; A2 $\}\}$

Verteilt werden soll die türkisch- und kurdischsprachige Zeitung an möglichst viele Kurden in Mecklenburg-Vorpommern und der ganzen Bundesrepublik.

An eine deutsche Übersetzung künftiger Ausgaben sei gedacht, doch das wäre auch ein finanzielles Problem, sagt Erginci. Die 60 nordischen Kurden, die im Berndshofer Heim untergebracht sind, fühlen sich genauso wie viele andere ihrer Landsleute in den Heimen in Mecklenburg-Vorpommern nicht genügend vertreten durch die zahlreichen kurdischen Vereine und Zentren. (NKU00/NOV.00925 Nordkurier, 03.11.2000, Sachgebiet: Lokales, Originalressort: Lokales Ueckermnde; Berndshofer Kurden geben Zeitung heraus)

92) $\{\{2$; als; attr. Adj.; A‘; A4 $\}\}$

Die wenigsten wissen, dass die Regisseurin von inzwischen acht Filmen auch sieben Romane geschrieben hat. Ihr dritter, der 1974 unter dem Titel "Le Soupirail" erschien, wurde in der Verfilmung "Une vraie jeune fille" erst im Vorjahr für die französischen Kinos freigegeben. Als "Ein Mädchen" liegt er nun in deutscher Übersetzung vor. Der Roman ist charakterisiert durch dieselbe Brisanz und Provokanz wie Breillats Filme, die als pornografisch und antifeministisch verunglimpft werden. Er handelt von der pubertierenden Alice, die ihre Ferien in der Provinz verbringt. (NKU01/JUL.09219 Nordkurier, 28.07.2001, Sachgebiet:

Nachrichten, Originalressort: Nachrichten Mecklenburg-Vorpommern; Provozierender Versuch)

93) $\{\{1$; für + Akk.; A2 $\}\}$

Am kommenden Sonnabend nun ist es soweit: Ingrid Brun, Veranstaltungskoordinatorin des Klosters, konnte "Tierra Flamante" für den 10. August verpflichten. Für ihren Namen haben die neun Tänzerinnen der Gruppe eine sehr freie Übersetzung gefunden. Von der wörtlichen aus dem Spanischen übertragenen "Glänzende Erde" leiten sie mit einem Augenzwinkern das "Neuland" ab. Neuland betraten die Frauen - zwischen 29 und 66 Jahre alt - als sie sich zusammenfanden, um gemeinsam mit Profis aufzutreten. (NKU02/AUG.01712 Nordkurier, 06.08.2002, Sachgebiet: Lokales, Originalressort: Lokales Prenzlau; Flamenco verzaubert erneut im Friedgarten)

94) $\{\{0\}\}$

Es war dann der Tod von Heinrich II. nach einer Verwundung während eines Turniers im Jahre 1559, der Nostradamus schließlich zu einem gewaltigen Ruhm verhalf. Das tragische Ende des Königs wurde mit einer seiner Centurien in Zusammenhang gebracht, die nach einer Übersetzung verkündet hatte: "Der junge Löwe wird den älteren überwinden. / Auf einer Kampfbahn im Einzelduell. / In einem goldenen Käfig wird er seine Augen durchstechen. (NKU03/DEZ.04479 Nordkurier, 13.12.2003, Sachgebiet: Nachrichten, Originalressort: Nachrichten Mecklenburg-Vorpommern; Der Mann dunkler Prophezeiung) 
95) $\{\{1$; attr. Adj.; A4 $\}\}$

Darmstadt (ddp). Zum 25-jährigen Bestehen von R.E.M. erscheint am 2. Mai das Buch "Inside Out" von Craig Rosen in deutscher Übersetzung. Der Autor erzählt in dem Buch die Geschichte der einzelnen R.E.M.-Songs, wie die Agentur Promoteam Schmitt \& Rauch am Dienstag in Darmstadt mitteilte. "Inside Out" basiert auf Originalinterviews mit den R.E.M.Musikern. (NKU05/APR.01181 Nordkurier, 06.04.2005, Buch über R.E.M. erscheint auf Deutsch)

96) $((1$; in + Akk.; A3))

Sabine Lange ist 1953 in Stralsund geboren, hat Musik sowie Literatur studiert und sich seit 1984 in Feldberg intensiv mit dem Werk von Hans Fallada beschäftigt. Aus diesem biografischen Kreislauf ergeben sich die Pole, manchmal Antipoden, ihres dichterischen Strebens, die sie umgebende Landschaft in eine poetische Landschaft zu holen, eine Wortmalerei zu betreiben, die entwirft und nicht nur schöne Wortbilder anhäuft: "Heute ein Tag/ an dem Jugend/ zurückgewonnen wird/ Land/ dem Meer abgerungenà" Die Landschaft, die Natur, das Tier sind die Fluchtpunkte im Guten. Ihr lyrischer Ton ist längst gefestigt, es entsteht ein Sound, was etwa Jenny Williams bei der Übersetzung ins Englische bemerkte, als die irische Germanistin die Sammlung "Immer zu Fuß" (1994) im vorigen Jahr unter dem Titel "The Fishermen Sleep" herausgab. Diese Sprache verbietet sich alle Ausschweifungen, diese Lyrik strebt nach Konzentration und nach der Lust auf ins Kleinste gedrängte Mehrfachbedeutungen, betreibt Äquilibristik mit Metaphern. Man spürt beim Lesen das unbedingt Existenzielle der Verse für die Dichterin. (NKU06/JAN.05268 Nordkurier, 26.01.2006, Alle Elfenbeintürme belegt)

97) $\{\{2$; attr. Adj.; für + Akk.; A4; A2 $\}\}$

Die Universität Greifswald will nicht in die Pferdezucht einsteigen - obwohl im neu gebauten Institut für Physik ein Mustang steht. Die Wissenschaftler haben sich die Bezeichnung einer Pferderasse für eine Testanlage entliehen. Die Wortschöpfung MuSTAnG bringt die englische Übersetzung für Myonen-Weltraumwetter-Teleskop in Greifswald auf einen griffigen Punkt. "Wir wollen künftig als Teil eines internationalen Netzwerks zwischen Deutschland, Brasilien, Japan und Australien vorhersagen können, wenn die Erde durch Teilchenstürme der Sonne bedroht wird", erläutert Professor Rainer Hippler. "So ganz passend ist die Bezeichnung Teleskop allerdings nicht", räumt der 59-jährige Physiker ein.

(NKU07/JUN.00247 Nordkurier, 01.06.2007, Im Wettlauf mit dem Sonnensturm)

98) $\{\{2 ;$ attr. Adj.; Gen.; A4; A2 $\}\}$

Der Angeklagte hatte zunächst die Tat zugegeben, später aber sein Geständnis widerrufen. Mehrere Anträge der Verteidiger sorgten für einen zähen Auftakt. Nach der dritten Verhandlungsunterbrechung stellte Anwalt Oliver Wallasch einen Befangenheitsantrag gegen die Kammer, weil unter anderem die Anträge auf eine russische Übersetzung der Anklageschrift und die Hinzuziehung eines Dolmetschers abgelehnt worden waren. Der Vorsitzende Richter Sebastian Bührmann schloss danach die Sitzung. (NKU08/NOV.01307 Nordkurier, 05.11.2008, Prozess um Klotz-Angriff)

99) $\{\{1$; attr. Adj.; A4 $\}\}$

Es geht zurück auf Anregungen, die Rollenhagen 1566 in Wittenberg durch die Vorlesungen von Veit Windsheim (15011578) erhielt. Der hatte die griechische Batrachomyomachia (Krieg der Frösche und Mäuse) vorgestellt eine epische Parodie, die man lange Zeit Homer zuschrieb, die aber sicher erst später entstand. Nun regte Windsheim seine Studenten an, 
dieses Werk in deutscher Übersetzung bearbeitet herauszubringen. Die danach von Rollenhagen vorgelegte Variante fand die Anerkennung seines Dozenten. Bereits damals wollte Rollenhagen das Material erweitern, was jedoch aus von ihm nicht mitgeteilten Gründen erst später geschah und dann 1595 zu ersten Drucklegung führte.

(NKU09/MAI.03561 Nordkurier, 11.05.2009, Froschkönig Bausback im Krieg Vor 400 Jahren starb der Schulmann und Dichter Georg Rollenhagen, der Schöpfer des Froschmeuseler)

100) ((2; Gen.; in + Akk.; A2; A3))

Darüber informierte Torsten Gertz, Vorsitzender des Heimatverbandes. Im Malchiner Museum soll dann eine Präsentation über Jacob Schimmelmann zu sehen sein, der 1712 in Demmin geboren wurde. Bekannt wurde er durch die Übersetzung der altisländischen EddaDichtung ins Hochdeutsche. (NKU10/FEB.03483 Nordkurier, 13.02.2010, Präsentation angekündigt) 
1) $\{\{1+2$; mit + Dat.; Subj.; Dat.; A3; A1;A2 $\}\}\{\{\text { SVG; Dat. A. geben }\}\}^{230}$

Die Zähmung der Naturkräfte in den industriellen Produkten ging mit dem Verlust vom geheimen Wissen um ihre Beschaffenheit einher. Das Feuer war nur noch Energie und kein Wesen mehr.

Die Malerin Vija Celmins hat der Sorge der Pythia mit ihren Bildern Ausdruck gegeben. Sie wurde 1938 im lettischen Riga geboren, erlebte die Besatzung durch die Nazis, den Einmarsch der Kommunisten, floh mit ihren Eltern 1945 ins zerstörte Deutschland und von dort 1949 weiter in die USA. Später lebte sie 18 Jahre lang in Los Angeles, das bereits damals nicht gerade als friedliebende Stadt bekannt war. (A97/MAI.02742 St. Galler Tagblatt, [Tageszeitung], 10.05.1997, Jg. 53. Originalressort: TB-KUL (Abk.); * Gerhard mack: Die Herrin des Feuers, [Bericht])

\section{2) $\{\{0\}\}$}

In seinem Gespräch mit dem Präsidenten Walter Moser war herauszuhören, dass er durch die Musik Gott erlebe, der nicht nur Komponisten inspiriere, sondern auch Musikanten und Dirigenten.

Subtile Gestaltung

Der Posaunenchor steht unter der Leitung von Sabine Hasler-Egli, die es verstand, den Gehalt der einzelnen Werke subtil in Ausdruck und Dynamik zu gestalten. Das swinghafte Element in «Three Kings Swing» des Heilsarmee-Komponisten William Himes liess das Thema aus der «Arlesienne-Suite» von Georges Bizet in neuem Licht und Zauber erstrahlen. «The Reason» von Ernest Rance gab dem Solocornettisten Peter Weber Gelegenheit zu brillieren. (A97/NOV.33186 St. Galler Tagblatt, [Tageszeitung], 03.11.1997, Jg. 53. Originalressort: TB-GO (Abk.); * Rolf Schnelli: Durch die Musik Gott loben und preisen, [Bericht])

\section{3) $\{\{1$; davon + Nebensatz; A2 $\}\}$}

Der 3. November kam, und an eben diesem Tag, dem Einsendeschluss, ging Erika Remund nach St.Gallen. Sie hatte ihre Entwürfe, drei an der Zahl, «ganz speziell eingepackt. Für mich war auch das Ausdruck davon, dass für mich die Wettbewerbsteilnahme etwas Spezielles war». Sie lächelt: «Die Einpackerei gestaltete sich beinahe wie ein Ritual.»

Yolanda gewann (A97/NOV.37833 St. Galler Tagblatt, [Tageszeitung], 26.11.1997, Jg. 53. Originalressort: TB-GO (Abk.); * Marianne Bargagna: «Yolanda III» mit drei Kuhköpfen gewann, [Bericht])

4) $\{\{1+1$; damit; dass-Subjektsatz; A3; A2 $\}\}\{\{$ SVG; zum A. bringen + Passiv $\}\}$ «Der Regierungsrat beabsichtigt ferner, eine Erhöhung der Amtsdauer für die Kantonalen Behörden von drei Jahren (vgl. Art. 65 Abs. 1 der Kantonsverfassung) auf vier Jahre zu beantragen. Mit Blick auf eine möglichst einheitliche Regelung für die Amtsdauer auf kantonaler Ebene schlägt er vor, in Art. 7 Abs. 1 des Entwurfs für ein Gemeindegesetz, wo die Amtsdauer in den Gemeinden geregelt ist, auf die Regelung für die kantonalen Behörden

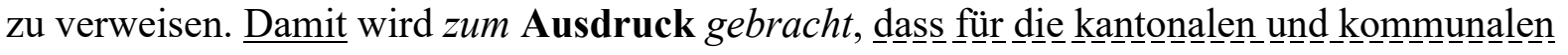

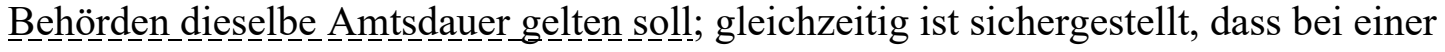

\footnotetext{
${ }^{230}$ Die unterschiedlichen Klammern stehen für die einzelnen Bedeutungen von Ausdruck: $\{\{$ Bed. I $\}\} ;[[$ Bed. II]].
} 
Verlängerung der Amtsdauer auf kantonaler Stufe keine Änderung des Gemeindegestzes nötig wird.»

5 «Auf dem Weg zur

» (A98/FEB.07551 St. Galler Tagblatt, [Tageszeitung], 06.02.1998, Jg. 54.

Originalressort: TB-KAP (Abk.); Regierungsrat AR: Bericht und Antrag an den Kantonsrat für die 2. Lesung des Gemeindegesetzes, [Bericht])

5) $\{\{1+1$; damit; Subj.; A3; A2 $\}\}\{\{$ SVG; zum A. bringen + Passiv $\}\}$

Viele modernen Inszenierungen sind derart unmotiviert, als ginge es einzig darum, zu zeigen, dass jemand sich auf der Bühne extrem zu bewegen und dabei gleichzeitig zu singen vermag. Wofür? Wa a soll damit zum Ausdruck gebracht werden? Macht es die Rolle stärker? Von Wieland Wagner lernte ich, selbst Gesten nur sehr sparsam einzusetzen. (A98/MAI.29972 St. Galler Tagblatt, [Tageszeitung], 08.05.1998, Jg. 54. Originalressort: TB-KUF (Abk.); Interview: Adalbert Reif: Oper ist: singen, singen, singen, [Interview])

6) $\{\{0+2$; keine Real.; Subj.; Akk; kein Argument; A1; A2 $\}\}\{\{$ SVG; zum A. bringen $\}\}$ Eine literarische Motivation stand also am Anfang - und eine politische. Die erste Veranstaltung am 4. Juni 1992 wurde vom thurgauischen Ständerat Thomas Onken moderiert und hatte «Bodensee - Euroregion?» zum Thema. Ulrike Läng_nle aus __ Vorarlbergerg, Jochen

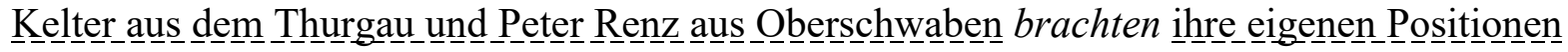
und Erfahrungen zum Theme-

In Wochen- oder Monatsabständen folgten dann weitere Veranstaltungen, immer mit der gleichen Grundstruktur: Drei Vortragende, zu einer übergreifenden Thematik versammelt und von einem vierten moderiert. An Themen war in den letzten sechs Jahren kein Mangel: «Mundart heute», gab es, oder: «Kriminale Geschichten», «Literarische Experimente», «Kinderliteratur», «Obsessionen». (A98/JUL.47111 St. Galler Tagblatt, [Tageszeitung], 14.07.1998, Jg. 54. Originalressort: TB-THG (Abk.); Bernadette Conrad: Zur Grenzöffnung beitragen, [Bericht])

7) $\{\{1+2$; mit + Dat.; Subj.; Akk; A3;A1;A2 $\}\}\{\{$ SVG; zum A. bringen $\}\}$ Auch als er zu seiner beruflichen Weiterbildung in Paris weilte, kam Meier in Kontakt mit der Malerei. Meier hätte sich schon damals gerne mit Malerei beschäftigt, aber sein Beruf ging ihm vor, und er hatte ja damals auch noch nicht genügend Zeit für sein Hobby. Mit

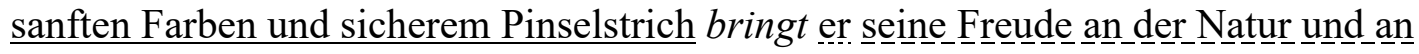
Landsch haften zum Ausdruck. Die Vernissage ist am 24. Oktober um 14 Uhr. Zur Eröffnung der Ausstellung spricht Vizeammann Josef Senn. (A98/OKT.64627 St. Galler Tagblatt, [Tageszeitung], 14.10.1998, Jg. 54. Originalressort: TT-NEC (Abk.); Blues Night mit Andy Egert, [Bericht])

8) $\{\{0+2$; keine Real.; Subj.; dass-Objektsatz; kein Argument; A1; A2 $\}\}\{\{$ SVG; zum A. bringen $\}\}$

Malcolm Green will das Verdienst nicht für sich selbst reklamieren. Seine Erklärung ist lapidar: das bei vielen Leuten ausgeprägte Bedürfnis nach gemeinsamem Singen sei die Grundlage; am Leitenden liege es nur mehr, den Anstoss zu geben. Die_Worte_Greens bringen

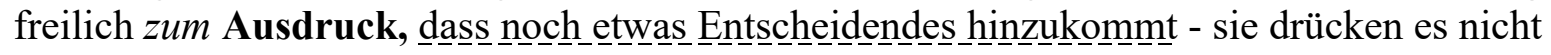
buchstäblich, aber atmosphärisch aus: in jedem Wort bebt Begeisterung und mit ihr der Glaube an die Kraft der Musik. Sie ist nicht zu übertreffen, wo Musik aus dem Herzen kommt. Davon ist Malcolm Green fest überzeugt. (A98/DEZ.77698 St. Galler Tagblatt, [Tageszeitung], 01.12.1998, Jg. 54. Originalressort: TB-SG (Abk.); hannes nussbaumer: Musik als Angelegenheit des Herzens, [Bericht]) 
9) $\{\{1+2$; mit + Dat.; Subj.; Akk.; A3; A1;A2 $\}\}\{\{$ SVG; zum A. bringen $\}\}$

Geweckt und gefördert wurde er durch zwei Onkels, die in Peru arbeiten. Anlässlich einer Reise nach Peru 1997 wurde der Wunsch wieder lebendig, die Menschen und ihre Kultur näher kennenzulernen und mit ihnen gemeinsam unterwegs zu sein. Mit ihrem Einsatz möchte

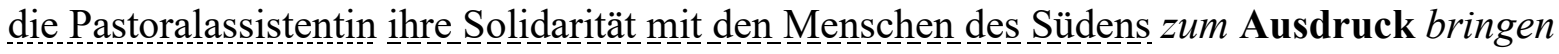
und selbst einen Beitrag leisten, dass die Welt ein wenig gerechter wird.

Wichtig ist ihr auch die Vernetzung mit den Menschen in der Schweiz, der Brückenschlag vom Norden in den Süden und umgekehrt. «Denn nur wenn sich auch hier etwas verändert, kann es in den ärmeren Ländern auch zu dauerhaften Veränderungen kommen», sagt sie.

(A99/FEB.11318 St. Galler Tagblatt, [Tageszeitung], 16.02.1999, Jg. 55. Originalressort: TB-SG (Abk.); Für eine gerechtere Welt, [Bericht])

10) $\{\{0+2$; keine Real.; Subj.; Akk; kein Argument; A1; A2 $\}\}\{\{$ SVG; zum A. bringen $\}\}$ Viele zeigten Lust auf Farbe

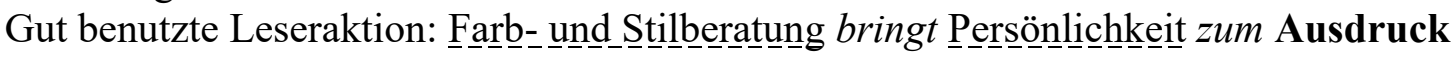
«Wiler Zeitung · Volksfreund» lud zum Vortrag «Lust auf Farbe - aber welche?» ein. Modeberaterin Luzia Leuenberger zeigte im Hof, worauf beim Ankleiden zu achten ist und welche Stilbrüche zu vermeiden sind. (A99/MAR.23167 St. Galler Tagblatt, [Tageszeitung], 31.03.1999, Jg. 55. Originalressort: WV-WIL (Abk.); Gisela Legler: Viele zeigten Lust auf Farbe, [Bericht])

11) $\{\{0+2$; keine Real.; Subj.; Akk; kein Argument; A1; A2 $\}\}\{\{$ SVG; zum A. bringen $\}\}$ Es täte den Menschen sehr wohl, wenn sie von Friedensbotschaften aus den Kriegs- und Krisengebieten hören könnten, und wenn Friede in Familien aufkäme, wo Spannungen und Zwietracht überhand genommen hätten. Alle seien eingeladen, beimHerrn Ruhe zu finden und

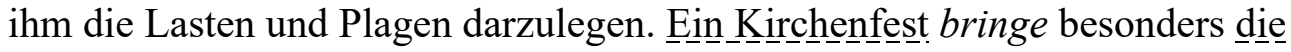
Zusammen-ngehörigkeit zum Ausdruck.

Vorbildliche Frau

Margaretha wurde in der Zentraltürkei geboren. (A99/JUL.47657 St. Galler Tagblatt, [Tageszeitung], 06.07.1999, Jg. 55. Originalressort: RT-ORT (Abk.); RÖsli Zeller: Eine Botschaft, die aufhorchen lässt, [Bericht])

12) $\{\{0+2$; keine Real.; Subj.; Dat; kein Argument; A1; A2 $\}\}\{\{$ SVG; Dat. A. verschaffen $\}\}$ Appenzellerfest 2000

Am Wochenende vom 1. bis 3. September 2000 soll auf der Linie Gonten-Appenzell-GaisTeufen ein Appenzeller Fest für alle Einwohnerinnen und Einwohner der beiden Halbkantone stattfinden. Diesses einmalige Fest will dem Gé Gefühl der_Freude am Leben Len und der Gegenwart Ausdruck verschaffen und sich nicht den heutigen Schwierigkeiten beugen. Die Initianten setzen sich aus öffentlich engagierten Persönlichkeiten aus Politik und Wirtschaft der beiden Halbkantone zusammen.

Alle sind eingeladen, Mitglied des «Vereins Appenzellerfest» mit einem einmaligen Beitrag von 10 Franken zu werden. (A99/AUG.58348 St. Galler Tagblatt, [Tageszeitung], 26.08.1999, Jg. 55. Originalressort: AT-VOR (Abk.); Öffentliche Parkplätze bewirtschaften?, [Bericht])

13) $\{\{0+1$; keine Real.; Subj.; kein Argument; A2 $\}\}\{\{$ SVG; zum A. kommen $\}\}$ Evangelisch Birwinken feierte Dankgottesdienst für Ernte und Kirchensanierung 
Ein grosser Erntesegen und eine Kirche, die in neuem Glanz erstrahlt: In Evangelisch Birwinken herrscht Grund zur Freude und Dankbarkeit. Im Festgottesdienst kam beides zum Ausdruck.

Urs Müller

Birwinken. (A99/OKT.71164 St. Galler Tagblatt, [Tageszeitung], 11.10.1999, Jg. 55.

Originalressort: TB-ABI (Abk.); Urs Müller: Dankbar, wer die Werte schätzt, [Bericht])

14) $\{\{1+2$; mit + Dat.; Subj.; Akk.; A3; A1;A2 $\}\}\{\{$ SVG; zum A. bringen $\}\}$

Ein Medley, arrangiert von Willi Valotti, machte die Tango-Rhythmen im Kirchenraum fast spürbar. Zwei Walzer von Antonin Dvorak entführten sodann in die Welt der Klassik. Einenen Hauch von melancholischer_Fröhlichkeit brachten die Spielenden mit dieser Musik zum Ausdruck, was an die Heimatliebe des tschechischen Komponisten erinnerte.

Südlich der Alpen

Nach Dvorak fand man sich in einer Suite von Ernst Fischer «Südlich der Alpen» wieder. (A00/JAN.03892 St. Galler Tagblatt, [Tageszeitung], 18.01.2000, Jg. 56. Originalressort: AT-INN (Abk.); SIBYLLE SPEISER: Akkordeon, wie man es selten hört, [Bericht])

15) $\{\{0+2$; keine Real.; Subj.; Akk; kein Argument; A1; A2 $\}\}\{\{$ SVG; zum A. bringen $\}\}$ Anderseits hat kein Papst so viel für die Verständigung zwischen Christen und Juden und die Anerkennung Israels durch den Vatikan unternommen wie Johannes Paul II. Auch er hat somit, wie Sadat, einen Weg geöffnet: jenen des gegenseitigen Respekts. Die _politis $\underline{\text { ch }} \underline{\text { hen }}$ un-

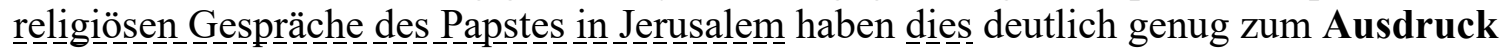
gebracht.

Christian Schwarz (A00/MAR.22450 St. Galler Tagblatt, [Tageszeitung], 24.03.2000, Jg. 56. Originalressort: TB-AKT (Abk.); Das Schweigen bleibt, [Bericht])

16) $\{\{1$; Gen.; A2 $\}\}$

Damit beginnt für Alfons Haag nach 18 Jahren im Amt am 1. Juli die vierte Wahlperiode. Nach der Gratulationscour bedankte sich der Wahlsieger und ließ durchblicken, dass ihn die Sechs vor dem Ergebnis persönlich freue. Sicher sei die Wahlbeteiligung von 55,4 Prozent verbesserungsfähig, aber durchaus ein Vertrauensbeweis, Ausdruck breiter Zustimmung und eine Basis, um vernünftig weiter zu arbeiten. Dies werde er mit gleichem Engagement und Freude tun, versprach er.

Kritisch bewertete er den Wahlkampf, den es so in Bürstadt noch nie gegeben habe. (M07/MAR.02913 Mannheimer Morgen, [Tageszeitung], 12.03.2007, Jg. 62, Ausgabe Südhessen Morgen (Viernheim). - Sachgebiet: Lokales, Originalressort: Viernheim; Von unserem Redaktionsmitglied Bernhard Zinke: Haag nimmt 60-Prozent-Hürde)

17) $\{\{0+2$; keine Real.; Subj.; Dat.; kein Argument; A1; A2 $\}\}\{\{S V G ;$ Dat. A. geben $\}\}$ Mit einem festlichen Einzug und der Orgelbegleitung durch Karin Fend wurde der Gottesdienst mit der Amtseinsetzung von Marie-Louise Mäder eröffnet. Doris und Marco Nigg gaben der Feier mit den musikalischen Einlagen eine besonders festliche Note. Pfarrer Lorenz Wüst überreichte der neuen Mitarbeiterin (eine Spätberufene) drei Geschenke, und zudem gab er der Hoffnung Ausdruck, dass ihr seelsorgerliches Wirken Früchte tragen werde. Die Pastoralpraktikantin versprach, Pfarrer Wüst dort zu unterstützen, wo es nötig sei, und zudem sprach sie all jenen Dankesworte aus, die ihr den Einstieg in die Pfarrei

Diepoldsau-Schmitter erleichtert haben. 
Licht und Schatten (A00/SEP.61291 St. Galler Tagblatt, [Tageszeitung], 12.09.2000, Jg. 56. Originalressort: RT-URT (Abk.); RÖsli Zeller: Zur Lichtbringerin im Alltag werden, [Bericht])

18) $\{\{0+1$; keine Real.; Subj.; kein Argument; A2 $\}\}\{\{$ SVG; zum A. kommen $\}\}$ «Es ging uns darum, Theorie und Praxis zu verbinden.» Und noch etwas ist für sie charakteristisch: die Verbindung zwischen der lateinischen und der deutschen Schweiz. «Das zeigt sich auch in der Wahl des Nachfolgers von Nicolas Druey.» Gewählt wurde der Romand François Dessemontet. Auf sein Engagement für den Schweizerischen Juristenverein angesprochen, erklärte er: «Es ist mir ein grosses Anliegen, dass wir uns gemeinsam um die künftigen Entwicklungen auf dem Gebiet des internationalen Rechts kümmern.» Das kam vor allem in unseren Gesprächen mit den «Stars» der Tagung zum Ausdruck. Peter Forstmoser, neuer VR-Präsident der Swiss-Re, erklärte, dass der Einfluss der amerikanischen Rechtssprechung auch bei uns unverkennbar sei. «Das gilt ebenfalls für das EU-Recht», ergänzte Peter Nobel. (A00/OKT.67527 St. Galler Tagblatt, [Tageszeitung], 02.10.2000, Jg. 56. Originalressort: TB-OST (Abk.); MELANIE RIETMANN: «Recht ist ein Stück Kultur", [Bericht])

19) $\{\{2+1$; Posspr.; attr. Adj; Subj.; A2; A3; A2 $\}\}\{\{$ SVG; Poss. Ausdruck erhalten $\}\}$ Im «Gloria» und «Credo» sind die Texte übereinandergeschichtet, deshalb die ungewöhnliche Kürze. Im «Credo» werden nur die ersten Glaubenssätze gleichzeitig in den verschiedenen Chorstimmen vorgetragen. Im «Incarnatus» erhält jeder_Ged_anke- seinen eigenen musikalischen Ausdruck. Als Höhepunkt wird das «Benedictus» angesehen, mit der ausgedehnten Arie für Sopran, der konzertierenden Orgel und dem Streichertrio. Die Stärke der «Missa brevis» liegt im Ausdruck, der den Hörer mit magischer Gewalt in seinen Bann zwingt. (A01/JAN.03601 St. Galler Tagblatt, [Tageszeitung], 17.01.2001, Jg. 57. Originalressort: RT-ORT (Abk.); Walter LÜchinger: Meisterwerke von Haydn und Franck erklingen, [Bericht])

20) $\{\{1$; Gen.; A2 $\}\}$

Dem Planungsstart zur Ladenvergrösserung waren bereits klare Ziele vorgegeben, nämlich ein zweckmässiges und genügendes Raumangebot für die nächsten Jahre und eine moderne Verkaufsinfrastruktur zu schaffen. Zudem galt es, einen optimalen Kundendienst wäh- rend der Bauzeit zu gewährleisten, der Kundschaft einen noch besse-ren Dienst und die Kostensicherheit zu bieten. Diese Ziele sind auch Spiegelbild und Ausdruck der hiesigen Gesellschaft und Kultur. Man erkennt darin Geschichte, Träume und Visionen. Ein endgültiges Bild erhalten diese Ziele aber erst in der Bau- und Ausführungsphase. (A01/MAR.12638 St. Galler Tagblatt, [Tageszeitung], 30.03.2001, Jg. 57. Originalressort: RT-EXT (Abk.), [Bericht])

21) $\{\{0+1$; keine Real.; Subj.; kein Argument; A2 $\}\}\{\{$ SVG; zum A. kommen $\}\}$

Die Jugendriege dürfe auf die grosse Unterstützung durch den örtlichen Turnverein zählen und sei auch im Bereich der Suchtprävention nicht zu unterschätzen.

Den Schülern verbunden

Die_Begeisterung für de_ den_Leh_rberuf kommt deutlich zum Ausdruck, wenn Nina Storchenegger lebhaft von ihren Schülerinnen und Schülern erzählt. Sie unterrichtet die vierte und fünfte Primarschulklasse in Ganterschwil und ist froh um die Erfahrungen, die sie während ihrer Tätigkeit als Jugi-Leiterin mit dieser Altersgruppe gemacht hat. Sie möchte diese Zeit nicht missen und hofft, dass vermehrt Jugendliche im Turnverein mitwirken und insbesondere bereit sind, Leitungsfunktionen zu übernehmen. (A01/OKT.37034 St. Galler 
Tagblatt, [Tageszeitung], 20.10.2001, Jg. 57. Originalressort: WV-UZW (Abk.); Bea Näf: Die Jugi wesentlich mitgeprägt, [Bericht])

22) $\{\{0+2$; keine Real.; Subj.; Akk; kein Argument; A1; A2 $\}\}\{\{$ SVG; zum A. bringen $\}\}$ Tragbar für die Stadt Wil

Christof Gämperle (FDP), Präsident der zuständigen vorberatenden Kommission, erklärte zur Eintretensdebatte, man habe sich in der Kommission grundlegende Fragen gestellt: «Kann sich Wil so etwas leisten?» Als «lohnenswert» und «für die Stadt Wil durchaus tragbar» wurde das Vorhaben in der Folge von verschiedenen Votanten bezeichnet. Andere Sprecher brachten allerdings ihre Bedenken zum Ausdruck: Man dürfe diese finanzielle Belastung nicht einfach geringschätzen oder ignorieren.

Verfahren beschleunigen

$\mathrm{Zu}$ reden gab in der parlamentarischen Beratung auch der Faktor Zeit: Einerseits seien die Zustände im Bergholz nicht mehr sehr viel länger halt- und zumutbar, die Sanierung sei dringend, wurde etwa betont. (A07/SEP.14839 St. Galler Tagblatt, [Tageszeitung], 28.09.2007, Nr. 226, S. 45. - Sachgebiet: Regionales, Originalressort: WV-Aufschlag; s.hauser: «Ja» zum Gesamtkonzept Bergholz)

23) [[1; Apposition; A']]

Wir haben das Schweizervolk, ein Drittel des Schweizervolkes verarscht, und das darf nicht passieren. Die CVP hat das verschuldet, und das muss sie auch tragen müssen, und ich wünsche ihnen viel Glück dazu, denn die SVP in der Opposition, das wird nicht einfach.» Nicht einfach - für wen? Eine schöne Drohgebärde am Schweizer Fernsehen, gepaart mit dem charmanten Ausdruck «verarscht», das macht Eindruck. Da weiss man gleich, in diesem Rheintal leben einfache Leute mit einfacher Ausdrucksweise, etwas viel Mist und Gülle. Die SVP spricht viel von Demokratie, von Volkswille und Bewahrung der Freiheit: Wo Gift drauf steht, muss auch Gift drin sein. (A07/DEZ.07932 St. Galler Tagblatt, [Tageszeitung], 18.12.2007, Nr. 295, S. 36. - Sachgebiet: Regionales, Originalressort: RT-Eingesandt; Brave Untertanen)

24) $\{\{0+1$; keine Real.; Subj.; kein Argument; A2 $\}\}\{\{$ SVG; zum A. kommen $\}\}$ Wenn alles rund läuft, steht dem Baubeginn der Umfahrung Bütschwil und eventuell Wattwil im Jahr 2013 nichts mehr im Weg. Politischer_Wille für_Lobbyarbeit kam am

Podiumsgespräch am Mittwochabend zum Ausdruck.

Sylvia Baumann

Eines ist klar: Alle Propagandisten für die Umfahrungen Bütschwil und Wattwil hoffen, dass der Kantonsrat das vorliegende Bauprojekt in das Strassenbauprogramm 2008 aufnimmt.

(A08/FEB.09361 St. Galler Tagblatt, [Tageszeitung], 23.02.2008, Nr. 45, S. 53. Sachgebiet: Regionales, Originalressort: WV-Untertoggenburg; m.forster: Hoffen auf die Favoritenrolle)

25) $\{\{0\}\}$

Die Blätter von der linken in die rechte wechselnd, um besser gestikulieren zu können, las er mit lebhafter Mimik Texte aus der Gedenkschrift zum 100. Geburtstag von Walther Wahrenberger aus Lütisburg (1899-1949).

Es hielt Furrer nicht lange auf dem Stuhl, er musste sich bewegen, stieg sogar auf die Sitzgelegenheit, um Spannung und Ausdruck zu verstärken. Er streute Dialektsätze ein, erging sich in italienischem oder französischem Redeschwall; die Lesung steigerte sich zum Ein-Mann-Stück. Nein, da war noch eine zweite Komponente, die Musik: Willi Häne, ein gebürtiger Kirchberger, der heute in St. Gallen lebt, spielte Akkordeon. (A08/APR.11079 St. 
Galler Tagblatt, [Tageszeitung], 26.04.2008, Nr. 97, S. 51. - Sachgebiet: Regionales, Originalressort: WV-Untertoggenburg; m.forster: Das hätte Wahrenberger gefallen)

26) [[1; Apposition; A']]

Auf Anfrage der Wiler Zeitung erklärt Beat Weibel, von der Arbeitsgruppe RIST, dass die Gründungsversammlung auf den 6. August verschoben worden sei. Hauptsächlich verantwortlich für diesen neuen Termin seien Anträge auf Namensänderung. So werde zum einen gewünscht, dass der Name der Standortgemeinde Münchwilen Aufnahme finden müsste und zum anderen sei der Ausdruck Tannzapfenland einigen zu wenig bekannt. (nas.)

(A08/JUN.06975 St. Galler Tagblatt, [Tageszeitung], 24.06.2008, Nr. 145, S. 40. Sachgebiet: Regionales, Originalressort: WV-Hinterthurgau; n.stricker: Gründung RIST verschoben)

27) [[1; Apposition; A']]

Das Restaurant selbst ist allerdings keine Idylle, hier dominiert zeitloser 08/15-Stil, und beim Essen fühlt man sich schon fast wie in Österreich.» Weiter wird erwähnt, dass der Geschäftsführer, Küchenchef und weitere Mitarbeiter aus dem «östlichen Nachbarland» stammen.

Neben der Abstufung von 13 Punkten im Jahr 2007 auf 12 in diesem Jahr ärgert sich Mandl vor allem über die Bezeichnung «08/15-Stil». Ein weiteres Ärgernis sei der Ausdruck «östliches Nachbarland»: «Wir fassen dies als einen verdeckten Seitenhieb auf unsere Nationalität auf.» Seinen Gästen teilte der Geschäftsführer daher mit, er sei nicht daran interessiert, weiterhin im Gault Millau aufgeführt zu werden.

Bild: Marc Ulrich (A08/OKT.02479 St. Galler Tagblatt, [Tageszeitung], 08.10.2008, Nr. 235, S. 42. - Sachgebiet: Regionales / Nachrichten, Originalressort: SG-Schauplatz-OS; c.zweili: Korb für Gault Millau)

28) $\{\{0+2$; keine Real.; Subj.; Dat; kein Argument; A1; A2 $\}\}\{\{$ SVG; Dat. A. geben $\}\}$ Kontakte wichtig

Klee unterstrich die Wichtigkeit der Schulbesuche durch die Schulratsmitglieder: «Kontakte zu unseren Lehrpersonen sind für eine gute Zusammenarbeit ein Muss.» Jedes Ratsmitglied hat deshalb die Aufgabe, bei den sechs Lehrpersonen, die ihm zugeteilt sind, jährlich mindestens einen Unterrichtsbesuch zu machen und darüber einen Bericht zu verfassen. Die

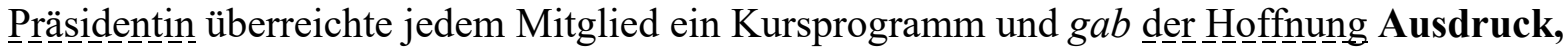
dass die Chance zur Wissenserweiterung genutzt werde. Im gegenseitigen guten Einvernehmen konnten die zahlreichen Aufgaben, Ämter und Delegationen verteilt werden. Neuer Vizepräsident ist Sandro Pizzingrilli. (A09/JAN.03510 St. Galler Tagblatt, [Tageszeitung], 19.01.2009, Nr. 14, S. 31. - Sachgebiet: Regionales, Originalressort: RTUnterrheintal; s.miara: Die Ziele gemeinsam erreichen)

29) $\{\{0+2$; keine Real.; Subj.; Dat; kein Argument; A1; A2 $\}\}\{\{$ SVG; Dat. A. geben $\}\}$ Gemäss seinem Votum ist der mit dem neu zur Verfügung stehenden Finanzinstrument gewonnene Handlungsspielraum vor allem im Hinblick auf die kommenden, beträchtlichen Investitionsvorhaben wie der Sportpark Bergholz ein grosser Vorteil. Auch die SP hiess die

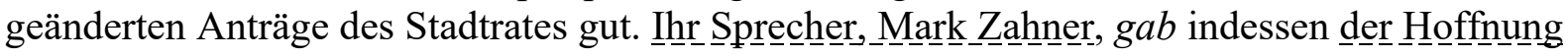
Ausdruck, dass das neue Steuerausgleichskonto nicht für ungerechtfertigte Steuersenkungen missbraucht wird.

«Leitplanken setzen»

Dass es sich diesbezüglich um eine grundlose Sorge handelt, brachte Fraktionspräsident Norbert Hodel (FDP) in seinem Votum zum Ausdruck. (A09/MAI.00407 St. Galler 
Tagblatt, [Tageszeitung], 01.05.2009, Nr. 100, S. 31. - Sachgebiet: Regionales, Originalressort: WV-Stadt-Wil; r.ammann: Ja zu einer Steuerausgleichsreserve)

30) $\{\{2+1$; Posspr.; mit + Dat.; Subj.; A2; A3; A2 $\}\}\{\{$ SVG; Poss. A. finden $\}\}$

Im Gloria wird dem harmonisch geprägten Chorsatz ein Instrumentalmotiv entgegengestellt. Dramatische Passagen prägen den Mittelteil des selten aufgeführten «Credo», begleitet von Violinen mit Arpeggio-Figuren. Der innige Charakter der ganzen_Messe findet in der SopranArie des «Benedictus» mit konzentrierender Orgelbegleitung seinen reinsten Ausdruck: Von zartem Klangreiz und rokokohafter Feinheit ist es seit jeher als mystischer Kern des Werks gedeutet worden. Hell, leicht und leuchtkräftig intonierte die Sopranistin Felizitas Hetzel dieses Benedictus, eine glanzvolle Leistung, die einen enthusiastischen Applaus hervorrief. Das abschliessende «Agnus Dei» mit seiner flehenden Bitte um Frieden «Dona nobis Pace» sang der grosse Chor mit Hingabe, sorgfältiger Intonation und lyrischer Wärme.

(A09/SEP.05074 St. Galler Tagblatt, [Tageszeitung], 15.09.2009, Nr. 214, S. 40. Sachgebiet: Regionales / Sport, Originalressort: TT-Regionalsport; h.kugler: Starkes Jubiläumskonzert)

31) $\{\{0\}\}$

Mit ausgezeichnetem Musikgespür wurden die Radschläge in die Diagonale der Bühne gesetzt, eine sinfonische Version von Queens «We Will Rock You» gab unter anderem den akustischen Boden dazu.

Die Begrüssung durch die Kleinsten bezauberte schlichtweg. Durch die Arbeit mit dem Körper gefördert, strahlte jedes einzelne der kleinen Mädchen bereits eine ungeheure Individualität aus, was sich auch durch sämtliche nachfolgenden Einzelstücke zog: charmante Strassenkehrer, die mit witzigen Standfiguren eine originelle Interpretation einer ebenso originellen Sandpapier-Komposition von Leroy Anderson hinlegten; «Rouge» oder «In der Geisterbahn» - auf Ausdruck und Licht ausgerichtetes Bewegungstheater mit expressionistischen Zügen; dann aber auch wieder kleine, wuslige Kobolde, neckisch mit den Zipfelmützen wackelnd.

Faszination des Augenblicks

Der kleine Gärtner mit seinen Blumen eroberte im Sturm die Herzen des Publikums, stämmig-stampfig begeisterte auch der Bauerntanzstil von «Tempi Diversi».

(A09/NOV.04939 St. Galler Tagblatt, [Tageszeitung], 16.11.2009, Nr. 267, S. 34. Sachgebiet: Regionales, Originalressort: WV-Stadt-Wil; a.bruelisauer: Den Körper zum Sprechen gebracht)

32) [[1; für + Akk.; A2]]

Anscheinend versprechen Turngeräte besseren Erfolg.

Derweil bleibt die Verkündigung unter anderem mit der Erklärung schwieriger Bibelstellen auf der Strecke. «Wie schade» ist gar kein Ausdruck für das, was uns an faszinierender Glaubenshilfe vorenthalten bleibt und wir somit weiterhin mit einem vielfach gespaltenen

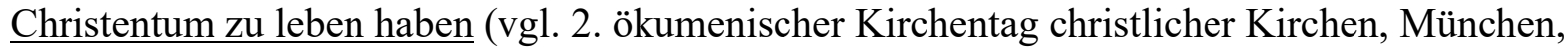
12.-16.5.2010, wo man sich einmal mehr nicht zu einem gemeinsamen Abendmahl durchzuringen vermag...).

Dr. oec. Wolfgang Eisenbeiss

Goethestr. (A10/MAR.02977 St. Galler Tagblatt, 10.03.2010, Nr. 57, S. 30. - Sachgebiet: Sonstiges, Originalressort: traueranzeigen; y.stadler: Erfolg mit Turngeräten?)

33) $\{\{1+1$; mit + Dat.; Subj.; A3; A2 $\}\}\{\{$ SVG; zum A. kommen $\}\}$

Unterstützung in der Krise 
Die Vielfalt der Verbandstätigkeit zeigte sich im reichen Kursangebot und den Impulstagen. In besonderem Masse zeigt sie sich seit 30 Jahren in der Solidarität mit Frauen und Familien in Krisensituationen verschiedenster Art. In der Jahresrechnung kam diesese_Aufga $\underline{\text { abe }}$ mit dem grössten Posten zum Ausdruck.238 500 Franken wurden für die Beratungsstelle inklusive Verbandssekretariat aufgewendet; bei Gesamtausgaben von 562000 Franken. Die Beratungsstelle wird mit verschiedenen Beiträgen finanziert. (A10/MAI.06092 St. Galler Tagblatt, 21.05.2010, Nr. 116, S. 47. - Sachgebiet: Regionales / Regionales, Originalressort: WV-Region-Flawil; b.marks: «Vielfalt ist Gemeinsamkeit»)

34) $\{\{1+1$; mit + Dat.; dass-Subjektsatz; A3; A2 $\}\}\{\{$ SVG; zum A. bringen + Passiv $\}\}$ Da fällt ein als religiöses Symbol getragenes Kopftuch besonders auf.

Was ist denn aus religiöser Sicht der Sinn einer Kopfbedeckung?

Pahud de Mortanges: Mit der religiösen Kleidung wird vereinfacht gesagt zum Ausdruck gebracht, dass $\underline{\text { man }}$ sich in besonderer Weise seiner Religion verpflichtet weiss. Im einzelnen gibt es aber für moslemische Frauen unterschiedliche Motivationen, eine Kopfbedeckung zu tragen. Kopfbedeckungen gibt es nicht nur im Islam, sondern auch zum Beispiel im Judentum und bei den Sikh. (A10/SEP.03455 St. Galler Tagblatt, 22.09.2010, Nr. 221, S. 3.

Originalressort: thema; «Das Kopftuch macht den Islam sichtbar»)

35) $\{\{0+2$; keine Real.; Subj.; Akk; kein Argument; A1; A2 $\}\}\{\{$ SVG; zum A. bringen $\}\}$ Am Samstag, 8. Januar, um 20 Uhr lädt die evangelische Kirchgemeinde die Balgacher Bevölkerung und weitere Interessierte zur Neujahrsbegrüssung ins Kirchgemeindehaus Breite ein. Die Foolish Freylach Band aus St. Gallen spielt Klezmermusik; dazwischen erzählt Batja Guggenheim Geschichten und gibt Einblicke in die jiddische Kultur. Klezmer ist Musik, die

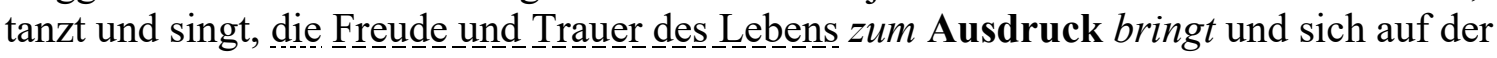
ganzen Welt grosser Beliebtheit erfreut. Sie ist so vielfältig wie die osteuropäische jüdische Kultur, aus der sie entstanden ist. Es ist Musik, die eindeutig zu erkennen ist und sich doch verändert. (A11/JAN.01558 St. Galler Tagblatt, 07.01.2011, Nr. 5, S. 45. - Sachgebiet: Regionales / Unterhaltung/Kultur, Originalressort: RT-Weekend; m.vonderlinden: Neujahrsgrüsse mit Klezmer)

36) $\{\{0+2$; keine Real.; Subj.; Dat; kein Argument; A1; A2 $\}\}\{\{$ SVG; Dat. A. geben $\}\}$ Werner Hofmann informierte über die Riga 12, die im Juni des kommenden Jahres in Uzwil stattfindet. Aus Oberuzwil hätten sich bis dato 24 Betriebe angemeldet. Hofo-nanann $g a b$ der

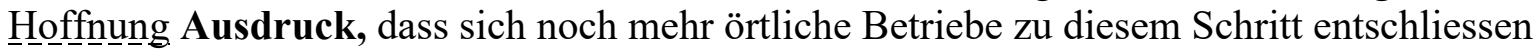
können.

Zunahme der Gewaltdelikte

Im zweiten Teil referierte Regierungsrätin Karin Keller-Sutter zur Sicherheitspolitik. (A11/APR.06263 St. Galler Tagblatt, 18.04.2011, Nr. 91, S. 39. - Sachgebiet: Regionales / Regionales, Originalressort: WV-Region-Uzwil; p.stutz: Ein positives Bild über das Gewerbe gezeichnet)

37) $\{\{0+2$; keine Real.; Subj.; Akk; kein Argument; A1; A2 $\}\}\{\{$ SVG; zum A. bringen $\}\}$ Wohl nicht zuletzt weil Leclerc das Drehbuch zusammen mit seiner algerischstämmigen Lebensgefährtin Baya Kasmi geschrieben hat, werden die Perspektiven beider Protagonisten ausgewogen vermittelt. Vertrauen kann der 46jährige Regisseur auch auf seine beiden

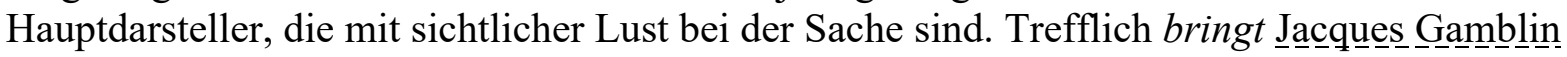
die Unsicherheit Arthurs zum Ausdruck, während Sara Forestier Bahia freizügig und mit vollem Körpereinsatz spielt.

Die Zukunft den Mischlingen 
Verpackt in die Liebesgeschichte führt Michel Leclerc gleichzeitig leichthändig einen Diskurs über Fragen der Identität, stellt der Verdrängung eine offene Aufarbeitung der

Familiengeschichte gegenüber und erzählt in der privaten Geschichte vom Umgang der französischen Gesellschaft mit diesen Themen. (A11/AUG.00004 St. Galler Tagblatt, 02.08.2011, Nr. 177, S. 30. - Sachgebiet: Regionales / Kultur, Originalressort: SGst.galler kultur; a.stock: Sex, Politik und Traumata)

38) $\{\{0+2$; keine Real.; Subj.; Akk; kein Argument; A1; A2 $\}\}\{\{$ SVG; zum A. bringen $\}\}$ Haben Sie schon viele Glückwünsche erhalten?

Christian Lohr: Es ist unglaublich, wie viele Glückwünsche als SMS, E-Mail und auf Facebook gekommen sind. Es ist sehr schön, zu spüren, wive_viele und ihre Freude auf eine besonders schöne Art zum Ausdruck bringen .

Fürchten Sie, jetzt der Alibi- oder Vorzeige-Behinderte zu werden?

Lohr: Man entwickelt mit der Zeit eine harte Haut und Kraft, um die Aufgabe zu meistern, Hoffnungsträger für andere zu sein. (A11/NOV.06506 St. Galler Tagblatt, 15.11.2011, Nr. 267, S. 25. - Sachgebiet: Regionales, Originalressort: TZ-kanton thurgau; martin.knoepfel: «Viele Leute boten spontan Hilfe an»)

39) $\{\{0+2$; keine Real.; Subj.; Akk; kein Argument; A1; A2 $\}\}\{\{$ SVG; zum A. bringen $\}\}$ Judo-Olympiasiegerin Yvonne Bönisch boykottiert die Teilnahme an der Eröffnungsfeier der Olympischen Spiele in Peking

- Potsdam (dpa/sid). Immer mehr Sportler machen sich Gedanken, wie sie sich während der

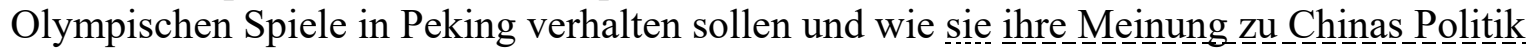
zum Ausdruck bringen können. Als erste aktive deutsche Olympiasiegerin hat sich JudoKämpferin Yvonne Bönisch jetzt entschlossen, die Eröffnungszeremonie der Sommerspiele zu boykottieren: „Ich werde ein Zeichen setzen und nicht an der Eröffnungsfeier teilnehmen“, sagte sie gestern.

Ein Boykott der Wettkämpfe komme für sie aber nicht infrage, sagte die 27-Jährige, die 2004 in Athen als erste deutsche Judokämpferin Olympiagold holte und im August erneut zu den deutschen Medaillenhoffnungen zählt. (HAZ08/APR.02957 Hannoversche Allgemeine, 16.04.2008, S. 28; Leises Zeichen des Protests [Ausführliche Zitierung nicht verfügbar])

40) $\{\{1+2$; mit + Dat.; Subj.; Akk; A3; A1;A2 $\}\}\{\{$ SVG; zum A. bringen $\}\}$

Wir können uns des Eindrucks nicht erwehren, dass unser neues Symbol ein wenig scheu in der Gegend herumsteht und nicht viel reißt. Aber egal: Für die Zukunft der Orchidee gibt die Entscheidung zu schönsten Hoffnungen Anlass. Wir wollen das $\underline{\text { da }}$, bevor wir zu einer Gratulationsexkursion in die Kalkflachsümpfe aufbrechen, mit einem eilig zusammengeschusterten Vierzeiler zum Ausdruck bringen: Sah ein Knab“ ein Kräutlein stehen / von des Fleisches Farben / jetzt, da's einen Paten hat / wird's wohl nicht mehr darben.

se (HAZ08/JUN.03801 Hannoversche Allgemeine, 20.06.2008, S. 17; Unser Kraut [Ausführliche Zitierung nicht verfügbar])

41) $\{\{1 ;$ Gen.; A2 $\}\}$

»Nur jene Erbanlagen bleiben auf Dauer erhalten, die konsequent eigennützlich für ihre eigene maximal mögliche Verbreitung sorgen. Ein gleichsam >moralisches Selbstbeschränkungs-Gen ‘ hätte unter den Wettbewerbsbedingungen der natürlichen Selektion keine Chance. [...]

Elterliche Aufopferung zugunsten der Kinder - für viele geradezu der Prototyp einer altruistischen Moralität - entpuppt sich deshalb ebenfalls als Ausdruck des genetischen 
$>$ Prinzips Eigennutz $\$. Folglich ist unsere Moral auch in dieser Hinsicht $>$ die Moral unserer Gener. [...]

Tatsächlich wurde die soziale Evolution ganz wesentlich durch Täuschung, Manipulation und Ausbeutung vorangetrieben, und das betrifft auch die Evolution des Eltern/KindVerhältnisses, also jene Szenerie, in der die Gewissensentstehung verankert ist. [...]

Genetische Konflikte wie zwischen Parasit und Wirt bestehen auch zwischen Eltern und ihren Kindern. [...] (FSP/BRT.00000 Baladur, Rigo: Der stille Tod. Menschheit zwischen Demenz und Dementi. - Oberhausen: ATHENA-Verlag, 2001 [S. 60])

42) $\{\{1$; Gen.; A2 $\}\}$

Der Eidg. Datenschützer Hanspeter Thür will am Bankgeheimnis festhalten. Er stehe zu 100 Prozent hinter dem Bankgeheimnis als Schutz der Privatsphäre der Bankkunden, betonte er im Gespräch mit der «NZZ am Sonntag», das auch im «Schweizer Monat» erscheint. Das Bankgeheimnis sei Ausdruck eines fundamentalen Prinzips des helvetischen Rechtsstaates: des Schutzes der Privatsphäre, zu der auch die finanzielle Privatsphäre gehöre. Der Datenschützer präzisiert allerdings: «Genau jene, die das Prinzip in Anspruch nehmen, haben es mit ihrem Geschäftsmodell ad absurdum geführt.»

Punkto automatischem Informationsaustausch warnte er: «Wenn der Staat in jedem Bürger einen potenziellen Steuerhinterzieher sieht, dann traut er seinen Bürgern nicht mehr.

(A12/APR.08542 St. Galler Tagblatt, 23.04.2012, Nr. 94, S. 5. - Sachgebiet: Lokales, Originalressort: inland; Widmer-Schlumpf sieht Entspannung im US-Streit)

43) $\{\{0+1$; keine Real.; Subj.; kein Argument; A2 $\}\}\{\{$ SVG; zum A. bringen + PASSIV $\}\}$

Die Unterstützung durch die FDP, die Walter Grob vor vier Jahren noch genoss, ist somit dahin. Parteipräsident Markus Rohner sagt auf Anfrage, die Bürgervorversammlung habe dieses Jahr im Dorf hohe Wellen geworfen. Innerhalb der FDP sei in jüngster Zeit die

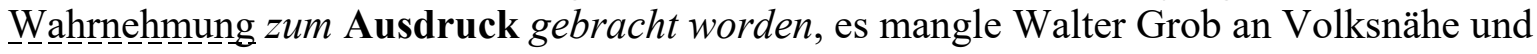
an der nötigen kommunikativen Fähigkeit, die gerade bei wichtigen Themen zwingend sei. Spricht man mit weiteren Bürgern, wird bald einmal klar: Walter Grobs Kritiker attestieren ihm zwar Sachkompetenz, halten jedoch seine Sozialkompetenz - gemessen an den hohen Ansprüchen an einen Gemeindepräsidenten - für ungenügend.

$\mathrm{Ob}$ die SVP Au-Heerbrugg hinter Grob steht, entscheidet sich heute an einer Vorstandssitzung. (A12/JUL.00582 St. Galler Tagblatt, 03.07.2012, Nr. 153, S. 29. Sachgebiet: Regionales, Originalressort: RT-Aufschlag; g.bruderer: Stefan Suter fordert Grob heraus)

44) $\{\{1$; dass-Satz; A2 $\}\}$

«Aber es geht auch viel Wissen verloren», betonte er.

Besonders strich Elmar Steiger an der Wahlfeier das «sensationelle» Resultat vom

Schulratspräsidenten Norbert Stieger hervor, der mit fast 99 Prozent der gültigen Stimmen gewählt worden ist. «Dies ist ein Ausdruck, dass die Bürger seine intensive Arbeit schätzen», ist der FDP-Präsident überzeugt. Auch weitere Kandidaten der FDP konnten auf glanzvolle Wahlresultate anstossen.

Investitionen braucht es (A12/SEP.11347 St. Galler Tagblatt, 25.09.2012, Nr. 224, S. 31. Sachgebiet: Regionales, Originalressort: TT-Neutoggenburg; sa.schmid: «Es lohnt sich, wenn man etwas tut»)

45) [[2; Apposition; für + Akk.; A‘; A2]]

«Das war vor etwa 20 Jahren. Mich störte, dass die Höhenangaben, wie beispielsweise $<$ bis auf 600 Meter> nicht immer ganz stimmten und so für Verwirrung bei den Bauern sorgten», 
erklärt Michel. Aus dem Österreichischen kannte er den Ausdruck «höhere Tallagen» und so habe er eines Tages einem Meteorologen in Zürich vorgeschlagen, den Ausdruck «tiefere Lagen» für den Bereich zwischen 600 und 1000 Meter einzuführen - mit Erfolg.

Meteorologisches Gedächtnis

Wie aus der Pistole geschossen, kann Otto Michel aussergewöhnliche Wetterereignisse aus seiner Erinnerung abrufen. (A12/DEZ.11351 St. Galler Tagblatt, 29.12.2012, Nr. 304, S. 46. - Sachgebiet: Regionales, Originalressort: WV-Hinterthurgau; s.dudle: Erfinder der «Tieferen Lagen»)

46) $\{\{0+1$; keine Real.; dass-Subjektsatz; kein Argument; A2 $\}\}\{\{$ SVG; zum A. kommen $\}\}$ RORSCHACHERBERG. 2011 haben die Kantonsräte Felix Bischofberger und Felix Gemperle beim Kanton eine Interpellation eingereicht mit dem Titel «Seeuferanstoss in Neuseeland, Rolle des Kantons?». In der Antwort sei klar zum Ausdruck gekommen, das $\underline{\text { s }}$ die $\underline{\text { e }}$ Regierung einem umfassenden öffentlichen Zugang zu den Ufern der st. gallischen Seen eine

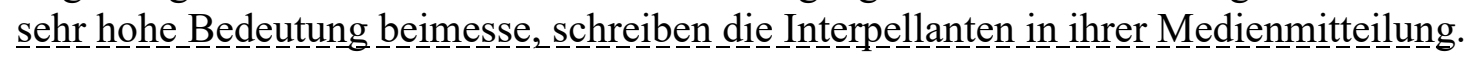
Hauptstrasse keine Alternative

Beim öffentlichen Podium im Seerestaurant habe die Idee eines durchgehenden Seeuferweges im Bereich Neuseeland sehr grosse Unterstützung erhalten. (A13/MAR.13188 St. Galler Tagblatt, 30.03.2013, Nr. 74, S. 39. - Sachgebiet: Regionales, Originalressort: OTrorschach; r.hirtl: Erste Variante entspricht nicht dem Volkswillen)

47) $\{\{1+1$; in + Dat.; Subj.; A3; A2 $\}\}\{\{$ SVG; zum A. gelangen $\}\}$

Weshalb? Im Kern geht es bezüglich dieser Initiative nicht primär um die Armee, sondern es geht vielmehr um die Pflicht, für den Staat, für Land und Volk einen Dienst zu leisten. Es geht also auch um die Bürgerpflicht, sich für eine beschränkte Zeit für die Mitbürgerinnen und Mitbürger zu wehren, was ja im Wort Wehrpflicht zum Ausdruck gelangt.

Die Linke möchte sich also vom Staat, an den sie hohe Erwartungen hat - angefangen bei der Bildung, über den gesicherten Grundlohn sowie die Gesundheitsvorsorge bis hin zu einer gesicherten Altersvorsorge -, nicht in die Pflicht nehmen lassen. Oder wenn es denn ein Dienst in einer freiwilligen Miliz, im Zivildienst oder im Zivilschutz wäre, so nur mit einer sehr guten Entlöhnung, so ganz nach dem Grundsatz: Zuerst möchte ich vom Staat etwas erhalten, bevor ich mich für einen Dienst an der Gesellschaft bereit erkläre. (A13/AUG.12051 St. Galler Tagblatt, 12.08.2013, Nr. 185, S. 29. - Sachgebiet: Unterhaltung, Originalressort: Forum; Wehrpflicht ist wichtig)

48) $\{\{1+1$; in + Dat.; Subj.; A3; A2 $\}\}\{\{$ SVG; zum A. kommen $\}\}$

Er lässt nicht auf sich warten wie Godot. Peter Ries sitzt schon da; er hat die Energie und Euphorie des schöpferischen Prozesses mitgebracht aus der Vormittagsprobe. Ein glücklicher Mensch, den das Unglück unseres Daseins umtreibt: ob es in Text und Setting eines Theaterstücks zum Ausdruck kommt, ob es uns im Sekundentakt über digitale Nachrichtendienste mit Bildern von Flüchtlingen oder Kriegsopfern überflutet oder in unmittelbarer Nähe sichtbar wird.

Wie neulich, am Rande einer Probe: als Polizei, Notarzt und Schaulustige schräg vis-à-vis vor dem Hochhaus der Fachhochschule standen und bange nach oben schauten. Auch davon wird Ries erzählen, nach seiner Erfahrung mit Samuel Beckett gefragt. (A14/NOV.01208 St.

Galler Tagblatt, 04.11.2014, Nr. 257, S. 22. Originalressort: Focus; a.stock: Beckett über die Schulter geblickt)

49) $\{\{0+1$; keine Real.; Akk.; kein Argument; A2 $\}\}\{\{$ SVG; zum A. bringen $\}\}$ Sechs Weinregionen 
Tatsächlich ist die Sorte kein Kraftpaket mit unverwechselbarer Aromatik wie etwa Riesling oder Sauvignon blanc. Ihre Leistung ist es, die Eigenheiten der jeweiligen Lage optimal zum Ausdruck zu bringen. Das macht sie in der Waadt mit ihrer vielfältigen Boden- und Klimastruktur und 13580 Weinparzellen zum Erlebnis. Die sechs Waadtländer Weinregionen verteilen sich auf die alpinen Zonen des Chablais, die Steillagen des Lavaux, die sanften Hügel der La Côte zwischen Genfersee und Jura sowie auf drei kleinere Gebiete im Norden des Kantons. (A14/NOV.10635 St. Galler Tagblatt, 27.11.2014, Nr. 276, S. 14. Originalressort: Lokal; Die Weinidentität der Waadt)

50) $\{\{1$; Gen.; A2 $\}\}$

„Zeichen der Zeit" - so das Motto des Weltgebetstags - setzten die Wolfenbütteler Frauen mit ausgewählten Texten und Lobpreisungen. Bewegende Momente erlebten die Besucher in der Hauptkirche, wo Antonio Donga und seine Trommelgruppe rhythmische Akzente setzten. Von diesem Ausdruck afrikanischer Lebensfreude ließen sich zum Schluss des Gottesdienstes alle Besucher mitreißen, die einen großen Kreis bildeten und im Takt mit den Händen klatschten. Im Nachgang hatten sie Gelegenheit, Exotisches aus der Küche Südafrikas zu kosten. Die Backrezepte stammen von den dortigen Weltgebetstagsfrauen. (BRZ06/MAR.03358 Braunschweiger Zeitung, 06.03.2006; Gebetstag: Bewegender Gottesdienst in BMV [Ausführliche Zitierung nicht verfügbar])

51) $\{\{1+2$; attr. Adj.; Subj.; Dat.; A3; A1;A2 $\}\}\{\{$ SVG; Dat. A. verleihen $\}\}$ Nach seinem letzten Konzert in Braunschweig, auf der Feldschlößchen Bühne, sprang Achim Reichel wie ein adoleszenter Rockmusiker ins Becken des Raffteichbads und holte sich nach einem musikalischen Marathon die wohlverdiente Erfrischung.

Zwei Jahre später ist der Rahmen ein anderer, doch der Protagonist noch immer der Inbegriff eines ewig Junggebliebenen. In der Beton-Burg Stadthalle präsentierte der „Urvater der deutschen Rockmusik“ gestern Abend sein neues Album „Volxlieder“, das seiner_Neigung.

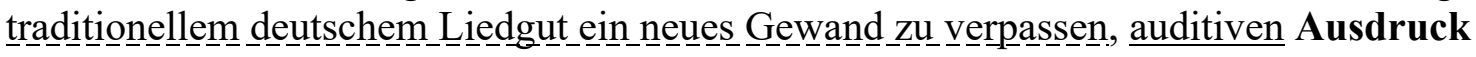
verleiht.

Bestuhlung, hie und da ergrautes Haar, verhaltene Ovationen - Reichels Publikum ist mit ihm durch die Jahre gegangen, reifer geworden und scheint auch sein neues Steckenpferd Volksmusik zu goutieren, denn ein freier Sitzplatz ließ sich im weiten Hallen-Rund kaum ausmachen.

Wer weiß, was wäre, hätte der 62-Jährige nicht vor ein paar Jahren in einem irischen Pub gesessen und auf die Aufforderung hin, ein schönes deutsche Lied auf der Gitarre vorzutragen, große Augen gemacht. (BRZ06/OKT.08965 Braunschweiger Zeitung, 19.10.2006; \&\#8222;Angst ist ein schlechter Partner!\&\#8220; [Ausführliche Zitierung nicht verfügbar])

52) $\{\{1+1$; durch + Akk.; Subj.; A3; A2 $\}\}\{\{$ SVG; zum A. kommen $\}\}$

Tuniken, Chasubles, gehrockartige Jacken und Kimono-Schnitte bringen neue Formen in die Kollektionen.

Einfarbige Outfits leben durch die Vielfalt der Oberflächen und deren unterschiedliche Lichteffekte. So kann die Wiederbelebung der_Eleganz durch luxuriöse Materialien besonders zum Ausdruck kommen.

Das Kleid steht bei den Damen wieder hoch im Kurs. Im Frühjahr treffen Sie mit Wickelkleidern aus Jersey oder Hemdblusen-Typen die richtige Wahl fürs Büro und besonders für kurze Geschäftsreisen. (BRZ07/FEB.15129 Braunschweiger Zeitung, 17.02.2007; Frühjahrs-Kleider: Gute Wahl für\&\#8217;s Büro [Ausführliche Zitierung nicht verfügbar]) 
53) $\{\{1$; Gen.; A2 $\}\}$

Im Moment geht bei der Qualitätsfindung noch einiges durcheinander.

Am IHK-Brief haben offensichtlich etliche mitgestrickt. In seinen ängstlichsten Passagen ist er Ausdruck einer Unsicherheit und Unentschiedenheit, die der Stadt noch nie gut getan hat. Schöner sind immer jene Städte, wo die Bürger stets das Detail und das Ganze betrachten. Dieser Aspekt wird in dem Brief glücklicherweise auch betont. (BRZ07/SEP.20168 Braunschweiger Zeitung, 13.09.2007; Bohlweg liegt nicht an der Alster [Ausführliche Zitierung nicht verfügbar])

54) $\{\{1$; Gen.; A2 $\}\}$

Gleichzeitig eröffnete er damit die Fortsetzung der Vortragsreihe „Bei Lessing zu Gast“. Friedrich von Hagedorn, den der 20-jährige Lessing einst „den größten Dichter unserer Zeit““ nannte, wurde am 23. April 1708 geboren. Seine lebensbejahende Dichtung sprach der jungen Generation nach dem 30-jährigen Krieg aus dem Herzen und war Ausdruck ihres Lebensgefühls. Von Hagedorn selbst wurde nur 46 Jahre alt. Durch Alkoholexzesse und übermäßiges Essen hatte er sich seine Gesundheit vorzeitig ruiniert. (BRZ08/APR.15340 Braunschweiger Zeitung, 29.04.2008; Auch heute noch belebend und amüsant [Ausführliche Zitierung nicht verfügbar])

55) $\{\{1$; Gen.; A2 $\}\}$

Und deswegen ist die eigentliche Stadt eine der menschlichen Bindungen und Emotionen. Eine Grafik, die dieses Geflecht wiederspiegelte, sähe ungefähr aus wie ein Schnittmusterbogen. Die dicken Striche wären Ausdruck unserer starken Gefühle, die dünnen Striche entsprächen den schwächeren Bindungen, etwa zu Bekannten, Nachbarn und Arbeitskollegen.

Und wir definieren uns immer wieder über die vertrauten Ansichten der Stadt. Wir wollen uns $\mathrm{zu}$ ihr bekennen, und wir wollen stolz auf sie sein, besonders auf ihre unverwechselbaren Seiten. (BRZ08/JUN.14852 Braunschweiger Zeitung, 28.06.2008; Eine Stadt ist dann schön, wenn jeder auf den anderen achtet [Ausführliche Zitierung nicht verfügbar])

56) $\{\{1+2$; mit + Dat.; Subj.; Akk.; A3;A1;A2 $\}\}\{\{$ SVG; zum A. bringen $\}\}$

Das wurde gestern in Budapest und Berlin mitgeteilt. Beim „Paneuropäischen Picknick“ in Sopron war es am 19. August 1989 erstmals einer größeren Zahl von DDR-Bürgern gelungen,

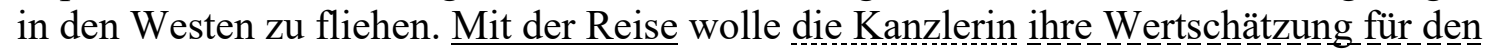
besonderen Beitrag Ungarns zur Freiheit_und Einheit_Deuts_chlands zum Ausdruck bringen, hieß es.

Gabriele Pauli (52)

bleibt trotz interner Kritik Bundesvorsitzende ihrer Partei Freie Union. (BRZ09/AUG.06674

Braunschweiger Zeitung, 15.08.2009; Gordon Bajnai (41) [Ausführliche Zitierung nicht verfügbar])

57) $[[0]]$

Im Gegenteil: Zum ersten Mal sehe ich klar, wenigstens in dieser Beziehung. Bisher nämlich habe ich noch nie darüber nachgedacht. Wie alle meinesgleichen und auch andere, so habe auch ich diesen Ausdruck gebraucht, ganz einfach so, gedankenlos. Bloß über neue Ausdrücke, da habe ich mich lüstig gemacht, die habe ich nicht so einfach hingenommen. Doch jetzt bewirkte eine kurze Bemerkung, dass mir die Schuppen von den Augen fielen, so dass sie - siehe oben - klarer sehen können. (BRZ10/JAN.12485 Braunschweiger Zeitung, 
30.01.2010; Die Zeit als unabänderliche Größe [Ausführliche Zitierung nicht verfügbar])

58) $\{\{0+2$; keine Real.; Subj.; dass-Objektsatz; kein Argument; A1; A2 $\}\}\{\{$ SVG; zum A. bringen $\}\}$

Sie gründet sich darauf, dass die internationalen Finanzmärkte eine Sanierung Griechenlands ohne die Zustimmung der wichtigen Wirtschaftsmacht Deutschland sowieso nicht akzeptieren würden. Gleichzeitig leitet sie einen Richtungswechsel in der deutschen Europapolitik ein. Der_größte Mitgliedsstaat, der lange mit viel_Geld der stille Garant der EU E offen zum Ausdruck gebracht, das $\underline{\text { s }}$ er nicht jeden Preis_zahlt. zugleich die zunehmende euroskeptische Haltung der Deutschen und deren historisch bedingte latente Angst vor der Geldentwertung.“ (BRZ10/MAR.11912 Braunschweiger Zeitung, 25.03.2010; [Ausführliche Zitierung nicht verfügbar])

59) $\{\{0+2$; keine Real.; Subj.; Akk.; kein Argument; A1; A2 $\}\}\{\{$ SVG; zum A. bringen $\}\}$

Die Anklage wegen viermaligen Verstoßes gegen das Gewaltschutzgesetz sowie

Sachbeschädigung wurde komplettiert durch eine weitere Anklage wegen Trunkenheit am Steuer. Dazu war es gekommen, nachdem der Mann auf der Calberlaher Hauptstraße mit einem Blutalkoholwert von 2,73 Promille, auf einem roten Damenrad entgegen der Fahrtrichtung unterwegs war und der Polizei quasi in die Arme fuhr.

„Die Frauen haben sich von mir getrennt“, brachte der geständige Angeklagte auf richterliche

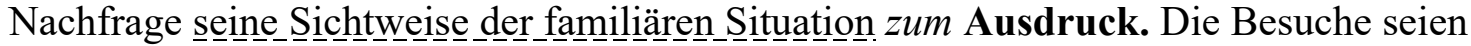
erfolgt, um die Frau zu ärgern, der Fernseher sei aus Versehen heruntergefallen und die volltrunkene Radtour sei nicht der Rede wert gewesen, argumentierte der Mann mit wegwerfender Handbewegung.

„Sein Problem ist der Alkohol“, sagte der Richter, und die Noch-Ehefrau bestätigte diese Vermutung. (BRZ10/OKT.07458 Braunschweiger Zeitung, 15.10.2010; Ehemann schlägt mit Bratpfanne auf Frau ein [Ausführliche Zitierung nicht verfügbar])

60) $\{\{1 ; \mathrm{Gen} ; \mathrm{A} 2\}\}$

3500 Karten stehen für das sommerliche Zwischenspiel im architektonisch reizvollen alten Kraftwerk zur Verfügung (siehe Service-Kasten).

Peter Maffay verzichtet auf ein Honorar. Das Konzert ist Ausdruck der engen Verbundenheit von Künstler, Unternehmen und vor allem VW-Betriebsrat. Dessen Vorsitzendem Bernd Osterloh war es ein besonderes Anliegen, den beliebten Musiker zu einem Zusatzkonzert zu bewegen. „Bernd Osterloh hat tatsächlich großen Anteil am Zustandekommen des Konzerts. (BRZ11/MAI.06023 Braunschweiger Zeitung, 13.05.2011; Maffay rockt das Kraftwerk [Ausführliche Zitierung nicht verfügbar])

61) $\{\{0\}\}$

Im Klinikum steht „Der Morgen“, eine jüngere, 1993 bis 1996 entstandene Arbeit aus Carrara-Marmor. Aus weißem Marmor hat Hermann Kracht noch in seiner letzten Werkphase mehrere Skulpturen geschaffen: Daphne, Kassandra; Figuren aus der Antike. Vertraut waren ihm die antiken Mythen so wie die antiken, von der Renaissance erneuerten Anforderungen an einen Bildhauer: Maß, Proportionalität und Ausdruck.

Holz war sein überwiegend genutztes Material, auch so schwierige Hölzer wie die der Lärche. Dazu gehört das ,lesende Kind“, 2002 für die Wohltberg-Schule gefertigt, viele Jahrzehnte vorher schon „Geschwister“ in der Grundschule Alt-Wolfsburg. (BRZ12/MAR.11969 Braunschweiger Zeitung, 21.03.2012; Skulpturen, die zurück bleiben [Ausführliche Zitierung nicht verfügbar]) 
62) $\{\{0+2$; keine Real.; Subj.; Dat.; kein Argument; A1; A2 $\}\}\{\{S V G ;$ Dat. A. verleihen $\}\}$ Paulas Engagement für die Wale kommt nicht von ungefähr. „Ich mag Tiere gern“, verrät sie. Jetzt konnte sie dieser_Liebe einmal Ausdruck verleihen. Sonst profitieren von dieser Tierliebe zu Hause sieben Schafe, eine Katze und zwei Hunde. 800 Argumente für den Walschutz: Paula aus Rothemühle hat mit viel Fleiß und großelterlicher Hilfe Papier-Wale gefaltet, die über ein Tierschutz-Magazin der US-Botschaft zugestellt werden sollen. (BRZ12/AUG.12378 Braunschweiger Zeitung, 24.08.2012; Ein Herz und ein Händchen für Wale [Ausführliche Zitierung nicht verfügbar])

63) $\{\{1$; von + Dat.; A2 $\}\}$

In jungen Jahren erlernte er wohl tatsächlich das "Handwerk" der Malerei als Beruf. Der künstlerischen Umsetzung widmete er sich erst, seit er seinem offiziellen Berufsleben 2004 den Rücken gekehrt hat.

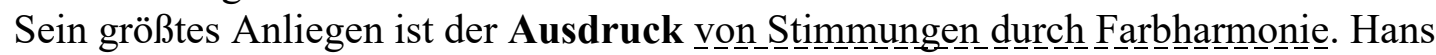
Wilfling arbeitet vorwiegend mit Acryl- und Dispersionsfarbe. Durch raue Schichtungen und der Beifügung von verschiedenen Materialien entstehen einzigartige Strukturen.

(BVZ07/FEB.00965 Burgenländische Volkszeitung, [Wochenzeitung], 14.02.2007, Nr. 07, Jg. 2007, Oberwart, S. 40. - Sachgebiet: Kultur, Originalressort: KULTUR; Die Bilder ohne Titel)

64) $\{\{1+2$; mit + Dat.; Subj.; Akk.; A3; A1; A2 $\}\}\{\{$ SVG; zum A. bringen $\}\}$

Die Führung passierte aus einem Corner an die "lange Stange", wo Ladislav Szijjarto ungehindert einnetzen konnte. Parndorf antwortete prompt, doch Daniel Vasic scheiterte in aussichtsreicher Position.

Nach der Pause war es wieder Vasic, der mit einem Lattenschuss das Kräfteverhältnis an dies_em_-_ A $\underline{b}$ end zum Ausdruck brachte. Auf den Ausgleich musste die 1B aber bis zur Nachspielzeit warten. Bei einem Vorstoß von Matthias Novak wurde dieser per Ellbogencheck im Strafraum niedergestreckt. (BVZ08/SEP.02044 Burgenländische Volkszeitung, [Wochenzeitung], 17.09.2008, Nr. 38, Jg. 2008, Neusiedl, S. 70. Sachgebiet: Sport, Originalressort: FUSSBALL; Volle Ausbeute für Tadtener RumpfElf)

65) $\{\{1$; Gen.; A2 $\}\}$

der Rechtssozialist, der für diesen Posten kandidierte, fiel durch, da ihm die Kommunisten ihre Stimmen verweigerten, weil er es ablehnte, sich gegen die EVG-Verträge auszusprechen. der Druck der Wählermassen daß der Widerstand des französischen Volkes gegen die EVG-Verträge auch im parlamentarischen Leben Frankreichs so stark sichtbar wird, ist ein Ausdruck der Breite dieser Bewegung im ganzen Lande. der Druck der Wählermassen ist so gewaltig, daß buchstäblich jeder einzelne Abgeordnete von seinen Wählern zur Stellungnahme gedrängt wird. im ganzen Lande ergießt sich ein ununterbrochener Strom von Delegationen zu den Behörden, von Gemeinderäten bis zu Regierungsstellen. (BZK/D54.00065 Neues

Deutschland, [Tageszeitung], 04.02.1954, Jg. 9, Berliner Ausgabe "Vorwärts", S. 7. Sachgebiet: Politik, Originalressort: POLITIK; Michel, Rosa: Nationale Erhebung in Frankreich gegen EVG)

66) $\{\{0+2$; keine Real.; Subj.; dass-Objektsatz; kein Argument; A1; A2 $\}\}\{\{$ SVG; zum A. bringen $\}\}$ 
die Sozialistische Einheitspartei Deutschlands und die in der Nationalen Front des demokratischen Deutschland vereinigten Parteien und Massenorganisationen der DDR sind fest entschlossen, auch in Zukunft alles zu tun, um die Freundschaft zwischen unseren Völkern zu hüten und auf der Grundlage des im Juni dieses Jahres abgeschlossenen Vertrages über Freundschaft, gegenseitigen Beistand und Zusammenarbeit zwischen der Sowjetunion und der DDR weiter zu vertiefen.

brüderliche Kampfgemeinschaft

die Sozialistische Einheitspartei Deutschlands hat in Verbindung mit den Ergebnissen des Oktoberplenums des ZK der KPdSU ihr volles Vertrauen zum Leninschen ZK der KPdSU

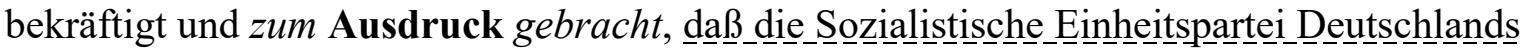

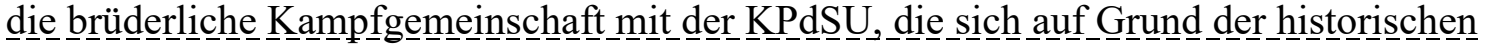
Veränderungen im Leben des Sowjetvolkes zur Partei des ganzen Volkes entwickelt hat, weiter festigen wird. jeder, der mit den grundlegenden Ideen und Prinzipien des MarxismusLeninismus und den neuen Erscheinungen im Leben des Sowjetvolkes vertraut ist, muß die im Programm der KPdSU enthaltene historisch bedeutsame Feststellung bestätigen, daß "durch den Sieg des Sozialismus in der UdSSR und die Festigung der Einheit der Sowjetgesellschaft" die KPdSU zur Partei des ganzen Volkes geworden ist. diese neue qualitativ höhere Etappe in der Entwicklung der KPdSU ergibt sich aus der unbestreitbaren Tatsache, daß der Sowjetstaat entsprechend der Veränderung der Klassenstruktur der Sowjetgesellschaft sich von einem Staat der Diktatur des Proletariats zu einem Staat des ganzen Volkes entwickelt hat; sie widerspiegelt die großen Leistungen, die da

(BZK/D64.00558 Neues Deutschland, [Tageszeitung], 07.11.1964, Jg. 19, Berliner Ausgabe, S. 7. - Sachgebiet: Politik, Originalressort: POLITIK; Honecker, Erich: Die KPdSU - die Partei des ganzen Volkes)

67) $\{\{1$; Gen.; A2 $\}\}$

Nicht jeder Islamist ist jedoch zwangsläufig auch ein Terrorist.

Das Bin-Laden-Netz - in 60 Ländern verankert - ist also kein Verein von Psychopathen oder Kriminellen, sondern Ausdruck einer politisch-religiösen Strömung, die mit Anhängern innerhalb der islamischen Zivilisation rechnen kann. Gemäßigte Muslime machen Front gegen den Bin-Laden-Terrorismus und ergreifen Partei für den Westen im Kampf gegen dieses Netz-werk. Die islamische Orthodoxie und selbst nicht zum Terrorismus gehörende Islamisten hingegen haben sich gegen Amerika und hinter bin Laden gestellt.

(FOC01/OKT.00197 FOCUS, 08.10.2001, S. 082-082, Originalressort: DEUT/Deutschland; Deutsche Behörden haben viel verschlafen)

68) $\{\{0\}\}$

Er trank ja gern und war schon ein wenig beschwipst. Es fiel sogleich auf, wie ungemein belesen und gebildet er war. Blickte man in seine Augen, dann überwältigte ein finsterer, ruhiger und beklemmender Ausdruck.

FOCUS: Klingt irgendwie "existenziell"? (FOC06/APR.00182 FOCUS, 10.04.2006, S. 066068, Sachgebiet: Kultur, Originalressort: KULT/Kultur; "Nenn das Unglück nicht beim Namen")

69) [[1; Apposition; A']]

Und, der klare Sache: Natuurlich hat er eine große Familie, die an den Hits mitgeschrieben hat - Janusz Popolski etwa, die trübste aller Tassen, Andrzej, der Botschafter der Liebe und Porno-King, oder Dorota, Popolskis bezaubernde rote Kusinetschka, die Heißeste der Heißen und 14-fache Miss Zabrze, wohlgemerkt in Folge. 
Der Name "Popolski" verballhornt den Ausdruck "auf Polnisch" (po polsku), und selbstverständlich endet in der Show alles auf -ski: Die Musikinstrumentski baute Stradiwarski, das Polyester-Outfitski stifteten Dolski und Gabbanski. Im Internets-ki gibt's das T-Shirt-Shopski. Und selbst dem guten Roland-Keybord der Popolski-Band wird ein Stück des Schriftzugs wegradiert, denn es stammt - klar - aus "Poland". (FOC10/FEB.00291 FOCUS, 13.02.2010, S. 110-111, Sachgebiet: Medien, Originalressort: Medien; Hoppla, "Der Popolski-Show"!)

70) $\{\{1$; Gen.; A2 $\}\}$

Ihr offizielles Urteil über den Parlamentarismus bleibt auf Nachfrage vernichtend, so, wie es der Parteilinie entspricht. Wegner gehört seit mehr als 40 Jahren der kommunistischen DKP an, und die möchte das herkömmliche politische System überwinden. Parlamente, meint die DKP, seien sowieso nur Ausdruck der Herrschaft des Kapitals - und die sei von übel und müsse enden durch den Sieg der Arbeiterklasse. Aber Theorie und praktische Arbeit sind eben zwei verschiedene Dinge. Als kürzlich während einer Anhörung des Sozialausschusses die Vertreter von CDU und FDP anfingen, laut über eine notwendige bessere Qualifikation von Pflegedienstkräften nachzudenken, hatte Wegner, die frühere Krankenschwester, ein AhaErlebnis. (HAZ09/DEZ.03124 Hannoversche Allgemeine, 21.12.2009; Klassenkampf aus der letzten Reihe [Ausführliche Zitierung nicht verfügbar])

71) [[1; Apposition; A']]

Sagnol geht fest von Kahns Abtritt als Kapitän am Ende der Saison aus

Der französische Fußball-Nationalspieler Willy Sagnol vom deutschen Rekordmeister Bayern München hat in einem Interview mit dem Wochenend-Magazin der französischen Zeitung "L'Equipe" verraten, dass Torwart Oliver Kahn bereits 2007 seine Kapitänsbinde abgeben und er diese übernehmen wird. "Ich werde definitiv in einem Jahr Kapitän in München sein, wenn Oliver Kahn aufhört", sagte der Vizeweltmeister in dem auf Französisch geführten Gespräch und benutzte dabei den Ausdruck "wenn" als zeitlichen Begriff und nicht die im Deutschen mögliche Verwendung als "falls".

Damit geht der Rechtsverteidiger fest von Kahns Abtritt als Kapitän nach Ablauf dieser Saison aus. Unklar an der Formulierung Sagnols blieb allerdings, ob Kahn möglicherweise sogar sein Karriereende für 2007 ins Auge gefasst haben könnte. (HMP06/NOV.00488 Hamburger Morgenpost, 06.11.2006, S. 8; Willy "Ich werde der neue Bayern- Boss" [Ausführliche Zitierung nicht verfügbar])

72) $\{\{1$; von + Dat.; A2 $\}\}$

Hafize Dogan, Langenhorn

Betr.: GAL offen für Schwarz-Grün

Anja Hajduk meint, nicht mit der CDU zu verhandeln, wäre ein Ausdruck von Schwäche. Ich meine, wer glaubt, mit Ole von Beust grüne Inhalte umsetzen zu können, hat den Schuss nicht gehört. Olaf Platen, Wilhelmsburg (HMP08/MAR.00012 Hamburger Morgenpost, 01.03.2008, S. 7; LESERBRIEFE [Ausführliche Zitierung nicht verfügbar])

73) $\{\{0+2$; keine Real.; Subj.; Akk.; kein Argument; A1; A2 $\}\}\{\{$ SVG; zum A. bringen $\}\}$ Außerdem shaken die Mädels zu diesem Lied gern ihre Hüften - und Mann kommt auf seine Kosten."

",Wake me up' von Avicii ist derzeit der coolste Hit"

Hula-Kette, Feder-Ohrringe, knallbunte Shirts: Diese Mädels haben die gute Laune richtig zum Ausdruck gebracht. Moni (26), Julie (25), Nessi (33), Dani (24), Anja (27) und Melli (34) aus Horst: "Ganz klar, ,Wake me up' von Avicii ist derzeit der coolste Titel zum 
Abfeiern. Da kommt man gleich in Bewegung. (HMP13/JUL.05020 Hamburger Morgenpost, 28.07.2013, S. 6; So klingt der Sommer [Ausführliche Zitierung nicht verfügbar])

74) $\{\{1+1$; in + Dat.; Subj.; A3; A2 $\}\}\{\{$ SVG; zum A. kommen $\}\}$

Bei strömendem Regen fügte die Iseltaler Elf Landskron die erste Heimniederlage zu. Über die gesamten 90 Minuten gaben die zweikampfstarken Matreier den Ton an. Sie waren läuferisch und spielerisch überlegener, als es im Ergebnis zum Ausdruck kommt. Hätten Matreis Stürmer alle herausgespielten Torchancen genützt, wäre Landskron/VSV um eine deutliche Abfuhr nicht herumgekommen. (I00/APR.18907 Tiroler Tageszeitung, [Tageszeitung], 03.04.2000. Originalressort: Regional Osttirol; Punktezuwachs für)

75) $\{\{0\}\}$

Orchestermusiker an den Sesselkanten

Dann die Siebte als Auftakt zum Bruckner-Schwerpunkt des Musikfestes. Muti entwickelt die Brucknerschen Dimensionen aus dem Inneren, läßt das Hauptthema des 1. Satzes bedachtsam, geradezu rezitativisch anheben, zwingt die Musik und die Musiker zum kammermusikalisch konzentrierten Ausdruck, entwickelt die prächtigen Klangeruptionen aus der gewaltigen Innenspannung der Symphonie. Der Ausbruch mit dem Beckenschlag im Adagio, der folgende Abgesang auf den Tod Richard KRITIK

Wagners, das mit viel klanglicher Raffinesse drängende Scherzo oder der hymnische Schluß: Muti leitet Bruckners spirituelle Ausdruckssphären konsequent aus seinem genialen Klangempfinden ab und behält den formalen Spannungsbogen bis zuletzt fest im Griff. (I96/MAI.17513 Tiroler Tageszeitung, [Tageszeitung], 06.05.1996. - Sachgebiet: Kultur, Originalressort: Kultur; Eleganz und Spiritualität)

76) $\{\{2+1$; Posspr.; in + Dat.; Subj.; A2; A3; A2 $\}\}\{\{$ SVG; Poss. A. finden $\}\}$

Um diesem Horror vorzubeugen, pflegt man zu mächtigen Zeitungen und Magazinen beste Beziehungen, indem ihre RedakteureInnen im Staatsfunk schon einmal auch Sendungen moderieren und gestalten dürfen. Dies schafft ein Klima gegenseitigen Verständnisses und Umgangsformen, die jenen in einem englischen Golfklub des 19. Jahrhunderts in nichts nachstehen.

Die Folge solch brüderlicher Verhältnisse ist naturgemäß eine geistige Stagnation, die ihren besten Ausdruck im kontinuierlichen Niedergang der heimischen Film-, Unterhaltungs-, Verlags- und Musikindustrie findet. Wenn man bedenkt, daß England allein mit seiner Popmusik mehr Exporterlöse erzielt als mit seiner Stahlindustrie, wird klar, daß es nicht nur aus Gründen der geistigen Hygiene, sondern auch aus wirtschaftlichen Überlegungen heraus nicht gleichgültig sein kann, wenn eine der wichtigsten Branchen der Zukunft als Wiener Erbhof nur noch sich selbst reproduziert.

Denn nicht nur bei den General- und Normalintendanten ist die Auswahl auf ein paar Namen zwischen Parteizentralen und Medienhofräten beschränkt. (I98/JUL.28702 Tiroler

Tageszeitung, [Tageszeitung], 18.07.1998. Originalressort: Tirol aktuell; Nachrichten aus der Welt der Saurier)

77) $\{\{1$; Gen.; A2 $\}\}$

CHRISTOPH BAGDY

Die Stadt Fürstenfeld sagte Dank- für unermüdliches Bemühungen um wirtschaftliche, kulturelle oder sportliche Entwicklung ebenso wie für herausragende Leistungen im Sozialbereich. Als sichtbarer Ausdruck der Wertschätzung standen Urkunden und Ehrenzeichen zur Verfügung. Diese wurden im neuen Mediensaal der Hauptschule 
Schillerplatz an 20 verdiente Personen des Fürstenfelder Stadtlebens überreicht. "Die Schwierigkeit der Auswahl lag in der numerischen Begrenzung", betonte Vizebürgermeister Josef Rath, dass dem Gemeinderat die "Vorauswahl" nicht leichtgefallen war, jede Ehrung dann jedoch einstimmig beschlossen wurde. (K00/FEB.08436 Kleine Zeitung, [Tageszeitung], 01.02.2000. Originalressort: Feldbach; Dank \& Anerkennung)

78) $\{\{1$; Gen.; A2 $\}\}$

Das Gespür für Farben und Formen hat den gelernten Handelskaufmann, der von 1933 bis zu seiner Pensionierung in Sachsenburg tätig war und nun in Spittal lebt, von Kindheit an begleitet; das künstlerische Sehen und Umsetzen wird seit 1985 in von namhaften Künstlern geleiteten Kursen und Seminaren und in der vor zehn Jahren gegründeten "Mal-Gruppe Rojachhof" (zu deren Initiatoren er gehört) geschult.

Die beständige Auseinandersetzung mit der Kunst ermöglicht Kohl den Blick für die kleinen Dinge, für das Erleben der Natur; in der Begegnung mit Gleichgesinnten vor allem bei den monatlichen Treffen der Malgruppe hat er "Toleranz gegenüber den Menschen und ihrem Anderssein" gelernt und erfahren.

So sieht Roland Kohl dann auch die Ausstellung im Schloss als reinen Ausdruck der Bereitschaft, sich der "Herausforderung Kunst" zu stellen.

Roland Kohl in seinem kleinen Atelier in Spittal.

Der 94-Jährige initiierte die Mal-Gruppe Rojachhof mit RIE-PRESS (K00/SEP.71755 Kleine Zeitung, [Tageszeitung], 27.09.2000. - Sachgebiet: Kultur, Originalressort: Kultur; Der Blick für kleine Dinge wird abstrakt umgesetzt)

79) $\{\{1$; Posspr.; A2 $\}\}$

REINHOLD REITERER

Einen faulen, nachlässigen und närrischen Menschen erkennt man, wenn er eine lange, flache Hand hat mit kurzen und dicken Fingern", heißt es im "Kurzen Tractat von der Chiromantie des sinnreichen Philosophen Johann Weber" aus dem Jahr 1710. "Es ist mit den Händen wie mit den Gesichtern: ihr Ausdruck wechselt unablässig", notierte 1950 der Kunsthistoriker Carl Burkhardt nach eingehender Betrachtung der Skulpturen von Auguste Rodin.

Die Hand: Symbol, Signal, Werkzeug. Anatomisch beeindruckend. (K97/JAN.00023 Kleine Zeitung, [Tageszeitung], 01.01.1997. Originalressort: Lokal; In diesem Ort sprechen Hände Bände)

80) $\{\{0+2$; keine Real.; Subj.; Akk.; kein Argument; A1; A2 $\}\}\{\{$ SVG; zum A. bringen $\}\}$ Laut Information des Umweltministeriums blieb dieser Fonds jedoch bis heute leer. Daß die slowenische Regierung angesichts der Mißachtung der gesetzlichen Vorgaben untätig bleibt, läßt sich nur dahingehend erklären, daß auch seitens der politischen Vertretung kein ernsthaftes Interesse an einer vorzeitigen Schließung des Atomreaktors besteht. "Wenn Österreicher ihr Unbehagen zum Ausdruck bringen, kann ich das verstehen. Andererseits importiert Österreich selbst Atomstrom aus Frankreich. Wenn im AKW Kr ko in moderne Technologie investiert wird, dann ist uns ein sicheres Kraftwerk lieber als Panikmache", meint Danilo Siter, Bürgermeister von Kr ko. (K97/JUN.48296 Kleine Zeitung, [Tageszeitung], 26.06.1997. Originalressort: Lokal; Kapitel "Ausstieg" in Krko beendet)

81) $\{\{1 ;$ Gen.; A2 $\}\}$

Warum die ganz gewöhnlichen Männerschlapfen über so ein saumäßiges Image verfügen, ist mir schleierhaft. Modeleute sagen, Männer in Sandalen wären lächerlich, Schwächlinge, Spießer, eine ästhetische Beleidigung für das Auge. Ein Ausdruck selbstvergessener Geschmacksverwirrung. Wir lassen uns nicht länger von selbsternannten Modepäpsten 
beleidigen, wir gönnen dem gemeinen Fußvolk ganz unten verschiedene Treter, die oben praktisch ohne sind. Frische Zugluft für den Schweißfuß. (K98/JUN.42534 Kleine Zeitung, [Tageszeitung], 02.06.1998. - Sachgebiet: Wirtschaft, Originalressort: Wirtschaft; Die tollsten Cabrios für das gemeine Fußvolk)

82) $\{\{1$; Gen.; A2 $\}\}$

Zum Höhepunkt: "Um diese Zeit ereignete sich ein schneller Übergang von der Helligkeit zur Dunkelheit: der ganze Eindruck der Szene auf die auserlesene Versammlung der Zuschauer hatte etwas Schauerliches: Die Gesichter erschienen in fahler Farbe, die Berge waren in tiefes Schwarz gehüllt. Wenige Sekunden vor dem Verschwinden ward der Wind heftiger." Und weiter: "Unter der versammelten Menge ward ein allgemeiner Ausdruck des Erstaunens laut; einzelne Lichter erschienen in der in ein magisches Dunkel gehüllten Stadt.. ."

Der gesamte, ungekürzte Text und andere historische und praktische Hinweise finden sich unter http://www.joanneum.ac.at/ sonnen- finsternis. (K99/JUL.53270 Kleine Zeitung, [Tageszeitung], 17.07.1999. Originalressort: Lokal; "Die Gesichter in fahler Farbe")

83) $\{\{1$; von + Dat.; A2 $\}\}$

Es gehe darum, Macht zu erleben, sich Respekt zu verschaffen, weiß die Psychologin Bettina Schuster vom Landesschulamt. Die Beute sei weniger wichtig. Anders als früher sei Gewalt zur üblichen Form von Konfliktbewältigung geworden, konstatiert Jugendstaatsanwältin Kerstin Deike und sieht das als Ausdruck von Verwahrlosung und Wertverlust. Besonders bei brutalen Übergriffen, wie der Folterung wehrloser Kinder oder Obdachloser, prüfe sie die verminderte Schuldfähigkeit. Doch die Täter seien oft voll zurechnungfähig.

(L99/MAR.08907 Berliner Morgenpost, [Tageszeitung], 03.03.1999, S. 11.

Originalressort: BERLIN; Von Vera Fischer: "Das hatn Kick": Warum Jugendliche zuschlagen)

84) $\{\{1$; Gen.; A2 $\}\}$

"Ich trete wieder an, garantiert", sagte der 72jährige gestern. Unterdessen stieß die von Bubis in Interviews gezogene Negativbilanz seiner Amtszeit auf Widerspruch. Die Selbstkritik sei ungerechtfertigt und Ausdruck seiner Bescheidenheit, sagte das Präsidiumsmitglied des Zentralrats, Michel Friedman.Bubis reagierte dankbar auf den positiven Zuspruch. Er brauche diese Ermunterung, um die Kraft zum Weiterarbeiten zu finden. In den vergangenen Jahren habe er bei offiziellen Stellen möglicherweise viel bewirkt. (L99/JUL.46388 Berliner Morgenpost, [Tageszeitung], 29.07.1999, S. 2. Originalressort: POLITIK; BM/AP: Bubis will trotz bitterer Bilanz wieder antreten)

85) $\{\{0+3$; keine Real.; Akk.; Subj.; Pronadv. damit; kein Argument; A2; A1; A3 $\}\}\{\{$ SVG; zum A. bringen $\}$ \}

»Das ist wieder typisch«, maulte Anita. »Für die anderen hast du immer ein gutes Wort und

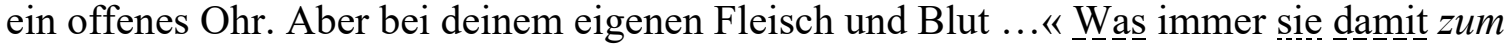

Ausdruck hatte bringen wollen, Anita behielt es für sich und zog beleidigt ab in den Kleinen Salon.

Wilma blickte auf die Uhr. Es war kurz vor 21 Uhr. (DIV/EPS.00001 Emme, Pierre:

Schneenockerleklat . Palinskis neunter Fall , (Erstveröffentlichung 2009) - Meßkirch: Gmeiner-Verlag, 2011)

86) $[[0]]$ 
Auch vom Schwerpunkt Bierkneipe bin ich weg und habe die Richtung „Speiserestaurant“ eingeschlagen - als Küchenmeister liegt mir das sowieso viel mehr - und mittlerweile bin ich wieder recht zufrieden mit meinem Betrieb."

„Voll die richtige Linie, man sieht es an unseren Tellern“, lobte Paul Wellmann das Essen, aber Lindt wollte die Gelegenheit nutzen, den offensichtlich gut informierten Wirt noch etwas auszufragen: „Haben die dann irgendwann doch noch neue Kunststoffpressen angeschafft oder wie läuft das jetzt bei >Blanco<?“،

„Ach wo“, der Wirt schüttelte den Kopf, ,es kommen ja immer noch ein paar Mitarbeiter zu mir an den Stammtisch, daher weiß ich recht genau, welche Schweinereien - Entschuldigung, aber für mich ist das absolut der richtige Ausdruck - da abgingen. Nachdem die Mehrzahl der Belegschaft entlassen war, kamen die ganzen Maschinen fort. Klar, die alten Teile zur Verschrottung, dachte jeder. (DIV/LBB.00001 Leix, Bernd: Bucheckern . Oskar Lindts erster Fall , (Erstveröffentlichung 2005) - Meßkirch: Gmeiner-Verlag, 2011)

87) $\{\{0+2$; keine Real.; Subj.; Akk.; kein Argument; A1; A2 $\}\}\{\{$ SVG; zum A. bringen $\}\}$ "Mär sinn amgaangen, eis eege Wuerzelen ze kappen", schreibt Spautz. Und: "Unsere Glaubwürdigkeit geht den Bach runter."

Die Politikerin erklärt weiter, dass sie es nicht als normal empfinde, dass bestimmte wirtschaftsliberale Personen, die im Besitz einer LSAP-Mitgliedskarte seien, ohne Hemmungen und mit einer gewissen "Effiezienz" ihre Thesen nach innen und nach außen vertreten würden. Sie jedenfalls werde ihre Position als Gewerkschafterin zukünftig noch konsequenter verteidigen und dies auch bei den künftigen Abstimmungen zum Ausdruck bringen. "Ich weiß", gibt sich Spautz überzeugt, "dass meine Haltung von vielen Parteimitgliedern, Wählerinnen und Wählern und von den Gewerkschaftlern verstanden wird."

Aber Vera Spautz kritisiert nicht nur die LSAP-Oberen, die Abgeordnete übt auch Selbstkritik. (LTB10/SEP.00576 Luxemburger Tageblatt, 25.09.2010, Sachgebiet: Politik, Originalressort: POL - POLITIK; LSAP vor der Zerreißprobe?)

88) $\{\{0+1$; keine Real; Subj; kein Argument; A2 $\}\}\{\{$ SVG; zum A. bringen + PASSIV $\}\}$ Raus aus der Dunkelkammer

Die LSAP habe bis zum letzten Freitag viel Geduld in dem Cargolux-Dossier aufgebracht. Die Fragen und Skepsis $\underline{\text { die }}$ man hatte, wurden auch am Freitag in der Sitzung der Nachhaltigkeitskommission zum Ausdruck gebracht, meinte gestern LSAP-Fraktionschef Lucien Lux in einer Reaktion auf die letzten Entwicklungen in Sachen Cargolux. "Das Projekt muss jetzt aus der Dunkelkammer heraus", forderte Lux. Bereits das Vorprojekt, das am Montag geleakt wurde, zeige, dass die Verhandlungen "knallhart" geführt werden und dass die Konsequenzen für das Personal und den Standort ebenso "knallhart" sein könnten. (LTB13/OKT.00832 Luxemburger Tageblatt, 09.10.2013, Raus aus der Dunkelkammer)

89) $\{\{1 ;$ Gen.; A2 $\}\}$

Das Großherzogtum hat sich in den letzten zwei Jahren mit seinem nicht-ständigen Sitz im UN-Sicherheitsrat Respekt auf dem internationalen Parkett verschafft. Außenminister Jean Asselborn und das Diplomatenteam um UN-Botschafterin Sylvie Lucas haben in zahlreichen Dossiers das nötige Fingerspitzengefühl bewiesen: Wichtige Resolutionen wurden etwa im Sicherheitsrat im Zusammenspiel mit anderen Staaten beschlossen. Dass Asselborn in seiner gestrigen Erklärung zu Luxemburgs Außenpolitik Lucas und ihrer Mannschaft dankte, war demnach nicht übertrieben, sondern Ausdruck intakter diplomatischer Strukturen im Außenministerium. Dies ist besonders im Hinblick auf das kommende Jahr wichtig: 
Luxemburg wird 2015 zum zwölften Mal die EU-Ratspräsidentschaft übernehmen. (LTB14/NOV.02325 Luxemburger Tageblatt, 26.11.2014, Diplomatisches Profil)

90) $\{\{1$; Gen.; A2 $\}\}$

Die wachsende Bedeutung des Pazifikraums verbietet es der EU gleichwohl, sich in den Schmollwinkel zurückzuziehen. Vor allem Bundeskanzler Helmut Kohl wird nicht müde zu betonen, welche Chancen sich den wirtschaftlich allesamt angeschlagenen europäischen Staaten in Asien bieten. Die Häufigkeit seiner Besuche in China, wo er sich derzeit zum vierten Mal in seiner Amtszeit aufhält, ist Ausdruck der wachsenden Bedeutung Asiens für die deutsche (und europäische) Außenpolitik.

Die Apec umfaßt sechs Jahre nach ihrer Gründung ein Gebiet von mehr als der Hälfte des Erdballs, in dem mehr als zwei Milliarden Menschen leben. Mit Ausnahme der EU sind alle Industrieländer und auch die wichtigsten Schwellenländer Mitglied. (M95/511.29267 Mannheimer Morgen, [Tageszeitung], 16.11.1995, Jg. 50. - Sachgebiet: Wirtschaft, Originalressort: WIRTSCHAFT; Europa muß Türen nach Asien weiter öffnen)

91) $\{\{1+2$; mit + Dat.; Subj.; Dat.; A3; A1;A2 $\}\}\{\{$ SVG; Dat. A. verleihen $\}\}$ Der Münchner Regisseur Hans-Christian Schmid und sein in Berlin studierender irischer Kollege Eoin Moore haben zu gleichen Teilen den mit 80000 Mark dotierten RegieFörderpreis der Hypo-Bank erhalten. Die Auszeichnung wurde den beiden jungen Regisseuren auf dem Münchner Filmfest für ihre Kinofilme "23" und "plus-minus null" verliehen. Die Preisträger seien Talente, die mit ihren Arbeiten ihrer groß_en Lieb-be zum Ausdruck verliehen hätten, befand die Jury. Hans-Christian Schmid erzählt in "23" die Geschichte eines Computerfreaks, der den Bezug zur Realität verliert, vereinsamt und zugrunde geht. Der Hypo-Förderpreis für junge Nachwuchsregisseure ist der höchstdotierte private Filmpreis in Deutschland. (M98/JUL.56040 Mannheimer Morgen, [Tageszeitung], 07.07.1998, Jg. 53. - Sachgebiet: Kultur, Originalressort: Feuilleton; Umschau, [Feuilleton])

92) $\{\{0+2$; keine Real.; Subj.; Dat.; kein Argument; A1; A2 $\}\}\{\{$ SVG; Dat. A. geben $\}\}$ Über die Tätigkeit des von Schaub als "Hauptarbeiter des Vereins" bezeichneten Langendörfer konnten die Revisorinnen Ingrid Dreier und Annemarie Zimmermann-Serr "nur Gutes" berichten. Deshalb erfolgte die Entlastung des Vorstands auf Vorschlag Dreiers

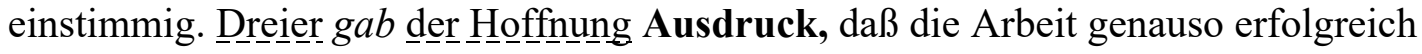
fortgeführt werden könne wie bisher.

"Nur durch die Unterstützung des Fördervereins ist es möglich, Dinge zu fördern, für die sonst kein Geld da ist", bemerkte Gerlitz dankbar. Ihre vorgetragene Idee, mehr Literatur auf Tonträgern bereitzustellen, da deren Bedeutung wachse, war ein weiterer Hinweis für die Notwendigkeit, neue Mitglieder zu werben. (M99/MAI.31364 Mannheimer Morgen, [Tageszeitung], 17.05.1999, Jg. 54. Originalressort: Rhein-Neckar; Steigende Benutzerzahlen in der Stadtbücherei)

93) $\{\{1$; Gen.; A2 $\}\}$

Zur Kandidatur hatte sich Stumpf nur zögernd bereit erklärt: "Schließlich weiß ich aus meiner Zeit als Stellvertreter, was da auf mich zu kommt", begründet er. Außerdem müsse auch der Lebenspartner grünes Licht geben, bevor man ein solches Amt übernehme.

Dass es eine ganze Reihe von CDU-Mitgliedern abgelehnt hat, den Posten zu übernehmen, ist für den 55-Jährigen Ausdruck eines gesellschaftlichen Problems: Auch in seiner Funktion als Abteilungsleiter beim SV "Vorwärts" Bobstadt erlebt Stumpf, dass immer weniger Menschen sich in Organisationen langfristig engagieren wollen. "Viele scheuen sich einfach, 
Verantwortung zu übernehmen." Da sei ja auch viel einfacher, die Aktiven zu kritisieren, wenn's nicht so läuft, wie man sich das vorstellt, merkt er ironisch an.

Obwohl das Amt viel Arbeit und Verantwortung mit sich bringt, hat ihn die Aufgabe schließlich doch interessiert: "Die Ge staltungsmöglich- keiten haben mich gereizt", erzählt Stumpf, "ich möchte etwas bewegen." (M99/DEZ.79872 Mannheimer Morgen, [Tageszeitung], 03.12.1999, Jg. 54. Originalressort: Lokal Bürstadt / Biblis; Von unserem Redaktionsmitglied Andreas Rother: In Teamarbeit etwas bewegen)

94) $\{\{1+2$; in + Dat.; Subj.; Akk.; A3; A1; A2 $\}\}\{\{$ SVG; zum A. bringen $\}\}$ Gleiche Eigenschaften attestierte auch Bürgermeister Alfred Kappel dem politisch" stets fairen" Ortsvorsteher. Und auch Gerhard Maier, Vorsitzender der Bibliser Gemeindevertretung, betonte die gute und von persönlicher Freundschaft geprägte Zusammenarbeit -" auch wenn wir politisch von unterschiedlicher Coleur sind". Fraktionsmitglied Hildegard Cornelius-Gaus hatte ihre Laudatio auf den "König von Nordheim". in Prosa-Form zum Ausdruck gebracht, und die Gäste aufgefordert, mit ihr anzustoßen auf den "besten Ortsvorsteher, den wir in Nordheim haben".

"Was soll ich dazu noch sagen?", fragte Fiedler in die Runde. Er dankte Dieter als Redner, dankte Kappel für die "gute Zeit miteinander", dankte Maier für den "menschlich sehr guten Umgang untereinander", und dankte Gimbel, der die kleine Feier arrangiert hatte.

(M00/AUG.46384 Mannheimer Morgen, [Tageszeitung], 14.08.2000, Jg. 55. Originalressort: Lokal Bürstadt / Biblis; Dem "König von Nordheim" aufgewartet)

95) $\{\{1$; Posspr.; A1 $\}\}$

MARTINA GEDECK: Ich habe sehr viel mit Kindern gespielt, die können auch sehr verkrampft und unbegabt sein. Tatjana Trieb war für mich ein großes Glück, weil sie intuitiv genau das gemacht hat, worauf es ankommt. Sie hat reagiert, mit ihrem Gesicht, mit ihrem Ausdruck. Das war sehr aus der Situation heraus, was für dieses Mutter-Tochter-Verhältnis wichtig ist. "Grüne Wüste" ist ein Film über Beziehungsprobleme, aber keine Komödie, über erste Liebe, aber keine Teenie-Romanze... (M01/JAN.02290 Mannheimer Morgen, [Tageszeitung], 12.01.2001, Jg. 56. - Sachgebiet: Kultur, Originalressort: Feuilleton; Von unserem Mitarbeiter Manfred Müller: "Man sollte wesentliche Filme machen", [Feuilleton])

96) $\{\{1+2$; durch + Akk.; Subj.; Akk.; A3; A1; A2 $\}\}\{\{$ SVG; zum A. bringen $\}\}$

Als Multitalent beherrscht er nicht nur das Saxophon oder die Jazzklarinette, sondern steuert hin und wieder auch Gesangsnummern bei. Sein "La Fiesta"-Arrangement brachte sogar Latin-Groove ins Zentrum. Als große Jazzhoffnung angekündigt und dies auch bestätigend: der 24-jährige Münchner Trompeter Axel Schlosser, der drei Jahre lang in Mannheim lebte und seine Fre zum Ausdruck brachte. Mit Peter Feil (Posaune), Tom Schlüter (Piano), Wolfgang Mörike (Bass) und Oliver Strauch (Schlagzeug) hat die Formation weitere, gleichrangige Musiker zu bieten.

Wunderbar relaxter Kammerjazz, der ohne Knalleffekte auskommt und den großen Schillerplatz auch bis in die hintersten Ränge erfüllt. (M01/JUL.55745 Mannheimer Morgen, [Tageszeitung], 30.07.2001, Jg. 56. Originalressort: Lokal Lampertheim; Von unserem Redaktionsmitglied Uwe Rauschelbach: Die "Sultans" lassen den Schillerplatz swingen)

97) $\{\{0+1$; keine Real.; Akk.; kein Argument; A2 $\}\}\{\{$ SVG; zum A. bringen $\}\}$ Gestiftet hat sie die Gemeinde, 3000 Mark hat sie für das Klettergerät bereitgestellt. 
"So alle zwei bis drei Jahre lassen wir den Kindern was Gutes zukommen", bestätigte Bürgermeister Roos, der zusammen mit Haupt- und Personalamtsleiter Arno Hilsheimer im Kindergarten in der Mozartstraße war und sich davon überzeugte, dass die Kinder zufrieden sind.

Um die Fre das Lied von der Schlangenjagd geübt. Mit großen Bewegungen und vielen Geräuschen sangen sie dem Bürgermeister vor, wie sie durch das Schlangengras und über den See das Schlangennest finden und am Schluss trotz allem Mut Reißaus nehmen.

Das hölzerne Reptil im Garten wirkt allerdings alles andere als bedrohlich. (M01/NOV.82984

Mannheimer Morgen, [Tageszeitung], 01.11.2001, Jg. 56. Originalressort: Lokal Bürstadt / Biblis; Hölzernes Reptil erfreut Kinder)

98) $\{\{2+1$; Posspr.; in + Dat.; Subj.; A2; A3; A2 $\}\}\{\{$ SVG; Poss. A. finden $\}\}$

Der englische Künstler benutzte für dieses Konzept die gleichen hochgezüchteten technischen Möglichkeiten der Medien, um deren Manipulationsfähigkeit dem Betrachter vor Augen zu führen.

1997 überzeugte er bei der documenta X mit seinen "Seven rooms", die er zusammen mit dem Informationstheoretiker Ecke Bonk entwickelte und für die er den Arnold-Bode-Preis der Stadt Kassel erhielt. Seine Auseinandersetzung mit Schrift und Sprache fand in seinen Illustrationen zu James Joyces "Ulysses" seinen Ausdruck, die er bereits 1948 begann und bis 1982 zum 100. Geburtstag des Autors in verschiedenen digitalen Drucktechniken vollendete. Erst von Joyce, so sagte Hamilton in einem Interview, habe er das Malen gelernt.

(M02/FEB.14362 Mannheimer Morgen, [Tageszeitung], 22.02.2002, Jg. 57. - Sachgebiet: Kultur, Originalressort: Feuilleton; Mit kritischer Distanz, [Feuilleton])

99) $\{\{2+1$; Posspr.; in + Dat.; Subj.; A2; A3; A2 $\}\}\{\{$ SVG; Poss. A. finden $\}\}$ "Abwegig", Kommentar vom 30. 11. 2002 Frau Ball gibt einen sehr einseitigen Kommentar zu einer sehr verkürzten Meldung ab, die unter dem Tenor steht "Ärzte wollen mehr Pillen verordnen". Der Kommentar ist deswegen abwegig, da er in keiner Weise die differenzierten Bemühungen der Ärzteschaft, die sich unter dem Thema "Perspektiven und Chancen in einer zukunftsorientierten Arzneimittelpolitik" geäußert hat, erwähnt. Im Kommentar fehlt der Hinweis darauf, dass der Arzt dem Patienten gegenüber die Pflicht hat, die geschuldeten Leistungen nach einem bestimmten Anforderungsmaß - auch im Arzneimittelbereich -, welchen heute seinen Ausdruck im Wort "medizinischer Standard" findet, zu erbringen. Da die Vertragsärzte (Kassenärzte) unverändert allein die gesamte Verantwortung für die Arzneimittelversorgung tragen im Bereich der gesetzlichen Krankenversicherungen, geht die aktuelle Diskussion um die Arzneimittel an den Ärzten nicht vorbei. Die Verquickung mit der Veröffentlichung der Stiftung Warentest über die Selbstmedikation ist ebenso abwegig.

(M02/DEZ.93583 Mannheimer Morgen, [Tageszeitung], 12.12.2002, Jg. 57. Originalressort: Briefe an den Herausgeber; Ärzte kommen zu kurz, [Leserbrief])

100) $\{\{2+1$; Posspr.; in + Dat.; Subj.; A2; A3; A2 $\}\}\{\{$ SVG; Poss. A. finden $\}\}$

"Fire \& Rain". Unsere Welt beherbergt eine Vielzahl unterschiedlicher Kulturkreise, Religionen, Völkergruppen. Deren_Selbstdefinitition findet ihren Ausdruck in teils Jahrtausende alten Sagen, Mythen, Weltanschauungen - bis heute immer wieder aufs Neue überliefert in den schier unzähligen Sprachen und Dialekten der Welt. Doch so unterschiedlich dies in Inhalt und äußerer Form auch sein mag, etwas ist ihnen gemein: die Sehnsucht nach und die Furcht vor der Anziehungskraft hin zu den elementarsten Kräften unseres Erdballs: Feuer und Wasser. 
Die neue Gymmotion-Produktion "Fire \& Rain" bringt die verschiedenen Ausprägungen dieser Elemente in eindrucksvollen Show-Bildern auf die Bühne. (M03/NOV.73796

Mannheimer Morgen, [Tageszeitung], 06.11.2003, Jg. 58. - Sachgebiet: Sport,

Originalressort: Ried-Sport; Weltklasse erstmals auch in Bensheim) 
1) $\{\{1$; Gen.; A1 $\}\}$

«Wir können erst eingreifen, wenn etwas passiert ist», heisst im Pensionskassenwesen eine von ihnen. Deshalb gelte es, vermehrt präventive Instrumente einzubauen, zum Beispiel eine vorgängige Bewilligung für gewisse Transaktionen. Vorprogrammiert ist eine Verspätung der Kontrolleure ferner durch den Umstand, dass eine Vorsorgeeinrichtung ihre Jahresrechnung erst nach neun Monaten eingereicht haben muss.

Mehr Interesse nötig Einen wesentlichen Beitrag zur Sicherheit ihrer Pensionskassengelder könnten aber auch die Arbeitnehmer selber leisten, ist Huber überzeugt. (A97/NOV.34021 St. Galler Tagblatt, [Tageszeitung], 07.11.1997, Jg. 53. Originalressort: TB-THG (Abk.); * Christian Kamm: Öfters ein Auge aufs Sparschwein, [Bericht])

2) $\{\{0\}\}$

Die Folge: Viele Spanier hatten sich an den Trauben verschluckt. Japan begrüsste das «Jahr des Tigers», das auf das «Jahr des Ochsen» folgte.

In der ehemaligen britischen Kronkolonie Hongkong haben die Neujahrs-Feiern mit einer Verspätung begonnen. Erst vier Minuten nach Mitternacht wurde das Jahr mit einem Kanonenschuss begrüsst, weil die Kugel im Kanonenlauf steckengeblieben war. Der Salutschuss ist eine Tradition aus britischer Kolonialzeit. (A98/JAN.00032 St. Galler Tagblatt, [Tageszeitung], 03.01.1998, Jg. 54. Originalressort: TB-SPL (Abk.); Willkommen 1998!, [Bericht])

3) $\{\{1 ;$ Messwert; A2 $\}\}$

Dorfverein-, Schüler- und Grümpelturnier

fussball.

Über das kommende Wochenende meint es der Wettergott mit dem FC St.Margrethen besser und so kann das Grümpeli mit einer Woche Verspätung durchgeführt werden.

Drei Tage Hochbetrieb erwarten alle am St.Margrether Dorfvereins-, Schüler- und Grümpelturner 1998. Bereits morgen Freitagabend ab 17.30 Uhr geht mit dem Turnier der St.Margrether Dorfvereine die Jagd auf den beliebten Ball loss. (A98/JUN.40650 St. Galler Tagblatt, [Tageszeitung], 18.06.1998, Jg. 54. Originalressort: RT-SPO (Abk.); Dorfverein-, Schüler- und Grümpelturnier, [Bericht])

4) $\{\{1 ;$ Messwert; A2 $\}\}$

Für Müller wäre daher sogar eine Zusammenarbeit mit dem HC Thurgau denkbar. Thurgau: Liquiditätsproblem

Der Nachbar hätte zunehmend Grund, solche Vorschläge ernsthaft zu prüfen: Sinkende Zuschauerzahlen (pro Match 330 Besucher weniger als letzte Saison), fehlende

Werbeeinnahmen und sportlicher Sturzflug (am Dienstag verloren die Thurgauer bei Servette 1:10) haben zu einer prekären Situation geführt: Der HCThurgau hat Liquiditätsprobleme und konnte im Oktober erstmals in der Vereinsgeschichte die Löhne erst mit einigen Tagen Verspätung auszahlen. Trotzdem ist Präsident Paulus Grolimund kategorisch: «Eine Zusammenarbeit mit dem SCHSäntis ist kein Thema.» Während die beiden Ostschweizer Vereine nach Punkten und Zuschauern in den unteren Tabellenregionen klassiert sind, kommen aus der Westschweiz positivere Signale. 
(A98/NOV.72267 St. Galler Tagblatt, [Tageszeitung], 12.11.1998, Jg. 54. Originalressort: TB-SPO (Abk.); Eine Liga ohne Ausweg?, [Bericht])

\section{5) $\{\{0\}\}$}

Regierungsrat Willy Hermann: «Jede staatliche Wirtschaftsförderung greift ins Leere, wenn sie nicht auf priva-ter Initiative und unternehmerischem Wagemut gründet.» Dass gerade dies im Rheintal gegeben sei, lasse die wirtschaftliche Zukunft der Region Rheintal Gutes hoffen. Die Rhema wird als Zeichen der Solidarität und als Beweis dafür bezeichnet, dass es das Gewerbe verstanden habe, mit der rasanten Entwicklung Schritt zu halten. Beim Eröffnungsrundgang halten sich die Gäste offensichtlich «in den verschlungenen Korridoren so lange auf», dass sie erst mit Verspätung zum Mittagessen kommen, wie die Chronisten berichten.

An der Messe selbst sind «so ziemlich alle Branchen vertreten. Es gibt keinen Stand, dessen Inhaber sich nicht die grösste Mühe gegeben hätte, sein An-gebot wirkungsvoll und gluschtig vor Augen zu führen. (A99/MAR.17893 St. Galler Tagblatt, [Tageszeitung], 13.03.1999, Jg. 55. Originalressort: RT-ORT (Abk.); stefan kern*: Rhema '99, [Bericht])

6) $\{\{1$; Indefpr.; A2 $\}\}$

Bekanntlich obsiegt sie zu guter Letzt dank des Zauberbuchs, das ihr der Rabe Abraxas bringt.

Im Kino(k) ist das nicht anders: Gezeigt wird - morgen letztmals - die gelungene Verfilmung des erfolgreichen Kinderbuches durch ein tschechisches Animationsteam unter der Regie von Zdenek Smetana. Die Produktion entstand 1983 in den legendären Trnka-Studios in Prag und läuft mit einiger Verspätung zum ersten Mal in den Schweizer Kinos. Die amüsante Lektion über die verschiedenen Bedeutungen des Wortes «gut» ist in diesem Film eine Verbindung aus kindergerechter Unterhaltung und poetischer Zeichentrickkunst. Morgen Sonntag, Kinok, 15 Uhr (A00/MAR.22679 St. Galler Tagblatt, [Tageszeitung], 25.03.2000, Jg. 56. Originalressort: TB-SGK (Abk.); Sonntagstipp, [Bericht])

7) $\{\{1 ;$ Messwert; A2 $\}\}$

Der Millennium-Dome, der für das neue Jahrtausend stehen sollte, wurde für die Briten eine der grössten Pleiten des Jahres. Der Komplex hatte mit seinem kulturellen Angebot nicht die erwarteten Besucher an die Themse gelockt.

\section{Ein Jahr Verspätung}

(ap)

Ein Jahr später als erwartet hat bei der norwegischen Eisenbahngesellschaft das Jahr-2000-

Problem zugeschlagen. (A01/JAN.00082 St. Galler Tagblatt, [Tageszeitung], 03.01.2001, Jg. 57. Originalressort: TB-SPL (Abk.); Letzte Schaulustige, [Bericht])

8) $\{\{0+1$; keine Real.; Subj.; kein Arg.; A1 $\}\}\{\{S V G ;$ V. haben $\}\}$

Stadion-Projekt hat Verspätung

Eröffnung auf Sommer 2004 angekündigt - Fahrplan soll weiterhin gelten

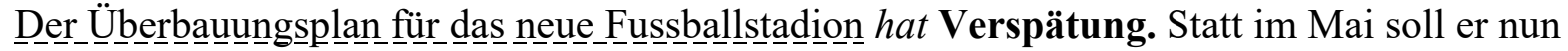
im August eingereicht werden. Knackpunkt des Projektes ist die Zahl der Parkplätze.

(A01/JUL.17375 St. Galler Tagblatt, [Tageszeitung], 14.07.2001, Jg. 57. Originalressort: TB-SGF (Abk.); Andreas Kneubühler: Stadion-Projekt hat Verspätung, [Bericht])

9) $\{\{1$; attr. Adj.; A2 $\}\}$

Durch Platzen des Injektors entströmte im Führerstand der Maschine eine Menge Dampf. Führer und Heizer kamen glücklicherweise ohne bedeutende Verletzungen davon. In einer 
schwachen Viertelstunde war der Schaden wieder so weit repariert, dass der Zug mit einer kleinen Verspätung die Fahrt fortsetzen konnte.

Vor 50 Jahren

6. September: Lichtensteig. (A07/SEP.01782 St. Galler Tagblatt, [Tageszeitung], 05.09.2007, Nr. 206, S. 43. - Sachgebiet: Regionales / Lokales, Originalressort: TTAgenda; r.riehm: Seilzieher: Schweizer Meister ohne Punkteverlust)

10) $\{\{1 ;$ attr. Adj.; A2 $\}\}$

lanz des gestrigen Wahlsonntages aus Sicht der Regionen Wil und Hinterthurgau.

Stefan Hauser

Warten, so lautete zunächst die Devise am gestrigen Wahlsonntag auch für die 57

Kandidierenden im St. Galler und Hinterthurgauer Einzugsgebiet der «Wiler Zeitung» Diese Wartezeit dehnte sich zumindest anfangs zusätzlich: Infolge technischer Probleme wurden aktualisierte Ergebnisse mit erheblicher Verspätung bekannt.

Sitzverschiebung mit Folgen

Im Kanton St. Gallen zeichnete sich im Verlauf des Nachmittages ab, dass die SVP im Nationalrat auf Kosten der FDP einen Sitz gewinnen könnte. (A07/OKT.10272 St. Galler

Tagblatt, [Tageszeitung], 22.10.2007, Nr. 246, S. 49. - Sachgebiet: Regionales, Originalressort: WV-Aufschlag; s.hauser: «Zum zweiten Mal ärgerlich knapp»)

11) $\{\{1 ;$ Messwert; A2 $\}\}$

Das Angebot auf dem «grössten Event-Schiff in europäischen Binnengewässern» richtet sich an grössere Unternehmen ab 500 Mitarbeitern, nationale und internationale Institutionen, Event-Agenturen und die Tourismusindustrie in einem Umkreis von 300 Kilometern, Mitteleuropa oder gar weltweit. Für Anfang Oktober gebe es bereits Buchungen ab dem Heimathafen Bregenz, sagt Rupp, «und fast täglich gibt es weitere Anfragen». Die «Sonnenkönigin» startet am 18. September mit einem halben Jahr Verspätung zur Jungfernfahrt ab Bregenz. Die technische Herausforderung beim Prototyp sei gross gewesen. Unternehmer Walter Klaus habe viele Details selber kontrollieren wollen, und man habe Rückschläge hinnehmen müssen, hiess es gestern. (A08/JUL.01052 St. Galler Tagblatt, [Tageszeitung], 04.07.2008, Nr. 154, S. 9. - Sachgebiet: Lokales, Originalressort: Ostschweiz; t.walliser: See wird zur Bühne)

12) $\{\{0+1$; keine Real.; Subj.; kein Arg.; A2 $\}\}\{\{$ SVG; V. haben $\}\}$

Die rund 40 Kilometer auf einem harten Velosattel spürten auch die geübten Velofahrerinnen am entsprechenden Körperteil. In Rorschach gaben die Toggenburgerinnen die Velos wieder zurück und hatten noch Zeit, sich etwas umzusehen, bis der nächste Zug via St. Margrethen, Bregenz nach Dornbirn führte.

Da der Zug Verspätung hatte, erreichten sie den roten Bus leider nicht mehr. Das hiess, 45 Minuten zusätzliches Tippeln bis zur Seilbahnstation. Die Gondel führte auf den Karren, und in weniger als fünf Minuten eröffnete sich ein unvergleichliches Panorama mit den Schweizer Bergen, dem Rheintal und dem Bodensee. (A08/OKT.03673 St. Galler Tagblatt, [Tageszeitung], 11.10.2008, Nr. 238, S. 37. - Sachgebiet: Regionales, Originalressort: WV-Untertoggenburg; a.buechi: Auf Umwegen nach Österreich)

13) $\{\{1$; Komp; A1 $\}\}$

Bahnreisende bekommen bei Zugverspätungen ab einer Stunde künftig einen Teil des Fahrpreises zurück. Der Bundestag wird heute ein Gesetz beschließen, dass erstmals einen gesetzlichen Anspruch auf Entschädigung bei Bahnverspätungen regelt. 
„Wir schaffen mehr Rechtssicherheit und machen Bahnfahren attraktiver“, sagt Justizministerin Brigitte Zypries (SPD). (BRZ09/APR.09773 Braunschweiger Zeitung, 24.04.2009; Bahn muss bei Verspätungen künftig zahlen [Ausführliche Zitierung nicht verfügbar])

14) $\{\{1$; Messwert; A2 $\}\}$

Viel zu schnell verfloss die Zeit und schon standen die beiden Busse zur Rückkehr bereit. Ein Unfall im Limmattal und ein launischer Petrus liess die Heimfahrt nur zögernd gewähren. Mit einer Stunde Verspätung wurde der Ausgangspunkt in Hurnen erreicht. Heidi hatte schon aufgetischt und der feine selbstgemachte Kartoffelsalat mit «Sturzis Chämibraten» mundete ausgezeichnet.

«Die Nacht ist ohne Ende», sagte sich der harte Kern der Senioren und liess den verregneten, aber fröhlichen Tag gemütlich ausklingen. (A09/AUG.03885 St. Galler Tagblatt, [Tageszeitung], 15.08.2009, Nr. 188, S. 45. - Sachgebiet: Regionales, Originalressort: WV-Hinterthurgau; Auf den Spuren Wilhelm Tells)

15) $\{\{1$; Indefpr.; A2 $\}\}$

Mehr Konfetti und Papierschnitzel denn je hüllten die Besucherinnen und Besucher des diesjährigen Fasnachtsumzugs in bunte Wolken. Wer sich von den langen Schlangen vor den Eingangspforten nicht hatte abschrecken lassen und sich einen möglichst guten Standort gewählt hatte, kam aber auch in den Genuss von frechen Sujets, lautstarker Musik und meist süssen Gaben der Umzugsteilnehmer. Zuvor war allerdings einiges an Geduld gefragt: Denn gestartet wurde der Umzug mit einiger Verspätung und auch dann wollte er zunächst nicht so recht in Gang kommen. Oft wurden die Wagen beim Einbiegen in die Umzugsroute von der Menschenmenge behindert, so dass es immer wieder zu Stockungen kam. Aber als die 46. und letzte Nummer die Strassen entlangzog, war das Geduldspiel vom Anfang längst vergessen. (A10/MAR.00235 St. Galler Tagblatt, 01.03.2010, Nr. 49, S. 37. - Sachgebiet: Regionales, Originalressort: TG-Oberthurgau; r.kohn: Bad in bunten Punkten)

16) $\{\{1+1$; Messwert; Subj.; A2; A1 $\}\}\{\{$ SVG; V. haben $\}\}$

Sorgen müssen wir uns keine machen - wir haben Tickets gelöst. Dafür müssen sich die beiden Kontrolleurinnen von den Fahrgästen einiges anhören: «Sie sollten lieber schauen, dass die Bahn pünktlich kommt. Am Marktplatz hat sie immer eine Minute Verspätung», echauffiert sich ein älterer Herr.

In Trogen wechseln wir den fahrbaren Untersatz. Pk ist enttäuscht, dass zwei Rentnerinnen die vordersten Panoramasitze des Postautos ergattert haben. (A10/JUL.07735 St. Galler Tagblatt, 31.07.2010, Nr. 176, S. 37. - Sachgebiet: Regionales, Originalressort: ATAufschlag; m.faessler: Regen, Kontrollen und ein abgefahrener Seitenspiegel)

17) $\{\{0\}\}$

Ursprünglich war der Spatenstich für den Neu- und Erweiterungsbau schon auf Anfang 2010 geplant. Die Baubewilligung wurde dann aber erst etwa Mitte Dezember erteilt. «Das Konzept wurde noch einmal überarbeitet», erklärt Koller die Verspätung.« Weil wir noch aktuelle Erkenntnisse im Bereich Alterswohnen berücksichtigen wollten.» Vor allem Erkenntnisse über Demenz seien noch zusätzlich in die Planung eingeflossen. So soll für die Demenzkranken nun ein eigener Bereich entstehen. (A11/JAN.00506 St. Galler Tagblatt, 04.01.2011, Nr. 2, S. 35. - Sachgebiet: Regionales, Originalressort: GO-Gossau; r.rohner: «Entwurzelung vermeiden»)

18) $\{\{1$; Messwert; A2 $\}\}$ 
Beim Rückweg vom Hauptbahnhof St. Gallen nach Abtwil um 17.18 Uhr ist die Masse an Passagieren, welche den Bus erwischen wollen, meist sehr gross, da ja alle Arbeitnehmer nach Hause wollen. Die Verspätungen der Busse sind dann teilweise einfach nur noch untragbar. Und obwohl die Zahl wartender Passagiere sicherlich um die 50 bis 60 beträgt, kommt der Bus mit siebenminütiger Verspätung und ist auch noch randvoll.

Ich finde, für den Preis, den wir zahlen, um die öffentlichen Verkehrsmittel zu benutzen, sollte auch gewährleistet sein, dass es darin zu den Stosszeiten genügend Platz für alle wartenden Passagiere hat. Mein Appell an die VBSG: Sie sollen doch bitte erwägen, Sonderbusse einzusetzen, falls dies zu den Stosszeiten notwendig sein sollte.

(A11/MAR.03971 St. Galler Tagblatt, 11.03.2011, Nr. 59, S. 44. - Sachgebiet: Regionales, Originalressort: SG-stadt; r.voneschen: VBSG-Busse: Unpünktlich und voll)

19) $\{\{0\}\}$

Doch keine Zeit für Kleingedrucktes. Knapp reicht nach der Baustellen-Tour die Lücke für den Zugang in ein zurückversetztes Haus. Der Gastgeber hat Verständnis für die Verspätung: «Eine Frau ist bei mir im Garten auch schon umhergeirrt.»

Bauschutt weg, Bahn frei

Nach dem Treffen bietet er sogar eine Autofahrt in die Stadt an. (A11/JUL.06711 St. Galler

Tagblatt, 22.07.2011, Nr. 169, S. 35. - Sachgebiet: Regionales, Originalressort: SG-stadt; m.kessler: Baustellenslalom in den Hinterhof)

20) $\{\{1$; Indefpr.; A2 $\}\}$

Ursprünglich wollte von der Leyen eine Novelle des Jugendschutzgesetzes am Mittwoch im Kabinett beraten lassen. Nach der Intervention der Kanzlerin wird der Vorschlag nun am Montag von den Staatssekretären der Ministerien erst einmal genauer unter die Lupe genommen. Und mit einiger Verspätung will von der Leyen selbst Expertenrunden organisieren. „Ich will jetzt nichts durchpeitschen, sondern glaube, dass uns eine Atempause hilft, über wirksame Schritte im Jugendschutz zu diskutieren“, sagte von der Leyen laut einem Vorabbericht der „Bild“-Zeitung (Montagausgabe). An einer Lösung soll nun ein größerer „runder Tisch“ arbeiten: „Von dem erwarte ich aber auch, dass konkrete Vorschläge zur Verbesserung des viel zu laschen Jugendschutzes vorgelegt werden, die von allen mitgetragen werden können.“ (HAZ07/OKT.04226 Hannoversche Allgemeine, 15.10.2007, S. 3; Zu jung zum Trinken \&\#8211; und zu jung zum Spionieren [Ausführliche Zitierung nicht verfügbar])

21) $\{\{0\}\}$

Grundsätzlich muss dann die Fluggesellschaft für den Schaden aufkommen. Allerdings sind die Summen begrenzt. Nach dem international gültigen Luftverkehrs-Übereinkommen von Montreal haftet die Fluggesellschaft bei Verlust, Beschädigung und Verspätung von Gepäck mit maximal 1000 sogenannten Sonderziehungsrechten pro Passagier. Das entspricht etwa 1060 Euro für Koffer und Inhalt. Mehr Geld gibt es nur, wenn man nachweisen kann, dass die Fluggesellschaft grob fahrlässig oder vorsätzlich gehandelt hat - das aber gelingt selten.

(HAZ08/MAI.05732 Hannoversche Allgemeine, 31.05.2008, S. 1; Fluggepäck weg \&\#8211; was ist es wert? [Ausführliche Zitierung nicht verfügbar])

22) $\{\{1 ;$ Messwert; A2 $\}\}$

Alte Fahrensleute, die in der altehrwürdigen SPD schon manch tränenreichen Rücktritt erlebt haben, sprechen am Sonntag völlig konsterniert von einer ,sensationellen“ Entwicklung.

Teilnehmer der Klausurtagung von Parteipräsidium, Fraktionsvorstand, SPD- 
Bundesministern und SPD-Ministerpräsidenten erlebten eine Konferenz der Parteiführung von nie dagewesener Konfusion.

Parteichef Kurt Beck beginnt mit anderthalbstündiger Verspätung gegen $12.30 \mathrm{Uhr}$ seine Ausführungen. Alle sind guten Mutes. Jedem Teilnehmer ist klar, dass nun die Kür des SPDKanzlerkandidaten für 2009 ansteht. (HAZ08/SEP.01578 Hannoversche Allgemeine, 08.09.2008, S. 3; \&\#8222;Die Selbstachtung überschritten\&\#8220; [Ausführliche Zitierung nicht verfügbar])

23) $\{\{1$; attr. Adj.; A2 $\}\}$

Nach HAZ-Informationen bleiben Sendungen oft tagelang liegen, weil die Briefträger ihr Pensum nicht mehr schaffen. Klagen von Postkunden kommen etwa aus dem Zooviertel, der List und der Südstadt. Auch Bürger aus Sehnde und Gehrden haben sich bei der HAZ darüber beschwert, dass sie an manchen Tagen überhaupt keine Post zugestellt bekämen, beziehungsweise die Sendungen mit deutlich erkennbarer Verspätung einträfen. Die Post verweist zur Erklärung auf einen hohen Krankenstand bei ihren Zustellern, der derzeit bei sieben Prozent liege.

Ein wesentlich dramatischeres Bild der Lage zeichnet die Dienstleistungsgewerkschaft ver.di. (HAZ08/OKT.02841 Hannoversche Allgemeine, 16.10.2008, S. 17; Briefe kommen zu spät \&\#8211; Ärger über Post wächst [Ausführliche Zitierung nicht verfügbar])

24) $\{\{0\}\}$

«Man kann sich vorstellen, dass der Kundenstrom in einem halbfertigen Einkaufszentrum nicht berauschend ist.» Die im November wiedereröffnete Migros-Filiale sei gut gestartet und bei den Kunden auf gute Akzeptanz gestossen, heisst es bei Migros Ostschweiz. Die Verzögerung der Gesamteröffnung sei zwar bedauerlich, man freue sich aber dennoch auf September.

Auch für Denner ist die Verspätung ärgerlich. Die geplante Filiale kann erst im September eröffnen. «Das tangiert natürlich unsere Planung», sagt Paloma Martino, Leiterin Unternehmenskommunikation bei Denner. (A12/APR.09757 St. Galler Tagblatt, 25.04.2012, Nr. 96, S. 33. - Sachgebiet: Regionales, Originalressort: SG-st.gallen aufschlag; r.streule: Silberturm öffnet erst im Herbst)

25) $\{\{1$; attr. Adj; A2 $\}\}$

Als dieser eintraf gab es aber doch eine Schrecksekunde, denn da war ein Sitzplatz zu wenig. Nach einem Telefonanruf konnte dieses Missgeschick kurzerhand behoben werden und die Reise über den Ricken Richtung Innerschweiz konnte losgehen. Von Biberbrugg ging es Richtung Ratenpass, wo trotz kleiner Verspätung ein Kaffeehalt angesagt war. Dabei konnte die vielfältige, schöne Landschaft und ein Blick in die Berge genossen werden. Ab und zu gab es auch ein Bienenhaus zu entdecken. (A12/JUN.14214 St. Galler Tagblatt, 30.06.2012, Nr. 151, S. 47. - Sachgebiet: Regionales, Originalressort: TT-Obertoggenburg; sa.schmid: Den emsigen Bienen auf der Spur)

26) $\{\{1$; Messwert; A2 $\}\}$

«Ich denke, die Jury hat einen Fehlentscheid gefällt. Es war nicht sicher», sagte Vonn.

Skaardal und sein Team hatten für den Super-G am 5. Februar nach mehreren

Verschiebungen mit dreieinhalb Stunden Verspätung trotz widriger Bedingungen grünes Licht gegeben. Vonn stürzte bei der Landung nach einem Sprung und zog sich dabei am rechten Knie einen Kreuzband- und Innenbandriss sowie einen Bruch des Schienbeinkopfs zu. Sie habe während ihrer Fahrt kaum glauben können, wie schlecht die Verhältnisse waren, sagte Vonn in einer Telefonkonferenz. (A13/FEB.10086 St. Galler Tagblatt, 25.02.2013, 
Nr. 46, S. 21. - Sachgebiet: Sport, Originalressort: sport; c.krapf: Vonn kritisiert Rennleitung und Skaardal)

27) $\{\{0\}\}$

Bei der neuen Velowegverbindung von der Froheimstrasse zur Sulzstrasse fehlt nur noch der Belag. Mitte August soll er für die Velofahrer und Fussgänger freigegeben werden. Wegen des schlechten Wetters im Frühling mit Verspätung.

ANDREA STERCHI

GOLDACH. (A13/JUL.06569 St. Galler Tagblatt, 17.07.2013, Nr. 164, S. 29. -

Sachgebiet: Regionales, Originalressort: OT-rorschach; a.sterchi: Nasser Frühling verzögert Bauarbeiten)

28) $\{\{1$; Messwert; A2 $\}\}$

12 Tore in Appenzell

FUSSBALL. Am Sonntag spielten die Frauen des FC Appenzell auf dem Zielplatz gegen den FC Wil 2. Da die Unparteiische nicht erschien, wurde die Partie durch einen

Ersatzschiedsrichter mit über einer Stunde Verspätung angepfiffen. Schon in der 8. Minute

fiel das erste Tor für Appenzell. Drei Minuten später erfolgte ein Eckball, der durch Melissa

Schenk sauber im Netz versenkt wurde. (A14/JUN.00140 St. Galler Tagblatt, 03.06.2014,

Nr. 127, S. 39. - Sachgebiet: Sport, Originalressort: Regionalsport; volontaer-apz: 12

Tore in Appenzell)

29) $\{\{1$; Messwert; A2 $\}\}$

Trotz grösserer Spielanteile des Heimteams gelang den Gästen ein früher Führungstreffer, welcher über die Zeit gerettet werden konnte.

Fehlender Schiedsrichter

Da der aufgebotene Unparteiische nicht erschien, startete die Partie mit halbstündiger

Verspätung. Beide Mannschaften schienen aber wach und bissig. Trotz ausgeglichenem Ballbesitz in den ersten Minuten spielte sich der FCB die besseren Möglichkeiten heraus.

(A14/OKT.06811 St. Galler Tagblatt, 21.10.2014, Nr. 245, S. 34. - Sachgebiet: Sport, Originalressort: Regionalsport; b.lanzendorfer: Vermeidbare Niederlage)

30) $\{\{1$; Messwert; A2 $\}\}$

Unsanfte Landung als schlechtes Omen?

Eine harte Landung erlebten die VfL-Profis bei ihrer Rückkehr auf dem Flughafen Hannover. Mit fast einstündiger Verspätung setzte die Boeing-737 ziemlich unsanft auf. Ein schlechtes Omen? Nicht dass die „Wölfe“ auch in der Bundesliga hart landen... Heute haben sie aber erst einmal frei. (BRZ06/JAN.09487 Braunschweiger Zeitung, 19.01.2006; Unsanfte Landung als schlechtes Omen? [Ausführliche Zitierung nicht verfügbar])

31) $\{\{1$; Messwert; A2 $\}\}$

Die Bahn kommt mehr als eine halbe Stunde zu spät, die Anschlusszüge warten nicht und der Reisende verpasst womöglich noch das Flugzeug - Verspätungen im Bahnverkehr sind ärgerlich und können den Fahrgast viel Geld kosten. Die Bundesregierung will deshalb durchsetzen, dass Fahrgäste in solchen Fällen höher entschädigt werden.

Nach Medienberichten will die große Koalition die Deutsche Bahn per Gesetz dazu verpflichten, bei einer halben Stunde Verspätung 30 Prozent des Fahrpreises zurückzuerstatten. Bei einer Stunden sollen demnach 60 Prozent, bei 90 Minuten 90 Prozent zurückgezahlt werden. 
Die Bahn fürchtet starke finanzielle Belastungen, sollte die Regierung ihre Pläne durchsetzen. (BRZ06/MAI.08533 Braunschweiger Zeitung, 16.05.2006; \&\#8222;Mehr Geld bei ZugVerspätung\&\#8220; [Ausführliche Zitierung nicht verfügbar])

32) $\{\{1$; Messwert; A2 $\}\}$

Mit einer starken kämpferischen Leistung holte der SV Nordsteimke seinen ersten Zähler in der Fußball-Bezirksliga 2. Die Elf von Trainer Ralf Zauske spielte bei dem favorisierten SV Kralenriede 2:2 (1:1).

Obwohl rechtzeitig in Nordsteimke gemeinsam mit Fans im gecharterten Reisebus aufgebrochen, kamen die Wolfsburger nach einer Irrfahrt durch Braunschweig verspätet in der Kralenriede an. Deshalb wurde die Partie mit 20-minütiger Verspätung angepfiffen. Orientierungslos war in den ersten Minuten der SVN auch auf dem Spielfeld. Bei einem Freistoß der Gastgeber stand die Mauer schlecht und Ersatzkeeper Lars Jendrossek zeigte keine Reaktion. (BRZ06/SEP.01811 Braunschweiger Zeitung, 04.09.2006; André Krause trifft doppelt [Ausführliche Zitierung nicht verfügbar])

33) $\{\{0\}\}$

TWIEFLINGEN.

Fast vom Winde verweht wurde die Spielpremiere von Trainer Matthias Brömse bei seinem neuen Verein SG Hoiersdorf/Twieflingen. Aufgrund von starken Windböen mit prasselnden Regen wurde das Testspiel gegen seinen alten Verein FC Schöningen 08 erst mit Verspätung angepfiffen.

Unter den widrigen Wetter- und Platzverhältnissen litten die spielerischen Aktionen. Kampf war deshalb meistens Trumpf. (BRZ07/JAN.15040 Braunschweiger Zeitung, 22.01.2007; FC Schöningen 08 mit 3:0-Sieg [Ausführliche Zitierung nicht verfügbar])

34) $\{\{1$; Messwert; A2 $\}\}$

Das Verletzungsrisiko für die Läufer wäre zu hoch gewesen.

Kurz vor 22 Uhr schließlich gab die Polizei die Strecke wieder frei. Der vierte Lauf des Abends wurde mit 30 Minuten Verspätung gestartet. $\mathrm{Zu}$ diesem Zeitpunkt wusste aber noch niemand, dass jede Hilfe für den 69-jährigen Mann zu spät gekommen war.

Niemand im Publikum ahnte etwas vom Todesfall. (BRZ07/JUN.20711 Braunschweiger Zeitung, 16.06.2007; Organisatoren verschweigen Todesfall [Ausführliche Zitierung nicht verfügbar])

35) $\{\{0\}\}$

VfL mit Verspätung nach Duisburg

Mit 33 Minuten Verspätung begann gestern Nachmittag die Zugfahrt der VfL-Profis nach Duisburg. So lange mussten sie warten, bis ihr ICE im Wolfsburger Bahnhof eintraf.

(BRZ07/AUG.09486 Braunschweiger Zeitung, 18.08.2007; VfL mit Verspätung nach Duisburg [Ausführliche Zitierung nicht verfügbar])

36) $\{\{1$; Messwert; A2 $\}\}$

Die Rentner geben sich entspannt. „Wir haben viel Zeit.“ Ihr Blick geht nach oben, zur Wandtafel. Der ICE 940 nach Düsseldorf hat 30 Minuten Verspätung. 30 Minuten Zwischenstopp in Wolfsburg: Carmen Wolff und Charlotte.

In der Eingangshalle des Wolfsburger Hauptbahnhofs geht es meist entspannt zu.

(BRZ07/NOV.19113 Braunschweiger Zeitung, 03.11.2007; Wo Reisende nur abends rennen [Ausführliche Zitierung nicht verfügbar]) 
37) $\{\{0\}\}$

Von Stephanie Peißker

FÜMMELSE.

„Die Sonnenfinsternis hat ja Verspätung!“ , ruft Dieter Nitsche und schaut ungeduldig auf die Uhr. „Weißt Du nicht, dass der Mond in der Gewerkschaft ist?“, scherzt Hans Hubner. Beide beobachten bei Fümmelse das Himmelsspektakel. (BRZ08/AUG.01090 Braunschweiger Zeitung, 02.08.2008; Die Sonne sieht aus wie ein angeknabberter Keks [Ausführliche Zitierung nicht verfügbar])

38) $\{\{1$; Messwert; A2 $\}\}$

Hoffmann-von-Fallersleben-Schule: Die Sanierung des Kellergeschosses beginnt nach Abstimmung mit der Schulleitung nach den Ferien

Gymnasium Kleine Burg: Arbeiten an Fenstern und Fassade finden während der Schulzeit und in den Herbstferien statt

Gymnasium Martino-Katharineum: Die neuen Sanitäranlagen in der Sporthalle werden mit zwei Tagen Verspätung fertiggestellt wegen unvorhersehbarer Änderungen bei der Trassenführung von Wasserleitungen. Die Toiletten werden zum Schuljahresbeginn nicht fertig sein, Ersatz wird bereitgestellt, die Turnhalle wird auch nutzbar sein Gymnasium Neue Oberschule: Der Umfang der Fenster-Erneuerung war laut Stadt in den Sommerferien nicht zu schaffen. (BRZ08/AUG.10312 Braunschweiger Zeitung, 21.08.2008; Bauarbeiter bleiben zum Schulstart [Ausführliche Zitierung nicht verfügbar])

39) $\{\{0\}\}$

Jahresversammlung der Feuerwehr Linden

LINDEN.

Die Jahresversammlung der Freiwilligen Feuerwehr Linden musste mit Verspätung eröffnet werden. Grund: Viele Mitglieder waren bei einem schweren Verkehrsunfall zwischen Linden und Wendessen im Einsatz. Um 21 Uhr gab Ortsbrandmeister Rüdiger Hartmann dann das Startsignal. (BRZ09/JAN.07737 Braunschweiger Zeitung, 20.01.2009; Unfall verhindert den pünktlichen Beginn [Ausführliche Zitierung nicht verfügbar])

40) $\{\{1$; Messwert; A2 $\}\}$

Ab 60 Minuten Verspätung am Ziel

Ab 120 Minuten Verspätung am Ziel

$\mathrm{Ab} 60$ Minuten Verspätung

in der Nacht

(zwischen 0 und 5 Uhr) (BRZ09/JUL.26469 Braunschweiger Zeitung, 29.07.2009; Die neuen Rechte der Bahnkunden [Ausführliche Zitierung nicht verfügbar])

41) $\{\{1+1$; Messwert; Subj.; A2; A1 $\}\}\{\{$ SVG; V. haben $\}\}$

Erst nach 90 Minuten Wartezeit konnte der Regionalexpress die Fahrt aus eigener Kraft fortsetzen. Die Höhe des Sachschadens könne erst in der Werkstatt festgestellt werden, berichtet ein Bahnsprecher. Der_Gegenzug von Hanno Verspätung. Betroffen waren auch die Reisenden, die in Ringelheim den Zug 10.19 Uhr nach Hannover benutzten. Sie mussten 40 Minuten warten. (BRZ09/SEP.06374 Braunschweiger Zeitung, 12.09.2009; Regionalexpress erfasst Auto [Ausführliche Zitierung nicht verfügbar])

42) $\{\{1$; Indefpr.; A2 $\}\}$ 
Von Maike Neupert

BROME.

Mit einiger Verspätung sind sie dann doch angekommen: Die Gäste aus La Guerche-deBretagne, der Partnergemeinde Bromes. Mit Sekt und Knabbereien wurden sie standesgemäß vor dem Rathaus empfangen.

Seit 27 Jahren besteht die Partnerschaft zwischen den beiden Gemeinden.

(BRZ10/MAI.06074 Braunschweiger Zeitung, 15.05.2010; Brome hat Besuch aus Frankreich [Ausführliche Zitierung nicht verfügbar])

43) $\{\{0+1$; keine Real.; Subj.; kein Arg.; A1 $\}\}\{\{$ SVG; V. haben $\}\}$

Dann kennt Ihr sicher auch die Hinweistafeln an den Haltestellen, auf denen man immer ganz aktuell ablesen kann, wie lange es noch dauert, bis ein Bus oder eine Bahn kommt. Über einen Satelliten im Weltraum wird diese Zeit errechnet. Das ist ganz praktisch, wenn ein B Bus mal Verspätung hat, weil er zum Beispiel im Stau steht. Auf der Anzeige sieht man dann, wie viele Minuten man noch warten muss. Die Angaben werden von einem Computer errechnet. (BRZ10/JUL.11827 Braunschweiger Zeitung, 28.07.2010; Tafeln zeigen, wann Bus oder Bahn kommen [Ausführliche Zitierung nicht verfügbar])

44) $\{\{1$; Messwert; A2 $\}\}$

Dass es nicht zu einer weiteren Verschiebung kam, verdankte der VfB den Isenbüttelern. Die Verantwortlichen des MTV II erklärten sich kurzfristig bereit, die Partie in Isenbüttel auszutragen. So konnten [sic] die Partie mit einer 60-minütigen Verspätung doch noch angepfiffen werden.

Im Spiel 1 nach dem Rücktritt von VfB-Coach Christian Politze (wir berichteten) waren es auch die Hausherren, die von Beginn an den Ton angaben - und dank eines Eigentores nach nur zwölf Minuten in Führung gingen. MTV-Angreifer Alexander Dinus erhöhte in der 35 . Minute auf 2:0, die Vorentscheidung. (BRZ10/OKT.06949 Braunschweiger Zeitung, 14.10.2010; VfB geht auch in Isenbüttel leer aus [Ausführliche Zitierung nicht verfügbar])

45) $\{\{0\}\}$

Sie können sich vom Anbieter eine alternative Reisemöglichkeit organisieren lassen allerdings muss das keine Flugverbindung sein. Während längerer Verzögerungen haben Sie Anspruch auf Verpflegung, wenn Sie an einem Ort festsitzen, auch auf eine Übernachtungsmöglichkeit. Falls Sie zurückbleiben, weil das Unternehmen den Flug überbucht hat, dann stehen Ihnen je nach Entfernung und Verspätung zwischen 250 und 600 Euro Entschädigung zu.

Was kann ich tun, wenn mein Flug Verspätung hat?

$\mathrm{Ab}$ drei Stunden Verzögerung gelten die gleichen Bedingungen wie bei einem Ausfall - es sei denn, die Fluglinie kann beweisen, dass sie keine Schuld trifft. (BRZ10/DEZ.11871

Braunschweiger Zeitung, 27.12.2010; So bekommen Reisende ihr Recht [Ausführliche Zitierung nicht verfügbar])

46) $\{\{0+1$; keine Real.; Subj.; kein Arg.; A1 $\}\}\{\{$ SVG; V. haben $\}\}$

In der ganzen bitterkalten Welt gab es nicht einen einzigen Ort, an dem er sich niederlassen konnte.

Ralph Truitt sah auf seine silberne Uhr. Ja, der Zu herum starrten ihn schweigend an. Sie wussten es. (BRZ11/APR.13442 Braunschweiger Zeitung, 30.04.2011; Eine verlässliche Frau [Ausführliche Zitierung nicht verfügbar]) 
47) $\{\{1$; Messwert; A2 $\}\}$

Von Stephanie Knostmann BRAUNSCHWEIG. Mit sieben Jahren Verspätung muss sich seit gestern ein 32-jähriger Berliner wegen einer Serie schwerer Einbruchdiebstähle vor dem Landgericht Braunschweig verantworten.

Der Mann, der sich der Strafverfolgung bislang durch Flucht ins Ausland entzogen hatte, zeigte sich am ersten Verhandlungstag geständig. „Ich stehe zu allem“, sagte er.

(BRZ11/OKT.08774 Braunschweiger Zeitung, 18.10.2011; Später Prozess für SerienEinbrecher aus Berlin [Ausführliche Zitierung nicht verfügbar])

48) $\{\{1$; attr. Adj.; A2 $\}\}$

Von Georg Fiedler

WOLFSBURG. Mit leichter Verspätung, dafür umso intensiver hat die Rückkehr der Weißstörche begonnen. Seit vergangener Woche hat die Hälfte der erwarteten zehn Storchenpaare die Horste bezogen.

Auf der Westseite des Ilkerbruchs und am Weyhäuser Weg sind die angestammten Paare eingetroffen, wobei die jeweils beringten Männchen seit etlichen Jahren nesttreu sind.

(BRZ12/MAR.08407 Braunschweiger Zeitung, 15.03.2012; Die Störche kehren auf ihre Nester zurück [Ausführliche Zitierung nicht verfügbar])

49) $\{\{1$; Messwert; A2 $\}\}$

SCHLADEN. 70 Jahre und noch kein bisschen müde ist der Sänger Gunter Gabriel, der auf Bühne der Schladener Schlangenfarm vor 300 begeisterten Konzertbesuchern mit seiner Band auftrat. Vorher hatte der Sänger den Schlangenfarmer Jürgen Hergert in Atem gehalten, weil er mit zehnminütiger Verspätung eintraf und direkt auf die Bühne ging.

Hergert hatte Gabriel angekündigt als Mann, der mehr als 1000 Lieder geschrieben habe. Warmmachen brauchte sich der Künstler nicht. (BRZ12/JUL.03963 Braunschweiger Zeitung, 09.07.2012; Vom Auto direktauf die Bühne: Gunter Gabriel [Ausführliche Zitierung nicht verfügbar])

50) $\{\{1$; Messwert; A2 $\}\}$

HILLERSE. Der SSV Kästorf hat Geschmack am Gewinnen gefunden - dank Tino

Gewinner: Drei Tage nach dem ersten Saisonsieg in der Fußball-Landesliga durften die RotWeißen auch gestern Abend im Bezirkspokal jubeln - üben einen 2:1 (1:1)-Erfolg beim Staffelrivalen TSV Hillerse.

Zunächst rieben sich die Zuschauer rund um den Hillerser B-Platz verwundert die Augen, als die Partie mit zehn Minuten Verspätung angepfiffen wurde. Denn der TSV begann nur zu zehnt und mit Innenverteidiger Moritz Stubbe zwischen den Pfosten. Erst nach 15 Minuten traf Torwart Kai Krüger ein, und fortan durfte Stubbe wieder auf seine Stammposition vorrücken. (BRZ12/SEP.10486 Braunschweiger Zeitung, 20.09.2012; Gewinner trifft wieder \&\#8211; und Kästorf gewinnt [Ausführliche Zitierung nicht verfügbar])

51) $\{\{1$; Messwert; A2 $\}\}$

Viel Geduld mussten Autofahrer auf Braunschweigs Straßen mitbringen. Für viele Pendler ging es im Berufsverkehr mit dem Auto nur im Schritttempo stadteinwärts. Wegen der Sperrungen der Schunterbrücke in Querum sowie der Ebertallee hatten mehrere Buslinien bis zu 45 Minuten Verspätung.

An den Autobahnkreuzen Süd und Ölper war nach fast zwei Tagen Teilsperrung zumindest am Nachmittag wieder freie Fahrt. 
Mindestens 38 Einsätze fuhr Salzgitters Feuerwehr. (BRZ13/MAI.10270 Braunschweiger Zeitung, 28.05.2013, Braunschweiger Zeitung. Originalressort: 1Titel; Land unter in Wolfenbüttel)

52) $\{\{1$; Messwert; A2 $\}\}$

Mit einem Patschen konnte er nicht weiterfahren.

Schließlich schaffte er es doch, die Kostüme und Utensilien nach St. Andrä zu bringen. Mit einer Stunde Verspätung um 18 Uhr konnte die Perchtengruppe mit ihrer Vorstellung beginnen. (BVZ08/DEZ.00542 Burgenländische Volkszeitung, [Wochenzeitung], 03.12.2008, Nr. 49, Jg. 2008, Neusiedl, S. 32. Originalressort: SEEWINKEL; Der Perchtenlauf unter einem Unglücksstern)

53) $\{\{1+1$; Messwert; Subj.; A2; A1 $\}\}\{\{$ SVG; V. haben))

Doch nicht etwa ein Banküberfall oder gar Schlimmeres war passiert. Nein, in Bruckneudorf wurde eine Lok im Polizei-Design präsentiert. Ein bisschen peinlich war nur, dass der Z Zug dann gute 15 Minuten Verspätung gehabt hat. So schön die Lok in Silber mit ihren blauen und roten Streifen auch ist, den Einsatz als Dienstwagen sollte die Polizei noch mal gut überlegen. Bei der Pünktlichkeit der Züge hätten die Verbrecher einen schönen Vorsprung, schmunzelt ihr Gmoatrommler (BVZ12/APR.02915 Burgenländische Volkszeitung, 26.04.2012, Neusiedl. - Sachgebiet: Lokales, Originalressort: STADT NEUSIEDL AM SEE; REITEPIA)

54) $\{\{1$; Messwert; A2 $\}\}$

da Toulouse-Lautrec seine Karriere als Plakatmaler begann, hat die Nationalbibliothek eine Auswahl aus dem graphischen Werk des Künstlers beigesteuert.

ausgezeichnete Kritiken und lebhaften Applaus beim Publikum hat das Brecht-Stück

"Trommeln in der Nacht" im kleinen Theatre de Lutece. die Premiere fand mit zweiwöchentlicher Verspätung statt, da Helene Weigel die Genehmigung zur Aufführung zunächst verweigert hatte. die flotte Inszenierung besorgte Andre Steiger, der bereits im Vorjahr von sich reden machte, als er die sarkastische Komödie "der Hofmeister" von Lenz auf die Bretter brachte. man empfindet das Brecht-Stück als so wohltuend, da es nicht zum "theatre engage" dieses Autors gehört. (BZK/W59.00001 Die Welt, [Tageszeitung], 02.01.1959, Deutschland-Ausgabe, S. 6. Originalressort: FEUILLETON; Weber, Gerhard Werner: was Paris in diesen Tagen bot / Ein Brief, [Feuilleton])

55) $\{\{1$; eine ... von; A2 $\}\}$

Denen stehen die Probleme, die wir inzwischen bereits gelöst haben, noch ins Haus.

CZ: Entscheidend für das Überleben von Borland ist sicherlich der Erfolg von Dbase für

Windows. Das Produkt kommt mit einer Verspätung von über zwölf Monaten auf den

Markt. Warum diese Verzögerung?

Kahn: Dbase ist das erste Produkt einer völlig neuen Generation von Windows-Produkten.

(C94/JUN.02102 COMPUTER ZEITUNG, [Wochenzeitung], 30.06.1994, Nr. 26, Jg. 29, S. 2, [Interview])

56) $\{\{1$; Messwert; A2 $\}\}$

Zwei Etagen tiefer, im Operation Control Center der Frankfurter Lufthansa-Basis, geht es in kritischen Momenten zu wie beim Parketthandel an der Börse. An die 50 Mitarbeiter je Schicht betreuen von dort die täglich 1200 Flüge von 230 Jets. Jede Phase eines Fluges - vom Anlassen der Triebwerke bis zum Wiederandocken am Gate des Zielorts - ist auf Dutzenden Bildschirmen abrufbar - inklusive jeder Sekunde Verspätung. 
"Schlechte Nachrichten sind besser als keine Nachrichten", betont Michael Spors, 47, Leiter von Verkehrszentrale und Flugdienstberatung. (FOC00/MAR.00547 FOCUS, 27.03.2000, S. 060-062, Originalressort: DEUT/Deutschland; LUFTVERKEHR)

57) $\{\{0\}\}$

Almunia: Die Lösung für das deutsche Wachstumsproblem liegt nicht in Brüssel, sondern in den Arbeitsmarkt- und Sozialreformen der Regierung. Deren Erfolge zeigen sich erst mit Verspätung.

FOCUS: Das Herumgeschraube am Pakt ist kein abgekartetes Spiel zwischen

Sozialdemokraten in Brüssel und Berlin? (FOC05/JAN.00353 FOCUS, 24.01.2005, S. 138138, Sachgebiet: Wirtschaft, Originalressort: WIRT/Wirtschaft; "Nein, nein, nein!")

58) $\{\{1$; attr. Adj.; A2 $\}\}$

Nett oder konsequent?

Die Partei sucht mit reichlich Verspätung einen neuen Ansatz, um ihre Reformen an den verunsicherten Wähler zu bringen

Wissen Sie noch, was Sie vor vier Monaten alles falsch gemacht haben? Die CDU versucht ein solches Gedächtnisexperiment diesen Montag. (FOC05/DEZ.00032 FOCUS, 05.12.2005, S. 038-038, Originalressort: DEUT/Deutschland; CDU)

59) $\{\{1$; Messwert; A2 $\}\}$

Unglücklicher als McNerney, der seit fast sechs Jahren Konzernchef ist, konnte ein

Luftfahrtmanager 2010 kaum agieren: Er hat keine einzige B 747 verkauft und vom anderen

Oldtimer B 777 weniger abgesetzt als erwartet. Dafür kommt der vergrößerte Jumbo-Jet 747-

8 erst mit eineinhalb Jahren Verspätung auf den Markt und der - einstige - Hoffnungsträger

B 787 nach sieben Verspätungsrunden frühestens im dritten Quartal.

Die Folgen: Dem Luftfahrt-Giganten fehlen nicht nur Einnahmen. (FOC11/JAN.00584

FOCUS, 31.01.2011, S. 116-117, Sachgebiet: Wirtschaft, Originalressort:

WIRTSCHAFT; MONTAG IST ZEUGNISTAG)

60) $\{\{1$; Messwert; A2 $\}\}$

So moderat wird es aber wohl auch hier nicht weitergehen. Nach Angaben des VDA geht es mit den Bestellungen steil abwärts: Aus dem Inland seien im Dezember 21 Prozent weniger Neuwagen bestellt worden, aus dem Ausland kam sogar ein Drittel weniger Aufträge.

Aus den Aufträgen werden erst mit einigen Wochen Verspätung Neuzulassungen, und so rechnet der VDA mit einem, ,sehr schwachen Neuzulassungsgeschehen zu Beginn des Jahres“, die Lage sei „besorgniserregend“. Die Politik müsse „klare Rahmenbedingungen schaffen, um das Vertrauen der Verbraucher zu stabilisieren und deren Kaufbereitschaft wieder zu stärken“, fordert der VDA mit Blick auf die lange diskutierte Reform der KfzSteuer.

Während teure Oberklassewagen und Sportwagen 2008 bei den Händlern stehen blieben, griffen die Käufer nach Angaben des Kraftfahrtbundesamtes zu Kleinwagen. So brachte vor allem der neue Fiat 500 den jahrelang gebeutelten Italienern ein Verkaufsplus von 19,4 Prozent im Gesamtjahr. (HAZ09/JAN.00928 Hannoversche Allgemeine, 07.01.2009, S. 9; Export spielt nicht mehr mit [Ausführliche Zitierung nicht verfügbar])

61) $\{\{1$; Messwert; A2 $\}\}$

Man denke nur an die Olympischen Spiele von München 1972 und das Attentat auf die israelische Mannschaft: „The games must go on“, verkündete Avery Brundage, damals Präsident des Internationalen Olympischen Komitees. Nach dem Tod von 17 Menschen, 
darunter elf israelische Athleten, hielt der Sport einen halben Tag inne. Nach der HeyselStadion-Katastrophe in Brüssel mit 39 Toten und 454 Verletzten, ausgelöst von randalierenden Fans, wurde das Endspiel um den Europapokal der Landesmeister 1985 mit eineinhalb Stunden Verspätung angepfiffen. Und selbst am 11. September 2001 schaffte es der Fußball nicht, innezuhalten: Am Tag der Terroranschläge auf die USA hielt die Europäische Fußball-Union am Spielbetrieb fest; Erst am nächsten Morgen sagte sie alle Partien in Champions League und UEFA-Cup ab. Die Bundesligisten kickten weiter, mit Trauerflor. (HAZ09/NOV.01728 Hannoversche Allgemeine, 13.11.2009; Die seltene Stille [Ausführliche Zitierung nicht verfügbar])

62) $\{\{0\}\}$

An beiden Tagen waren insbesondere Züge nach Köln und Düsseldorf betroffen. Hier mussten die Reisenden witterungsbedingt auch schon mal eine Stunde auf ihre Bahn warten. Nicht selten war Blitzeis in Tschechien der Grund für das Ausharren auf den hannoverschen Bahnsteigen: „Züge aus Tschechien kommen verspätet in Berlin an, da startet dann auch der innerdeutsche Anschlussverkehr mit Verspätung“, sagt Brunkhorst. Die Reisenden nahmen es gelassen und wärmten sich mit dampfendem Kaffee und Kakao. Auch Harzreisende hatten mit Verzögerungen und Umwegen zu kämpfen. (HAZ10/JAN.01483 Hannoversche Allgemeine, 11.01.2010; Acht Flüge fallen wegender Schneemassen aus [Ausführliche Zitierung nicht verfügbar])

63) $\{\{1$; eine ... von; A2 $\}\}$

Eine Woche ist seit dem Zugunglück im japanischen Amagasaki vergangen. Jetzt erwägt die Bahngesellschaft eine Änderung der engen Fahrpläne. Grund: Der Lokführer war zu schnell gefahren, um eine Verspätung von 90 Sekunden aufzuholen. Die Zahl der Toten ist inzwischen auf 107 gestiegen.

INTERNET-STUDIE (HMP05/MAI.00104 Hamburger Morgenpost, 02.05.2005, S. 43; NEWS [Ausführliche Zitierung nicht verfügbar])

64) $\{\{1$; Messwert; A2 $\}\}$

Die Besatzung hatte den Nager kurz vor dem Start durch die Erste Klasse huschen sehen. Die eingeleitete Rattenjagd war allerdings vergeblich. Mit eineinhalb Stunden Verspätung startete die Maschine nach Bahrain. Dort angekommen wurde die Maschine dann gründlich ausgeräuchert.

ERWISCHT (HMP06/MAR.01086 Hamburger Morgenpost, 11.03.2006, S. 45; ERZWUNGEN [Ausführliche Zitierung nicht verfügbar])

65) $\{\{1$; Indefpr.; A2 $\}\}$

"Ist doch wunderschön hier, oder?"

Er sagt es mit der Besessenheit eines Vergessenen, der alle überraschen will. Eben nur mit etwas Verspätung.

»Als der Ägypter zu mir ins Krankenhaus kam, habe ich ihn rauswerfen lassen« Oliver Hampel (HMP06/JUL.00825 Hamburger Morgenpost, 07.07.2006, S. 29; Der „vergessene« Profi ist zurück [Ausführliche Zitierung nicht verfügbar])

66) $\{\{1$; Messwert; A2 $\}\}$

Es fehlen: eine Hose und der Regisseur. Beide haben eine Entschuldigung: Er ist bei der Hochzeit seines Bruders, die Hose beim Regisseur. Als sie schließlich mit einer Stunde Verspätung kommen, gehts los: die Backstage-Gruppe von Philipp Meier von Rouden probt ihr Stück "Denkste". 
Backstage ist der Jugendclub des Schauspielhauses. Unter dem "Oberbefehl" von Theaterpädagoge Michael Müller haben Jugendliche zwischen 12 und 24 Jahren in acht Gruppen eine Saison lang getextet und geprobt. (HMP07/JUN.00037 Hamburger Morgenpost, 01.06.2007, S. 20-21; Starke Stücke von Theater-Fans [Ausführliche Zitierung nicht verfügbar])

67) $\{\{1$; Messwert; A2 $\}\}$

HSV-KURZPÄSSE

SPÄT: Mit 80 Minuten Verspätung, um 1.15 Uhr Ortszeit, erreichte der HSV in der Nacht zu Sonntag das Wüsten-Emirat. Bitter für Joris Mathijsen, dass zu allem Überfluss seine Zimmerkarte nicht funktionierte. Noch um kurz vor drei Uhr geisterte der Niederländer dezent genervt durch die Lobby des "JW Marriott". (HMP09/JAN.00260 Hamburger Morgenpost, 05.01.2009, S. 2; HSV-KURZPÄSSE [Ausführliche Zitierung nicht verfügbar])

68) $\{\{0\}\}$

"Fristlos" muss auch gleich sein

Will der Arbeitgeber einen Mitarbeiter wegen einer groben Verfehlung fristlos kündigen, muss er das besser gleich tun. Das Landesarbeitsgericht Rheinland-Pfalz erklärte jetzt nämlich eine fristlose Entlassung wegen Verspätung für unzulässig: Bei der Schwere des Vorwurfs wäre der Rauswurf zwar berechtigt gewesen, meinten die Richter - aber der Arbeitgeber hätte sie unverzüglich aussprechen müssen, keinen weiteren Tag warten dürfen (Az. 6 Sa 709/08).

(HMP09/NOV.02953 Hamburger Morgenpost, 29.11.2009, S. 45; URTEILE [Ausführliche Zitierung nicht verfügbar])

69) $\{\{0\}\}$

Sommerplatte mit Verspätung "Jamaica"-Debüt mit tanzbarem Elektro-Pop

Schlechtes Timing: Wäre "No Problem", das Debüt der beiden Franzosen Antoine Hilaire und Florent Lyonnet, drei Monate früher erschienen, es wäre wohl der Soundtrack des superheißen Sommers 2010 gewesen. Macht nichts, dann ist "No Problem" eben das Hitalbum des Herbstes -auch wenn das weit weniger sexy klingt. (HMP10/AUG.01760 Hamburger Morgenpost, 20.08.2010, S. 23; Sommerplatte mit Verspätung "Jamaica"Debüt mit tanzbarem Elektro-Pop [Ausführliche Zitierung nicht verfügbar])

70) $\{\{1$; Messwert; A2 $\}\}$

BU: Merkel-Sprecher Steffen Seibert (Mitte) erklärt Journalisten die Verspätung.

BU: Wollte der deutschen Kanzlerin wohl eins auswischen und stoppte den Kanzlerinnen-

Airbus: Irans Diktator Mahmud Ahmadinedschad

BU: Zwei Stunden Verspätung: Merkel mit Indiens Premier Manmohan Singh

(HMP11/JUN.00069 Hamburger Morgenpost, 01.06.2011, S. 09; Affront in der Luft Iran schickt Merkel in die Warteschleife Kanzlerinnen-Airbus muss zwei Stunden lang kreisen / Teheran verbietet Überflug / Westerwelle "Respektlosigkeit" / Botschafter einbestellt [Ausführliche Zitierung nicht verfügbar])

71) $\{\{1$; Messwert; A2 $\}\}$

Weniger Verspätungen bei der Hamburger S-Bahn

Die Hamburger S-Bahn holt auf. 95,4 Prozent aller Züge hatten im vergangenen Jahr weniger als drei Minuten Verspätung. Damit hat das Unternehmen erstmals seit Einführung der Qualitätssteuerung (2008) den vom Hamburger Verkehrsverbund (HVV) geforderten Pünktlichkeitswert von 94,7 Prozent eingehalten. 2010 lag man bei 94,5 Prozent. 
(HMP12/JAN.01183 Hamburger Morgenpost, 13.01.2012, S. 09; Weniger Verspätungen bei der Hamburger S-Bahn [Ausführliche Zitierung nicht verfügbar])

72) $\{\{1$; Messwert; A2 $\}\}$

-> Gibt es Vergütungen bei Verspätungen? Bei Flügen bis zu 1500 Kilometern haben

Fluggäste ab zwei Stunden Verspätung Anspruch auf freie Telefonate, Getränke, Mahlzeiten, gegebenenfalls auch Hotel-Übernachtungen. Bei Strecken bis zu 3500 Kilometern gilt dies nach drei Stunden Verspätung, ab 3500 Kilometern nach vier Stunden.

-> Was sollte man sonst noch bedenken? Wer im Anschluss an den Flug einen Mietwagen gebucht hat, muss sich selbst um Umbuchung oder Stornierung kümmern.

(HMP12/SEP.00636 Hamburger Morgenpost, 07.09.2012, S. 02, 03; THEMA DES

TAGES Allein in Hamburg fallen 120 Flüge aus Er stürzt heute die Lufthansa ins Chaos Er ist 39 -und Chef der mehr als 10000 organisierten Flugbegleiter in Deutschland. Heute legt Nicoley Baublies unsere Airports lahm. Über 1000 Lufthansa- Flüge werden gestrichen. Viel Frust und Ärger für die Passagiere. [Ausführliche Zitierung nicht verfügbar])

73) $\{\{0\}\}$

Polettos doppelte Premiere

im St. Pauli-Theater genossen sie das neue Stück in vollen Zügen

Es ging (fast) ohne Verspätung los. Und das bei der Bahn! Nun ja, gestern Abend fegte die "Linie S1" ja auch über die Bühne. (HMP13/SEP.00701 Hamburger Morgenpost, 09.09.2013, S. 12; Polettos doppelte Premiere [Ausführliche Zitierung nicht verfügbar])

74) $\{\{1$; Messwert; A2 $\}\}$

Knapper in doppelter Hinsicht lief's für Eintracht Norderstedt. Erst stand die Mannschaft von Thomas Seeliger auf dem Weg zum TSV Havelse im Stau. Das Spiel begann mit 30 Minuten Verspätung und gar nicht gut für die Eintracht: Halstenberg (9.) traf früh für die

Niedersachsen.

In der Folgezeit fanden die Holsteiner zwar ins Spiel, litten jedoch unter einem Tor-Stau, der alle höheren Ziele zunichte machte. Jan Lüneburg (49.) verpasste die beste Chance zum Ausgleich, bevor El-Helwe (71.) und Zakrzewski (83.) zum schließlich deutlichen 3:0-Erfolg der Havelser trafen. (HMP14/AUG.01284 Hamburger Morgenpost, 18.08.2014, S. AB28; Chancenlos gegen Bremens Talente [Ausführliche Zitierung nicht verfügbar])

75) $\{\{1$; Messwert; A2 $\}\}$

Allerdings muss angemerkt werden, dass Norwegen, Finnland, Schweden und Italien bei weitem nicht mit ihren stärksten Teams am Start waren, die Vor-WM in Ramsau war beispielsweise wesentlich stärker besetzt gewesen, damals war Österreich Dritter geworden. Doch dies soll die Freude über den Sieg der Österreicher nicht schmälern. Startläufer Urain hatte seine Aufgabe, mit möglichst wenig Rückstand an Botwinow zu übergeben, gut erfüllt und kam nach den ersten 10 Kilometern mit knapp 27 Sekunden Verspätung ins Ziel. "Der Boti ist dann ein Super-Rennen gelaufen, ist auf die Führenden aufgelaufen und hat als Dritter übergeben", schildert Franz Weingartner den Verlauf des Rennens. Walcher hielt dann das Dreierpaket mit Finnland und Russland beisammen, während die viertplatzierten Norweger zurückfielen. (I00/MAR.12600 Tiroler Tageszeitung, [Tageszeitung], 06.03.2000. Sachgebiet: Sport, Originalressort: Sport; Saisonsieg Nr.1 für den Weltmeister)

76) $\{\{1$; Gen.;A1 $\}\}$ 
Corriere dello sport (I): "Hermann Maier hat versucht, den Schlauen zu spielen, und ist von der Jury hart bestraft worden. Hujara hat bewiesen, dass er die österreichische Übermacht nicht fördert, wie es andere Athleten behaupten. Bereits vor einer Woche hatte Kristian Ghedina in Val d'Isere wegen der Verspätung Maiers protestiert. Nichts war passiert." Liberation (F): "Hermann Maier ist durch die FIS-Jury erbarmungslos disqualifiziert worden. (I00/DEZ.73647 Tiroler Tageszeitung, [Tageszeitung], 19.12.2000. - Sachgebiet: Sport, Originalressort: Sport; PRESSE-STIMMEN ZUM EKLAT UM HERMANN MAIER")

77) $\{\{1 ;$ attr. Adj.; A2 $\}\}$

Dort heißt es dann umsteigen auf die Außerfernbahn. Das gleiche gilt für die Gegenrichtung: Wer am Weg in die Landeshauptstadt die um eine Stunde schnellere Post benutzen will, muß von Reutte zuerst mit der Bahn bis Ehrwald fahren, erst dann darf umgestiegen werden. Unangenehm wird es für den Fahrgast, wenn die Post über den Fernpaß kommend eine geringfügige Verspätung aufweist und der Zug bereits abgefahren ist. Dann heißt es zwei Stunden warten, bis die nächste Diesellok Richtung Reutte fährt, während zwischenzeitlich die leeren Postbusse zur Wartung in die Postgarage Reutte überstellt werden. Auch die Linie Reutte Ehrwald Imst soll eingestellt werden, die Post hätte im Zugspitztalkessel lediglich Zubringerfunktion. (I96/MAI.18647 Tiroler Tageszeitung, [Tageszeitung], 13.05.1996. Originalressort: Regional Innsbruck und Umgebung; Zukunftsszenario Umsteigen)

78) $\{\{1$; Gen.; A1 $\}\}$

Wenn es auch nicht das erste Buch ist, das sich mit der überfälligen Modernisierung Österreichs auseinandersetzt, bietet es doch einen guten Überblick über den hohen Veränderungsbedarf in vielen Bereichen.

(kapp)

Die Journalistin Trautl Brandstaller sucht als Herausgeberin des Bandes Zuflucht bei Robert Musil, der in seinem "Mann ohne Eigenschaften" die chronische Verspätung Österreichs in allen Lebensbereichen beschrieben hat. Musils Kakanien wo es "Fortschritt, aber nicht zuviel Fortschritt" gegeben hat unterscheide sich kaum von jenem "Postkakanien", in dem wir heute leben, schreibt Brandstaller. Und sie verhehlt auch nicht, daß der Titel des Buches "Österreich 2 " eine Gegenthese zum FPÖ-Verfassungskonzept einer "Dritten Republik" symbolisieren soll, das für sie ein "autoritäres Trugbild" ist. (I96/DEZ.48700 Tiroler Tageszeitung, [Tageszeitung], 17.12.1996. - Sachgebiet: Kultur, Originalressort: Kultur; Fortschritt, aber lieber nicht zuviel davon)

79) $\{\{0\}\}$

Sie war nach ihrer Verhaftung am Timmelsjoch 1995 wegen der Beteiligung am Sprengstoffanschlag 1984 auf einen Strommasten im Burggrafenamt zu sieben Monaten Haft verurteilt worden. Schon 1992 war sie in Abwesenheit wegen Beteiligung an einer Serie von Sprengstoffanschlägen zwischen 1986 und 1988 zu 12 Jahren Haft verurteilt worden.

Der Prozeß begann mit Verspätung. Der Gefängniswagen, mit dem Frau Unterkircher aus Mailand nach Bozen gebracht worden war, war an einer Kreuzung mit einem Pkw zusammengeprallt. Unterkirchers Anwalt, Alessio Cuccurullo, bemühte sich vergeblich, die Glaubwürdigkeit des Kronzeugen Adalbert Holzer in Zweifel zu ziehen. (I97/OKT.38551 Tiroler Tageszeitung, [Tageszeitung], 03.10.1997. Originalressort: Tirol aktuell; Karola Unterkircher: Urteil wurde bestätigt)

80) $\{\{0\}\}$

Nach der Prämierung in der ersten Septemberwoche werden die Karikaturen vom 8. bis zum 20. September im ArtCult Center im Tabakmuseum in Wien gezeigt. 
Vier Türen für den Audi A3

Was beim Golf selbstverständlich ist, kommt beim kleinen Audi mit Verspätung: Der beliebte Kompaktklaßler wird ab Frühling 1999 mit vier Türen zu haben sein. Gezeigt wird der Viertürer-A3 am Genfer Automobilsalon, gleich danach wird er bei den Händlern stehen. Acht Häferl für den Audi A6 (I98/AUG.32582 Tiroler Tageszeitung, [Tageszeitung], 14.08.1998. - Sachgebiet: Auto / Motor, Originalressort: Motor; Karikaturen rund ums Auto)

81) $\{\{1$; Messwert; A2 $\}\}$

Voll anlaufen wird der Banknotendruck dann ab September. Die Herstellung wäre an und für sich aber wesentlich früher vorgesehen gewesen. Weil aber die Europäische Zentralbank (EZB) nicht rechtzeitig die Papierspezifikation für die Geldscheine abgestimmt hat sowie die Sicherheitsmerkmale der Banknoten, wird mit zumindest zweimonatiger Verspätung angedruckt. Europaweit werden 13 Milliarden Scheine im Wert von 520 Mrd. Euro (7,2 Billionen S) gedruckt: Ab 1. Jänner 2002 werden die neuen Noten ausgegeben.

(I99/AUG.31148 Tiroler Tageszeitung, [Tageszeitung], 11.08.1999. Originalressort: Seite 1; Euro-Druck mit einiger Verzögerung)

82) $\{\{1$; Messwert; A2 $\}\}$

AUGUST KUHN

AUS KITZBÜHEL

Rippen oben und unten, im Mittelteil Neuschnee, der beim allerbesten Willen nicht hinausgebracht werden konnte - so präsentierte sich gestern die Streif 2000 mit zwei Tagen Verspätung. Dienstag schon war das erste Training geplant, dass es gestern so weit war, darf als mittleres Wunder gelten. Denn nach viel Neuschnee in den letzten 48 Stunden war Schwerarbeit angesagt. (K00/JAN.05228 Kleine Zeitung, [Tageszeitung], 21.01.2000. Sachgebiet: Sport, Originalressort: Sport; Ghedina fürchtet Lawinengefahr)

83) $\{\{1$; Messwert; A2 $\}\}$

Mehr als 40.000 Menschen drängten sich gestern in der Villacher Innenstadt - um zu feiern, um anzuprosten, um den besten und längsten Faschingsumzug Kärntens zu sehen. Dabei wäre der Faschingshöhepunkt beinahe ohne "Chef" über die Bühne gegangen: Faschingsprinz Egon Putzi hatte seinen letzten großen Treffpunkt mit der Gilde um 9 Uhr einfach verschlafen. "Der lebt halt nach amerikanischer Zeit", seufzte Kanzler Gernot Bartl. Mit einstündiger Verspätung kam Putzi dann doch noch...

Rekordverdächtig. Beim Umzug selbst gab es dann einen neuen Rekord zu vermelden: 145 motorisierte Fahrzeuge, 16 Kapellen, 3500 maskierte Teilnehmer, davon 550 Kinder.

(K00/MAR.18430 Kleine Zeitung, [Tageszeitung], 05.03.2000. Originalressort: Villach; 8 Grad - und doch so heiß!)

84) $\{\{1$; Indefpr.; A2 $\}\}$

Die Falle heißt SK Sturm

Mit etwas Verspätung setzte gestern die Maschine mit der 150-köpfigen Delegation aus Monaco am Flughafen Graz-Thalerhof auf. Bei den Spielern herrschte dennoch gute Stimmung. Grund dafür sind die vergangenen Ergebnisse. (K00/OKT.76721 Kleine Zeitung, [Tageszeitung], 17.10.2000. - Sachgebiet: Sport, Originalressort: Sport; Die Falle heißt SK Sturm)

85) $\{\{1$; Messwert; A2 $\}\}$

Kundenfreundliche ÖBB 
3. 1.: Der EuroCity "Johann Strauß" hatte in Passau 25 Minuten Verspätung. Besorgt fragte ich den Schaffner, ob ich in Linz den Anschluß nach Graz erreichen würde. Da wurde über Lautsprecher angesagt, daß sich Reisende nach Selzthal - Graz beim Schaffner melden sollten. (K97/JAN.03377 Kleine Zeitung, [Tageszeitung], 16.01.1997. Originalressort: Leserbriefe, [Leserbrief])

86) $\{\{1$; Messwert; A2 $\}\}$

Damals hätte der Final-Gegner Ivan Lendl geheißen, am Ostersonntag hieß er Sergi

Bruguera. Und in diesem Match war der Erfolg nur eine Frage der Zeit.

In den Tennis-Annalen dürfte dieser mit acht Jahren Verspätung geglückte und wohl auch ersehnte Titelgewinn einmalig sein. Ein Beweis von Musters Qualitäten als Dauerbrenner im Tennis-Circuit und eine Bestätigung seiner außergewöhnlichen Willensstärke.

360.000 US-Dollar sackte der Österreicher in Florida ein. (K97/APR.24339 Kleine Zeitung, [Tageszeitung], 01.04.1997. - Sachgebiet: Sport, Originalressort: Sport; Toms "weiße Weste" steht in Graz auf dem Spiel)

87) $\{\{0\}\}$

RUNDSCHAU

Niklasdorf.

Heute, Samstag, beginnt um 19 Uhr im Veranstaltungszentrum das Kabarett "Entschuldigen Sie bitte die Verspätung" mit Josi Prokopetz.

Eisenerz.

Heute, Samstag, findet in Eisenerz der "14. Eisenerzer Stadtrundlauf" statt. (K97/SEP.71310 Kleine Zeitung, [Tageszeitung], 20.09.1997. Originalressort: Leoben; RUNDSCHAU)

88) $\{\{1$; Messwert; A2 $\}\}$

Ich gehöre jetzt dazu."

Bis Hermann Maier sein Sieger- Interview geben konnte, hat es gestern lange gedauert. Mit mehr als einer Stunde Verspätung kam es zum "ersten Versuch", diese Abfahrt (mit dem Slalom in Veysonnaz am Sonntag als LauberhornKombination) über die Bühne zu bringen. Nach vier Läufern aber bereits die erste Unterbrechung. Da lag Hans Knauß vorne, knapp dahinter Stefan Eberharter, der zwar durch zwei "Fußgänger" im oberen Streckenteil etwas behindert war, aber auf einen Neustart verzichtete. (K98/JAN.04108 Kleine Zeitung, [Tageszeitung], 17.01.1998. - Sachgebiet: Sport, Originalressort: Sport; Maier beendete die 13jährige)

89) $\{\{1$; Messwert; A2 $\}\}$

Beide verletzten sich Mittwoch beim 2:0 Frankreichs gegen Andorra.

Ehrung.

Mit einer Stunde Verspätung begann Donnerstag die Verleihung der Goldenen Teekanne für die beliebtesten Schisportler. Schuld war Hermann Maier, der als Ausrede ein Meeting mit Pete Sampras parat hatte. Die Preise erhielten neben Maier auch Renate Götschl und Kombinierer Mario Stecher. (K98/OKT.78395 Kleine Zeitung, [Tageszeitung], 17.10.1998. - Sachgebiet: Sport, Originalressort: Sport)

90) $\{\{1$; attr. Adj.; A2 $\}\}$

JOHANNA BAINSCHAB

"High noon" gestern in der Spittaler Innenstadt - ab 12 Uhr war beim traditionellen Faschingsumzug wieder Narrenfreiheit angesagt. Und trotz einer beachtlichen Verspätung von einer dreiviertel Stunde, die die Hunderten Schaulustigen im eisigen Wind ausharren 
mußten, waren beim Umzug von allen Seiten begeisterte "He-Lei"- Rufe zu vernehmen. Denn die Spittaler Faschingsnarren haben wieder keine Kosten und Mühen gescheut, ihrem Publikum, das zu beiden Seiten die Gehsteige säumte, ihre einfallsreichen und vor allem witzigen Ideen zu präsentieren. Die Palette reichte von "Peace\&Love"-Relikten aus den Siebzigern über einen fahrbaren Tennisplatz bis hin zu einem überdimensionalen Kinderwagen, in welchem mehr als ein Narrenbaby Platz fand. (K99/FEB.11790 Kleine Zeitung, [Tageszeitung], 14.02.1999. Originalressort: Oberkärnten; "He-Lei" ist Narren-sicher)

91) $\{\{1$; Messwert; A2 $\}\}$

So etwa bei der Neugestaltung Griesplatz- Nord, die ja bis zur Eröffnung der

Landesausstellung 2000 fertig sein sollte, oder bei der Umgestaltung des Volksgartens, dem geplanten Beitrag der Stadt Graz zur Internationalen Gartenschau (IGS) 2000 in

Unterpremstätten.

Und eben jenes "Blumenschaufenster" für die IGS 2000 könnte für die Murmetropole eine ordentliche Bauchlandung werden. Denn für die Sanierung der Parkanlage im Bezirk Lend haben die Mannen des Stadtgartenamtes unter Führung ihres politischen Referenten Stadtrat Walter Ferk rund ein halbes Jahr Verspätung "aufgerissen". Der Spatenstich war bereits für April dieses Jahres geplant, im nächsten Frühling sollte man schon im völlig neu gestalteten Garten flanieren können.

Im Büro Ferk betont man, dass die Umsetzung des 12- Millionen- Schilling-Projektes nun zügig vorangehen solle. (K99/AUG.61167 Kleine Zeitung, [Tageszeitung], 18.08.1999. Originalressort: Graz; Grazer Bauprojekten droht "Zitterpartie")

92) $\{\{1$; Messwert; A2 $\}\}$

Ankunft mit Verspätung - aber die Griechen zeigten wenig Respekt

Mit 50 Minuten Verspätung kletterte der Tross von Panathinaikos Athen gestern Mittag auf dem Grazer Thalerhof aus dem Flugzeug - und man hatte gleich den Eindruck, dass die Gäste vor dem österreichischen Gegner nicht recht zittern. "Wir sind hergekommen, um nicht zu verlieren. Wir hatten in der Meisterschaft einen guten Start, sind auch Zweiter", tönte der deutsche Spielmacher Karl- Heinz Pflipsen, als Ex-Kollege von Toni Polster aus Gladbach nach Athen übersiedelt. (K99/OKT.79873 Kleine Zeitung, [Tageszeitung], 21.10.1999. Sachgebiet: Sport, Originalressort: Sport; Ankunft mit Verspätung - aber die Griechen zeigten wenig Respekt)

93) $\{\{1$; Messwert; A2 $\}\}$

Die rot-weiß-rote Mannschaft trat in Valencia wie ein Kegelklub auf, dafür holten die Spanier "alle Neune". "So mies waren wir noch nie", titelte Täglich Alles, während der Kurier schrieb: "Raul nahm Österreich mit vier Toren auf die Hörner."Der entsetzte österreichische Teamchef Herbert Prohaska verweigerte direkt nach dem Spiel jeglichen Kommentar und schickte an seiner Stelle Präsident Beppo Mauhart zur Pressekonferenz. Gestern morgen stellte sich "Schneckerl" Prohaska zerknirscht nach nur drei Stunden Schlaf der Presse. Die Österreicher waren erst mit zweieinhalb Stunden Verspätung um 6.45 Uhr in Wien angekommen. "Wir waren nicht einmal Trainingspartner für die Spanier. Es war ein kollektives Versagen der Mannschaft", resümierte Prohaska, der über seine Zukunft noch keine Aussage machte. (L99/MAR.15608 Berliner Morgenpost, [Tageszeitung], 29.03.1999, S. 25. Originalressort: SPORT; sid: Alle Neune: Kein Debakel, sondern eine Hinrichtung)

94) $\{\{1+1$; Messwert; Subj.; A2; A1 $\}\}\{\{$ SVG; V. haben $\}\}$ 
Drei von vier Auskünften waren demnach deutlich überteuert.Auch die Pünktlichkeit habe im Vergleich zu 1997 leicht nachgelassen. Von den überprüften 11000 Zügen auf acht Testbahnhöfen erreichten nur noch 55 Prozent das Ziel pünktlich oder mit einer Minute Differenz. Jederer fünftㅡㄹ Z Zug habe mehr als sechs Minuten Verspätung gehabt. Das Flaggschiff ICE habe am schlechtesten abgeschnitten, weil die Züge durch Baustellen ausgebremst werden. Nur jeder dritte ICE-Zug sei pünktlich gewesen. (L99/AUG.57390 Berliner Morgenpost, [Tageszeitung], 27.08.1999, S. 25. Originalressort: WIRTSCHAFT; BM: Schlecht beraten und zu teuer verkauft)

95) $\{\{0\}\}$

Selbstbeherrschung ist eine Tugend, die Stars und Prominenten besonders schwer fällt. Eine Probe aufs Exempel lieferte jetzt Prinz Charles (51). Als er im Auto bei der Fahrt zum Flughafen erfuhr, dass seine Maschine mit Verspätung abfliegen würde, tobte er wie Rumpelstilzchen. In einem Wutanfall packte er sein Handy und schleuderte es aus dem Fenster seines Bentley.Solches sinnlose Ausrasten ist typisch für Menschen, die kraft ihrer besonderen Stellung in der Gesellschaft im Rampenlicht der Öffentlichkeit stehen. Dieses Ausraster- Syndrom beschrieb jetzt in einem Buch Winston Fletcher, ehemaliger Chef einer Werbeagentur, der langjährige einschlägige Erfahrungen im Umgang mit Stars sammeln konnte.Der königliche Wutanfall des Prinzen, der jetzt bekannt wurde, hatte ein peinliches Nachspiel. (L99/NOV.85421 Berliner Morgenpost, [Tageszeitung], 29.11.1999, S. 8. Originalressort: AUS ALLER WELT; SAD: Wenn Prominente vor Wut ausflippen)

96) $\{\{1$; attr. Adj.; A2 $\}\}$

Schließlich stand er unter Bewährung.

Brigittes Patienten mussten an diesem Morgen auf ihre Hausärztin warten. Als sie schließlich mit einer noch nie da gewesenen Verspätung in die Praxis fuhr, holte er sich die Landeszeitung und das Albblatt aus dem Briefkasten.

4

Im Lokalblatt war der Ballonabsturz bereits den zweiten Tag nacheinander das Aufmacherthema. (DIV/WPB.00001 Wark, Peter: Ballonglühen , (Erstveröffentlichung 2003) - Meßkirch: Gmeiner-Verlag, 2011)

97) $\{\{1$; Messwert; A2 $\}\}$

Von ihren Kollegen dort empfangen, ging die Reise der luxemburgischen Rettungskräfte dann per Bus zur "Base nationale de support" (BNS) in Lintgen, wo ein Debriefing auf dem Programm stand. Die Ankunft in der BNS war für 23.00 Uhr geplant. Weil das Flugzeug aber mit mehr als 90 Minuten Verspätung auf der Landebahn in Melsbroek aufsetzte, verzögerte sich auch die Ankunft des 15-köpfigen Teams in Lintgen. Die Heimreise der beiden restlichen Croix-Rouge-Mitarbeiter wird, wie das Außenministerium gestern mitteilte, samt Material in Zusammenarbeit mit den französischen Autoritäten organisiert. sz (LTB10/JAN.00420 Luxemburger Tageblatt, 18.01.2010, Originalressort: FD - FAITS DIVERS; Aus Katastrophengebiet zurück)

98) $\{\{1+1$; Messwert; Subj.; A2; A1 $\}\}\{\{$ SVG; V. haben $\}\}$

Vier Flüge ausgefallen

Am Luxemburger Flughafen sind gestern Morgen die Flüge von und nach Madrid, Frankfurt am Main, Paris und London ausgefallen, der Fl Flug n_a Verspätung, wie ein Luxair-Sprecher erklärte. Gegen 12.00 Uhr hatte sich die Lage wieder weitestgehend normalisiert. (LTB11/DEZ.00529 Luxemburger Tageblatt, 17.12.2011, Originalressort: FD - FAITS DIVERS; Sturm erfordert 215 Rettungseinsätze) 
99) $\{\{1$; Indefpr.; A2 $\}\}$

Kurz vor dem ersten Start um 11.00 Uhr fand ein Warm-up statt, an dem die Kinder teilnehmen konnten, um sich auf die Rennen vorzubereiten.

Die Kleinsten der Jahrgänge 2005/06 wurden mit etwas Verspätung wegen des großen Andrangs bei den Nachmeldungen auf die Strecke geschickt. Alleine bei diesem Rennen waren rund 120 kleine Läufer und Läuferinnen am Start. Insgesamt vermerkten die Organisatoren über 220 Teilnehmer, was wiederum einen Teilnehmerrekord für dieses noch junge Rennen bedeutet. (LTB12/SEP.01271 Luxemburger Tageblatt, 24.09.2012, Teilnehmerrekord mit 220 Kindern)

100) $\{\{1 ;$ Messwert; A2 $\}\}$

Der einzige Luxemburger Teilnehmer, Laurent Didier, kam gestern mit einem dritten Feld in Saint-Vallier an. Er klassierte sich direkt hinter seinem Mannschaftskollegen Grégory Rast auf dem 100. Rang mit 8'26" Verspätung. In dieser Gruppe befand sich vom RadioShackTeam auch Jens Voigt (102.). Laurent Didier liegt in der Gesamtwertung nun auf Platz 131. Sein Rückstand auf Talansky beträgt 18'47". (LTB13/MAR.00492 Luxemburger Tageblatt, 08.03.2013, Die Ruhe vor dem Sturm?) 
1) $\{\{0\}\}^{231}$

Die Tessiner sprachen sich 1803 für ihre Zugehörigkeit zur Schweiz aus, obwohl sie vorher 300 Jahre lang Untertanen der «Gnädigen Herren» nördlich der Alpen gewesen waren. Kein selbstverständlicher Entscheid also. Doch die Tessiner versprachen sich im Schweizerischen Bundesstaat Freiheit und Selbständigkeit. Diese bilden den Inhalt des Tessiner Schweizertums.

Bereit zur Verteidigung (A97/AUG.19728 St. Galler Tagblatt, [Tageszeitung], 22.08.1997, Jg. 53. Originalressort: TB-INL (Abk.); * Kurt Huber: Wo das lateinische Temperament regiert, [Bericht])

2) $((1 ;$ attr. Adj.; A1))

Die Freiheit gefeiert

200 Jahre freier Thurgau - Festakt in Frauenfeld mit Grussworten von Arnold Koller und Erwin Teufel

Die Thurgauer Freiheit hat einen Geburtstag: Am 3. März 1798 wurde das damalige Untertanengebiet in die Freiheit entlassen. Mit der Bevölkerung feierten gestern Bundesrat Arnold Koller und der baden-württembergische Ministerpräsident Erwin Teufel.

Christian Kamm (A98/MAR.13324 St. Galler Tagblatt, [Tageszeitung], 04.03.1998, Jg. 54. Originalressort: TB-OST (Abk.); Christian Kamm: Die Freiheit gefeiert, [Bericht])

\section{3) ((1; Komp.; A1))}

Für Chinas Führung brachte der Besuch des amerikanischen Präsidenten Prestige. Das Reich der Mitte ist nicht mehr Gegner, sondern Partner der USA, ein Garant für Frieden, Stabilität, Sicherheit und Wohlstand in der Welt.

Zwar wird China nicht sofort Mitglied der Welthandelsorganisation - aber Clinton «bedauert»; zwar werden die USA ihre Waffenlieferungen an Taiwan nicht einstellen - aber Clinton bestätigte die «Ein-China-Politik» der USA; zwar forderte Clinton zum Dialog mit dem Dalai Lama auf und verlangte Religionsfreiheit für die Tibeter - er sagte aber auch: «Ich erkenne an, dass Tibet ein Teil Chinas ist, eine autonome Region Chinas.»

Auch Clinton kann mit seinem Besuch im Reich der Mitte zufrieden sein. Die Chinesen haben ihm bestätigt, dass sie Partnerschaft wollen und dafür auch zu Konzessionen bereit sind: China verbreitet keine Massenvernichtungswaffen weiter, es übernimmt Verantwortung zur Beilegung von Krisen, es ist bereit, mit Hilfe der USA sein Justizsystem umzugestalten. (A98/JUN.43947 St. Galler Tagblatt, [Tageszeitung], 30.06.1998, Jg. 54. Originalressort: TB-AKT (Abk.); Der amerikanische Traum in China, [Bericht])

4) $\{\{1+1$; NS; Dat.; A2; A1 $\}\}\{\{$ SVG; Dat. F. lassen $\}\}$ «Bauherr behält Freiheit»

Führt hier die Ortsgemeinde aber nicht durch die Hintertür eine Art Protektionismus für das Diepoldsauer Gewerbe ein? Frei verneint entschieden: «Wir lassen ja dem $\underline{\text { Bauherrn }}$ die Freiheit, wem er die Aufträge vergeben will. Da reden wir nicht drein.» In der Tat hat sich der erste und bisher einzige Bauherr für ein auswärtiges Baugeschäft entschieden. «Ganz

${ }^{231}$ Die unterschiedlichen Klammern stehen für die unterschiedlichen Bedeutungen von Freiheit: ((Bed. I)); [[Bed. II]]; \{\{Bed. III $\}$. 
offensichtlich spielt also die Konkurrenz doch», sagt Anton Frei, verweist aber darauf, dass man noch wenig Erfahrungen habe. (A99/MAI.37277 St. Galler Tagblatt, [Tageszeitung], 28.05.1999, Jg. 55. Originalressort: RT-FRO (Abk.); Hanspeter Thurnherr: Lob im Dorf - Kritik von auswärts, [Bericht])

5) ((0))

Freiheit bewahren

Wollen wir uns etwas bewahren, was uns von aussen nie wieder gegeben werden könnte? Ich meine die Freiheit. Am allerwenigsten würde sie uns von jenen zugestanden, die ihre eigene schon längst verloren haben. Ich meine die Freiheit der eigenen Entscheidungsfindung, der eigenen Richter und nicht zuletzt des freien Wortes. (A99/NOV.77915 St. Galler Tagblatt, [Tageszeitung], 05.11.1999, Jg. 55. Originalressort: TB-FOR (Abk.); Freiheit bewahren, [Bericht])

6) $[[0]]$

Insgesamt wurden über 700 Frondienststunden für Hegearbeit, Baustellen-Abfischen und Arbeiten am Fähweiher, in dem Elterntiere gehalten und im Frühwinter zur Laichgewinnung gefangen werden, geleistet, weiss Grob zu berichten.

Gewässerbewirtschaftung

Im Frühling und im Herbst wurden die 16 Aufzuchtbäche abgefischt und dabei 6842 junge

Bachforellen gefeumert und in den Necker in die Freiheit entlassen. Gemäss Statistik waren es 1971 Sömmerlinge, 3917 Jährlinge, 785 2-Jährige und 167, die bereits das Fangmass von 25 Zentimeter aufwiesen.

Die Hitparade der Aufzuchtbäche wird vom Aachbach (18,5 Prozent), vom unteren Trämel (12,1 Prozent) und vom Spreitenbach (11,6 Prozent) angeführt. (A00/MAR.18807 St. Galler Tagblatt, [Tageszeitung], 11.03.2000, Jg. 56. Originalressort: TT-ALT (Abk.); KARL DIEM: Wartelisten werden kürzer und kürzer, [Bericht])

7) $\{\{2$; Komp.; Gen.; A2; A1 $\}\}$

Bei den Schwellenwerten für die verschiedenen Verfahren schlagen die Verbände beim freihändigen Verfahren vor, den Wert von bisher 100000 auf 50000 Franken zu senken. Der tiefere Wert öffne den Marktzutritt für mehr Firmen, begründen die Verbände die Reduktion. Sie enge im Gegenzug aber die Entscheidungsfreiheit des öffentlichen Auftraggebers ein.

Würde der höhere Schwellenwert gewählt, setzen die Verbände voraus, dass trotzdem für die Vergabe von Aufträgen unter 100000 Franken das Einladungsverfahren die Regel bleibe. Mindestens drei Kriterien (A00/JAN.06165 St. Galler Tagblatt, [Tageszeitung], 26.01.2000, Jg. 56. Originalressort: AT-APP (Abk.); Gewerbeverband und öffentliche Beschaffung, [Bericht])

8) $\{\{1$ Infsatz; A2 $\}\}$

Dazu gehört auch die Gesundheit, soweit sie durch andere bedroht wird», schreibt die Allianz in einer Medienmitteilung. Jeder mündige Mensch sei alleiniger Eigentümer seines Körpers; er dürfe seine Gesundheit erhalten oder schädigen - jedoch mit seinem Tun nicht andere mitschädigen. «Damit steht die Freiheit, nicht zwangsweise mitrauchen zu müssen, über der Freiheit, überall und jederzeit rauchen zu dürfen.»

Allgemeiner Trend

Neben dem Tessin und Solothurn, wo entsprechende Regelungen bereits in Kraft sind, wird am gleichen Tag wie Ausserrhoden auch die Bevölkerung Graubündens über rauchfreie Restaurants entscheiden. (A07/NOV.04741 St. Galler Tagblatt, [Tageszeitung], 10.11.2007, 
Nr. 263, S. 13. - Sachgebiet: Regionales / Lokales, Originalressort: Ausser-Innerrhoden; d.scarano: Allianz für Rauchverbot in der Gastronomie)

9) $\{\{0+1$; keine Real.; Subj.; kein Arg.; A1 $\}\}\{\{$ SVG; F. haben $\}\}$

Lees Ziel ist, im nächsten Jahr einen Waldkindergarten in seiner Stadt Incheon zu gründen. «Koreas Kinder brauchen mehr Bewegung und mehr Natur», ist er überzeugt. «Im Wald können sie spielerisch lernen und haben mehr Freiheit, hier hat es keine Wände und keine Türen.»

In Korea sei die Idee fast unbekannt, er wolle sie dort aber verbreiten. «Ich bin schon völlig infiziert.» Im asiatischen Land zählten allerdings schon bei den Kleinsten Leistung und Disziplin. (A08/JUL.01243 St. Galler Tagblatt, [Tageszeitung], 04.07.2008, Nr. 154, S. 43. - Sachgebiet: Regionales, Originalressort: SG-Stadt; k.mueller: Im Fokus)

10) ((1; Gen.; A1))

Ihr Motto: „Moral ist nur die Entschuldigung all derer, die sich nicht trauen, ihre Wahrheit zu leben." Zum Kinostart zeigen Regisseur und Hauptdarstellerinnen den Film um 20Uhr im Cinecittà.

BÜHNE

Die Freiheit des Poeten

Das Beat-Generationen-Projekt „Cosmic Baseball -

Das Blut des Poeten Jack Kerouac" taucht ein in den Mythos des ,,angry young man“, der sich gegen Autoritäten auflehnt und die Freiheit auf der Landstraße sucht. (NUN10/MAR.00512

Nürnberger Nachrichten, 04.03.2010, S. 3; [Ausführliche Zitierung nicht verfügbar])

11) ((1; Komp.; A1))

Konkret habe die Regierung das Nachbarland Indien um Helikopter und Nachtsichtgeräte gebeten. Indiens Premier Vajpayee sicherte König Gyanendra telefonisch zu, Nepal zu helfen. Nepals Polizei soll mindestens acht Journalisten verhaftet haben, nachdem unter dem Ausnahmezustand die Pressefreiheit eingeschränkt wurde. (A01/NOV.47549 St. Galler Tagblatt, [Tageszeitung], 29.11.2001, Jg. 57. Originalressort: TB-AUS (Abk.); Nepal fordert indische Militärhilfe, [Bericht])

12) ((1; für + Akk.; A1))

Obama: Seit Jahren sage ich schon, dass es Zeit ist, den Weg direkter Diplomatie zu beschreiten, und zwar ohne Vorbedingungen, sowohl mit Freunden als auch mit Feinden. Allerdings bin ich nicht daran interessiert zu reden um des Redens willen. Im Falle Kubas sollte eine derartige Diplomatie Möglichkeiten schaffen, die Interessen der USA und die Sache der Freiheit für das kubanische Volk voranzubringen.

Sánchez: Wären Sie bereit, unser Land zu besuchen?

Obama: Niemals würde ich Schritte ausschliessen, welche die Interessen der USA und die Sache der Freiheit für das kubanische Volk fördern könnten. (A09/DEZ.05036 St. Galler Tagblatt, [Tageszeitung], 17.12.2009, Nr. 294, S. 2. Originalressort: seite zwei; DOKUMENTATION)

13) $((0))$

In den kurzen Pausen zwischen den Liedern schnappte ich einige Gesprächsfetzen vom Nebentisch auf. Grosse Themen wurden da verhandelt. «Was heisst denn das: Freiheit?» Der Mitzwanziger, der diese Frage aufgeworfen hatte, lehnte sich auf seinem Stuhl zurück, die Arme hinter dem Kopf verschränkt, beugte sich aber sogleich wieder nach vorne, weil er keine Chance hatte, die Antwort, die sein Gegenüber ihm geben wollte, zu verstehen. «Du bist 
frei, wenn du nichts mehr zu verlieren hast. So ähnlich hat das doch Janis Joplin mal gesungen», meinte er. (A10/MAR.05311 St. Galler Tagblatt, 17.03.2010, Nr. 63, S. 35. Sachgebiet: Regionales, Originalressort: AT-Appenzellerland; uw.ferrari: Zwei Schildkröten)

14) $((0))$

Eigentlich. Doch wenn Frau Holle mit dem Pfadschlitten kooperiert, wird's heikel - wie hier in Thal. Doch Hüter dieser Freiheit gibt es überall: mit einer Schaufel verhalf jemand der Presse wieder zu ihrem Recht. Schlimm wär's ja nicht, wenn «Sonntags-Zeitung» und «Sonntags-Blick» mal nicht verfügbar wären. Das Tagblatt kommt bestimmt - am Montag.

(A10/DEZ.06563 St. Galler Tagblatt, 22.12.2010, Nr. 299, S. 35. - Sachgebiet:

Regionales, Originalressort: OT-rorschach; r.hirtl: NAMEN \& NOTIZEN Vom Ende des «Sulzbergs», fleissigen Schülerinnen und Schülern sowie Frau Holles versuchter Zensur der Presse.)

15) $((0))$

Mit Salbei - der Pflanze der Dankbarkeit - bedankt sich die Präsidentin Gisela Schönenberger für das Mitmachen und Mittragen der verschiedenen Anlässe und lädt die Mitglieder ein, auch im neuen Jahr zur Vielfalt des Vereines beizutragen.

Ein Beet und ein Vogel

Symbolisch für die Vielfalt, die Angela Fässler während acht Jahren Vorstandsarbeit in den Verein gebracht hat, wird ihr das Pflanzen-quer-Beet überreicht und dazu ein Vogel, der die Freiheit symbolisieren soll, die Angela nun geniessen darf.

Als Nachfolgerin kann Heidi Krämer mit Applaus begrüsst und kurz darauf gewählt werden. Mit Marianne Seliner darf auch das vakante Revisorenamt wieder besetzt werden.

(A11/MAR.02097 St. Galler Tagblatt, 05.03.2011, Nr. 54, S. 51. - Sachgebiet: Regionales, Originalressort: TT-Neckertal; c.oberholzer: Frauengemeinschaft mit neuem Logo)

16) $\{\{1+1$; Infsatz; Dat.; A2; A1 $\}\}\{\{$ SVG; Dat. F. lassen $\}\}$

Die neusten Entscheidungen der SBB kriminalisieren die treusten Kunden. Ich besitze seit vielen Jahren ein GA, obwohl ich den Kaufpreis nicht «herausfahre». Dies weil es mir ein Stück Freiheit lässt, spontan doch eine andere Strecke zu fahren oder gegen einen Aufpreis die Klasse zu wechseln. Sei es, weil ich mir den Luxus gönnen möchte oder um der überfüllten 2. Klasse zu entgehen.

Mit absolut inakzeptablen Argumenten werden hier Massnahmen ergriffen, den Service weiter zu senken. (A11/JUN.06499 St. Galler Tagblatt, 21.06.2011, Nr. 142, S. 30. Sachgebiet: Unterhaltung, Originalressort: forum TB; y.stadler: GA \&\#8211; Zahlen Ja, Service Nein)

17) $((0))$

Die Erfahrungen des Göttinger Politikwissenschaftlers sagen viel über die Kluft zwischen dem Islam und dem Westen aus: Einerseits ist Tibi in der Tradition und mit dem Stolz einer Familie aufgewachsen, die in der syrischen Hauptstadt seit dem 13. Jahrhundert Richter und Rechtsgelehrte stellte, mit der Würde und dem Gepränge alter Damaszener Aristokratie - von den Dienstboten bis zum unnahbaren Patriarchen. „Mein Vater hat mich zum ersten Mal umarmt, als ich 1962 nach Deutschland abflog“, erzählt er. „Bis dahin dosierte er jede Zuwendung streng nach Leistung - mein Fahrrad durfte ich beispielsweise nur in genau dem Maß benutzen, wie ich gute Schulnoten erhielt.“ Andererseits begeisterte sich Tibi für James Dean, Elvis Presley und Pat Boone und fürs amerikanische Versprechen von Freiheit und Glücksseligkeit. 
Als Tibi nach Europa kommt, ruht er noch in der Tradition: „Am Flughafen habe ich Leute gesehen, die sich in aller Öffentlichkeit küssten - für mich war das damals ein Kulturschock: Sex auf der Straße!" Sexuelle Freizügigkeit empfand er zwar als faszinierend, aber zunächst zugleich als ehrlos. Doch als sein Vater nach einem der zahlreichen Putsche in Syrien enteignet wurde und Tibi sich das Geld fürs Studium als Tellerwäscher, als Post- und Lagerarbeiter verdienen musste, lernte er neu über den Westen nachzudenken.

(HAZ08/JUN.00067 Hannoversche Allgemeine, 02.06.2008, S. 13; Der umtriebige Damaszener [Ausführliche Zitierung nicht verfügbar])

18) ((1; attr. Adj; A1))

Schriftsteller lesen aus Protest

Ärger gibt es trotzdem: Schriftsteller, die in Jaipur zu Gast waren, lasen aus Protest Auszüge aus den «Satanischen Versen» vor. Die Aktion kam bei einem Publikum, das sich über die Einschränkung der literarischen Freiheit erregte, gut an. Weniger zufrieden waren die Veranstalter. Diese fürchten um die Existenz ihres fünf Jahre alten Festivals.

(A12/JAN.08258 St. Galler Tagblatt, 25.01.2012, Nr. 20, S. 11. Originalressort: focus; p.reichen: Angebliche Rushdie-Killer wohl eine Lüge)

19) ((1; Komp.; A1))

Damit ist Schluss. Der Kanton hat die Schulpflicht nun auf den Kindergarten ausgedehnt. Fällt damit die Wahlfreiheit weg? «Ja, aber», erklärt Etterlin dazu: «Wenn Eltern ihr Kind trotz erreichtem Alter noch nicht in den Kindergarten schicken wollen, bieten wir Beratung und Unterstützung an.» Zum einen sind in Rorschach zwei Einschulungsberaterinnen im Einsatz, und der Schulpsychologische Dienst sei ebenfalls darauf vorbereitet. Im Zweifelsfall rät Etterlin zu einem pragmatischen Vorgehen: «Wir probieren es in Zusammenarbeit mit den Eltern, wie das Kind auf den Kindergartenbesuch reagiert, und suchen dann nach individuellen Lösungen.» (A08/FEB.08654 St. Galler Tagblatt, [Tageszeitung], 22.02.2008, Nr. 44, S. 57. - Sachgebiet: Regionales, Originalressort: OT-Rorschach; f.bichsel: Trendwende: Wieder mehr Kinder)

20) $\{\{1 ;$ Infsatz; A2 $\}\}$

Die gebürtige Teufnerin lebt seit 25 Jahren in Sevilla, Flamenco ist die Sprache der Tänzerin. «Flamenco ist fest, hat seine klaren Regeln, die ich respektiere», sagt Bettina Castaño, die heute auf vielen Bühnen der Welt erfolgreich tanzt. Flamenco ist ihr Abc, aber mit diesem Alphabet hat sie sich die Sprachen vieler anderer Musikstile erschlossen: Flamenco, das Gesetz, und daneben die Freiheit, sich in ganz verschiedenen Welt-Musiken zu bewegen. Und dabei verwandelt sie sich in eine Zigeunerin, geht indischer Musik nach, zeigt klassische spanische Musik mit Orchester oder tanzt im Moment sehr erfolgreich mit den Alder-Buebe Appenzeller Musik. «Mein Heimweh-Programm», wie sie sagt. (A12/NOV.02661 St. Galler Tagblatt, 08.11.2012, Nr. 262, S. 45. - Sachgebiet: Regionales / Kultur, Originalressort: SG-st.galler kultur; M.Preisser: Kastanienbrauner Bach)

\section{1) $\{\{0+1$; keine Real.; Dat.; kein Argument; A1 $\}\}\{\{$ SVG; Dat. F. lassen $\}\}$}

Der künstlerische Leiter des Sandskulpturen-Festivals in Rorschach, Urs Koller, hat das diesjährige Motto in einem Gedichtband des deutschen Schriftstellers Hans Magnus Enzensberger entdeckt. Dass es immer einen gebe, der grösser oder kleiner ist als du, sei ein universelles Thema, das die Menschen tagtäglich bewege. «Es lässt den__Künnsstlernn sehr viel Freiheit.»

Plattform für Künstler 
Neu ist am Festival vom 10. bis 18. August aber nicht nur das Motto. (A13/JUN.01778 St.

Galler Tagblatt, 05.06.2013, Nr. 128, S. 38. - Sachgebiet: Regionales / Regionales, Originalressort: SG-region st.gallen; r.rohner: Sandskulpturen-Festival hat ein neues

Motto)

22) $\{\{0\}\}$

In seinem Unterrichtsfach Latein, so Spreckels weiter, habe es in Lüneburg nur eine dreistündige Fortbildung zum Zentralabitur gegeben: „Da konnten nicht einmal die wichtigsten grundlegenden Fragen ausreichend abgearbeitet werden.“

Die Gewerkschaft Erziehung und Wissenschaft fürchtet, dass wegen der zentral gesteuerten Fragen der Unterricht methodisch und inhaltlich verflachen könnte.

„Wir wissen, dass einige Pädagogen unter dem Mantel der Freiheit ihre thematischen Steckenpferde im Unterricht bis zur Pensionierung ritten“, räumt der GEW-

Landesvorsitzende Eberhard Brandt zwar Mängel des alten Systems ein. Die Gefahr der Einengung sei jedoch so groß, dass die Gewerkschaft das Zentralabitur ablehne. Auch Dagmar Richter, Leiterin des Gymnasiums Salzgitter-Bad, sieht weniger Raum für das Vertiefen individueller Schwerpunkte seitens der Lehrer. (BRZ06/APR.09051

Braunschweiger Zeitung, 20.04.2006; Premiere für das neue Abitur [Ausführliche Zitierung nicht verfügbar])

23) $((0))$

Melanie Thiemann hält es für ein „sehr wichtiges Thema“. Die 17-jährige, angehende Werkzeugmacherin muss sich hier jedoch mit sehr filigranen Materialien und mit der Figur aus fernöstlichen Kulturen auseinander setzen. Friedrich-Wilhelm Wessargas lehrt die jungen Auszubildenden von Neuland, Stadtwerke, Schnellecke, VW Immobilien, VW Coaching und Volkswagen AG, wie man Drachen baut und auf ihnen mit Tuschen und Pinseln Botschaften vermittelt. „Mit Verständnis und Toleranz können alle leben“, verkündet da ein Drache, ein anderer noch knapper: „Freiheit, Zeit, Leben“.

Von einer interessanten Erfahrung spricht auch Julian Schlichting. Der 22-Jährige muss sich in seiner Lehre mit verbundenen Materialien, auch mit Kautschuk befassen, hier sind es Lettern aus Blei und schwarze Tinte. (BRZ06/SEP.03828 Braunschweiger Zeitung, 07.09.2006; Diese Botschaften hängen an der Leine [Ausführliche Zitierung nicht verfügbar])

24) ((1; Komp.; A1))

Die Sozialdemokraten verlangen deshalb eine Vertretung des Konvents in der Rektorenkonferenz und die Wahl der Rektoren sowie Prorektoren durch den Mittelschulrat. Für ein solches Gremium wird sich auch die FDP einsetzen. Gerade weil in den Mittelschulen die Lehr- und Methodenfreiheit gross sei, brauche es entsprechende Kontrollen. Auch fördere ein solches Gremium die Weiterentwicklung der Strategie der Mittelschulen. «Reform ist steckengeblieben» (A11/MAI.10840 St. Galler Tagblatt, 30.05.2011, Nr. 125, S. 25. - Sachgebiet: Lokales, Originalressort: ostschweiz; Kaum Chancen für Mittelschulrat)

25) $[[0]]$

„Ich bin überzeugt davon, dass das Tier versehentlich eingesperrt worden ist. Wir haben sie nur zwei Häuser entfernt von uns gefunden, ihre Pfoten waren ganz sauber", berichtet Gudrun Thiele. Wie das Tier den Weg zurück in die Freiheit gefunden hat, wird wohl ein Geheimnis bleiben. Die Katzendame hat zweieinhalb Kilo Gewicht verloren. Jetzt wird Joselie liebevoll 
aufgepäppelt. (BRZ07/OKT.12840 Braunschweiger Zeitung, 16.10.2007; Von der großen Freiheit hat Katzendame Joselie genug [Ausführliche Zitierung nicht verfügbar])

26) ((1; attr. Adj.; A1))

Das Reich der Mitte hat sich Reformen verordnet, die mit einer zentralen Planung nicht lösbar sind. Der Trend geht zu mehr Pluralismus und mehr Teilhabe. Jedes Quäntchen an persönlicher Freiheit vereinnahmen die Menschen für sich. Sie fordern mehr Mitsprache. Aber ein Mehrparteiensystem steht nicht zur Debatte. (BRZ08/AUG.09793 Braunschweiger Zeitung, 21.08.2008; China sucht seinen Weg [Ausführliche Zitierung nicht verfügbar])

27) ((1; attr. Adj.; A1))

Ein Klassiker für eine Woche im Fokus - mit Eigenproduktionen, Gastspielen, Filmen, Vorträgen, Diskussionen - das ist ein Bildungsprogramm, mit dem das Theater anspruchsvoll und unterhaltsam seine Unentbehrlichkeit unter Beweis stellen kann.

Schauspieldirektorin Katja Ott und Dramaturg Johannes Blume skizzierten Kleist bei der Vorstellung des Programms als einen seinerzeit erfolglosen Außenseiter zwischen Klassik und Romantik. Als Dichter, der dem goethischen Humanismus misstraute und an der klassischen Vorstellung, die Welt könne an Vernunft und innerer Freiheit genesen, (ver)zweifelte.

„Kleist schildert das Scheitern der menschlichen Prozesse“, so Blume. „Seine Stücke handeln von der Nicht-Kommunikationsfähigkeit, vom Nicht-Zusammenkommen der Menschen.

(BRZ09/JAN.02591 Braunschweiger Zeitung, 08.01.2009; Ein poetischer Extremist [Ausführliche Zitierung nicht verfügbar])

28) $\{\{1+1$; Inf(satz); Subj.; A2; A1 $\}\}\{\{$ SVG; F. haben $\}\}$

Zu den Artikeln und Leserbriefen zum Thema Nichtwähler:

Der Leserbriefschreiber Stephan Schalow hat völlig Recht mit seiner Ansicht, dass es legitim sei, nicht wählen zu gehen. In einem demokratischen Gemeinwesen sollte ma- $-\underline{\text { na }}$ auch die Freiheit haben an Wahlen nicht teilzunehmen, aus welchen Gründen auch immer. Aber ich habe Schwierigkeiten mit dem Begriff „volksnahe Politik“. Was ist das? Jeder hat vermutlich eigene Vorstellungen darüber. (BRZ09/OKT.02633 Braunschweiger Zeitung, 07.10.2009; Die Wähler wollten Frau Reimann [Ausführliche Zitierung nicht verfügbar])

29) ((1; Komp.; A1))

Dafür sei der Markt zu stark reguliert. Dass die Versicherten jedes Jahr die Kasse wechseln können, führe ausserdem zu einem enormen Verwaltungsaufwand, sagt der ehemalige FDPPräsident. «Dieser Wettbewerb kostet uns 300 Millionen Franken pro Jahr.» Er würde es deshalb begrüssen, wenn die Wahlfreiheit zumindest eingeschränkt würde, so dass nur noch alle drei Jahre gewechselt werden könnte.

$\mathrm{Ob}$ er die Einheitskasse-Initiative befürwortet, lässt Steinegger offen. Er betont lediglich: «Im Bereich der Krankenversicherung kann man sicher von der Suva lernen - jedenfalls eher als umgekehrt.» (A14/AUG.08052 St. Galler Tagblatt, 25.08.2014, Nr. 196, S. 3.

Originalressort: Schweiz; d.wirth: Kann die Suva Vorbild sein?)

30) ((1; Posspr.; A1))

„Das ist ein Freudentag“, sagt der Priester. „Aber er enthält auch Traurigkeit. Es tut weh, dass so viele, die für unsere Freiheit kämpften, diesen Tag nicht erleben konnten.“

Ein beinamputierter Veteran, der noch einmal die alte Uniform aus dem Bürgerkrieg angezogen hat, nickt. Rund zwei Millionen Menschen starben in dem jahrzehntelangen Bürgerkrieg, der erst 2005 beendete wurde. (BRZ11/JUL.04613 Braunschweiger Zeitung, 
11.07.2011; Jubel in Juba: Endlich sind wir frei [Ausführliche Zitierung nicht verfügbar])

\section{1) $((0))$}

Seine ersten Rollen: allesamt B-Movies. Doch dann kam „Easy Rider“ (1969). Dieser epochale Film über Freiheit und Abenteuer, über das Lebensgefühl der 60er Jahre. Aber auch ein Film, der polarisierte, der Amerikas Gesellschaft den Spiegel vorhielt. Der Untertitel: „Ein Mann suchte Amerika, doch er konnte es nirgends mehr finden." Nicholson mimt darin kraftvoll den alkoholsüchtigen Anwalt George Hanson. (BRZ12/APR.09696

Braunschweiger Zeitung, 21.04.2012; Der Exzentriker [Ausführliche Zitierung nicht verfügbar])

\section{2) ((1; Komp.; A1))}

Genau diesen Verdacht hegen vor allem die unionsregierten Länder. Die Wunschlisten an den einzigen Ländervertreter im Konvent, den Thüringer Europaminister Jürgen Gnauck (CDU), sind denn auch lang. Ob Minderheiten- oder Familienschutz, Medienfreiheit, Asylrecht oder Umweltschutz - eine Forderung steht stets im Mittelpunkt: dass durch die Charta keine neuen Souveränitätsrechte auf die europäischen Organe übertragen werden.

Kein Problem, beschwichtigt Gnauck. (FOC00/SEP.00372 FOCUS, 18.09.2000, S. 060-062, Originalressort: DEUT/Deutschland; EU)

33) ((1; attr. Adj.; A1))

Anstatt als Geologe für die Deutsche Erdöl AG nach Nigeria zu gehen, startete er seine berufliche Karriere als Vertriebsassistent bei IBM. Der Branche ist er seitdem treu geblieben und sich selbst auch.

Unternehmerische Freiheit im Job motiviert ihn. Es macht ihm Spaß, Trends im Markt zu erkennen und zukunftsorientierte Strategien zu entwickeln. Da "die damalige hierarchische Organisation bei IBM wenig Gestaltungsraum zuließ", wechselte er als Leiter Vertrieb und Marketing zur Software AG. (C97/SEP.04043 COMPUTER ZEITUNG, [Wochenzeitung], 11.09.1997, Nr. 37, Jg. 32, S. 7. - ZUR PERSON, Dr. Volker Dietz, Tandem, [Bericht])

34) ((0))

Gut, sie müsste sich wohl aus gesellschaftlichen Gründen hinter einem Pseudonym verstecken. Den Zwang hatte ich nicht. Aber diese Freiheit habe ich mir durch meine Arbeit erkämpft. Ich bin mit anderen Partnern Co-Besitzerin der Zeitschrift "Art press". Wir haben etwa 30000 Leser, verdienen nicht viel, aber sind finanziell autonom. (FOC01/SEP.00096 FOCUS, 03.09.2001, S. 194-199, Sachgebiet: Mode, Originalressort: MODL/Modernes Leben; Eine öffentliche Frau)

35) ((1; Komp.; A1))

Aber insgesamt gesehen boomen die Busreisen mit allem Drum \& Dran. Ist man erst einmal eingestiegen, so braucht man sich wirklich um nichts mehr zu kümmern. Kein hektisches Kartenlesen und auf die richtige Autobahnabfahrt warten, genügend Beinfreiheit, um sich auch ausstrecken zu können, und ein deutschsprachiger Reiseleiter, der einem immer darauf hinweist, wo man gerade ist, und was es hier zu sehen gibt.

Wer fährt? Das Angebot wird hauptsächlich von älteren Menschen in Anspruch genommen. (K98/MAR.18955 Kleine Zeitung, [Tageszeitung], 11.03.1998. - Sachgebiet: Reise, Originalressort: Reise \& Ziele; Das Buspaket)

36) ((1; für + Akk.; A1)) 
Das gelingt nicht immer; manche Szenen wie die Stadtansicht des mittelalterlichen Hamburg wirken reichlich piefig. Dafür aber haben Szenenbildner Peter Menne und Kamerafrau Daniela Knapp sonst wunderbare Bilder und Ansichten geschaffen; oft in norddeutsches Blaugrau getaucht. Das Leben auf und an der Nordsee, wo die Freibeuter gern den Schlachtruf „Freiheit für Friesland“" anstimmen, ist nicht ganz so heiter wie bei den Kollegen aus „Fluch der Karibik“. Taddickens Film ist zudem ungleich bescheidener als die amerikanische Großproduktion mit Johnny Depp als Jack Sparrow. Doch „12 Meter ohne Kopf" hat durchaus einen rauen Charme. Und gegen ein freies Friesland ist ja nichts zu sagen. (HAZ09/DEZ.01328 Hannoversche Allgemeine, 10.12.2009; Freiheit für Friesland [Ausführliche Zitierung nicht verfügbar])

37) $\{\{0\}\}$

WEISHEIT DES TAGES

"Freiheit heißt Verantwortung. Deshalb wird sie von den meisten Menschen gefürchtet" George Bernard Shaw (1856-1950), irischer Schriftsteller (HMP07/JUN.01114 Hamburger Morgenpost, 11.06.2007, S. 40; WEISHEIT DES TAGES [Ausführliche Zitierung nicht verfügbar])

38) ((1; Gen.; A1))

Hohe Geldstrafen für Kunstexperten Moskauer Kuratoren entgehen dem Arbeitslager Menschenrechtler, Künstler und Museumschefs sind empört: In einem umstrittenen Prozess um die Freiheit der Kunst hat ein Moskauer Gericht den international renommierten Experten Andrej Jerofejew zu einer Geldstrafe verurteilt. Mit der Ausstellung "Verbotene Kunst" hätten Jerofejew und der Mitangeklagte Juri Samodurow 2007 zu "religiösem Hass aufgewiegelt", urteilte das Gericht gestern.

Jerofejew hatte in der Schau religiöse und politische Tabus in der russischen Kunstwelt thematisiert. (HMP10/JUL.01324 Hamburger Morgenpost, 13.07.2010, S. 18; Hohe Geldstrafen für Kunstexperten Moskauer Kuratoren entgehen dem Arbeitslager [Ausführliche Zitierung nicht verfügbar])

39) $\{\{1+1$; Gen.; Dat.; A2; A1 $\}\}\{\{$ SVG; Dat. bleibt die F. $\}\}$

Nie wird man das ganze Stück erleben, und dennoch ist es so geschickt gebaut, daß man, ein Minimum an Wissen über Alma vorausgesetzt, jederzeit einsteigen und sich von der Intensität der Szenen fesseln lassen kann. Das Totenmahl für Mahler wird als opulentes Pausenbüfett zelebriert, seine Musik erklingt zu den verschiedenen Lebensstationen seiner berühmten Witwe, die hier sehr liebevoll gezeichnet wird und groteske Züge nur in der Projektion ihrer berüchtigten Autobiografie "Mein Leben" annimmt.

Manker, der selbst den rabiaten Kokoschka spielt, hat aus Sobols poetisch-pittoreskem Bilderbogen eine Theateraufführung der anderen Art gemacht: spannend in der perfekten Erarbeitung der Szenen und zugleich entspannend, weil dem Bes Besucher die Freiheit des Schauens, Lauschens und Staunens bleibt. (I96/JUN.21852 Tiroler Tageszeitung, [Tageszeitung], 07.06.1996. - Sachgebiet: Kultur, Originalressort: Kultur; Frau Alma bittet zum Fest)

40) $[[0]]$

Für Aufregung sorgte eine Katze gestern nachmittag in Mayrhofen. "Minki" hatte sich den Motorraum eines abgestellten Autos freiwillig als Gefängnis ausgesucht. Das Tier kroch am Parkplatz vor einem Hotel unter den Wagen, gelangte unter die Motorhaube und fand den Weg nicht mehr zurück in die sichere Freiheit. Das laute Katzengejammere lockte viele Passanten an. "Das arme Tier muß doch gerettet werden", schlug die deutsche Urlauberin Lilo 
Brückner verzweifelt bei der Gendarmerie Alarm. (I98/AUG.34494 Tiroler Tageszeitung, [Tageszeitung], 29.08.1998. Originalressort: Regional Unterinntal; "Minki" steckte hilflos im Motorraum)

41) ((1; Posspr.; A1))

Dabei müsste der umstrittene Kulturbeauftragte und Kolumnist Andreas Mölzer selbst das größte Interesse daran haben, seines Auftrages ledig zu werden. Wirkt doch eine quasiamtliche Aufgabe auf die journalistische Freiheit wie ein Sarg. Und welcher Publizist will schon seine eigene Freiheit zu Grabe tragen? Klare Aufgabentrennung ist hier eine Frage der Kultur. (K00/APR.28362 Kleine Zeitung, [Tageszeitung], 08.04.2000. Originalressort: Lokal; Kulturschatten)

42) $\{\{1$; Komp.; A2 $\}\}$

Kulturfragen kann man nicht mit den Maßstäben des Binnenmarktes messen. Das ist eine Frage der nationalen Identität. Hier benötigen wir mehr Spielraum und mehr

Handlungsfreiheit. (K98/OKT.80514 Kleine Zeitung, [Tageszeitung], 24.10.1998.

Originalressort: Hintergrund; Klima: "Der Euro ist nicht das Ende der Entwicklung")

43) ((1; Komp.; A1))

Frau Hecht-Galinski sagte, sie werde sich äußern, wenn das Urteil schriftlich vorliegt. Sie erwägt Berufung. Weil es um Pressefreiheit geht, könnte das Verfahren bis zum

Bundesverfassungsgericht gehen.Das Gericht hatte unter anderem argumentiert, es sei üblich, daß für Ehrendoktorhüte Gegenleistungen erfolgten. Der Senat hatte der Bar-Ilan-Universität 1984100000 Mark für die Einrichtung eines Lehrstuhls zur Erforschung der Rolle der Juden in Preußen und Berlin gespendet. (L98/MAI.01611 Berliner Morgenpost, [Tageszeitung], 24.05.1998, S. 14. Originalressort: BERLIN; BM: Etappensieg im Streit um die unbotmäßigen Galinski-Worte)

44) ((0))

Drei Tage vor seinem 75. Geburtstag tummelte er sich ganz in Weiß und unter der Fallschirmherrschaft eines Profis im Himmel über Texas - eine schöne Bescherung. Aus 4000 Metern Höhe flog der Himmelsstürmer - und landete heil in den Armen zweier Helfer. Halb zog es ihn, halb sank er nieder: Ex-Präsident Bush überprüfte erneut, ob die Freiheit über den Wolken grenzenlos ist. Gattin Barbara (auf dem rechten Bild links) empfing ihn nach der glücklichen Landung mit einem Kuß. rtr (L99/JUN.31071 Berliner Morgenpost, [Tageszeitung], 11.06.1999, S. 8. Originalressort: POLITIK; BM: George Bush zum dritten Mal flügge)

45) ((1; attr. Adj.; A1))

darin predigte er die völlige Selbstgenügsamkeit des Staates, vor allem in wirtschaftlicher Beziehung. der Staat soll dafür sorgen, daß alle Bedürfnisse seiner Bürger durch inländische Produktion befriedigt werden. freie Berufswahl, wirtschaftliche Freiheit, Außenhandel, ja sogar private Auslandsreisen sollen verboten werden. von der Freiheit des einzelnen bleibt nicht mehr viel übrig. diese Ideen sind von der Staatsrechtslehre seiner Zeit und der unmittelbaren Nachwelt abgelehnt worden. (LIM/LI1.00454 Klimmnich, Otto: Deutsche Verfassungsgeschichte, (= Lehrbücher des Öffentlichen Rechts, Bd. 5). Frankfurt a.M.: Athenäum Verlag, 1970, S. 289-? (auszugsweise))

46) ((1; Gen.; A1)) 
Der Staat stellte mit seinen Beamten fest, welcher Artikel wie teuer sein durfte. "Ein Unding", meint Gabriel Bleser. Auch jetzt, vier Jahre nach dem Gesetz über die Freiheit des Marktes, ist Bleser davon überzeugt, dass in vielen Köpfen in Luxemburg noch die Reglementierung der Preise vorhanden ist.

Bleser war Co-Autor des Gesetzes, ist nach eigenen Worten erst spät zu diesem Projekt gestoßen. (LTB08/JUL.00304 Luxemburger Tageblatt, 10.07.2008, Sachgebiet: Wirtschaft, Originalressort: WIFI - Wirtschaft; Der Mann des Wettbewerbsrechtes)

47) ((1; Komp.; A1))

Die Erste Kammer des Ersten Senats nahm damit eine Verfassungsbeschwerde eines mit einem Tagungszentrum ausgestatteten Hotels gegen den Südwestrundfunk (SWR) nicht zur Entscheidung an. Das Hotel hatte die technische Sperrung der öffentlich-rechtlichen Programme für seine 114 Fernsehgeräte und eine Befreiung von der Gebührenpflicht verlangt. Nach den Worten der Richter enthält die im Grundgesetz garantierte Informationsfreiheit keine "Garantie kostenloser Information". Dieses Recht wäre nur dann verletzt, wenn die staatlich festgesetzten Entgelte so hoch wären, dass Interessenten von Informationsquellen fern gehalten würden. (Aktenzeichen: 1 BvR 1013/99) (M99/SEP.64870 Mannheimer Morgen, [Tageszeitung], 29.09.1999, Jg. 54. - Sachgebiet: Fernsehen / Hörfunk, Originalressort: Fernsehen und Hörfunk; Auch wer Privat-TV sieht, muss zahlen)

48) ((1; für + Akk.; A1))

Janukowitsch lässt zwei Polit-Häftlinge frei

Janukowitsch lässt zwei Polit-Häftlinge frei

"Freiheit für Julia!", schreien Tausende von Kehlen im Kiewer Schwetschenko-Park. Neben dem Porträt des überraschend am Morgen freigelassenen "orangen" Volkshelden Juri Luzenko halten sie Bilder von Julia Timoschenko in ihren blonden Haarkranz hoch. "Freiheit für Julia!", schreien Tausende von Kehlen im Kiewer Schwetschenko-Park.

(LTB13/APR.00284 Luxemburger Tageblatt, 08.04.2013, Janukowitsch lässt zwei PolitHäftlinge frei)

49) $[[0]]$

Angeklagt in elf Punkten des Betrugs, der Veruntreuung und der Urkundenfälschung, drohen dem Spekulanten theoretisch 14 Jahre Haft. Leeson pokert um seine Strafe. Er muß sich als "Bauernopfer" verkaufen, wenn er bald wieder in Freiheit sein will.

Zwar hat er recht eigenverantwortlich riesige Beträge auf die japanische Börse gesetzt und nach dem Kobe-Erdbeben alles verloren. Aber genaugenommen war der Derivatenhändler nur ein Buchhalter und für dieses komplizierteste aller Börsengeschäfte gar nicht qualifiziert.

(M95/511.30763 Mannheimer Morgen, [Tageszeitung], 24.11.1995, Jg. 50. - Sachgebiet: Wirtschaft, Originalressort: WIRTSCHAFT; Rainer Köhler: Luxus-Flug ins Kittchen)

50) $((0))$

Station macht sie in der Römerstadt bis zum 20. September.

In einer Art Rollenspiel wurde Hecker bei seiner Ankunft von keinem geringeren als

Bürgermeister Rolf Reble begrüßt, der den Abgeordneten des Wahlkreises

Weinheim/Ladenburg als den "Hoffnungsträger von vielen" in der Römerstadt willkommen hieß. "Er hat für uns alle, für Wohlstand, Bildung und vor allem die Freiheit gekämpft und gelitten", brachte ihm Reble seine Huldigung entgegen. Ganz so, wie wohl das damalige Stadtoberhaupt auch, als Heckers auf seiner Reise nach Mannheim einen kurzen Zwischenstopp in Ladenburg einlegte. 
Zurück in der Gegenwart zeigte sich Reble sehr erfreut über die Tatsache, daß die bereits am 27. Februar gestartete "ZeitZug"-Ausstellung anläßlich des 150. (M98/SEP.74678

Mannheimer Morgen, [Tageszeitung], 10.09.1998, Jg. 53. Originalressort: RheinNeckar; Von unserem Redaktionsmitglied Achim Horak: Nach 150 Jahren ist Hecker wieder da)

\section{1) ((1; Posspr.; A1))}

Heute setzt SAP weit über zehn Milliarden Mark im Jahr um und beschäftigt weltweit rund 22000 Mitarbeiter.

Tschira hat sich inzwischen wie sein Vorstandskollege Dietmar Hopp aus dem operativen Geschäft verabschiedet und begleitet jetzt die Geschicke des Konzerns im Aufsichtsrat. Seine neue Freiheit nutzt der Wissenschafts-, Kunstfreund und Bücher- wurm auf produktive Art: "Ich bin Stifter". Den größten Teil seines Vermögens steckte er in die 1995 gegründete gemeinnützige Klaus Tschira Stiftung (KTS). "Was ich verdiente", erzählt der gebürtige Freiburger, "wurde etwa zur einen Hälfte von der Einkommensteuer aufgefressen und die andere von der Vermögensteuer." (M01/MAR.16877 Mannheimer Morgen, [Tageszeitung], 09.03.2001, Jg. 56. Originalressort: Welt und Wissen; Von unserem Redaktionsmitglied Michael Schröder: Klaus Tschira legt die Hände nicht in den Schoß: Der SAP-Mitbegründer ist ein guter "Unruhe-Stifter")

\section{2) $((0))$}

Ein Begriff auf Messers Schneide also. In der Philosophiegeschichte ein Problem, das den Schritt vom schönen Schein zum wahren Mitgefühl ausmacht und die Moral auf den Plan ruft. Noch ganz nach Kant sieht Schiller zwar die sinnliche Überwältigung durch dargestelltes Leiden, doch auch - durch Kraft der eigenen Intellektibilität- die Möglichkeit zur Freiheit. Man leidet - kann sich durch Rückschlüsse und Taten aber davon befreien. Schopenhauer hatte das Mitleid später als letzte ethische Motivation zumindest noch in Betracht gezogen und der Menschheit Restwürde belassen, doch nicht nur Gott, auch der moralische Mensch ist seit Nietzsche tot, den Himmel hat er verloren, doch als ästhetischer Mensch die Erde gewonnen. (M03/JUN.39828 Mannheimer Morgen, [Tageszeitung], 18.06.2003, Jg. 58. Sachgebiet: Kultur, Originalressort: Kultur; Von unserem Mitarbeiter Ralf-Carl Langhals: Die Spaßgesellschaft leidet nicht gerne, [Feuilleton])

\section{3) ((1; Komp.; A1))}

Die neue Kultusministerin Nicola Beer (FDP) stellte sich hinter den Vorschlag des Ministerpräsidenten. „Wir sind immer für die selbstständige Schule gewesen. Und die Wahlfreiheit zwischen G8 und G9 ist eine Möglichkeit, diese Wahlfreiheit weiterzuentwickeln“, sagte sie. Eine Umstellung sei aber nach eingehender Prüfung frühestens für das Schuljahr 2013/2014 zu erwarten. „Wir werden hier kein Durcheinander machen. (M12/JUN.05982 Mannheimer Morgen, [Tageszeitung], 19.06.2012, Jg. 67, Südhessen Morgen (Lampertheim), S. 5. - Sachgebiet: Regionales, Originalressort: Hessen; Bouffier pocht auf Wahlfreiheit, [Bericht [Bericht ; Nachrichten]])

\section{4) $((0))$}

Pressereise auf Schillers Spuren

In Schillers Namen wird die Freiheit gefeiert. Wie sehr die Parole des Nationaltheaters in Mannheim gilt, erfuhren am Wochenende 15 Journalisten aus ganz Deutschland bei einer Reise auf den Spuren des Dichters auf Einladung der Tourismus-Marketing Gesellschaft Baden-Württemberg mit Blick auf das kommende Gedenkjahr zu Friedrich Schillers 200. 
Todestag am 9. Mai. (M04/SEP.67087 Mannheimer Morgen, [Tageszeitung], 27.09.2004, Jg. 59. Originalressort: Mannheim; Pressereise auf Schillers Spuren)

55) $\{\{0\}\}$

Tilla lernte er in einem kalten Winter beim Schlittenfahren auf dem nahe gelegenen Kohlhof kennen: "Es war Liebe auf den ersten Blick." Sie war 18 Jahre jung und er keine 20 Jahre. "So viel Freiheit wie heute gab es zu unserer Zeit nicht", mischt sich die Braut ein. "Wir Mädels haben die Schlitten an die der Jungen festgemacht, und weil für mich kein Platz mehr war, habe ich mich auf seinen Rücken gesetzt", lacht sie und sieht ihn dabei verliebt an. Mit der Hochzeit warteten sie nicht allzu lange. (M05/JUN.50538 Mannheimer Morgen, [Tageszeitung], 21.06.2005, Jg. 60. Originalressort: Rhein-Neckar / Bergstraße; Goldene Hochzeit im Hause Pröll)

56) $((0))$

Die Gesellschaft in Deutschland braucht nach Überzeugung des Freiburger Erzbischofs Robert Zollitsch eine gemeinsame Vision, um die Herausforderungen zu bestehen. Dazu gehöre eine "Kultur der Achtsamkeit", die auch "für die oft leisen Töne des Guten und Segensreichen in unserem Alltag hellhörig werde", sagte der Oberhirte gestern in Freiburg beim Neujahrsempfang für die Vertreter von Kirche, Politik und des öffentlichen Lebens. Zollitsch äußerte jedoch die Befürchtung, die Gesellschaft drifte auseinander, weil sie sich zu wenig mit den tragenden Säulen von Demokratie und Freiheit beschäftige. Nur wer wisse, wofür und woraufhin er spare, sei zu Einschränkungen bereit; die Politiker seien jedoch zu sehr damit beschäftigt, Lasten zu verteilen, "statt aus einem großen überzeugenden Konzept die Sehnsucht nach einer Gesellschaft in Frieden, Solidarität und Gerechtigkeit lebendig zu halten". Ob es uns gut gehe und wir uns glücklich fühlten, sei eine Frage der Perspektive, weniger des Besitzens und Habens, betonte Zollitsch. (M06/JAN.02435 Mannheimer Morgen, [Tageszeitung], 11.01.2006, Jg. 61. - Sachgebiet: Regionales, Originalressort: Südwest; Furcht vor Zerfall der Gesellschaft)

57) $((1 ;$ Gen.; A1))

Dabei macht er sich geschickt die neuen Möglichkeiten zunutze, die das Zeitalter der elektronischen Kommunikation bietet. Ob Maut-Daten, Passfotos oder eingescannte Fingeabdrücke: Die Staatsgewalt, suggeriert Schäuble, der von 1989 bis 1991 unter Helmut Kohl schon einmal Innenminister war, muss im Kampf gegen den Terror oder bei schweren Verbrechen einen schnellen und möglichst ungehinderten Zugriff auf Informationen haben. Deshalb beschneide der Rechtsstaat, wie er ihn sich vorstelle, auch nicht die Freiheit des Einzelnen. Im Gegenteil: "Der Rechtsstaat bemüht sich, die Freiheit zu schützen." Zwei Reportern des "Stern", die ihm vor kurzem entrüstet vorwarfen, er wolle das Land in einen Hochsicherheitstrakt verwandeln, entgegnete er ruhig: Der nächste Anschlag in Deutschland sei keine Frage des Ob, sondern nur noch eine Frage des Wann.

(M07/APR.03902 Mannheimer Morgen, [Tageszeitung], 19.04.2007, Jg. 62, Stadtausgabe. - Sachgebiet: Politik, Originalressort: Politik; Von unserem Korrespondenten Rudi Wais: Auf dem Kreuzzug gegen den Terror)

58) $((0))$

Könnte sie heute auf die Hilfe ihrer Mitschüler, der Nachbarskinder, der Freunde ihrer Eltern hoffen? „Erkennt die Gesellschaft heute die Gefahren des Faschismus?“ Die Ausstellung will keine Antworten sondern Anstöße geben und auch das Heute auf den Prüfstand stellen, wie Thomas Heppner, Direktor des Anne-Frank-Zentrum Berlin, in seiner Ansprache verdeutlichte. Demokratie, Freiheit, Menschenwürde müssten immer wieder von Neuem 
verteidigt werden, wie die Ereignisse vom Juni 2006 in Sachsen-Anhalt zeigten. Der „Heimatbund Ostelbien“ veranstaltete dort ein gespenstisches Sommersonnwend-Spektakel. Junge Neonazis verbrannten während einer Feuerzeremonie ein Exemplar des Tagebuchs von Anne Frank. (M08/JAN.04537 Mannheimer Morgen, [Tageszeitung], 18.01.2008, Jg. 63, Stadtausgabe, S. 19. - Sachgebiet: Lokales, Originalressort: Mannheim; Von unserem Redaktionsmitglied Susanne Räuchle: Grauen zeigt sich auf Knopfdruck, [Bericht [Bericht ; Nachrichten]])

59) $((0))$

Friedrich Merz, der profilierteste Unionskopf in Wirtschafts- und Finanzdingen, ist weg und niemand ist in der Lage, diese Leere zu füllen. Von Stuttgart wird kein Signal ausgehen, inhaltlich hat die CDU nichts zu bieten, Wohltaten sollen erst im Wahlprogramm im nächsten Sommer verkündet werden. Im Zeichen der Krise deutet sich dagegen eine erste inhaltliche Diskrepanz zwischen der CDU-Chefin und ihrer Partei an: In ihrem Herzen ist Merkel noch immer die Reformerin des Leipziger Parteitags, sie setzt auf die Kraft der Freiheit und die Freiheit des Marktes. Für die CDU hingegen war Leipzig nur Mittel zum Zweck, um Schröder vorzuführen. Nun erschallt auch in der Union wieder der Ruf nach dem starken Staat, der Schutzschirme spannt und Risiken abfängt. (M08/DEZ.93298 Mannheimer Morgen, [Tageszeitung], 01.12.2008, Jg. 63, Stadtausgabe, S. 2. - Sachgebiet: Politik, Originalressort: Politik; In der Defensive, [Bericht [Bericht ; Nachrichten]])

60) $((0))$

„Vielleicht werden ich und meine Freunde heute nicht mehr heimkehren. Doch wir sind bereit, unser Leben der Demokratie zu opfern." Solche und ähnliche Sätze dringen über Facebook aus dem Iran in die freie Welt. Wie jener Schreiber, der sich selbst für „die Freiheit und meine Rechte“ opfern will, trotzten am Wochenende Tausende Iraner der ungeheuerlichen Drohung des Geistlichen Führers Ajatollah Ali Chamenei, der in einer schicksalhaften Rede den Sicherheitskräften, den Revolutionsgarden und vor allem der paramilitärischen Bassidsch-Miliz grünes Licht zur direkten Attacke auf friedlich demonstrierende Menschenmassen gegeben hatte.

Und die Prügeltruppen der iranischen Führung schlugen von Motorrädern brutal zu, nachdem es Tausenden Polizisten nicht gelungen war, den Menschenstrom, der den Drohungen trotzte und seinen Frustrationen Luft zu machen suchte, vollends zu zerschlagen.

Chamenei scheut kein Blut, um seine Macht abzusichern. (M09/JUN.47955 Mannheimer Morgen, [Tageszeitung], 22.06.2009, Jg. 64, Stadtausgabe, S. 3. - Sachgebiet: Politik, Originalressort: Politik; Von unserer Korrespondentin Birgit Cerha, [Bericht [Bericht ; Nachrichten]])

\section{1) $((0))$}

Hinter dem neuen Namen steckt auch ein neues Gesamtkonzept, deshalb wurde aus dem bisherigen „Gesundheitszentrum Olymp“ in der Industriestraße 39a nun „My life“. Am Wochenende feierte die frisch renovierte Einrichtung mit vielen Attraktionen ihre Neueröffnung.

Mit dem vor über 20 Jahren eröffneten „Olymp“ hätten viele Kunden gedanklich noch das reine Krafttraining verbunden, doch das Angebot beinhalte inzwischen sehr viel mehr, begründete Geschäftsführerin Simone Schmidt gegenüber dem „Südhessen Morgen“ den Namenswechsel. „Gesundheit erhalten, Freiheit genießen, Ausgleich und innere Ruhe finden", laute deshalb nun die neue Philosophie.

Neues Vacu-Step-Gerät 
Neben dem großen Gerätepark, zu dem sich neu ein Vacu-Step-Gerät gesellte, gibt es auch ein vielfältiges Kursangebot, das von Selbstverteidigung über Aerobic bis zu

Präventionsprogrammen reicht. (M10/MAI.37102 Mannheimer Morgen, [Tageszeitung], 17.05.2010, Jg. 65, Südhessen Morgen (Lampertheim), S. 18. - Sachgebiet: Lokales, Originalressort: Lampertheim; Entspannung in der Saunalandschaft, [Bericht [Bericht ; Nachrichten]])

62) $((0))$

Überzeugt davon, dass „kein Künstler jemals das Monopol über die Vollendung seines Kunstwerks beanspruchen dürfe“, lädt der Bildhauer sein Publikum ein, die Gestalt „mit Schlägen, Tritten, Werkzeugen oder, falls notwendig, sogar mit Waffen" in die perfekte Form zu bringen. Nach einer angemessenen Frist der Scheu machen sich die Menschen grimmig im Dienste der Kunst ans Werk. Abends, nach Büroschluss, zieht auch Lea los, um hemmungslos zuschlagend „ein besserer Mensch zu werden“. Setz' Figuren sehnen sich nach Freiheit, deren Grenzen sie nicht kennen. Der Autor versteht es, beim Leser Mitgefühl für seine verzweifelten Helden hervorzurufen, die nur eines wollen: glücklich sein. Das Buch ist im Suhrkamp Verlag erschienen (350 S., 19,90 Euro). (M11/APR.00007 Mannheimer Morgen, [Tageszeitung], 01.04.2011, Jg. 66, Stadtausgabe, S. 28. Originalressort: Bücher, [Feuilleton [Bericht ; Feuilleton]])

63) ((1; Posspr.; A1))

Allerdings sind Symptome schon vor Jahrhunderten beschrieben worden - beispielsweise in einem Buch, das der flämische Arzt und Philosoph Joostens anno 1561 veröffentlichte. Und zwar mit dem anschaulichen Titel: „Über das Würfelspiel oder die Heilung der Leidenschaft, um Geld zu spielen“. Dass Menschen Glück erzwingen wollen und sich dabei Unglück einhandeln, ist schon aus der Antike überliefert. Jedenfalls notierte Tacitus, dass unsere Vorfahren, die Germanen, beim Würfeln „die Leidenschaft im Gewinnen und Verlieren“ derart „hemmungslos“ betrieben hätten, dass manche Hab und Gut - und damit auch ihre Freiheit - verspielten. Beim Würfeln blieb es bekanntlich nicht. Wie jeder weiß, hat der Kick um Glück und Geld so manch eine Erfindung initiiert: Von der zum Rotieren gebrachten Unberechenbarkeit des selbstherrlichen Zufalls, Roulette genannt, bis zum ,einarmigen Banditen". Übrigens war es ein in die USA ausgewanderter schwäbischer Maschinenbauer, der Ende des 19. Jahrhunderts der „slot machine“ mit Hebelarm und Münz-Schlitz zum Siegeszug verhalf. (M11/DEZ.02200 Mannheimer Morgen, [Tageszeitung], 07.12.2011, Jg. 66, Stadtausgabe, S. 15. - Sachgebiet: Lokales, Originalressort: Mannheim, [Bericht [Bericht ; Nachrichten]])

64) $((1 ;$ Gen.; A1))

Unerwartetes geschieht, vielleicht auch Ungewolltes. Ich muss Ja sagen zur Veränderung, zur Verwandlung und in zuerst zum Leben selbst.

Und die Freiheit des Geistes gehört dazu. Mit „Kleingeisterei“ ist noch nie ein Aufbruch gelungen, jedenfalls nicht nachhaltig.

Vielleicht geht es auch zunächst einfach um ein Sich-Öffnen in eine neue Lebendigkeit:

„Öffne Dich! (M12/JAN.03964 Mannheimer Morgen, [Tageszeitung], 14.01.2012, Jg. 67, Stadtausgabe, S. 0. - Sachgebiet: Weltwissen, Originalressort: Welt und Wissen; Aufbruch wagen, [Bericht [Bericht ; Reportage]])

65) ((1; Posspr.; A1)) 
„Die Freiräume werden enger. Ist es uns das Wert“, fragte Stadtverordnetenvorsteher Dr. Jörn Ritterbusch. Auch wenn er grundsätzlich dazu bereit sei, ,setzen wir die Unterschrift unter ein Dokument, das unsere Freiheit als Parlamentarier und Bürger einschränken wird.“ „Vorstadium einer Insolvenz“

Allerdings, so Kämmerei-Leiter Herbert Scholz und Bürgermeister Baaß, habe das RP über die Kommunalaufsicht des Kreises Bergstraße auch jetzt schon die Möglichkeit, in den Viernheimer Haushalt einzugreifen. (M13/JAN.05762 Mannheimer Morgen, [Tageszeitung], 19.01.2013, Jg. 68, Südhessen Morgen (Viernheim), S. 17. - Sachgebiet: Lokales, Originalressort: Viernheim; Von unserem Redaktionsmitglied Bertram Bähr: Minister billigt Viernheims Sparvorschläge, [Bericht [Bericht ; Nachrichten]])

66) ((1; attr. Adj.; A1))

Philosophiekritik

Antiidealistisch war er gewiss gesinnt, und ebenso wäre seine Kunstauffassung zu charakterisieren. Die Gedankenübungen der idealistischen Philosophie, von Fichte, Schelling, Hegel, das Denken also, das sich auf sich selbst bezieht und nicht auf Wirkliches reagieren, sondern es prägen und erst wahrhaft schaffen soll, als vollendeter Beweis menschlicher Freiheit und Handlungsmacht - Büchner macht sich darüber lustig: In „Leonce und Lena“ sieht der denkende König Peter sein System durcheinander geraten, weil er sich beim Ankleiden mit dem Zuknöpfen vertan hat. Und Prinz Leonce nennt es tatsächlich ein Ideal, sich selbst auf den Kopf zu sehen. Büchner, den Mediziner, der seine Dissertation über das Nervensystem der Fische schrieb, schlicht einen Materialisten zu nennen, der allein Stoffliches für wirklich hält, wäre indes gleichfalls voreilig. (M13/OKT.06985 Mannheimer Morgen, [Tageszeitung], 23.10.2013, Jg. 68, Stadtausgabe, S. 27. - Sachgebiet: Kultur, Originalressort: Kultur; Von unserem Redaktionsmitglied Thomas Groß: Leben schaffen, dann ist\&\#8217;s gut, [Feuilleton])

67) ((1; attr. Adj.; A1))

Von unserem Mitarbeiter Alfred Huber

Zum enthusiastischen Jubeln gab es bei der Uraufführung 1824 in Wien eigentlich wenig Grund. Die Napoleonischen Befreiungskriege waren längst beendet, doch die erhoffte persönliche Freiheit hatten sie den Bürgern weder in Preußen noch in Österreich gebracht. Statt dessen kam es, geschürt von den Revolutionsängsten der Herrschenden, zu jenen Karlsbader Beschlüssen von 1819, die beide Länder gewissermaßen in Polizeistaaten verwandelten. Vorbei somit all die revolutionären Hoffnungen, denen Schiller noch in seiner um 1785 verfassten Ode „An die Freude“, diesem „Primärdokument für die künftige Politik der Begeisterung“ (Peter Sloterdijk), gehuldigt hatte. (M14/JAN.00455 Mannheimer Morgen, [Tageszeitung], 03.01.2014, Jg. 69, Stadtausgabe, S. 27. - Sachgebiet: Kultur, Originalressort: Kultur; Von unserem Mitarbeiter Alfred Huber: Aufbruch ins Gelobte Land, [Feuilleton])

68) $((0))$

Dass seine mahnenden Worte in der Gegenwart noch immer aktuell sind, zeigt der aktuelle Freiheitsindex, den das John-Stuart-Mill-Institut für Freiheitsforschung gestern vorgestellt hat.

Wertschätzung sinkt

Denn, so das Résumé des 46 Seiten umfassenden Papiers: Die Wertschätzung der Freiheit in der Bevölkerung ist leicht gesunken. In diesem Jahr ging das Heidelberger Institut zum vierten Mal der Frage nach, ob den Bürgern Selbstbestimmung oder Sicherheitsbedürfnis stärker am Herzen liegen. Bei der Beantwortung dieser Frage half unter anderem das Institut 
für Demoskopie Allensbach, das insgesamt mehr als 1500 Personen interviewte.

(M14/OKT.01995 Mannheimer Morgen, [Tageszeitung], 08.10.2014, Jg. 69, Stadtausgabe, S. 4. - Sachgebiet: Politik, Originalressort: Politik; Von unserem Redaktionsmitglied Julia Lauer: Freiheit kontra Sicherheit, [Bericht ; Nachrichten])

69) ((1; attr. Adj; A1))

es gibt nicht die allgemeine Freiheit, sondern diese nur in ihren geschichtlichen Abwandlungen. Freiheit ist noch nicht, wo der Form nach eine sogenannte Demokratie als Organisationsform einer Bevölkerung auferlegt wird, die gar nicht weiß, was Freiheit ist, und nur unter deren Namen faktisch diktatorisch und, auf der Stufe des technischen Zeitalters, gar totalitär regiert wird. politische Freiheit ist nicht identisch mit abendländischer Freiheit, sondern ihre Bedingung. wo politische Freiheit preisgegeben wurde, da scheint uns auch die Freiheit überhaupt, Europa und das Abendland preisgegeben. Europa hat die Freiheitsidee in seiner Wurzel. (MK1/WJA.00000 Jaspers, Karl: Die Atombombe und die Zukunft des Menschen. Politisches Bewußtsein in unserer Zeit, [Sachbuch]. München: R. Piper \& Co Verlag, 37.-44. Tausend 1962 (1. Auflage 1958), 505 S. [S. 134])

70) $((0))$

Peter Fischer,

Völkerrechtler in Wien, sagte, die Vorgangsweise der 14 EU-Staaten widerspreche dem Geist des Amsterdam-Vertrags. Dieser sehe zwar Sanktionen gegen einzelne Mitglieder vor, aber nur, wenn sich diese "schwere und anhaltende Verletzungen" der Prinzipien von Freiheit, Achtung der Menschenrechte, Demokratie und Rechtsstaatlichkeit schuldig machen. Darüber hinaus müsste die österreichische Regierung im Rahmen eines Verfahrens zu einer Stellungnahme aufgefordert werden, was nicht passiert sei. Martin Nettersheim, (N00/FEB.04490 Salzburger Nachrichten, [Tageszeitung], 02.02.2000. Originalressort: Kopf Story; EXPERTEN EINIG)

71) $((0))$

Ideale, die wenig mit der Wirklichkeit zu tun haben

Das sieht "Betty" (Sonja Romei) ähnlich: "Im Vordergrund der Geschichte stehen für mich die Träume, denen die beiden Frauen nachjagen; Ideale und Idole, die ziemlich wenig mit der Wirklichkeit zu tun haben. So besitzen sie eine völlig utopische Vorstellung von Amerika als Schlaraffenland, als Inbegriff von Freiheit, ohne sich zu überlegen, was sie dort tatsächlich erwarten würde, denn so weit wird nicht geträumt, weil das nicht wichtig ist. Es geht darum, in dieser künstlich geschaffenen Seifenblase, in der alles glitzert, zu leben."

"Ternitz Tennessee" ist nach den Kurzfilmen "Speak Easy" (1997 ebenfalls im Programm der Viennale) und "Mehr oder weniger" (1999) der erste abendfüllende Film von Mirjam Unger. (N00/OKT.48255 Salzburger Nachrichten, [Tageszeitung], 19.10.2000. - Sachgebiet: Kultur, Originalressort: Kultur; Tennessee, südlich von Wien)

72) ((1; Gen.; A1))

Die heimischen Gewerkschaften sind sich auch im klaren darüber: Die Teilnahme am EWR ist ein Schritt Richtung EG-Beitritt. Erst dort wird Österreich in allen Fragen gleichberechtigt mitbestimmen können.

Im Zuge der Freiheit des Personenverkehrs wird es möglich sein, innerhalb des EWR Arbeit zu suchen, ohne gegenüber den jeweils Ortsansässigen benachteiligt zu werden. Ein übermäßiger Zustrom ausländischer Arbeitnehmer aus dem Titel EWR nach Österreich ist nicht zu erwarten; übrigens arbeiten jetzt schon mehr Inländer im Ausland als umgekehrt. 
Es wird also für österreichische Unternehmen leichter, eigenes Personal in EG-Filialen mitzunehmen. (N91/OKT.17700 Salzburger Nachrichten, [Tageszeitung], 28.10.1991. Internationaler Wettbewerb schafft Arbeitsplätze Grenzkontrollen:)

73) ((1; Gen.; A1))

Ab 1. Jänner 1993 soll sie endlich kommen, die große Freiheit des schrankenlosen Handels. Waren aller Art sollen zwischen EG-Ländern die Grenzen passieren können - ohne Zölle, ohne Kontrollen, ohne Papierkram. Doch noch bevor diese große Freiheit des Binnenmarktes Wirklichkeit wird, gibt es bereits Ausnahmen: für manche Produkte wird der Handel zwischen EG- Ländern eingeschränkt, wenn nicht sogar ganz verboten. So beschlossen die Umweltminister der EG am Dienstag eine Verordnung über die "Verbringung von Abfällen", wonach Müll - voraussichtlich ab 1994 - nicht mehr als Handelsware oder Wirtschaftsgut betrachtet werden darf (siehe Seite 14). Müll unterliegt somit nicht dem Handelsrecht, sondern dem Umweltrecht. (N92/OKT.39316 Salzburger Nachrichten, [Tageszeitung], 22.10.1992. - Grenzen dicht für Müll und Kunst!)

74) ((1; Gen.; A1))

Er glaubt, daß eine solche Maßnahme eine Qualitätsverbesserung nach sich ziehen könnte. Ein Verbot des Verkaufs unter dem Einstandspreis gab es in Österreich bereits Ende der achtziger Jahre. Im Herbst 1990 wurde dieses im § 3a des Nahversorgungsgesetzes festgelegte Verbot allerdings vom Verfassungsgerichtshof aufgehoben, weil dieser gegen die Freiheit der Erwerbsausübung verstoße. Schon damals hatte Schüssel angekündigt, das Gesetz reparieren zu wollen. Seine Vorstöße scheiterten allerdings bisher immer am Koalitionspartner SPÖ. (N93/AUG.30550 Salzburger Nachrichten, [Tageszeitung], 24.08.1993. - Lebensmittelhändler für Schleuderverbot:)

75) $((0))$

Sitzstreik in Salzburg, Mahnwache in Graz - Botschaft der Inhaftierten an Unterstützer SALZBURG, GRAZ (SN).

Durch einen Besuch ihres Anwaltes Gerhard Mory haben die vier in Salzburg in Schubhaft festgehaltenen Kosovo-Albaner am Samstag von jenem Sitzstreik erfahren, der seit Freitag unter dem Motto "Freiheit statt Schubhaft" vor der Polizeidirektion in der Alpenstraße abgehalten wird. "Wir finden das ganz nett von Ihnen", ließen die Schubhäftlinge in einer an Mory überreichten Botschaft wissen. Namentlich bedankte sich das Quartett bei Anwalt Mory, Pfarrer Heinrich Wagner und Erzbischof Georg Eder sowie "amnesty international" (ai). (N94/MAI.18010 Salzburger Nachrichten, [Tageszeitung], 16.05.1994. - KosovoAlbaner: Höchstgerichte befaßt)

76) ((1; Gen.; A1))

Ein Gespräch mit dem Schriftsteller.

Aber anders als Rushdie ist der türkische Schriftsteller nicht in den Untergrund gegangen. Er tritt weiter in der Öffentlichkeit auf, verteidigt unerschrocken die Freiheit des Worts wie bei der Solidaritätsveranstaltung "1000 Tage im Ballon" Anfang dieser Woche im Salzburger Landestheater. Er ist froh, daß er an diesem Abend hat teilnehmen können, und er wünscht sich, daß solche Veranstaltungen für die weltweit wachsende Zahl verfolgter Autoren auch in anderen westeuropäischen Ländern und in der Türkei stattfinden.

"Ich lebe ständig unter dem Schutz der Polizei", erzählt er im SN-Gespräch.

(N95/MAR.10347 Salzburger Nachrichten, [Tageszeitung], 18.03.1995. - Kultur-Kampf in der Türkei Islamischer Fundamentalismus oder) 
77) $((0))$

"Eine Überzeugung, über die man nachdenkt und für die man argumentieren will, verliert ipso facto ihre Selbstverständlichkeit." Relativierung zwinge zum Wählen. Für manche bedeute dies Freiheit, andere erlebten dies als Entfremdung, Absturz und Vereinsamung. Leben in verschiedenen Kulturen werde oft mit Toleranz bewältigt. Allerdings könnte diese in Nihilismus übergehen: "Alles wird akzeptabel." (N97/JUL.27155 Salzburger Nachrichten, [Tageszeitung], 04.07.1997. - Sachgebiet: Gesundheit / Umwelt / Wissenschaft, Originalressort: Gesundheit / Umwelt / Wissenschaft; Was ist ein Wiener, der Richtung Mekka betet?)

78) $\{\{0\}\}$

f.).Ab 19. Februar 1999 dürfen 75 Industriefirmen (ab 40 Mill. kWh Jahresbedarf) ihre Stromlieferanten EU-weit frei wählen. Diese Freiheit droht aber an prohibitiven Durchleitungstarifen zu scheitern: Sie sind im Extremfall doppelt so hoch wie in Bayern. Erich Haas, Österreich-Repräsentant der Bayernwerk AG, schätzt die Mehrkosten der 75 Unternehmen aus diesem Titel auf 200 bis 300 Mill. S pro Jahr. (N98/DEZ.51575 Salzburger Nachrichten, [Tageszeitung], 30.12.1998. - Sachgebiet: Wirtschaft, Originalressort: Wirtschaft; "Stromwettbewerb wird von Amts wegen ausgeschaltet")

79) ((1+1; Posspr.; Dat.; A1; A1)) ((SVG; Dat. F. lassen))

Ein Land - zwei Systeme

China lässt 므ongkong - mit Abstrichen - tatsächlich seine Freiheit. Der Verwalter Tung Chee Hwa benimmt sich dafür wie ein Pekinger Vize- Minister.

VIKTOR HERMANN Den letzten Termin auf dem Boden der alten Volksrepublik China in Shanghai hatte einem Multimillionär gegolten, einem 41-jährigen Selfmademan, der zwar ein höflicher, umgänglicher Mensch geblieben war, aber es sichtlich genoss, mit seinem RollsRoyce Eindruck zu schinden. (N99/DEZ.53829 Salzburger Nachrichten, [Tageszeitung], 17.12.1999. Originalressort: Kopf Story; Ein Land - zwei Systeme)

80) $[[0]]$

Illig berichtet auch von einem Projekt auf polnischer Seite, demzufolge

Wölfe ausgewildert werden sollen. Diese brauchen aber große zusammenhängende

Waldgebiete für ein Leben in Freiheit. (NKU00/SEP.09739 Nordkurier, 29.09.2000, Sachgebiet: Lokales, Originalressort: Lokales Ueckermnde; Verschiedene Meinungen zur Grenzöffnung)

81) $[[0]]$

Was es da so in der Nacht anstellt, beweist der Käfig, der schon mehrmals verstärkt werden musste. Allzu gern ist Gismo in Freiheit und daher sind Ausbruchversuche keine Seltenheit. Mit Hilfe seiner kleinen, aber kräftigen Zähne nagt er bevorzugt Flucht-Löcher. Wenn Gismo draußen ist, geht er sogar an Kabel und Hauswände. (NKU01/SEP.01444 Nordkurier, 05.09.2001, Sachgebiet: Lokales, Originalressort: Lokales Prenzlau; Gismo weckt junges Ehepaar jede Nacht)

82) ((1; Gen.; A1))

Herr zu werden. Ob die Verordnung jedem Einzelfall gerecht wird, darüber lässt sich trefflich streiten. Wie über jedes Gesetz, denn die haben es so an sich, dass sie die Freiheit des Einzelnen eingrenzen. Aber wo die Vernunft endet, muss der Staat regulierend eingreifen sonst brauchten wir ihn nicht. Mehr als unglücklich ist es indes, wenn Gesetze und 
Verordnungen nicht bis (NKU02/APR.02175 Nordkurier, 08.04.2002, Sachgebiet: Lokales, Originalressort: Lokales Usedom; Gewissensfrage)

83) $[[0]]$

Die Blinker sind im Kühlergrill eingebaut. Wenn sich übers Aussehen auch streiten lässt, so haben die runden Formen einen wichtigen Vorteil: den Reisenden steht trefflich Platz in diesem Kleinwagen zur Verfügung. Selbst wenn Fahrer und Beifahrer größer sind als 1,75 Meter, gibt es nach oben und zur Seite hin ausreichend Freiheit. Auch die Beine können so richtig lang gemacht werden. Und selbst bei weit nach hinten gestellten Vordersitzen drücken die Knie der Hinterbänkler nicht in die Vorderlehnen. (NKU04/OKT.00696 Nordkurier, 02.10.2004, Originalressort: Verkehr; Knuddeliger Flitzer mit beachtlichem Platzangebot)

84) $((0))$

Weltoffene Menschen auf beiden Seiten haben kaum die Möglichkeit, zusammenzukommen: "Palästinenser dürfen nicht ohne triftigen Grund nach Israel, und nur wenige meiner israelischen Freunde haben sich trotz Angst vor Anschlägen nach Beit Jala gewagt." Esther Heinke wollte den Menschen vor Ort helfen. Ein gutes Sprachdiplom in deutscher Sprache erhöht die Chance, ein Studium in Deutschland beginnen zu können, das weltweit anerkannt ist und ein Stück Freiheit bedeutet. Die junge Deutsche hofft, die Kinder von der Talitha Kumi dem ein wenig nähergebracht zu haben. Einen Markt mit Teppichen, Seide und Gewürzen wie in 1001 Nacht konnte Esther Heinke nicht durchstöbern. (NKU07/JAN.00835 Nordkurier, 05.01.2007, Deutschunterricht im Krisengebiet)

85) $((0))$

Man müsse sich also auch immer fragen, was uns unfrei macht, gefangen nimmt. "Vielleicht ist es für mehr Menschen, als wir oft wahrnehmen, die Sorge um die äußere Existenz - die schlichte Sorge, über die Runden zu kommen. Da wird dann die Frage nach der Freiheit leicht zu einem Luxus, den man sich gar nicht leisten kann", so Meckel. Eingeengt sein könne man aber auch in Arbeit und Pflichten. Aus dieser Perspektive werde Freiheit leicht zum Traum. (NKU07/NOV.00611 Nordkurier, 02.11.2007, Frage nach der Freiheit erneut gestellt)

86) $((0))$

Verschiedene Fragen werden dabei aufgeworfen: Wo liegen die Ursachen? Wie soll man jugendlichen Gewalttätern gegenübertreten? Wie jugendlicher Gewalt vorgebeugt und sie wirksam bekämpft werden kann ist das Thema bei dem Schülerwettbewerb "Aufhören!" Freiheit lernen, Gewalt verhindern - der Friedrich-Naumann-Stiftung. Schüler ab der 10. Klasse können bis zum 30. November alleine oder in Gruppen Beiträge einreichen. Alles von Texten, Karikaturen und Postern über Rollenspiele und Videos bis hin zu virtuellen Projekten ist erlaubt. (NKU08/SEP.06505 Nordkurier, 23.09.2008, Den Ursachen von Jugendgewalt auf der Spur)

87) $((0))$

Aber nun besteht die Gefahr, dass wird den aufrechten Gang wieder verlernen. Denn wir erleben jetzt auch, wie im Gegenteil die missverstandene und missbrauchte Freiheit, die keine Grenzen mehr respektieren will, uns in neue Zwänge führt. Paulus schreibt in dem Briefe an die Galater: So besteht nun in der Freiheit, zu der uns Christus befreit hat aber sehet zu, dass ihr durch die Freiheit nicht dem Fleisch (d.h. der zügellosen Gier) Raum gebt (Gal. 5,1 +13). Hier wird die Wurzel der gegenwärtigen weltweiten Wirtschaftskrise aufgedeckt. Die 
missbrauchte Freiheit verkehrt sich in ihr Gegenteil und führt uns in neue schwere Abhängigkeiten. (NKU09/MAI.11189 Nordkurier, 30.05.2009, Besinnung zum Sonntag)

88) $[[0]]$

Er schrieb fünf Stücke und zählte zu den bedeutendsten politischen Schriftstellern Ísterreichs. 1937 wird er verhaftet, das ist zunächst eine Verwechslung, doch da sich herausstellt, dass er selbst politische Schriften verfasst, wird er inhaftiert. Eine Amnestie bringt ihm kurz die Freiheit, bei einem Fluchtversuch in die Schweiz wird er erneut verhaftet und im Juni 1938 ins Konzentrationslager Dachau transportiert, 1939 stirbt er im KZ Buchenwald an Typhus. (NKU10/DEZ.10852 Nordkurier, 29.12.2010, Originalressort: Lokalredaktion; Insel geflüster)

89) $((1 ;$ Gen.; A1))

Und trotzdem möchte ich mit meiner Kandidatur die Bandbreite von Personen der liberalen Partei aufzeigen. So bedeutet für mich weniger an Staat auch gleichzeitig mehr an bürgerschaftlichem Tun. Entsprechend meiner aktuellen Lebenslagen fand man mich als ehrenamtlichen Bürgermeister, als Mitbegründer der Elterninitiativschule in Remplin, als Organisator der Malchiner Umwelttage oder wie in jüngster Zeit als Mitglied eines etwas anderen Dorfvereins in Gessin. 2 Es ist für mich auch in der Neuzeit das Leitziel des Liberalismus, die Freiheit des Einzelnen vornehmlich gegenüber staatlicher Gewalt zu schützen. Und das ist gut so! Bürger können es mit ihrer geballten Macht an Erfahrungen immer besser als der Staat. (NKU11/AUG.08415 Nordkurier, 23.08.2011, Originalressort: Lokalredaktion; Thomas Krüger, SPD)

90) $((0))$

Spielen da auch eigene Erlebnisse und Erfahrungen mit?Ja, das lässt sich bei so einem Thema kaum vermeiden. "Auf den Wiesen meiner Kindheit", "Immer bei mir" und "Die Stadt die keiner kennt" - alle drei haben unmittelbar mit meiner Rückkehr nach 30 Jahren in meine Geburtsstadt Halle zu tun. Heimkommen, in den Ort der Kindheit, in der alles noch so leicht ist, wo Freiheit eine Selbstverständlichkeit ist, über die niemand nachdenkt. Dann war mir der Dank an meine viel zu früh verstorbenen Eltern ein Bedürfnis, denen ich so gern noch so vieles gesagt hätte. Eltern und ihre Fürsorge sind ebenfalls Selbstverständlichkeiten, die viel zu oft einfach hingenommen werden. (NKU12/OKT.06196 Nordkurier, 18.10.2012, Originalressort: Lokalredaktion; Falkenberghat die Freiheitim Gepäck)

91) [[1; Posspr.; A1]]

Illegaler Fischzug endet im Netz der Kameras

Da hilft auch nicht mehr, alle Hühneraugen zuzudrücken. Wenn einer schon 13 Kerben auf seiner Vorstrafen-Latte hat und daraus nicht lernen wollte, muss er trotz eines vergeblichen Einbruchs dafür mit seiner Freiheit büßen.

Waren. Ein fairer Sportsmann stemmt sich gegen seine Niederlage. (NKU14/APR.00413

Nordkurier, 04.04.2014, S. 17, Illegaler Fischzug endet im Netz der Kameras)

92) $((1 ;$ Gen.; A1))

Etwa die verschlungenen, formal präzise geformten Schriftzüge dokumentieren nicht nur eine Botschaft, sie überzeugen durch Form und Struktur.

Trotz der textlichen Botschaft und der klaren "Tonschrift" bleibt eine Balance zum Meditativen. Diese ist eingebettet in Freiheit der Formgebung und dem individuellen Mitteilungsbedürfnis. Ihre Botschaften wirken direkt ins Leben hinein. Die Arbeiten von Charlotte Wiesmann sind in vielen Ausstellungen und Sammlungen vertreten. 
(NON07/OKT.15730 Niederösterreichische Nachrichten, 23.10.2007, S. 50; TonBotschaften zum Nachdenken [Ausführliche Zitierung nicht verfügbar])

93) $[[0]]$

Weit kamen sie nicht. Dem 46-Jährigen ging rasch die Puste aus, am nahe gelegenen „Zielpunkt“-Parkplatz wurde er mit einer geballten Ladung Pfefferspray außer Gefecht gesetzt.

Sein Komplize war Minuten länger in Freiheit. Den 35-Jährigen, der seine Muskeln in einem slowakischen Gefängnis gestählt hatte, ließ ein Warnschuss in der Munggenaststraße kalt. In der Birkengasse schlug schließlich die große Stunde von Schäferhund „Restan“ und von seinem Herrl Gruppeninspektor Franz Rausch. (NON10/MAR.00607 Niederösterreichische Nachrichten, 02.03.2010; Held heißt \&\#8222;Restan\&\#8220; [Ausführliche Zitierung nicht verfügbar])

94) $((0))$

Immerhin ist bereits rund jeder vierte Führerscheinprüfling ein L17-Schüler.

Und die Unfallzahlen bei der betroffenen Altersgruppe seien damit „massiv zurückgegangen“, betont Fachverbandsobmann Wiedermann.

Der L17-Führerschein bedeutet für viele junge Lenker die große Freiheit. Allerdings setzt diese Freiheit auch die Reife voraus, Verantwortung zu übernehmen und Gefahren richtig einzuschätzen. Nicht angepasste Geschwindigkeit und Unfälle beim Überholen kommen bei jungen Lenkern signifikant häufiger vor als bei älteren Autofahrern. (NON12/OKT.16692 Niederösterreichische Nachrichten, 25.10.2012; Erfolgsmodell trotz [Ausführliche Zitierung nicht verfügbar])

95) $[[0]]$

Vortrag. Am Mittwoch, dem 26. Februar, um 20 Uhr erzählt der Bergführer, Fotograf und Weltreisende Hans Thurner im Manker Stadtsaal über seine Reise zu Fuß über die Alpen von Wien nach Nizza. Sein Vortrag ,2.000 km Freiheit" verspricht ein wahres Wechselspiel der Natur von den Felsen der Dolomiten bis zur mediterranen Landschaft Frankreichs. Vier Monate voller Höhen und Tiefen. (NON14/FEB.08777 Niederösterreichische Nachrichten, 20.02.2014; KURZ NOTIERT [Ausführliche Zitierung nicht verfügbar])

96) ((0))

Nachholbedarf an Freiheit

Als "Skandal" hat es der scheidende Präsident des Lutherischen Weltbundes (LWB), der bayerische Landesbischof Johannes Hanselmann, bezeichnet, daß Menschenrechte und Freiheit für Millionen von Menschen noch immer nicht Wirklichkeit geworden seien. Auch wenn die "Signale der Zeit" heute gerade etwa im Ostblock auf Freiheit stünden, bestehe noch ein "ungeheuer großer" Nachholbedarf an Freiheit, nicht zuletzt auch in Lateinamerika, betonte Hanselmann zur Eröffnung der VIII. Vollversammlung des Lutherischen Weltbundes im südbrasilianischen Curitiba. Aufgabe der Kirche sei es, einen "besonderen Raum der Freiheit" anzubieten. (NUN90/JAN.02263 Nürnberger Nachrichten, 31.01.1990, S. 4; [Ausführliche Zitierung nicht verfügbar])

97) $[[0]]$

PRAG(dpa/AP) - Zum engagierten Kampf um die demokratische Revolution hat der tschechoslowakische Staatspräsident Vaclav Havel seine Mitbürger aufgerufen.

Vor etwa 100000 Menschen, die erstmals in Freiheit der Niederschlagung des Prager Frühlings durch Panzer der Warschauer Paktstaaten 1968 gedachten, sagte Havel: "Wir 
müssen alle zur Verfügung stehenden rechtlichen und politischen Mittel nützen, um die unfähige oder sabotierende Nomenklatura des alten Regimes zu bekämpfen." In scharfen Worten prangerte Havel vor allem die Widerstände der "Mafia" gegen Wirtschaftsreformen an. (NUN90/AUG.01418 Nürnberger Nachrichten, 22.08.1990, S. 1; Prag: Havel ruft zum Kampf aufPrag: Havel ruft zum Kampf auf [Ausführliche Zitierung nicht verfügbar])

98) $((0))$

Im Jahr 1945 und bald danach begannen die Deutschen, Demokratie zu lernen und - im Westen vor allem - auch zu üben. Jene Generationen, die gerade noch dem Naziterror und Hitlers Kriegsmaschine entronnen waren, begriffen damals mit diesen Erfahrungen und angesichts kommunistischer Praktiken im Osten sehr bald, daß die Herrschaft durch Diskussion und Abstimmung zwei Voraussetzungen hat: die Toleranz anderer mit abweichender Meinung und die Abwehr aller, die eine solche Haltung nicht bekunden. Freiheit also für alle - außer für die Feinde der Freiheit. Grundsätze verraten Dies hat, alles in allem, trotz einiger bedenklicher Entgleisungen, vier Jahrzehnte lang in Westdeutschland einigermaßen funktioniert. (NUN91/OKT.00098 Nürnberger Nachrichten, 02.10.1991, S. 2; Einmischung gefragt - Vor dem Ausländerhaß darf der Staat nicht kapitulieren Die Uno, Haiti und das Elend der Dritten Welt [Ausführliche Zitierung nicht verfügbar])

99) ((0))

Verpflichtet uns das nicht? Wenn wir die Überwindung der Teilung Europas im Jahr 1989 der Strahlkraft der Demokratie zuschreiben, müssen wir uns dann nicht auch um Demokratie bei der Vollendung der Europäischen Einigung bemühen?

Der Kollektivismus ist nicht am militärischen oder wirtschaftlichen Übergewicht des Westens zugrunde gegangen, sondern am Mangel an Freiheit. Auch in Zukunft muß das Motto also heißen: Freiheit ist das höchste Gut. Sie muß auch Vorrang vor nationalen Kollektiven haben. (REI/RBP.00065 Roman Herzog: Artikel zum Thema "Die Rechte des Menschen" in der Wochenzeitung "Die Zeit", Hrsg: Bundespäsidialamt [Ausführliche Zitierung nicht verfügbar])

100) ((1; attr. Adj.; A1))

$\mathrm{Ob}$ es so kommt, weiß niemand. Und China hat in den 5000 Jahren seiner Geschichte vielfach scharfe Brüche erlebt. Spannungen gibt es auch heute zuhauf: Wirtschaftlicher Freiheit steht politische Unfreiheit gegenüber. Der gewaltige Sog der Wirtschaftszentren auf die jungen und erfolgshungrigen Menschen verschärft die soziale Situation auf dem Land. Die Industrialisierung und der rasant wachsende Energiebedarf führen zu schweren Schäden an Umwelt und Natur. (REI/RBP.00787 Horst Köhler: Weihnachtsansprache von Bundespräsident Horst Köhler, Hrsg: Bundespäsidialamt [Ausführliche Zitierung nicht verfügbar]) 


\section{1) $\{\{0\}\}$}

Der Bundesrat entscheidet demnächst über eine abgeschwächte Sparvorlage.

beat waber

Das Problem harrt seit Jahren einer Lösung: Die freiwillige AHV/IV, der ausgewanderte Schweizer beitreten können, ist hoch defizitär. 59 Millionen Franken haben die knapp 50000 Versicherten 1996 einbezahlt. Die Rentenleistungen sind indes dreimal so hoch.

(A98/JAN.01649 St. Galler Tagblatt, [Tageszeitung], 12.01.1998, Jg. 54. Originalressort: TB-INL (Abk.); beat waber: Erfolg der Auslandschweizer-Lobby, [Bericht])

2) $\{\{1 ;$ Gen.; A' $\}\}$

Wäre 1500-Seelen-Gemeinde

Für den Erler Kirchenpräsidenten Fredy Wirth läge der Vorteil einer Gebietsumteilung in den übereinstimmenden Grenzen von Kirchgemeinde und Politischer Gemeinde. Zudem wäre beim Religionsunterricht das Problem der Stundenplan-Friktionen gelöst. Evangelisch Erlen, das vor Jahren Riedt aus der evangelischen Kirchgemeinde Sulgen übernommen hat, würde kommt ein Wechsel zustande - danach rund 1500 Seelen zählen. Eine Zahl, die für die eine vollamtliche Pfarrstelle an der oberen Belastungsgrenze zu liegen käme, wie Fredy Wirth sagte. (A98/MAR.14380 St. Galler Tagblatt, [Tageszeitung], 07.03.1998, Jg. 54.

Originalressort: TB-ABI (Abk.); Urs Müller: In Amriswil oder Erlen zu Hause?, [Bericht])

3) $\{\{1+1$; App.; Subj.; A‘; A1 $\}\}\{\{$ SVG; P. haben $\}\}$

Al-Anon kann nicht alle Probleme aus der Welt schaffen, doch der Austausch hilft, frei, glücklich und zufriedener zu werden. Davon profitiert die ganze Familie, einschliesslich der alkoholkranken Person. In der Selbsthilfegruppe Al-Anon sind alle willkommen, welche das Problem Alkoholismus in ihrer Familie oder im Freundeskreis haben. Die Gruppenbesucher und Gruppenbesucherinnen bleiben anonym und sprechen sich mit dem Vornamen an.

Al-Anon (A99/FEB.13321 St. Galler Tagblatt, [Tageszeitung], 24.02.1999, Jg. 55.

Originalressort: TT-NEU (Abk.); Ökumenischer Weltgebetstag, [Bericht])

4) $\{\{0\}\}$

Mit fünfundzwanzig Jährchen wusste Mutter plötzlich in den Augen der zwei über das und jenes wieder Bescheid.

Als Fünfunddreissigjährige waren sie froh, Mutter vor wichtigen Entscheidungen nach ihrer Meinung fragen zu können.

Mit fünfundvierzig wollten sie wieder vermehrt wissen, wie ihre Mutter über dieses oder jenes Problem denkt.

Jetzt sind die beiden fünfundsechzig. Sie hätten nur einen einzigen Wunsch:

(A99/MAI.33183 St. Galler Tagblatt, [Tageszeitung], 10.05.1999, Jg. 55. Originalressort: TT-SER (Abk.); Seitenblick, [Bericht])

5) $\{\{0\}\}$

Seelhofer ist Präsident der Stiftung Licht-Heilzentrum St. Germain, deren Gründung im Handelsamtsblatt vom 9. November 1999 publiziert wurde. 
Noch befindet sich das Grundstück in Privatbesitz. Laut Seelhofer ist das kein Problem. Die Stiftung habe mit dem Bauern einen Optionsvertrag abgeschlossen. «Aber wir kaufen erst, wenn die Baubewilligung rechtsgültig vorliegt», sagt er. (A00/FEB.08870 St. Galler Tagblatt, [Tageszeitung], 04.02.2000, Jg. 56. Originalressort: TB-OST (Abk.); Silvan lüchinger: Noch ist mehr Dunkelheit als Licht, [Bericht])

6) $\{\{0\}\}$

Sie hemmen sogar und lenken ab von der harten Wirklichkeit. Wer also übernimmt die Verantwortung, zeigt Mut? Wie heisst doch ein Sprichwort: «Wenn jemand ein Problem erkannt hat und nichts zur Lösung des Problems beiträgt, ist er selbst ein Teil des Problems.. !!» Freuen wir uns also auf eine künftig wieder erfolgreiche Arboner Mannschaft, die miteinander und nicht gegeneinander für die Stadt der Ideen und Chancen kämpft. Mit einem neuen Trainer vielleicht?

Patrick Köppel, (A00/APR.25240 St. Galler Tagblatt, [Tageszeitung], 13.04.2000, Jg. 56. Originalressort: TB-ZUS (Abk.); Gedanken zur politischen Kultur in Arbon, [Bericht])

7) $\{\{1$ Komp.; A2 $\}\}$

Krammers Vorschlag: In Kapfenberg müßten wieder Schnellzüge halten, und zwar die täglich sieben Intercity-Zugpaare auf der Strecke Graz- Wien.

Krammers Logik ist einleuchtend: "Jetzt kommen die Kapfenberger entweder mit dem Auto nach Bruck, oder sie lassen sich zum Brucker Bahnhof bringen. Kommen sie selbst, verschärfen sie das Parkplatzproblem, lassen sie sich bringen, verstärken sie den ohnehin schon enormen Autoverkehr zwischen Bruck und Kapfenberg." Da laut Krammer zumindest die Hälfte der Fahrgäste, die in Bruck ein- oder aussteigen, aus dem Bereich Kapfenberg kommt, wäre das nicht nur eine spürbare Entlastung der Brucker Bahnhofsumgebung. Krammer zweifelt auch nicht daran, daß mehr Kapfenberger die Bahn benützen würden, wenn sie dafür nicht vorher nach Bruck fahren müßten. (K98/APR.28199 Kleine Zeitung, [Tageszeitung], 12.04.1998. Originalressort: Mürztal; Aufwertung für Kapfenberg?)

8) $\{\{0\}\}$

Tagesvignette: Selbsthilfe ist nötig

Mit dieser Unterschriftensammlung will das Petitionskomitee auf keinen Fall, dass sich der Verkehr weiterhin durch Bregenz hindurchzwängt. Aber es darf auch nicht sein, dass mit einer Tagesvignette das ganze Problem nach Diepoldsau - Hohenems verschoben wird. Ebenfalls käme es dem sogenannten St.-Florian-Prinzip entgegen, die ganze Verkehrslawine einfach nach Götzis - Kriessern zu verschieben. Zugegeben, die kürzeste Verbindung zwischen Chur und München ist über Kriessern, aber ebenso wäre die schnellste Verbindung von St. Gallen nach München über St. Margrethen, wenn da nicht das Nadelöhr Bregenz wäre. (A07/SEP.11982 St. Galler Tagblatt, [Tageszeitung], 25.09.2007, Nr. 223, S. 44. Sachgebiet: Regionales, Originalressort: RT-Eingesandt; Diepoldsau, durchtrenntes Dorf am Rhein)

9) $\{\{0\}\}$

Eine der Schwierigkeiten liege darin, dass im Kanton Zürich die Hälfte der Waldfläche Privaten gehöre (im appenzellischen Verbandsgebiet sind es 60 Prozent). Da fehlt laut Keller der Ansprechpartner. So versuche man in Zürich, die Waldeigentümer durch Information und Motivation für das Problem zu sensibilisieren. Angestrebt werde eine

Bewirtschaftungsvereinbarung, dank der die privaten Eigentümer ihre Kompetenzen bündeln und an die Förster delegieren könnten. 
Im Kanton Zürich gibt es hundert Revierförster. (A07/NOV.13256 St. Galler Tagblatt, [Tageszeitung], 28.11.2007, Nr. 278, S. 43. - Sachgebiet: Regionales, Originalressort: ATAppenzellerland; b.gaemperle: Holz ist hoch im Kurs)

10) $\{\{1 ;$ für + Akk.; A1 $\}\}$

«Für das OK kein Problem»

Aufgefallen sind einige Läufer auch Christoph Tobler, dem OK-Präsidenten der

Veranstaltung. «Mich haben sie nicht gestört, sie taten mir eher leid, weil sie sich so abmühten, während es Velofahrer und Inlineskater gemütlich nehmen konnten», erinnert er sich und ergänzt: «Es war aber für die Organisation kein Problem.»

Zweitauflage 2009?

Er könne sich nicht vorstellen, «dass die Läufer nicht wieder kommen dürfen.» Damit spricht er im Sinne von Lohrer, der sagt: "Wir würden es gerne wieder machen.» Auf Unterstützung des Organisationskomitees darf er aber kaum hoffen, denn Tobler liess keine Zweifel offen: «Fördern werden wir die Läufer nicht.» (A08/SEP.00579 St. Galler Tagblatt, [Tageszeitung], 02.09.2008, Nr. 204, S. 35. - Sachgebiet: Regionales, Originalressort: TG-Arbon; m.eichenberger: Willkommen \&\#8211; nicht gefördert)

11) $\{\{0\}\}$

Die Schweizer Delegation hat den Wunsch von Disziplinenchef Franco Giovanoli mit drei Top-drei-Klassierungen damit schon nach dem ersten WM-Tag zu zwei Dritteln erfüllt. «Ich wäre nicht traurig, wenn weitere Spitzenrangierungen dazukommen würden», sagte

Giovanoli. Nationaltrainer Harald Benselin wird in einem Jahr, vor den Olympischen Spielen von Vancouver, vor einem Problem stehen. Die Schweizer Boardercross-Frauen stellen das weltweit stärkste Team, Giovanoli wird aber nur maximal vier Quotenplätze zur Verfügung haben. Wie vor der WM 2005 (Frieden nicht berücksichtigt) oder Olympia 2006 (Frei) drohen unpopuläre Entscheide. (A09/JAN.03397 St. Galler Tagblatt, [Tageszeitung], 19.01.2009, Nr. 14, S. 19. - Sachgebiet: Sport, Originalressort: sport; Tagblatt: Fast perfekter WMAuftakt)

12) $\{\{0\}\}$

Wildtiere als Grenzgänger werden zum Problem

Die Ausserrhoder Hochwildjäger befassten sich an ihrer Hauptversammlung mit Jagdwild, das nach dem Schuss über die Grenze in einen Nachbarkanton oder ins Jagdbanngebiet wechselt.

Urnäsch. (A09/APR.05584 St. Galler Tagblatt, [Tageszeitung], 22.04.2009, Nr. 92, S. 37. Sachgebiet: Regionales, Originalressort: AT-Appenzellerland; m.faessler: Wildtiere als Grenzgänger werden zum Problem)

13) $\{\{0\}\}$

Auch für Eltern gedacht

Im September besuchte der Bus das Torkelfest in Berneck. Bei solchen Veranstaltungen werde der Alkoholkonsum oft zum Problem, erzählt Claudia Tobler. Daher wurden Jugendliche dazu eingeladen, eine Rauschbrille aufzusetzen. Zudem stellte das Netzwerk einen Parcours auf. (A09/OKT.02440 St. Galler Tagblatt, [Tageszeitung], 09.10.2009, Nr. 235, S. 37. - Sachgebiet: Regionales, Originalressort: RT-Aufschlag; a.ruedisueli: Das Jugendnetzwerk ist mobil)

14) $\{\{1 ;$ für + Akk.; A1 $\}\}$ 
Nur 28 Prozent aller Stimmberechtigten erwägen in der Krankenversicherung ein ManagedCare-Modell. Dies zeigt der Gesundheitsmonitor 2010. Claude Longchamp vom Forschungsinstitut GFS ortet deshalb «ein massives Problem» für die Politik. «Hier ist eine riesige Lücke», sagte Longchamp gestern vor den Medien. Denn die Politik setze grosse Hoffnungen in Managed-Care-Modelle. (A10/JUN.08044 St. Galler Tagblatt, 25.06.2010, Nr. 145, S. 7. - Sachgebiet: Lokales, Originalressort: inland; d.angst: Managed Care im Volk unbeliebt)

15) $\{\{1+1$; Komp.; Subj.; A2; A1 $\}\}\{\{$ SVG; P. haben $\}\}$

Gestern hat die Arbeitsmarktverwaltung ihren Jahresbericht für 2007 vorgestellt. Neben Strukturproblemen gibt es in Sachen Arbeitslosigkeit seit September 2007 erstmals Anzeichen für eine positive Trendwende. Und doch gibt es keinen Grund zur Entwarnung, denn Luxemburg hat ein handfestes Bildungsproblem. Über die Hälfte der gemeldeten Arbeitslosen kommt über die niedrigste Bildungsstufe nicht hinaus. (Foto: Isabella Finzi)GSeite 15 (LTB08/MAR.00285 Luxemburger Tageblatt, 11.03.2008, Sachgebiet: Politik, Originalressort: POL - Politik; Luxemburg hat ein Bildungsproblem)

16) $\{\{1 ;$ für + Akk.; A1 $\}\}$

Dank dem grösseren Platzangebot hätten in der Bibliothek auch verschiedene Aktivitäten durchgeführt werden können, die vorher nicht möglich gewesen seien. So würden Bewohner des Alterszentrums Sonnenhof regelmässig die Bibliothek besuchen. Dank des Liftes sei das kein Problem für die zum Teil gehbehinderten Pensionäre.

«Leider haben wir im vergangenen Jahr knapp die Schallgrenze von 100000 Ausleihungen verpasst», bedauerte Irène Häne. Gegenüber 2009 könne aber praktisch in allen Bereichen ein Wachstum verzeichnet werden. (A11/FEB.03112 St. Galler Tagblatt, 09.02.2011, Nr. 33, S. 34. - Sachgebiet: Regionales, Originalressort: WV-Stadt-Wil; n.stricker: Schallgrenze knapp verpasst)

17) $\{\{0\}\}$

Allerdings glaube ich nicht daran, dass dieser in den nächsten zehn Jahren kommen wird. Manuela Baumann, 48, Verkäuferin, Flawil

Ich bin zwar keine Gossauerin, aber ich sehe das Problem. Der Verkehr auf der Hauptstrasse, besonders wenn es dem Feierabend zugeht, ist schlimm. Trotzdem sehe ich keine baldige Lösung, die Gossauer sind stur. (A11/SEP.07258 St. Galler Tagblatt, 17.09.2011, Nr. 217, S. 53. - Sachgebiet: Regionales, Originalressort: GO-Gossau; m.loher: «Wir haben genug vom Verkehr»)

18) $\{\{1$; Gen.; A1 $\}\}$

Hildesheims Bischof Norbert Trelle hat die neuen Bleiberechtsregelungen für Flüchtlinge als viel zu eng bezeichnet. „Wir dürfen nicht alle Flüchtlinge unter Generalverdacht stellen“, sagte Trelle dieser Zeitung. Die Bleiberechtsregelungen befriedeten das Problem der lange Zeit in Deutschland nur Geduldeten nicht. „Die Gesetzeslage scheint so zu sein, dass alles restriktiv ausgelegt wird.“ Trelle wies darauf hin, dass nicht alle Flüchtlinge selbst für ihren Lebensunterhalt aufkommen könnten.

P Trelle im Interview Seite 9 (HAZ07/AUG.01943 Hannoversche Allgemeine, 25.08.2007, S. 1; Bischof Trelle rügt das Bleiberecht als zu eng [Ausführliche Zitierung nicht verfügbar])

19) $\{\{1+1$; Inf.-Satz; Subj.; A2; A1 $\}\}\{\{$ SVG; P. haben $\}\}$ 
Das Potenzial ist da, er kann es in der Bundesliga schaffen.“ Etwa gleich auf Schalke zum Auftakt? „Man soll niemals nie sagen“, antwortet Balogun. Das klingt großmäulig, ist es aber nicht, weil er vorausgeschickt hat: „Ich will von Cherundolo lernen und habe kein Problem, mich unterzuordnen. Ich habe nicht den Anspruch, sofort Stammspieler zu sein.“ Von der ersten Elf ein Stück weg, aber schon erprobt in der Fußball-Bundesliga ist auch Salvatore Zizzo, seit einem Jahr bei den 96ern. (HAZ08/JUL.01767 Hannoversche Allgemeine, 09.07.2008, S. 25; Ein Trio auf Heckings Fahndungs-Radar [Ausführliche Zitierung nicht verfügbar])

20) $\{\{1+1$; dass-Satz; Subj.; A'; A1 $\}\}\{\{$ SVG; P. haben $\}\}$ «Mental hat es plötzlich einen Bruch gegeben», schildert Marcel Baumann, «und von da an hatte nichts mehr funktioniert.» Die letzten zwei Drittel schloss man mit 2:7 und 1:5. Dabei hatte der UHC United Toggenburg Bazenheid die vergangene Saison doch noch besser als dieser Club abgeschlossen.

Gefestigte Spieler fehlten

Zwei Gründe seien ausschlaggebend für den aktuellen Stand der Toggenburger, erläutert Trainer Marcel Baumann: «Erstens hatten wir zu Beginn das Problem, dass mehrere Spieler wegen Auslandaufenthaltes oder Rekrutenschule abwesend waren. Zweitens haben einige Gegner auf diese Saison hin ihr Team mit ehemaligen NLA-Spielern und Ausländern verstärkt.»

Mittlerweile seien die bisher abwesenden Spieler zurückgekehrt und bereits ein Spiel oder zumindest das Training mit der eigenen Mannschaft absolviert. (A12/JAN.00986 St. Galler Tagblatt, 07.01.2012, Nr. 5, S. 51. - Sachgebiet: Regionales / Sport, Originalressort: WVRegionalsport; u.nobel: Toggenburger in Abstiegsgefahr)

\section{1) $\{\{0\}\}$}

Mit zwei Rohrbriden spannte er links und rechts der Kette Abschnitte kleinerer Rohre ein, die das erste Zahnrad von Schmutz abschirmen. Bei dieser Art von Arbeit habe ihm seine Erfahrung als «Töfflibueb» sehr geholfen, meint Thomas Hofstetter. «Da ich früher oft an Mofas geschraubt habe, wusste ich in etwa, wie ich das Problem anpacken muss.» Trotz der Erfahrung war das Projekt sehr zeitaufwendig. Wie viel Spass ihm die Arbeit bereitet hat, wird sofort klar, als er die Anzahl investierter Arbeitsstunden deutlich unterschätzt: «Ich würde sagen, das waren rund zehn Stunden», sagt Thomas Hofstetter.

(A12/APR.06989 St. Galler Tagblatt, 19.04.2012, Nr. 91, S. 41. - Sachgebiet: Regionales, Originalressort: AT-Appenzellerland; t.zuest: Fahrzeug aus Abflussrohr und TrimmerMotor)

22) $\{\{1+1$; bei + Dat.; Subj.; A2; A1 $\}\}\{\{$ SVG; P. haben $\}\}$ In den vergangenen Jahren zeigten die Schweizer starke Leistungen bei den Junioren und im Europacup. Warum hat kaum einer der Nachwuchsfahrer den Sprung in den Weltcup geschafft?

Beim Übergang vom Nachwuchs in den Weltcup haben wir ein Problem. Unsere Juniorenförderung funktioniert nur bis zum Alter von 20 Jahren. Ich habe Vermutungen, was in diesem Bereich nicht zusammenpasst. (A13/JAN.02300 St. Galler Tagblatt, 09.01.2013, Nr. 6, S. 17. - Sachgebiet: Sport, Originalressort: sport; c.krapf: NACHGEFRAGT)

23) $\{\{0\}\}$

Im ganzen Dorf blieben die Strassenlampen bis etwa um 19.45 Uhr dunkel. Ein Stromausfall war nicht der Grund, denn in den Privathäusern waren Lichter zu sehen. «Das Problem lag bei der Rundsteuerung der Strassenbeleuchtung», erklärte Thomas Rütsche, Betriebsleiter der 
Dorfkorporation Ebnat-Kappel auf Anfrage. An der Steuerung sei ein Netzteil ausgefallen, so dass die Trafostationen den Befehl zum Einschalten der Strassenlampen nicht erhalten hätten. Im Zuge der Problembehebung (A13/MAR.05978 St. Galler Tagblatt, 13.03.2013, Nr. 60, S. 33. - Sachgebiet: Regionales, Originalressort: TT-Aufschlag; sa.schmid: EbnatKappler Strassenlampen blieben dunkel)

24) $\{\{0\}\}$

Ein Verbot wäre seiner Meinung nach völlig unverhältnismässig. Rechtsgerichtete Kreise seien mit derartigen Vorstössen in erster Linie auf Stimmenfang aus. Es gehe ihnen nicht darum, ein konkretes Problem zu lösen. «Sie zetteln eine Stellvertreterdiskussion an, um eine Anti-Islam-Stimmung zu schaffen.» Valentina Smajli, Vize-Präsidentin des Forums für einen fortschrittlichen Islam, bezeichnet die Initiativpläne als «Schritt in die richtige Richtung». Die Verschleierung werde von patriarchal geprägten Gruppierungen genutzt, um die niedere Stellung der Frau zu demonstrieren. (A13/SEP.02764 St. Galler Tagblatt, 24.09.2013, Nr. 222, S. 2. Originalressort: Thema; «Die Initiative ist pfannenfertig»)

25) $\{\{0+2$; keine Real.; Dat.; Subj.; kein Argument; A1; A' $\}\}$ \{\{SVG; Dat. P. bereiten $\}\}$ Die Holzwirtschaft ist defizitär - es kann die Zeit kommen, in der der Wald gar nur noch gepflegt wird, kaum mehr bewirtschaftet.»

Ein Abflussrohr im Biberdamm

Ein immer grösseres Problem bereitet den Förstern auch der Biber. Anhand eines Beispiels bei Reuti erklärt Guntersweiler: «Der Biber setzt ganze Parzellen unter Wasser, Eschen und Tannen sterben ab.» Nur dank eines Rohres, das er durch den Staudamm des Bibers geschoben hat, fliesse das Wasser nun wieder ab. Carmen Haag sagt zur wachsenden Biberpopulation im Thurgau: «Das Problem ist erkannt. (A14/AUG.07851 St. Galler Tagblatt, 23.08.2014, Nr. 195, S. 45. Originalressort: Weinfelden; mario.testa: Carmen Haag besucht den Biber)

26) $\{\{1$; mit + Dat.; A2 $\}\}$

Bis zum Ise-Seitenarm ist bereits ein Weg angelegt. Wrobel plant aber eine lange Allee mit Bäumen und einer kleinen Brücke, die direkt in Russland gebaut werden soll. „Ich hoffe, dass sich das Problem mit dem privaten Grundstück in Kürze lösen lässt", hofft er auf weitere verständnisvolle Gifhorner. Ebenso kritisch sieht er das Gerücht, die Rhön-Kliniken wollten auf dem städtischen Grundstück jenseits der B 188 einen Krankenhaus-Neubau errichten. „Das wäre eine Schande. (BRZ05/NOV.11534 Braunschweiger Zeitung, 09.11.2005; Vergoldete Wetterfahnen zum Dank [Ausführliche Zitierung nicht verfügbar])

27) $\{\{0\}\}$

Seine Leistenverletzung hat der Mittelfeldspieler überstanden. „Ich fühle mich gut. Laufen ist kein Problem. Jetzt muss ich abwarten, wie es mit dem Ball klappt.“

Autounfall: Biliskov im Glück (BRZ06/JUN.14881 Braunschweiger Zeitung, 29.06.2006; [Ausführliche Zitierung nicht verfügbar])

28) $\{\{0\}\}$

Das Vorhaben gestaltet sich aber weit schwieriger als angenommen. Gegenwärtig gelten die Bemühun-gen der Gemeinde der Suche nach geeigneten Räumlichkeiten, nach-dem sich die naheliegendste Mög-lichkeit zerschlagen hat. „Wenn man nach marktwirtschaftlichen Gesichtspunkten entscheiden könnte, also nach Angebot und Nachfrage, wäre das Problem leichter lösbar", sagt Hannig. Denn ihre Aufstellung der Einwohner der Samtgemeinde 
Papenteich nach Alter (und Geschlecht) weist aus, dass für die kontinuierlich wachsende Bevölke-rung in Meine und dem Papenteich eine nahe ärztliche Versorgung günstig wäre. Angedacht sind Augenarzt, Kinderarzt und Neurologe. (BRZ07/FEB.11670 Braunschweiger Zeitung, 28.02.2007; Ehrgeiziges Ziel: Mehr Ärzte in den Papenteich holen [Ausführliche Zitierung nicht verfügbar])

29) $\{\{0\}\}$

Doch ob das Blütenfest überleben wird, scheint fraglich zu sein.

„Wir haben nur noch 22 Mitglieder, der Altersdurchschnitt ist recht hoch“, sagt Bernd Witte, Vorsitzender der 1979 gegründeten Interessengemeinschaft. Ein weiteres Problem sei der sinkende Wasserspiegel des Sandteiches. Schon jetzt seien große Flächen des Teiches verlandet.

Kassenwart Jürgen Werner ist überzeugt, dass bei den Bauarbeiten im Baugebiet nördlich des Teiches wasserführende Schichten, die den Teich gespeist hätten, zerstört wurden.

(BRZ07/MAI.03399 Braunschweiger Zeitung, 31.05.2007; Blütenfest in Gefahr: Wasser und Mitglieder schwinden [Ausführliche Zitierung nicht verfügbar])

30) $\{\{0\}\}$

Ganz entschieden tritt die sonst recht zurückhaltende Osterloh bei der Frage auf, wie man am besten den Neonazis entgegentreten solle. „Keinerlei Zusammenarbeit mit Extremisten, egal ob links oder rechts“, sagt sie. Dass Extremisten nicht sagten, was hinter ihnen stecke, das sei das große Problem.

Bei der Frage, was in Sachen Schacht Konrad zu unternehmen sei, versucht sie Einigkeit zwischen ihr und den anderen Kandidaten herzustellen: „Wir müssen in dieser Frage zusammenarbeiten“, so Osterloh. Einen Ausgleich für Salzgitter müsse es in jedem Fall geben. (BRZ07/NOV.11886 Braunschweiger Zeitung, 30.11.2007; Osterloh: Mein Schwerpunkt sind die Familien [Ausführliche Zitierung nicht verfügbar])

31) $\{\{0\}\}$

Nach zehn Partien wartet der neue Coach des Fußball-Regionalligisten immer noch auf den ersten Sieg. Die erwartete Verbesserung nach dem Trainerwechsel blieb aus.

Das Problem: Die Wolfsburger fungieren in der gesamten Rückrunde als Aufbaugegner. Die „kleinen Wölfe“ treffen immer auf die Mannschaft, die einen Spieltag später gegen Eintracht Braunschweig antritt. Damit beschäftigt sich Houbtchev jedoch nicht. (BRZ08/APR.13008 Braunschweiger Zeitung, 24.04.2008; Philipp Kreuels verspricht: Wir gewinnen noch [Ausführliche Zitierung nicht verfügbar])

32) $\{\{0\}\}$

Zurzeit sind zudem vor allem Gesundheitssportarten sehr beliebt.

Haben die Vereine die Zeichen der Zeit nicht erkannt?

Das Problem ist komplexer. Um die Mitglieder im Verein zu halten, versucht man, die Beiträge so gering wie möglich zu halten, dadurch sind aber Investitionen vor allem bei kleineren Dorfvereinen äußerst schwierig. Für zehn bis zwölf Sportler eine neue Abteilungen zu schaffen, ist einfach nicht zu finanzieren. (BRZ08/SEP.01211 Braunschweiger Zeitung, 03.09.2008; \&\#8222; Trendsportarten gezielt fördern\&\#8220; [Ausführliche Zitierung nicht verfügbar])

33) $\{\{1$; attr. Adj.; A2 $\}\}$

In einigen Bereichen tappen die Planer im Dunkeln. „Wohin die Reise beim Tischlerhof hingehen soll, ist noch völlig offen“, sagte Pfaff. 
In der Seilerstraße sahen Planer und Anwohner ein wachsendes strukturelles Problem. Der Laden-Leerstand, die Parkplatzsituation und die mögliche Finanzierung der Sanierung waren Fragen, die die Zuhörer interessierten. Hier musste Thomas sie vertrösten: „Frühestens in einem halben Jahr werden Zahlen auf den Tisch liegen“, prognostizierte sie.

(BRZ09/FEB.13359 Braunschweiger Zeitung, 27.02.2009; Aus Hinterhofgebiet wird Szeneviertel [Ausführliche Zitierung nicht verfügbar])

34) $\{\{0\}\}$

„Die wussten auch alle von unserem Mädchenprojekt und hätten ihre Schwestern mitbringen können. Aber die Hemmschwelle ist wohl doch noch zu hoch, weil das in deren Familien nicht zur Tradition gehört.“

Britta Carlson kennt das Problem bereits, hat aber bei einem viertägigen Camp mit Türkinnen in Leipzig auch schon gute Erfahrungen gemacht. „Ausländische Mädchen zu kriegen ist schwer, das ist ja nochmal eine Kategorie schärfer, als überhaupt Mädchen zu Fußball zu holen", sagt die 31-Jährige, die auch als Co-Trainerin beim Bundesligisten VfL Wolfsburg arbeitet. Dabei sei Mannschaftssport der einfachste Weg, Kinder dazu zu bringen, miteinander zu kommunizieren. (BRZ09/MAI.03643 Braunschweiger Zeitung, 08.05.2009; Fußball schlägt Deutschstunde [Ausführliche Zitierung nicht verfügbar])

35) $\{\{1+1$; attr. Adj.; Subj.; A2; A1 $\}\}\{\{$ SVG; P. haben $\}\}$

Da könnte Sänger Glitter glatt minutenlang sein Mikrofon weglegen, die Besucher übernähmen den Gesangspart.

Leider nahm die Kuba-Halle diesen Punkt etwas zu wörtlich. Mindestens ein Mikrofon hatte ein technisches Problem. Hinzu kam, dass die Aussteuerung der Band nicht stimmte prompt erklärten Stammgäste von Sweety den Auftritt zur schlechtest verständlichen Nummer der vergangenen Jahre.

Der prächtigen Stimmung tat das aber keinen Abbruch. (BRZ09/DEZ.03576

Braunschweiger Zeitung, 07.12.2009; Tanzen zur Musik der Eltern [Ausführliche Zitierung nicht verfügbar])

36) $\{\{0\}\}$

Ja, das ist möglich. Solche Flächen haben wir auch, zum Beispiel in Neudorf-Platendorf und anderswo. Das war auch schon 1975 ein Problem. Wenn man dort hineingeht, sieht man nichts - und steht auf einmal in der Glut.

Kann man gegen solche Moor- und Torfbrände Vorsorge treffen? (BRZ10/AUG.04210

Braunschweiger Zeitung, 11.08.2010; \&\#8222;Flammen können 200 Meter weit über die Baumwipfel springen\&\#8220; [Ausführliche Zitierung nicht verfügbar])

37) $\{\{1+1$; in + Dat.; Subj.; A2; A1 $\}\}\{\{$ SVG; P. haben $\}\}$

Dort wird deshalb auch häufig gewechselt und noch nach der besten Formation gesucht", meint Neururer.

Im Mittelfeld sieht der diplomierte Sportlehrer den VfL einen Tick besser besetzt als die Schalker. „Im defensiven Mittelfeld hat S04 ein Problem. Dort wird zuviel umgestellt. In diesem Bereich sehe ich den VfL mit Josué einfach im Moment etwas besser aufgestellt." (BRZ10/NOV.07061 Braunschweiger Zeitung, 13.11.2010; \&\#8222;Im Sturm ist S04 defizitär belastet\&\#8220; [Ausführliche Zitierung nicht verfügbar])

38) $\{\{0+1$; keine Real.; Subj.; kein Argument; A1 $\}\}\{\{$ SVG; P. haben $\}\}$

Es war insgesamt ganz schön schwierig und ich hatte lange mit Lewis Hamilton zu tun. 
Bis zum letzten Abschnitt war es nie leicht, aber dann hatte ich einen beruhigenden Vorsprung. Lewis hatte wohl ein Problem und auf Jenson Button hatte ich genügend Vorsprung."

Was war denn mit dem Energierückgewinnungssystem in Ihrem Wagen los? „Das war ein bisschen ein An und Aus. (BRZ11/APR.05011 Braunschweiger Zeitung, 11.04.2011; \&\#8222;Das waren zwei wirklich perfekte Rennen\&\#8220; [Ausführliche Zitierung nicht verfügbar])

39) $\{\{1$; für + Akk.; A1 $\}\}$

Viel Spaß gemacht hat ihnen auch die Buchstabensuche. Jedes Kind sollte aus den Überschriften in der Zeitung Buchstaben schneiden und seinen eigenen Namen kleben. Für die meisten kein Problem, nur Alexia musste Geduld aufbringen. Erzieherin Kathrin Bunk: „Es gab einfach kein X - da mussten wir lange suchen.“(BRZ11/SEP.13961 Braunschweiger Zeitung, 23.09.2011; Spaß bei Buchstabensuche [Ausführliche Zitierung nicht verfügbar])

40) $\{\{1$; Komp.; A2 $\}\}$

"Da ist vielen der Aufwand und das Risiko zu groß", ist er sich bewusst. Um das Fest auf eine breitere Basis zu stellen und aufzuwerten, sind inzwischen auch der Innenstadtbeirat und die Citymanagerin Rita Koob mit im Boot. Während es 2006 noch Abstimmungsprobleme gab, klappte das Zusammenspiel in diesem Jahr besser. "Wir haben uns alle 14 Tage getroffen", so Berg. Dabei kümmert sich die Citymanagerin vorwiegend um das Tagesprogramm, während die Gastwirte das Abendprogramm bestreiten.

(M07/MAI.00810 Mannheimer Morgen, [Tageszeitung], 04.05.2007, Jg. 62, Ausgabe Südhessen Morgen (Lampertheim). - Sachgebiet: Lokales, Originalressort: Lampertheim; Auch Vereine dürfen beim Stadtfest auftischen)

41) $\{\{0\}\}$

Er lässt noch viel rotieren im Training. Jeder muss mal zuschauen. „Zum jetzigen Zeitpunkt ist das noch kein Problem, man kann viele Stabilitätsübungen machen. Außerdem wäre es fahrlässig, wenn ich mich nur auf elf Spieler festlegen würde. Mit Naldo und Josué fehlen uns im nächsten Spiel zwei Leute, die sich berechtigte Hoffnungen machen, zu spielen“, sagt Hecking. (BRZ13/JAN.01385 Braunschweiger Zeitung, 05.01.2013, Braunschweiger Zeitung. - Sachgebiet: Sport, Originalressort: Sport; Perisic-Transfer kurz vor dem Abschluss)

42) $\{\{0\}\}$

Außerdem gebe es eine Selbstverpflichtung der Industrie, sagt VfA-Sprecher Rolf Hömke. Man müsse nur sicherstellen, dass sich die Unternehmen daran halten.

Doch genau das ist das Problem. Was ist, wenn sie es nicht tun? „Uns ist nicht bekannt, dass es in einem Land Bußgelder oder dergleichen gäbe für nicht rechtzeitige Publikation von Studienergebnissen“", räumt Hömke ein. (BRZ13/MAR.06902 Braunschweiger Zeitung, 19.03.2013, Braunschweiger Zeitung. Originalressort: 1Antw; \&\#8222;Unnötiges Risiko für die Patienten\&\#8220;)

43) $\{\{0\}\}$

Wenn es eine berufsbildende Schule ist, ist dies natürlich auch sinnvoll und wahrscheinlich gleich attraktiv wie ein Lehrberuf.

Reinhard Deutsch, Malermeister in Jennersdorf: 
Das Problem ist: Es bleiben relativ wenige gute Schüler. Der Großteil der Jugendlichen, der nach der Pflichtschule an weiterführende Schulen geht, hätte früher einen Handwerksberuf erlernt. (BVZ08/FEB.01441 Burgenländische Volkszeitung, [Wochenzeitung], 20.02.2008, Nr. 08, Jg. 2008, Oberwart, S. 3. Originalressort: THEMA DER WOCHE; Lebschy, Günther: Ist die Lehre nun wieder attraktiver?)

44) $\{\{0\}\}$

Für das kommende Heimspiel gegen Purbach ist Bernd Kager mit der fünften Gelben gesperrt. Für ihn dürfte Peter Lehner, der gegen Oberpullendorf geschont wurde, nachrücken. Manager Michael Benedek rechnet damit, dass in den kommenden Wochen noch andere gelbgefährdete Spieler zwangspausieren müssen: „Aber alles kein Problem! Wir haben unsere Jungen, die in die Bresche springen können."Wie beispielsweise Thomas Herrklotz, der gegen Oberpullendorf statt des verletzten Sinisa Baksa stürmte.

PURBACH - KOHFIDISCH 1:1. Die Kohfidischer entführten bei Titelaspirant Purbach einen Punkt. (BVZ10/MAI.00855 Burgenländische Volkszeitung, 13.05.2010, Oberwart. Sachgebiet: Sport, Originalressort: FUSSBALL; OWZRED: Erfolge in der Fremde)

45) $\{\{0\}\}$

Selbstentzündung: Stroh geriet plötzlich in Brand

Gegen 7.42 Uhr wurden die Florianis zum Einsatzort gerufen, wie Gerald Kammerhofer erklärt: „Die Strohballen dürfte durch Selbstentzündung in Brand geraten sein. Darin finden stets Gärprozesse statt, normalerweise sind die Temperaturen aber kein Problem. Tritt aber etwa zu viel Sauerstoff hinzu oder wird es zu heiß, so kann dies zu brennen beginnen." Einsatz nach Ballabend: 35 Mann am Einsatzort (BVZ13/JAN.01807 Burgenländische Volkszeitung, 17.01.2013, Neusiedl. - Sachgebiet: Lokales, Originalressort: Lokales; NSDRED: Strohballen in Brand)

46) $\{\{0\}\}$

Ein Parallelrechner besteht heute aus 16 bis etwa 10000 solcher Mikrorechner, die über spezielle Verbindungseinrichtungen kommunizieren. Solche Parallelrechner sind nicht nur weitaus leistungsfähiger, sondern auch wesentlich kostengünstiger. Das Problem dieser Parallelrechner, das bisher ihrer Verbreitung im Wege steht, ist die für normale Benutzer zu komplizierte Programmierung. Die Parallelrechnerentwicklung erfordert daher nicht nur Innovationen bei der Hardware und der Systemsoftware, sondern auch für die Benutzung, was vor allem die Entwicklung neuartiger Programmiermodelle erfordert. Dies ist ein wichtiges Forschungsthema, da auch das leistungsfähigste Rechnersystem nichts nützt, wenn die Benutzer damit nicht umgehen können. (C93/AUG.02886 COMPUTER ZEITUNG, [Wochenzeitung], 26.08.1993, Nr. 34, Jg. 28, S. 15. - Neue Impulse für die Parallelrechnertechnik, [Bericht])

47) $\{\{0\}\}$

CZ: Warum kommt der NC, bestehend aus marktüblichen Standardbauteilen, so spät?

Dies: Die Schwierigkeit ist ja nicht, eine Network Station zu bauen - vorausgesetzt, man hat sich auf einen Satz von Standards geeinigt. Das Problem in einer solchen "Thin-client"-Welt ist vielmehr die Server-Software, die anders als bisher die benutzerindividuellen Arbeitsumgebungen übergreifend verwalten muß. Wenn jemand mit einem Browser vom PC aus durch die Homepages surft, kann er seine Favoriten in ein Kurzwahlverzeichnis aufnehmen. So entsteht eine Benutzerumgebung mit Dateien und verfügbaren Programmen in individuellen Einstellungen, die lokal gespeichert ist. (C96/DEZ.04788 COMPUTER 
ZEITUNG, [Wochenzeitung], 05.12.1996, Nr. 49, Jg. 31, S. 14 - Interview. - Bob E. Dies, IBM, [Interview])

48) $\{\{0\}\}$

$\mathrm{Ob}$ man dann noch mental die richtige Fitness hat, ist eine andere Frage. Da liegt das entscheidendere Gewicht, das auf die Leistung drückt. Ich habe bislang noch keine psychischen Ermüdungserscheinungen an mir festgestellt, bin aber darauf vorbereitet, dass dieses Problem irgendwann an mir knabbern wird.

FOCUS: Psychisch und physisch sind Sie noch so stark wie ein Twen? (FOC03/APR.00208

FOCUS, 14.04.2003, S. 132-134, Sachgebiet: Sport, Originalressort:

MODL/SPRT/Sport; "Ich bin kein Getriebener")

49) $\{\{1$ Komp.; A2 $\}\}$

Ganzheitlich betrachtet kostet das die Kunden jedoch jede Menge Geld. Da ist allein schon die Sprach- und Kulturbarriere. Mitarbeiter beispielsweise in der Buchhaltung oder der Produktion können sich scheuen, bei einem Softwareproblem einen englischsprachigen Helpdesk anzurufen. Das kann zu Ausfällen und Verzögerungen im Arbeitsalltag oder der Qualität der Produkte führen.

Was machen Sie anders? (M14/NOV.03783 Mannheimer Morgen, [Tageszeitung], 14.11.2014, Jg. 69, Stadtausgabe, S. 9. - IT-Service mit dem Menschen im Mittelpunkt)

50) $\{\{1$; für + Akk.; A1 $\}\}$

"Wir rechnen mit einer Energiebilanz, die in etwa dem Verbrauch eines 2-Liter-Autos entspricht", sagt Kampker.

Dass seine Spartransporter nur 80 Kilometer Reichweite haben und es mit ihren 61 PS gerade mal auf eine Höchstgeschwindigkeit von 85 Kilometern in der Stunde bringen, ist für die Post kein Problem. Das reicht für die Touren aus. Auch teure Schnellladetechnik brauchen die robusten Dienstwagen nicht. (FOC13/AUG.00351 FOCUS, 26.08.2013, S. 90, Sachgebiet: Wirtschaft, Originalressort: WIRTSCHAFT; Viel Post, wenig Fenster, kein Radio)

51) $\{\{0+1$; keine Real.; Subj.; kein Argument; A1 $\}\}\{\{$ SVG; P. haben $\}\}$

Die Richtlinien geben den Bundesministern seit langer Zeit vor, den Dienstwagen zur dienstlichen und privaten Verwendung nutzen zu dürfen, bei entsprechender Versteuerung der Privatfahrten gemäß einem Fahrtenbuch. Die Kanzlerin, der Vizekanzler sowie die Innen- und Verteidigungsminister dürfen sogar aus sehr einleuchtenden Sicherheitsgründen nie mit Privatwagen unterwegs sein. SPD-Kanzlerkandidat Frank-Walter Steinmeier hat jetzt ein Problem. Er hat dem Druck des vermeintlich aufgewühlten Wahlvolks nachgegeben und die Gesundheitsministerin aus dem Kompetenzteam der SPD ,,vorerst“ hinauskomplimentiert. Jetzt, wo bekannt wird, dass das halbe Kabinett den Dienstwagen im Urlaub in Anspruch genommen hat, stellt sich die Frage, ob auch Tiefensee, Gabriel oder Wieczorek-Zeul das Kompetenzteam verlassen müssen. (HAZ09/AUG.00687 Hannoversche Allgemeine, 05.08.2009; Steinmeiers Problem [Ausführliche Zitierung nicht verfügbar])

52) $\{\{0\}\}$

Beide Regelungen werden von Flüchtlingsorganisationen heftig kritisiert. Die Dublin-IIVerordnung diene allein der Abwehr von Flüchtlingen, erklärt zum Beispiel die Bundesarbeitsgemeinschaft Asyl in der Kirche. Staaten wie Deutschland schöben das Problem damit auf die Länder Südeuropas ab, die damit aber zunehmend überfordert seien: Gerade in Italien seien die Lebensverhältnisse für Flüchtlinge oft katastrophal. tof 
(HAZ13/JUN.00461 Hannoversche Allgemeine, 08.06.2013; Dublin-II-Regel [Ausführliche Zitierung nicht verfügbar])

53) $\{\{0\}\}$

DFB-KURZPÄSSE

ENTWARNUNG: Sowohl Torwart Oliver Kahn als auch Stürmer Miroslav Klose werden gegen die Türkei spielen können. Kahn ("Es gibt kein Problem") konnte trotz seiner Knieprellung die Leistungsüberprüfungen mitmachen und wird nach Michael Ballacks Ausfall erstmals wieder als Kapitän auflaufen. Klose (fiebrige Grippe) wird heute zum Team stoßen und morgen mit nach Istanbul reisen.

PAUSE: Lukas Podolski pausierte gestern wegen muskulärer Probleme bei den morgentlichen Sprinttests, stieg aber am Nachmittag voll mit ein. (HMP05/OKT.00294 Hamburger Morgenpost, 05.10.2005, S. 32; DFB-KURZPÄSSE [Ausführliche Zitierung nicht verfügbar])

54) $\{\{1$; Komp.; A2 $\}\}$

Er sagt, dieses Ziel sei in dieser kurzen Zeit nicht erreichbar. Daher mache sich unter den Studierenden eine gewisse Hilflosigkeit breit: "Auch wenn man sich noch so anstrengt, man schafft es nicht in fünf Semestern."

Den Grund sieht Markus in zu vielen Lehrveranstaltungen und massiven

Organisationsproblemen."Übungen für ein ganzes Semester wie das Studio Gebäudelehre werden an nur zwei Tagen ausgehängt. Wer da krank ist oder den Anschlag nicht findet, hat Pech, ist draußen. (N97/MAR.09276 Salzburger Nachrichten, [Tageszeitung], 03.03.1997. - 600 TU-Studenten und ein Problem: "In fünf Semestern schafft es)

55) $\{\{0\}\}$

Brisantes Spiel.

Schon bei den Verkäufen der Damenturniere in Hamburg und Berlin war "Clifford Chance" im Boot. Beim DTB will man kein Problem im "gemischten Doppel" des Präsidenten sehen. "Das hatte nur Vorteile. Wir haben nicht die üblichen Stundensätze gezahlt.

(HMP08/OKT.01893 Hamburger Morgenpost, 20.10.2008, S. 1-20; Der Tennis-Boss und sein doppeltes Spiel [Ausführliche Zitierung nicht verfügbar])

56) $\{\{0\}\}$

Doch schon bald nervt mich, dass mir dauernd Äste im Weg sind und sich in mein Gesicht drücken. Auch sitzen manche Kirschen so fest, dass ich ganze Äste mitpflücke. Solange die Kirschen auf Augenhöhe hängen, bleibt das mein einziges Problem. Doch sobald ich mich bücken oder strecken muss, wird's anstrengend. Es dauert nicht lange und ich merke die Arbeit in den Armen und im Rücken. (HMP09/JUL.02019 Hamburger Morgenpost, 20.07.2009, S. 12-13; Knallharte Arbeit für die süßen Früchtchen [Ausführliche Zitierung nicht verfügbar])

57) $\{\{1+1$; mit + einander; Subj.; A2; A1 $\}\}\{\{$ SVG; P. haben $\}\}$

Moritz auch", sagt Pfad und meint Moritz Hillebrand, den einflussreichen Eishockey-Mann bei Anschutz. "Und Moritz lügt mich nicht an."

Sollte aber doch ein wahrer Kern hinter der Geschichte stecken, "dann", so Pfad, "hätten

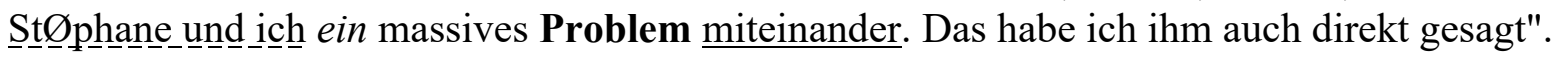
Momentan aber sei ihm das alles "viel zu viel Konjunktiv", sagt Pfad zum Abschluss noch. (HMP10/DEZ.02695 Hamburger Morgenpost, 28.12.2010, S. 37; Jetzt droht der nächste 
Umbruch Boss Pfad schlägt Alarm "Die Zeit arbeitet gegen uns" Floppt Laporte, fliegt Richer! [Ausführliche Zitierung nicht verfügbar])

58) $\{\{0\}\}$

Er sei unglücklich in London, will zurück in die Bundesliga, so war es in der englischen Zeitung "Metro" zu lesen. Mit Tottenham-Boss Daniel Levy soll es demnach bereits ein Gespräch gegeben haben. Das Problem: Unter 10 Millionen Euro würde man van der Vaart, der noch Vertrag bis 2014 hat, wohl kaum abgeben. (HMP12/JUL.03212 Hamburger Morgenpost, 04.07.2012, S. 33; Van der Vaart bittet um Freigabe [Ausführliche Zitierung nicht verfügbar])

59) $\{\{1$; attr. Adj; A2 $\}\}$

Und RSC-Torhüter Silvio Proto bestätigte: "Er hat großen Anteil am Titel."

In dieser Woche reiste Troost aus dem niederländischen Nijmegen in den Volkspark, wollte seinen Landsmann Bert van Marwijk überzeugen, mit ihm zu arbeiten. "Er hat gesagt, dass er keine Zeit für so etwas hat", sagt Troost - und erklärt stattdessen der MOPO, wie er die HSVVerlierer zu Gewinnern machen würde: "Das Problem ist kein taktisches, es ist ein mentales. Ich würde das Geheimtraining abschaffen, die Jungs brauchen die Fans. Und ich würde laute Musik beim Training laufen lassen. (HMP14/FEB.01020 Hamburger Morgenpost, 12.02.2014, S. 30; "Gebt mir 20 Minuten mit dem Team ..." [Ausführliche Zitierung nicht verfügbar])

60) $\{\{0\}\}$

So ist der Datenverkehr nicht zurückverfolgbar.

Eine junge Frau surft in einem Straßencafé mit ihrem Laptop im Internet. Mit kostenlosem WLAN kein Problem.

Der SPD-Abgeordnete Hansjörg Schmidt (HMP14/NOV.01336 Hamburger Morgenpost, 17.11.2014, S. 10; Bald gibt's Gratis-WLAN in der ganzen City [Ausführliche Zitierung nicht verfügbar])

61) $\{\{1$; Gen.; A' $\}\}$

Der Nationale Widerstandsrat des Iran hat am Sonntag den Besuch von Nationalratspräsident Heinz Fischer in Teheran in scharfer Form verurteilt. Die Oppositionsgruppe kritisierte insbesondere, dass Fischer das Mausoleum von Revolutionsführer Ayatollah Khomeini besucht und einen Kranz auf dem Grab niedergelegt habe. Fischer hält sich derzeit an der Spitze einer Parlamentarierdelegation im Iran auf und brachte nach Angaben seines Sprechers gegenüber Staatspräsident Khatami das Problem der Pressefreiheit im Iran zur Sprache. Er setzte sich für den inhaftierten Chefredakteur Hassan Youssefi Eshkevari ein und erörterte weiters die Probleme der kurdischen Minderheit im Iran.

Zwischenfall bei einer Demo (I00/OKT.63242 Tiroler Tageszeitung, [Tageszeitung], 30.10.2000. - Sachgebiet: Politik, Originalressort: Innenpolitik)

62) $\{\{0\}\}$

Angeblich soll sich der Wiener Finanzstadtrat Edlinger darunter befinden. Da der aber am Kongreß referieren soll, wird der Weltverband seine Reisekosten übernehmen. Im übrigen sieht Pramböck kein Problem, "ein Weltverband tagt nun einmal in verschiedenen Kontinenten". Man habe in Mauritius, aber auch in Südafrika ein "fundiertes Programm". (I97/JAN.01613 Tiroler Tageszeitung, [Tageszeitung], 16.01.1997. - Sachgebiet: Politik, Originalressort: Innenpolitik; Politiker-"Dienstreise" ins Urlauberparadies) 
63) $\{\{1$; Gen.; A' $\}\}$

Über weite Strecken ging es nur um zwei Personen. Ist mehr geblieben als der Eindruck direkter Konfrontation zwischen dem Älteren und dem Jüngeren, dem Einheitskanzler mit einer blaß gewordenen Bilanz und dem Medienstar als Mann wohltuender

Absichtserklärungen? Beim zentralen Problem der Arbeitslosigkeit rangen alle Wahlkämpfer um die richtigen Worte.

"16 Jahre sind genug", proklamierte der SPD-Kanzlerkandidat. Schröder machte die schlechte Stimmung im Land zu seinem wichtigsten Argument. (I98/SEP.38466 Tiroler Tageszeitung, [Tageszeitung], 26.09.1998. - Sachgebiet: Politik, Originalressort: Innenpolitik; Deutschlands Zukunft?)

64) $\{\{0\}\}$

"Viele können es gar nicht glauben, daß ich diese Arbeit umsonst mache" erzählt die Rinnerin, die Ehrenamtlichkeit und vor allem das Zuhörenkönnen werde sehr hoch eingeschätzt. Geschätzt wird die Arbeit auch vom Personal: "Die Patienten sind ruhiger und fühlen sich gut aufgehoben, wenn jemand vom Besuchsdienst bei ihnen ist" so die Aussagen einer Krankenschwester.

Wenn die Konfrontation mit Krankheit und Tod zum Problem wird, gibt es für "KlinikBrücke"-Mitarbeiter die Möglichkeit der Supervision. In regelmäßigen Abständen stehen auch Fortbildungsmaßnahmen u.a. finanziert von TILAK und Land auf dem Programm. Vereinsobfrau Hribar hegt indes schon Expansionspläne: "Besuchsdienste sollten auch an anderen Krankenhäusern und Kliniken organisiert werden". (I99/MAR.10862 Tiroler Tageszeitung, [Tageszeitung], 23.03.1999. Originalressort: Tirol aktuell; Einfach nur zuhören)

65) $\{\{0\}\}$

Zusätzlich gibt es pro Jahr etwa 1000 Einsätze, bei denen Verstopfungen in privaten Haushalten behoben werden müssen.

Eine groß angelegte Informationskampagne soll die Grazer nun darüber aufklären, was man nicht ins WC werfen darf. Die Rückseite der April-Ausgabe der Zeitschrift BIG bietet eine übersichtliche Information zu dem Thema, und auch die Videowand am Jakominiplatz und Steiermark 1 werden auf das Problem aufmerksam machen. "Es ist wie bei der Mülltrennung - man muss das Bewusstsein in der Bevölkerung wecken", so die zuständige Stadträtin Maxie Uray- Frick. Für den Akutfall hat man eine Ver stopfungs-Hotline eingerichtet: \& $(0664) 49$ 83 872. (K00/APR.27759 Kleine Zeitung, [Tageszeitung], 06.04.2000. Originalressort: Graz; Müllentsorgung über das WC gefährdet die Umwelt)

66) $\{\{0\}\}$

Durchgreifen ist nötig

Ob Leistungen für ausländische Flüchtlinge oder Sozialhilfe für einheimische Bedürftige wenn Fälle von Abzockern bekannt werden, die auf Staatskosten leben, sind viele Bundesbürger empört. Da dieses Problem jetzt in verstärktem Maße auftritt, ist ein Durchgreifen vonnöten.

Ing. Leo F. Jungmeier, Villach (K00/NOV.80120 Kleine Zeitung, [Tageszeitung], 02.11.2000. Originalressort: Leserbriefe, [Leserbrief])

67) $\{\{0\}\}$

Es werden Ankündigungen gemacht, die zu falschen Erwartungen führen.

Was sind die Folgen der enttäuschten Erwartungshaltung? 
LACHER: Dieses Problem spürt die gesamte Gesellschaft. Aber auch unser Unternehmen hätte weit größere Exportmöglichkeiten, wenn wir wenigstens bei den Exportfinanzierungen durch die Kontrollbank die gleichen Bedingungen wie unsere Mitbewerber aus anderen europäischen Ländern hätten.

MIT PAGO-DIREKTOR GERHARD LACHER SPRACH ADOLF WINKLER

(K97/JAN.07811 Kleine Zeitung, [Tageszeitung], 31.01.1997. - Sachgebiet: Wirtschaft, Originalressort: Wirtschaft; Behinderung statt Stütze bei Export)

68) $\{\{1 ;$ attr. Adj.; A1 $\}\}$

Zielscheiben

HARALD HOFER

Schimpfen und meckern ist kein typisches Hartberger Problem - nur hier hat man eine verfeinerte Variante gefunden. Hier schafft man es, aus einem Opfer gleichzeitig einen Täter und umgekehrt zu machen.

Zielscheibe ist der Hartberger Tourismusverband, dem von seinen rund 400 zwangsverpflichteten Beitragszahlern Inaktivität vorgeworfen wird. (K97/SEP.72889 Kleine Zeitung, [Tageszeitung], 25.09.1997. Originalressort: Hartberg; Zielscheiben)

69) $\{\{1$; Komp.; A2 $\}\}$

Der lange und kalte Winter hat zu einem rund vierwöchigen Rückstand auf den ohnehin engen Zeitplan geführt, und dieser Rückstand liess sich leider nicht mehr aufholen. Die Baukommission wollte in dieser Situation nicht an einem unrealistischen Ziel festhalten, vor allem auch im Hinblick auf die beteiligten Unternehmen und im Interesse einer weiterhin sorgfältigen Bauausführung.

Wegen der Verschiebung entstehen keine Raumprobleme und keine Kostenfolgen. Die Pavillons bleiben einfach bis zu den Herbstferien stehen, denn der Umzug soll erst dann erfolgen, damit der Schulbetrieb nicht leidet. Dies hat wiederum zur Folge, dass die Umgebungsarbeiten erst zu jenem Zeitpunkt in Angriff genommen werden können. (A09/JUN.08240 St. Galler Tagblatt, [Tageszeitung], 26.06.2009, Nr. 146, S. 47. Sachgebiet: Regionales / Regionales, Originalressort: WV-Region-Wil; a.buechi: Bezug wird verschoben)

70) $\{\{0\}\}$

Aus allen Berufs- und Altersschichten setzt sich das Team zusammen, das pro Jahr über 100.000 Stunden im Einsatz steht. Bei ihren 15.000 Ausfahrten pro Jahr legen die RotkreuzFahrzeuge rund eine halbe Million Kilometer zurück. "Weil aber unsere Tätigkeiten immer mehr ausgebaut werden, ist ein erhöhter Personalbedarf im Rettungswesen notwendig", nennt der Rettungskommandant des Bezirkes, Heinz Gritzner, das Problem: "Wir brauchen dringend neue ehrenamtliche Mitarbeiter!" Ein Engpaß, der sich bislang noch nicht dramatisch ausgewirkt hat. "Die Leute dürften sich die Arbeit bei uns zu kompliziert und schwierig vorstellen", meint der Bezirksschulungsreferent Werner Orasch. (K99/FEB.09430 Kleine Zeitung, [Tageszeitung], 06.02.1999. Originalressort: Mittelkärnten; Rotes Kreuz sucht dringend ehrenamtliche Mitarbeiter)

71) $\{\{1+1$; mit - Pronadv.; Subj.; A2; A 1$\}\}$

Wer steht hinter dem GI?

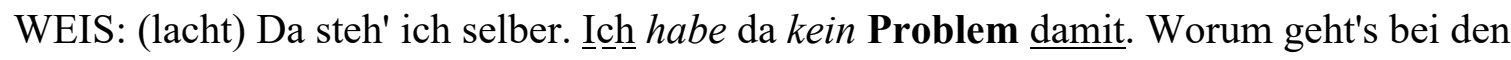
ganzen Interventionen? Es geht darum, daß wir es mit Politikern zu tun haben, die in ORFGremien sitzen und die naturgemäß alles tun, um ihrer Partei zu Geltung zu verhelfen. 
(K99/AUG.57911 Kleine Zeitung, [Tageszeitung], 05.08.1999. Originalressort: Hintergrund; "Es geht nicht darum, dass wir entpolitisiert werden")

72) $\{\{0+1$; keine Real.; Subj.; kein Arg.; A1 $\}\}\{\{$ SVG; P. haben $\}\}$

Verkäuferinnen, Fensterputzer, Taxifahrer, Aushilfskellner und -lehrer, Sargträger und

Zeitungsboten, Studenten und Hausfrauen - sie alle lasten es ihm an, aus ihrer kleinen

Zuverdienstoase vertrieben zu werden. Sie fühlen sich einfach unfair behandelt - tödlich für eine Partei, die vorgibt, für die Arbeitnehmer zu kämpfen.Walter Riester sei, so meinte einmal sein Parteigenosse Peter Glotz, ein wenig naiv. Aber der eher treuherzig wirkende Sch hat ein anderes Problem: Er ist nicht aus dem Holze des Politischen geschnitzt. Sein Mikrokosmos war die gewerkschaftliche Welt, in der taktierende und trickreiche Unterhändler das Geben und Nehmen am Verhandlungstisch mit den Arbeitgebern ritualisieren. Derartige Taktiken findet man häufiger im diplomatischen Gewerk der Außenpolitik. (L99/MAI.23094 Berliner Morgenpost, [Tageszeitung], 10.05.1999, S. 3. Originalressort: IM BLICKPUNKT; Von Peter Gillies: Nicht aus dem Holz des Politischen)

73) $\{\{0\}\}$

Bergholz-Rehbrücke - 25000 Kraftfahzeuge fahren täglich durch den Ort Bergholz-

Rehbrücke vor den Toren der Landeshauptstadt. Anwohner klagen seit Jahren über Lärm, Gestank und Erschütterungen. "Der Verkehr ist unser größtes Problem", sagt auch Bürgermeisterin Annerose Hamisch-Fischer (PDS).Doch sicher ist: Die Ortsumgehung für Drewitz (L 79), die auch Bergholz-Rehbrücke entlasten soll, wird für etwa 100 Millionen Mark gebaut. Die Pläne sind noch bis 28. Juli im Amt Rehbrücke ausgelegt und am 8. Juli findet dazu eine Bürgerversammlung statt.Heftige Kritik gibt es bereits an der Trassenführung: Entgegen ursprünglicher Planung führe sie quer durch die Nuthewiesen mit geschützten Feuchtbiotopen, darunter ein Niedermoor, einen Meter tief. Die Gemeindevertretung und eine Bürgerinitiative fühlen sich vom Brandenburgischen Straßenbauamt getäuscht: "Im Rahmen der Umweltverträglichkeitsstudie hatten wir einen Kompromiß gefunden, von dem jetzt keine Rede mehr ist", klagt die Bürgermeisterin.

(L99/JUL.39610 Berliner Morgenpost, [Tageszeitung], 02.07.1999, S. 36.

Originalressort: 1; BM: Die Furcht vor dem Verkehrskollaps)

74) $\{\{0\}\}$

Die Praxis von Doktor Stein war rappelvoll. Andreij hatte sein Mäppchen und seinen Zeichenblock mit, legte sich auf den Boden des Wartezimmers und malte. Außer, dass alle über ihn wegsteigen mussten, war er kein Problem.

Nach einer Stunde fiel Sandra ein, dass nun bald Ralf und Dimitrij zurückkommen würden, und sie jetzt eigentlich Mittagessen kochen müsste. »Ich fahr jetzt heim«, sagte sie zu Nina. (DIV/SRS.00001 Ramge, Sigrid: Strahlenkinder. 20 Jahre nach Tschernobyl. Föritz: Amicus, 2006, S. [S. 93])

75) $\{\{1 ;$ attr. Adj.; A1 $\}\}$

Lediglich in der konservativen EVP-Fraktion gibt es noch ein Zögern, die anderen großen politischen Gruppierungen im EP wollen, dass sich die EU an einer Neuansiedelung der Guantanamo-Gefangenen beteiligt. "Wir wollen Sicherheitsbedenken oberste Priorität geben", erklärte Hartmut Nassauer im Namen der EVP-Fraktion. Der Vorsitzende der SPE-Fraktion, Martin Schulz, hingegen will die Grundrechte der Gefangenen höher ansiedeln und meinte, es sei "die falsche Botschaft, wenn wir in dem Moment, wo ein Unrechtszustand beendet wird, sagen: Das können wir nicht". "Europa kann sich nicht zurücklehnen und sagen, das ist ein 
amerikanisches Problem", sagte seinerseits Graham Watson. "Es wäre falsch, Nein zu sagen", so der Vorsitzende der Liberalen weiter. (LTB09/FEB.00101 Luxemburger Tageblatt, 04.02.2009, Originalressort: EU - EU-INSTITUTIONEN; "Es wäre falsch, Nein zu sagen")

76) $\{\{0\}\}$

Gerard Lopez in "Die Welt"

"Bernie ist nicht das Problem"

In einem Interview mit Die Welt (welt.de) äußerte sich der Vorsitzende des Lotus-F1-Teams, Gerard Lopez, zu seiner Präsenz in der Formel 1 und deren Zukunft - natürlich nicht ohne zu Beginn des Gesprächs noch einmal auf den Premieren-Sieg von Abu Dhabi zurückzukommen: "Wir haben das ganze Jahr schon mit einem Kimi-Sieg gerechnet. (...)Gegen Ende der Saison wuchsen jedoch unsere Zweifel, weil die ganzen Teams, die um die WM kämpfen, extrem aufgerüstet haben. (LTB12/NOV.00929 Luxemburger Tageblatt, 17.11.2012, "Bernie ist nicht das Problem")

77) $\{\{0\}\}$

In einem Leitartikel vom 28. November 1964, übertitelt mit "Für eine Deontologie der Presse", äußert sich der neue Tageblatt-Direktor Jacques Poos, erst seit wenigen Monaten im Amt, in verärgertem und polemischem Tonfall über das journalistische Schaffen des Républicain Lorrain:

"Jene, die es hauptsächlich angeht, verstehen wohl eher Französisch. Das Problem, das wir uns hier aufzuwerfen genötigt sehen, geht jedoch alle an. (\&) Jene Presse (\&), die Tatbestände wahllos und ziellos ausschlachtet' und sich darüber in unkontrollierbaren Gerüchten und zusammenhanglosen Mutmaßungen ergeht, verstößt groberweise gegen die drei (\&) Prinzipien [der Presse; Anmerkung des Autors: 1. Intellektueller Gedankenaustausch, 2. Informationsaufgabe, 3. Interpretation und Kommentar]. Sie benützt die Freiheit der Presse zu einem antisozialen Zweck. Sie opfert das Recht auf Information einer eigennützigen Geschäftemacherei. (LTB13/OKT.03205 Luxemburger Tageblatt, 31.10.2013, ohne Titel)

78) $\{\{1+1$; mit + Dat.; Subj.; A2; A1 $\}\}\{\{$ SVG; P. haben $\}\}$

Das Eidgenössische Finanzdepartment (EFD) hatte bereits klargemacht, dass Bern zunächst vor allem den Austausch mit Ländern prüfen will, "mit denen enge wirtschaftliche und politische Beziehungen bestehen" und die "aufgrund ihres Marktpotenzials für die Schweizer Finanzindustrie als wichtig und zukunftsweisend erachtet werden". Mit dem Start des AIA sei dabei nicht vor $2018 \mathrm{zu}$ rechnen. Lichtenstein dagegen hat mit der Unterzeichnung kein Problem, nachdem das Umsteuern in Richtung auf einen transparenten Finanzsektor lange beschlossene Sache ist.

Appell an (LTB14/OKT.02652 Luxemburger Tageblatt, 30.10.2014, Abschied vom Bankgeheimnis)

79) $\{\{0\}\}$

Verfolgte Schriftsteller

Die Verfolgung von Schriftstellern und Verlegern nimmt weltweit zu und gewinnt für die Arbeit der internationalen Autorenvereinigung PEN laut dem westdeutschen PENGeneralsekretär Johano Strasser immer mehr an Bedeutung. Im Vorfeld des morgen beginnenden Kongresses der Schriftstellervereinigung in Dresden wies Strasser noch auf ein anderes Problem hin: "Wenn wir erfolgreich waren und Schriftsteller freibekommen haben, stellt sich zunehmend die Frage, was mit den Autoren in unserem Land geschehen soll, 
getrennt von ihrer Sprache." Es müsse dafür gesorgt werden, daß sie

Publikationsmöglichkeiten erhielten, damit sie als Schriftsteller leben könnten.

dpa (M98/OKT.84435 Mannheimer Morgen, [Tageszeitung], 28.10.1998, Jg. 53. -

Sachgebiet: Kultur, Originalressort: Feuilleton; Verfolgte Schriftsteller, [Feuilleton])

80) $\{\{0\}\}$

Pläne aus der Mitte der neunziger Jahre, ein weiteres Haus in Mannheim zu errichten, sind nicht völlig vom Tisch, sagte van Diedenhoven im Gespräch mit unserer Zeitung. In Walldorf sei mit der momentanen Erweiterung das Ende der Fahnenstange erreicht, es stünde kein weiteres Gelände mehr zur Verfügung. Außerdem seien zwei Häuser im selben Einzugsgebiet kein Problem, in Stuttgart beispielsweise gebe es eine ähnliche Situation. Wenn sich die Situation weiterhin so positiv entwickelt wie im Moment, sei der Standort Walldorf in fünf bis sechs Jahren kapazitätsmäßig ausgereizt. Spätestens dann müsse man über Alternativen nachdenken. (M00/MAI.16512 Mannheimer Morgen, [Tageszeitung], 04.05.2000, Jg. 55. Sachgebiet: Wirtschaft, Originalressort: Wirtschaft; Von unserem Redaktionsmitglied Ulla Cramer: Ikea richtet sich neu ein)

81) $\{\{0\}\}$

Im Vatikan gilt es als schweren Tabubruch, solche Sätze öffentlich zu äußern. Der Vorsitzende der Bischofskonferenz, Karl Lehmann, hatte einmal den Ärger des Vatikans erregt, weil er über die theoretische Möglichkeit zum Papst-Rückzug auch nur laut nachgedacht hatte.

Zwei "Schulen" gibt es zu dem Problem im Vatikan. Die einen sagen, gerade dieser Papst trete niemals zurück, viel zu willensstark sei der Pole. "Es gibt keinen pensionierten Papst", soll er einmal vor Jahren gesagt haben. (M00/DEZ.80886 Mannheimer Morgen, [Tageszeitung], 27.12.2000, Jg. 55. - Sachgebiet: Politik, Originalressort: Politik; Von dpa-Korrespondent Peer Meinert (Rom): Noch nicht alle Träume erfüllt)

82) $\{\{0\}\}$

Spruch des Tages

"Kein Problem, von mir aus können die Düsseldorfer Peter Maffay hören, wenn sie dann besser spielen. Nur darauf kommt es an" - Campino, Sänger der Punkband Tote Hosen, die gestern als neuer Hauptsponsor des einstigen Renommierklubs Fortuna Düsseldorf präsentiert worden ist. Fortuna ist inzwischen bekanntlich in die Regionalliga abgestiegen.

(M01/JUL.51061 Mannheimer Morgen, [Tageszeitung], 12.07.2001, Jg. 56. - Sachgebiet: Sport, Originalressort: Sport; Spruch des Tages)

83) $\{\{0\}\}$

Aufgrund arbeitsrechtlicher Bestimmungen wird das Gros der zu fast 100 Prozent aus

Osteuropa stammenden Arbeiter jedes Jahr zum selben Termin nach Lampertheim einbestellt. Der Nachteil: Geht die Ernte früh los, sind die Helfer noch nicht da, geht sie spät los, sind sie wieder weg, bevor die letzten weißen und grünen Stangen eingebracht sind. Ein jedes Jahr wiederkehrendes Problem, das die Bauern genauso einkalkulieren wie Regenwetter.

Während Steinmetz' Erntehelfer alle aus Polen kommen, sind 70 Prozent der Arbeiter von Willi Billau Rumänen, der Rest Polen. "Ich habe seit elf Jahren einen festen Stamm", berichtet der stellvertretende Vorsitzende des Kreisbauernverbands. (M02/APR.26540 Mannheimer Morgen, [Tageszeitung], 09.04.2002, Jg. 57. Originalressort: Lokal Bürstadt / Biblis; Von unserem Redaktionsmitglied Alexandra Schorre: Leckermäuler haben Lust auf Lampertheimer Spargel) 
84) $\{\{1$; Inf.-Satz; A` $\}\}$

Warum hat das noch keiner versucht?

SPRENGLER: Für mich ist das alles denkbar. Es ist aber nicht das Problem, so etwas zu versuchen. Das Problem ist, dass man dabei an Widrigkeiten stößt, die man stadtintern lösen muss. Dass die Pop-Elite auf dem Marktplatz auftritt, Motto "Mannheim deluxe", so etwas hätte ich schon gern. (M02/JUL.55122 Mannheimer Morgen, [Tageszeitung], 24.07.2002, Jg. 57. Originalressort: Lokal Mannheim; Von unserem Redaktionsmitglied Georg Spindler: "Bei meinen Konzerten werden keine Bands verheizt")

85) $\{\{0\}\}$

Dies ist zwar keine richtige Lösung. Aber wenigstens kehrt für wenige Monate eine Besserung ein", sagte Oberstudiendirektor Robert Wunn von der Berufsbildenden Schule Technik II. Eine Wirkung von der Plakatserie verspricht sich auch Schulleiterkollege Wilhelm Kirsch von der BBS Technik I. "Allein kann die Schule das Problem aber nicht lösen." Dies sei ein Zeichen des allgemeinen Werteverfalls. (M02/DEZ.94092 Mannheimer Morgen, [Tageszeitung], 14.12.2002, Jg. 57. Originalressort: Lokal Ludwigshafen; Von unserem Redaktionsmitglied Thomas Schrott: Sauberkeit in Schulen soll sich bessern)

86) $\{\{1$; Gen.; A' $\}\}$

Selbst am Vorabend eines Rücktritts würde er diesen nicht bestätigen, hat der machtbewusste Ministerpräsident einmal betont. Der Abgeordnete Andreas Hoffmann etwa sieht in dieser Botschaft "alle Chancen für einen vorzeitigen Wechsel angelegt".

Bleibt das Problem der potenziellen Nachfolger. "Unser politisches System taugt nicht für einen planmäßigen Generationswechsel", erklärt ein langgedienter Abgeordneter. Die Kandidaten müssten sich eigentlich profilieren. (M03/DEZ.87857 Mannheimer Morgen, [Tageszeitung], 31.12.2003, Jg. 58. Originalressort: Südwest; Von unserem Korrespondenten Peter Reinhardt (Stuttgart): Des Teufels Jahr liest sich gar wechselhaft)

87) $\{\{0\}\}$

Befreit von jahrzehntealter Patina, bezaubert das Zusammenspiel von weißem Sandstein in den Obergeschossen und rotem Sandstein im Kellerbereich. Die seitlichen Backsteinwände wurden abgestrahlt.

"Ein großes Problem stellte die Restaurierung der Original-Jugendstilerker dar. Zum einen erfüllte die Einfachverglasung nicht mehr die Ansprüche auf komfortables Wohnen, zum anderen war es schwierig, kompetente Fachhandwerker für die Restaurierung und gleichzeitige Verbesserung der Wohnansprüche zu finden", erklärt Annelie Steiner. In joint venture-Manier konnten schließlich die Firmen Rutsch (Meckesheim) und Meysen (Heidelberg) eine Lösung durch Setzen eines zweiten Fensters vor die alten Holzfenster mit den kunstfertig restaurierten Bleiverglasungen anbieten, die sowohl den Vorgaben des Denkmalschutzes als auch dem Wunsch nach modernem Wohnkomfort entsprach.

(M04/SEP.62004 Mannheimer Morgen, [Tageszeitung], 08.09.2004, Jg. 59. Originalressort: Stadtteilausgabe Mitte; Von unserer Mitarbeiterin Sylvia Osthues: Vom Keller bis unter den Dachstuhl reiner Jugendstil)

88) $\{\{0\}\}$

"Das ist unsere Anschubfinanzierung." Auch haben die Gewerkschaften den laufenden Tarifvertrag nicht gekündigt und dürfen nicht streiken.

Erstmals beteiligen sich die Länder nicht an den Verhandlungen, obwohl fast eine Million der bundesweit 2,6 Millionen Beschäftigten im öffentlichen Dienst (ohne Beamte) bei den 
Ländern arbeiten. Das ist ein Problem, denn selbst wenn in Potsdam ein vorbildliches Tarifrecht herauskommt - es gilt für Angestellte des Bundes und der Städte - nicht aber für Landesbedienstete.

Schon seit zwei Jahren verhandeln die Beteiligten hinter den Kulissen. Alle Seiten haben eingesehen, dass das heutige Tarifrecht von gestern ist. (M05/FEB.10224 Mannheimer Morgen, [Tageszeitung], 08.02.2005, Jg. 60. - Sachgebiet: Politik, Originalressort: Politik; Von unserem Redaktionsmitglied Heiko Brohm: Mehr Geld für mehr Leistung)

89) $\{\{1$ Komp.; A2 $\}\}$

Finanzdecke hängt zu tief

Im nächsten Schuljahr wird die Hochsteig mit 43 Kindern wieder «übervoll» und vermehrt müssen Kinder abgewiesen werden. Wie alle Sonderschul-Institutionen im Kanton hat auch das Schulheim Hochsteig mit Budgetproblemen zu kämpfen. Das Bildungsdepartement verlangt von allen Institutionen Sparanstrengungen und hat die Budgets gekürzt. Für das Schulheim Hochsteig bedeutete dies: Im 2009 wurde das Budget dreimal zurückgewiesen, jedes Mal mussten erneut Kürzungen vorgenommen werden, erläuterte Heimleiter Markus Egger: "Wir sehen im Moment keine andere Möglichkeit, als anstehende Unterhaltsarbeiten an den Gebäuden zurückzustellen, dies im Wissen, dass später höhere Kosten anfallen.

(A10/MAI.01454 St. Galler Tagblatt, 05.05.2010, Nr. 103, S. 35. - Sachgebiet: Regionales, Originalressort: TT-Neutoggenburg; h.kugler: «In Regelklassen nicht therapierbar»)

90) $\{\{0\}\}$

Der Mann hatte mit seiner Ankündigung, allen 3700 Sabines aus dem belgischen Telefonbuch einen Brief zu schreiben, Schlagzeilen gemacht. Ein in Zeitungen publiziertes Foto, das ihn mit seiner Urlaubsbekanntschaft auf Kuba zeigt, ließ bei den Bürgern von La Glanerie keinen Zweifel: Das sei die Betreiberin der einzigen Tankstelle samt Kneipe und Supermarkt im Ort, meinten sie. Doch dann gab es ein unüberwindbares Problem: "Ich bin nicht die Frau auf dem Foto, ich sehe ihr nur ähnlich", sagte die mutmaßliche Gesuchte der Zeitung "Het Laatste Nieuws". "Ich bin auch in Kuba gewesen, ja. Aber ich kenne den Kanadier nicht." Zu einem Treffen sei sie nicht bereit. (M06/FEB.08929 Mannheimer Morgen, [Tageszeitung], 02.02.2006, Jg. 61. Originalressort: Aus aller Welt; Urlaubsliebe wiedererkannt)

91) $\{\{1$; Komp.; A2 $\}\}$

Damit dürfte der Däne den Kampf um einen Stammplatz im offensiven Mittelfeld vorerst verloren haben.

Gleiches gilt für den ebenfalls angeschlagenen Rechtsverteidiger Peter Pekarik. Wegen Leistenproblemen absolvierte der Slowake gestern zusammen mit Makoto Hasebe (Innenbanddehnung) nur ein Sondertraining unter der Anleitung von Reha-Coach Wolfhard Savoy.

Damit stehen Trainer Armin Veh im Spiel gegen die Spanier 23 gesunde Akteure (darunter 3 Torhüter) zur Verfügung. Die erste Elf sieht derzeit so aus: Benaglio - Riether, Simunek, Barzagli, Schäfer - Ziani, Josué, Gentner - Misimovic - Grafite, Dzeko. (BRZ09/JUL.25723 Braunschweiger Zeitung, 27.07.2009; Ohne Kahlenberg heute im Test gegen Mallorca [Ausführliche Zitierung nicht verfügbar])

92) $\{\{1 ;$ dass-Satz; A` $\}\}\{\{$ +bestehen dabei $\}\}$

Bereits heute kann sich der Einsatz der kleinen Kraftwerke in Mehrfamilienhäusern rechnen, wie Hohmann erläutert. Dies müsse im Einzelfall errechnet werden. Für Vermieter bestehe 
dabei allerdings das Problem, dass sie die Investitionskosten nicht an die Mieter weitergeben dürfen - obwohl diese davon profitieren. gms

Die Mannheimer MVV testet das Modell Kraftwerk im Keller seit Ende 2006 in einem Pilotprojekt mit 20 ausgewählten Kunden. (M07/APR.01260 Mannheimer Morgen, [Tageszeitung], 07.04.2007, Jg. 62, Stadtausgabe. - Sachgebiet: Wohnen, Originalressort: Immobilien / Wohnungen; Das eigene Kraftwerk im Keller)

93) $\{\{0\}\}$

Der Hinweis auf die schöne Erinnerung an die ehemaligen Flussbäder, die ich in meiner Jugend selbst oft besucht habe, geht an der Realität vorbei. Hätte es zu diesen Zeiten schon das Auto als Beförderungsmittel für Jedermann gegeben, wären die Flussbäder schon damals nicht möglich gewesen. An diesem Problem scheitert ja auch die Strandbadwiederaufwertung seit vielen Jahren, es sei denn, man würde dort den halben Waldpark abholzen. So blieben für die nun mal nötigen Autoabstellplätze beim geplanten „Riwwerside“ nur die Wiesen im Schlossgarten umzuwandeln, die dann allerdings schnell von den Studenten der Uni und berufstätigen Pendlern belegt wären. Das Auto hat uns viel Freizügigkeit ermöglicht, aber auch viel Lebensqualität genommen, womit wir uns abfinden müssen. (M08/MAR.16666 Mannheimer Morgen, [Tageszeitung], 03.03.2008, Jg. 63, Stadtausgabe, S. 24. Sachgebiet: Leserbriefe, Originalressort: Leserforum; Parkproblem unlösbar, [Leserbrief])

94) $\{\{0\}\}$

„Nur ein voller See ist ein toller See“, sagt Liane Makowski. Ihr Hotel „Talblick“ ist derzeit, obwohl August, alles andere als ausgebucht. „Der niedrige Wasserstand ist ein echtes Problem“, sagt sie. „Die Bootsverleiher und Segelschulen sitzen nächste Woche buchstäblich auf dem Trockenen. Und das betrifft natürlich auch alle Hotels und Pensionen.“

(M08/AUG.65090 Mannheimer Morgen, [Tageszeitung], 21.08.2008, Jg. 63, Südhessen Morgen (Lampertheim), S. 6. - Sachgebiet: Regionales, Originalressort: Hessen; Von dpa-Korrespondent Chris Melzer: \&\#8222;Für uns eine Katastrophe\&\#8220; [Bericht [Bericht ; Nachrichten]])

95) $\{\{0\}\}$

Welches ist das dringlichste Problem, vor dem Bolivien noch steht?

Kiefer:

Es ist meine persönliche Sicht, aber ich halte die Umweltzerstörung für ein großes Problem. Vor allem im Norden und Osten des Landes werden noch immer Regenwald und Weideland abgebrannt. Das sind schon mittlere bis große Umweltkatastrophen, die sich jedes Jahr abspielen. (M09/JUL.51253 Mannheimer Morgen, [Tageszeitung], 03.07.2009, Jg. 64, Stadtausgabe, S. 3. - Sachgebiet: Weltwissen, Originalressort: Welt und Wissen; Von unserem Redaktionsmitglied Fabian Busch: \&\#8222;Indigene Völker fühlen sich jetzt ernst genommen\&\#8220;, [Bericht [Bericht ; Reportage]])

96) $\{\{0\}\}$

Ganzheitliche Prozesse seien beim Lernen aber von Bedeutung, das Schlagwort Kopf-HandHerz vor allem im Werken.

Jeder Schüler habe Wünsche, Träume, Vorstellungen. "Die Umsetzung ist das Problem", meint Stauber. Es sei schwer, diese Spannung zu ertragen. Deshalb bilde gerade Werken ein starkes Gegengewicht zu Konsum und Passivität. (N95/MAR.10351 Salzburger Nachrichten, [Tageszeitung], 18.03.1995. - Polster und Spitzersackerl Pausenfüller für müden Schuldirektor) 
97) $\{\{0\}\}$

Die Verteidigerin der Frau hatte die Anklage aufgerufen, das Verfahren wegen der beiden ältesten Söhne fallenzulassen. Dem folgte der Staatsanwalt nicht. Der Richter betonte, das Urteil sei keine Lösung für das Problem. Er forderte die Beteiligten auf, den Streit zwischen dem Ehepaar und dem Schulamt vor dem Verwaltungsgericht klären zu lassen. Das Strafgericht sei der falsche Ort. (M11/FEB.08747 Mannheimer Morgen, [Tageszeitung], 26.02.2011, Jg. 66, Südhessen Morgen (Lampertheim), S. 5. - Sachgebiet: Regionales, Originalressort: Hessen, [Bericht [Bericht ; Nachrichten]])

98) $\{\{1$; Gen.; A' $\}\}$

Doch im Namen bleiben sie lokalpatriotisch: „Team M-M-W“ nennen sie sich nach den Anfangsbuchstaben der Vornamen.

Am 25. Februar fällt der Startschuss in Dresden, das M-M-W-Team wird sich in dieser Gegend anschließen und Zimmer schätzt, dass der Konvoi an der französischen Grenze auf 30 bis 35 Fahrzeuge angewachsen sein wird. Gängige Modelle werden es sein, bei denen auch in Afrika sich das Problem der Ersatzteilbeschaffung in Grenzen hält. Denn ein weiterer gravierender Unterschied zur Profi-Veranstaltung „Paris - Dakar“ ist, dass die Autos am Ziel einem karitativen Zweck dienen.

Die Fahrzeuge älteren Baujahrs, die die Rallyeteilnehmer für diese Reise benutzen, werden kurz nach Ankunft in der gambischen Hauptstadt versteigert. (M11/NOV.05260

Mannheimer Morgen, [Tageszeitung], 16.11.2011, Jg. 66, Rhein-Neckar, S. 15. Sachgebiet: Regionales, Originalressort: Rhein-Neckar; Von unserem Mitarbeiter Achim Wirths: Am Ziel werden die Autos versteigert, [Bericht [Bericht ; Nachrichten]])

99) $\{\{0\}\}$

Es gibt ein Kriegerdenkmal, einen Weiher und einen Fußballclub. Und es gibt eine Bahnstrecke, die den Ort teilt. Genau hier liegt das Problem. Die einzige öffentliche Straße zum Ortsteil Weißenbach führt über diese Gleise. Knapp 400 Anwohner müssen über den Bahnübergang, wenn sie mit dem Auto wegfahren wollen. (M12/MAI.04257 Mannheimer Morgen, [Tageszeitung], 14.05.2012, Jg. 67, Stadtausgabe, S. 27. - Sachgebiet: Regionales, Originalressort: Metropolregion; Von unserem Redaktionsmitglied Kevin Hagen: Eingeschränkt \&\#8211; Protest im Ort, [Bericht [Bericht ; Nachrichten]])

100) $\{\{0\}\}$

Denn eine weitere Bedingung besteht darin, dass in einer Stadt von der Größe Lampertheims sieben Geschäfte und vier Gastronomiebetriebe mindestens zwei fair gehandelte Produkte anbieten müssen. Gaststätten und Cafés seien aber oft vertraglich an bestimmte Röstereien gebunden, die auch die Kaffeemaschinen stellten, weiß Meisel. Das sei aber kein Problem, die Gastronomen könnten ihren Gästen alternativ auch fair gehandelten Tee, Kakao, Zucker, Fruchtsaft oder Gebäck offerieren. (M12/AUG.10276 Mannheimer Morgen, [Tageszeitung], 31.08.2012, Jg. 67, Südhessen Morgen (Lampertheim), S. 18. Sachgebiet: Lokales, Originalressort: Lampertheim; Von unserer Mitarbeiterin Bärbel Jakob: Auch Gastronomen gefordert, [Bericht [Bericht ; Nachrichten]]) 
1) $\{\{1$; Gen.; A1 $\}\}$

Ein Augenzeuge berichtete, er habe aus rund zehn Kilometern Entfernung gesehen, wie das brennende Flugzeug in den Sumpf krachte. Die Leichen der Passagiere waren bis zur Unkenntlichkeit verbrannt. Vertreter der uruguayischen Luftwaffe sagten, Ursache des Absturzes sei vermutlich, dass die Maschine zu schwer geworden sei, weil sich am Rumpf Eis gebildet habe. (A97/OKT.29311 St. Galler Tagblatt, [Tageszeitung], 13.10.1997, Jg. 53. Originalressort: TB-SPL (Abk.); Flugzeug abgestürzt und explodiert, [Bericht])

2) $\{\{1$; für - Pronadv.;A1 $\}\}$

Die Steurabrechnung zeigt, dass in der Gemeinde Widnau realistisch budgetiert wurde. Bei den natürlichen Personen wurde das Budget um knapp 200000 Franken unterschritten. Die Ursache dafür sind Abgänge von Steuerpflichtigen. Der Ertrag beläuft sich auf rund 12,167 Millionen Franken. Gegenüber dem Budget ergaben sich grössere Mehrerträge bei den Quellensteuern aus Vorsorge, Handänderungssteuern, Reinertrags- und Eigenkapitalsteuern sowie den Erbschafts- und Schenkungssteuern. (A98/JAN.05262 St. Galler Tagblatt, [Tageszeitung], 28.01.1998, Jg. 54. Originalressort: RT-URT (Abk.); Steuern knapp über Budget, [Bericht])

3) $\{\{1$; Gen.; A1 $\}\}$

In beiden Zügen befanden sich etwa 1700 Passagiere.

Technischer Defekt?

Die Ursache des Unglücks ist noch unklar. Der Polizeichef des Staates, Pooran Chand Dogra, schloss einen Anschlag oder Sabotage aus. Die Nachrichtenagentur PTI zitierte Mitarbeiter der Bahn, wonach in der Mitte des Zuges die Verbindung zwischen zwei der 19 Waggons abgerissen sei. (A98/NOV.76740 St. Galler Tagblatt, [Tageszeitung], 27.11.1998, Jg. 54. Originalressort: TB-SPL (Abk.); Zug rast in entgleiste Wagen, [Bericht])

4) $\{\{1$; Gen.; A1 $\}\}$

Deswegen, weil die Kelag eine viel günstigere Abnehmerstruktur hat: Sie hat mehr Großabnehmer und auch ein kürzeres Leitungsnetz, die Stadt hingegen viel lange Leitungen und sehr viele Kleinabnehmer, also viele kleine Haushalte mit einem relativ geringen Verbrauch.

Sowohl die Kelag als auch die Stadt beliefern ihre Kunden mit Erdgas, das die Kelag zur Verfügung stellt, das heißt, die Stadt kauft das von ihr benötigte Erdgas der Kelag ab, und zwar zu einem Preis, den die Kelag einheitlich für alle ihre Kunden in ganz Kärnten hat. Eine zweite Ursache der Preis- Zweiteilung ist auch die verschiedene Verrechnungsart. Die Stadt stellt - wie seit jeher - einen fixen Grundpreis und einen Arbeitspreis (Preis für die tatsächlich verbrauchte Gasmenge) in Rechnung. Das System der Kelag ist transparenter: Es gibt nur eine Zählermiete und den Preis für die tatsächlich verbrauchte Gasmenge.

(K96/NOV.27611 Kleine Zeitung, [Tageszeitung], 16.11.1996. Originalressort: Villach; Teurer als Kelag - Gaspreis der Stadt erhitzt Gemüter)

5) $\{\{0\}\}$

SIG. 
Das Unternehmensergebnis des SIG-Konzerns ist im ersten Halbjahr 1999 gegenüber dem Vorjahr um 7 Mio. Fr. auf 39 Mio. Fr. zurückgegangen. Ursache waren die wirtschaftlichen Schwierigkeiten in Osteuropa und Südostasien. Der Umsatz sank um 13 auf 923 Mio. Franken, das Betriebsergebnis reduzierte sich - auch wegen fehlender ausserordentlicher Erträge - von 72 auf 58 Mio. Franken. Für das ganze Jahr dürften die Ertragswerte voraussichtlich unter den Zahlen von 1998 bleiben. (A99/SEP.61478 St. Galler Tagblatt, [Tageszeitung], 06.09.1999, Jg. 55. Originalressort: TB-WIR (Abk.); Tiefste Arbeitslosigkeit der USA seit 29 Jahren, [Bericht])

6) $\{\{1$ Komp.;A1 $\}\}$

Pfadihütte abgebrannt. In der Nacht auf Dienstag ist oberhalb von Poschiavo eine unbewohnte Pfadfinder-Hütte aus Holz niedergebrannt. Die Brandursache ist Teil einer Untersuchung. Der Sachschaden beträgt rund 150000 Franken. (A00/NOV.74677 St. Galler Tagblatt, [Tageszeitung], 01.11.2000, Jg. 56. Originalressort: TB-OAK (Abk.); St. Gallen, [Bericht])

7) $\{\{1 ;$ Gen.; A1 $\}\}$

Die Behörden baten dringend um mehr Boote zur Verteilung von Hilfsgütern.

Abholzung schuld

Das Rote Kreuz und die Vereinten Nationen halten die rigide Abholzung in der Region für die Ursache der regelmässigen Überschwemmungen durch das Mekong-Hochwasser. Diese Entwicklung könne sich in den kommenden Jahren noch verschärfen.

Das Kinderhilfswerk Unicef fürchtet, dass bei einem Rückgang der Fluten Krankheiten wie Cholera, Malaria und Dengue-Fieber zunehmen. (A00/OKT.67425 St. Galler Tagblatt, [Tageszeitung], 02.10.2000, Jg. 56. Originalressort: TB-SPL (Abk.); Massenevakuierung in Vietnam, [Bericht])

8) $\{\{1$; für - Pronadv.; A1 $\}\}$

In den Achtziger- und Neunzigerjahren nahm die Stadtplanung erneut Anläufe, um für das Entwicklungsgebiet Platztor am Rand der Altstadt ein Projekt zu finden. Daraus wurde wieder nichts. Ursache dafür waren vor allem die komplizierten Eigentumsverhältnisse. Sie wurden aber vor einiger Zeit bereinigt. Heute gehört das Areal vor dem Platztor zum grossen Teil der Stadt St. Gallen. (A01/SEP.24659 St. Galler Tagblatt, [Tageszeitung], 01.09.2001, Jg. 57. Originalressort: TB-SGF (Abk.); reto voneschen: Wie wird das Platztor attraktiv?, [Bericht])

9) $\{\{1 ;$ für + Akk.; A1 $\}\}$

Nur wenige Meter entfernt fand zu dieser Zeit eine Chilbi statt. Mehrere explosionsartige Geräusche sorgten bei Chilbi-Besucherinnen und -Besuchern sowie Passanten für Schrecksekunden. Die Ursache für diese Geräusche waren das Platzen der Autoreifen und einer im Bereich des Autounterstandes gelagerten Gasflasche.

Zur genauen Ermittlung dieser Brandursache wurde gestern der Kriminaltechnische Dienst der Kantonspolizei St. Gallen beigezogen. (sda) (A07/AUG.00786 St. Galler Tagblatt, [Tageszeitung], 27.08.2007, Nr. 198, S. 52. - Sachgebiet: Regionales, Originalressort: RTPiazza; Hoher Sachschaden nach Brand)

10) $\{\{1$; für - Pronadv.;A1 $\}\}$

Durch vermehrte Kontrollen und sofortige Anzeigen bei entsprechenden Vorfällen wolle der Stadtrat eine harte Handschrift deutlich machen. «Wir werden Nachtruhe erzwingen und Vandalismus entschieden bekämpfen.» Trotz der Ankündigung repressiver Massnahmen 
macht der Stadtpräsident auch klar, dass Besucher der Innenstadt nicht bestimmten Lokalen zugeordnet werden können. Sollten bei Ruhestörungen jedoch Restaurants als Ursache dafür ermittelt werden, würden die Betreiber zur Rechenschaft gezogen. (rtl) (A08/DEZ.01684 St. Galler Tagblatt, [Tageszeitung], 05.12.2008, Nr. 285, S. 37. - Sachgebiet: Regionales, Originalressort: OT-rorschach; r.hirtl: Vom Hooligan-Phänomen auf dem falschen Bein erwischt worden)

11) $\{\{0\}\}$

Wir hatten beispielsweise eine Patientin, die einen Raubüberfall überstanden hat. Nach dem Überfall hatte sie das Broken-Heart-Disease. Da konnte man präzise sagen, das war die Ursache. Die Bedrohung durch den Überfall war der Auslöser. Aber bei vielen entdeckt man die genaue Ursache gar nicht. (A09/APR.04463 St. Galler Tagblatt, [Tageszeitung], 18.04.2009, Nr. 89, S. 39. - Sachgebiet: Regionales, Originalressort: ATAppenzellerland; m.faessler: «Das Herz ist der Mittelpunkt»)

12) $\{\{1$; Komp; A1 $\}\}$

Die angebaute Scheune konnte grösstenteils gerettet werden. Das Haus brannte bis auf die Grundmauern nieder. Die Spezialdienste der Kantonspolizei haben die Ermittlungen zur Brandursache aufgenommen. (kapo) (A10/JAN.04613 St. Galler Tagblatt, 20.01.2010, Nr. 15, S. 35. - Sachgebiet: Regionales, Originalressort: TT-Neutoggenburg; c.oberholzer: Haus vollständig niedergebrannt)

13) $\{\{1$; für + Akk.; A1 $\}\}$

Stundenlang war man mit dem Wegschaufeln der Schlammassen beschäftigt. "Leider war es nicht die erste Überschwemmung dieser Art, bereits im Juni waren unsere Keller unter Wasser. Schuld daran ist, daß Dachrinnen und die Lichtschächte der Keller gemeinsam in einen Regenwasserkanal entsorgt werden, der ohnehin schon überlastet ist", meinen die Wohnungseigentümer die Ursache für ihren Ärger zu kennen.

Weil man befürchtet, daß die ständige Nässe im Keller - auch im Stiegenhaus ist die Wand bereits feucht - nicht nur die dort gelagerten Sachen zerstört, sondern auch die Bausubstanz angreift, forderte man von der zuständigen Wohnbaugenossenschaft schon im Juni rasche Abhilfe. "Uns wird vorgeworfen, wir seien überempfindlich. (K97/SEP.69078 Kleine Zeitung, [Tageszeitung], 11.09.1997. Originalressort: Feldbach; Ende der "Wasserspiele"?)

14) $\{\{1 ;$ für + Akk.; A1 $\}\}$ «Unbestritten ist die Materie hochkomplex», sagt der Ausserrhoder Gesundheitsdirektor Matthias Weishaupt. An der Volksdiskussion nach der ersten Lesung mochte sich jedenfalls niemand beteiligen. Weishaupt indes vermutet die Ursache für dieses Stumm-Bleiben der Bevölkerung in einer derart wichtigen Sache nicht in der Überforderung, sondern in der guten Qualität der Vorlage. Will heissen: Man hat aus einer eidgenössischen Vorlage noch das Beste herausgeholt. Dieses Beste - was bedeutet das nun für die Patientinnen und Prämienzahler? (A11/SEP.07535 St. Galler Tagblatt, 17.09.2011, Nr. 217, S. 49. Sachgebiet: Regionales, Originalressort: AT-Appenzellerland; g.berlinger: Risiken und Nebenwirkungen)

15) $\{\{1 ;$ für + Akk.; A1 $\}\}$

Im Sommer schrumpft sie immer. Aber vor 30 Jahren waren auch dann noch 7,5 bis acht Millionen Quadratkilometer von Eis bedeckt - im vergangenen Jahr lediglich vier Millionen. 
Der Klimawandel und die globale Erwärmung werden als Ursache für den Rückgang des Arktiseises gesehen. Die Eisschmelze wiederum hat aber selbst beträchtliche Auswirkungen auf das Weltklima, weil die Arktis dieses beeinflusst. Umweltschutzverbänden zufolge bedroht die mit der Eisschmelze einhergehende Erhöhung des Meeresspiegels zunächst vor allem die Lebensgrundlagen der in der Arktis lebenden Bevölkerung, deren Siedlungen und Dauerfrostböden von Wasser und Morast überflutet werden könnten. (HAZ07/SEP.05137 Hannoversche Allgemeine, 17.09.2007, S. 5; Der Weg ist frei: Fluch oder Segen? [Ausführliche Zitierung nicht verfügbar])

16) $\{\{0\}\}$

„Eine Gefahr für Leib und Leben hat nicht bestanden, weil der Zug nicht auf einem Gegengleis unterwegs war", sagt Üstra-Sprecher Udo Iwannek.

Die Irrfahrt ereignete sich bereits am Dienstag vergangener Woche, sie wurde aber erst gestern bekannt. Ursache war nach Angaben der Üstra eine Computerpanne. In Tunnelstrecken werden Weichen und Signale ausschließlich per Rechner gesteuert, die Fahrer können dort im Gegensatz zu manchen oberirdischen Abschnitten nicht selbst eingreifen. Dabei gibt es nach Angaben der Üstra zwei Systeme. (HAZ08/JAN.05476 Hannoversche Allgemeine, 29.01.2008, S. 18; Stadtbahn biegt falsch ab [Ausführliche Zitierung nicht verfügbar])

17) $\{\{1 ;$ für + Akk.; A1 $\}\}$

Die Rettungskräfte entdeckten ein Feuer im Keller des Hauses, das schnell unter Kontrolle gebracht war. Das Gebäude wurde evakuiert. Die Ursache für die Explosion war zunächst ebenso wie die Höhe des Schadens unklar.

lni (HAZ08/SEP.03333 Hannoversche Allgemeine, 17.09.2008, S. 5; Explosion in Wohnhaus [Ausführliche Zitierung nicht verfügbar])

18) $\{\{1$; Gen.; A1 $\}\}$

Die Detonation riss die Giebelwand des Hauses ab, Trümmerteile begruben einen Lieferwagen unter sich und beschädigten vier weitere Autos. Die Feuerwehr evakuierte das Haus, insgesamt 22 Bewohner mussten die Weihnachtsfeiertage bei Bekannten oder in Ausweichwohnungen verbringen. Die Ursache des Unglücks ist bisher unklar, als wahrscheinlich gilt jedoch eine Gasexplosion. Den Mittwoch verbrachte die Feuerwehr damit, das Haus abzusichern. Noch immer ist das Gebäude in der Philipsbornstraße nicht bewohnbar. Seite 15 (HAZ08/DEZ.04844 Hannoversche Allgemeine, 27.12.2008, S. 1; 23Jähriger nach Gasexplosion in Lebensgefahr [Ausführliche Zitierung nicht verfügbar])

19) $\{\{1 ;$ für + Akk.; A1 $\}\}$

«Die Nachfrage nach solchen Bohrtürmen ist im Moment einfach sehr gross, besonders in Deutschland wird viel gebohrt.»

Nun verzögert sich das Projekt - ursprünglich wollte die Stadt mit den Bohrungen Ende 2011 beginnen - weiter. Das just nachdem der Rechtsstreit um den Bohrauftrag, Ursache für die erste Verzögerung des Projekts, vorbei ist.

Bohrauftrag definitiv vergeben

Der 15-Millionen-Auftrag im Sittertobel war von Bohrfirmen umkämpft. (A12/MAI.04537

St. Galler Tagblatt, 10.05.2012, Nr. 109, S. 25. - Sachgebiet: Lokales, Originalressort: ostschweiz; m.scherrer: Auftrag vergeben, Bohrturm gesucht)

20) $\{\{1$; Komp.; A1 $\}\}$ 
Auf einem Parkplatz ist am späten Sonntagabend ein Auto ausgebrannt. Es wurde vollständig zerstört. Die Brandursache wird noch untersucht. Der Lenker des Autos hatte bereits während der Fahrt Brandgeruch wahrgenommen. Er stellte seinen Wagen auf dem Parkplatz ab. (A11/NOV.06423 St. Galler Tagblatt, 15.11.2011, Nr. 267, S. 44. - Sachgebiet: Regionales / Nachrichten, Originalressort: SG-schauplatz-OS; k.meier: Parkiertes Auto ausgebrannt)

21) $\{\{1$; für + Akk.; A1 $\}\}$

Allergien als Folge?

Durch die Veränderungen des ursprünglichen Verhältnisses des Menschen zu seinen mikrobiellen Untermietern wird vielen Studien zufolge die Entwicklung unseres Immunsystems beeinflusst. Dies könnte die Ursache für die steigende Häufigkeit von Allergien und Autoimmun-Erkrankungen sein, sagen Experten. (A12/DEZ.07223 St. Galler Tagblatt, 18.12.2012, Nr. 296, S. 11. Originalressort: focus; r.app: Was der Kot über uns erzählt)

22) $\{\{0\}\}$

Laut Gossaus Kommunikationsbeauftragtem Urs Salzmann könnten mehr Quartiervereine nützlich sein. «Diese müssen aber aus den Quartieren wachsen.» Adrian Beeli vom Quartierverein Rosenau vermutet, dass das Bedürfnis dazu in Gebieten mit vielen Mietwohnungen kleiner ist. Auch die «Interessenlosigkeit», sich für die Allgemeinheit einzusetzen, könne eine Ursache sein. (A14/MAR.01079 St. Galler Tagblatt, 22.03.2014, Nr. 68, S. 51. Originalressort: Appenzellerland; j.nehmiz: Lobbyieren für Nachbarn)

23) $\{\{0\}\}$

Gibt es auch Härtefälle, wenn jemand mit Ihrer Expertise nicht einverstanden ist?

Ottolini: Ja, das gibt es. Etwa bei Wasserschäden, wenn eine undichte Gebäudehülle die Ursache ist. Sind Böden, Wände oder das Dach nicht dicht, kommt die Privatversicherung zum Zug - ausser es sei das halbe Dach wegen eines Sturmschadens abgedeckt worden. (A14/AUG.03577 St. Galler Tagblatt, 13.08.2014, Nr. 186, S. 16. - Sachgebiet: Lokales, Originalressort: Ostschweiz; c.zweili: «Unsere Arbeit beginnt erst jetzt»)

24) $\{\{0\}\}$

Heiser? Viel trinken, nicht flüstern!

Infektionen, Überbelastung oder Allergien können Ursache sein - Trockene Luft ist schlecht für die Schleimhaut

Von Martin Wöhler, Apotheker aus Braunschweig

Heiserkeit entsteht, wenn die Stimmbänder nicht mehr frei schwingen können.

(BRZ05/NOV.08057 Braunschweiger Zeitung, 15.11.2005; Heiser? Viel trinken, nicht flüstern! [Ausführliche Zitierung nicht verfügbar])

25) $\{\{0\}\}$

WESTLICHER RING. Dicke Rauchwolken sorgten mittags in einem Mehrfamilienhaus in der Hugo-Luther-Straße für Aufregung. Ursache war ein Brot, das eine 20-Jährige in ihrer Mikrowelle vergessen hatte. Der Laib verbrannte im eingeschalteten Gerät und löste sich in Rauch auf. Noch vor Eintreffen der Feuerwehr hatte die Wohnungsinhaberin den Brand gelöscht. (BRZ06/MAR.05591 Braunschweiger Zeitung, 10.03.2006; Brot in Flammen [Ausführliche Zitierung nicht verfügbar])

26) $\{\{1$; von + Dat.; A1 $\}\}$ 
Folgt man ihrer Argumentation halten christliche Werte für Sie Chancen bereit die Krankheit einzudämmen. Fraglich bleibt dann jedoch wie der katholische Teil der christlichen Gemeinde das Benutzen von Kondomen, als einzige wirksame Maßnahme zur Vorbeugung von AIDS, verbieten kann... Würde in Ihrer Argumentation nicht auch die christliche (katholische) Kirche als Ursache von AIDS gelten?

Da die Leserin sich in Ihrem Leserbrief auf den Kinofilm „Brokeback Mountain“ beziehen, möchte ich Ihnen den Film „V wie Vendetta“ empfehlen. In ihm wird eindrucksvoll aufgezeigt was in einer Gesellschaft passiert, in der Meinungen wie Ihre mehrheitsfähig werden. (BRZ06/MAR.13463 Braunschweiger Zeitung, 24.03.2006; Lieben Sie Ihren Nächsten wie sich selbst [Ausführliche Zitierung nicht verfügbar])

27) $\{\{1$; Komp.; A1 $\}\}$

Während die Nachbarlaube vor einem größeren Schaden durch die Feuerwehrkräfte geschützt werden konnte, brannte die andere Laube vollständig aus. Mindestens 2000 Euro Schaden waren entstanden. Die Brandursache ist noch unbekannt. (BRZ09/SEP.07032

Braunschweiger Zeitung, 14.09.2009; Gartenlaube ausgebrannt [Ausführliche Zitierung nicht verfügbar])

28) $\{\{1$; Gen.; A1 $\}\}$

Viele Insassen konnten sich über die Notausgänge retten. Einer der Überlebenden sagte, kurz nach dem Aufsetzen der Maschine habe es einen heftigen Knall gegeben: „Und dann war überall Feuer, und in der Kabine brach eine Panik aus." Nach Angaben des Auswärtigen Amtes in Berlin waren keine deutschen Passagiere an Bord.

Die Ursache des Unglücks ist noch unklar. Medienberichten zufolge hatte die Unglücksmaschine eine zu hohe Geschwindigkeit. Ein Regierungssprecher hatte jedoch einen Sabotageakt ins Spiel gebracht, weil Überlebende von einer Explosion berichtet hatten.

(BRZ07/MAR.02063 Braunschweiger Zeitung, 08.03.2007; Boeing geht in Flammen auf: 23 Menschen sterben [Ausführliche Zitierung nicht verfügbar])

29) $\{\{1 ;$ Gen.; A1 $\}\}$

„Alle Verfahren der Energiegewinnung werden gebraucht - auch Kernenergie“

Die Nutzung fossiler Brennstoffe bildet heute das Rückgrat der weltweiten

Energieversorgung. Das dabei entstehende CO2 wird als wesentliche Ursache des

Klimawandels angesehen.

Dies und die Endlichkeit der Lagerstätten erfordern die Entwicklung einer grundsätzlich neuen Energiepolitik. Dabei müssen alle sinnvollen Optionen zur effektiven Nutzung und Erzeugung von Energie geprüft und eingesetzt werden, auch die Kernenergie.

(BRZ07/OKT.03147 Braunschweiger Zeitung, 12.10.2007; [Ausführliche Zitierung nicht verfügbar])

30) $\{\{0+1$; keine Real.; Subj.; kein Arg.; A1 $\}\}\{\{$ SVG; U. haben $\}\}$

Die großen Palastzentren der mykenischen Kultur im heutigen Griechenland werden zerstört. Das Reich der mächtigen Hethiter in Kleinasien zerbricht und rätselhafte Seevölker dringen gewaltsam in die Levante und Ägypten ein. Da diese "Katastrophen" auf einem relativ großen Gebiet innerhalb nur weniger Jahre stattfanden, liegt die Vermutung nahe, sie alle könnten eine gemeinsame Ursache haben. Und tatsächlich haben Paläoklimatologen eine Klimaveränderung in der Region feststellen können. Nach David Kaniewski beispielsweise, Paläoklimatologe an der Universität in Toulouse, fallen die großen Umwälzungen mit dem Beginn einer 300-jährigen Phase verstärkter Trockenheit zusammen. (B18/JUN.02347 
Berliner Zeitung, 30.06.2018. - Sachgebiet: Lokales, Originalressort: MAG; Felix Firme: Aufstieg und Fall)

31) $\{\{0\}\}$

Häufig sind Becken- und Beinvenen betroffen. Ursachen können sein: Gefäßwandschäden nach Operationen oder Traumen, verlangsamte Blutströmung durch Bettruhe oder Gipsverband, bei beengter Sitzhaltung auf Reisen oder Krampfaderprobleme. Auch erhöhte Blut-Gerinnungsneigung bei Protein-C- und Protein-S-Mangel, Faktur-V-Leiden, Tumore oder die Pille kommen als Ursache in Betracht.

Bei bettlägerigen Patienten verursacht eine akute Beinvenenthrombose kaum Beschwerden. Bei mobilen Patienten treten Schmerzen und Schwellungen auf. (BRZ08/JUL.02883

Braunschweiger Zeitung, 05.07.2008; Wie vermeide ich auf Flugreisen eine Thrombose? [Ausführliche Zitierung nicht verfügbar])

32) $\{\{1$; Gen.; A1 $\}\}$

Nach dem Airbus-Absturz vor der französischen Mittelmeerküste herrscht weiter Rätselraten über die Ursache. Taucher bargen zwar den zweiten Flugschreiber und die Leiche eines weiteren Opfers. Die Ursache des Unglücks sei jedoch noch völlig unklar, sagte der Staatsanwalt in Perpignan. Zurzeit werde gegen unbekannt wegen fahrlässiger Tötung ermittelt. Der A 320 war am Donnerstag mit sieben Menschen an Bord abgestürzt.

(BRZ08/DEZ.00731 Braunschweiger Zeitung, 02.12.2008; Weitere Leiche nach Airbus Absturz geborgen [Ausführliche Zitierung nicht verfügbar])

33) $\{\{1$; Gen.; A1 $\}\}$

Ein junger Mann kommt bei einem Brand in seinem Haus ums Leben; er hinterlässt eine Frau und zwei kleine Kinder. Das geht uns allen sehr, sehr nahe.

Doch dann am nächsten Tag in der Salzgitter-Zeitung: Vermutungen über Ursache des

Feuers, über die Geschehnisse in diesem Haus.

Warum dieser Artikel? Was sollte die Öffentlichkeit für ein Interesse an derartigen - nicht einmal gesicherten - Details haben? (BRZ09/MAR.15678 Braunschweiger Zeitung, 31.03.2009; Gründe gehen uns nichts an [Ausführliche Zitierung nicht verfügbar])

34) $\{\{1$; Komp.; A1 $\}\}$

Unfallursache noch ungeklärt, die Beteiligten erlitten leichte Verletzungen.

ST.ANDRÄ Am Sonntag krachte es im Ortsgebiet von St. Andrä - gegen halb sieben Uhr abends wurde die Ortsfeuerwehr zur Ortsausfahrt Richtung Wallern gerufen, nachdem dort zwei PKW an der Kreuzung zusammengestoßen waren. Die Unfallursache ist derzeit noch ungeklärt, so ein Polizist der Polizeiinspektion Frauenkirchen gegenüber der BVZ am Montag.

Kollision: Lenkerin erlitt

leichte Verletzungen (BVZ12/MAR.03932 Burgenländische Volkszeitung, 29.03.2012, Neusiedl. - Sachgebiet: Lokales, Originalressort: CHRONIK; NSDRED: Zwei PKW krachten aneinander)

35) $\{\{0\}\}$

Bereits 270 Unfälle wurden gemeldet. Davon waren 81 auf Glatteis zurückzuführen. Vor zwei Jahren waren es im selben Zeitraum insgesamt 242 Unfälle (7-mal war Glatteis die Ursache) und im vergangenen Jahr 244 (43).

Erstaunlich sei, heißt es in einer Mitteilung der Polizei, dass sich trotz der gestiegenen Unfallzahlen die Personen- und Sachschäden reduziert hätten. Offensichtlich passten die 
Verkehrsteilnehmer ihre Fahrweise den Verhältnissen an und fahren entsprechend langsam und vorsichtig. (BRZ10/FEB.05014 Braunschweiger Zeitung, 10.02.2010; Unfallrekord im Januar auf spiegelglatten Straßen [Ausführliche Zitierung nicht verfügbar])

36) $\{\{1$; für + Akk.;A1 $\}\}$

Jetzt auch Pascal. Der 19-Jährige war Kapitän der VfL-A-Junioren - erhielt aber kein Angebot.

„Die haben gesagt, er sei zu klein“, berichtet Marcel über die Ursache für den Wechsel des 1,73 Meter großen Pascal. „Aber Lionel Messi ist auch nicht groß“, fügt er kritisch an und wirkt dabei ein bisschen wie der große, beschützende Bruder.

„Ich denke, dass wir uns gegenseitig pushen“, freut sich Marcel jedenfalls auf die sportliche Zusammenkunft. (BRZ10/JUL.08494 Braunschweiger Zeitung, 20.07.2010; Für Wolfsburg zu klein \&\#8211; ab zum großen Bruder! [Ausführliche Zitierung nicht verfügbar])

37) $\{\{0\}\}$

Fest steht, dass am frühen Abend des Unglückstags eine Wetterlage mit Gewitterfront im Raum Hannover herrschte, die in Richtung Braunschweig zog und der Pilot laut DWD „keine zeitnahe individuelle Flugwetterbewertung eingeholt hat".

Es fehlten aber auch noch Auswertungen zum technischen Zustand des Ballons.

„Erfahrungsgemäß gibt es für einen solchen Unfall keine zentrale Ursache“, weiß Eisenreich aus seiner Tätigkeit zu berichten. Deshalb halte er sich mit Einschätzungen zurück. „Es wäre allerdings sehr hilfreich, wenn wir mit dem Piloten direkt sprechen könnten, um sein Erleben des Unglücks miteinbeziehen zu können“, sagt Eisenreich. (BRZ10/DEZ.07372

Braunschweiger Zeitung, 15.12.2010; Pilot kannte die Wetterlage nicht [Ausführliche Zitierung nicht verfügbar])

38) $\{\{1$; Gen.; A1 $\}\}$

Der Baum blockierte drei Fahrbahnen. Die Feuerwehr war eine gute Stunde damit beschäftigt, dass Gewächs zu zersägen. Zu Ursache der Entwurzelung konnten die Einsatzkräfte nichts sagen. Foto: Norbert Kampermann (BRZ11/MAI.10560

Braunschweiger Zeitung, 23.05.2011; Baum stürzte gestern Abend auf den Sackring [Ausführliche Zitierung nicht verfügbar])

39) $\{\{0\}\}$

Sollte das Nervensystem befallen werden, kann es zu plötzlichen Lähmungen, aber auch zu „diffusen neurologischen Folgen“wie Müdigkeit und Konzentrationsschwierigkeiten kommen. Der Experte: „Hierzu gibt es konträre Diskussionen in Fachkreisen. In jedem Fall sollte ein Facharzt aufgesucht werden, da die Ursache schwer festzustellen ist."

Werner Sieling daheim bei der Gartenarbeit. Er weiß: Die Zecke gibt eine betäubende Substanz ab, so dass ihr Stich nicht zu spüren ist. (BRZ11/JUL.09104 Braunschweiger Zeitung, 21.07.2011; \&\#8222; Viele Ärzte haben von Zecken keine Ahnung\&\#8220; [Ausführliche Zitierung nicht verfügbar])

40) $\{\{1$; für + Akk.; A1 $\}\}$

„Ich bin überrascht, wie gedankenlos die Entwicklung der Strompreise hingenommen wird“, sagte Oettinger auf dem internationalen Wirtschaftsforum in Baden-Baden.

Schon heute sei Strom fast nirgends so teuer wie in Deutschland. Dies sei eine Ursache für eine stetige De-Industrialisierung - energieträchtige Produktionen würden ins Ausland verlagert, sagte Oettinger. 
Deutschland müsse deshalb seine Subventionen für Solarenergie überdenken. „Irgendwann müssen wir erkennen, dass die Sonne in anderen Ländern stärker und länger scheint.“

Hintergrund dieser Kritik: Obwohl die Solarbranche fast ebenso viel Förderung erhält wie die Windkraft, ist ihr Anteil an der Stromerzeugung deutlich geringer. (BRZ11/OKT.11681 Braunschweiger Zeitung, 24.10.2011; \&\#8222; In anderen Ländern scheint die Sonne eben stärker\&\#8220; [Ausführliche Zitierung nicht verfügbar])

41) $\{\{0\}\}$

Richtig sauer. Der Grund: Die Heizkostenabrechnung von ihm und seinen Nachbarn scheint ihm ungewöhnlich hoch. Und er hat auch schon eine Ursache ausgemacht: Sie heißt Fernwärme-Contracting, also Heizwärme als Dienstleistung (siehe Kasten).

Dazu muss man wissen, dass Krügers Wohnung sowie etwa 1500 weitere in der Braunschweiger Weststadt mit Fernwärme aus dem Kraftwerk von BS Energy beheizt werden. Zwar ist Fernwärme im Vergleich zu anderen Heizmethoden, etwa Öl und Gas, ohnehin etwas teurer. (BRZ12/DEZ.10194 Braunschweiger Zeitung, 22.12.2012; Heizpreise \&\#8211; Mieter beklagen Mehrkostendurch Fernwärme-Dienstleister [Ausführliche Zitierung nicht verfügbar])

42) $\{\{0\}\}$

Ursache eines Brandes unklar

Bisher weiß noch niemand, wie es zu dem Brand gekommen ist, der am Donnerstagabend ein ganzes Haus in Heere zerstört hat. Spezialisten der Polizei arbeiten aber fieberhaft daran, die Ursache zu klären. Sie vermuten, dass das Feuer in der Garage ausgebrochen war.

In dem Haus wohnten zwei Senioren, eine 81-jährige Frau und ihr 83-jähriger

Lebensgefährte. (BRZ13/JUN.00179 Braunschweiger Zeitung, 01.06.2013,

Braunschweiger Zeitung. Originalressort: 1Leute; Ursache eines Brandes unklar)

43) $\{\{1$; Gen.; A1 $\}\}$

Über 270 Millionen Euro konnten seit 1955 ersungen werden. Im Vorjahr wurden 13,4

Millionen Euro gesammelt.

Sternsingersolidarität konkret: Armut und Unterdrückung sind gleichzeitig Ursache und Folgen des Unrechts, das Millionen von Menschen in den Entwicklungsländern widerfährt. Ihnen gilt der Einsatz der "Hilfe unter gutem Stern". Mit den Spenden werden Menschen unterstützt, die an einer gerechten und lebenswerten Welt für alle arbeiten.

(BVZ09/JAN.00726 Burgenländische Volkszeitung, [Wochenzeitung], 31.12.2008, Nr. 01, Jg. 2009, Neusiedl, S. 14. - Sachgebiet: Religion, Originalressort: DIÖZESE EISENSTADT; Gerechtigkeit für die Welt!)

44) $\{\{0\}\}$

Umweltdienst: Müll fing Feuer

In der Vorwoche entstand beim Abladen von Müll ein Brand, die Ursache ist noch unklar.

Die Stadtfeuerwehr Oberwart rückte aus und löschte mit tatkräftiger Unterstützung durch die Mitarbeiter des Umweltdienstes das Feuer. ZVG/STF OBERWART (BVZ12/MAI.01710

Burgenländische Volkszeitung, 17.05.2012, Oberwart. - Sachgebiet: Lokales, Originalressort: CHRONIK; OWZRED: Umweltdienst: Müll fing Feuer)

45) $\{\{1$; für + Akk.; A1 $\}\}$

Hundebiss als Ursache für Todesfall?

Ursache noch ungeklärt 
62-jährige Hundeliebhaberin verstarb sechs Tage, nachdem sie von einem Hund angefallen worden war, im Spital. (BVZ14/MAI.01835 Burgenländische Volkszeitung, 15.05.2014, Mattersburg. - Sachgebiet: Lokales, Originalressort: Lokales; MATRED: Hundebiss als Ursache für Todesfall?)

46) $\{\{0\}\}$

Die Marktanalysten der britischen Gartner Group haben herausgefunden, daß die Unternehmen für den Computereinsatz am Arbeitsplatz zu viel bezahlen.

Trotz gesunkener Preise für Hard- und Software - so ermittelten die Analysten - hätten sich die Nutzungskosten eines Computers wegen der teurer gewordenen Arbeitszeit innerhalb von sieben Jahren mehr als verdoppelt und würden nun 40000 Dollar pro Jahr und Arbeitsplatz betragen. Ursache: Rund die Hälfte seiner Arbeitszeit am Computer verbringe ein Angestellter damit, spezifische Hard- oder Softwareprobleme zu lösen. Die beste Einsparmöglichkeit sei ein Just-in-time-Training an neu installierten Produkten.

(C95/MAR.00827 COMPUTER ZEITUNG, [Wochenzeitung], 16.03.1995, Nr. 11, Jg. 30, S. 6. - Computerarbeit, [Bericht])

47) $\{\{0\}\}$

Kein Sender ließ die Tragödie aus. Überall Querschnitte komatöser Hirne und Wissenschaftler, die sich vor laufenden Kameras Gedanken über die Obuchi verbliebenen geistigen Fähigkeiten machten. Als Ursache machten sie Karo aus: der Premier sei ein Opfer von Überarbeitung und Überforderung bis zur völligen Erschöpfung.

Die Tage vor Obuchis Schlaganfall hatten es in sich. (FOC00/APR.00320 FOCUS, 10.04.2000, S. 334-337, Sachgebiet: Politik, Originalressort: AUSL/Ausland; JAPAN)

48) $\{\{1$; für + Akk.; A1 $\}\}$

"Aber es stimmt nicht." Fettleibigkeit sei in den meisten Fällen keine Erbkrankheit. "Ebenso sind Hormonstörungen, etwa eine Schilddrüsenunterfunktion, nur selten die Ursache für das Übergewicht." Paul Trayhurn, Direktor des Zentrums für Ernährungsgenomik an der Universität Liverpool, betont: "Hunderte Gene sind an der Regulierung des Körpergewichts beteiligt." Auch bei Tieren sei die einzig wirksame Abnehmstrategie: "Weniger essen und mehr Bewegung." (FOC05/MAR.00347 FOCUS, 21.03.2005, S. 082-083, Originalressort: FORT/MEDI/Medizin; TIERMEDIZIN)

49) $\{\{1$ Komp.; A1 $\}\}$

Alle zehn Sekunden stirbt ein Mensch an den Folgen des Rauchens - das sind rund vier Millionen Tote weltweit pro Jahr. Bleiben die gegenwärtigen Verhaltensmuster - jeder dritte Erwachsene raucht - bestehen, erhöht sich die Zahl der Toten bis zum Jahre 2030 auf zehn Millionen. Rauchen ist dann weltweit Todesursache Nummer 1! Diese Zahlen wurden jetzt beim Europäischen Krebskongress in Wien bekannt gegeben. An dem Kongress, der morgen zu Ende geht, nehmen rund 9000 Krebsforscher, Ärzte und Krankenpfleger teil.

(L99/SEP.64061 Berliner Morgenpost, [Tageszeitung], 15.09.1999, S. 46. Originalressort: HOCHSCHULE \& WISSENSCHAFT; groe)

50) $\{\{0\}\}$

Depressive Zustände können auch durch Hormonungleichgewichte ausgelöst werden, die in Zusammenhang mit einer Schilddrüsenerkrankung oder einer Erschöpfung der

Nebennierenrinde stehen. Der Therapieansatz sollte dann ein ganz anderer sein.

Psychopharmaka würden in solchen Fällen nur Symptome bekämpfen, aber nicht an der eigentlichen Ursache ansetzen. 
90574 ROSSTAL CHRISTA KÜHNE (FOC09/NOV.00272 FOCUS, 16.11.2009, S. 78-78, Originalressort: Redaktion; Wie in einer eisernen Lunge)

51) $\{\{0\}\}$

Ich glaube, man braucht sogar noch etwas anderes: Wenn man richtig verzeihen will, muss man sich dafür Zeit nehmen. Du musst zu einem Standpunkt gekommen sein, du musst den Vorgang wirklich noch einmal zerlegen und fragen, was ist Schuld, was ist lediglich Ursache? Das sind zwei verschiedene Dinge. Ursache meint bestimmte Kausalitäten, warum sich etwas so oder so verhält. (FOC12/MAR.00296 FOCUS, 26.03.2012, S. 104-108, Sachgebiet: Kultur, Originalressort: KULTUR UND LEBEN, MEDIEN; »Ohne Freude bist du eine arme Sau«)

52) $\{\{1 ;$ Gen.; A1 $\}\}$

Mutter und zwei Kinder sterben bei Wohnhausbrand

Ursache des Feuers in Lübecker Altstadt noch unklar

- Lübeck (dpa). Bei einem Feuer in einem Wohnhaus in der Lübecker Altstadt sind in der

Nacht zum Sonntag eine Frau und zwei Kinder ums Leben gekommen. (HAZ09/FEB.02702

Hannoversche Allgemeine, 16.02.2009, S. 10; Mutter und zwei Kinder sterben bei Wohnhausbrand [Ausführliche Zitierung nicht verfügbar])

53) $\{\{1$; für + Akk.; A1 $\}\}$

Bahn sucht weiternach der Ursache für Kurzschlüsse

Hannover (tm). Die Deutsche Bahn hat sich einen Tag nach dem Zusammenbruch des Zugverkehrs rund um Hannover zur Kritik an ihrer Informationspolitik geäußert.

(HAZ10/JUN.00240 Hannoversche Allgemeine, 04.06.2010; Bahn sucht weiternach der Ursache für Kurzschlüsse [Ausführliche Zitierung nicht verfügbar])

54) $\{\{0\}\}$

Hier finden sich bis zu 11 Millionen Keime pro Quadratzentimeter, Tausend Mal mehr als auf dem Küchenboden. Das hat vor allem mit offenen Lebensmitteln, umgekippten Behältern zu tun. Eine mögliche Ursache: Das Ausräumen und Säubern des Kühlschranks ist aufwendig und wird zu oft hinausgezögert. $1 / 2$ Gegenmaßnahme: Lebensmittel immer verschlossen halten, Verdorbenes täglich aussortieren, wöchentlich reinigen,

Mindesttemperatur: fünf Grad!

Staubsauger (HMP08/AUG.03230 Hamburger Morgenpost, 29.08.2008, S. 32-33; Fiese, kleine Biester [Ausführliche Zitierung nicht verfügbar])

55) $\{\{1$; Gen.; A1 $\}\}$

Aber auch wenn man alles richtig macht, kann es sein, dass sich Schimmelpunkte an der

Wand bilden. In solchen Fällen sagt der Vermieter häufig: "Sie haben zu wenig geheizt oder gelüftet." Oft ist aber die Gebäudesubstanz Ursache des Schimmels.

Und das sind Indizien dafür, dass die Schimmelbildung nicht vom Bewohner verschuldet ist:

Die Schäden waren schon beim Vormieter vorhanden oder sind bald nach Bezug der

Wohnung zum Vorschein gekommen. (HMP10/SEP.00963 Hamburger Morgenpost, 10.09.2010, S. 26, 27; Kampf den Schimmelpilzen - Im schlimmsten Fall gefährden sie die Gesundheit - Gegen die Plagegeister hilft regelmäßiges Lüften [Ausführliche Zitierung nicht verfügbar])

56) $\{\{1$; Gen.;A1 $\}\}$ 
Doch genau die hat nach Informationen der "Financial Times Deutschland" ein Antrag der Deutschen bei der EU im Auge: Ein "Haushalts-Beauftragter" soll in Athen die Finanzaufsicht übernehmen, die Griechen sollen "für einen bestimmten Zeitraum" ihre Souveränität an die EU abtreten. Künftig würde Griechenlands Haushalt nicht mehr in Athen, sondern in Brüssel beschlossen.

$\mathrm{Ob}$ das beim Kassensturz überhaupt hilft, ist aber völlig offen: Ursache des dramatischen neuen 15-Milliarden-Haushaltsloches ist nämlich die Schwäche der griechischen Wirtschaft. Und die geht vor allem wegen des schon bestehenden drastischen EU-Spardiktats endgültig in die Knie.

Nach "Spiegel"-Informationen ist man in der Troika pessimistisch, ob Griechenland diese 15 Milliarden aus eigener Kraft zusammenbekommt. (HMP12/JAN.02817 Hamburger Morgenpost, 29.01.2012, S. 02; Berlin fordert von der EU Entmündigt die Griechen! Geheimplan aus Berlin will das bankrotte Land unter Brüsseler Kontrolle stellen [Ausführliche Zitierung nicht verfügbar])

57) $\{\{1$; Komp.; A1 $\}\}$

Der Autofahrerclub bezeichnet den vorgelegten Strafenkatalog als "undifferenzierten Vorschlag zum Abkassieren, der in dieser Form unakzeptabel ist". Fritz Tippel, Jurist des Autofahrerclubs: "Es wird nur nach absoluten Tempoüberschreitungen gestraft. Auf die Hauptunfallursache, die nicht angepasste Geschwindigkeit, wird nicht Rücksicht genommen."

Für das Tempoverhalten der Autofahrer sei nicht die Höhe der Strafen ausschlaggebend, vielmehr trügen die Sinnhaftigkeit der Tempolimits und die verstärkte Präsenz der Exekutive sowie die Unmittelbarkeit der Bestrafung zur Änderung des Verhaltens bei.

(K00/OKT.78790 Kleine Zeitung, [Tageszeitung], 25.10.2000. Originalressort: Lokal; Rasen soll viel teurer werden)

58) $\{\{1$; Gen.; A1 $\}\}$

Die Passagiere starben nach Angaben von CNN, als der Vorortzug gestern in der Bronx entgleiste. Wie viele Verletzte es gab, war auch Stunden nach dem Unglück noch unklar. Ebenso die Ursache des Unfalls. Mindestens vier der sieben Wagen waren aus den Schienen gesprungen. Das Ende des Zuges habe im Wasser gehangen. (HMP13/DEZ.00111

Hamburger Morgenpost, 02.12.2013, S. 54; Zug in New York entgleist: Vier Tote [Ausführliche Zitierung nicht verfügbar])

59) $\{\{1+1$; Posspr.; dass-Subjsatz.; A1; A1 $\}\}\{\{$ SVG; U. haben $\}\}$

Lehrlinge zeigten Spitzenleistung

Dasss Tiroler Tourismus-Fachkräfte in der ganzen WEelt gefragt sind, hat seine Ursache nicht zuletzt im hohen Niveau der Lehrausbildung. Bei den jüngsten Wettbewerben glänzten wieder viele Jugendliche.

INNSBRUCK. (I00/FEB.11360 Tiroler Tageszeitung, [Tageszeitung], 29.02.2000.

Originalressort: Allgemein; Lehrlinge zeigten Spitzenleistung)

60) $\{\{1$; Gen.; A1 $\}\}$

Der Firmenchef von Stolt Offshore, Julian Thomson, plädierte für eine Hebung des gesamten 150 Meter langen Wracks aus 100 Meter Tiefe. An den Plänen werde noch gearbeitet.

Zur Ursache des Untergangs in der Barentssee gibt es Spekulationen: Während die russische Militärstaatsanwaltschaft in Richtung einer Kollision mit einem fremden U-Boot ermittelt, schlossen norwegische Experten eine gewaltige Gasexplosion in der "Kursk" nicht aus. Bisher wurden jedoch keine Aufnahmen von dem zerstörten Bug der "Kursk", die nähere 
Aufschlüsse zur Ursache bringen könnten, seitens der Russen veröffentlicht. Inzwischen sind die 118 toten Seemänner von Präsident Wladimir Putin für ihre Tapferkeit ausgezeichnet worden. (I00/AUG.49640 Tiroler Tageszeitung, [Tageszeitung], 28.08.2000. Originalressort: Allgemein; Bergung der Leichen aus "Kursk")

61) $\{\{1$; für + Akk.; A1 $\}\}$

Diese Rückgänge sind vor allem auf die "drastischen Rückgänge" im Tourismus zurückzuführen, sagt die EU.

Strukturveränderung ist notwenig

Als Ursache für den Einbruch werden die "schlechte preisliche Wettbewerbsfähigkeit" sowie die "unzureichende Qualität des Angebots" festgehalten. Dies gilt in erster Linie für den Sommertourismus, konstatiert Brüssel. Darüber hinaus leidet der österreichische Fremdenverkehr an "erheblichen Überkapazitäten". (I96/MAR.09372 Tiroler Tageszeitung, [Tageszeitung], 08.03.1996. - Sachgebiet: Wirtschaft, Originalressort: Wirtschaft; Überkapazitäten, Angebotsmängel: EU-Rüffel für den Tourismus)

62) $\{\{1$; für + Akk.; A1 $\}\}$

Wettergott Petrus kennt heuer wenig Gnade, bisherige Sommerbilanzen im Tourismus sind entsprechend: $15 \%$ Nächtigungsminus z. B. in Imst, 10 bis $12 \%$ in St. Anton, auch der TVB Längenfeld erwartet für den Juli "eine zweistellige Minuszahl". Immerhin: Mit dem Mai und Juni waren Orte wie Imst (plus 53,2 \%, vor allem dank der vielen Feiertage), Längenfeld oder Galtür zufrieden. Ursache für Rückgänge kann die Wirtschaftslage im Hauptherkunftsland Deutschland sein. Doch auch schlechte Wetterberichte schrecken ab: "Durch die elektronischen Medien sind die Gäste bestens informiert", weiß Landecks TVB-Dir. Klaus Koller. (I97/JUL.28938 Tiroler Tageszeitung, [Tageszeitung], 26.07.1997. Originalressort: Regional Oberinntal, Außerfern; Petrus sorgt für Minuszahlen)

63) $\{\{1$; Gen.; A1 $\}\}$

Zum Vergleich: In Innsbruck kommt ein Exekutivbeamter auf 198 Einwohner, in Salzburg einer auf 216. Auf die Frage, ob es an der Dienstordnung der Polizei liegen könnte, daß vergleichsweise wenig Polizisten auf den Straßen zu sehen sind, hält Knapp sich bedeckt, räumt aber ein, "daß sich hier manches machen ließe". Eine mögliche Ursache der hohen Verbrechensrate kann man aber mit Sicherheit ausschließen: die oft strapazierte

"Ausländerkriminalität". 12,6 Prozent der in Innsbruck ermittelten Straftäter waren Ausländer mehr als die Hälfte davon Touristen. Der Anteil jener Gruppe an den Straftaten in Innsbruck, die im allgemeinen Sprachgebrauch mit Ausländerkriminalität assoziiert wird, also Ausländer mit Wohnsitz in Österreich, ist gering: 6,2 Prozent. Und aus dieser Zahl läßt sich ein einfacher Schluß ziehen. (I97/DEZ.48421 Tiroler Tageszeitung, [Tageszeitung], 09.12.1997. Originalressort: Regional Unterinntal; 13.000 Vergehen in Innsbruck)

64) $\{\{1$; für + Akk.; A1 $\}\}$

Sportmaschine abgestürzt

GRAZ.

Die Ursache für den Absturz einer Sportmaschine am Sonntag nachmittag in der Breitenau (Bezirk Bruck an der Mur) bleibt vorerst ungeklärt. Bei dem Unglück waren beide Insassen, ein 44jährigen Techniker aus Leoben und ein 40jähriger Angestellter aus Kapfenberg, ums Leben gekommen. Wenige Minuten nach dem Start um 15.18 Uhr in Kapfenberg war der Funkkontakt abgebrochen. (I98/FEB.04461 Tiroler Tageszeitung, [Tageszeitung], 03.02.1998. Originalressort: Journal; Sportmaschine abgestürzt) 
65) $\{\{1$; Gen.; A1 $\}\}$

Am Tag zuvor hatte Prof. Leopold Wagner, Geologe des Wirtschaftsministeriums, die Plombe angebracht. Zuletzt waren (wie berichtet) Experten erstmals seit den Felsstürzen am Eiblschrofen in den Stollen, um Beweise zu sichern und Erkundungen durchzuführen. Davon erhofft man sich weitere Aufschlüsse über die Ursache der Felsstürze. Nach jeder Ausfahrt wird verplombt. Ursache für die Aufregung der Anrainer dürfte Freitag ein am Bergwerksgelände in Betrieb genommener "Nachbrecher" gewesen sein. (I99/SEP.35695 Tiroler Tageszeitung, [Tageszeitung], 11.09.1999. Originalressort: Tirol aktuell; Anrainer vermuteten illegale Arbeit im Schwazer Bergwerk)

66) $\{\{1$; für - Pronadv.; A1 $\}\}$

180 würden gerne "Geschichte" und 140 "Geografie" unterrichten, wenn es einen Arbeitsplatz für sie gebe.

Trotz dieser Zahlen zeigt die Ge samtbilanz eine leichte Entspannung: Die Zahl der arbeitslosen Lehrer ist seit dem August des Vorjahres um 162 zurückgegangen. Die Ursache dafür ist eine Art Generationenwechsel, der Platz macht für den Lehrernachwuchs.

Weil Lehrer oft glauben, dass sie sich beim Arbeitsmarktservice nicht melden müssen (weil sie keinen Anspruch auf Arbeitslosenunterstützung haben), verzichten viele arbeitslose Lehrer nach Angaben des AMS auf Förderprogramme und finanziell unterstützte Fortbildung, erläutert Hermann Gössinger, AMS-Pressesprecher. So gebe es zum Beispiel ein Qualifizierungsprogramm gemeinsam mit der "Solidaritätsaktion arbeitslose Lehrer und Lehrerinnen", über das laufend Jobs im Umwelt- und Sozialbereich angeboten werden. (K00/SEP.67561 Kleine Zeitung, [Tageszeitung], 12.09.2000. Originalressort: Lokal; Viele Lehrer bleiben zu Schulbeginn sitzen)

67) $\{\{1$; Gen.; A1 $\}\}$

Eine reine Nervensache

Das Gutachten fiel negativ aus, für die Ursache des plötzlichen Gebrechens fand der Trainer allerdings keine eindeutige, plausible Erklärung. Walter Knaller war nach dem 1:1 des FC Austria/VSV in Stockerau jedenfalls heilfroh, ohne gröbere Schäden davongekommen zu sein. "Das war sicher die spielerisch schlechteste Leistung der Saison, trotzdem haben wir nicht verloren." (K98/AUG.65749 Kleine Zeitung, [Tageszeitung], 31.08.1998. Sachgebiet: Sport, Originalressort: Sport; Eine reine Nervensache)

68) $\{\{1$; Gen.; A1 $\}\}$

Reitstallbrand: Buben hatten gezündelt

Linz. -

Geklärt wurde die Ursache jenes Brandes, der Sonntag nachmittag in Puchenau bei Linz einen Reitstall vernichtete. Zwei Buben hatten mit einem Feuerzeug gezündelt und dabei das Gebäude in Brand gesetzt. Der Schaden dürfte rund 1,5 Millionen Schilling ausmachen. (K99/JAN.02334 Kleine Zeitung, [Tageszeitung], 12.01.1999. Originalressort: Lokal; Reitstallbrand: Buben hatten gezündelt)

69) $\{\{1$; für - Pronadv.; A1 $\}\}$

Es gibt Momente, in denen auch unsereiner von einer Art schaudernder Ehrfurcht erfaßt wird. Mir geschah das zuletzt am Samstag abend in der lauschigen Osteria Montin zu Venedig. Die Ursache hierfür war der amerikanische Schauspieler und Regisseur Dennis Hopper, an dessen Tisch ich saß.

Ein mittelgroßer Herr, in feines Tuch gewandet, der mit dunkler Samtstimme wohlgewählte Worte von sich gab. Er trank Wasser statt Wein und nahm Erdbeereis statt Grappa. 
(K99/JUN.43355 Kleine Zeitung, [Tageszeitung], 14.06.1999. - Sachgebiet: Fernsehen, Originalressort: Fernsehen; Mr. Dennis Hopper)

70) $\{\{1+2$; Posspr.; Subj.; in + Dat.; A1; A1; A’ $\}\}\{\{$ SVG; Poss. U. finden $\}\}$

Die Staatsanwaltschaft sei selbst für "Insider" unübersichtlich. Um Abhilfe zu schaffen, wird die zügige Umsetzung von Maßnahmen innerhalb der Verwaltungsreform vorgeschlagen. So sollte eine der acht Hauptabteilungen der Staatsanwaltschaft in ein Leistungs- und Verantwortungzentrum (LUV) als Träger dezentralisierter Fach- und Ressourcenverantwortung umgewandelt werden, um möglichst viele neue Steuerungselemente und Arbeitsabläufe erproben zu können. Gefordert werden daneben ein besseres Personalmanagement und eine gezielte Führungskräfteschulung. "Vielele Defizizite der jetzigen Situation finden ihre Ursache in der Überbetonung fachlich juristischer

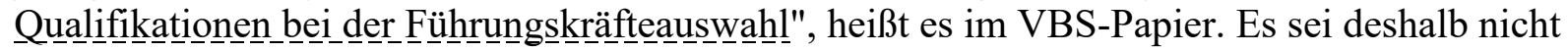
einzusehen, "daß Standards anderer Berliner Verwaltungen, die einschlägige Fortbildung und Erprobung solcher Fähigkeiten zur Bewerbungsvoraussetzung für Beförderungsstellen machen, nicht auch innerhalb der Justiz angewendet werden". (L99/JUL.42633 Berliner Morgenpost, [Tageszeitung], 12.07.1999, S. 11. Originalressort: 1; Von Jörg Meißner: Selbst "Insider" finden sich nicht zurecht)

71) $\{\{1$; für + Akk.; A1 $\}\}$

$\mathrm{BM} / \mathrm{dpa}$

Berlin -

Der Ministerpräsident von Mecklenburg-Vorpommern, Harald Ringstorff (SPD), sieht in dem strikten Abgrenzungskurs der sächsischen SPD gegenüber der PDS eine Ursache für die erlittene Schlappe bei der Landtagswahl. Es sei ein Fehler gewesen, in der Opposition die PDS stärker zu attackieren als die CDU-Regierung, sagte Ringstorff der "Freien Presse".Eine Umfrage in Mecklenburg-Vorpommern, wo Ringstorff eine rot-rote Koalition führt, zeigt indes, dass die Einbindung der PDS in die politische Verantwortung bei den Wählern deutlich an Zustimmung verliert und sich die SPD um ihr eigenes Ansehen sorgen muss. Ein Jahr nach der Landtagswahl bescheinigen drei Fünftel der Bürger der Landesregierung schlechte Arbeit, wie Emnid für die "Schweriner Volkszeitung" ermittelte. (L99/SEP.67695 Berliner Morgenpost, [Tageszeitung], 25.09.1999, S. 2. Originalressort: POLITIK; BM/dpa)

72) $\{\{1$; für + Akk.; A1 $\}\}$

Trotz der angesprochenen Entwicklung liegt Luxemburg damit immer noch deutlich unter dem europäischen Durchschnitt (vier Prozent).

Als eine Ursache für diese Situation führte Landwirtschaftsminister Fernand Boden gestern vor Journalisten an, dass in den letzten drei Jahren kaum landwirtschaftliche Betriebe auf ökologischen Landbau umgestellt hätten. Es bestehe auch wenig Interesse auf Ebene der verarbeitenden Betriebe daran, weitere Betriebe zu "veredeln".

300.000 Euro (LTB09/FEB.00385 Luxemburger Tageblatt, 13.02.2009, Sachgebiet: Politik, Originalressort: POL - POLITIK; 6.000 Hektar bis 2013 heißt das Ziel)

73) $\{\{1$; Gen.; A1 $\}\}$

bei Bränden in pakistanischen Sweatshops

Bei verheerenden Bränden in zwei pakistanischen Fabriken sind mindestens 314 Menschen ums Leben gekommen. Ursache der erschreckend hohen Opferzahl war auch, dass es vor Ort an Notausgängen, Alarm- und Sprinkleranlagen mangelte, hieß es gestern.

Allein aus der niedergebrannten Textilfabrik in Karatschi wurden nach offiziellen Angaben 289 Leichen geborgen. Es handelt sich um eines der folgenschwersten Unglücke in einem 
Industriebetrieb in der 65-jährigen Geschichte des Landes. (LTB12/SEP.00631

Luxemburger Tageblatt, 13.09.2012, Über 300 Menschen sterben)

74) $\{\{1$; Gen.; A1 $\}\}$

Wenige Sekunden nach dem Start im Raumfahrtzentrum in Cape Canaveral ging die USRaumfähre Challenger am 28. Januar 1986 in einem Feuerball auf. Alle sieben

Besatzungsmitglieder kamen dabei ums Leben. Die Ursache des Unglücks waren kaputte Dichtungsringe an einer Feststoffrakete. Nach der Challenger-Katastrophe wurden alle Flüge von Raumfähren der NASA bis 1988 ausgesetzt. Das Tageblatt machte am 29. Januar 1986 den Wettlauf um die Dominanz im All für dieses Unglück verantwortlich.

(LTB13/DEZ.00128 Luxemburger Tageblatt, 03.12.2013, ohne Titel)

75) $\{\{1$; für - Pronadv.; A1 $\}\}$

mw) Ohne PCB-Dämpfe hat für zwei Klassen von Schulanfängern der Unterricht in Waibstadts Realschule begonnen. Wie berichtet war die Schule im Dezember 1994 von Eltern und Schülern bestreikt und danach geschlossen worden, als besorgniserregend hohe PCBWerte (polychlorierte Biphenyle) von bis zu 17000 Nanogramm in den Klassenräumen gemessen worden waren. Ursache dafür waren Fugen und Decken, aus denen das Gift ausströmte. Nach Beseitigung der Fugen im Frühjahr geht es nun an den zweiten Sanierungsschritt. Die drei Millionen Mark teure Entgiftung des Schulgebäudes übersteigt allerdings die Finanzkraft der Stadt, die bisher rund ein Drittel der Kosten aus dem Ausgleichsstock des Landes erstattet bekommt. (M95/509.17642 Mannheimer Morgen, [Tageszeitung], 12.09.1995, Jg. 50. Originalressort: NACHBARSCHAFT; Unterricht ohne PCB-Dämpfe)

76) $\{\{1$; für + Akk.; A1 $\}\}$

Die Grundlagen für die Schmerzbewältigung werden nach Ansicht einer kanadischen Expertin in der frühen Kindheit gelegt. Wie ernst oder besorgt die Reaktion der Eltern auf die Schmerzen des Kindes reagierten, entscheide über den Umgang des Kindes damit, erklärte Patricia McGarth. Die Ursache für die unterschiedliche Schmerzempfindlichkeit dagegen liege vermutlich in den Genen: Experimente mit Mäusen hätten ergeben, daß das weibliche Hormon Östrogen und das männliche Testosteron für das Schmerzempfinden verantwortlich seien.

AP (M98/APR.31640 Mannheimer Morgen, [Tageszeitung], 14.04.1998, Jg. 53. Originalressort: Aus aller Welt; Frauen können mit dem Schmerz besser umgehen)

77) $\{\{1$; Gen.; A1 $\}\}$

Doch "Chiapas - Chronik eines angekündigten Massakers" trug wenig zur Erhellung bei. Eine chronologische Abfolge wäre hilfreicher gewesen, als es die ständigen Zeitsprünge waren. Vor allem aber wurde die Ursache des Konflikts, bei dem es nicht nur um ethnische Differenzen ging, nie deutlich. Die Frage, inwiefern die mexikanische Regierung mitverantwortlich am Tod der Bewohner im Dorf Acteal war, blieb ebenso im dunklen wie die Philosophien der politischen Kontrahenten, die den Indios unermeßliches Leid zugefügt haben. Nur eines wurde in der Reportage klar herausgearbeitet: die andauernde Verzweiflung unschuldiger Menschen, die für wenige Tage ins öffentliche Bewußtsein gerückt waren und daraus ebenso schnell wieder verschwanden. (M98/MAI.40311 Mannheimer Morgen, [Tageszeitung], 13.05.1998, Jg. 53. - Sachgebiet: Fernsehen / Hörfunk, Originalressort: Fernsehen und Hörfunk; Von Bettina Kozana: Im dunklen)

78) $\{\{1 ;$ für + Akk.;A1 $\}\}$ 
Gondelunglück führt zu Spekulationen und Ratlosigkeit

Grenoble.

Die Ursache für den Absturz einer Seilbahn-Gondel in der Nähe von Grenoble mit 20 Toten ist gestern rätselhaft geblieben. Die Ermittler lehnten unter Hinweis auf die laufenden Untersuchungen jede Auskunft ab. Sie überprüften nahe des kleinen Ortes Saint-Etienne en Devoluy in den französischen Alpen weiter die Trümmer der Gondel, die am Vortag 80 Meter tief abgestürzt war. (M99/JUL.43180 Mannheimer Morgen, [Tageszeitung], 03.07.1999, Jg. 54. Originalressort: Aus aller Welt; Gab es schon vorher Probleme?)

79) $\{\{1$; für + Akk.; A1 $\}\}$

Außerdem wurden 70000 Solarwärme-Anlagen gebaut. Nach aktuellen UVS- Schätzungen wurde auf dem Deutschen Solarmarkt ein Umsatz in Höhe von rund 1,5 Milliarden Mark (767 Millionen Euro) ausgelöst.

Ursache für den jüngsten Solarboom seien verbesserte Förderbedingungen und die gestiegenen Preise für Öl und Gas, stellte die Vereinigung fest. Das Jahr 2000 werde von Solarunternehmern deshalb als "Einstieg ins Solarzeitalter" gewertet. Die Branche rechnet auch für 2001 mit einem deutlichen Marktzuwachs. (M01/JAN.04985 Mannheimer Morgen, [Tageszeitung], 23.01.2001, Jg. 56. - Solarenergie legt zu)

80) $\{\{1+1$; Posspr.; Subj.; A1; A1 $\}\}\{\{$ SVG; Poss. U. haben $\}\}$ Erneut verletzt ist dagegen mit Laviani ein weiterer junger Profi. Seine Muskelzerrung aus dem Bochumspiel ist wieder aufgebrochen. "Unsere aktuelle__S Situation hat ihre Ursache sicher nicht im mangelnden Willen der Spieler", bilanzierte Rapolder die vergangene Woche. "Aber die Identifikation mit dem Verein muss sich im Engagement und der Konzentration deutlicher niederschlagen."

ve (M01/OKT.82035 Mannheimer Morgen, [Tageszeitung], 29.10.2001, Jg. 56. Sachgebiet: Sport, Originalressort: Sport; Waldhof: Die Mischung macht's)

81) $\{\{1$; für + Akk.; A1 $\}\}$

Menschliches Versagen

Biblis.

Vermutlich war menschliches Versagen die Ursache für den tödlichen Unfall, der sich am Freitagmorgen im Kernkraftwerk Biblis ereignet hat. Wie Sachverständige des hessischen Landeskriminalamtes ermittelten, war bei den Wartungsarbeiten im Schaltanlagen-Gebäude von Block A eine Schutzvorrichtung entgegen der Sicherheitsvorschriften entfernt worden. Dies teilte die Bergsträßer Polizei gestern mit. (M02/MAR.23480 Mannheimer Morgen, [Tageszeitung], 26.03.2002, Jg. 57. Originalressort: Umlandseite(n); Menschliches Versagen)

82) $\{\{1$; Gen.; A1 $\}\}$

Polizeibericht

Mysteriöse Ampel

Noch ungeklärt ist die Ursache eines Unfalls, der sich - wie die Polizei erst jetzt mitteilt bereits am vergangenen Donnerstag um 8.15 Uhr auf der Kreuzung L 6/L 4 ereignete. Dort stießen zwei Autos zusammen, deren Fahrer behaupten, bei grünem Ampellicht losgefahren zu sein. Bei der Karambolage entstand ein Schaden von rund 3000 Euro. (M02/AUG.59401 Mannheimer Morgen, [Tageszeitung], 10.08.2002, Jg. 57. Originalressort: Lokal Mannheim; Polizeibericht)

83) $\{\{0\}\}$ 
Das kostbare Lebensmittel kommt denn auch nicht vollständig beim Verbraucher an: In den letzten zehn Jahren hat sich der Wasserverlust von 5,8 auf 10,4 Prozent nahezu verdoppelt. Von unserem Redaktionsmitglied Uwe Rauschelbach

Die Frage nach der Ursache löst beim Geschäftsführer der Stadtwerke GmbH Gelächter aus: "Wenn wir die Lösung hätten, hätten wir keine Wasserverluste", erklärt Frank Kaus auf Anfrage dieser Zeitung. "Das versickert, ohne dass Sie's wirklich verhindern können." Wenn man bedenke, dass durch einen tropfenden Wasserhahn allein 150 Liter am Tag verschwendet würden, seien zehn Prozent von 1,7 Millionen Kubikmeter Wasser, die im vergangenen Jahr abgegeben wurden, zudem nur ein Tropfen auf den heißen Stein. (M02/NOV.85183

Mannheimer Morgen, [Tageszeitung], 15.11.2002, Jg. 57. Originalressort: Lokal Lampertheim; Von unserem Redaktionsmitglied Uwe Rauschelbach: Immer mehr Wasser geht verloren)

84) $\{\{1$; für + Akk.;A1 $\}\}$

Ursachenforschung wegen Brand dauert

Schriesheim.

"Es wird wohl noch mehrere Tage dauern", antwortete Jörg Hofer, Mitarbeiter der Heidelberger Polizei-Pressestelle, gestern auf die Frage des "MM", ob die Kripo inzwischen die Ursache für den Brand auf dem Reiterhof der Familie Urban ermittelt hat.

Es handle sich, so Hofer, um die Suche nach der berühmten Nadel im Heuhaufen: Wegen der Größe des Feuers und der erheblichen Ausdehnung des Brandorts sei es für die Ermittler der Weinheimer Kriminalpolizei recht schwierig, schnell etwas herauszufinden.

Am Samstagnachmittag hatte das Feuer auf dem Urban-Anwesen in Schriesheim einen Schaden in Höhe von 380000 Euro angerichtet. (M03/MAR.20075 Mannheimer Morgen, [Tageszeitung], 27.03.2003, Jg. 58. Originalressort: Umlandseite; Ursachenforschung wegen Brand dauert)

85) $\{\{1$; Gen.; A1 $\}\}$

Ihr Alter hatte die Schauspielerin geheim gehalten. Selbst das Produktionsteam der

"Lindenstraße" weiß ihr Geburtsdatum nicht. Die Ursache des plötzlichen Todes ist ebenfalls nicht bekannt. Die Angehörigen wünschten darüber keine Berichterstattung, hieß es. Beim "Lindenstraßen"-Team hat man nach Angaben einer Sprecherin der Schauspielerin nichts von einer etwaigen Erkrankung angemerkt. (M03/SEP.57967 Mannheimer Morgen, [Tageszeitung], 04.09.2003, Jg. 58. Originalressort: Aus aller Welt; "Lindenstraße"Team trauert)

86) $\{\{1$; für + Akk.; A1 $\}\}$

Umfragen zeigten jedoch eindeutig: "Schülerinnen wie Schüler lehnen getrennten Unterricht ab, weil er stinklangweilig wäre."

Mehrere Landespolitiker fordern, das zahlenmäßige Übergewicht der Lehrerinnen vor allem in den Grundschulen abzubauen. Denn sie sehen eine "Feminisierung des Unterrichts" als Ursache für das schlechtere Abschneiden von Jungen. So fordert etwa die Stuttgarter Bildungsministerin Annette Schavan, den Anteil der männlichen Lehrer auf mindestens 30 Prozent in den Grundschulen zu erhöhen.

Solche Forderungen hält Kraus für unrealistisch, "weil nur zwei Prozent der Bewerber für ein Lehramt in der Grundschule Männer sind". (M03/DEZ.81151 Mannheimer Morgen, [Tageszeitung], 03.12.2003, Jg. 58. Originalressort: Aus aller Welt; Mädchen hängen ihre Mitschüler ab)

87) $\{\{1$; Komp.; A1 $\}\}$ 
Einen beabsichtigten Schlag habe es nie gegeben.

Der Richter vertagte den Prozeß. Ein ärztliches Gutachten soll die genaue

Verletzungsursache klären helfen. (N95/JUL.26771 Salzburger Nachrichten, [Tageszeitung], 18.07.1995. - Brauchtumsangelegenheiten)

88) $\{\{1$; Komp.; A1 $\}\}$

Am Montagabend gegen 20 Uhr hat es am Stettiner Platz in Pasewalk einen Unfall gegeben, bei dem zwei Autos beschädigt worden sind. Wie die Polizei mitteilte, ist bei der Kollision ein Gesamtschaden von etwa 600 Euro entstanden. Unfallursache war ein Fahrfehler beim Ausparken. (NKU08/APR.06516 Nordkurier, 23.04.2008, Werkzeug aus einer Garage gestohlen)

89) $\{\{1$; für + Akk.; A1 $\}\}$

Kanal wird nun saniert

Ursache für Straßenloch

Von unserem Redaktionsmitglied Sybille Burmeister

Das Loch in der Von-Kieffer-Straße schlägt hohe Wellen in der Stadtentwässerung.

(M05/MAI.42007 Mannheimer Morgen, [Tageszeitung], 24.05.2005, Jg. 60.

Originalressort: Ludwigshafen; Von unserem Redaktionsmitglied Sybille Burmeister:

Kanal wird nun saniert)

90) $\{\{1$; Gen.; A1 $\}\}$

Hat man David Cronenberg häufig vorgeworfen, seine Werke seien zu verklausuliert, gibt es hier nichts, was es nicht zu verstehen gibt. An der Oberfläche "unterhält" der Regisseur, spult seinen Plot konsequent ab, überrascht durch Gewalt-Ausbrüche und befriedigt des Zuschauers Bedürfnis nach "Gerechtigkeit". Doch zwischen den Bildern, wenn er über Ursache und Wirkung der Gewalt räsoniert, Familienstrukturen erforscht und in seelische Abgründe abtaucht, dann erweist er sich als existenzialistischer Autorenfilmer reinsten Wassers. Dann gibt es keine Eindeutigkeiten mehr, kein Recht und kein Unrecht. Die Welt ist kompliziert, komplex, das biblische Prinzip von Gut und Böse hat sich seit Kain und Abel nicht mehr verändert. (M05/OKT.83005 Mannheimer Morgen, [Tageszeitung], 12.10.2005, Jg. 60. Sachgebiet: Kultur, Originalressort: Kultur; Von unserem Mitarbeiter Gebhard Hölzl: Biedermann oder Brandstifter?, [Feuilleton])

91) $\{\{1$; Gen.; A1 $\}\}$

20000 Euro Brandschaden

Schriesheim/Rhein-Neckar-Kreis.

Unklar ist noch die Ursache eines Feuers, das am vergangenen Sonntagabend, kurz nach 20 Uhr, die Scheune eines Aussiedlerhofs in Schriesheim fast vollständig zerstörte.

Die angerückte, 55 Mann starke Schriesheimer Feuerwehr, hatte den Brand schnell gelöscht, so der Polizeibericht.

Die Brandermittler der Weinheimer Kriminalpolizei haben nun die Ermittlungen zur Brandursache aufgenommen. (M06/JUN.46032 Mannheimer Morgen, [Tageszeitung], 14.06.2006, Jg. 61. - Sachgebiet: Lokales, Originalressort: Ladenburg; 20000 Euro Brandschaden)

92) $\{\{1$; für + Akk; A1 $\}\}$

Weniger Lohn heiße es für breite Teile der Bevölkerung - bei "explodierenden Gewinnen der Konzerne und unvorstellbaren Vermögen der Reichen und Superreichen". Bei 85600 Euro 
liege der Gewinn pro Stunde für jeden der zwei Aldi-Brüder. "Der wachsende Reichtum des Kapitals ist die Ursache für die wachsende Armut."

Wer arm ist, stirbt früher. Im Schnitt sechs bis acht Jahre. (M06/OKT.79832 Mannheimer Morgen, [Tageszeitung], 13.10.2006, Jg. 61. - Gewerkschaft schlägt kämpferische Töne an)

93) $\{\{1$; von + Dat.;A1 $\}\}$

Besonders spendenfreudig und hilfsbereit sind die Einwohner Lampertheims, wenn es darum geht, blinden Menschen in Entwicklungsländern zu helfen. Wie die Christoffel-

Blindenmission (CBM) in Bensheim mitteilt, erhielt sie im vergangenen Jahr von 147

Lampertheimer Bürgern insgesamt 8230 Euro an Spenden. Mit diesem Geld können rund 274

Menschen in Asien, Afrika oder Lateinamerika am Grauen Star, der häufigsten Ursache von Blindheit, operiert und wieder sehend gemacht werden.

Während in Deutschland die Patienten in der Regel vor der Erblindung operiert werden, ist die Situation in Entwicklungsländern laut CBM viel dramatischer: Rund die Hälfte der 37 Millionen blinden Menschen ist am Grauen Star erblindet. Hauptgründe: Es fehlt an Krankenhäusern und Augenärzten. (M07/MAR.05349 Mannheimer Morgen, [Tageszeitung], 21.03.2007, Jg. 62, Ausgabe Südhessen Morgen (Lampertheim). Sachgebiet: Lokales, Originalressort: Lampertheim; Ein Herz für blinde Menschen)

94) $\{\{1$; Gen.; A1 $\}\}$

Wie die Polizei gestern mitteilte, hatte der Mann am Dienstag alleine am Vereinsheim seines Sportvereins gearbeitet. Am Mittag hörte ein Vereinsmitglied den Sturz und fand den 67Jährigen schwerverletzt. Die genaue Ursache des Unfalls ist unklar. 1rs Aus der Region (M07/SEP.03370 Mannheimer Morgen, [Tageszeitung], 14.09.2007, Jg. 62, Stadtausgabe. - Sachgebiet: Regionales, Originalressort: Nachbarschaft; Kampfhund verletzt Frauchen)

95) $\{\{0\}\}$

Er fährt selbst Motorrad, weiß aus eigenem Erleben, wie verwundbar man sich ohne jede Knautschzone zuweilen fühlt. Und er verweist auf Statistiken, wonach es mehrheitlich nicht die Motorradfahrer sind, die solche Unfälle verursachen. Die gestrige Ursache ist noch unklar. Zeugen werden gebeten, sich bei der Polizei Heidelberg (Tel. 06221/99-1870) zu melden. (M08/JUN.49513 Mannheimer Morgen, [Tageszeitung], 27.06.2008, Jg. 63, Bergstrasse, S. 17. - Sachgebiet: Lokales, Originalressort: Bergstrasse; Von unserem Redaktionsmitglied Konstantin Groß: 40-jähriger Motorradfahrer stirbt vor Ort, [Bericht [Bericht ; Nachrichten]])

96) $\{\{1$; für + Akk.; A1 $\}\}$

21-jähriger Lübecker stirbt durch Methanol

Hamburg.

Eine Methanolvergiftung ist die Ursache für den Tod eines 21 Jahre alten Lübecker Schülers, der am vergangenen Donnerstag in der Türkei nach einem Trinkgelage gestorben war. Dies habe die Obduktion des Leichnams am Universitätsklinikum Hamburg-Eppendorf (UKE) ergeben, teilte die Staatsanwaltschaft Hamburg gestern mit. Die Rechtsmedizin des UKE war in Amtshilfe für die Lübecker Staatsanwaltschaft tätig geworden. (M09/APR.25524

Mannheimer Morgen, [Tageszeitung], 02.04.2009, Jg. 64, Stadtausgabe, S. 16. Originalressort: Aus aller Welt; 21-jähriger Lübecker stirbt durch Methanol, [Bericht [Bericht ; Nachrichten]]) 
97) $\{\{1$; Gen.; A1 $\}\}$

Drei Airbusse müssen notlanden

Paris.

Eineinhalb Wochen nach dem Absturz des Airbus, bei dem 228 Menschen ums Leben kamen, liegt die Ursache der Katastrophe weiter im Dunkeln. Air France wehrt sich gegen

Spekulationen, dass der Ausfall der Geschwindigkeitsmesser Ursache des Unglücks war. Der Unglücks-Airbus A330 hatte vor dem Absturz den Ausfall mehrerer Systeme gemeldet, darunter eine Fehlfunktion der Pitot-Sonden zur Geschwindigkeitsmessung.

(M09/JUN.45104 Mannheimer Morgen, [Tageszeitung], 12.06.2009, Jg. 64, Stadtausgabe, S. 14. Originalressort: Aus aller Welt; Drei Airbusse müssen notlanden, [Bericht [Bericht ; Nachrichten]])

98) $\{\{1$; für + Akk.; A1 $\}\}$

Dazu sollte immer Expertenrat eingeholt werden. Gründer und Gründerinnen schätzen ihren Beratungsbedarf oft falsch ein. Informationsdefizite sind die zweithäufigste Ursache für das frühzeitige Aus junger Unternehmen.

Aber auch für ,gestandene“ kleine und mittlere Unternehmen gilt es mehr denn je, sich umfassend über die aktuellen Förder- und Finanzierungsangebote des Staates zu informieren. Auch wenn sich die Anzeichen einer wirtschaftlichen Erholung verdichten, ist die Krise nach Ansicht der meisten Fachleute 2010 noch nicht vorbei und viele Mittelständler sind auf der Suche nach Krediten oder Förderhilfen. (M10/APR.30581 Mannheimer Morgen, [Tageszeitung], 21.04.2010, Jg. 65, Stadtausgabe, S. 6. - Sachgebiet: Wirtschaft, Originalressort: Wirtschaft; Von unserem Redaktionsmitglied Bettina Eschbacher: Expertentipps für Gründer, [Bericht [Bericht ; Nachrichten]])

99) $\{\{0\}\}$

In einem Tunnel des Leipziger Hauptbahnhofs ist ein ICE mit 310 Fahrgästen an Bord fast zwei Stunden lang liegengeblieben. Grund sei eine technische Störung am Zug gewesen, sagte Bahnsprecher Jörg Bönisch am Wochenende. Nähere Angaben zur Ursache machte der Sprecher nicht. Der defekte Superschnellzug wurde von einer Diesellok in den Bahnhof gezogen. Verletzt wurde niemand. (M10/OKT.71309 Mannheimer Morgen,

[Tageszeitung], 18.10.2010, Jg. 65, Stadtausgabe, S. 15. Originalressort: Aus aller Welt, [Bericht [Bericht ; Nachrichten]])

100) $\{\{1$; Gen.; A1 $\}\}$

Statt die Voraussetzungen hierfür zu verbessern und einen Gegentrend einzuleiten, werden Ausbildungs- und Versorgungsstrukturen abgerissen. Die Mittelschicht wird abgeschmolzen, während andere Staaten die Mittelschicht aufbauen und stabilisieren.

Zwei zentrale strukturelle Defekte der deutschen Demokratie sind sowohl Ursache als auch Lösungsansätze zur Bewältigung der Situation: die mangelnde Wahrnehmung einer nachhaltigen Wachstumsperspektive und unzureichende Strukturen der Information und Mitbestimmung.

1) Wahlrecht für Familien - Sicherung der Zukunftsfähigkeit Deutschlands: Um die Zukunftsfähigkeit Deutschlands zu sichern, wird Familien ein erhöhtes Gestaltungsrecht gegeben. Familien/eingetragene Lebenspartnerschaften/Alleinerziehende sollen ein erhöhtes Stimmrecht bekommen, das der Anzahl der Kinder entspricht, für die sie erziehungsberechtigt sind. (M11/OKT.09520 Mannheimer Morgen, [Tageszeitung], 29.10.2011, Jg. 66, Stadtausgabe, S. 8. - Sachgebiet: Leserbriefe, Originalressort: Leserforum, [Leserbrief]) 